\title{
Accuracy in Powder Diffraction II
}

Proceedings of the International Conference May 26-29, 1992

E. Prince and J. K. Stalick, Editors

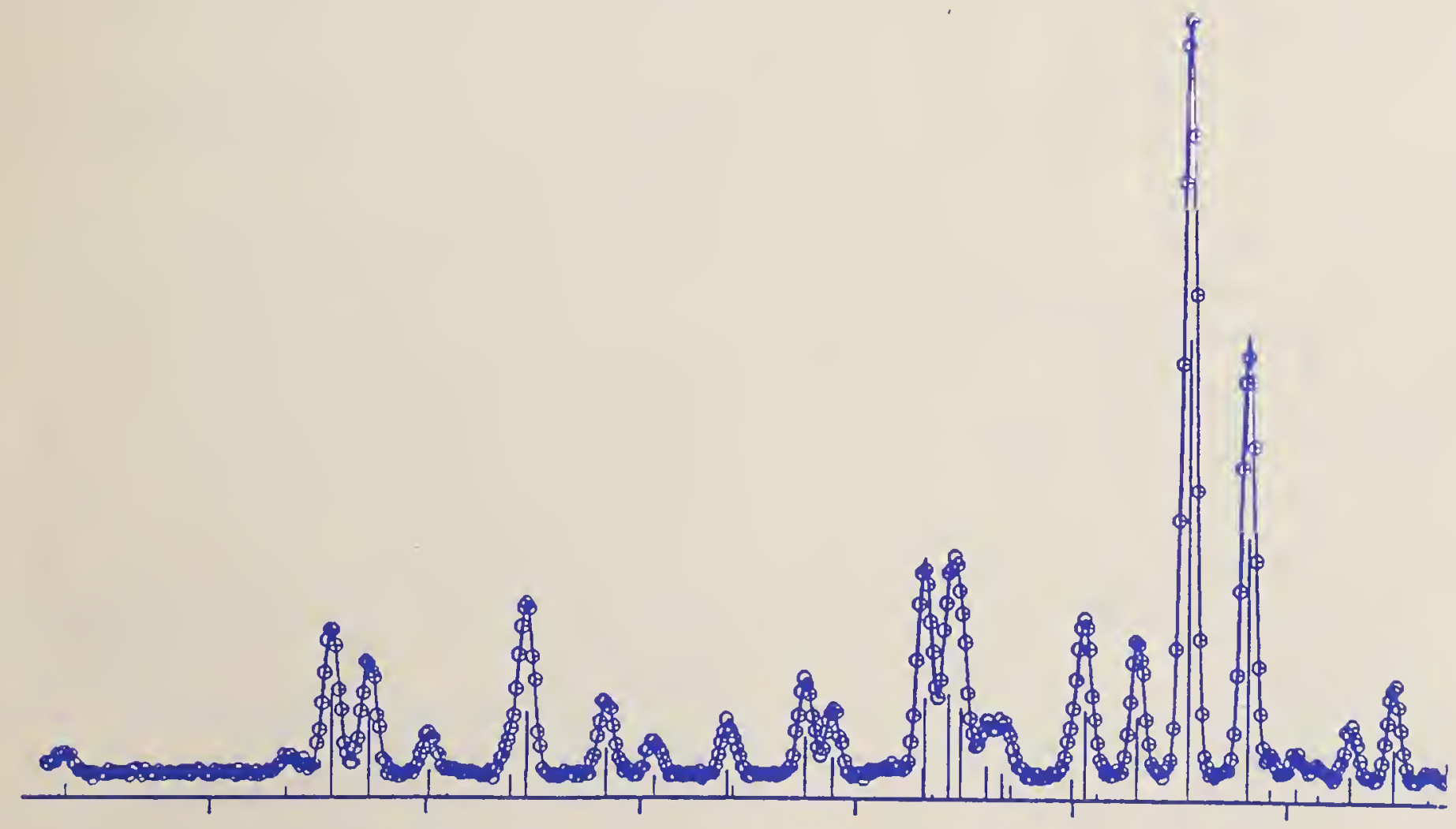

$\mathrm{QC}$ 
$T$

he National Institute of Standards and Technology was established in 1988 by Congress to "assist industry in the development of technology ... needed to improve product quality, to modernize manufacturing processes, to ensure product reliability ... and to facilitate rapid commercialization ... of products based on new scientific discoveries."

NIST, originally founded as the National Bureau of Standards in 1901, works to strengthen U.S. industry's competitiveness; advance science and engineering; and improve public health, safety, and the environment. One of the agency's basic functions is to develop, maintain, and retain custody of the national standards of measurement, and provide the means and methods for comparing standards used in science, engineering, manufacturing, commerce, industry, and education with the standards adopted or recognized by the Federal Government.

As an agency of the U.S. Commerce Department's Technology Administration, NIST conducts basic and applied research in the physical sciences and engineering and performs related services. The Institute does generic and precompetitive work on new and advanced technologies. NIST's research facilities are located at Gaithersburg, MD 20899, and at Boulder, CO 80303. Major technical operating units and their principal activities are listed below. For more information contact the Public Inquiries Desk, 301-975-3058.

\section{Technology Services}

- Manufacturing Technology Centers Program

- Standards Services

- Technology Commercialization

- Measurement Services

- Technology Evaluation and Assessment

- Information Services

\section{Electronics and Electrical Engineering}

Laboratory

- Microelectronics

- Law Enforcement Standards

- Electricity

- Semiconductor Electronics

- Electromagnetic Fields ${ }^{1}$

- Electromagnetic Technology

\section{Chemical Science and Technology}

Laboratory

- Biotechnology

- Chemical Engineering ${ }^{1}$

- Chemical Kinetics and Thermodynamics

- Inorganic Analytical Research

- Organic Analytical Research

- Process Measurements

- Surface and Microanalysis Science

- Thermophysics ${ }^{2}$

\section{Physics Laboratory}

- Electron and Optical Physics

- Atomic Physics

- Molecular Physics

- Radiometric Physics

- Quantum Metrology

- Ionizing Radiation

- Time and Frequency ${ }^{1}$

- Quantum Physics ${ }^{1}$
Manufacturing Engineering Laboratory

- Precision Engineering

- Automated Production Technology

- Robot Systems

- Factory Automation

- Fabrication Technology

Materials Science and Engineering Laboratory

- Intelligent Processing of Materials

- Ceramics

- Materials Reliability ${ }^{1}$

- Polymers

- Metallurgy

- Reactor Radiation

Building and Fire Research Laboratory

- Structures

- Building Materials

- Building Environment

- Fire Science and Engineering

- Fire Measurement and Research

Computer Systems Laboratory

- Information Systems Engineering

- Systems and Software Technology

- Computer Security

- Systems and Network Architecture

- Advanced Systems

Computing and Applied Mathematics

Laboratory

- Applied and Computational Mathematics ${ }^{2}$

- Statistical Engineering ${ }^{2}$

- Scientific Computing Environments ${ }^{2}$

- Computer Services ${ }^{2}$

- Computer Systems and Communications ${ }^{2}$

- Information Systems

\footnotetext{
${ }^{1}$ At Boulder, CO 80303.

${ }^{2}$ Some elements at Boulder, CO 80303.
} 


\section{NIST Special Publication 846}

\section{Accuracy in Powder Diffraction II}

\section{Proceedings of the International Conference May 26-29, 1992}

E. Prince and J. K. Stalick, Editors

Materials Science and Engineering Laboratory

National Institute of Standards and Technology

Gaithersburg, MD 20899

Organized by:

Commission on Powder Diffraction of the

International Union of Crystallography

\section{Sponsored by:}

JCPDS - International Centre for Diffraction Data

International Union of Crystallography

National Institute of Standards and Technology

Issued October 1992

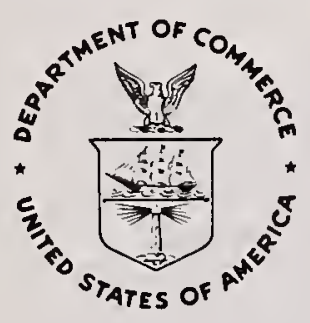

\section{U.S. Department of Commerce}

Barbara Hackman Franklin, Secretary

Technology Administration

Robert M. White, Under Secretary for Technology

National Institute of Standards and Technology

John W. Lyons, Director 
National Institute of Standards and Technology

Special Publication 846

Natl. Inst. Stand. Technol.

Spec. Publ. 846

241 pages (Oct. 1992)

CODEN: NSPUE2
U.S. Government Printing Office

Washington: 1992
For sale by the Superintendent of Documents

U.S. Government Printing Office Washington, DC 20402 


\section{Foreword}

Few areas of research endeavour in the physical sciences have experienced a renaissance on such a scale, and few have had such an impact on related disciplines during the past 15 years, as has powder diffraction. Sensing this imminent revolution back in 1979, the U. S. National Bureau of Standards (now NIST) hosted a conference entitled Accuracy in Powder Diffraction. The proceedings of this symposium were subsequently published as NBS Special Publication 567 and this document is still widely quoted in the literature, more than 10 years later!

In the years since this benchmark meeting, new generations of high resolution and intense sources of $\mathrm{X}$-rays and neutrons have been designed and built, new and exciting methods for data collection and analysis have been developed, and powerful computers have come within reach of, if not on, scientists' desks. Never before have so many previously neglected materials come under such close scrutiny, and never before has it been possible to study these materials under such a wide range of non-ambient conditions. Diffraction patterns previously collected only for phase identification or unit cell determination are now used to study such things as the subtleties of electron density distribution, the mechanisms of phase transformations, and the distribution of cation ionization states.

In the light of this renaissance, the IUCr Commission on Powder Diffraction (CPD), in conjunction with the National Institute of Standards and Technology, JCPDS-International Centre for Diffraction Data, and the International Union of Crystallography, organized a second conference, Accuracy in Powder Diffraction II, as a continuation of the series started in 1979. Six sessions provided the framework for APD-II: phase identification and quantification; accuracy and standards; new developments in software and data analysis; profile fitting, decomposition, and microstructural effects; novel applications and structural science; new developments in hardware, including detectors, and studies under non-ambient and time-resolved conditions.

The scientific sessions incorporated keynote, invited, oral and poster papers, a tribute to the late W. Parrish, and two workshops organized by the JCPDS-ICDD, entitled ICDD round robin on powder diffractometer sensitivity and Indexing methods. In addition, the program for the last session of the meeting was specifically designed to interface smoothly with the Workshop on X-ray and Neutron Diffraction at High Pressure, organized by the High pressure Group of the IUCr, which was held in Washington, DC, at the Geophysical Laboratory of the Carnegie Institution of Washington. Many attendees obtained substantial benefit from attending both of these meetings.

Generous support for the attendance of young scientists and invited speakers was obtained from the IUCr and the JCPDS-ICDD, respectively. It need hardly be added that the continued financial support of both of these organizations is extremely valuable to, and very much appreciated by the powder diffraction community in general and the APD-II Organizing Committee in particular. The cooperation and generosity of the NIST in providing support in the form of both personnel and facilities was essential for the organization of the meeting, and for this we are also very grateful.

I take the opportunity here to express my thanks to the members of the Scientific Program Committee for their hard work and cooperation; their efforts resulted in an enlightening and fruitful meeting.

\section{R.J. Hill \\ Chairman, Scientific Program Committee}

\section{Acknowledgments}

The timely publication of this volume of proceedings was made possible by the cooperation of the authors, who provided machine-readable text, many of them in $\mathrm{IAT}_{\mathrm{E}} \mathrm{X}$ or Wordperfect, so that the labor involved in producing camera-ready copy was minimal. We are grateful to Carol O'Connor for invaluable assistance in preparing the text for the printer. The success of the conference was assured by the efforts of the international program committee, ably chaired by Rod Hill, and for the smooth running of the conference we are indebted to Kathleen Kilmer and her staff in the NIST conference office, particularly Tammie Grice and Lori Phillips. The conference would not have occurred at all without the impetus of Ray Young and the IUCr Commission on Powder Diffraction and the generous sponsorship of the International Union of Crystallography and the JCPDS-International Centre for Diffraction Data.

E. Prince

J. K. Stalick, Editors 
Commission on Powder Diffraction

R. A. Young (USA), Chairman

Z. Bojarski (Poland)

D. E. Cox (USA)

J. Fiala (Czechoslovakia)

A. W. Hewat (France)

R. J. Hill (Australia)

J. I. Langford (UK)

D. Louër (France)

P.-E. Werner (Sweden)

T. Yamanaka (Japan)

D. K. Smith (USA), Consultant

L. Frevel (USA), JCPDS-ICDD representative

International Program Committee

R. J. Hill (Australia), Chairman

D. E. Cox (USA)

J. Fiala (Czechoslovakia)

H. Fuess (Germany)

D. Louër (France)

R. J. Nelmes (UK)

E. Prince (USA)

D. K. Smith (USA)

H. Toraya (Japan)

L. Zevin (Israel)

\section{Local Committee}

E. Prince, Co-chairman

J. K. Stalick, Co-chairman

T. Grice

K. Kilmer

C. O'Connor

L. Phillips

J. E. Post

C. T. Prewitt

Special thanks to:

Blake Industries, Inc.

Charles Supper Company

Fein-Marquart Associates, Inc.

Materials Data Inc.

Molecular Simulations, Inc.

Philips Electronic Instruments Company

Polycrystal Book Service

Scintag, Inc.

Siemens Analytical X-ray Instruments 


\section{Table of Contents}

T. C. Huang. Contributions of William Parrish to Powder Diffraction $\ldots \ldots \ldots \ldots \ldots \ldots \ldots \ldots$.

B. L. Davis. Quantitative Phase Analysis with Reference Intensity Ratios . . . . . . . . . . . . 7

L. Zevin. Standardless Method of Quantitative X-ray Diffractometry $\ldots \ldots \ldots \ldots \ldots \ldots \ldots \ldots .17$

R. L. SNYDER. Reference Intensity Ratios, Whole Pattern Fitting and Standardless X-ray Quanti-

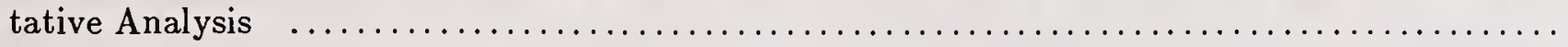

J. K. Stalick. Quantitative Phase Analysis with the Rietveld Method .................. 34

H. Hermann and M. Ermrich. Microabsorption Corrections ..................... 38

M. Nakhmanson. Computer-Aided Phase Identification $\ldots \ldots \ldots \ldots \ldots \ldots \ldots \ldots \ldots \ldots \ldots \ldots . \ldots$

P.-E. WERnER. On the Relative Merits of Cameras and Diffractometers for XRD .......... 51

J.-F. BÉrar. Data Optimization and Propagation of Errors in Powder Diffraction .......... 63

J. P. Cline. NIST XRD Standard Reference Materials: Their Characterization and Uses ..... 68

L. B. McCusker. Integrated Software for Structure Solution from Powder Data ........... 75

J. M. Newsam, M. W. Deem and C. M. Freeman. Direct Space Methods of Structure Solution

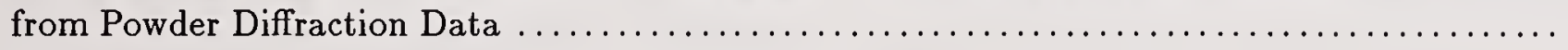

D. Loü̈r. Automatic Indexing Procedures and Applications $\ldots \ldots \ldots \ldots \ldots \ldots \ldots \ldots \ldots \ldots . \quad 92$

B. H. Toвy and T. Egami. Accuracy in Pair Distribution Function Analysis for Crystalline and

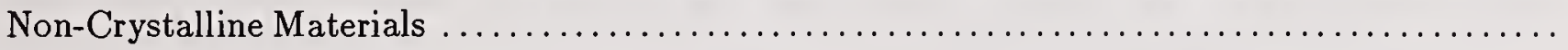

J. I. Langford. The Use of the Voigt Function in Determining Microstructural Properties from

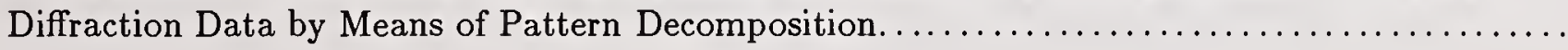

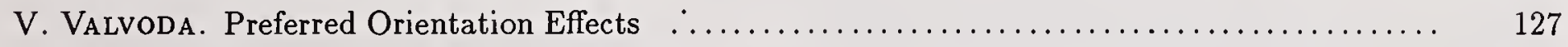

T. M. Holden and J. H. Root. Residual Stress Measurements with Neutrons $\ldots \ldots \ldots \ldots \ldots \quad 136$

A. LE BaIL. Modelling Anisotropic Crystallite Size/Microstrain in Rietveld Analysis .......... 142

D. L. Bish. Structure Building with Rietveld Analysis .......................... 154

H. Boysen. Anharmonic Thermal Parameters, Disorder and Phase Transitions ............. 165

J. P. Attfield. Recent Advances in the Use of Anomalous Dispersion Effects ............. 175

L. W. Finger. Instrumentation for Studies at the Powder/Single-crystal Boundary $\ldots \ldots \ldots \ldots \quad 183$

M. Sutton, S. Brauer, Y. S. Yang, H. E. Fischer, J. O. Ström-Olsen and G. B. Stephenson. Time-resolved X-ray Diffraction Studies of Crystallization in Metallic Glasses .. 
R. J. Nelmes, J. S. Loveday and J. M. Besson. New Developments in High-Pressure Neutron

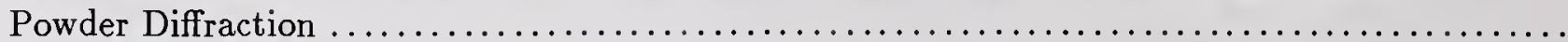

T. Yamanaka. Crystal Structure Analysis by Fitting of Powder Diffraction Data on Energy Dis-

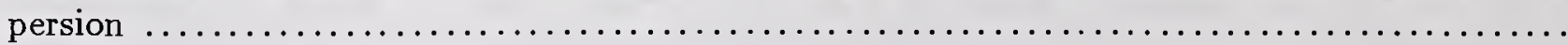

Abstracts. Session 1: Phase Identification and Quantification ........................ 206

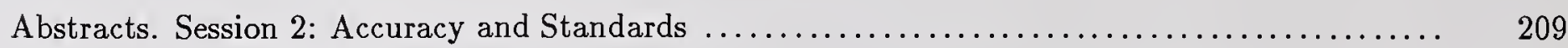

Abstracts. Session 3: New Developments in Software and Data Analysis ................ 211

Abstracts. Session 4: Profile Fitting, Decomposition and Microstructural Effects ............. 215

Abstracts. Session 5: Novel Applications and Structural Science ...................... 219

Abstracts. Session 6: New Developments in Hardware, Including Detectors, and Studies under

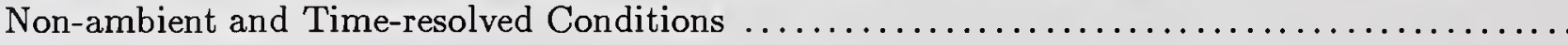

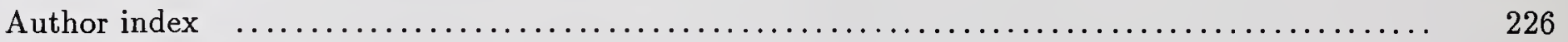

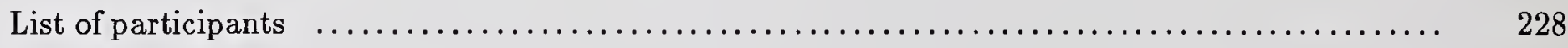

\title{
Disclaimer
}

Certain trade names and company products are identified in order to adequately specify the experimental procedure. In no case does such identification imply recommendation or endorsement by the National Institute of Standards and Technology, nor does it imply that the products are necessarily the best available for the purpose.

\begin{abstract}
The proceedings of the international conference Accuracy in Powder Diffraction II present the invited papers and abstracts of the papers contributed to the conference, which was held at NIST, Gaithersburg, Maryland, during May 26-29, 1992. The conference was organized by the Commission on Powder Diffraction of the International Union of Crystallography, and was jointly sponsored by NIST, JCPDS - International Centre for Diffraction Data and the International Union of Crystallography. The proceedings contain 25 invited papers and 73 contributed abstracts. The program of the conference was divided into six topics: Phase Identification and Quantification; Accuracy and Standards; New Developments in Software and Data Analysis; Profile Fitting, Decomposition and Microstructural Effects; Novel Applications and Structrual Science; and New Developments in Hardware, Including Detectors, and Studies under Non-ambient and Time-resolved Conditions. A ceremonial session was devoted to a tribute to the late William Parrish and his contributions to powder diffraction. In addition, there were two tutorial workshops organized by the JCPDS - International Centre for Diffraction Data, one on diffractometer sensitivity and one on automatic indexing methods.
\end{abstract}

Keywords: Accuracy; Instrumentation; Microstructure; Phase analysis; Powder diffraction; Software; Standards; Structural science. 
National Institute of Standards and Technology Special Publication 846. Proceedings of the international conference Accuracy in Powder Diffraction II, held at NIST, Gaithersburg, MD, May 26-29, 1992. (Issued October 1992)

\title{
Contributions of William Parrish to Powder Diffraction
}

\author{
T. C. Huang \\ IBM Research Division, Almaden Research Center \\ 650 Harry Road, San Jose, CA 95120-6099, U.S.A.
}

\begin{abstract}
This paper summarizes some of the important contributions of the late Dr. William Parrish to powder diffraction. He was responsible for developing many of the instruments and methods that became commercial products widely used for powder diffraction analysis. His most important achievement was the 1947 invention of the "Norelco" diffractometer using a new X-ray optics geometry that gave high intensity and resolution with good profile shape. It is the basic instrument on which most powder work has been done for almost half a century, and more than 10,000 powder diffractometers are now in use throughout the world. After joining IBM in 1970, he developed diffractometer automation methods that eventually evolved into a series of IBM X-ray products. A large number of analytical programs were developed that added new capabilities to X-ray analysis. In the early 1980 s he began a systematic study of synchrotron powder diffraction, and this synchrotron research provides the basis for a new and exciting era of powder diffraction. Many important advances will be possible based on his pioneering efforts. Parrish was not only a great powder diffractionist, but also a wonderful person, who will be greatly missed.
\end{abstract}

\section{Introduction}

Ever since its discovery by Debye and Scherrer more than 75 years ago, the powder diffraction method has played a significant role in the development of modern science and technology. The name of Bill Parrish is linked closely to many of the most important steps in the evolution of powder diffraction instrumentation, from relatively primitive powder cameras to the highly sophisticated and automated diffractometers we now use.

\section{Invention of the powder diffractometer}

Parrish's most important achievement involved the invention of the "Norelco" powder diffractometer in

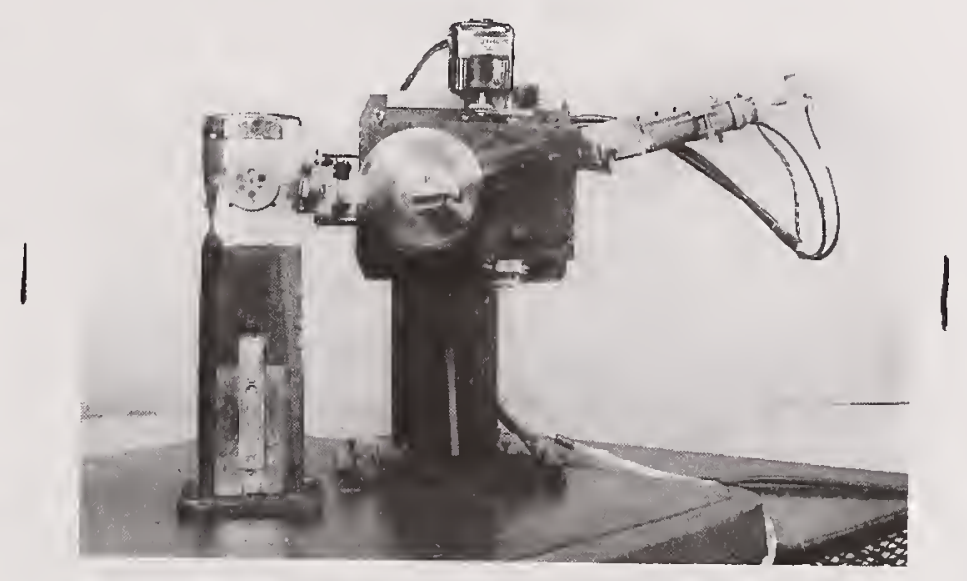

Figure 1: The first "Norelco" powder diffractometer invented by W. Parrish at Philips Laboratories, 1947.

1947 (figure 1). The instrument used a "new" Xray optics geometry to greatly increase the quality of powder diffraction patterns over those of an earlier diffractometer assembled by H. Friedman in 1945 . The latter device used the spot focus with an effective size of 0.24 by $2.5 \mathrm{~mm}$; the dimension of the line focus in Parrish's device was only 0.06 by $10 \mathrm{~mm}$. To improve asymmetric profile broadening due to the large axial divergence in the Friedman diffractometer, Parrish used a set of long parallel equally-spaced thin Mo foils (Soller slits) in the incident beam. The long X-ray line source thus became effectively a number of short overlapping narrow sources parallel to the specimen axis of rotation and had the additional advantages of high intensity and high resolution. A second set of parallel slits was placed in the diffracted beam, and the large increase in resolution decreased the profile widths of the peaks from about $0.35^{\circ}$ to $0.10^{\circ}(2 \theta)$. Figure 2 shows the optics and line profiles of the two instruments.

It is unfortunate that the Parrish diffractometer 

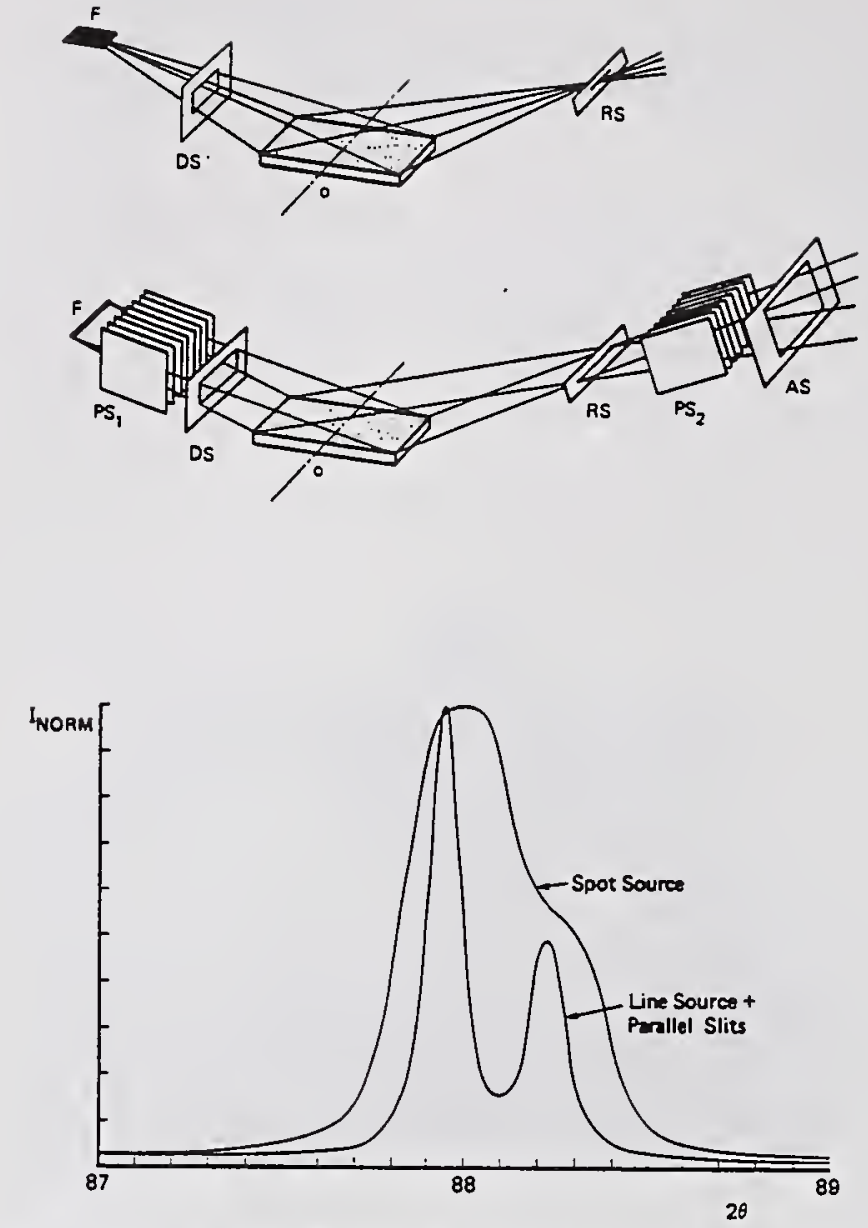

Figure 2: X-ray optics of the early and new diffractometers (top) and their profiles: $\mathrm{Si}(422)$ and $\mathrm{Cu}$ $\mathrm{K} \alpha$. (bottom).

is often incorrectly referred as a Bragg-Brentano device. The counter tube diffractometer geometry was uniquely Parrish's, and he was awarded a U.S. Patent in 1951. More than 10,000 are now in use throughout the world; this alone could be recognized as a most important achievement because it became the basic instrument on which most powder work has been done for almost half of a century.

Within a few years, the new instrument had revolutionized powder diffraction techniques and made possible major improvements in the measurement of powder patterns. Following those developments, Parrish devoted himself to many years of intensive research aimed at improving both the instrumentation of the diffractometer and the understanding of the basic principles of the new instrument. He was a pioneer in the utilization of scintillation and proportional counters with pulse amplitude discrimination, making possible large improvements in $\mathrm{X}$-ray detection (Parrish and Kohler, 1956). Both detectors had dead times of the order of $0.25 \mu \mathrm{sec}$, and the pulse amplitudes were proportional to the energies of the X-ray quanta. This made possible the use of a pulse height analyzer to accept only pulses of amplitude within preselected limits and thereby greatly reduced the background with only a few percent loss of characteristic line intensity. The combined action of the $\mathrm{Ni}$ filter and electronic discrimination increased peak-to-background ratio well beyond the range of the Geiger counter used in early models. Figure 3 shows the effects of pulse height discrimination with a scintillation counter on the spectrum of a Cu X-ray tube.
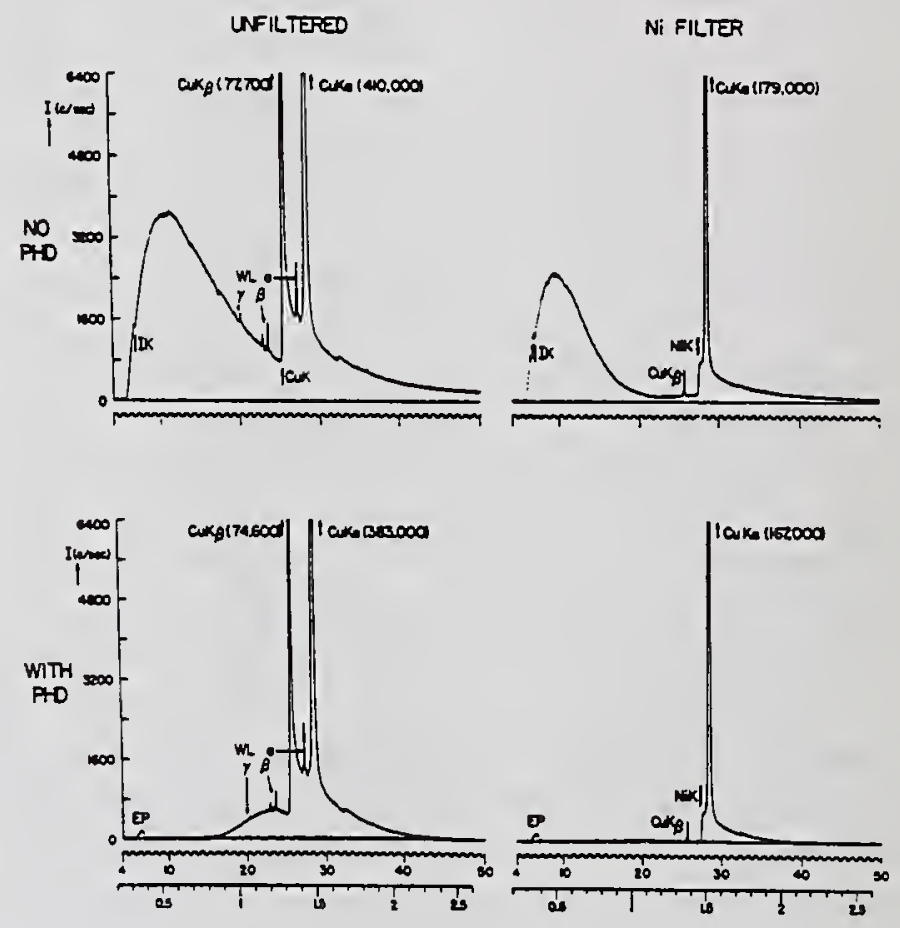

Figure 3: Spectrum of Cu X-ray tube recorded with a scintillation counter.

Parrish and his co-workers at Philips developed principles and mechanical devices to aid in simplifying the alignment and calibration of the diffractometer (Parrish \& Lowitzsch, 1959). A flat specimen spinner for rotating the specimen in its own plane was introduced to reduce the effect of crystallite size statistics and in-plane preferred orientation on absolute and relative intensities (de Wolff, Taylor \& Parrish, 1959). He was one of the first to investigate the sources of systematic errors in counter diffractometry (Parrish \& Wilson, 1959) and the use of the centroid method for handling aberrations in precision lattice parameter determination (Taylor, Mack \& Parrish, 1964).

Parrish also extended the applications of the diffractometer and made modifications of the stan- 


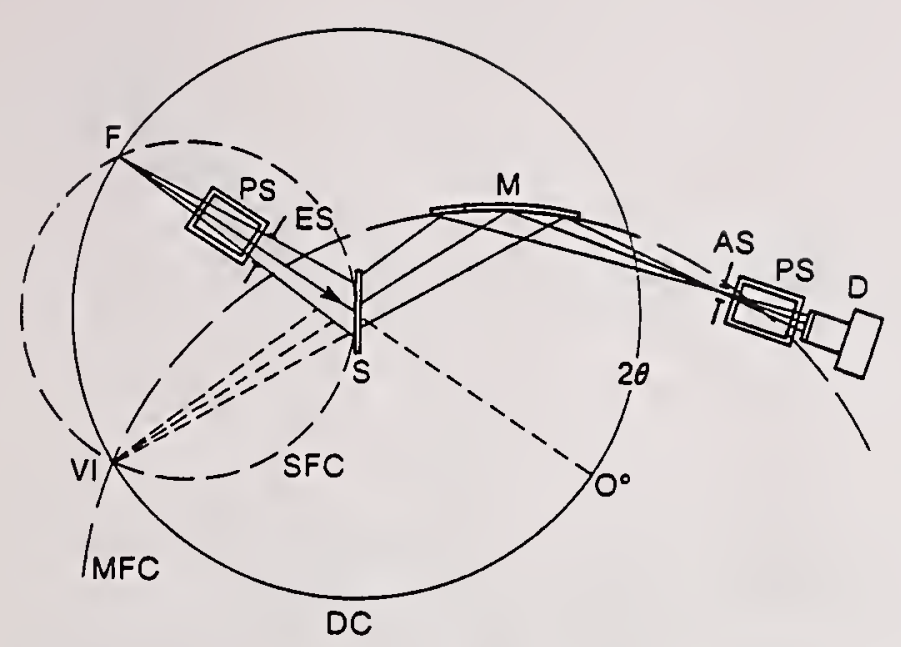

Figure 4: X-ray optics of the transmission specimen with a diffracted-beam focusing monochromator.

dard reflection diffractometer to permit transmission and Seemann-Bohlin measurements. The X-ray optical arrangement of the diffractometers, modified for transmission specimen and curved focusing crystal monochromator, is shown in figure 4. The transmission geometry is very useful for measuring large $d$ spacings and as a supplementary technique to the reflection method for measuring preferentially oriented samples such as clay minerals and polymers (Parrish, 1958). The X-ray optics for the SeemannBohlin method using an incident-beam monochromator is shown in figure 5 (Parrish \& Mack, 1967). This method with a small fixed incident angle is most suitable for the measurement of thin films because of a large path length and small penetration depth by the incident beam.

Further advances in reflection specimen powder diffractometry for recording complex powder patterns arising from low crystallographic symmetry and large unit-cell dimensions, multiple-phase specimens and structural imperfections were also made by Parrish (1968). A long fine focus X-ray tube was used; the anode was set at a relatively large angle $\left(\geq 10^{\circ}\right)$ to reduce target self-absorption for high incident intensity. The length and spacing of the incident-beam parallel slits were increased so that the total intensity reaching the specimen was high, the distribution was more uniform, and at the same time there was no loss of resolution. The divergence slit and the antiscattering slit were set close to the specimen to effectively eliminate unwanted scattered radiation. A simple vacuum chamber eliminated most of the airpath absorption losses. Cr K $\alpha$ X-ray radiation was used to increase dispersion and to reduce the difficulties caused by overlapping reflections.

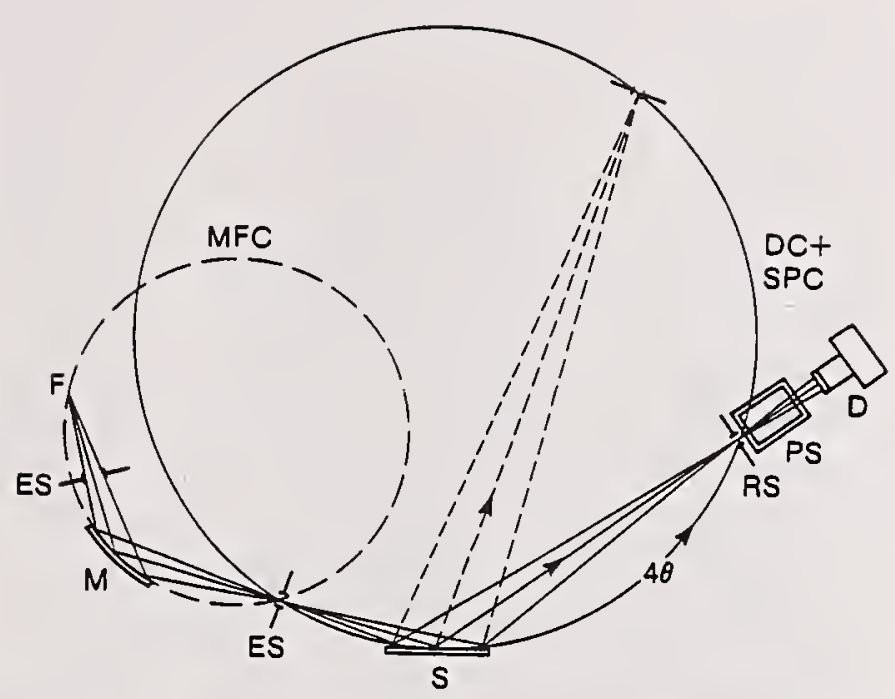

Figure 5: X-ray optics for the Seemann-Bohlin method with an incident-beam focusing monochromator.

Parrish somehow found time to write a large number of papers; many of these are contained in his book X-ray Analysis Papers, which, for many years, served as the Bible of powder diffractometry (Parrish, 1965).

\section{Computer automation}

Parrish was quick to recognize the potential power of computers, and, in collaboration with his coworkers at IBM, he developed diffractometer automation methods in the 1970s. A large number of analytical programs were developed, these added new capabilities to X-ray powder diffraction analysis. The work eventually evolved into a series of IBM Xray products and was recognized by IBM Outstanding Contribution Awards.

The profile fitting method developed for the reduction of experimental patterns made possible the separation of the instrument function from the specimen diffraction contribution, an essential step for obtaining accurate diffraction data. The precision of the profile fitting method was found to be $\pm 0.0004^{\circ} 2 \theta$ and $0.2 \%$ intensity (Parrish and Huang, 1980). Overlapping peaks could be resolved with better resolution than the original experimental data (Huang \& Parrish, 1975). Figure 6 shows the results of profile fitting analysis of the quartz cluster at $67^{\circ}-69^{\circ}$.

In collaboration with Professor G. Will of Germany, powder structures were refined using the twostage method in which the integrated intensities were determined by profile fitting and used in powder least squares refinements (POWLS) (Will, 1979). The method was tested with $\mathrm{Si}$, quartz, and corundum 


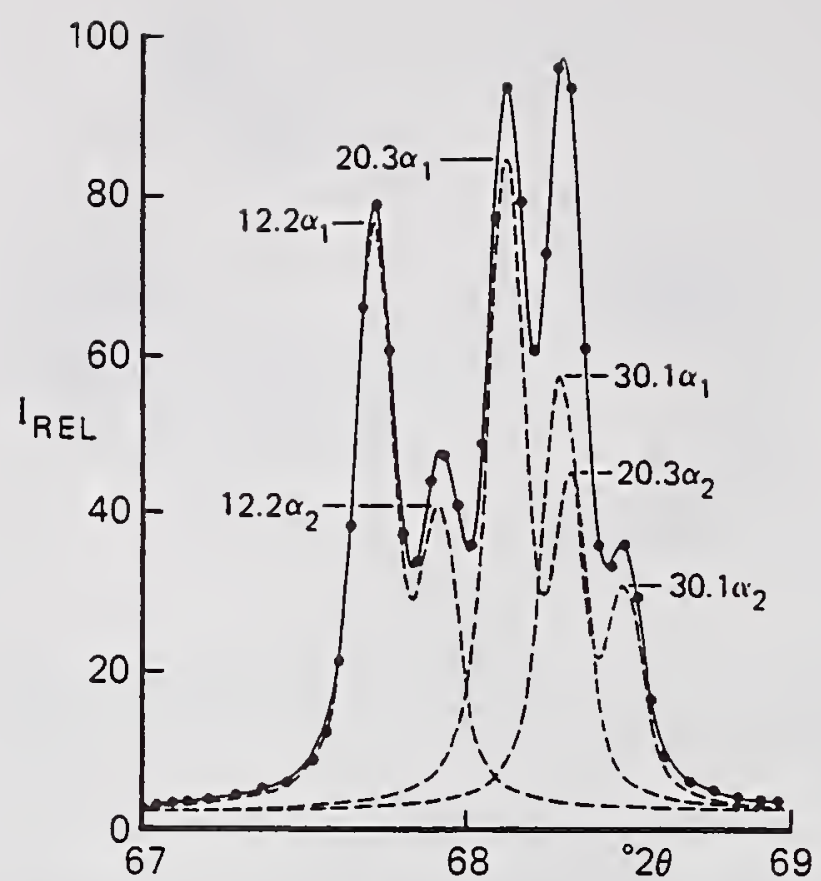

Figure 6: Profile fitting analysis of the quartz cluster of (12.2), (20.3), and (30.1) reflections.

gave $\mathrm{R}$ factors below $1 \%$ (Will, Parrish \& Huang, 1983). The method is different from the Rietveld method, which refines the entire pattern and determines the structure in one step.

An accurate computer peak search program which used first and second derivatives was also developed for rapid determination of peak positions and intensities with accuracy up to $0.001^{\circ}$ (Huang \& Parrish, 1984).

An advanced search/match algorithm was developed for automatic searching of the JCPDS Powder Diffraction File (PDF) and accurate phase identification of an unknown mixture (Huang \& Parrish, 1982). It was also demonstrated that only twelve dspacings in the PDF standard patterns were needed for automatic phase identification (Huang, Parrish \& Post, 1983).

\section{Synchrotron powder diffractometry}

Parrish became interested in the remarkable new storage ring radiation sources and began studies at the Stanford Synchrotron Radiation Laboratory in 1977. In collaboration with Professor M. Hart of England, new parallel-beam optics with an incidentbeam channel monochromator and a set of horizontal parallel slits in the diffracted beam were developed (figure 7). High intensity patterns with narrow symmetrical profiles were obtained (Parrish, Hart, Erickson, Masciocchi \& Huang, 1986; Parrish, Hart,

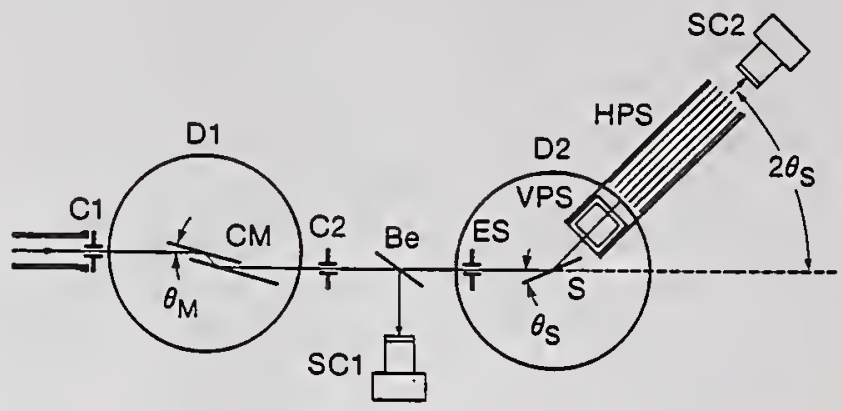

Figure 7: Synchrotron radiation parallel beam X-ray optics used for powder diffractometry.

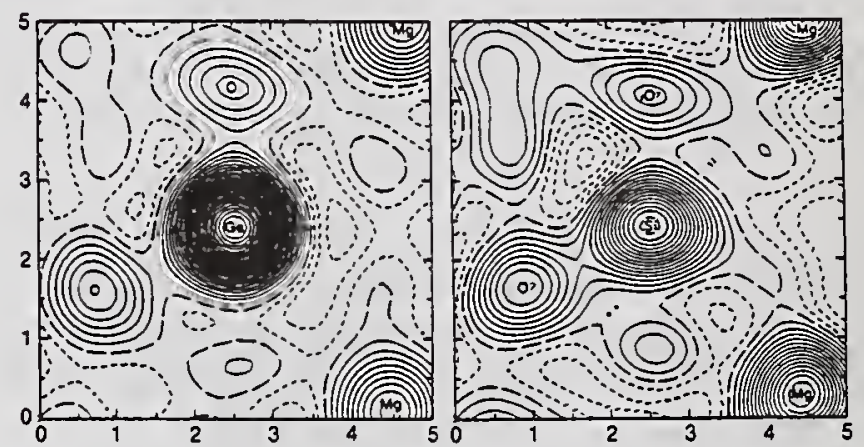

Figure 8: Fourier maps of a section of $\mathrm{MgGeO}_{4}$ (left) and $\mathrm{MgSiO}_{4}$ (right).

Huang \& Bellotto, 1987). The method could also be modified for energy dispersive diffraction using a selected, fixed $\theta-2 \theta$ and step scanning the incidentbeam monochromator. Some of the finest X-ray powder diffraction patterns recorded to date were obtained with the synchrotron method (figure 9).

Synchrotron diffraction data were used successfully for powder-structure analysis. Figure 8 shows electron density maps of orthorhombic $\mathrm{MgGeO}_{4}$ calculated using Fourier coefficients taken directly from the synchrotron intensities. These yielded excellent agreement with electron density of $\mathrm{MgSiO}_{4}$ calculated from a single-crystal data set containing 1349 reflections.

The advantages of using monochromatic and parallel synchrotron X-rays for the microstructural analysis of powder materials were also reported (Huang, Hart, Parrish \& Masciocchi, 1987). It was shown that three dimensional crystallite-size and microstrain determinations of $\mathrm{Pd}$ powders were made successfully using $1 \AA$ synchrotron radiation. The symmetrical instrument profile shape had major advantages in the correction of instrumental broadening and in the determination of a low level $\left(10^{-4}\right.$ range) of stacking-fault probabilities. 

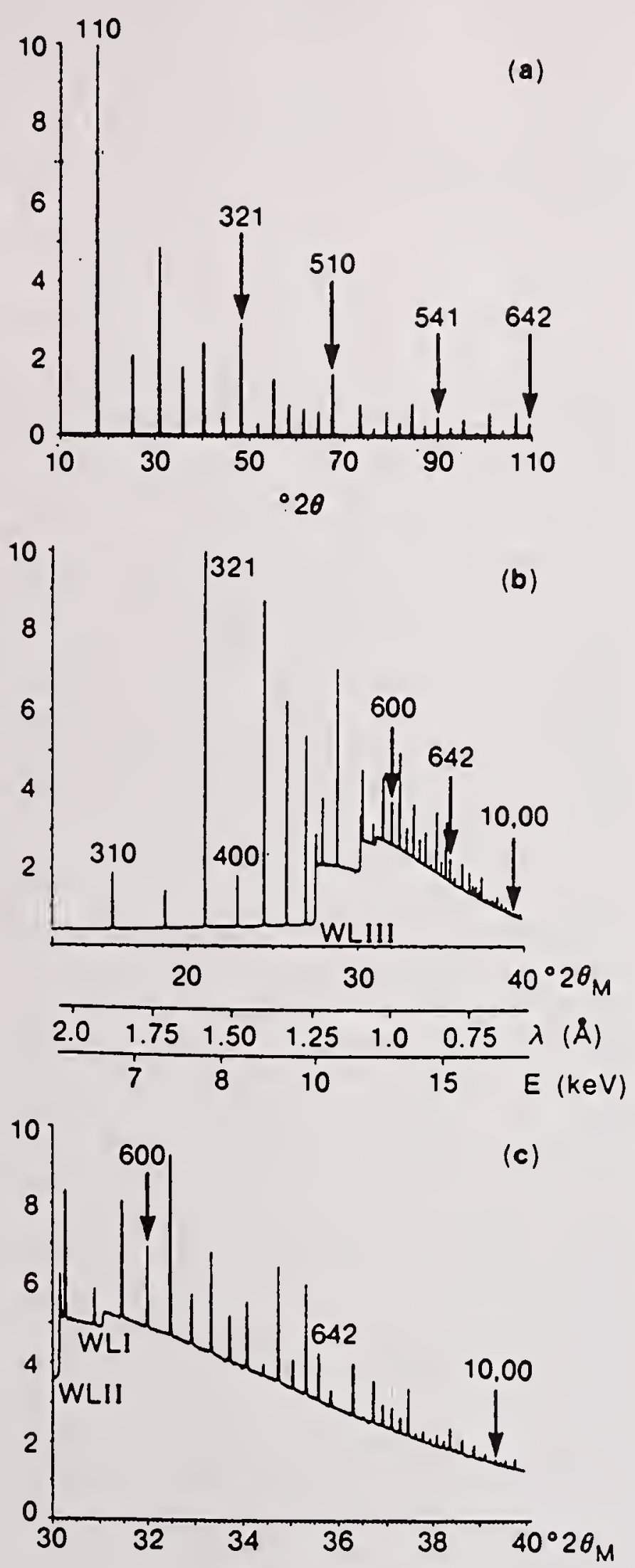

Figure 9: Synchrotron diffraction patterns of $\mathrm{W}$ powders: (a) $\theta-2 \theta$ scan with $\lambda=0.688 \AA$.; (b) energy dispersive scan with $2 \theta=135^{\circ}$; and (c) expansion of the energy dispersive pattern.
The precision of the derivation of d-values and the accuracy of determination of lattice parameters from symmetric and single-wavelength synchrotron diffraction peaks were also reported (Huang, Parrish, Masciocchi \& Wang, 1990). The precision in determining $d$-values from synchrotron peaks in the front reflection region was high with $\Delta \mathrm{d} / \mathrm{d}$ in the low $10^{-5}$ range. Using the precision lattice parameters obtained from $\mathrm{Cu} \mathrm{K} \alpha_{1}$ diffraction peaks in the back reflection region as references, the accuracy in the lattice parameter determination from alumina synchrotron peaks reached the $1-2 \times 10^{-6}$ range.

This synchrotron research provides the basis for a new and exciting era of powder diffraction. Many important advances will be possible based on his pioneering efforts.

In recognition of his outstanding contributions to powder diffraction, the JCPDS - International Centre for Diffraction data awarded Parrish the Hanawalt Award in 1987, and the Annual Denver X-Ray Conference presented him the first Barrett Award in 1988.

Parrish was an indefatigable researcher. In a 1987 letter to the late Dr. J. D. Hanawalt, Parrish wrote "I have always worked hard, but these past few years on the synchrotron research have been extremely difficult because of the very long hours and the multitude of papers to be written. It is so exciting I don't realize how tired I am".

Parrish was not only a great and dedicated powder diffractionist, but also a nice person, who will be greatly missed.

\section{References}

Friedman, H. (1945a). Electronics 18, 132.

Friedman, H. (1945b). U. S. Patent 2,286,785.

Huang, T. C., Hart, M., Parrish, W. \& Masciocchi, N. (1987). J. Appl. Phys. 61, 2813.

Huang, T. C. \& Parrish, W. (1975). Appl. Phys. Lett. 27, 123.

Huang, T. C. \& Parrish, W. (1982). Adv. X-Ray Anal. 25, 213.

Huang, T. C. \& Parrish, W. (1984). Adv. X-Ray Anal. 27, 45.

Huang, T. C., Parrish, W., Masciocchi, N., \& Wang, P. W. (1990). Adv. X-Ray Anal. 33, 295.

Huang, T. C., Parrish, W., \& Post, B. (1983). $A d v$. $X$-Ray Anal. 26, 93.

PARrish, W. (1958). 7th National Conf. on Clays and Clay Minerals, 230, New York: Pergamon.

PARrish, W. (1965). X-Ray Analysis Papers. Eindhoven: Centrex. 
Parrish, W. (1968). In $X$-ray and Electron Methods of Analysis, H. van Alphen \& W. Parrish, eds. 1, New York: Plenum.

Parrish, W. \& Hamaker, E. A. (1951). U. S. Patent $2,549,987$.

Parrish, W., Hart, M., Erickson, C. R., MasciocChI, N. \& Huang, T. C. (1986). Adv. X-Ray Anal. 29, 243.

Parrish, W., Hart, M., Huang, T. C. \& Bellotto, M. (1987). Adv. X-Ray Anal. 30, 373.

Parrish, W. \& Huang, T. C. (1980). Nat. Bur. Stand. Spec. Pub. 457, 95.

Parrish, W. \& Kohler, T. R. (1956). J. Appl. Phys. 27, 1215.

Parrish, W. \& Lowitzsch, K. (1959). Am. Mineral. 44, 765.

Parrish, W. \& Mack, M. (1963). Acta Cryst. 23, 687.

Parrish, W. \& Wilson, A. J. C. (1959). Inter. Table of X-Ray Cryst. II, 216.

TAYLOR, J., Mack, M. \& Parrish, W. (1964). Acta Cryst. 17, 1229.

WILL, G. (1979). J. Appl. Cryst. 12, 483.

Will, G., Parrish, W. \& Huang, T. C. (1983). J. Appl. Cryst. 16, 611.

Will, G., Masciocchi, N., Hart, M. \& Parrish, W. (1988). J. Appl. Cryst. 21, 182.

DE Wolf, P. M. TAYLOR, J. \& PARRISH, W. (1959). J. Appl. Phys. 30, 63. 
National Institute of Standards and Technology Special Publication 846. Proceedings of the international conference Accuracy in Powder Diffraction II, held at NIST, Gaithersburg, MD, May 26-29, 1992. (Issued October 1992)

\title{
Quantitative Phase Analysis with Reference Intensity Ratios
}

\author{
B. L. DAVIS \\ $X$-Ray Diffraction Laboratory, Engineering and Mining Experiment Station \\ South Dakota School of Mines and Technology \\ Rapid City, South Dakota 57701, U.S.A.
}

\section{Introduction}

The concept of introducing a ratio of analyte intensity to standard reference material intensity began with the report of de Woolf and Visser (1964) which in effect recommended establishment of a file of "reference intensities" in the Joint Committee on Powder Diffraction Standards manuals (Berry, 1970). This early paper has recently been formally published (de Woolf and Visser, 1988). This ratio has been given the notation $I / I_{c}$, meaning analyte intensity I over corundum standard intensity $I_{c}$. More recently the notation RIR (Reference Intensity Ratio) has become accepted with a somewhat broader implication that analyte peak intensities may be referenced to intensities from other than corundum standards (i.e., rutile, quartz).

Much earlier the internal standard method of quantitative X-ray diffraction (XRD) analysis was placed on a solid theoretical and experimental foundation by Alexander and Klug (1948). All subsequent work, including the various developments of multicomponent schemes for quantitative XRD analysis, have this development as a foundation. However, the explicit RIR form was not apparent in either the Alexander and Klug or de Woolf and Visser presentations of quantitative analysis. Indeed, many workers in the period from 1948 to 1974 developed their methods around a proportional constant, or combination of constants, connecting mass absorption, density, and scattering power (through fundamental calculable structure and scattering parameters) with the weight fraction of the analyte. These methods worked, and within their mathematical framework the RIR may be identified, but not always obviously. Table 1 presents a summary of the notation and equivalent parameters that define an implicit RIR approach in methods for those workers in quantitative multicomponent analysis whose work is available in the literature during the past 40 years.

While these methods provided a means for arriving at the desired result, namely the weight fraction of a given analyte in a mixture of several components, they did not provide the simple and powerful framework for direct application of the RIR that came with the contribution of F. Chung in his series of papers published in 19741975. Chung's papers (where the notation $k_{\mathbf{i}}$ is equivalent to RIR) demonstrated several of the powerful features of RIR analysis that, in the opinion of this author, had not been demonstrated prior to this time: (1) that an internal standard need not be added to the sample to be analysed in order to determine uniquely, through a knowledge of predetermined RIR's, all component weight fractions in the absence of unidentified or amorphous components, (2) that with known RIR values and the addition of an internal standard individual weight fractions could be determined without regard to whatever else might be in the sample and when all crystalline component weight fractions were determined, amorphous quantities (but not their composition) could be determined as well; and (3) the RIR, by nature of the base standard used for all materials, "flushed" out absorption effects. This simply means that the use of the RIR in any sample will correctly yield the weight fraction of that component regardless of the nature of the sample matrix. Chung (1975) also demonstrated that several RIR values could be determined simultaneously from a single sample mixture. Many of the features of the reference intensity ratio analysis method are also summarized by Hubbard and Snyder (1988).

Recent work now includes the use of RIR's in Rietveld analysis (Bish and Howard 1988; Hill, 1991) as well as in whole-pattern analysis (Smith et al., 1988; Smith, 1992). These techniques are very powerful for phase analysis and structure refinement but may not be the method of choice for routine analytical work simply because of their greater dependence on sophisticated computer algorithms and data input requirements; some discussion of these methods will be presented below but the emphasis of this paper is on application of basic 
Table 1. Equivalent Reference Intensity Ratio (RIR) notation

\begin{tabular}{|c|c|c|}
\hline REFERENCE & SYMBOL & EQUIVALENT \\
\hline Klug \& Alexander, 1948 & None & $\left(\mathrm{K}_{1} / \rho_{1}\right) /\left(\mathrm{K}_{\mathrm{s}} / \rho_{\mathrm{s}}\right)$ \\
\hline $\begin{array}{l}\text { Copeland \& Bragg, } 1958 \\
\text { Karlak \& Burnett, } 1966\end{array}$ & None & $\left(\mathrm{K}_{\mathrm{ij}} / \rho_{\mathrm{i}}\right) /\left(\mathrm{K}_{\mathbf{k l}} / \rho_{\mathrm{j}}\right)$ \\
\hline Bezjak, 1961 & {$\left[\alpha_{\mathrm{j}, 9}\right]^{-1}$} & $\left(\mathrm{~K}_{\mathrm{j}} / \rho_{\mathrm{j}}\right) /\left(\mathrm{K}_{\mathrm{s}} / \rho_{\mathrm{s}}\right)$ \\
\hline de Woolf \& Visser, 1964, 1988 & $\mathbf{C}$ & $C^{\prime} C^{n}=\left(I_{1} / W_{1}\right) /\left(I_{2} / W_{2}\right)$ \\
\hline Jahanbagloo \& Zoltai, 1966 & None & $\left(\left[\mathrm{INFI}_{\mathrm{i}} / \rho_{\mathrm{i}}\right) /\left([\mathrm{INFI}]_{\mathrm{c}} / \rho_{\mathrm{c}}\right)\right.$ \\
\hline Chung, 1974; Davis, 1980 & $\mathbf{k}_{\mathbf{i}}$ & $\mathrm{I}_{\mathbf{i}}^{0} \mu_{\mathrm{i}} / \mathrm{I}_{\mathrm{c}}^{\mathbf{0}} \mu_{\mathrm{c}}=\left(\mathrm{I}_{\mathrm{i}} / \mathrm{X}_{\mathrm{i}}\right) /\left(\mathrm{I}_{\mathrm{c}} / \mathrm{X}_{\mathrm{c}}\right)$ \\
\hline Hubbard, et al., 1976 & $\mathbf{k}_{\mathbf{i}}$ & $\left(\mathrm{I}_{\mathrm{i}} / \mathrm{X}_{\mathrm{i}}\right) /\left(\mathrm{I}_{\mathrm{c}} / \mathrm{X}_{\mathrm{c}}\right)=\left(\mu^{*} \gamma / \rho\right) /\left(\mu^{*}{ }_{\mathrm{c}} \gamma_{\mathrm{c}} / \rho_{\mathrm{c}}\right)$ \\
\hline Hubbard \& Smith, 1977 & None & $(\mu \gamma / \rho) /\left(\mu_{\mathrm{c}} \gamma_{\mathrm{c}} / \rho_{\mathrm{c}}\right)$ \\
\hline Monshi \& Messer, 1991 & None & $\mathrm{K}_{\mathrm{ei}} / \rho_{\mathrm{i}}$ \\
\hline
\end{tabular}

simple relationships and sample preparation methods to quantitative multicomponent analysis using RIR values based on corundum as the standard.

\section{Brief summary of Rietveld and whole-pattern methods}

Both of these methods employ the matching of large ranges of the component diffraction patterns in the determination of quantities present. The Rietveld method requires input of the structural parameters of each phase and results in not only the component weight fractions as output but refined structure and composition as output. Disadvantages include the limited number of variables that may be adjusted, and complications and accuracy losses due to the presence of amorphous phases or poorly crystalline phases. The working equations given by Bish and Howard (1988) are analogous to the adiabatic and matrix flushing equations of Chung $(1974 a, b)$ when the scaling factor is included. The database for quantitation in the Rietveld method is a set of calculated patterns.

The whole-pattern method of Smith et al. (1988) can make use of either experimental pure-phase diffraction patterns or calculated ones. The fitting (minimization) equation is:

$$
\delta I(2 \theta)=I_{\text {unt }}(\Delta 2 \theta)-\Sigma W_{p} R I R_{p} I_{p}(\Delta 2 \theta)
$$

where the summation is over $\mathrm{p}$ profiles divided into small (e.g. $0.1^{\circ} 2 \theta$ ) intervals $\Delta 2 \theta . \mathrm{W}_{\mathrm{p}}$ is adjusted so that this summation approaches as close as possible to the observed intensity $I_{\text {unk }}$ of each $\Delta 2 \theta$ interval. The reference intensity ratios must be generated independently from the pure-phase traces and are equivalent to peak-height ratios, rather than integrated intensity ratios. As in the Rietveld method the quantitation consists of matching and scaling the purephase patterns to the observed pattern with scale factors proportional to the weight fractions.

Both methods require considerable operator set-up time and the Rietveld method also involves substantial computer time. Once the proper component phase traces have been determined the accuracy of quantitation should be on the order of $1-2 \%$ absolute; this is likely to exceed the accuracy of routine single-peak RIR analysis significantly.

\section{Summary of Reference Intensity Ratio methodology}

The important simple relationships so useful in RIR analysis and derived by Chung (1974a, 1974b, 1975) will be stated and discussed briefly, with examples given of their use. Moreover, the use of thin layer 
(filter) technology, mass absorption, and solid solution studies as used with RIR equations in our laboratory will be integrated into this presentation. The more compact Chung notation $\mathbf{k}_{\mathbf{i}}$, for reference intensity ratio will be used in the discussion.

\subsection{Analysis with added standard.}

The working equation, given in the notation of Chung (1974a) which provides the weight fraction $W_{j}$ of component $j$ is,

$$
W_{j}=\frac{W_{c} \cdot I_{j}}{k_{j} \cdot I_{c}}
$$

where $k_{j}$ is the reference intensity ratio for component $j$ of the mixture and $I_{j}$ and $I_{c}$ are intensities measured for the jth component peak and standard (corundum), respectively, and $W_{c}$ is the weight fraction of the corundum standard in the composite sample (original sample + added corundum). This equation is straightforward to apply, but requires the addition of standard material. The advantage of its use is that any component weight fraction can be determined independently of any other material, crystalline or amorphous, that may be in the original sample.

\subsection{Analysis without internal standard}

Chung's (1974b) "adiabatic" equation permits analysis of all identified components of a mixture without addition of a standard providing all reference intensity ratios, $\mathbf{k}_{\mathbf{i}}$, are available. This equation is

$$
W_{j}=\left[\frac{k_{j}}{I_{j}} \sum_{i=1}^{n} \frac{I_{i}}{k_{i}}\right]^{-1}
$$

where the notation is the same as for Eq. (1) above. Here the sum is taken for all components so that the equation is self-normalizing; i.e., all the $W_{i}$ will sum to unity. Consequently the absolute weight fractions will be correctly determined through use of Eq. (2) only if all components are identified and crystalline. However, the ratios of the weight fractions remain correct in any case.

\subsection{Measuring the reference intensity ratio}

For the highest accuracy of analysis it is vitally important that both reference intensity measurement and sample measurements be devoid of significant primary extinction, microabsorption, and preferred orientation. It has been argued by some that maintaining a consistent degree of preferred orientation in both $\mathbf{k}_{\mathbf{i}}$ and sample intensity measurements is a satisfactory procedure. Such an approach, however, will not result in the $\mathbf{k}_{\mathbf{i}}$ being a universal constant and cannot be recommended except perhaps for oriented clay particle analyses.

The obvious and most common procedure is to mix analyte and standard together in a 1:1 weight proportion and simply measure the intensities of the strongest peaks, or a series of peaks from each and convert each to the strongest reflection from calculated intensity ratios, if available. Using the absorption-intensity relationship of Leroux et al. (1953) it has been shown (Davis, 1988, p. 75) that the $k_{i}$ can also be measured by a pure phase, non-mixing method. The definition of the $\mathbf{k}_{\mathbf{i}}$ and equivalency to this latter procedure is:

$$
k_{j} \equiv\left[\frac{I_{j}}{I_{c}}\right]_{1: 1}=\frac{\mu_{j} \cdot I_{j o}}{\mu_{c} \cdot I_{c o}}
$$

where the subscripts jo and co refer to intensities collected from pure-phase preparations. Davis et al. $(1990)^{1}$ have demonstrated the equivalency of the two methods in measuring the reference intensity ratios and also provide experimental verification from actual analysis by both procedures for different absorbing materials. The value of Eq. (3) is that possible relative losses of analyte or standard during aerosol suspension on filters, or of inhomogeneous mixing of bulk preparations, is avoided; use of the second expression does require, however, that there be no significant differences between the conditions of the analyte and standard intensity measurements (including instrument alignment and performance).

\subsection{Use of filter loading to suppress preferred orientation}

Two procedures for eliminating or greatly supressing preferred orientation of crystallites of the sample have been proposed. Calvert et al. (1983) describe a method for encapsulating individual inequant particles into
Two printer's errors should be noted in this paper. In Eqs. (4) and (5) the leftmost $I_{j}$ should read $\mathbf{k}_{\mathfrak{J}}$. 
spherical chemical agents and collecting aggregates of the spheres for analysis. We recommend (Davis, 1986) suspension of fine particles in a vertical airstream for collection onto glass microfiber filters. In either method, any significant departure from "infinite" thickness, as well as the presence of enclosing chemical or filter element matrix, requires intensity correction. For the filter technique the penetration, density, and mass absorption properties of several filter substrates have been reported by Davis and Johnson (1982) and Davis (1988). The corrections required in loading thin aerosol layers onto filters are combined into the relation

$$
I_{j}^{\infty}=\frac{I_{j}^{\alpha} \mu_{H} \Omega(\theta)}{W_{H B} \mu_{B}\left[1-e^{-2 \mu_{H} M_{g} / W_{H} \sin \theta}\right]}
$$

where $I_{\mathbf{j}}^{\infty}$ is the intensity corrected from the raw intensity $\mathbf{I}_{\mathbf{j}}^{\alpha} ; \mathbf{W}_{\mathrm{HB}}$ is the weight fraction of sample material (B) in the "hybrid" layer, that layer containing both substrate and sample components down to the depth of penetration of the sample; $\mu_{B}$ and $\mu_{H}$ are the mass absorption coefficients of the pure sample material and hybrid layer material, respectively; $\mathbf{M}_{B}$ is the mass per unit area of the sample on and within the substrate hybrid layer; and $\Omega(\theta)$ is a function to convert intensities from automatic divergence slit to fixed slit conditions. The quantities $\mu_{\mathbf{H}}$ and $\mathbf{W}_{\mathbf{H B}}$ are readily computed from fundamental filter properties (Davis and Johnson, 1982) using the relations

$$
\mu_{H}=\mu_{B} W_{H B}+\mu_{G}\left(1-W_{H B}\right)
$$

and,

$$
W_{H B}=\left[1+\frac{t_{G} \rho_{G}}{M_{B}}\right]^{-1}
$$

where $t_{\mathrm{G}}$ is the effective thickness (pore space removed) of the substrate material within the hybrid layer, and $\boldsymbol{\rho}_{\mathbf{G}}$ and $\mu_{\mathbf{G}}$ are the density and mass absorption of that material, respectively.

The devices described by Johnson and Davis (1982) and Davis (1986) may be constructed from easily obtainable laboratory equipment. Another even simpler device, from our experience in the past few months, has shown to be as effective as the larger ones in producing thin uniform sample layers. Figure 1 illustrates this device; a $250-\mathrm{ml}$ aspirator bottle is taped to a $25-\mathrm{mm}$ DELRIN filter cassette. The inlet is fitted with a short rubber hose and plug (plastic $0.25^{\prime \prime}$ pipe cap works well) drilled with a small hole to admit a $1-\mathrm{mm}$ capillary tube (slightly bent at one end). The sample is placed within the inlet close to the plug. With the collecting pump operating the capillary is moved outward and rotated to dislodge the sample material.

Turbulance within the inlet tube disaggregates the clumps and produces a fine aerosol. Very little practice is required to produce uniform sample layers. Whatman GF/C filters for Bragg-Brentano diffraction and Metricel VM-1 for transmission diffraction are the filters of choice for this technique.

\section{Analysis of amorphous components}

The determination of component weight fractions using Eq. (1) is done independent of the presence of amorphous (or unidentified crystalline) materials. In this relationship, $\mathrm{W}_{\mathrm{c}}+\Sigma \mathrm{W}_{\mathrm{i}}=1$, where the $\Sigma \mathrm{W}_{\mathrm{i}}$ come from the original sample. The test for amorphous

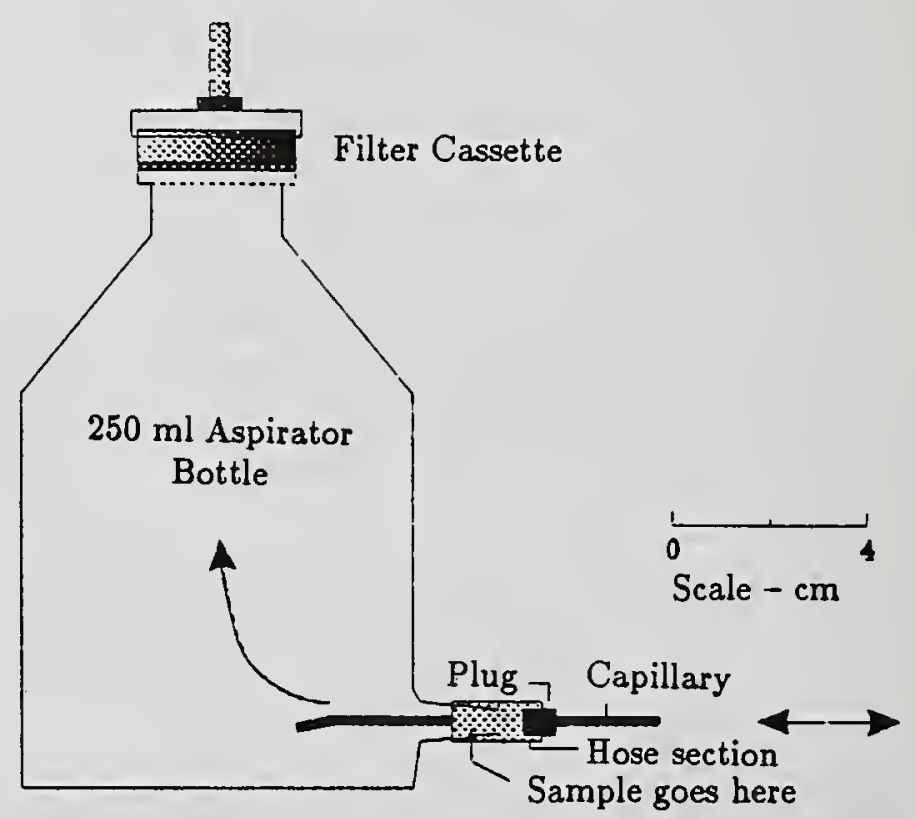

Figure 1. Small suspension chamber for loading of powders on filters.

material in the original sample is (Chung, 1974a)

$$
\sum_{\substack{i=1 \\ i \neq c}}^{n}\left(\frac{I_{i}}{k_{i}}\right)=\frac{\left(1-W_{c}\right)}{W_{c}} \cdot I_{c}
$$

where the summation term is simply the sum of all expressions of Eq. (1) for all components for which 
Table 2. Matrix flushing analysis of four-component sample

\begin{tabular}{|c|c|c|l|l|}
\hline Component & Intensity & $\mathrm{k}_{\mathbf{i}}$ & Known $\mathrm{W}_{\mathbf{i}}$ & Measured $\mathrm{W}_{\mathbf{i}}$ \\
\hline $\mathrm{ZnO}$ & 4661 & 4.35 & 0.3443 & 0.3641 \\
\hline $\mathrm{CaCO}_{3}$ & 2298 & 2.98 & 0.2800 & 0.2620 \\
\hline Silica Gel & --- & --- & 0.1613 & 0.1595 \\
\hline $\mathrm{Al}_{2} \mathrm{O}_{3}(\mathrm{c})$ & 631 & 1.00 & 0.2144 & $\ldots . .-$ \\
\hline
\end{tabular}

weight fractions can be measured. The left and right terms of Eq. (7) will be equal only if all components have been identified, are crystalline, and are included in the analysis. No information is generated about the amorphous components. What is actually happening when this "scattering power" balance is performed is that through division of measured intensities by the $\mathbf{k}_{\mathbf{i}}$ all intensity levels are converted to that value which would exist if all material were corundum! This then is compared with an empirically equivalent parameter computed from the corundum peak intensity and known added weight fraction.

\section{EXAMPLE}

F. Chung (1974a) illustrates his "Matrix Flushing" technique with added amorphous silica gel in a mixture of $\mathrm{ZnO}, \mathrm{CaCO}_{3}$, and $\mathrm{Al}_{2} \mathrm{O}_{3}$ (standard). Table 2 summarizes the data for the analysis. The added corundum is $\mathrm{W}_{\mathrm{c}}$ of Eq. (7) and the value of $\Sigma\left(\mathrm{I}_{\mathrm{i}} / \mathrm{k}_{\mathrm{i}}\right)$ for the two crystalline components, $\mathrm{ZnO}$ and $\mathrm{CaCO}_{3}$, is observed to be $1843 \mathrm{cps}$; this is compared to the right hand term of Eq. (7) which is 2312 . The intensity sum from the conversion of the sample intensities to equivalent corundum scattering power falls short, indicating the presence of unaccounted for material, in this case the silica gel. The weight fraction of silica gel is thus $1-W_{c}-\Sigma W_{i}=0.1595$, in good agreement with the known input value of 0.1613 . Experience in our lab using this procedure is not as good; while the method is theoretically sound, there are many sources for significant error which render the results more semiquantitative than often required.

The method frequently used at the EMES lab combines the "adiabatic" equation (Eq. 2) with mass absorption measurements to ascertain the amount of amorphous material present. This procedure provides, in our experience, more accurate results but requires a knowledge of the composition of the amorphous component so that its mass absorption can be calculated. Generally this is not too demanding a requirement, since materials such as fly ash, carbonaceous matter, limonite, and opal, to cite some very common ones, are generally well enough characterized chemically to allow their mass absorption to be computed within reasonable limits. Where the pure amorphous material is available this parameter may be measured experimentally. Experimental measurement is best done by direct beam transmission (Davis and Johnson, 1987; Davis, 1988, Ch. 9) or substrate diffraction (Frevel and Roth, 1982). The procedure for such analysis makes use of the simple mass absorption balance

$$
\mu_{B}=W_{z} \mu_{z}+W_{a n} \mu_{a m}=W_{z} \mu_{z}+\left(1-W_{z}\right) \mu_{a n}
$$

where the total sample bulk mass absorption, $\mu_{B}$, is measured by one of the above two methods, $\mu_{z}$, the mass absorption coefficient of the crystalline portion of the sample, is computed from the multicomponent crystalline analysis, using the relation $\mu_{\mathrm{z}}=\mathrm{W}_{\mathrm{i}} \mu_{\mathrm{i}}, \mathrm{W}_{\mathrm{i}}$ being the original sample component weight fractions; $\mu_{\mathrm{am}}$ is the known (measured or calculated) mass absorption coefficient of the amorphous component. $W_{z}$ and $\mathrm{W}_{\mathrm{am}}$ are the weight fractions of crystalline and amorphous portions of the whole sample, respectively, and are to be determined from the mass absorption balance.

\section{EXAMPLES}

\section{$\underline{\text { Single Amorphous Component }}$}

We recently analyzed a series of graphite samples of varying purity. Material from Sonora, Mexico contained significant clay, carbonaceous matter, 
Table 3. Analysis of an impure graphite

\begin{tabular}{||l|l|l|l|l||}
\hline Component & $\mathrm{k}_{\mathrm{i}}$ & $\mathrm{I}_{\mathrm{i}}^{\alpha}$ & $\mathrm{I}_{\mathrm{i}}^{\infty}$ & $\mathrm{W}_{\mathrm{i}}$ \\
\hline Chlorite & 0.73 & 2455 & 11254 & $0.0 \Sigma 0$ \\
\hline Kaolinite & 0.84 & 312 & 1440 & 0.010 \\
\hline Muscovite & 0.72 & 1620 & 9726 & 0.113 \\
\hline Quartz & 0.72 & 3192 & 8143 & 0.063 \\
\hline Pyrite & 1.62 & 979 & 2292 & 0.008 \\
\hline Orthoclase & 0.73 & 1599 & 4673 & 0.036 \\
\hline Graphite & 4.9 & 146691 & 380066 & 0.431 \\
\hline Carb (Amorph) & --- & --- & - & 0.253 \\
\hline
\end{tabular}

$\mathrm{M}_{\mathrm{B}}=0.00322 \mathrm{~g} \mathrm{~cm}^{-2} ; \mu_{\mathrm{B}}=20.48 \mathrm{~cm}^{2} \mathrm{~g}^{-1} ; \mu_{\mathrm{Z}}=25.4 \mathrm{~cm}^{2} \mathrm{~g}^{-1} ; \mu_{\text {Graphlte }}=6.0 \mathrm{~cm}^{2} \mathrm{~g}^{-1}$. All Data using CuK $\alpha$ radiation. Data courtesy of Superior Graphite Co., Chicago.

chlorite, quartz, and other impurities. Table 3 presents the results of the crystalline and amorphous component analysis of the sample loaded on a $4.25-\mathrm{cm}$ Whatman GF/C filter by aerosol suspension, using Eq. 2. The raw intensities $\left(I_{i}^{\alpha}\right)$ and the intensities corrected for transparency and matrix effects $\left(l_{i}^{\infty}\right)$, for peak overlap, and for automatic divergence slits are also given in the table.

The bulk sample mass absorption was measured by direct-beam transmission. Because of the presence of silicate impurities the value of $\mu_{\mathrm{B}}$ was substantially higher than that for pure carbon $\left(4.2 \mathrm{~cm}^{2} \mathrm{~g}^{-1}\right)$ but much lower than that $\left(\mu_{z}\right)$ for the crystalline portion of the sample. The mass absorption for the "pure" graphite, also by direct-beam transmission, was $6.0 \mathrm{~cm}^{2} \mathrm{~g}^{-1}$, substantially higher than that expected for pure carbon and is presumably due to adsorbed volatile gaseous materials such as water vapor, oxygen, nitrogen, and other minor impurities within the graphite layering. The results of the mass absorption balance reveals the presence of non-crystalline carbonaceous matter intimately mixed with the graphite.

\section{Two Amorphous Components}

Urban aerosols commonly contain both silicious fly ash (SFA) and carbonaceous fly ash (CFA). When two amorphous components exist in the sample Eq. 8 can be expanded to

$$
\mu_{B}=W_{z} \mu_{z}+W_{a n 1} \mu_{a n 1}+W_{a n 2} \mu_{a n 2}
$$

where $W_{a m 1}+W_{a m 2}=1-W_{z}$ is the conservation condition allowing solution of this equation if the crystalline fraction $\mathrm{W}_{\mathbf{z}}$ can be determined independently. In many cases clearing of the collection filter with immersion oils permits estimation of this parameter from polarizing optical microscopy. For the industrial region of Orem, Utah, several analyses of airborne particulate demonstrated both types of amorphous material. Table 4 illustrates one of the analyses (Davis 1981a) from this area. Note that the reference material in this earlier study is $\mathrm{CaF}_{2}$, not $\mathrm{Al}_{2} \mathrm{O}_{3}$. Variance errors $\mathrm{s}\left(\mathrm{W}_{\mathbf{i}}\right)$ are included in this example. They are calculated through a formal variance error propagation procedure (Davis, 1981b). 
Table 4. Analysis of ambient industrial aerosol containing two amorphous components

\begin{tabular}{||l|l|l|l|l||}
\hline \multicolumn{1}{|c|}{ Component } & $\mathrm{k}_{\mathrm{i}}\left(\mathrm{CaF}_{2}\right)$ & $\mathrm{I}_{\mathrm{i}}^{\infty}($ Counts $)$ & $\mathrm{W}_{\mathrm{i}}$ & $\mathrm{s}\left(\mathrm{W}_{\mathrm{i}}\right)$ \\
\hline Muscovite & 0.078 & 2986 & 0.098 & 0.015 \\
\hline Gypsum & 0.225 & 6367 & 0.061 & 0.011 \\
\hline Kaolinite & 0.172 & 957 & 0.012 & 0.004 \\
\hline Quartz & 0.829 & 48,812 & 0.128 & 0.020 \\
\hline Calcite & 0.537 & 17,586 & 0.090 & 0.036 \\
\hline Hematite & 0.342 & 4251 & 0.027 & 0.016 \\
\hline CFA & ---- & --- & 0.280 & 0.226 \\
\hline SFA & --- & --- & 0.320 & 0.234 \\
\hline
\end{tabular}

$\mu_{\mathrm{B}}=42.8 \mathrm{~cm}^{2} \mathrm{~g}^{-1} ; \mu_{\mathrm{Z}}=59.8 \mathrm{~cm}^{2} \mathrm{~g}^{-1} ; \mu_{\mathrm{CFA}}=8 \mathrm{~cm}^{2} \mathrm{~g}^{-1} ; \mu_{\mathrm{SFA}}=52 \mathrm{~cm}^{2} \mathrm{~g}^{-1}$ All data for CuK $\alpha$ radiation. Reference material for $\mathrm{k}_{1}$ is $\mathrm{CaF}_{2}$.

\section{Reference Intensity Ratio determination}

Undoubtedly the most critical parameter in quantitative XRD analysis is the RIR value. The RIR values published up until 1988 in the JCPDS volumes were provided by analysts without use of guidelines for uniform methodology. Hence, sample preparation, particle size and orientation, and source of standard $\mathrm{Al}_{2} \mathrm{O}_{3}$ were not controlled. Powder Diffraction published a table of selected RIR values and other parameters in 1988 with a second expanded table printed in Dec. 1989 (Davis et al, 1989).

A sample "type" is provided with each entry and measured mass absorption values are given, where available. Most workers are still using "Well" preparations, including "side drifting", which may only partially supress preferred orientation, and mass absorption measurements are as yet not commonly provided.

\section{Experimental and calculated RIR's for solid solutions}

Included in the following sections are brief descriptions of very recently published, or as yet unpublished experimental and calculated data from the SDSM\&T laboratory on $k_{i}$ and $\mu_{i}$ measurements for some solid solution minerals. These data illustrate with a few examples the utility of such data as well as the need for more experimental work on solid solution series compounds.

\subsection{Biotite minerals}

Figure 2 is taken from Kath et al. (1991). Eleven biotites were studied by XRD, XRF, and $\mathrm{AA} / \mathrm{ICP}$ to obtain species chemistry, observed and calculated mass absorption coefficients, and $k_{i}$ values, here denoted $L_{k}$ to distinguish the pure phase method of measurement from the mixing method. The value of these data lie in the ability to predict reasonably accurate $k_{i}$ values from a knowledge of $\mathrm{Fe} /(\mathrm{Fe}+\mathrm{Mg})$ ratios or mass absorption coefficients. The regression for $L_{k}$ vs. $W_{F e}[=$ $\mathrm{Fe} /(\mathrm{Fe}+\mathrm{Mg})]$ of Fig. 2 is:

$$
\mathrm{Lk}_{\mathrm{i}}=6.359 \mathrm{~W}_{\mathrm{Fe}}+1.513(\mathrm{R}=\mathbf{0 . 9 1})
$$

\subsection{Asbestiform amphiboles}

Figure 3 presents data obtained from Davis (1990a) and Davis (1992). The monoclinic asbestiform amphiboles demonstrate satisfactory correlation of both $\mu_{i}$ and $\mathrm{Lk}_{\mathbf{i}}$ with weight percent $\mathrm{FeO}$. Except for anthophyllite, with space group Pnma (hk $\ell=610)$, the amphibole symmetry is $C 2 / m$ with the strong reflection $\mathrm{hk} \ell=110$. Thus in the figure the anthophyllite points 


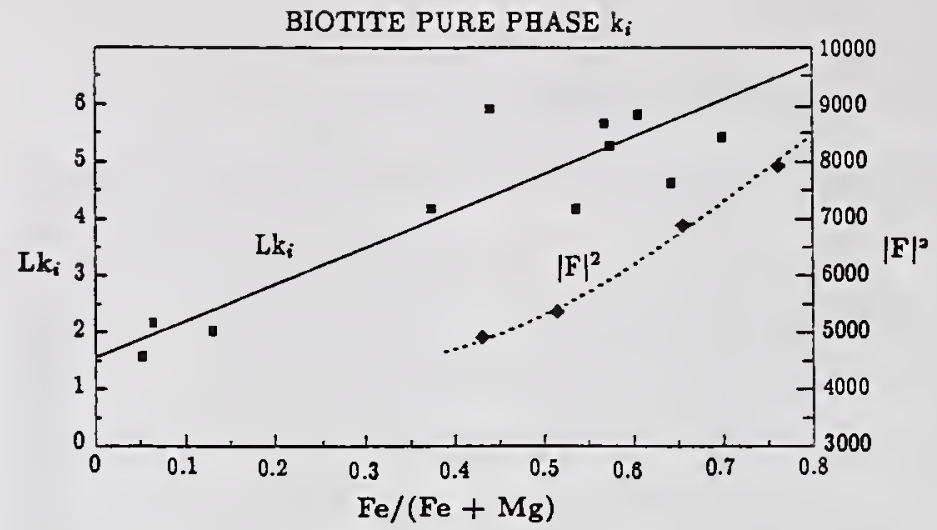

Figure 2. Reference constant and mass absorption data for biotites.

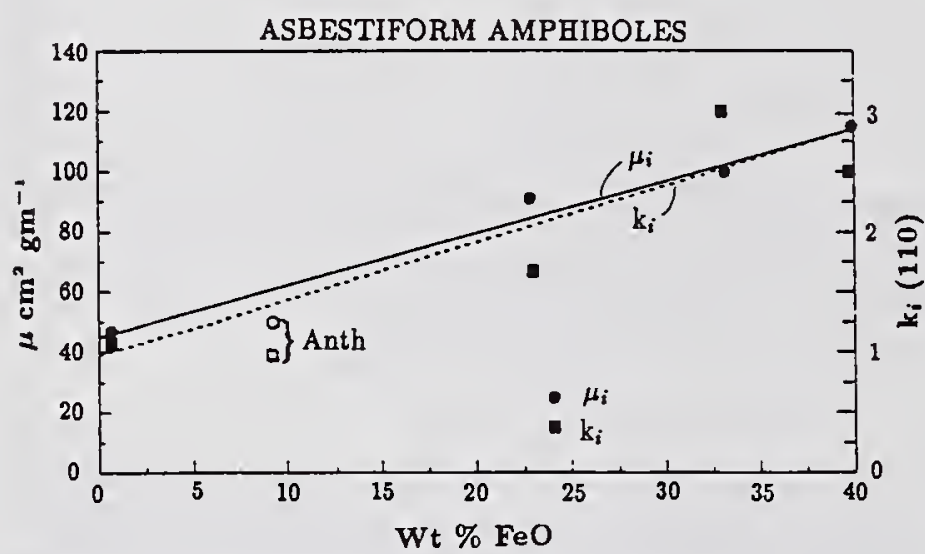

Figure 3. Reference constant and mass absorption data for asbestiform amphiboles.

are plotted for comparision but are not included in the regressions.

For the monoclinic amphiboles these regressions are:

$$
\begin{aligned}
\mathrm{Lk}_{\mathrm{i}} & =0.045 \mathrm{~W}_{\mathrm{FeO}}+0.982(\mathbf{R}=\mathbf{0 . 8 2}) \\
\mu_{\mathrm{i}} & =1.712 \mathrm{~W}_{\mathrm{FeO}}+45.9 \mathrm{~cm}^{2} \mathrm{~g}^{-1} \quad(\mathbf{R}=\mathbf{0 . 9 9 4})
\end{aligned}
$$

where $\mathrm{W}_{\mathrm{FeO}}$ is the weight percent $\mathrm{FeO}$ in the amphibole. These data should be regarded as preliminary, as far as establishing $\mathbf{k}_{\mathrm{i}}$ and $\mu_{\mathrm{i}}$ trends are concerned, since many more such values for amphibole subspecies should be obtained.

\subsection{Olivines}

Jahanbagloo and Zoltai (1966) completed a comprehensive study of the complete olivine solid solution series, including XRD spacing tables and intensity normalizing factors, from which $k_{i}$ values could be calculated. Additional intensities and absorption factors were calculated for olivines by Borg and Smith (1969). Using the method of Hubbard et al.
(1976) and Hubbard and Smith (1977), we converted the component intensities to $\mathbf{k}_{\mathbf{i}}$ values. Fig. 4 presents calculated density $(\rho)$, mass absorption $(\mu)$, and $\mathbf{k}_{\mathbf{i}}$ values, for the full $\mathrm{Mg} / \mathrm{Fe}$ variation of this series.

The linear regression for all 10 olivine members is:

$$
\mathrm{k}_{\mathbf{i}}=0.00754 \mathrm{Fa}+0.762(\mathbf{R}=\mathbf{0 . 9 8})
$$

where $\mathrm{Fa}$ is the mol percent fayalite, $\mathrm{Fe}_{2} \mathrm{SiO}_{4}$ in the member.

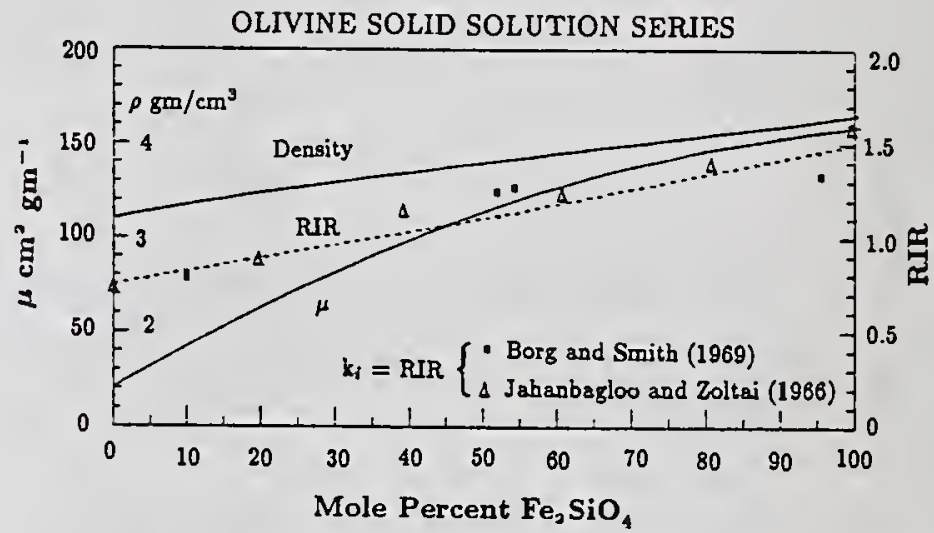

Figure 4. Calculated reference constant and mass absorption data for olivines.

\subsection{Plagioclase feldspars}

Several strong plagioclase feldspar peaks cluster together in the $27.5-28^{\circ} 2 \theta$ region ( $\mathrm{CuK} \alpha$ radiation). We have measured a special type of RIR consisting of, for example, the $202+220+040+002$ intensities of oligoclase peaks existing within this short spectral range. Similar constants were measured for other plagioclase members. The mass absorption coefficient and $\mathrm{k}_{\mathrm{i}}$ are plotted in Fig. 5 versus weight percent $\mathrm{CaO}$ $\left(\mathrm{W}_{\mathrm{CaO}}\right)$. Note from the $\mathrm{k}_{\mathrm{i}}$ scale of the figure that little variation of $\mathbf{k}_{\mathbf{i}}$ exists for this peak cluster over the $\mathrm{Na}$ Ca solid solution limits. Using an average or median $k_{i}$ value for a plagioclase analysis will closely determine its weight fraction in the mixture; however, the composition remains unknown, unless its mass absorption or $\mathrm{CaO}$ composition can also be measured in the pure phase.

The regression equations for $\mu_{i}$ and $k_{i}$ are:

$$
\begin{aligned}
& \mu_{\mathrm{i}}=0.877 \mathrm{~W}_{\mathrm{CaO}}+34.92(\mathrm{R}=\mathbf{0 . 9 9}) \\
& \mathrm{k}_{\mathrm{i}}=0.0098 \mathrm{~W}_{\mathrm{CaO}}+1.545(\mathrm{R}=\mathbf{0 . 9 5})
\end{aligned}
$$




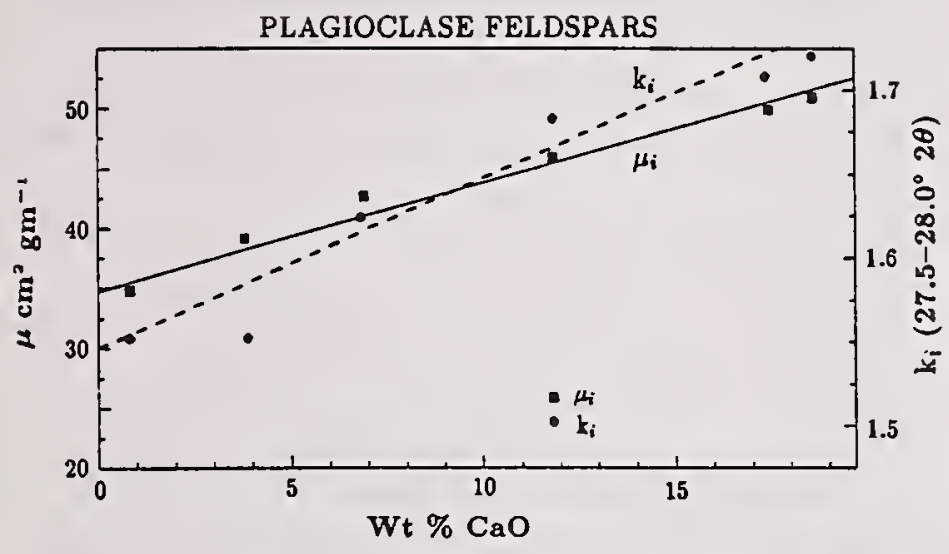

Figure 5. Reference constant and mass absorption data for plagioclase feldspars.

\section{References and Bibliography}

(* Indicates Direct RIR Application)

Alexander, L. \& Klug, H. P. (1948). Basic Aspects of X-ray Absorption in Quantitative Diffraction Analysis of Powder Mixtures. Anal. Chem. 20, 886889.

*Bayliss, P. (1986). Quantitative Analysis of Sedimentary Minerals by Powder X-ray Diffraction. Powd. Diff. 1, 37-39.

*Berry, L. G. (Editor) (1970). Establishment of a Reference Intensity. In Inorganic Index to the Powder Diffraction File; Joint Committee on Powder Diffraction Standards, (PD15-21).

Bezjak, A. (1961). X-ray Quantitative Analysis of Multiphase Systems. Croatica Chemica Acta 33, $197-$ 200.

*Bish, D.L. \& Howard, S.A. (1988). Quantitative Analysis Using the Rietveld Method. J. Appl. Cryst. 21, 86-91.

Borg, I.Y. \& Smith, D. K. (1969). Calculated X-ray Powder Patterns for Silicate Minerals. Memoir 122, Geological Society of America.

Calvert, L.D., Sirianni, A.F., \& Gainsford, G.J. (1983). A Comparison of Methods for Reducing Preferred Orientation. Adv. X-ray Anal. 26, 105-110.

Copeland, L.E. \& Bragg, R. H.(1958). Quantitative X-ray Diffraction Analysis. Anal. Chem. 30, 196201.

*Chung, F. H. (1974a). Quantitative Interpretation of X-ray Diffraction Patterns of Mixtures. I. Matrix Flushing Method for Quantitative Multicomponent Analysis. J. Appl. Cryst. 7, 519-525.

*Chung, F. H. (1974b). Quantitative Interpretation of X-ray Diffraction Patterns of Mixtures. II. Adiabatic
Principle of X-ray Diffraction Analysis of Mixtures. J. Appl. Cryst. 7, 526-531.

*Chung, F. H. (1975). Quantitative Interpretation of X-ray Diffraction Patterns of Mixtures. III. Simultaneous Determination of a Set of Reference Intensities., J. Appl. Cryst. 8, 17-19.

*Davis, B.L. (1981a). Quantitative Analysis of Crystalline and Amorphous Airborne Particulates in the Provo-Orem Vicinity, Utah. Atmos. Environ. 15, 613-618.

*Davis, B.L. (1981b) A Study of the Errors in X-ray Quantitative Analysis Procedures for Aerosols Collected on Filter Media. Atmos. Environ. 15, 291-296. Davis, B.L. (1986). A Tubular Aerosol Suspension Chamber for the Preparation of Powder Samples, for X-ray Diffraction Analysis. Powd. Diff. 1, 240-243. *Davis, B. L., Johnson, L.R., \& Mebrahtu, T. (1986). $\mathrm{X}$-ray Quantitative Analysis of Coal by the Reference Intensity Method. Powd. Diff. 1, 244-255.

*Davis, B.L. (1988). Reference Intensity Method of Quantitative X-ray Diffraction Analysis. Second Edition, Davis Consulting, 213 pp.

*Davis, B.L. (1990a). Quantitative Analysis of Asbestos Minerals by the Reference Intensity X-ray Diffraction Procedure. Am. Ind. Hyg. Assoc. J. 51, 297-303.

*Davis, B.L. (1990b). The Reference Intensity Ratio: Its Measurement and Significance. Powd. Diff. 5, 7679.

*Davis, B.L. (1992). Powder Patterns and Reference Intensity Ratios for Asbestos Amphiboles. Grants-InAid Final Summary Report, Jan. 1992. International Centre for Diffraction Data, Swarthmore, PA

Davis, B.L., \& Johnson, L.R. (1982). On the Use of Various Filter Substrates for Quantitative Particulate Analysis by X-ray Diffraction. Atmos. Environ. 16, 273-282.

Davis, B.L., \& Johnson, L.R. (1987). The Use of Mass Absorption in Quantitative X-ray Diffraction Analysis. Adv. X-ray Anal. 30, 333-342.

*Davis, B.L., Smith, D.K., \& Holomany, M.A. (1989). Tables of Experimental Reference Intensity Ratios: Table No. 2, December, 1989. Powd. Diff. 4, 201-205.

*Devlin, D.J., \& Amin, K.E. (1990). A Method for Quantitative Phase Analysis of Silicon Nitride by Xray Diffraction. Powd. Diff. 5, 121-124.

Dragoo, A.L. (1986). Standard Reference Materials for X-ray Diffraction Part I. Overview of Current and Future Standard Reference Materials. Powd. Diff. 1, 294-298. 
Flörke, O.W. \& Saalfeld, H. (1955). Ein Verfahren Zur Herstellung Texturfreier RöntgenPulverpraparate. Z. Krist. 106, 460-466.

Frevel, L.K. \& Roth, W.C. (1982) Semimicro Assay of Crystalline Phases by X-ray Powder Diffractometry. Anal. Chem. 54, 677-682.

*Goehner, R.P. (1982). X-ray Diffraction Quantitative Analysis Using Intensity Ratios and External Standards. Adv. X-ray Anal. 25, 309-313.

Hill, R.J. (1991). Expanded Use of the Rietveld Method in Studies of Phase Abundances in Multiphase Mixtures. Powd. Diff. 6, 74-77.

*Hubbard, C.R. \& Smith, D.K. (1977). Experimental and Calculated Standards for Quantitative Analysis by Powder Diffraction. Adv. X-ray Anal. 20, 27-39.

*Hubbard, C.R. \& Snyder, R.L. (1988). RIRMeasurement and Use in Quantitative XRD. Powd. Diff. 3, 75-77.

*Hubbard, C.R., Evans, E.H., \& Smith, D.K. (1976). The Reference Intensity Ratio, $\mathrm{I} / \mathrm{I}_{c}$, for Computer Simulated Patterns. J. Appl. Cryst. 9, 169-174.

Hughes, R. \& Bohor, B. (1970). Random Clay Powders Prepared by Spray Drying. Amer. Mineral. 55, 1780-1786.

Jahanbagloo, I.C. \& Zoltai, T. (1966). Calculated Xray Powder Diffraction Patterns. Department of Geology and Geophysics, University of Minnesota, Minneapolis, MN, $114 \mathrm{pp.}$

Jahanbagloo, I.C. \& Zoltai, T. (1968). Quantitative Analysis with the Aid of Calculated X-ray Powder Patterns. Anal. Chem. 40, 1739-1741.

*Johnson, L.R. \& Davis, B.L. (1982). X-ray Diffraction Analysis Using the Reference Intensity Method. Norelco Reporter, 29, 28-34.

Jonas, E.J. \& Kuykendall, J.R. (1966). Preparation of Montmorillonites for Random Powder Diffraction. Clay Mineral. Bull. 6, 232-235.

Karlak, R.F. \& Burnett, D.S. (1966). Quantitative Phase Analysis by X-ray Diffraction. Anal. Chem. 38, 1741-1745.

*Kath, R.L., Spilde, M.N., Davis, B.L., \& Smith, D.K. (1991). Reference Intensity Ratio and Mass Absorption Measurements of Eleven Biotites. Powd. Diff. 6, 183-186.

*Kim, H.S., Rawlings, R.D., \& Rogers, P.S. (1989). Quantitative Determination of Crystalline and Amorphous Phases in Glass-Ceramics by X-ray Diffraction Analysis. J. British Ceramic Soc. 88, 21-26. *Klages, M.G., \& Hopper, R.W. (1982). Clay Minerals in Northern Plains Coal Overburden as
Measured by X-ray Diffraction. Soil Sci. Soc. Amer. J. 46, 415-419.

Leroux, J., Lennox, D.H., \& Kay, K. (1953). Direct Quantitative X-ray Analysis. Anal. Chem. 25, 740743.

Maniar, P.D. \& Cooke, G.A. (1987). Modal Analyses of Granitoids by Quantitative X-ray Diffraction. Amer. Mineral. 72, 433-437.

Monshi, A. \& Messer, P.F. (1991). Ratio of Slopes Method for Quantitative X-ray Diffraction Analysis. J. Materials Sci. 26, 3623-3627.

*Nakamura, T., Sameshima, K., Okunaga, K., Sugiura, Y., \& Sato, J. (1989). Determination of Amorphous Phase in Quartz Powder by X-ray Powder Diffractometry. Powd. Diff. 4, 9-13.

Pétér, É., \& Kálmán, A. (1964). Quantitative X-ray Analysis of Crystalline Multicomponent Systems. Acta Chim Hung. Tomus, 41, 413-422.

*Raab, G.A. (1988). Semiquantitative Analysis by Xray Powder Diffraction (SQXRD) of the $<2 \mathrm{~mm}$ to $0.002 \mathrm{~mm}$ and $<0.002 \mathrm{~mm}$ Fractions of Soil. Powd. Diff. 3, 144-152.

*Raab, G. A. \& Dalheim, P. (1991). The Effects of Sampling Error on Detection Limits Determined for Quantitative X-ray Diffraction (Unpublished manuscript, Lockheed Company, Las Vegas, NV).

Smith, D.K. (1992). Particle Statistics and WholePattern Methods in Quantitative X-ray Powder Diffraction Analysis. Preprints for Adv. X-ray Anal. v35. Smith, D.K., Johnson, G.G., Jr., Scheible, A., Wims, A.M., Johnson, J.L., \& Ullmann, G. (1987). Quantitative X-ray Powder Diffraction Method Using the Full Diffraction Pattern. Powd. Diff. 2, 73-77.

Smith, S.T., Snyder, R.L., \& Brownell, W.E. (1979). Minimization of Preferred Orientation in Powders by Spray Drying. Adv. X-ray Anal. 22, 77-85.

*Smith, D.K., Johnson, G.G., Jr., \& Wims, A. M. (1988). Use of Full Diffraction Spectra, Both Experimental and Calculated, in Quantitative Powder Diffraction Analysis. Aust. J. Phys. 41, 311-321.

Taylor, J.C. (1991). Computer Programs for Standardless Quantitative Analysis of Minerals Using the Full Powder Diffraction Profile. Powd. Diff. 6, 1-9. *Woolf, P.M. de, \& Visser, J.W. (1988). Absolute Intensities-Outline of a Recommended Practice. Powd. Diff. 3, 202-204. (originally printed as Report 641.109, Technisch Physische Dienst, Delft, Holland, 1964). 
National Institute of Standards and Technology Special Publication 846. Proceedings of the international conference Accuracy in Powder Diffraction II, held at NIST, Gaithersburg, MD, May 26-29, 1992. (Issued October 1992)

\title{
Standardless Method of Quantitative X-ray Diffractometry
}

\author{
Lev Zevin
}

\author{
The Institutes for Applied Research, Ben-Gurion University, \\ P.O. Box 1025, Beer-Sheva, Israel
}

\begin{abstract}
Quantitative analysis of a system of $n$ phases can be carried out without analytical standards provided that at least $n$ mixtures of these phases are available. The solution to the "one peak one phase" case is given, as is that for the full-pattern approach. For the latter, cluster decomposition by profile fitting is recommended. Precision of the method is affected to the greatest extent by a lack of compositional diversity of the initial sample set and the probable presence of an undetected (amorphous) phase. A simple test is presented for estimating the suitability of the sample set for analysis and for evaluating the analysis error. Current and potential fields of application of the phase quantification technique are discussed.
\end{abstract}

\section{Introduction}

Some years ago Hermans \& Weidinger (1948) proposed a method for evaluating the crystalline fraction in cellulose that does not require a sample of a pure crystalline or a pure amorphous material. Their idea, which was rather simple, and is based on the following obvious relationships for the crystalline fractions, $x$ and $y$, in two samples of native and regenerated cellulose, respectively:

$$
\frac{x}{y}=\frac{\left(\mathrm{I}_{n}\right)_{c}}{\left(\mathrm{I}_{r}\right)_{c}} ; \quad \frac{1-x}{1-y}=\frac{\left(\mathrm{I}_{n}\right)_{a}}{\left(\mathrm{I}_{r}\right)_{a}}
$$

where $\left(I_{n}\right)_{c}$ and $\left(I_{n}\right)_{a}$ are the intensities of the diffraction lines for the crystalline and amorphous phases in native cellulose, and $\left(\mathrm{I}_{r}\right)_{c}$ and $\left(\mathrm{I}_{r}\right)_{a}$ are the same quantities for regenerated material. Equations (1) are easily solved for $x$ and $y$. This method was then extended to polyethylene and other polymers and has been used successfully for the past 40 years (Zevin \& Messalem, 1982).

The potential of this approach became better understood in the late 60 s and early 70 s, when quantification was extended to complicated mineral mixtures, and scientists realized the importance of scaling experimental data against proper, but not always available, analytical standards. The ideas of
Hermans \& Weidinger (1948) were revived in the works of Moore (1968), Zavyalova (1970), Driz \& Salin (1972) and Zevin \& Zavyalova (1974). More rigorous theory followed (Zevin, 1977; Fiala, 1980; Brown 1986) and completed the mathematical formalism of the method. However, despite the very attractive self-calibration aspect of this method, its application in practice has been rather sluggish as a result of a number of inherent drawbacks:

1. The method is severely affected by phase- or sample-related effects, such as preferred orientation, microabsorption, etc.

2. When the composition of the samples in the basic sample set are not diverse enough, precision is low; and

3. There may be technical problems in preparation of the basic sample set.

In the present work we propose ways to surmount the inherent difficulties of the method that hinder its wide implementation.

\section{Basic principles}

The intensity of the diffraction peak is related to the phase abundance by the Klug-Alexander equation:

$$
\begin{aligned}
\mathrm{J}_{s j} & =\frac{\mathrm{K}_{j} \mathrm{C}_{s j}}{\mu_{s}^{*}} \\
& =\frac{\mathrm{K}_{j} \mathrm{C}_{s j}}{\sum_{j=1}^{n} \mathrm{C}_{s j} \mu_{j}^{*}},
\end{aligned}
$$

where $\mathrm{J}_{s j}$ is the intensity diffracted by phase $j$ in the sample $s, \mathrm{~K}_{j}$ is a calibration constant, $\mathrm{C}_{s j}$ is the abundance of the corresponding phase, $\mu_{s}^{*}$ is the mass absorption coefficient of the sample $s$, and $\mu_{j}^{*}$ is the mass absorption coefficient of phase $j$. Suppose that $I_{s j}$ is the intensity corrected for sample absorption, and $\mu_{s}^{*}$ is obtained by an independent method. Then $\mathrm{I}_{s j}=\mathrm{J}_{s j} \mu_{s}^{*}$. If an internal standard is used, then $\mathrm{I}_{s j}=\mathrm{J}_{s j} /\left(\mathrm{J}_{s}\right)_{s t}$, where $\left(\mathrm{J}_{s}\right)_{s t}$ is the intensity 
diffracted by the internal standard. A simple equation holds for the corrected intensity:

$$
\mathrm{I}_{s j}=\mathrm{K}_{j} \mathrm{C}_{s j} .
$$

To obtain the calibration constant $K_{j}$, and to carry out an analysis of an $n$-phase sample by simple scaling against a pure phase (analytical standard), we need $n-1$ pure phases. The remaining $n$th phase is obtained from mass balance equation (4):

$$
\sum_{j=1}^{n} \mathrm{C}_{s j}=1, \quad 1 \leq s \leq n
$$

If we assign the first $n-1$ numbers to the pure phases and the number $s=n$ to the sample to be analyzed, then equations (3) and (4) will give matrix equation (5):

$$
\left(\begin{array}{cccc}
\mathrm{I}_{11} & 0 & \cdots & 0 \\
0 & \mathrm{I}_{22} & \cdots & 0 \\
\vdots & \vdots & \ddots & \vdots \\
\mathrm{I}_{n 1} & \mathrm{I}_{n 2} & \cdots & \mathrm{I}_{n n}
\end{array}\right)\left(\begin{array}{c}
\alpha_{1} \\
\alpha_{2} \\
\vdots \\
\alpha_{n}
\end{array}\right)=\left(\begin{array}{c}
1 \\
1 \\
\vdots \\
1
\end{array}\right)
$$

where $\alpha_{j}=1 / K_{j}$. Let us substitute the pure phases in the first $(n-1)$ rows of the data matrix with samples containing the same $n$ phases in different proportions:

$$
\left(\begin{array}{cccc}
\mathrm{I}_{11} & \mathrm{I}_{12} & \cdots & \mathrm{I}_{1 n} \\
\mathrm{I}_{21} & \mathrm{I}_{22} & \cdots & \mathrm{I}_{2 n} \\
\vdots & \vdots & \ddots & \vdots \\
\mathrm{I}_{n 1} & \mathrm{I}_{n 2} & \cdots & \mathrm{I}_{n n}
\end{array}\right)\left(\begin{array}{c}
\alpha_{1} \\
\alpha_{2} \\
\vdots \\
\alpha_{n}
\end{array}\right)=\left(\begin{array}{c}
1 \\
1 \\
\vdots \\
1
\end{array}\right) .
$$

Each row $s$ in the data matrix (6) represents a diffraction pattern of sample $s$, and each column represents the intensities of the diffraction peaks $j$ in the samples. Equations (6) can be solved for $\alpha_{j}$ coefficients, which emphasizes the principle of the standardless approach: we obtain calibration constants without the application of a pure phase.

If the number of samples $m$ exceeds the number of phases $n$, then the solution to equations (6) may be found by least-squares minimization:

$$
\alpha_{j}=\sum_{s=1}^{m} \sum_{k=1}^{n}\left[\mathbf{I}^{T} \mathbf{I}_{j k}^{-1} \mathrm{I}_{k s},\right.
$$

where $\mathrm{I}$ is the matrix whose elements are $\mathrm{I}_{s j}$, and $\mathrm{I}^{T}$ is its transpose. As soon as $\alpha_{j}$ are calculated, the weight fractions, $\mathrm{C}_{\boldsymbol{s}}$, may be obtained from equation (3). The results may be improved somewhat by appropriate weighting of the samples. Samples with one prominent phase, approaching the ideal pure phase situation, can be given greater weight than samples with even phase compositions or with a suspected undetected (amorphous) phase. Weighting may be achieved by simple repeating of rows of more reliable samples in the $\mathrm{I}_{s j}$ matrix.

Another form of analytical equation is obtained from equation (3), using the obvious relationships of the weight fractions of any phase $j$ in two samples $s$ and $r$, as follows:

$$
\mathrm{C}_{s j}=\mathrm{L}_{r s j} \mathrm{C}_{r j},
$$

where $\mathrm{L}_{r s j}=\mathrm{I}_{s j} / \mathrm{I}_{r j}$ is an intensity ratio.

After summation of the left and right hand sides of equation (8) over all phases $j$, we arrive at a system of $m$ linear equations with $n$ unknown weight fractions $\mathrm{C}_{r j}$ in the sample $r$, as expressed in the form:

$$
\sum_{j=1}^{n} \mathrm{~L}_{r s j} \mathrm{C}_{r j}=1 \text { for } 1 \leq s \leq m .
$$

Again, if the number of samples $m>n$, the solution to equation (9) for phase abundances $C_{r j}$ in sample $r$ may be found by least squares minimization:

$$
\mathrm{C}_{r j}=\sum_{l=1}^{m} \sum_{k=1}^{n}\left[\mathrm{~L}_{r}^{T} \mathrm{~L}_{r}\right]_{j k}^{-1}\left[\mathrm{~L}_{r}\right]_{k l},
$$

where $\mathrm{L}_{r s}$ is the matrix whose elements are $\mathrm{I}_{s j} / \mathrm{I}_{r j}$.

A situation that may arise in practice is that in which a sample's absorption is unknown, but the absorption coefficients of the component phases can be calculated. The solution to the standardless problem may also be found in this case. Starting from the basic equation (2), we obtain for the ratio of the uncorrected intensities corresponding to phase $j$ in samples $s$ and $r$ :

$$
\left(\mathrm{J}_{s j} / \mathrm{J}_{r j}\right) \mathrm{C}_{r j}=\mathrm{C}_{s j} \frac{\sum_{k} \mathrm{C}_{r k} \mu_{k}^{*}}{\sum_{k} \mathrm{C}_{s k} \mu_{k}^{*}}
$$

Multiplying both sides by $\mu_{j}^{*}$ and summing over all phases $j$ we arrive at:

$$
\sum_{j}\left(\mathrm{~J}_{s j} / \mathrm{J}_{r j}\right) \mathrm{C}_{r j} \mu_{j}^{*}=\sum_{j} \mathrm{C}_{r j} \mu_{j}^{*} .
$$

By variation of the sample number $1 \leq s \leq m$, and by including the mass balance equation, we obtain a system of linear equations for $n$ unknown weight fractions $\mathrm{C}_{r j}$ of phases $j$ in sample $r$ :

$$
\left\{\begin{array}{l}
\sum_{j} \mu_{j}^{*}\left[1-\mathrm{J}_{s j} / \mathrm{J}_{r j}\right] \mathrm{C}_{r j}=0,1 \leq s \leq m, \\
\sum_{j=1}^{n} \mathrm{C}_{r j}=1 .
\end{array}\right.
$$


Table 1: Data matrix for the 13 four-phase test samples

\begin{tabular}{|c|c|c|c|c|c|c|c|}
\hline \multirow[t]{2}{*}{ Sample } & \multicolumn{2}{|c|}{$\overline{\text { Quartz }}$} & \multicolumn{2}{|c|}{ Kaolin } & \multicolumn{2}{|c|}{ Pyrite } & \multirow{2}{*}{$\begin{array}{c}\text { Calcite } \\
104 \\
\end{array}$} \\
\hline & 101 & 100 & 001 & 002 & 311 & 200 & \\
\hline 1 & 0 & 0 & 56 & 44 & 0 & 0 & 100 \\
\hline 2 & 12 & 4.2 & 51 & 41 & 8 & 7 & 95 \\
\hline 3 & 20 & 7 & 49 & 38 & 12 & 11 & 99 \\
\hline 4 & 27 & 9 & 49 & 39 & 19 & 14.5 & 83 \\
\hline 5 & 30 & 12.5 & 44 & 35 & 21 & 19 & 69 \\
\hline 6 & 40 & 14 & 38 & 29 & 26 & 22 & 56 \\
\hline 7 & 46 & 16 & 34 & 26 & 34 & 28 & 43 \\
\hline 8 & 55 & 19 & 30 & 23 & 40 & 32 & 32 \\
\hline 9 & 64 & 23 & 18 & 14 & 48 & 42 & 23.5 \\
\hline 10 & 74 & 26 & 10 & 9 & 54 & 46 & 7.5 \\
\hline 11 & 34.5 & 12 & 55 & 45 & 12.5 & 11 & 17.5 \\
\hline 12 & 26 & 9 & 52 & 40 & 30 & 24 & 25 \\
\hline 13 & 17 & 6 & 47 & 37 & 33 & 29 & 46.5 \\
\hline
\end{tabular}

Table 2: Results of phase analysis of the 13 four-phase test samples

\begin{tabular}{|c|c|c|c|c|c|c|c|c|}
\hline \multirow[t]{2}{*}{ Sample } & \multicolumn{2}{|c|}{ Quartz, \% } & \multicolumn{2}{|c|}{ Pyrite, \% } & \multicolumn{2}{|c|}{ Calcite, $\%$} & \multicolumn{2}{|c|}{ Kaolinite, \% } \\
\hline & Actual & $\begin{array}{l}\text { Deter- } \\
\text { mined }\end{array}$ & Actual & $\begin{array}{l}\text { Deter- } \\
\text { mined }\end{array}$ & Actual & $\begin{array}{l}\text { Deter- } \\
\text { mined }\end{array}$ & Actual & $\begin{array}{l}\text { Deter- } \\
\text { mined }\end{array}$ \\
\hline 1 & $\overline{0}$ & 0.0 & 0 & 0.0 & 30 & 26.3 & 70 & 66.4 \\
\hline 2 & 7 & 7.4 & 6 & 4.6 & 27 & 24.3 & 60 & 59.8 \\
\hline 3 & 11 & 12.3 & 10 & 8.0 & 24 & 26.1 & 55 & 56.2 \\
\hline 4 & 15 & 16.6 & 14 & 11.7 & 21 & 21.9 & 50 & 57.2 \\
\hline 5 & 19 & 19.5 & 18 & 13.9 & 18 & 18.3 & 45 & 51.1 \\
\hline 6 & 23 & 24.8 & 22 & 16.7 & 15 & 14.6 & 40 & 43.2 \\
\hline 7 & 27 & 28.9 & 26 & 21.4 & 12 & 11.3 & 35 & 39.1 \\
\hline 8 & 31 & 34.6 & 30 & 25.2 & 9 & 8.4 & 30 & 34.2 \\
\hline 9 & 37 & 39.7 & 37 & 31.3 & 6 & 6.2 & 20 & 21.2 \\
\hline 11 & 20 & 21.3 & 10 & 8.4 & 5 & 4.6 & 65 & 64.9 \\
\hline 12 & 15 & 16.2 & 22.5 & 18.8 & 7.5 & 6.9 & 55 & 59.8 \\
\hline 13 & 10 & 10.5 & 27.5 & 21.5 & 12.5 & 12.2 & 50 & 54.9 \\
\hline
\end{tabular}

We have applied the standardless method to 13 four-phase mixtures of quartz, pyrite, calcite and kaolinite. Integrated intensities (corrected for absorption) are presented in Table 1. For phases with two measured diffraction peaks the sum of the two intensities was used in the analytical equations. Table 2 presents the phase abundances resulting from the implementation of equation (7). Most of the observed weight fractions fall within $10 \%$ (relative) limits around the actual values, although for pyrite the discrepancies are greater. The results reflect the main drawbacks of the technique, at least in its present state - only moderate precision.

\section{Full-pattern approach in the standardless technique}

The modern tendency in quantitative phase analysis is utilization of the full diffraction pattern of a sample for phase quantification (Smith et al. 1988; Madsen \& Hill, 1990). This approach aids in minimizing some sample- and phase-related problems that severely affect the precision of the analysis, such as preferred orientation and microabsorption. This approach also holds true for standardless methods.

There are two different techniques for processing digitalized patterns. In the first one, known as profile fitting, overlapping peaks are separated into components (or individual peaks) (Parrish et al., 1976; Will et al., 1983) This technique requires some knowledge of peak shape, but does not require any structural data. As soon as cluster decomposition has been car- 
ried out, and more than one peak is available for any phase $j$, then this phase is represented by combined intensity $\mathrm{I}_{s j}$ :

$$
\mathrm{I}_{s j}=\sum_{k} \mathrm{U}_{k}\left(\mathrm{i}_{s j}\right)_{k}
$$

where $\mathrm{U}_{k}$ is the weight given to the $k$-th peak of phase $j$. Combined intensities are handled in the conventional way, as outlined in the previous section.

In the second technique, known as the continuous pattern approach, the sample pattern is presented as a weighted sum of the patterns of the individual phases. For the standardless technique discussed here, this approach is expressed in the equation:

$$
\mathrm{I}_{s i}=\sum_{j=1}^{n} \mathrm{C}_{s j} \mathrm{~K}_{j i}
$$

where $s(=1,2, \ldots, m)$ is the sample index, $i(=$ $1,2, \ldots, t)$ is the angle index or step number, and $j(=1,2, \ldots, \mathrm{n})$ is the phase index. Each row $\mathbf{s}$ in the $\mathrm{I}_{s i}$ matrix represents the diffraction pattern of a sample, and each row of the $\mathrm{K}_{j i}$ matrix represents the diffraction pattern of a phase, $j$. The matrix $\mathbf{I}$ can be represented as the product of two matrixes $\mathbf{C}$ and $\mathbf{K}$ in an unlimited number of ways, and in the general case, with $\mathrm{C}_{s j}$ and $\mathrm{K}_{j i}$ unknown, there is no unique solution to equation (15). However, equation (15) can be solved in one particular case. Suppose that:

i) the number of samples $m$ is equal to the number of phases $n$; and

ii) there are at least $p$ zero elements in each row of the $\mathrm{K}_{j i}$ matrix. In other words, each phase does not contribute to at least $p$ diffraction peaks among $t$ diffraction peaks measured ( $t$ elements of a row in $\mathrm{K}$ ).

The $\mathbf{K}$ matrix is expressed as $\mathbf{K}=\mathbf{W I}$, where $\mathbf{W}=$ $\mathbf{C}^{-1}$. For each zero element in the $\mathbf{K}$ matrix:

$$
\sum_{s=1}^{n} \mathrm{~W}_{j s} \mathrm{I}_{s i}, i=1,2, \ldots p .
$$

The total number of equations (16) is $p \times n(n=$ the number of rows in $\mathbf{K}$.) We add $n$ mass balance equations:

$$
\sum_{j} \mathrm{C}_{j s}=1
$$

bringing the total number of equations to $p \times n+n$. If $p \geq n-1$, then the total number of equations is greater than or equal to $n^{2}$, and the number of unknown elements of $\mathrm{W}$ is $n \times n$. Inversion of $\mathrm{W}$ will give the required weight fractions $\mathrm{C}_{j s}$. The system of equations (16) and (17) is not linear and must be solved by iterations starting from some approximate solution. Data on zero-contribution angles can be acquired from the Powder Diffraction File or by calculation of the powder pattern from known structural data.

Let us now return to the general case expressed in equation (15) and consider the difference between the observed intensity at any scattering angle $2 \theta_{i}$, $I_{s i}$, and the sum of contributions of the individual phases:

$$
\varepsilon_{s i}=\mathrm{I}_{s i}-\sum_{j=1}^{n} \mathrm{C}_{s j} \mathrm{~K}_{j i}
$$

Our problem may be formulated as the minimization of the sum of squares over all $m$ samples and over all $t$ scattering angles:

$$
\min \sum_{s=1}^{m} \sum_{i=1}^{t}\left(\mathrm{I}_{s i}-\sum_{j=1}^{n} \mathrm{C}_{s j} \mathrm{~K}_{j i}\right)^{2} .
$$

The weight fractions $\mathrm{C}_{s j}$ and the coefficients $\mathrm{K}_{j i}$ are considered to be parameters of the minimization, subjected to the constraints:

$$
\begin{gathered}
0 \leq \mathrm{C}_{s j} \leq 1 ; \mathrm{K}_{j i} \geq 0 ; \sum_{j} \mathrm{C}_{s j}=1 ; \\
1 \leq s \leq m ; 1 \leq j \leq n ; 1 \leq i \leq t .
\end{gathered}
$$

It is possible that the problems can be solved by iterations, starting from some approximate concentration data $\mathrm{C}_{s j}^{(1)}$ (Fiala, 1980; Zevin et. al., 1981). The procedure is repeated up to the point where the difference between the successive iterations $\mathrm{C}_{s j}^{(k)}-$ $\mathrm{C}_{s j}^{(k+1)}$ falls below the expected error. The greatest uncertainty lies in the set of initial concentrations $\mathrm{C}_{s j}^{(1)}$. In order to ensure convergence to correct concentrations, $\mathrm{C}_{s j}^{(1)}$ must be sufficiently close to the global minimum of the sum. There is always some probability of falling into one of the local minima, which might not be close to the correct phase composition. Approximate phase abundances can sometimes be obtained from elemental chemical data, supposing some ideal chemical composition of the phases. Another way is implementation of conventional X-ray techniques, with analytical standards, which do not exactly correspond to the analyzed phases, but can nevertheless be used for scaling and evaluation of the starting values $\mathrm{C}_{s j}^{(1)}$.

We have applied this technique for the analysis of Israeli phosphorites consisting of three major phases, dolomite, calcite and francolite (carbofluor apatite 
Table 4: Analysis of the major phases in phosphate rock

\begin{tabular}{|c|c|c|c|c|c|c|c|c|c|}
\hline \multirow[t]{2}{*}{ Sample } & \multicolumn{3}{|c|}{ Francolite, \% } & \multicolumn{3}{|c|}{ Calcite, $\%$} & \multicolumn{3}{|c|}{ Dolomite, $\%$} \\
\hline & $\begin{array}{r}\text { Starting } \\
\text { value } \\
C^{(1)}\end{array}$ & $\begin{array}{l}\text { X-ray } \\
\text { anal. }\end{array}$ & $\begin{array}{l}\text { Chem. } \\
\text { anal. }\end{array}$ & $\begin{array}{r}\text { Starting } \\
\text { value } \\
C^{(1)}\end{array}$ & $\begin{array}{l}\text { X-ray } \\
\text { anal. }\end{array}$ & $\begin{array}{l}\text { Chem. } \\
\text { anal. }\end{array}$ & $\begin{array}{r}\text { Starting } \\
\text { value } \\
\mathrm{C}^{(1)}\end{array}$ & $\begin{array}{r}\text { X-ray } \\
\text { anal. }\end{array}$ & $\begin{array}{l}\text { Chem. } \\
\text { anal. }\end{array}$ \\
\hline 1 & 40 & 42.0 & 43.5 & 30 & $\overline{35.2}$ & 36.9 & $\overline{30}$ & $\overline{21.8}$ & 18.5 \\
\hline 2 & 20 & 18.0 & 15.2 & 60 & 72.7 & 76.8 & 20 & 9.2 & 7.6 \\
\hline 3 & 70 & 68.4 & 69.7 & 30 & 29.2 & 27.5 & 0 & 1.8 & 1.9 \\
\hline 4 & 50 & 51.4 & 50.9 & 30 & 36.4 & 37.8 & 20 & 10.3 & 9.0 \\
\hline 5 & 80 & 81.8 & 82.5 & 0 & 11.7 & 12.4 & 20 & 6.3 & 7.0 \\
\hline 6 & 50 & 37.1 & - & 50 & 47.5 & - & 0 & 12.1 & - \\
\hline
\end{tabular}

$\left.\mathrm{Ca}_{10}\left(\mathrm{PO}_{4}\right)_{6-x}\left(\mathrm{CO}_{3}\right)_{x} \mathrm{~F}_{2}\right)$, and several minor phases, quartz, gypsum and organic matter. Since the concentration of organic matter was less than $1 \%$, it was ignored. The gypsum was removed by water treatment, and quartz was analyzed by conventional scaling against a pure quartz sample. The remaining three phases were analyzed by applying minimization procedure (19), as follows. Twelve samples with different proportions of phases were prepared from several samples of phosphate rock by size separation. The absorption correction was acquired by measurements of Compton scattering (Bar On et al., 1981). The integrated intensity was collected in the 12 scat-

Table 3: Angular intervals and contributing phases in analysis of phosphates. Phases are 1 - Francolite, 2 - Calcite, 3 - Dolomite, 4 - Quartz

\begin{tabular}{lll}
\hline $\begin{array}{l}\text { Sample } \\
\text { no. }\end{array}$ & $\begin{array}{c}\text { Angular range } \\
2 \theta, \text { degrees }\end{array}$ & Phases \\
\hline 1 & $21.0-23.6$ & $1+2$ \\
2 & $25.0-26.2$ & $1+2$ \\
3 & $26.2-27.0$ & 4 \\
4 & $28.5-30.0$ & $1+2$ \\
5 & $30.2-31.2$ & 3 \\
6 & $31.2-34.6$ & 1 \\
7 & $35.0-36.4$ & $1+2$ \\
8 & $39.6-41.5$ & $1+2+3+4$ \\
9 & $42.6-44.0$ & 1 \\
10 & $44.0-49.10$ & $1+2$ \\
11 & $49.1-50.2$ & 1 \\
12 & $50.2-53.5$ & $1+3$ \\
\hline
\end{tabular}

tering angle intervals shown in table 3 , together with an overlapping pattern. Initial concentrations were obtained by scaling against pure phases, using separate diffraction peaks. Bulk chemical analysis was carried out for five samples, and the phase composition was derived from chemical data, taking into account $\mathrm{PO}_{4} \rightarrow \mathrm{CO}_{3}$ substitution in francolite (Zevin et al. 1988). Only a few iterations were required to reach the final results given in table 4 for some of the analyzed samples. We found that the minimization converged to a composition very close to that derived from bulk chemistry even, in the cases when the initial concentration was rather far from true values.

Two full-pattern approaches used in quantitative phase analysis, i.e., pattern decomposition and the continuous pattern approach, resemble two similar techniques applied in structure refinement from powder diffraction data. In the first approach, conventional "single crystal" methods of structure analysis are implemented after pattern decomposition into separate diffraction peaks. The second approach devised by Rietveld seems to be used overwhelmingly in structural studies, but the first one is more attractive in the standardless quantification techniques discussed here. Indeed, weak peaks that are difficult to separate have as much bearing in structural analysis as the strong ones. In the quantification procedure, on the other hand, weak peaks are less suitable for analysis, and greater weight is given to strong peaks, which can be separated with better accuracy. The continuous pattern approach, expressed in equations (15), is certainly overburdened with a large number $(n \times t)$ of $\mathrm{K}_{j i}$ constants, thus increasing the chance falling into a false minimum. In pattern decomposition the overlapping problem is treated independently of quantification, conferring an advantage on this technique. Unfortunately, very little has been done in experimental comparison of these two approaches, though full-pattern utilization must be the main path to further development and hopes for standardless quantification.

\section{Number of phases in analyzed samples}

Although the number and identity of the phases present in an analyzed sample are obtained by wellestablished manual or automatic identification procedures, it is sometimes useful to apply an independent 
check. Bezjak (1975) noted that if a set of $m$ samples each contains no more than $n<m$ identical phases, the intensity diffracted by any sample $s$ at any scattering angle $2 \theta_{i}$ is a linear combination of intensities diffracted at the same angle by $n$ other samples. The number of phases may be established applying these criteria. Relatively simple and easily programmable mathematical tests (factor analysis) are available for this purpose.

The number of factors (phases in our case) responsible for the variability of the intensities in the $\mathrm{I}_{s i}$ matrix is equal to the minimum number of linearly independent rows or columns, or to the matrix rank. Technically, it is simpler to determine the rank of symmetrical square $(m \times m$ or $t \times t)$ matrices $\mathbf{R}^{\prime}=\mathbf{I I}^{T}$ or $\mathbf{R}^{\prime \prime}=\mathbf{I}^{T} \mathrm{I}$, which is equal to the rank of the original matrix $\mathbf{I}$. The rank of $\mathbf{R}$ matrices is equal to the number of non-zero rows in the reduced triangular form (Wallace, 1960; Wallace \& Katz, 1969) and to the number of non-zero eigenvalues (Ritter et al., 1976).

Due to inevitable errors in intensities, which contribute to the variability of the $\mathrm{I}_{s i}$ matrix, its rank will be equal to $m$ or $n$, whichever is smaller. This is why when we separate major factors (phases) that affect the variability $\mathrm{I}_{s i}$ from insignificant factors caused by experimental errors, we have to take the latter into account. An element of the $\mathbf{R}$ matrix is expressed as:

$$
\mathrm{R}_{j k}=\sum_{i=1}^{t} \mathrm{I}_{i j} \mathrm{I}_{i k},
$$

and the standard deviation $\sigma\left(\mathrm{R}_{j k}\right)$ is obtained by the general rules of error propagation. As a rough estimate, suppose that the mean element of the $\mathbf{R}$ matrix $\langle\mathrm{R}\rangle \approx m\langle\mathrm{I}\rangle^{2}$, where $\langle\mathrm{I}\rangle$ is the mean intensity in the data matrix, and $m$ is the dimensionality of the $\mathrm{R}$ matrix. Then $\sigma(\langle\mathrm{R}\rangle) \approx 2 m\langle\mathrm{I}\rangle \sigma(\langle\mathrm{I}\rangle)=2\langle\mathrm{R}\rangle \varepsilon(\langle\mathrm{I}\rangle)$, where $\varepsilon(\langle\mathrm{I}\rangle)$ is the relative error in the intensity measurements, which can often be supposed to be a constant. The $\mathbf{R}$ matrix is reconstructed from eigenvectors $\mathbf{x}_{i}$ and eigenvalues $\lambda_{i}$. If there are $r$ non-zero eigenvectors, then

$$
\mathbf{R}^{r}=\sum_{i=1}^{r} \mathbf{x}_{i} \lambda_{i} \mathbf{x}_{i}^{T},
$$

where the superscript $r$ in $\mathbf{R}^{r}$ indicates that reconstruction was carried out with the full number of non-zero eigenvectors. Now suppose that only the first $h$ eigenvectors of the full row of $r$ eigenvectors, arranged in order of decreasing eigenvalues, correspond to statistically significant factors. Then the $\mathbf{R}^{h}$ matrix reconstructed by summation over only the first $h$ eigenvectors will be statistically equivalent to the original $\mathbf{R}$ matrix. If $n$ is the number of detected phases and $h>n$, then $h-n$ phases were undetected.

We have applied this test to the $13 \times 7 \mathrm{I}_{s i}$ matrix given in Table 1. Eigenvalues calculated for $\mathbf{R}_{7 \times 7}=$ $\mathbf{I}^{T} \mathbf{I}$ are shown graphically in figure 1 . The steep drop in eigenvalues from $\lambda_{4}$ to $\lambda_{5}$ emphasizes the presence of four major factors, in our case the four phases. The $\mathbf{R}^{4}$ matrix reconstructed from the first four eigenvectors is statistically indistinguishable from the original matrix.

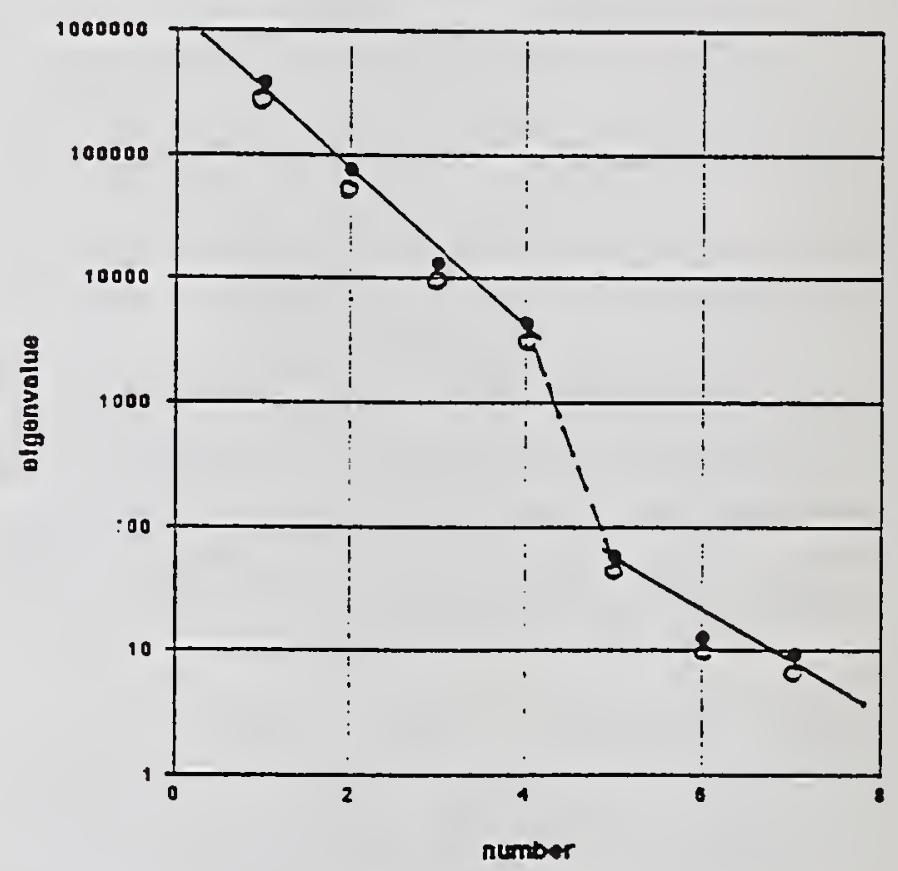

Figure 1: Eigenvalues of the product matrix $\mathbf{R}=$ II $^{T}$. - Nonvariant set of 134 -phase samples. Amorphous phase with maximal abundance of $30 \%$ is added to 8 of 13 samples.

This technique will not, however, reveal an amorphous phase that might otherwise go undetected. The amorphous phase will dilute the sample and reduce the intensities of all the crystalline phases in the sample, but it does not change the rank of the intensity matrix. Various amounts of amorphous phase were added artificially to 8 of 13 four-phase samples with the intensity matrix shown in table 1 . The abundance of the amorphous phase varied from $5 \%$ to $30 \%$. The character of the eigenvalues' distribution did not change (Fig. 1), emphasizing the presence of the same four crystalline phases. 


\section{Precision of the standardless technique}

Three factors affect the precision of the standardless technique:

\section{i. Intensity errors,}

ii. Uncertainty in the mass balance equation (4), and

iii. Diverse phase compositions in the samples.

It turns out that all three factors can be brought together in one compact formulation. Suppose the total weight fraction of all the detected phases in sample $s$ is equal to $b_{s} \leq 1$. Then a more general form of equation (6) is:

$$
\sum_{j} \mathrm{I}_{s j} \alpha_{j}=\mathrm{b}_{j}, s=1,2, \ldots, m .
$$

Estimation of the error in the elements of the $\alpha_{j}$ vector is given by Zevin \& Zevin (1989).

$$
\frac{\delta \alpha}{\alpha} \leq \mathrm{C}(\mathrm{I})\left[\frac{\varepsilon+\Delta \mathrm{b}_{s} / \sqrt{m}}{1-\varepsilon}\right]
$$

where $\varepsilon=\delta \mathrm{I} / \mathrm{I}$ is the relative error in intensity measurements, which is supposed to be constant through all $\mathrm{I}_{s i}, \Delta \mathrm{b}_{s}=\sqrt{\sum_{s}\left\langle\delta \mathrm{b}_{s}\right\rangle^{2}}$ is the deviation of the actual sum of the weight fractions from $1, m$ is the number of samples and $\mathrm{C}(\mathrm{I})$ is the condition number of matrix I, defined as:

$$
\mathrm{C}(\mathrm{I})=\sqrt{\lambda_{m}\left(\mathbf{I}^{T} \mathbf{I}\right) \times \lambda_{m}\left(\left[\mathbf{I}^{T} \mathbf{I}\right]^{-1}\right)},
$$

where $\lambda_{m}\left(\mathbf{I}^{T} \mathbf{I}\right)$ is the maximal eigenvalue of product matrix $\mathbf{I}^{T} \mathbf{I}$.

Three sources of error noted in the beginning are clearly separated in equation (24) with condition $\mathrm{C}(\mathrm{I})$ being responsible for the sample's diversity. Condition $\mathrm{C}(\mathrm{I})$ takes its minimal value of $\sqrt{(n-1) / n}(\approx 1$ for large $n)$ in the case of the most diverse samples, when we have one analyzed sample and $n-1$ pure phases (analytical standards) [equation (5)]. Substitution for the pure phases by unknown samples inevitably reduces the diversity of the sample set, increases the condition number $\mathrm{C}(\mathrm{I})$, and increases the analytical error.

Equation (24) provides us with an excellent tool for checking the "goodness" of the sample set. The following example confirms this premise. The experimental data presented in table 1 were used to calculate the "true" calibration constants $\left(\mathrm{K}_{j o}\right)$ as slopes of regression $\mathrm{I}_{s j}$ against true phase abundances $\mathrm{C}_{j}$. Subsets of four or more samples were chosen at random from the general group of 13 samples, and for each subset calibration constants $K_{j}$ were calculated from equation (7). The relative error in the calibration constants, $\delta \mathrm{K} / \mathrm{K}=\sum_{j}\left|\mathrm{~K}_{j}-\mathrm{K}_{j o}\right| / \mathrm{K}_{j o}$ was plotted against the condition number of the intensity matrix for the corresponding subset (Fig. 2). The correlation of the analytical error with the condition number is obvious. The value $\delta \mathrm{K} / \mathrm{K}$ remains within

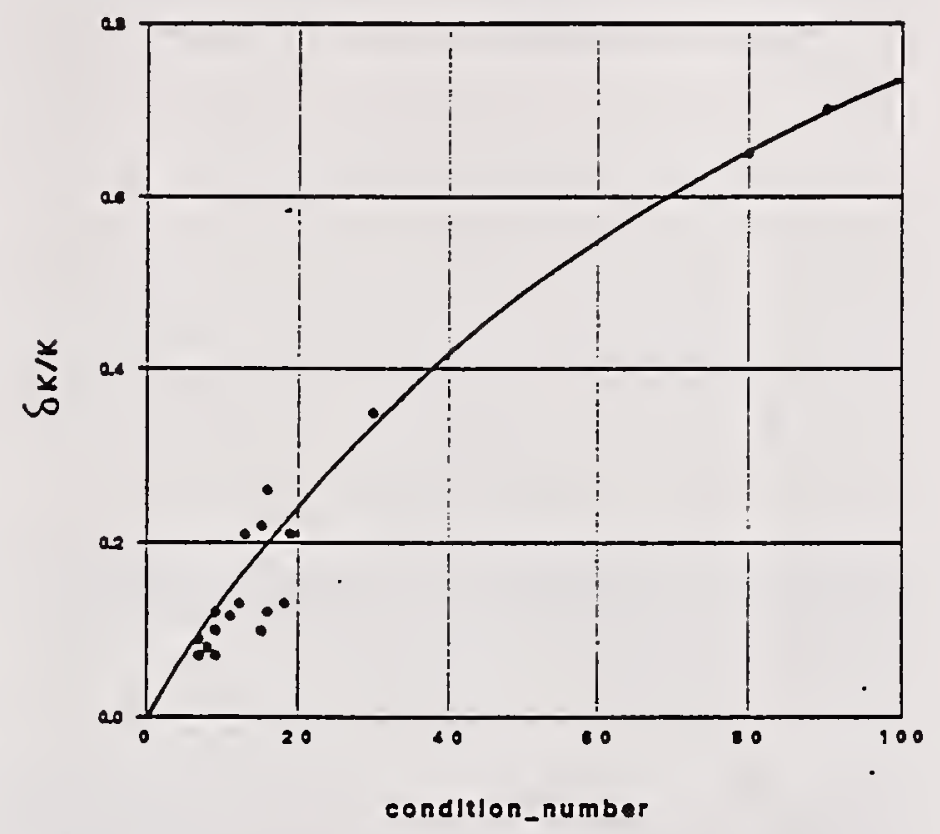

Figure 2: Precision of analysis as a function of the condition number of the intensity matrix.

the reasonable $10 \%$ range if the condition number does not exceed 10. The presence of an undetected (amorphous) phase was simulated by reducing the intensities in randomly chosen rows of the intensity matrix in table 1 . This resulted in an dramatic increase of the analysis error. The value of $\delta \mathrm{K} / \mathrm{K}$ increased from $\sim 0.09$ to an unacceptably high value of 0.18 when the presence of an amorphous phase with a maximum abundance of $>30 \%$ was simulated in 8 of 13 samples.

Intensity errors affect the results of standardless analysis to a lesser degree than variability of the samples. We can expect, however, that at an approximately constant condition number, the precision of the analysis will improve with increasing number of samples due to increased precision in intensities. An example is given in figure 3 . The initial set of four samples was well conditioned, with condition number $\sim 10$. Additional samples added subsequent to the initial set did not improve the condition number, but contributed to intensity precision and improved the results of analysis.

If the diversity of the sample set is unsatisfactory, it can be improved by using various materialhandling procedures known from mineral processing. They include size separation by sifting, gravity sepa- 


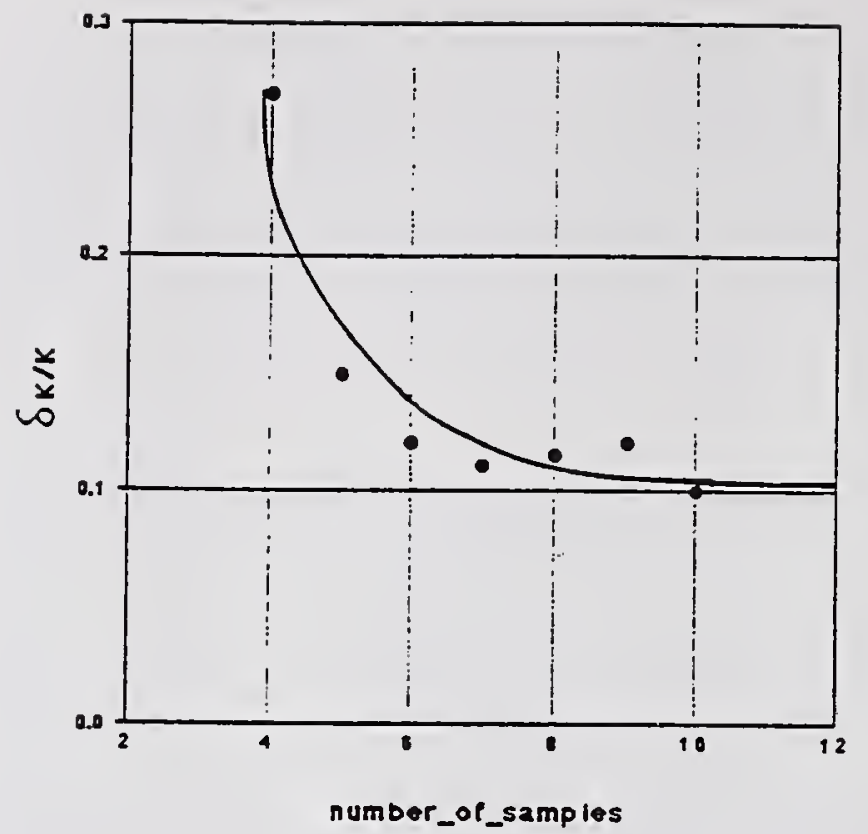

Figure 3: Improvement of precision of analysis by subsequent addition of samples to the well conditioned initial set.

ration by sedimentation in various liquids, magnetic separation, flotation, chemical treatment, etc. These procedures are usually selective for certain phases and produce samples with different proportions of the same phases.

\section{Conclusions}

Despite their long history standardless techniques have not yet found adequate application. Two-phase systems, such as ordered and disordered phases in polymers, are the sole object of its practical utilization. This can be partially explained by the lack of theoretical development and full understanding of the sources of main problems, which sometimes result in confusing outputs. It seems that by now the method has been more or less developed, including requirements to the initial group of samples and intensity precision. We can now outline the fields and modes of its application. It does, however require thorough selection and very often special preparation of the sample group used to evaluate calibration constants. The number of samples in this group must exceed the number of phases, and compositions of samples must themselves be compositionally variable. As soon as scaling has been achieved with a prepared sample group, and calibration (scaling) coefficients have been evaluated, analysis of other samples, composed of the same phases in different proportions, may be carried out by conventional techniques of phase quantification. Thus, the standardless meth- ods discussed here can be considered as methods for calibration or scaling of intensities, a necessary step in phase quantification.

Taking into account the labor and skill required for initial preparations, the standardless technique is suitable for large-scale analysis when many samples must be analyzed, except for the initial group. It is certainly unsuitable for sporadic analysis of a few samples. One cannot expect exclusive precision for the standardless technique due to the multiple intensity measurements needed for evaluation of single weight fraction. The virtues of this method lie in its potential accuracy, based on its self-calibrating ability.

\section{References}

Bar-ON, P., Zevin, L. \& LACH, Sh. (1981). X-ray Spectrom., 10, 57-60.

BezJaK A. (1975). Anal Chem. 47, 790-793.

Braun G. E. (1986). J. Appl. Cryst. 19, 217-221.

Driz, V. A. \& SaliN, A. L. (1972). Int. Clay Confer. Proceedings. 577-588, Madrid.

Fang J. H. \& Zevin, L. (1985). J. Sedim. Petrol. 55, 611-613.

Fiala, J. (1980). Anal. Chem. 52, 1300-1304.

Hermans P. H. \& Weidinger A. (1948). J. Appl. Phys. 19, 491-506.

Madsen, I. C. \& HiLl, R. J. (1990). Powd. Diffr. 5, 195-199.

Moore, C. A. (1968). Clays and Clay Minerals 16, 325336.

Parrish, W., Huang, T. C. \& Ayers, G. L. (1976). Trans. Amer. Cryst. Assoc. 12, 55-73.

RitTer, G. L., LOWRY, S. R., IsENHOUR, T. L. \& WiLkINS, C. L. (1976). Anal. Chem. 48, 591-595.

SMith, D. K., Johnson, G. G. \& WIMs, A. M. (1988). Austr. J. Phys. 41, 311-321.

Wallace, R. M. (1960). J. Phys. Chem. 64, 899-901.

Wallace, R. M. \& KatZ, S. M. (1964). J. Phys. Chem. 68, 3890-3892.

Will, G., Parrish, W. \& Huang, T. C. (1983). J. Appl. Cryst. 16, 611-622.

Zavyalova, L. L. (1970). Dissertation. Irkutsk Institute of Rare Metals, Russia.

ZeVIN, L. S. (1977). J. Appl. Cryst. 10, 147-150.

ZEVIN, L. S. Zavyalova L. L. (1974). Quantitative $X$ ray Phase Analysis, Nedra.

Zevin, L. LaCh S., Levi Y. \& Pregerson B. (1988). Int. J. Min. Process. 24, 235-245.

Zevin, L. \& Messalem, A. (1982). Polymer. 23, 601604.

ZEVIN, L. S., SHNECK, R. \& ZEVIN, I. M. (1981). Twelfth Intern. Congr. of Crystal. Collected Abstracts C-280-281.

Zevin, L. S. \& Zevin Sh. L. (1989). Powder Diffraction 4, 196-200. 
National Institute of Standards and Technology Special Publication 846. Proceedings of the international conference Accuracy in Powder Diffraction II, held at NIST, Gaithersburg, MD, May 26-29, 1992. (Issued October 1992)

\title{
Reference Intensity Ratios, Whole Pattern Fitting and Standardless X-ray Quantitative Analysis
}

\author{
ROBERT L. SNYDER \\ Institute for Ceramic Superconductivity, New York College of Ceramics at Alfred University \\ Alfred, NY 14802 USA
}

\begin{abstract}
A consistent set of notation is developed for each of the $R I R$ based methods for carrying out quantitative analysis by $\mathrm{X}$-ray powder diffraction. The so called "standardless" analysis procedures are shown to be a special case of the internal-standard method of analysis where the normalizing assumption is used. All analytical methods, other than whole pattern matching procedures, require the use of explicitly measured standards, typically in the form of RIR values; however, if only semi-quantitative results can be tolerated, the standards may be obtained by using published $R I R$ and relative intensity values. The exciting new techniques of whole pattern fitting and Rietveld constrained quantitative analysis are also described in $R I R$ notation and shown also to be the internal-standard method with the normalization assumption. The quantitative results obtained from Rietveld analysis are derived from computed standards in the from of computed, normalized, $R I R_{N}$ values. The normalization assumption in Rietveld analysis allows the exclusive use of computed standards and comes as close to a "standardless" analysis as one can achieve: completely relying on the absence of amorphous material and on the validity of the structural models. Relationships are given for obtaining quantitative analysis from the $R I R_{N}$ values obtainable from the least squares scale factors in the Rietveld and pattern matching procedures.
\end{abstract}

\section{Introduction}

The internal-standard method is the most general of any of the methods for quantitative phase analysis by X-ray powder diffraction. In recent years this method has been generalized and recast into Reference Intensity Ratio $(R I R)$ notation (see for example, Hubbard \& Snyder, 1988, and Snyder \& Bish, 1989). Previous to these developments
Bezjak (1961), Karlak \& Burnett (1966) and later Chung (1974a,b) introduced a misnamed "standardless" analysis procedure which Chung called the "adiabatic principle" or "matrix flushing method" which uses the $R I R$ concept, but not the notation. This method which we prefer to call the Normalized $R I R$ Method still relies on the $R I R$ values as standards. More recently (Snyder et al., 1981, 1982; Snyder \& Hubbard, 1984) the AUTO/NBS*QUANT84 system implemented the internal-standard method with Reference Intensity Ratio notation in addition to the normalized $R I R$ method. This system also implemented the use of overlapped lines and chemical constraints via an $R I R$ restatement of the Copland \& Bragg (1958) procedure. D. K. Smith and colleagues $(1986,1987,1988,1989)$ have described the use of the full pattern to even further enhance quantitative XRD analysis. The most recent developments have been the introduction of structural constraints while using the whole pattern via Rietveld analysis of multi-phase mixtures (Hill \& Howard, 1987, Bish \& Howard, 1988)

The purpose of this paper is to follow-up the Hubbard \& Snyder (1988) paper and develop a consistent set of notation to describe each of the $R I R$ based techniques in terms of this notation. It will also address the relatively recent misuse of the term "standardless analysis": the normalized internal-standard, whole pattern matching and Rietveld constrained methods, will all be shown to be similar forms of the internal-standard method, requiring the normalizing assumption that the sum of the crystalline phases contributing to the diffraction lines be unity. In addition, they must rely on standards in the form of previously measured $R I R$ values or crystal structure parameters.

\section{The internal standard method}

The intensity of a diffraction line $i$ from a pure phase 
$\alpha$ in the form of a flat plate on a powder diffractometer using Bragg-Brentano geometry is given by:

$$
I_{i \alpha}=\frac{K_{e} K_{i \alpha}}{\mu} .
$$

The intensity of a diffraction line $i$ from phase $\alpha$ in a mixture of phases is given by (Klug \& Alexander, 1974):

$$
I_{i \alpha}=\frac{K_{e} K_{i \alpha} X_{\alpha}}{\rho_{\alpha}\left(\frac{\mu}{\rho}\right)_{m}}
$$

where

- $\mu=$ the linear absorption coefficient,

- $\mathrm{X}_{\alpha}=$ weight fraction of phase $\alpha$,

- $\rho_{\alpha}=$ density of phase $\alpha$.

The mass absorption (i.e., mass attenuation) coefficient of the mixture is,

$$
\left(\frac{\mu}{\rho}\right)_{m}=\sum_{j=1}^{\# \text { of phases }} X_{j}\left(\frac{\mu}{\rho}\right)_{j} .
$$

$K_{e}$ is a constant for a particular experimental system,

$$
K_{e}=\frac{I_{0} \lambda^{3}}{64 \pi r} \cos ^{2} 2 \theta_{m}\left(\frac{e^{2}}{m_{e} c^{2}}\right)^{2}
$$

where

- $I_{0}=$ incident-beam intensity,

- $r=$ radius of the diffractometer,

- $\lambda=$ wavelength of the X-radiation,

- $\left(\frac{e^{2}}{m_{e} c^{2}}\right)^{2}$ is an electromagnetic radiation term required for $\mathrm{X}$-rays and omitted for neutrons,

- $c=$ speed of light,

- $e$ and $m_{e}=$ charge and mass of an electron,

- $2 \theta_{m}=$ the diffraction angle of the monochromator.

$K_{i \alpha}$ is a constant for each diffraction reflection $i$ from the crystal structure of phase $\alpha$,

$$
K_{i \alpha}=\frac{M_{i}}{V_{\alpha}^{2}}\left|F_{i \alpha}\right|^{2}\left(\frac{1+\cos ^{2} 2 \theta}{\sin ^{2} \theta \cos \theta}\right)_{i}
$$

where
- $M_{i}=$ Multiplicity for reflection $i$,

- $V_{\alpha}=$ volume of the unit cell of phase $\alpha$,

- $\left(\frac{1+\cos ^{2} 2 \theta}{\sin ^{2} \theta \cos \theta}\right)_{i}=$ the Lorentz and polarization corrections for the diffractometer $\left(L p_{i}\right)$,

- $F_{i \alpha}=$ the structure factor for reflection $i$, which relates the intensity to the crystal structure parameters:

$$
F_{h k \ell}=\sum_{j=1}^{\# \text { of atoms in cell }} f_{j} \exp 2 \pi i\left(h x_{j}+k y_{j}+\ell z_{j}\right)
$$

where

- $h k \ell=$ Miller indices of line $i$ of phase $\alpha$,

- $x_{j}, y_{j}, z_{j}=$ fractional coordinates for atom $j$,

- $f_{j}=$ the atomic scattering factor for atom $j$ :

$$
f_{j}=f_{c o r r} \exp -\frac{B_{j} \sin ^{2} \theta}{\lambda^{2}}
$$

where

- $f_{\text {corr }}=$ The atomic scattering factor corrected for anomalous dispersion namely:

$$
\left|f_{\text {corr }}\right|^{2}=\left(f_{0}+\Delta f^{\prime}\right)^{2}+\left(\Delta f^{\prime \prime}\right)^{2}
$$

where

- $B_{j}=$ Debye-Waller temperature factor for atom $i$ in phase $\alpha$,

- $\theta=$ Bragg diffraction angle.

The fundamental problem in quantitative analysis arises from the $\left(\frac{\mu}{\rho}\right)_{m}$ term in equation 2 . To solve for the weight fraction of phase $\alpha$ we must be able to compute $\left(\frac{\mu}{\rho}\right)_{m}$ and this in turn requires knowledge of the weight fractions of each phase. This cyclic argument can be stated in mathematical terms as the system being underdetermined. The internal-standard method is based on the elimination of the absorption factor $\left(\frac{\mu}{\rho}\right)_{m}$ by dividing two equations of type 2 giving equation 9 , which is linear in the weight fraction of phase $\alpha$ :

$$
\frac{I_{i \alpha}}{I_{j s}}=k \frac{X_{\alpha}}{X_{s}}
$$

Equation 9 is linear and forms the basis of the internal-standard method of analysis. The addition 
of a known amount, $\left(X_{s}\right)$, of an internal-standard to a mixture of phases, which may include amorphous material, permits quantitative analysis of each of the components of the mixture by first establishing the values of $\mathrm{k}$ for each phase, (i.e. the slope of the internal-standard plot of equation 9) from standards of known concentration.

\section{$I / I_{\text {corundum }}$}

The slope, $k$, of the plot of

$$
X_{s} \frac{I_{i \alpha}}{I_{j s}} v s . X_{\alpha}
$$

is a measure of the inherent diffracted intensities of the two phases. Visser \& DeWolff (1966) were the first to propose that $\mathrm{k}$ values could be published as materials constants if the concentration and the diffraction lines of the phases $\alpha$ and $s$ were specified and $s$ itself were agreed to by all researchers. Their proposal was to use corundum as the universal internal standard and to use the $100 \%$ lines of phase $\alpha$ and corundum mixed in a $50 \%$ by weight mixture. This $I / I_{\text {corundum }}$ or $I / I_{c}$ value has been widely accepted and over 4,400 are published with patterns for standards in the Powder Diffraction File of the International Centre for Diffraction data.

\section{The generalized Reference Intensity Ratio}

The concept of the $I / I_{\text {corundum }}$ value as a materials constant leads naturally to a broader definition which permits the use of reference phases other than corundum, more than just the $100 \%$ relative intensity lines and arbitrary concentrations of the two phases $\alpha$ and $s$. The idea of the $R I R$ has developed in the literature over the last 15 years (Chung, 1974a,b; Hubbard et al., 1976). The most general definition of it (Snyder \& Hubbard, 1984; Hubbard \& Snyder, 1988) is:

$$
R I R_{\alpha, s}=\frac{I_{i \alpha}}{I_{j s}} \frac{I_{j s}^{r e l}}{I_{i \alpha}^{r e l}} \frac{X_{s}}{X_{\alpha}}
$$

where $I_{j}^{r e l}$ is the relative intensity of line $\mathrm{j}$ scaled to the $100 \%$ line. In equation 10 we see that the $R I R$ is a function of $\alpha$ and $s$ only, not of $i, j$, or $X_{s}$. It, of course, remains the slope of the calibration curve for phase $\alpha$ with internal-standard $s$ but now has been scaled so that it may be computed from any pair of diffraction lines in a calibration mixture. We also see that $I / I_{c}$ values are simply $R I R$ values where $s$ is corundum. The $R I R$, when accurately measured for two well crystallized and prepared specimens, is a true constant and allows comparison of the absolute diffraction line intensities of one material to another. It also enables quantitative phase analysis in a number of convenient and useful ways.

The $R I R$ value in equation 10 may be obtained by careful calibration, in the traditional way that the slope of the internal-standard curve would be determined, or it may be derived from other $R I R$ values via,

$$
R I R_{\alpha, s}=\frac{R I R_{\alpha, s^{\prime}}}{R I R_{s, s^{\prime}}}
$$

where $s^{\prime}$ is any common reference phase. When $s^{\prime}$ is corundum, the $R I R_{\alpha, s^{\prime}}$ values are simply $I / I_{c}$ values. Hence, we can combine $I / I_{c}$ values for phases $\alpha$ and $s$ to obtain the Reference Intensity Ratio for phase $\alpha$ relative to phase $s$.

It is useful to express the generalized $R I R$ in terms of the fundamental constant $K_{i \alpha}$. This can be done by combining equation 2 with 10 to give

$$
R I R_{\alpha, s}=\frac{K_{i \alpha}}{K_{j s}} \frac{\rho_{s}}{\rho_{\alpha}} \frac{I_{j s}^{r e l}}{I_{i \alpha}^{r e l}} .
$$

The RIR may also be obtained from pure standards run under the same conditions by combining equations 2 with 12

$$
R I R_{\alpha, s}=\frac{I_{i \alpha}^{\circ}}{I_{j s}^{\circ}} \frac{\left(\frac{\mu}{\rho}\right)_{\alpha}}{\left(\frac{\mu}{\rho}\right)_{s}} \frac{I_{j s}^{r e l}}{I_{i \alpha}^{r e l}},
$$

where $I_{i}^{\circ}$ is the intensity of line $\mathrm{i}$ from a pure phase.

\section{Measurement of RIRs}

The simple 2-line procedure for measuring $I / I_{c}$ is quick but suffers from several drawbacks. Preferred orientation commonly affects the observed intensities unless careful sample preparation methods, such as spray drying, (Cline \& Snyder, 1983, 1985) are employed. Other problems include extinction and microabsorption (Cline \& Snyder, 1987), inhomogeneity of mixing, and variable crystallinity of the sample due to its synthesis, history and possible solid solution (Gehringer et al., 1983). Even the problem of having a representative number of crystallites in diffracting position is more serious than one would at first presume: Smith (1992) has recently expanded the classic particle statistics study of Alexander et al. (1948) showing that, even for a relatively low absorbing quartz sample diffracting $\mathrm{CuK}_{\alpha}$, crystallite sizes of less than $1.0 \mu$ are required to achieve a $1 \%$ 
relative error at a $2.3 \sigma$ confidence. All of these effects conspire to make the published values of $I / I_{c}$ subject to substantial error.

For greater accuracy Hubbard \& Smith (1976) recommended using multiple lines from both the sample $(\alpha)$ and the reference phase (corundum, in this case) and using multiple sample mountings. Such an approach often reveals when preferred orientation is present and provides realistic assessment of the reproducibility in the measurement of $I / I_{c}$. Traditional implementations of the internalstandard method usually use a single diffraction line from each of the phases to be analyzed. The AUTO/NBS*QUANT84 (Snyder et al. 1981, 1982; Snyder \& Hubbard, 1984) system implemented the use of multiple lines from each phase to improve statistics and help average out orientation errors. More importantly, this system rigorously propagated counting and statistical errors to evaluate their influence on the final analysis results.

Although comparison of the intensities in the observed pattern to those in a computed pattern will allow a quantitative measure of the amount of preferred orientation normal to the sample surface (Snyder \& Carr, 1974), it is never recommended to use a computational procedure in place of proper experimental technique. In general spray drying the samples (Smith et al., 1979a,b; Cline \& Snyder, 1985) is the best way to eliminate this serious source of error. In the spray-drying process the crystallites of the powder sample are agglomerated into spheres. The crystallites spread on the surface of these spheres expose all crystallographic surfaces to the X-ray beam. Effects of agglomerate inhomogeneity and microabsorption have been shown to be minimal (Cline \& Snyder, 1985). Spray dried powders have the disadvantage of acting like a somewhat viscous liquid but this is offset by their ability to fill a sample holder producing an X-ray infinite thick mount. Other techniques which minimize orientation, like powder on tape, air dispersions captured in filter paper, or powder dusted into grease on a zero background holder, introduce a $2 \theta$ dependent sample transparency error, due to the thin layer of diffracting specimen, which invalidates the general $R I R$ equation 10 .

\section{Quantitative analysis with $R I R s$}

The internal-standard method of quantitative analysis when stated in terms of an RIR comes from rearranging Equation 10 (Hubbard \& Snyder 1988),

$$
X_{\alpha}=\frac{I_{i \alpha}}{I_{j s}} \frac{I_{j s}^{r e l}}{I_{i \alpha}^{r e l}} \frac{X_{s}}{R I R_{\alpha, s}} .
$$

Taking $s$ to be an internal standard and substituting equation 11 into Equation 14 and rearranging we have

$$
X_{\alpha}=\frac{I_{i \alpha}}{I_{j s}} \frac{I_{j s}^{r e l}}{I_{i \alpha}^{r e l}} \frac{R I R_{s, c}}{R I R_{\alpha, c}} X_{s} .
$$

This equation is quite general and allows for the analysis of any crystalline phase with a known $R I R$ in an unknown mixture by the addition of a known amount of any internal standard. However, if all four of the required constants $\left(I_{i \alpha}^{\text {rel }}, I_{j s}^{\text {rel }}, R I R_{s, c}\right.$ and $\left.R I R_{\alpha, c}\right)$ are taken from the literature the results should be considered as only semi-quantitative, since each of them may contain significant error when compared to the actual phases in a particular unknown. Of course, the RIRs may also be calculated (Hubbard et al., 1976) when the crystal structures of the two phases are known. However, the calculated values show considerable variation from observed values depending on assumptions in the computational models. In addition the computational model cannot allow for specific specimen conditions like poor crystallinity, microabsorption, extinction and preferred orientation. In general, if the RIRs must be calculated the analysis should also be considered only semi-quantitative. These comments also apply to the Rietveld whole-pattern fitting method described below.

Equation 14 is valid even for complex mixtures which contain unidentified phases, amorphous phases, or identified phases with unknown RIRs. It, in fact, is simply a restatement of the internalstandard method in $R I R$ notation.

\section{The normalized RIR method}

Hubbard \& Snyder (1988) showed that the ratio of the weight fractions of any two phases whose RIRs, with respect to some reference $s$, are known may always be computed from

$$
\frac{X_{\alpha}}{X_{\beta}}=\frac{I_{i \alpha}}{I_{j \beta}} \frac{I_{j \beta}^{r e l}}{I_{i \alpha}^{r e l}} \frac{R I R_{\beta, s}}{R I R_{\alpha, s}} .
$$

If the $R I R$ values for all $\mathrm{n}$ phases in a mixture are known, then $n-1$ equations of type 16 may be written. It makes no difference if the reference phase, $s$, is one of the $\mathrm{n}$ phases being analyzed or if it is arbitrary like corundum. Bezjak (1961), Karlak \& Burnett (1966) 
and later Chung (1974a,b; 1975) pointed out that if no amorphous phases are present an additional equation holds:

$$
\sum_{k=1}^{n} X_{k}=1
$$

This normalization equation permits analysis, without the addition of any standard to the unknown specimen, by allowing us to write a system of $n$ equations and solve for the $\mathrm{n}$ weight fractions via (Hubbard \& Snyder 1988)

$$
X_{\alpha}=\frac{I_{i \alpha}}{R I R_{\alpha} I_{i \alpha}^{r e l}}\left[\sum_{k=1}^{\# \text { of phases }} \frac{I_{j, k}}{R I R_{k} I_{j, k}^{r e l}}\right]^{-1}
$$

Chung referred to the use of equation 17 as the matrix flushing or the adiabatic principle. The term "Standardless Analysis" is coming into frequent misuse or at least misleading use. Recent papers on the $R I R$ internal-standard method and its implementation in the whole pattern matching techniques have used the phrase "standardless" in ways that have caused some researchers to believe that the treatment of references, implicit in the $R I R$ and normalized $R I R$ approaches, are no longer required for quantitative analysis. In fact, standards are still required in all truly quantitative $\mathrm{X}$-ray analysis procedures, and the quality of the assumptions which go into the computation and measurement of $R I R s$ or their related pattern matching scale factors, along with the assumption that no amorphous phases are present, are often so poor as to make the method semi-quantitative at best.

It is important to emphasize that the presence of any amorphous or unidentified crystalline phase invalidates the use of equation 18 . All crystallites have some degree of amorphous character on their surfaces, due to the broken bonds in the surface. In some materials this amorphous layer penetrates quite deeply and produces a substantial concentration of non-diffracting or diffusely diffracting material, invalidating the normalization assumption. This effect is particularly important when calculated $R I R s$

are used, in that the computational model cannot allow for the effect of the bulk material's transformation into the amorphous layer. The loss of crystallinity in the surface of the crystallites, where the incident X-ray beam is the least absorbed, will strongly distort the $R I R$ from an ideal calculated value. Of course, any sample containing unidentified phases cannot be analyzed using the normalization assumption in that the required $R I R s$ will not be known.

When the $R I R$ values are known from another source, for example published $I / I_{c}$, or computed values, it may be tempting to use them along with the $\mathrm{I}^{r e l}$ values on the PDF card to perform a completely (incorrectly called), "standardless" quantitative analysis using the normalization equation 17 . These $I^{r e l}$ and $I / I_{c}$ values are seldom accurate enough to be used directly in quantitative analysis. The analyst should accurately determine the relative intensities and $R I R$ values for an analysis by careful calibration measurements from materials similar to those observed in the specimens to be analyzed. It needs to be stated that this method is far from standardless. The essential standard information is simply being obtained from the literature or computations, adding substantial uncertainty to any derived semi-quantitative results.

\section{Constrained XRD phase analysis: the generalized internal standard method}

A quantitative analysis combining both the $\mathrm{X}$-ray diffraction and chemical or thermal results with a knowledge of the composition of the individual phases can yield results of higher precision and accuracy than is generally possible with only one kind of observation. The analysis becomes more complex when several phases in a mixture have similar compositions and/or potential compositional variability, but it is possible, with appropriate constraints during analysis, to place limits on the actual compositions of the constituent phases.

The most general formulation of these ideas has been described by Copeland \& Bragg (1958). Their internal-standard equations for multicomponent quantitative analysis with possible line superposition and chemical constraints take the form of a system of simultaneous linear equations; each equation refers to the individual phase contributions to an integrated intensity over some $2 \theta$ range. In terms of $R I R$ values each equation is of the form

$$
\begin{array}{r}
\frac{I_{1}}{I_{j s}}=\left(\frac{I_{1 \alpha}^{r e l}}{I_{j s}^{r e l}} R I R_{\alpha, s}\right) \frac{X_{\alpha}}{X_{s}}+ \\
\quad\left(\frac{I_{1}^{r e l}}{I_{j s}^{r e l}} R I R_{\beta, s}\right) \frac{X_{\beta}}{X_{s}}+\ldots \\
+\left(\frac{I_{1 \omega}^{r e l}}{I_{j s}^{r e l}} R I R_{\omega, s}\right) \frac{X_{\omega}}{X_{s}}+\epsilon
\end{array}
$$


where

- $I_{i}$ is the integrated intensity of the $i^{\text {th }} 2 \theta$ range from the mixture of phases, which may contain contributions from one or more lines from one or more phases in the mixture,

- $I_{j s}$ is the intensity of a resolved line $j$ of the internal-standard $s$,

- $X_{\omega}$ is the weight fraction of phase $\omega$ in the mixture of sample plus internal-standard,

- $\epsilon$ is an error term.

Line overlap is allowed for by having each intensity ratio $\left(\frac{I_{i}}{I_{j_{1}}}\right)$ have contributions from multiple lines from multiple phases. As many terms involving contributions to the intensity ratio as needed are included in each linear equation. The Copland-Bragg analysis results in an overdetermined system of $n$ equations in $m$ unknowns, which may be solved for the weight fractions via least squares. Of course, the normalizing assumption can be imposed here also.

Quantitative elemental data, obtained from X-ray fluorescence analysis for example, can be added to the system of equations, without increasing the number of unknowns, making it overdetermined. Coupling both the elemental and diffraction data will result in more accurate quantitative analysis of multicomponent mixtures. More importantly, estimates of the standard deviations of the results will be more accurate. Both the line overlap and chemical analysis features of the Copeland-Bragg formalism have been included in the AUTO (Snyder et al. 1981, 1982) and NBS*QUANT84 systems (Snyder \& Hubbard, 1984). An example matrix equation for such an analysis might look like:

$$
\mathbf{Y}=\mathbf{R X}
$$

where

$$
Y=\left(\begin{array}{c}
\frac{I_{1}}{I_{n_{2}}} \\
\frac{I_{2}}{I_{3}} \\
\frac{I_{3}}{I_{1}} \\
\frac{I_{1}}{I_{n}} \\
\vdots \\
\% S i \\
\% A l \\
\% F e \\
\% O
\end{array}\right)
$$

$$
\mathbf{X}=\left(\begin{array}{c}
X_{\alpha} \\
X_{\beta} \\
X_{\gamma} \\
\vdots \\
X_{\omega}
\end{array}\right)
$$

and $\mathbf{R}$ is the matrix

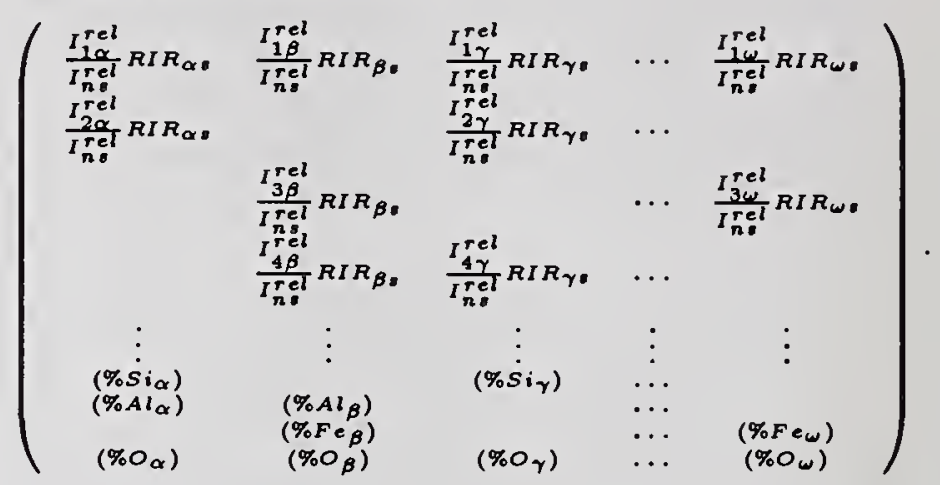

\section{Full-pattern fitting}

Quantitative analysis by X-ray powder diffraction has evolved since the 1930's starting with simple peak height measurements through the 1970's when diffractometers could measure background corrected integrated intensities. The arrival of automated diffractometers in the 1980's permitted the evolution of the AUTO/NBS*QUANT84 system which allowed the measurement of multiple lines per phase and resulted in a substantially enhanced statistical precision. The next logical step in this evolution is to use the whole digitized pattern to further enhance the statistical situation and average out some of the preferred orientation effects.

In recent years, D. K. Smith and his colleagues $(1986,1987,1988,1989)$ have developed whole pattern matching algorithms which have produced some of the best quantitative analyses ever reported. The use of the whole pattern involves collection of standard data on pure materials using fixed instrumental conditions. These standard patterns are processed to remove background and any instrumental artifacts. For standard materials that are unavailable in appropriate form, diffraction patterns can either be simulated from Powder Diffraction File data or calculated (Smith et al., 1987). In addition to collecting a data base of standard patterns, $R I R$ values for each are also measured or computed. Patterns are then measured for the unknown samples to be analyzed, using conditions identical to those used in obtaining the standard data. Background and artifacts are also removed from unknown-sample data. Lastly, the 
digital patterns are fit, point by point, to the standards with a least-squares procedure minimizing the expression

$$
\delta(2 \theta)=\left(I_{u n k}(2 \theta)-\sum_{k} X_{k} R I R_{k} I_{k}(2 \theta)\right)^{2},
$$

where $\mathrm{I}_{u n k}(2 \theta)$ and $\mathrm{I}_{k}(2 \theta)$ are the diffraction intensities at each $2 \theta$ interval for the unknown and each of the standard phases, $\mathrm{k}$, respectively.

In practice each of the reference patterns in the database is scaled point by point to its maximum intensity. The same is done with the pattern of the unknown mixture and the normalizing assumption is used to solve for the weight fractions.

This whole pattern fitting calibration procedure becomes a method for measuring RIRs from whole pattern standards. Once this is accomplished the method becomes a conventional $R I R$ quantitative analysis procedure, which uses calibration standards. Thus, the dilution of the unknown specimen with an internal standard may be avoided, as usual, only by applying the normalization assumption. The RIRs determined before the rescaling of the reference patterns act in the final least squares adjustment as normalized RIRs described below.

\section{Quantitative phase analysis using the Rietveld method}

At the same time that whole pattern matching procedures were developing, Hill \& Howard (1987) and Bish \& Howard (1988) began applying crystal structure constraints to the problem of quantitative analysis. The Rietveld method (Rietveld, 1969) was originally conceived as a method for refining crystal structures using neutron powder diffraction data. Least squares refinement is conducted by minimizing the sum of the weighted, squared differences between observed and calculated intensities at every step in a digital powder pattern. The variables are atomic positions, site occupancies, lattice parameters, scale factors, profiles, background and possibly a preferred orientation function. The method consists of fitting the complete experimental diffraction pattern with calculated profiles and backgrounds, and obtaining quantitative phase information from the scale factors for each phase in a mixture.

The intensity of reflection $i$ from a pure phase $\alpha$ was given by equation 1 which contains all of the structure sensitive parameters, which the Rietveld procedure optimizes, in the $K_{\mathbf{i} \alpha}$ term. The intensity at a given step is determined by summing the contributions from background and all neighboring Bragg reflections as

$$
y_{i}(c)=S \sum_{j} K_{j \alpha} G\left(\Delta \theta_{i, j \alpha}\right) P_{j}+y_{i b}(c),
$$

where $\mathrm{S}$ is the Rietveld scale factor which puts the computed intensities on the same scale as those observed, $\mathrm{P}_{j}$ is an optional preferred orientation function for the $\mathrm{j}^{\text {th }}$ Bragg reflection, $\mathrm{G}\left(\Delta \theta_{i, j \alpha}\right)$ is the profile shape function, and $y_{i b}(\mathrm{c})$ is the background (Wiles \& Young, 1981). Comparison of equations 1 and 22 shows that the Rietveld scale factor, $S$, is given by

$$
S=\frac{K_{e}}{\mu} .
$$

It is important to note that equation 23 will only be true for a correctly converged Rietveld refinement. Many discrepancies between the structural model and the actual structure will appear as errors in the scale factor and make the quantitative analysis incorrect. In the whole pattern-matching procedures the same scale error will be caused by differences between the observed pattern and the reference pattern due to such common effects as structural disorders or poorly modeled asymmetric profiles. It should be emphasized that the common use of computed symmetric profiles to model the asymmetric X-ray profiles will produce intensity mismatches which are directly incorporated into the scale factor and in turn the determined concentrations. For an exact modeling of the asymmetric X-ray peaks a convolution of the specimen profile shape function with a calibrated instrumental function must be carried out (Howard \& Snyder, 1989).

For a multi-phase mixture equation 22 can be rewritten summing over the $\mathrm{k}$ phases in a mixture as,

$$
y_{i c}=y_{i b}+\sum_{k} S_{k} \sum_{j} K_{j k} G_{i, j k} .
$$

Comparison of equations 2 and 24 allows us to write the scale factor for each phase as

$$
S_{\alpha}=K_{e} \frac{X_{\alpha}}{\left(\rho_{\alpha}\left(\frac{\mu}{\rho}\right)_{m}\right)},
$$

Therefore, in a Rietveld analysis of a multicomponent mixture, the scale factors contain the desired 
weight fraction information as,

$$
X_{\alpha}=\frac{\left(\frac{\mu}{\rho}\right)_{m}}{K_{e}} S_{\alpha} \rho_{\alpha} .
$$

However, the sample mass absorption coefficient is not known and we thus are forced to apply the usual internal-standard analysis, measuring the RIRs of the phases to be analyzed with an added internal standard, or to use $I / I_{c}$ values and apply the normalization assumption constraining the sum of the weight fractions of the phases considered to unity. Hill \& Howard (1987) were the first to show that the Rietveld scale factor acts in the role of a Reference Intensity Ratio permitting conventional quantitative analysis. We should note that each phase in the Rietveld refinement need not be modeled in order to obtain correct scale factors for those that are refined.

In order to analyze the relationship between the Rietveld scale factor and the $R I R$ let's first look at a system where we apply the normalizing equation 17 . In this case,

$$
X_{\alpha}=\frac{X_{\alpha}}{\left(X_{\alpha}+X_{\beta}+\ldots\right)} .
$$

Equation 27 can be solved for the weight fractions of each of the diffracting phases by substituting equation 26 into it,

$$
X_{\alpha}=\frac{S_{\alpha} \rho_{\alpha}}{\sum_{k} S_{k} \rho_{k}}
$$

We see that this procedure is exactly analogous to the normalization assumption in the internalstandard method where the Reference Intensity Ratios are measured prior to analysis. Instead of measuring Reference Intensity Ratios to put all intensities on an absolute scale, the Rietveld method calculates what we should call Normalized values, $R I R_{N}$, in that each is referred to a pseudo-phase with an RIR of 1.0. Comparison of equations 28 and 18 shows that the Rietveld scale factor is

$$
S_{\alpha} \rho_{\alpha}=\frac{I_{i \alpha}}{R I R_{N, \alpha} I_{i \alpha}^{r e l}} .
$$

The $\frac{I_{i \alpha}}{I_{i \alpha}^{\text {el }}}$ term permits the scaling of $R I R_{N, \alpha}$ so as to allow the computation of a conventional $R I R_{\alpha \beta}$ values by taking the ratio of two $R I R_{N, \alpha}$ values. Equation 29 permits the computation of normalized $R I R s$ from published or computed $R I R$ values.

It is clear that the weight fractions obtained from equation 28 will be incorrect when an amorphous or unmodeled phase is present, in that the sum in the denominator of equation 28 should contain terms for the unmodeled phases. From equation 28 we further see the analog to equation 18 ,

$$
X_{\alpha}=\frac{I_{i \alpha}}{R I R_{N, \alpha} I_{i \alpha}^{r e l}}\left[\sum_{k=1}^{\# \text { of phases }} \frac{I_{j, k}}{R I R_{N, k} I_{j, k}^{r e l}}\right]^{-1}
$$

The evaluation of weight fractions using equation 30 produces results similar to the normalized internal-standard method described above but without the need to measure RIR values in advance. If conventional RIRs are not to be used or mixed in to the set, then the somewhat artificial $\frac{I_{i \alpha}}{I_{i \alpha}^{\text {ci }}}$ terms can be ignored and the $R I R_{N, k}$ values will shift to another scale. The weight fractions resulting from the application of equation 28 or its $R I R$ equivalent equation 30 or a version of 30 without the scaling $\frac{I_{i a}}{I_{i a}^{\text {rel }}}$ terms, will all be correct as long as the normalizing assumptions hold. Thus, this procedure is much closer to the "standardless" analysis all researchers desire. It should be noted that the whole pattern matching procedures of Smith et al. can be cast in this same notation framework. Both procedures are subject to the systematic error inherent in the normalizing assumption as well as the additional errors, due to poor fitting between the reference and unknown due to poor profile modeling (e.g., due to anisotropic peak broadening and unmodeled peak asymmetries) and errors in the structural model, all of which will be swept in to the scale factors and, in turn, into the derived weight fractions.

\section{The internal standard method in pattern-fitting techniques}

To avoid the normalizing assumption, the conventional internal-standard method of analysis may be applied to Rietveld or pattern-matching quantitative analysis. This requires that a known weight fraction of a crystalline internal standard be present in, or added to, the unknown mixture. Thus, if $\mathrm{X}_{\mathrm{s}}$ is known, then the Normalized $R I R_{N}$, with or without the scaling $\frac{I_{i \alpha}}{I_{i \alpha}^{\text {rel }}}$ term, can be used directly, to determine the weight fractions for other phases in the sample. For example, the weight fraction for the $\alpha$ phase is determined by

$$
X_{\alpha}=\frac{R I R_{N, s}}{R I R_{N, \alpha}} X_{s}
$$


The normalized $R I R_{N}$ values are effectively refined parameters in the least squares, so the weight fraction of phase $\alpha$ can be directly determined. This internal-standard method does not constrain the sum of the weight fractions, as does the normalized $R I R$ or whole pattern fitting methods. The total weight fraction of any amorphous components can also be determined with the internal-standard Rietveld or pattern matching methods by explicitly fitting the pattern of the glass and using its $R I R_{N}$ value. Alternatively, if the amorphous pattern can be fit and subtracted using the background polynomial, then the difference between the sum of the weight fractions of the crystalline components and 1.0 is the total weight fraction of the amorphous components. O'Connor \& Raven (1969) used this method to conclude that their quartz contained an $18 \%$ amorphous component.

The only thing differentiating whole-pattern fitting $R I R$ method from the normal RIR-internalstandard method is its use of the whole pattern to establish the $R I R_{N}$ values greatly improving the statistical precision and averaging over some of the preferred orientation. When measured, conventional, $R I R s$ are incorporated into either whole-pattern fitting technique then the procedure becomes a conventional internal-standard $R I R$ procedure with the advantage of using all diffraction lines rather than a selected few. Despite the many potential problems with these whole pattern fitting methods the results reported to-date are among the best quantitative results in the literature.

\section{References}

Alexander, L. E. Klug, H. P \& Kummer, E. (1948). J. Appl. Phys. 19, 742.

Bish, D. L. \& Howard, S. A. (1988). J. Appl. Cryst. 21, 86-91.

BezjaK, A. (1961). Croatica Chemica Acta 33, 197-199.

Chung, F. H. (1974a). J. Appl. Cryst. 7, 519-525.

Chung, F. H. (1974b). J. Appl. Cryst. 7, 526-31.

Chung, F. H. (1975). J. Appl. Cryst. 8, 17-19.

ClINE, J. P. \& SNYDER, R. L. (1983). Adv. in $X$-ray Anal. 26, 111-118.

Cline, J. P. \& SNYDER, R. L. (1985). Advances in Material Characterization II, 131-144, R. L. Snyder, R. A. Condrate \& P. F. Johnson, eds., New York: Plenum Press.

Cline, J. P. \& SNYDER, R. L. (1987). Adv. in $X$-ray Anal. 30, 447-456.

Copeland, L. E. \& Bragg, R. H. (1958). Anal. Chem. 30, 196-206.

Gehringer, R. C., McCarthy, G. J., Garvey, R. G., \& SMITH D. K. (1983). Adv. $X$-ray Anal. 26, 119-128.

Howard, S. A. \& SNyder, R. L., (1989). J. A ppl. Cryst. 22, 238-243.
HLl, R. J. \& Howard, C. J. (1987). J. Appl. Cryst. 20, 467-474.

Hubbard, C. R., Evans, E. H. \& SMith, D. K. (1976). J. Appl. Cryst. 9, 169-174.

Hubbard C. R. \& SNYDER, R. L., (1983). Powder Diffraction 3, 74 .

Hubbard C. R., RobBins, C. R. \& SNYder, R. L. (1983). Adv. X-ray Anal. 26, 149-157.

Hubbard C. R. \& SnYder, R. L. (1988). Powder Diffraction 3, 74-78.

Karlak, F. \& Burnett, D. S. (1966). Anal. Chem. 38[12], 1741-1745.

Klug, H. P. \& AleXander, L. E. (1974). X-ray Diffraction Procedures, 2nd ed. New York: John Wiley and Sons

O'Connor, B. H. \& Raven, M. D. (1988). Powder Diffraction 3, 2-6.

Rietveld, H. M. (1969). J. Appl. Cryst. 2, 65-71.

SMith, D. K., Nichols, M. C. \& ZolensKy, M. E. (1982). POWD10. A FORTRAN IV program for calculating $X$-ray powder diffraction patterns - version 10 . The Pennsylvania State University, University Park, $\mathrm{Pa}$.

Smith, D. K., Johnson, Jr., G. G. \& Ruud, C. O., (1986). Adv. X-ray Anal. 29, 217-224.

Smith, D. K., Johnson, JR., G. G., Scheible, A., Wims, A. M., Johnson, J. L. \& UllmanN, G. (1987). Powder Diffraction 2, 73-77.

Smith, D. K., Johnson, JR., G. G. \& Wims, A. M. (1988). A ust. J. Phys. 41, 311-321.

Smith, D. K., Johnson, JR., G. G. Kelton, M. J. \& ANDERSon, C. A. (1989). Adv. X-ray Anal. 32 in press.

SmIth, D. K., (1992). Adv. $X$-ray Anal. 35, in press.

SMith, S. T., SNyder, R. L. \& BRownell, W. E., (1979a). Adv. X-ray Anal. 22, 77-88.

Smith, S. T., SNyder, R. L. \& Brownell, W. E. (1979b). Adv. $X$-ray Anal. 22, 181-191.

SNYDER, R. L. \& BISH, D. L. (1989). Modern Powder Diffraction, D. L. Bish \& J. E. Post ed. p101-144. Washington: Mineralogical Society of America.

SNyder, R. L. \& CARR, W. L. (1974). Surface. Surfaces and Interfaces of Glass and Ceramics, ed., V. D. Frechette p.85-99, New York: Plenum Press

Snyder, R. L., Hubbard, C. R. \& PanagiotopouLOS, N. C. (1981). AUTO: A Real Time Diffractometer Control System. NBSIR 81-2229, Gaithersburg: National Bureau of Standards.

Snyder, R. L., Hubbard, C. R. \& Panagiotopoulos, N. C. (1982). Adv. X-ray Anal. 25, 245-160.

SNyder, R. L. \& HubBard, C. R. (1984). NBS* QUANT84: A System for Quantitative Analysis by Automated $X$-ray Powder Diffraction, Internal Publication, Gaithersburg: National Bureau of Standards.

VisSER, J. W. \& DEWOLFF, P. M. (1964). Absolute Intensities Report 641.109 Delft: Technisch Physische Dienst.

Wiles, D. B. \& Young, R. A. (1981). J. Appl. Cryst. $14,149-151$. 
National Institute of Standards and Technology Special Publication 846. Proceedings of the international conference Accuracy in Powder Diffraction II, held at NIST, Gaithersburg, MD, May 26-29, 1992. (Issued October 1992)

\title{
Quantitative Phase Analysis with the Rietveld Method
}

\author{
J. K. Stalick \\ Materials Science and Engineering Laboratory, National Institute of Standards and Technology, \\ Gaithersburg, MD 20899, U.S.A.
}

\begin{abstract}
Quantitative phase analysis of multiphase mixtures using Rietveld refinement has recently been shown to be a viable alternative to conventional discretepeak methods, particularly for the analysis of mixtures with complex patterns or phases with broadened peaks. The analysis of the entire pattern reduces problems due to extinction, preferred orientation, and other systematic errors. The use of neutron rather than X-ray diffraction further enhances this technique, owing in part to the elimination of microabsorption and preferred orientation effects. Neutron Rietveld refinement has been used to determine low-concentration impurity phases in the high- $\mathrm{T}_{c}$ superconductor $\mathrm{YBa}_{2} \mathrm{Cu}_{3} \mathrm{O}_{7-\delta}$ by including the scattering contributions of the impurity phases as additions to background. In this system, $\mathrm{CuO}$ was detected at $0.36(6)$ wt. \%, even though no impurity peaks were visually observed.
\end{abstract}

\section{Introduction}

Rietveld analysis of powder diffraction data involves the fitting of the entire experimental diffraction pattern to a calculated profile (Rietveld, 1969) based on a known structural model. When used for quantitative phase analysis, the scale factors of two or more phases are refined along with background, unit cell parameters, peak widths and shapes, and perhaps crystal structure parameters. The recent review by Hill (1991) gives an excellent summary of the advantages of this technique, including (1) utilization of the entire diffraction profile, thus reducing systematic errors; (2) the ability to fit the background over the entire pattern; (3) the ability to correct for extinction and preferred orientation in each phase; (4) more efficient treatment of overlapping peaks; (5) the ability to refine crystal structure and peak profile parameters; and (6) the correct propagation of error into the results, using the standard deviations of the scale factors estimated from the least squares refinement.

Hill \& Howard (1987) have shown that for a mixture of crystalline materials, the mass of phase $p, m_{p}$, is given by

$$
m_{p} \propto S_{p}\left(Z_{p} M_{p} V_{p}\right)
$$

where $S_{p}$ is the Rietveld scale factor, $Z_{p}$ the number of molecules per unit cell, $M_{p}$ the molecular weight, and $V_{p}$ the unit cell volume. Bish \& Howard (1988) derived a similar relationship, $m_{p} \propto S_{p} \rho_{p}$, where $\rho_{p}$ is the calculated density with $\rho_{p}=Z_{p} M_{p} / N V_{p}$, thus giving $m_{p} \propto S_{p}\left(Z_{p} M_{p} / V_{p}\right)$. However, since most Rietveld software does not include the normalizing term $1 / V_{p}^{2}$ in the calculated intensity, but rather incorporates it as part of the scale factor, the formulation of Hill \& Howard is more generally applicable. Thus the weight fraction, $w_{p}$, of a component phase $p$ in a mixture of $n$ phases is given by

$$
w_{p}=\frac{S_{p}(Z M V)_{p}}{\sum_{i=1}^{n} S_{i}(Z M V)_{i}} .
$$

Hill (1990) refers to the quantity $Z M V$ (or $\rho V^{2}$ ) as the pattern intensity constant for a particular phase; this is the Rietveld equivalent of the more conventional Reference Intensity Ratio (RIR).

\section{The model}

The application of this technique is chiefly limited by the accuracy of the model used for the calculated diffraction patterns. The crystal structures of all phases must be known; while it is possible to refine the structures of one or more of the phases as part of the analysis, it is important that this refinement be both necessary (phases with variable composition) and warranted by the data (major constituents with reasonably well-differentiated reflections, and neutron diffraction data if possible). Using neutron data, Hill \& Howard (1987) were able to refine all crystal structure parameters along with 
Table 1: Theoretical and Experimental Phase Compositions (wt. \%)

\begin{tabular}{cccc}
\hline \hline & & \multicolumn{2}{c}{ Measured } \\
Mixture & Weighed & \multicolumn{1}{c}{ X-ray } & Neutron \\
& & & \\
\hline & & & \\
$\mathrm{TiO}_{2}$ & 70 & $69.0(20)$ & $70.5(3)$ \\
$\mathrm{Al}_{2} \mathrm{O}_{3}$ & 30 & $31.0(12)$ & $29.5(3)$ \\
& & & \\
$\mathrm{TiO}_{2}$ & 50 & $48.8(15)$ & $50.5(3)$ \\
$\mathrm{Al}_{2} \mathrm{O}_{3}$ & 50 & $51.2(19)$ & $49.5(3)$ \\
& & & \\
$\mathrm{TiO}_{2}$ & 30 & $26.5(7)$ & $30.4(3)$ \\
$\mathrm{Al}_{2} \mathrm{O}_{3}$ & 70 & $73.5(19)$ & $69.6(3)$ \\
& & & \\
$\mathrm{TiO}_{2}$ & 10 & $8.1(3)$ & $9.8(2)$ \\
$\mathrm{Al}_{2} \mathrm{O}_{3}$ & 90 & $91.9(21)$ & $90.2(2)$ \\
$\mathrm{TiO}_{2}$ & 50 & $48.9(18)$ & $50.7(3)$ \\
$\mathrm{SiO}_{2}$ & 50 & $51.1(21)$ & $49.3(3)$ \\
& & & \\
\hline
\end{tabular}

the phase abundances in $\mathrm{TiO}_{2} / \mathrm{Al}_{2} \mathrm{O}_{3}, \mathrm{TiO}_{2} / \mathrm{SiO}_{2}$, and $\mathrm{Si} / \mathrm{SiO}_{2}$ mixtures with good results. However, with X-ray data the structure parameters are not generally refined (Bish \& Howard, 1988; O'Connor \& Raven, 1988; Madsen \& Hill, 1990). Table 1, taken from Madsen \& Hill (1990), illustrates the results of neutron and X-ray refinements. The agreement between the weighed and measured wt. $\%$ is in general quite good, with the absolute errors and standard deviations being lower in the neutron refinements.

Other important considerations are primary extinction, preferred orientation, and microabsorption corrections, as well as the presence of amorphous or partly amorphous constituents (Hill, 1990). The use of the entire diffraction pattern minimizes the effects of extinction, and normalized preferred orientation corrections are included in most Rietveld software. Alternatively, sample preparation techniques (spray-drying etc.) may be used to eliminate or reduce preferred orientation. The Rietveld method also permits the determination of amorphous content through the addition of a standard of known crystallinity; for example, O'Connor \& Raven (1988) and Jordan, O'Connor \& Deyu (1990) have successfully determined the amorphous content of quartz specimens. The most serious remaining problem appears to be that of microabsorption corrections for phases with differing particle size distributions and mass absorption coefficients. Madsen \& Hill (1990) found reasonably good agreement between the theoretical and experimental X-ray Rietveld phase compositions for mixtures of $\mathrm{TiO}_{2}, \mathrm{Al}_{2} \mathrm{O}_{3}$, and $\mathrm{SiO}_{2}$ with mass absorption coefficents for $\mathrm{Cu} \mathrm{K} \alpha$ radiation of $129.3,31.1$, and $34.5 \mathrm{~cm}^{2} \mathrm{~g}^{-1}$, respectively (Table 1 ). However, mixtures of $\mathrm{LiF}$ and $\mathrm{Pb}\left(\mathrm{NO}_{3}\right)_{2}(\mu / \rho=20$ and $231 \mathrm{~cm}^{2} \mathrm{~g}^{-1}$ for $\mathrm{Co} \mathrm{K} \alpha$ radiation) resulted in large quantification errors without the application of a Brindley absorption correction (Taylor \& Matulis, 1991).

\section{Determination of impurity phases}

The problems arising from extinction, preferred orientation, and microabsorption are all effectively eliminated with neutron diffraction data. Furthermore, the large irradiated sample volume, along with the Gaussian line shape and greater angular range of data, permits the quantitative phase analysis of minor impurity phases.

This technique was applied in two studies of the high- $\mathrm{T}_{c}$ superconductor $\mathrm{YBa}_{2} \mathrm{Cu}_{3} \mathrm{O}_{7-\delta}$. The first study was undertaken to optimize processing conditions for the production of pure-phase $\mathrm{YBa}_{2} \mathrm{Cu}_{3} \mathrm{O}_{7}$ superconducting material. The second study involved the correlation of superconducting properties with structural changes upon partial substitution (up to $10 \%$ ) of other elements for $\mathrm{Cu}$. There are significant structural changes in this system with varying oxygen content and site substitution; for example, the crystallographic space group changes from tetragonal ( $\mathrm{P} 4 / \mathrm{mmm})$ to orthorhombic (Pmmm) with increasing oxygen content in the unsubstituted material. Crystallographic symmetry also varies with site substitution; furthermore, the determination of the amount of secondary phases is necessary as a check on the amount of substituent not incorporated in the superconductor. The Rietveld method provided the ability to refine the structure of the primary phase while simultaneously determining the phase abundance of impurity phases in these two studies.

Experimental- Potential impurity phases were identified from phase diagram studies (Roth, Rawn, Beech, Whitler \& Anderson, 1988), and experimental neutron diffraction patterns were obtained for $\mathrm{CuO}, \mathrm{BaCuO}_{2}, \mathrm{BaCO}_{3}$, and $\mathrm{Y}_{2} \mathrm{BaCuO}_{5}$ on the five-detector high resolution powder diffractometer at NIST. The structures of $\mathrm{CuO}, \mathrm{BaCO}_{3}$, and $\mathrm{Y}_{2} \mathrm{BaCuO}_{5}$ were refined using the Rietveld method in order to obtain the best values for the structural 
parameters (in particular the neutron thermal parameters); the structure of $\mathrm{BaCuO}_{2}$ could not be satisfactorily refined based upon published results (Kipka \& Müller-Buschbaum, 1977; Weller \& Lines, 1989), and the observed pattern was used in the impurity phase analyses. For all other potential impurity phases calculated patterns based upon known structures were used.

High-resolution neutron powder diffraction patterns were obtained for several preparations of $\mathrm{YBa}_{2} \mathrm{Cu}_{3} \mathrm{O}_{7-\delta}$ and $\mathrm{YBa}_{2}(\mathrm{Cu}, \mathrm{M})_{3} \mathrm{O}_{7 \pm \delta}, \mathrm{M}=\mathrm{Al}, \mathrm{Fe}$, $\mathrm{Ga}, \mathrm{Co}, \mathrm{Ni}, \mathrm{Mn}, \mathrm{Zn}, \mathrm{Au}$. The samples were contained in vanadium cans, and data were collected at room temperature with a $2 \theta$ angular range of $5-120^{\circ}$, with a step size of $0.05^{\circ}$ and a monochromatic wavelength of $1.5525(1) \AA$. Structures were refined using a modified version of the Rietveld program (Prince, 1980). The intensity contributions of impurity phases were added as a contribution to background and scale factors $S_{p}$ were refined for each potential impurity phase. The total calculated intensity for each point is given by

$$
(1 / c) Y_{i(c a l c)}+B_{i}+\sum_{p=1}^{n} S_{p} Y_{i p}
$$

where $1 / c$ is the primary phase scale factor and $B_{i}$ is the contribution at point $i$ of background as represented by a series of Chebychev polynomials. Whenever possible the impurity phase contributions $Y_{i p}$ were calculated from the known structure parameters; for $\mathrm{BaCuO}_{2}$ a background-subtracted observed pattern was used. The weight fractions $w_{p}$ were then calculated as previously described.

Results - The impurity phase found in nearly all samples studied was $\mathrm{CuO}$. Note that neutron diffraction is approximately twice as sensitive to the $\mathrm{CuO}$ phase as is X-ray diffraction, owing to the greater relative scattering of neutrons by atoms of low atomic number. Table 2 gives the results for a series of $\mathrm{YBa}_{2} \mathrm{Cu}_{3} \mathrm{O}_{7}$ samples prepared under different processing conditions. For sample $\mathbf{A}$ excess $\mathrm{CuO}$ was known to be present; however, samples $\mathbf{B}-\mathbf{E}$ appeared phase-pure based on X-ray powder patterns. Below about one wt. \% the $\mathrm{CuO}$ peaks were not discernable in the neutron patterns, even though the fraction of $\mathrm{CuO}$ was statistically significant. Figure 1 shows the experimental, calculated, and difference patterns in the region of the strongest $\mathrm{CuO}$ peak for sample $\mathbf{B}$, with $1.27(6)$ wt. \% $\mathrm{CuO}$. The $\mathrm{CuO}$ (111) reflection is clearly evident in the difference pattern, although it is smaller than the noise owing to approximations in the model for the $\mathrm{YBa}_{2} \mathrm{Cu}_{3} \mathrm{O}_{7}$ phase. Ta-
Table 2: Determination of $\mathrm{CuO}$ in $\mathrm{YBa}_{2} \mathrm{Cu}_{3} \mathrm{O}_{7}$ by Rietveld Refinement

\begin{tabular}{cccc}
\hline \hline Sample & $\begin{array}{c}\text { Scale Factor } \\
\mathrm{YBa}_{2} \mathrm{Cu}_{3} \mathrm{O}_{7}\end{array}$ & $\begin{array}{c}\text { Scale Factor } \\
\mathrm{CuO}\end{array}$ & $\begin{array}{c}\text { Weight } \\
\% \mathrm{CuO}\end{array}$ \\
\hline & & & \\
$\mathrm{A}$ & $6.90(3)$ & $2.93(3)$ & $8.7(2)$ \\
$\mathrm{B}$ & $7.29(3)$ & $0.42(2)$ & $1.27(6)$ \\
$\mathrm{C}$ & $7.81(2)$ & $0.19(2)$ & $0.54(5)$ \\
$\mathrm{D}$ & $3.73(2)$ & $0.06(1)$ & $0.36(6)$ \\
$\mathrm{E}$ & $3.24(2)$ & $-0.03(2)$ & 0 \\
\hline
\end{tabular}

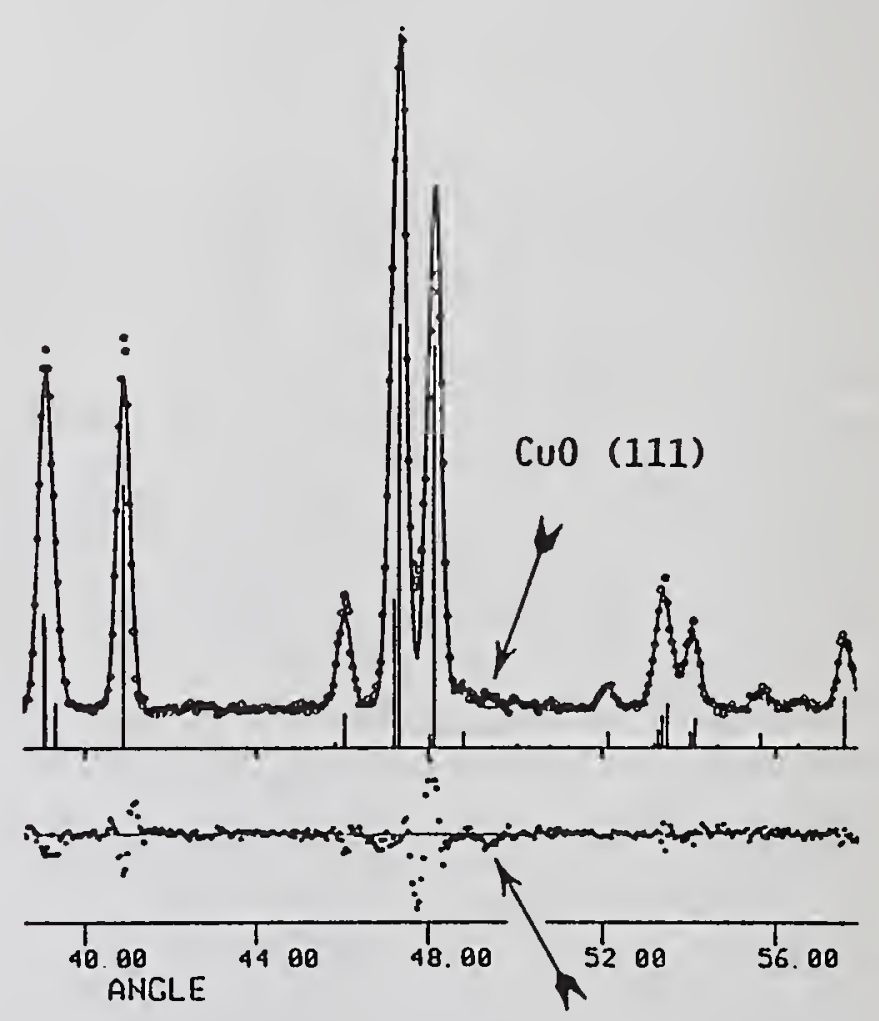

Figure 1: Experimental (circles), calculated (solid line), and difference (observed - calculated) patterns for $\mathrm{YBa}_{2} \mathrm{Cu}_{3} \mathrm{O}_{7}$ with $1.27(6)$ wt. $\% \mathrm{CuO}$ in the region 40-56 20. The $\mathrm{CuO}$ (111) reflection is indicated on the observed and difference patterns. 
Table 3: Refinement of Impurity Phase Scale Factors

\begin{tabular}{ccc}
\hline \hline Material & Scale Factor & Calc. Wt. \\
& & \\
\hline $\mathrm{YBa}_{2} \mathrm{Cu}_{3} \mathrm{O}_{7}$ & $7.81(2)$ & $99.35(13)$ \\
$\mathrm{CuO}$ & $0.19(2)$ & $0.54(5) \dagger$ \\
$\mathrm{Y}_{2} \mathrm{BaCuO}_{5}$ & $-0.001(1)$ & 0 \\
$\mathrm{BaCO}_{3}$ & $0.004(3)$ & $0.11(8)$
\end{tabular}

$\dagger$ Inclusion of $\mathrm{CuO}$ as an impurity phase decreased the agreement factors $R_{w p}$ and $R_{B}$ from 6.50 and 3.19 to 6.37 and 3.13 , respectively.

ble 3 presents the results for sample $\mathbf{C}$; the $\mathrm{X}$-ray and neutron diffraction patterns exhibited no impurity peaks. Only $\mathrm{CuO}$ was found to be present; the inclusion of $\mathrm{CuO}$ impurity scattering in the calculated model led to statistically significant improvements in the agreement factors for the Rietveld refinement.

The results for the site-substitution studies parallel those presented here for the processing of the pure superconductor material, and will not be discussed in detail. In general, the inclusion in the model of scattering from impurity phases lowered the standard deviations for the site occupancies, and in the case of the Au-substituted material confirmed the displacement of $\mathrm{Cu}$ atoms by $\mathrm{Au}$ atoms in the structure (Cieplak et al., 1990).

\section{Discussion}

Quantitative phase analysis with the Rietveld method has been shown to be a viable alternative to conventional discrete-peak methods. In the determination of impurity phases in the system discussed here, the Rietveld technique is far superior to other methods owing to the variability of the structure of the major superconductor phase and the very low concentration of impurity phases. Another system in which the Rietveld method appears to offer significant advantages is in the determination of mineral content of rock specimens. Two computer programs incorporating databases of known mineral structures have recently been reported. The program QPDA by Madsen \& Hill (1990) was designed for use by powder diffractionists with little or no experience in structure refinement. QPDA can be run in either single-phase or multi-phase refinement mode, and calculates phase abundance and size/strain information from the refined parameters. The database in this case also includes structures of common inorganic materials. The second system of computer programs, SIROQUANT, features corrections for amorphous content and for microabsorption using Brindley particle absorption contrast factors (Taylor, 1991). The database contains structure information on 90 common minerals, and new minerals can be added. Both programs include correction for preferred orientation based upon the March model. The Rietveld method for quantitative phase analysis has been shown to give good results in known mixtures of mineral specimens; thus the application of this technique to unknown mixtures appears to be forthcoming.

\section{Acknowledgments}

Many thanks to those who assisted with this project: E. Prince, J. J. Rhyne, and W. Wong-Ng of NIST, C. L. Chien and G. Xiao of The Johns Hopkins University, S. Johnson of SRI, C. Parker of Allied-Signal EMRC, and J. Hodge of CPS Superconductor Inc.

\section{References}

Bish, D. L. \& Howard, S. A. (1988). J. Appl. Crystallogr. 21, 86-91.

Cieplak, M. Z., Xiao, G., Chien, C. L., Bakhshai, A., Artymowicz, D., Bryden, W., Stalick, J. K. \& Rhyne, J. J. (1990). Phys. Rev. B 42, 6200-6208.

HILL, R. J. (1991). Powd. Diffr. 6, 74-77.

Hill, R. J. \& Howard, C. J. (1987). J. Appl. Crystallogr. 20, 467-474.

Jordan, B., O'ConNor, B. H. \& DeyU, L. (1990). Powd. Diffr. 5, 64-69.

KIPKa, R. \& Müller-Buschbaum, Hк. (1977). $Z$. Naturforsch. B 32, 121-123.

Madsen, I. C. \& Hill, R. J. (1990). Powd. Diffr. 5, 195-199.

O'Connor, B. H. \& Raven, M. D. (1988). Powd. Diffr. 3, 2-6.

PrINCE, E. (1980). U. S. Tech. Note 1117, 8-9.

Rietveld, H. M. (1969). J. Appl. Crystallogr. 2, 65-71.

Roth, R. S., RaWn, C. J., BeECh, F., Whitler, J. D. \& Anderson, J. O. (1988). In Ceramic Superconductors II (M. F. Yan, Ed.), Amer. Chem. Soc., 13-26.

TAYLOR, J. C. (1991). Powd. Diffr. 6, 2-9.

TaYlor, J. C. \& Matulis, C. E. (1991). J. Appl. Crystallogr. 24, 14-17.

Weller, M. T. \& Lines, D. R. (1989). J. Chem. Soc. Chem. Commun. 484-485. 
National Institute of Standards and Technology Special Publication 846. Proceedings of the international conference Accuracy in Powder Diffraction II, held at NIST, Gaithersburg, MD, May 26-29, 1992. (Issued October 1992)

\title{
Microabsorption Corrections
}

\author{
H. HermanN*AND M. ERmRich \\ Institute of Solid State and Materials Research, \\ D-O 8027 Dresden, Germany, \\ and \\ STOE \& CIE GmbH, \\ D-W-6100 Darmstadt, Germany
}

\section{Introduction}

The intensity of $\mathrm{X}$-rays diffracted by multiphase powder specimens is governed not only by the parameters of the X-ray device used for the experiment and the crystal structure and physical state of the scattering phase, but also by absorption processes that the $\mathrm{X}$-ray is subjected to as it passes through the sample. The magnitude of the absorption effect depends on the geometrical arrangement of X-ray source and detector, the macroscopic shape and size of the scattering sample, and the microstructural distribution of the absorbing constituents in the sample (Klug \& Alexander, 1974; Koch \& MacGillavry, 1985; Koopmans \& Rieck, 1985).

Here we consider the absorption of X-rays reflected from a flat specimen. For homogeneous materials, the processes are assumed to be described by the linear absorption coefficient, $\mu$, of the absorbing material and an exponential decay of the intensity with the path length of the ray in the material.

For heterogeneous materials the situation is much more complicated. The beam penetrates not only the scattering phase, $i$, in an $n$-phase material but also regions filled with particles of the other phases. If all particles are very small compared with the penetration depth of the X-rays, the beam, on average, will pass through many regions of all phases. Then the path length through particles of the scattering phase is given by the geometrical path length of the beam in the whole sample multiplied by the volume fraction of the scattering particles. Considering a series of samples of the same composition but with increasing particle size, the length of the path of the beam through the scattering phase exceeds the above-mentioned value more and more, and in the limit of very large particles the whole path of the

\footnotetext{
"Institut für Festkörper- und Werkstofforschung Dresden Helmholtzstr. 20, D-O-8027 Dresden, Germany
}

beam through the sample is within a single scattering particle at the surface of the sample. Supposing the linear absorption coefficients $\mu_{k}, k=1, \ldots, n$ to be different, it is obvious that the strength of the absorption effect varies with the size of scattering particles. In the following we analyze the absorption effect for flat samples and its dependence on bulk microstructure and surface morphology of the sample.

\section{Experimental evidence for microabsorption}

One of the first to investigate the microabsorption effect was Brindley (1945). He pointed out that in multiphase materials both the size of the scattering particles and the absorption contrast $\left(\mu_{i}-\bar{\mu}\right)$ (where $\bar{\mu}$ is the mean linear absorption coefficient of the sample) play a decisive role. The granular structure of powder specimens causes an angle-dependent contribution to the absorption of the incident and diffracted beam. This effect was studied by de Wolff (1956) and by Suortti (1972) who measured the reduction of specimen fluorescence radiation due to granularity. Trucano \& Battermann (1970) investigated the effect of porosity of amorphous powders, analyzing diffuse scattering.

Although the experimental results differed in some detail, the intensity reduction due to granularity could be described by the sum of a constant term proportional to the volume fraction of pores, the linear absorption coefficients and mean chord lengths of particles, and a smooth function of scattering angle. The former is determined by the bulk properties of the granular material, whereas the latter is essentially caused by the surface roughness of the sample.

Hermann \& Ermrich (1989) and Ermrich \& Hermann (1990) carried out quantitative phase analyses of silicon-molybdenum powder mixtures. They showed that the neglect of the microabsorption effect may cause a considerable deviation of the estimated volume fraction from the true value, which 
can reach the order of magnitude of the true value itself, whereas the error is well below $5 \%$ when the scattered intensities are corrected for microabsorption. Due to the region of scattering angle, $2 \theta$, considered ( $2 \theta$ not too small) and low degree of surface roughness it was sufficient to consider only the bulk contribution of the microabsorption effect as a correction to the quantitative phase analysis.

The surface roughness of scattering specimens has been shown to reduce the intensity of scattered Xrays especially in the low-angle region (Suortti, 1972; Sparks et al, 1991). As a consequence of this effect, structural refinements based on Rietveld analyses may result in unphysically low and even negative thermal parameters (Sparks et al., 1991; Masciocchi et al., 1991; Pitschke et al., 1991; Kumar et al., 1991).

The physical processes governing the attenuation of X-rays in matter are quite clear. Considering diffraction by flat specimens, the problem of estimating the microabsorption effect quantitatively by means of

$$
I_{i} / I_{0, i}=\int_{V} \exp \left[-\mu_{i} x_{i}-\mu_{(i)} x_{(i)}\right] d V / Q_{0}
$$

consists mainly in the mathematical description of the geometrical distribution of scattering and absorbing particles, in the calculation of the path length of a given ray through the regions covered by the scattering phase, and in averaging of all rays reflected by the sample. Here, the scattering phase is denoted by $i, x_{i}$ is the path length of a ray through phase $i, x_{(i)}$ is the same for the region outside phase $i$, and $\mu_{(i)}$ is the mean linear absorption coefficient of all regions of the sample that do not belong to phase $i . I_{0, i} / 2 \mu_{i}$ is the intensity from an $i$-type homogeneous smooth reference specimen obtained under the same experimental conditions as the scattered intensity $I_{i}$ from the heterogeneous sample. $Q_{0}$ is the cross section of the incident beam and $V$ is the sample volume. (See also figure 1.)

It is obvious that a mathematically well-defined structure model is needed to solve the integral (1) for representative classes of powder structures. The model should be variable with respect to volume fraction, and size and shape of scattering particles. The model should include both bulk properties and surface morphology, and it should be possible to calculate the geometrical characteristics required for the evaluation of (1). Furthermore, the model should be easy to handle. In the following we describe an approach to tackling the problem outlined above. It is based on methods that have been developed within the framework of the mathematical branch of Stochastic Geometry (see, e.g., Stoyan et al., 1987)

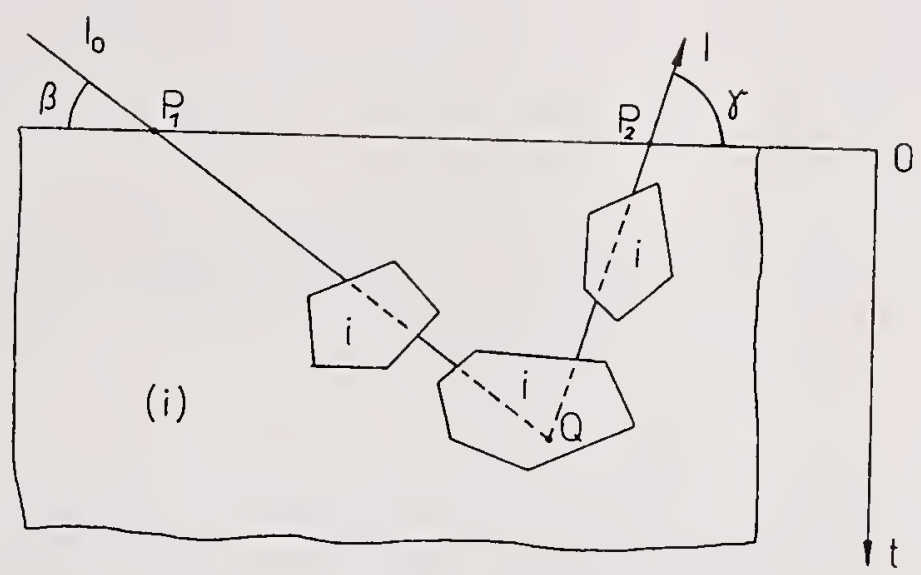

Figure 1: Scattering of a X-ray by a plane sample. The broken line represents the path length $x_{i}$ within phase $i$.

and have already proved to be suitable for practical applications (Hermann, 1991).

\section{Structure model for random powders}

\section{Bulk structure}

We simulate the powder sample by a random twophase model given by a random set $A$ and the complement $B=A^{c}$ of $A$. The one set represents the scattering particles and the other one the pores or the distribution of both pores and particles that do not belong to the scattering phase for respectively singleor multi-phase powders. The model is generated in two steps. In the first step a random (Poisson) field of points $P_{1}, P_{2}, \ldots$, with density $\lambda$ is generated. Then a sequence of isotropic, identically distributed, convex geometrical objects (for example spheres, random polyhedra etc.), $A_{1}, A_{2}, \ldots$, is produced. The $P_{k}^{\prime} s$ are the 'germs' and the $A_{k}^{\prime} s$ are the 'primary grains'. The $A_{k}^{\prime} s$ are shifted to the points $P_{k}$ whereby the grains may overlap. The union of all $A_{k}^{\prime} s$ forms a random set $A$. The model is given by all points in the space that are covered by one or more primary grains. Figure 2 illustrates the construction algorithm.

Generally the grains are characterized by the mean volume, $\bar{V}\left(A_{k}\right)$, the mean surface area, $\bar{S}\left(A_{k}\right)$, and the mean breadth $\bar{b}\left(A_{k}\right)$. (The breadth, $b_{k}$, of a grain, $A_{k}$, in a given direction is the distance between the two supporting parallel planes that are perpendicular to this direction. The mean value $\bar{b}\left(A_{k}\right)$ is the average of $b_{k}$ over all directions and all grains $A_{k}, k=1,2, \ldots$.

Furthermore, the distance probability function of 


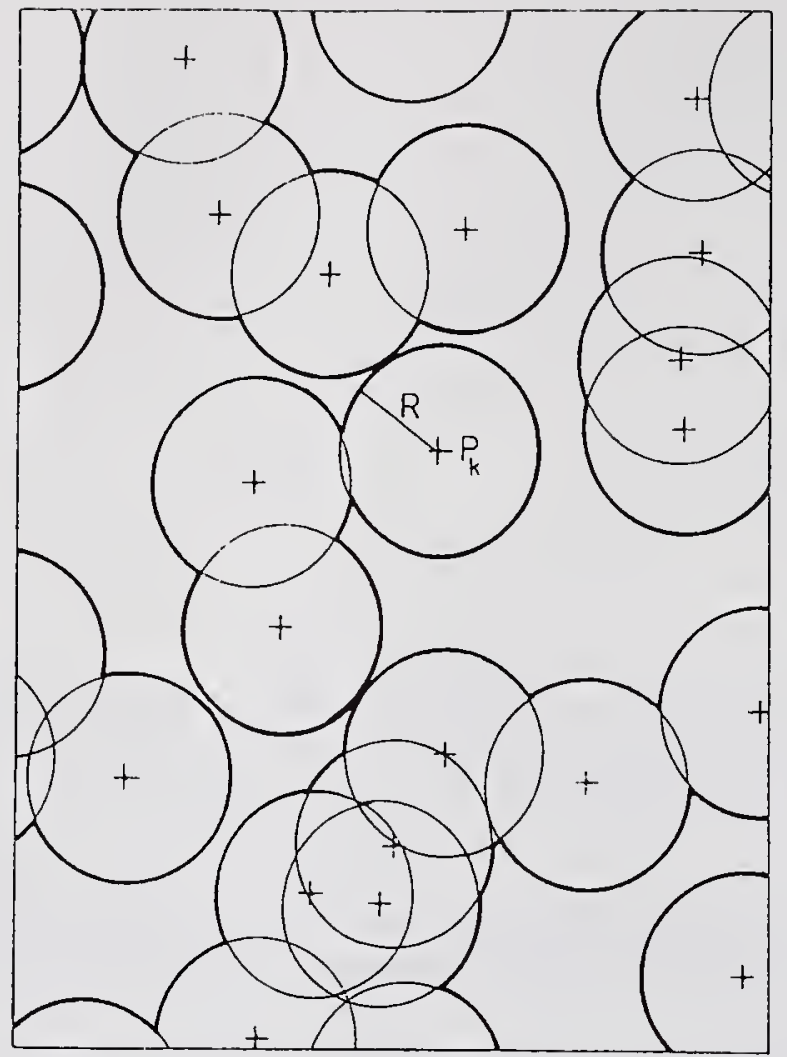

Figure 2: Generation of a planar Boolean model with identical circular grains. $R$ - radius of the grains, $P_{k}$ - germs of the model. The bold line marks the boundary of the random structure $A$ generated by the union of the grains.

a grain $A_{k}$ is

$$
\begin{gathered}
\gamma_{k}(r)=\frac{1}{2 \pi} \int_{\Omega} \int_{V} s_{k}(\vec{u}) s_{k}(\vec{u}+\vec{r}) d V d \Omega, \\
s_{k}(\vec{r})= \begin{cases}1, & \vec{r} \in A_{k} \\
0, & \vec{r} \notin A_{k}\end{cases}
\end{gathered}
$$

where the integration over $\Omega$ corresponds to the average over all spatial directions. The function

$$
\gamma^{0}(r)=\bar{\gamma}_{k}(r)
$$

is the mean distance distribution function averaged over all grains, $A_{k}$. The volume fraction of the random set $A$ is

$$
c_{A}=1-\exp \left[-\lambda \bar{V}\left(A_{k}\right)\right] .
$$

The covariance $C_{A}(r)$ of $A$, which is defined by the probability of finding two arbitrary points with distance $r$ both in region $A$, is

$$
C_{A}(r)=2 c_{A}-1+\left(1-c_{A}\right)^{2} \exp \left[\lambda \gamma^{0}(r)\right]
$$

$$
C_{A^{\mathrm{c}}}(r)=C_{A}(r)+1-2 c_{A} .
$$

The intersection of a Boolean model with straight lines generates a sequence of chords. The mean value of the chord lengths $x$ outside the random set $A$ is (Stoyan et al., 1987)

$$
\bar{l}_{B}=4 / \lambda \bar{S}\left(A_{k}\right)
$$

for three-dimensional models. For models with convex grains the chord lengths $x_{B}$ are random variables with an exponential distribution

$$
f\left(x_{B}\right)=\lambda_{1} \exp \left(-\lambda_{1} x_{B}\right), \quad \lambda_{1}=1 / \bar{l}_{B} .
$$

Here we use random Poisson polyhedra with mean breadth $b$ as grains. The following relations hold (Stoyan et al., 1987)

$$
\begin{aligned}
\gamma^{0}(r) & =\bar{V}\left(A_{k}\right) \exp (-3 r / 2 \bar{b}) \\
\bar{S}\left(A_{k}\right) & =32 \bar{b}^{2} / 3 \pi \\
\bar{V}\left(A_{k}\right) & =16 \bar{b}^{3} / 9 \pi
\end{aligned}
$$

The results for covariance and mean chord length are

$$
\begin{aligned}
C_{A}(r)= & 2 c_{A}-1+\left(1-c_{A}\right) \exp \left\{\left[-\ln \left(1-c_{A}\right)\right]\right. \\
& \times \exp (-3 r / 2 \bar{b})\}, \\
\bar{l}_{B}= & 2 \bar{b} /\left[-3 \ln \left(1-c_{A}\right)\right], \\
\bar{l}_{A}= & c_{A} \bar{l}_{B} /\left(1-c_{A}\right) .
\end{aligned}
$$

\section{Surface morphology}

The Boolean model is infinitely extended in all directions. A model with a planar surface is generated by a planar cut through the infinitely extended model. If the planar cut is carried out smoothly, the surface of the model is called smooth too. We consider the volume fraction $\alpha(t)$ of particles in the model bounded by a planar surface and its dependence on the distance $t$ from the surface. Then a sample with a smooth surface (Fig. $3 a$ ) is characterized by

$$
\alpha(t)=\left\{\begin{array}{rr}
\alpha_{0}, & t \geq 0 \\
0, & t<0
\end{array}\right.
$$

where $c_{A}=1-\alpha_{0}$ denotes the volume fraction of the pores if the random set $A$ is used to simulate the pore distribution. A rough surface is generated by a planar section of the infinitely extended model that is smooth at large dimensions but fuzzy at dimensions comparable to the size of the pores and the grains of the model (Fig. $3 b$ ). The volume fraction of a sample with a rough surface deviates from the bulk value $\alpha_{0}$ in the vicinity of the surface and may be described by the expression (Hermann \& Ermrich, 1987)

$$
\alpha(t)=\left\{\begin{aligned}
\alpha_{0}\left[1-\exp \left(-t / t_{0}\right)\right], & t \geq 0 \\
0, & t<0 .
\end{aligned}\right.
$$




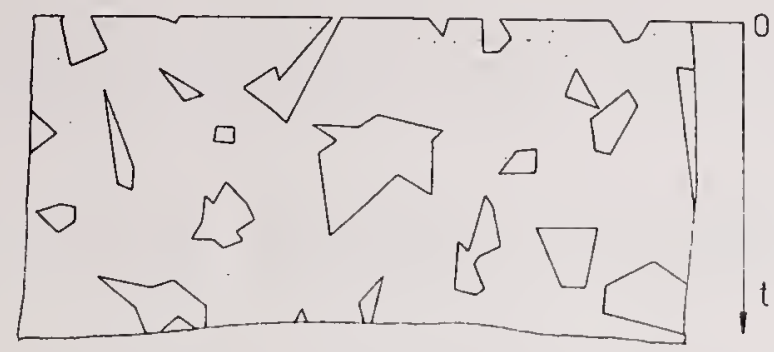

(a)

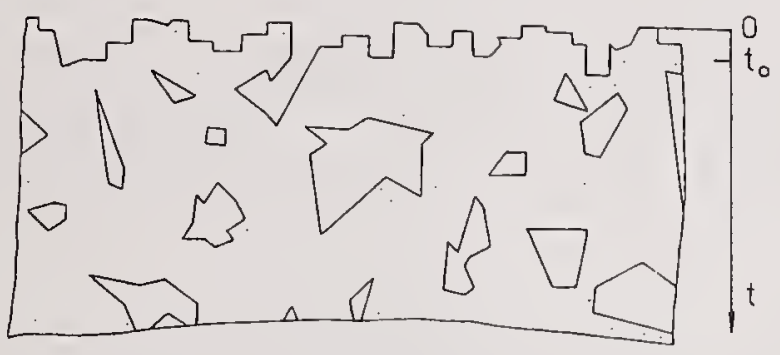

(b)

Figure 3: Simulation of surfaces of porous materials by intersecting a Boolean model. (a) Smooth surface produced by a smooth planar section through the infinitely extended model. (b) Rough surface generated by a fuzzy planar section of the Boolean model.

The difference between surfaces of the types shown in figures $3 a$ and $3 b$ will be discussed in some detail, since a precise definition of the term "surface roughness" is required for the correct interpretation of experimental data.

For this end, we calculate the mean profile depth, $\bar{t}=\langle\overline{P Q}\rangle$, and the mean fluctuation of the surface profile, $\overline{\Delta t}=\langle|\overline{P Q}-\bar{t}|\rangle$, of a smooth $\left(t_{0}=0\right)$, porous sample $(\langle\ldots)$ denotes the average over all points $P$ of the surface). We consider a point $P \in A\left(B=A^{c}\right)$ at the surface $(t=0)$ of the sample and the line $P Q$ that is parallel to the direction of the coordinate $t$ (Fig.4). The point $Q$ is the intersection point of that line with the boundary of $B$. The length $y$ of the line $P Q$ is randomly distributed. The density $h_{1}(y)$ of the probability distribution of $y=\overline{P Q}$ is approximated by

$$
\begin{aligned}
h_{1}(y) & =\lambda_{1} \exp \left(-\lambda_{1} y\right), \\
1 / \lambda_{1} & =\left(1-\alpha_{0}\right) \bar{l} / \alpha_{0}
\end{aligned}
$$

where $\bar{l}=\bar{l}_{B}$. (The exponential form of $h_{1}(y)$ applies exactly for $P \in B$. See Stoyan et al., 1987). Averaging over all points $P \in A$ and $P \in B$ and taking into account $(15,17)$ one obtains:

$$
\bar{t}=\left(1-\alpha_{0}\right) \int_{0}^{\infty} y h_{1}(y) d y
$$

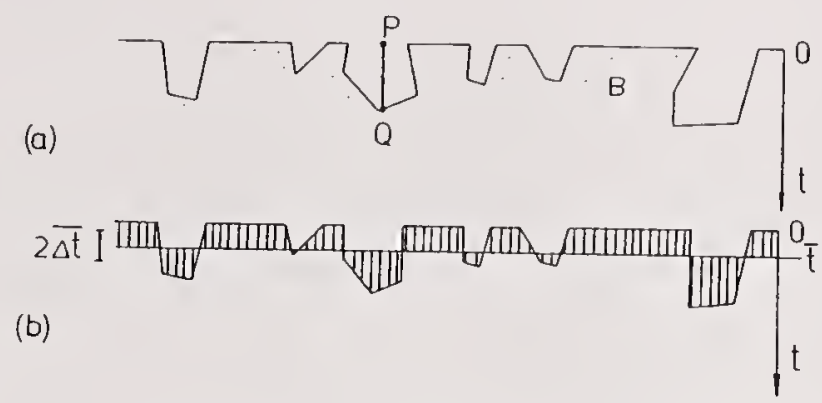

Figure 4: Surface (a) of a porous sample without surface roughness $\left(t_{0}=0\right)$ and its depth profile (b). The surface is situated at $t=0, \bar{t}$ is the mean profile depth, $\overline{\Delta t}$ is the mean depth fluctuation. $B$ represents the powder particles, and the complement of $B$ for $t>0$ describes the intersected pores.

$$
=\left(1-\alpha_{0}\right)^{2} \bar{l} / \alpha_{0} .
$$

The mean fluctuation $\overline{\Delta t}$ of the distance $\overline{P Q}$ is defined by

$$
\overline{\Delta t}=\alpha_{0} \bar{t}+\left(1-\alpha_{0}\right) \int_{0}^{\infty}|y-\bar{t}| h_{1}(y) d y .
$$

One obtains

$$
\overline{\Delta t}=2\left(1-\alpha_{0}\right)^{2} \bar{l} / \alpha_{0} \exp \left(1-\alpha_{0}\right) .
$$

The quantities $\bar{t}$ and $\overline{\Delta t}$ given by $(19,21)$ characterize the surface of a porous sample produced by a smooth planar section according to figure $3 a$. The surface roughness parameter $t_{0}$ defined in (16) vanishes for samples prepared in this way, so that, if the surface profile of a porous sample is characterized by $\bar{t}, \overline{\Delta t}$ and $(18,20)$, the degree of the surface roughness is zero. This definition is reasonable, since $\bar{t}$ and $\overline{\Delta t}$ are determined completely by the bulk parameters $\alpha_{0}$ and $\bar{l}$ of the sample.

A sample with the property $t_{0}>0$ can be simulated by the model shown in figure 5 . The quantity $z=\overline{P P^{\prime}}$ is assumed to be random with the probability density

$$
f(z)=\left(1 / t_{0}\right) \exp \left(-z / t_{0}\right), \quad z \geq 0
$$

according to (16). The probability density for the profile depth $x=y+z$ on the condition that $P^{\prime} \in B$ is

$$
\begin{aligned}
g(x)= & \int_{-\infty}^{+\infty} h_{1}(v) f(x-v) d v \\
= & \lambda_{1}\left[\lambda_{1} t_{0}-1\right]^{-1} \\
& \times\left[\exp \left(-x / t_{0}\right)-\exp \left(-\lambda_{1} x\right)\right] .
\end{aligned}
$$




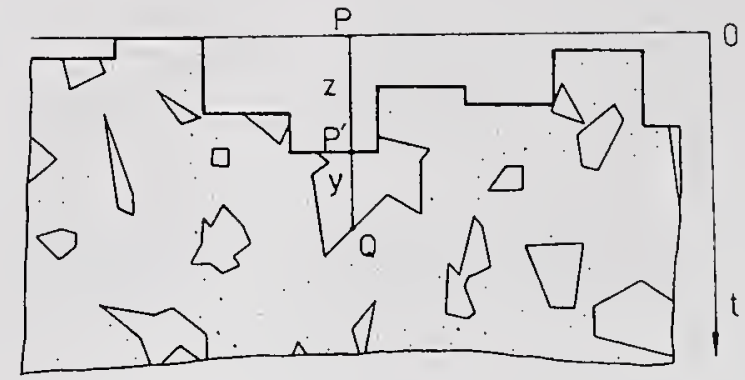

Figure 5: Model for a rough surface of a porous material. The surface is generated by a fuzzy section (bold line) through the Boolean model. Considering dimensions which are large compared to the size of pores and particles the section is planar and smooth.

The mean profile depth $\bar{t}$ of the rough surface of a porous material is

$$
\begin{aligned}
\bar{t} & =\alpha_{0} \int_{0}^{\infty} x f(x) d x+\left(1-\alpha_{0}\right) \int_{0}^{\infty} x g(x) d x \\
& =t_{0}+\left(1-\alpha_{0}\right)^{2} \vec{l} / \alpha_{0} .
\end{aligned}
$$

The mean fluctuation $\overline{\Delta t}$ is defined by

$$
\begin{aligned}
\overline{\Delta t}= & \alpha_{0} \int_{0}^{\infty}|x-\bar{t}| f(x) d x \\
& +\left(1-\alpha_{0}\right) \int_{0}^{\infty}|x-\bar{t}| g(x) d x .
\end{aligned}
$$

The integrals can be evaluated easily, but the corresponding expressions are somewhat complicated. An approximation is given by

$$
\overline{\Delta t}=2\left(1-\alpha_{0}\right)\left[\left(1-\alpha_{0}\right) \bar{l}+\alpha_{0} t_{0}\right]
$$

for

$$
t_{0} \ll \bar{l}, \quad 0.5<\alpha_{0}<1 .
$$

Now we estimate the order of magnitude of the quantities $t_{0}$ and $\bar{l}$ that can be expected for real samples. We use a hard-sphere structure (Fig.6), which is, of course, not a Boolean model, but yields limiting values for the ratio $t_{0} / \bar{l}$ for random structures. The model with vanishing surface roughness consists of all regions with $t>0$ including the spherical segments (shaded areas in figure 6). However, in a real sample prepared from equally sized spherical particles there would not be any intersected particle. Assuming tetrahedral packing, the height of the spherical segments is $R / 2$ ( $R$ is the sphere radius). If the intersecting particles are removed, the density of the sample is locally reduced in the vicinity of the surface. This reduction can be approximated by (16)

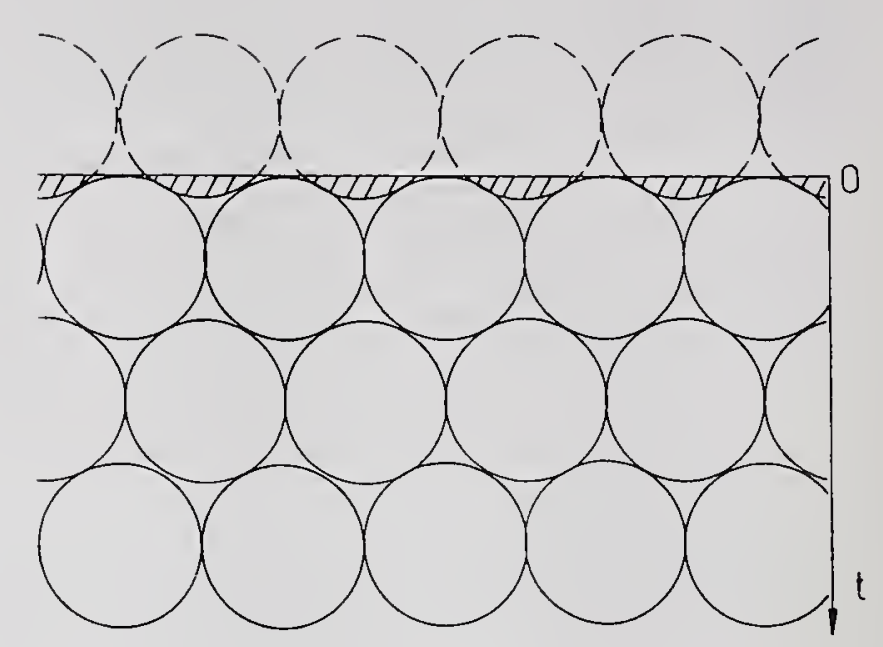

Figure 6: System of spherical particles with vanishing (shaded areas included) and non-vanishing (without shaded areas) surface roughness.

with parameter $t_{0}=0.3 R$. In a polydisperse system of spheres with mean radius $R$ the value $t_{0}=0.3 R$ is an upper limit, since holes generated by removing large intersecting particles situated at the surface can be filled by small particles. The mean chord length of the pores in a hard-sphere system is related to the mean chord length $\bar{l}_{s}=4 R / 3$ of a single sphere by $\bar{l}=\left(1-c_{A}\right) \bar{l}_{s} / c_{A}$. For a packing fraction of about 0.6 one obtains $\bar{l}=0.9 R$. The reduced surface roughness parameter $t_{0} / \bar{l}$ is therefore expected to be limited by

$$
t_{0} / \bar{l}<0.3
$$

for real powder samples with particles having approximately spherical shape. Consequently, (25) can be used to estimate $\overline{\Delta t}$ through the quantities $\bar{l}, \alpha_{0}$ and $t_{0}$.

\section{Absorption correction}

\section{Single-phase powders}

We consider X-rays diffracted symmetrically by a flat single-phase powder (Fig. 1 and $\beta=\gamma=\Theta$, where $2 \Theta$ is the scattering angle). Let $L$ be the geometrical length of a beam within the sample, and $L_{a}\left(x_{1}=L_{a}\right.$ in (1)) the corresponding path through the powder particles. $L_{a}$ is, even for fixed $L$ and scattering angle $2 \Theta$, a stochastic variable. It depends on the position of the volume element $d V$ within the plane parallel to the surface of the powder specimen. The mean value $\bar{L}_{a}$ is given by the integral over all increments $d r$ on $L$ lying in powder particles, on condition that 
the point of reflection, $Q$, (Fig. 1) is also in a powder particle. Simulating the distribution of pores by the random set $A$ explained in the previous section, one can write

$$
\begin{aligned}
\bar{L}_{a} & =\int_{P_{1}}^{Q} C_{A^{c}}(r) d r / \alpha+\int_{Q}^{P_{2}} C_{A^{c}}(r) d r / \alpha \\
& =2 \int_{0}^{L / 2} C_{A^{c}}(r) d r / \alpha .
\end{aligned}
$$

The width of the distribution of $L_{a}$ for fixed $L$ is of the order of the value $\bar{b}$ describing the mean linear dimension of the Poisson polyhedra from which the model is constructed.

Equation (1) can be written in terms of the variable $L$,

$$
I / I_{0}=\frac{1}{2} \int_{0}^{\infty} \alpha\left\langle\exp \left(-\mu L_{a}\right)\right\rangle d L
$$

where $\langle A\rangle$ denotes the average of the quantity $A$ over the distribution of $L_{a}$ for fixed $L$. If

$$
L_{a} \gg \bar{b}
$$

the width of the distribution of the random variable $L$ is small compared to $L$, and $\left\langle\exp \left(-\mu L_{a}\right)\right\rangle$ can be replaced by $\exp \left\langle-\mu L_{a}\right\rangle$. If, on the other hand, the condition (29) does not hold, one must require

$$
\mu \bar{b} \ll 1 .
$$

Then, expression $\left\langle\exp \left(-\mu L_{a}\right)\right\rangle$ can be approximated by $\left\langle 1-\mu L_{a}\right\rangle$ which is tantamount to the approximation $\left\langle\exp \left(-\mu L_{a}\right)\right\rangle=\exp \left\langle-\mu L_{a}\right\rangle$. Therefore

$$
I / I_{0}=\frac{1}{2} \int_{0}^{\infty} \alpha \exp \left(-\mu \bar{L}_{a}\right) d L
$$

is a good approximation for (28) in cases of 'fine' and 'medium' powders (cf. conditions $(29,30)$ according to the classification of powders introduced by Brindley, 1945). Expanding (12) for a low concentration of pores, $c_{A} \ll 1$, and inserting (12) and (16) into (27) one obtains (Hermann \& Ermrich, 1987)

$$
\begin{aligned}
\mu \bar{L}_{a} / \epsilon= & \tau z / \sin \Theta \\
& +\left(1-\alpha_{0}\right)[1-\exp (-\tau z / \sin \Theta)] / \alpha_{0} \\
& -(\tau / \sin \Theta)[1-\exp (-z)] \\
& +\frac{1-\exp [-(\tau+\sin \Theta) z / \sin \Theta]}{\tau+\sin \Theta} \\
\epsilon= & 2 \mu \beta \alpha_{0}, \tau=t_{0} / \beta, \beta=2 \bar{b} / 3 .
\end{aligned}
$$

Of course, the special cases $L_{a}=L$ (for $\alpha_{0}=1$ ) and $L_{a}=\alpha_{0} L$ (for $\beta / L \rightarrow 0$ ) follow from (32) for vanishing surface roughness $\left(t_{0}=0\right)$.
According to Harrison \& Paskin (1964) and Suortti (1972), we write

$$
I=I_{0}(1-P) / 2 \mu=I_{0} J,
$$

where $P$ is the term resulting from both bulk granularity and surface roughness $\left(I_{0}=\right.$ normalized intensity). Now the problem is reduced to the evaluation of the integral

$$
\begin{aligned}
J & =\int_{V} \exp \left(-\mu \bar{L}_{a}\right) d V / Q_{0} \\
& =\int_{0}^{\infty} \alpha(z) \exp \left[-\mu \bar{L}_{a}(z)\right] \beta \tau d z / \sin \Theta
\end{aligned}
$$

Assuming weak surface roughness and expanding (34) up to terms of order $\epsilon$ and $\tau^{2} / \sin ^{2} \Theta$ one obtains

$$
\begin{gathered}
P=P_{0}+P_{s}, \\
P_{0}=2 \mu \bar{l}\left(1-\alpha_{0}\right)^{2},
\end{gathered}
$$

and

$$
P_{s}=2 \mu \bar{l} \alpha_{0} \ln \frac{1}{\alpha_{0}} \frac{\tau}{\sin \Theta}\left(1-\frac{\tau}{\sin \Theta}\right) .
$$

Figure 7 illustrates the dependence of the bulk con-

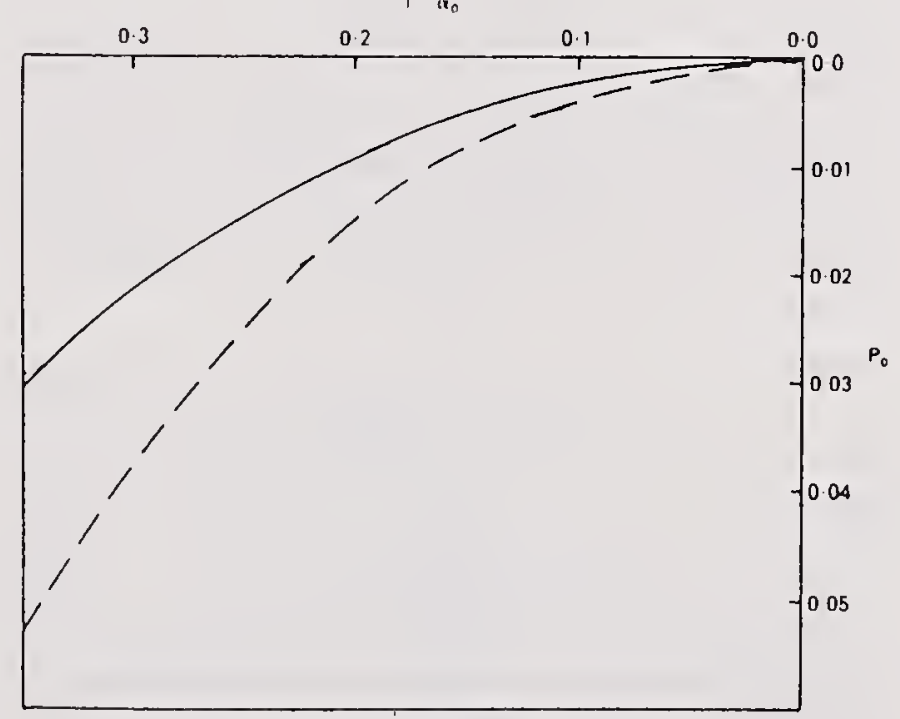

Figure 7: Dependence of bulk contribution, $P_{0}$, to absorption correction on volume fraction $\left(1-\alpha_{0}\right)$ of pores for different values $\mu \bar{l}$ (full curve $\mu \bar{l}=0.1$; broken curve $\mu \bar{l}=0.2$ ).

tribution, $P_{0}$, on the volume fraction of pores for different values of $\mu \bar{l}$, and figure 8 shows the surface contribution, $P_{s}$, and its variation with scattering angle for different degree of surface roughness. 


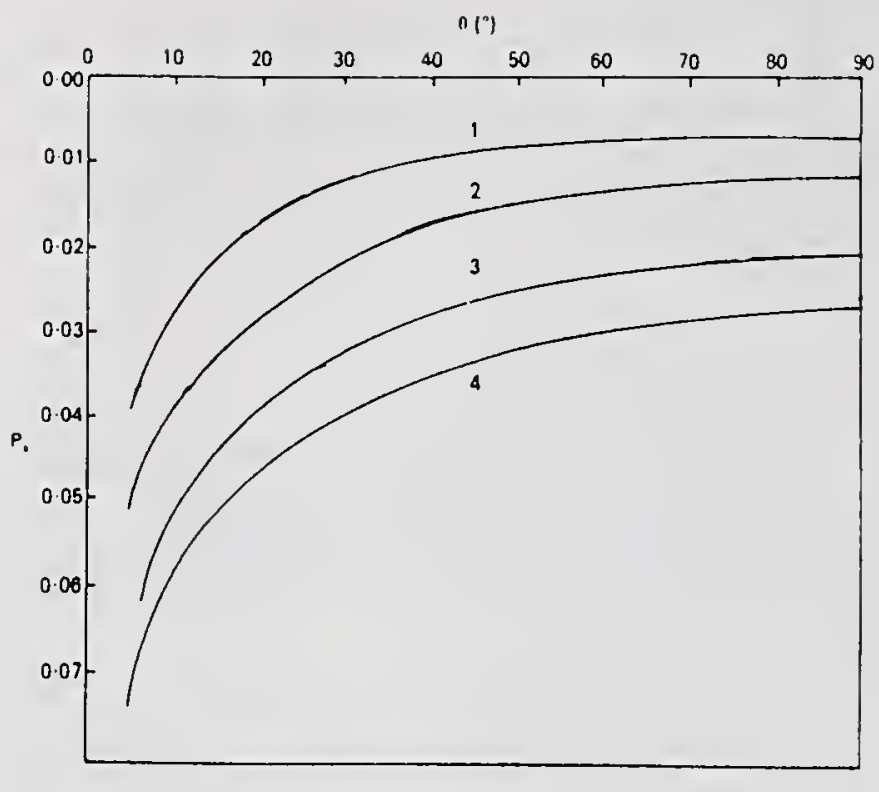

Figure 8: Dependence of surface contribution, $P_{s}(\Theta)$, on the degree, $\tau$, of surface roughness $(\mu \bar{l}=$ $\left.0.15, \alpha_{0}=0.70\right) . \quad 1: \tau=0.1 ; 2: \tau=0.2 ; 3:$ $\tau=0.4 ; \quad 4: \tau=0.6$.

\section{Multiphase powders}

The sample is assumed to consist of $n-1$ scattering phases, and the pores of the powder are denoted as phase $n$. We consider a beam diffracted by phase $i$. The distribution of $i$-type particles is described by a Boolean model with volume fraction $c_{i}$ and covariance $C_{i i}(r)$ given by $C_{A}(r)$ in (12). Taking into account that

$$
\mu_{(i)}=\frac{1}{1-c_{i}} \sum_{j \neq i} c_{j} \mu_{j},
$$

and

$$
\bar{\mu}=\sum_{j=1}^{n} c_{j} \mu_{j},
$$

Equation (1) can be evaluated in the same way as for single-phase powders but using

$$
\bar{L}_{i}=2 \int_{0}^{L / 2} C_{i i}(r) d r / c_{i}
$$

instead of (27) and replacing (30) by

$$
\left|\mu_{i}-\bar{\mu}\right| \bar{l} \ll 1 \text {. }
$$

Considering the bulk contribution one obtains for a low volume fraction, $c_{i}$, of phase $i$

$$
I_{i} / I_{0, i}=\frac{1}{2} c_{i} \int_{0}^{\infty} \exp \left[-\mu_{i} \bar{L}_{i}-\mu_{(i)}\left(L-\bar{L}_{i}\right)\right] d L,
$$

$$
\begin{gathered}
I_{i} / I_{0, i}=\frac{c_{i}}{2 \mu_{i}^{\prime}}, \\
\mu_{i}^{\prime}=\frac{\bar{\mu}}{1-2\left(1-c_{i}\right)\left(\mu_{i}-\bar{\mu}\right) \bar{l}_{i}},
\end{gathered}
$$

and

$$
\sum_{j=1}^{n} c_{j}=1
$$

Equations (43 to 45 ) can be used to correct for experimental scattering intensities obtained from samples with negligible surface roughness. However, generally both $c_{i}$ and $\bar{l}_{i}, i=1, \ldots, n-1$, are unknown quantities. That means that one set of scattered intensities, $I_{i}, i=1, \ldots, n-1$, will not be sufficient to carry out the complete microabsorption correction. Hermann \& Ermrich (1989) suggested recording a second set of intensities using radiation of a different wavelength. Then the absorption contrast, $\left(\mu_{i}-\bar{\mu}\right)$, will be modified, a second, independent set of equations can be established to eliminate the parameters $\bar{l}_{i}$ or to estimate both $c_{i}$ and $\bar{l}_{i}, i=1, \ldots, n-1$, and the microabsorption correction can be carried out. Ermrich \& Hermann (1990) applied the method of different wavelengths to study the microabsorption effect in quantitative phase analysis. Figure 9 illus-

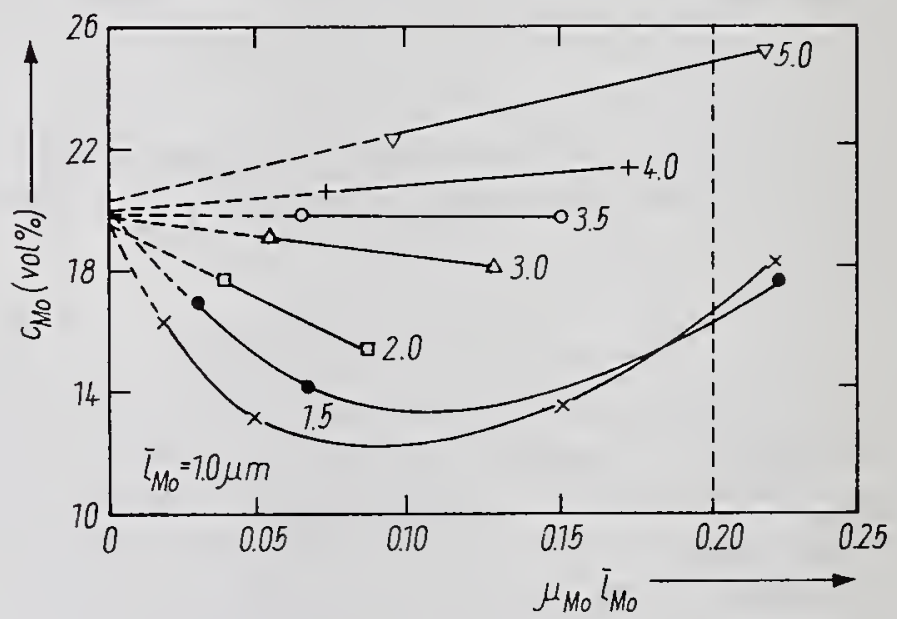

Figure 9: Volume fraction $c_{M o}$ versus $\mu \bar{l}_{M o}$ estimated by quantitative phase analysis of siliconmolybdenum powder including microabsorption correction.

trates the effect. A mixture of molybdenum and silicon powder was analyzed using five different wavelengths. The scattering intensities were corrected for by means of (43 to 45 ) assuming various values for $\bar{l}$. (The value for molybdenum was estimated from electron micrographs: $\bar{l}_{M o}=2.5 \pm 0.5 \mu \mathrm{m}$, particle size distribution from 0.1 to $30 \mu \mathrm{m}$ ). If the correct $\bar{l}$-value 
is tried, the estimated volume fraction should not depend on the wavelength used, but should approach the true value $\left(c_{M_{0}}=19.4 \mathrm{vol} \%\right.$ in figure 9$)$. Indeed, this is the case for assumed $\bar{l}_{M o}=3$ to $3.5 \mu \mathrm{m}$, and the result for the volume fraction of molybdenum particles obtained from the corrected intensities is $19.8 \mathrm{vol} \%$. (The results of the quantitative phase analysis without microabsorption correction but with assumed $\mu_{i}^{\prime}=\bar{\mu}$ in (43) varied from $7.5 \%$ for $\lambda=0.22911 \mathrm{~nm}$ to $15.2 \%$ for $\lambda=0.07107 \mathrm{~nm}$.)

\section{Influence of particle shape}

Since expression (44) has been derived for a special structure model, the question arises whether it is also typical for other random structures. Therefore, we consider a Boolean model with equally sized spheres as grains. This model represents a good contrast to the structure based on the polydisperse set of randomly shaped Poisson polyhedra used for the derivation of (35) and (44) The covariance for a Boolean model composed of spheres with radius $r_{i}$ is

$C_{i i}(r)=\left\{\begin{array}{l}2 c_{i}-1+\ldots \\ \ldots\left(1-c_{i}\right) \exp \left\{-\left[\frac{r^{3}}{16 r_{i}^{3}}-\frac{3 r}{4 r_{i}}\right] \ln \left(1-c_{i}\right)\right\} \\ r \leq 2 r_{i} \\ c_{i}^{2}, r>2 r_{i} .\end{array}\right.$

Inserting (46) into (40) and (42) one obtains for $c_{i} \ll$ 1 and condition (41)

$$
I_{i} / I_{0, i}=\frac{c_{i}}{2 \bar{\mu}}\left[1-k\left(1-c_{i}\right)\left(\mu_{i}-\bar{\mu}\right) \bar{l}_{i}\right],
$$

with

$$
k=\left\{\begin{aligned}
9 / 8, & \text { spheres } \\
2, & \text { polyhedra }
\end{aligned}\right.
$$

The mean chord length, $\bar{l}_{i}$, of particles is, in the limit of small volume fraction of phase $i\left(c_{i} \ll 1\right), \quad \bar{l}_{i}=$ $\bar{b}_{i} /\left(1-c_{i}\right)$ for Poisson polyhedra and $\bar{l}_{i}=4 r_{i} /\left(1-c_{i}\right)$ for spheres.

Expressions (47) and (48) show that the intensity of an X-ray beam reflected symmetrically by a planar sample does not only depend on the absorption contrast $\left(\mu_{i}-\bar{\mu}\right)$ and the mean chord length $\bar{l}_{i}$ but also on the shape of the scattering particles (Hermann et al., 1991). Because of the restriction $c_{i} \ll 1$, within the random set produced by the Boolean model, only a few overlaps of grains appear. Therefore, the shape of the scattering regions of the present structure model is similar to that of the grains used for the construction of the random set, and we may conclude from (47) and (48) that the absorption caused by a random arrangement is remarkably enhanced with increasing polydispersity and irregularity of the particles if systems with equal mean chord lengths of scattering particles are considered. The typical dependence of the absorption effect on the linear absorption coefficients, the volume fraction and the size of $i$-type particles is, however, retained.

\section{Grazing incidence}

For non-symmetric geometry of incident and reflected X-rays (Fig.1: $\beta \neq \gamma$ ) and vanishing surface roughness $(\tau=0)$ Eq.(1) yields the well-known formula

$$
I / I_{0}=1 /[\mu(1+\sin \beta / \sin \gamma)] .
$$

Considering a single-phase powder of packing fraction $\alpha_{0}$ and $\tau=0$ one obtains

$$
I / I_{0}=(1-P) /[\mu(1+\sin \beta / \sin \gamma)]
$$

and

$$
P=P_{o}=\mu \bar{l}\left(1-\alpha_{0}\right)^{2}, \quad \sin \beta \ll \mu \bar{l} .
$$

That means the bulk contribution, $P_{0}$, of the microabsorption effect takes half the value of $P_{0}$ for symmetric (Bragg-Brentano) geometry. The following arguments show that, compared to the symmetric beam geometry, the strength of the surface contribution is reduced as well when using the grazing incidence method: If the angle of incidence is very small,

$$
\sin \beta \ll \mu \bar{l}
$$

the sample is irradiated down to the depth, $t_{s}$, of the order of magnitude of $t_{s}=\sin \beta / \alpha_{0} \mu$. Taking into account (52) and the estimate (26) for the maximum value of surface roughness one obtains

$$
t_{s} \ll 3 t_{0} .
$$

That means, at least for strong surface roughness, that the diffracted $\mathrm{X}$-ray does not feel the total width, $\overline{\Delta t}=2\left(1-\alpha_{0}\right) \alpha_{0} t_{0}$, of the surface roughness profile fluctuation (see (25)) on its path through the sample. Consequently, at grazing incidence the systematic error of the scattering intensity caused by the $\Theta$-dependent surface roughness contribution of the microabsorption must also be expected to be well below the error appearing for Bragg-Brentano geometry. The grazing incidence geometry is therefore proposed as a tentative method of minimizing the bulk and surface roughness microabsorption effect.

\section{Conclusion}

Microabsorption affects the intensity of X-rays diffracted by highly absorbing granular materials considerably. The deviation of the measured intensity from the intensity expected for vanishing microabsorption may amount to the order of magnitude 
of the latter itself. This has been proved experimentally both for the bulk contribution and the surface roughness effect. The systematic error of scattering intensities due to microabsorption can be estimated by means of analytical expressions derived for random powders and variable microstructural parameters.

One should try to reduce the effect by suitable sample preparation (e.g. powder particles as fine as possible) and minimization of the absorption contrast (suitable choice of the X-ray wavelength). The grazing incidence method is also expected to bring about a weakening of the microabsorption effect.

For high-accuracy measurements and for those cases were the microabsorption effect cannot be avoided the analytical expressions presented above should be used to correct for the measured X-ray intensities. Then, the microstructural parameters 'mean chord length of particles', 'volume fraction' and 'surface rouhgness' must be determined by other experimental techniques, e.g. electron microscopy and quantitative metallography, or by carrying out scattering experiments at different wavelengths. It should also be possible to incorporate the expression describing the surface roughness contribution into Rietveld programs and to fit the unknown parameters.

Of course, one can also make use of the microabsorption effect to estimate microstructural parameters of granular bulk materials and rough surfaces.

\section{References}

Brindley, G. W. (1945). Philos. Mag. 36, 347-369.

Ermrich, M. \& Hermann, H. (1990). Phys. Status Solidi A118, K1-4.

Harrison, R. J. \& Paskin, A. (1964). Acta Cryst. 17, 325-331.

HermanN, H. (1991). Stochastic Models of Heterogeneous Solids. Zurich: Trans Tech Publ. Ltd.

HermanN, H. \& ERMrich, M. (1987). Acta Cryst. A43, 401-405.

Hermann, H. \& Ermrich, M. (1989). Powder Diffraction 4, 189-195.

Hermann, H., Pitschke, W. \& Mattern, N. (1992). Phys. Status Solidi A132, 103-114.

HermanN, H., Röske, K. \& Hentschel, M. (1991). Acta Cryst. A47, 446-447

Klug. H. P \& AlexANDER, L. E. (1974). X-Ray Diffraction Procedures 2nd Ed. New York: Wiley.

KoCH, B. \& MACGILLAVRY, C. H. (1985). International Tables for X-Ray Crystallography, C. H. MacGillavry \& G. D. Rieck, eds. 3, 157-192.

KoopMans, K. \& RIECK, G. D. (1985). International Tables for X-Ray Crystallography, C. H. MacGillavry \& G. D. Rieck, eds. 3, 194-199.

Kumar, R., Sparks, C. J., Shiraishi, T., Specht, E. D., Zschack, P., Ice, G. E. \& Hisatsume, K. (1991). Mat. Res. Soc. Symp. Proc. 213, 369-374.

Masciocchi, N., Toraya, H. \& Parrish, W. (1991). Mater. Sci. Forum79-82, 245-250.

Pitschke, W., HermanN, H. \& Mattern, N. (1991). Powder Diffraction, to appear.

Sparks, C. J., Kumar, R., Specht, E. D., Zschack, P. \& ICE, G. E. (1991). Advances in X-Ray Analysis - PIXAM Conf., Hawaii, to appear.

Stoyan, D., Kendall, W. S. \& Mecke, J. (1987). Stochastic Geometry and Its Applications. Chichester: Wiley.

SuORTti, P. (1972). J. Appl. Cryst. 5, 325-331.

Trucano, P. \& Batterman, B. W. (1970). J. Appl. Cryst. 41, 3949-3953.

WoLfF, P. M. DE (1956). Acta Cryst. 9, 682-683. 
National Institute of Standards and Technology Special Publication 846. Proceedings of the international conference Accuracy in Powder Diffraction II, held at NIST, Gaithersburg, MD, May 26-29, 1992. (Issued October 1992)

\title{
Computer-Aided Phase Identification
}

\author{
M. NAKHMANSON \\ Comphys Lab Enterprise, NPO "Bourevestnik" \\ St. Petersburg, 195272 Russia
}

Considered in this paper are two problems connected with the use of powder diffraction standards base: identification and classification. Identification of $\mathrm{X}$ ray patterns or qualitative phase analysis for a long time was considered a single problem deserving contemplation. As a matter of fact the PDF file has been created just for solution of this problem. The available database which is a result of many-year efforts of JCPDS usually allows solution of some other non-traditional problems as well. One of these problems, which is directly connected with identification, is considered in the second part of the paper.

\section{Identification}

Let us consider mathematical formulation of identification problem. Assume that there is a set $\mathbf{A}$ of some objects $a_{1}, a_{2}, \cdots, a_{i}, \cdots, a_{n}$, where $n$ is the number of objects:

$$
\mathbf{A}=\left(a_{i}\right)=\left(a_{1}, a_{2}, \cdots, a_{i}, \cdots, a_{n}\right)
$$

In our case objects $\left(a_{i}\right)$ are various substances (compounds). Each object can be described by a collection of attributes or parameters $\left(p_{i k}\right)$ :

$$
\mathbf{P}_{i}=\left(p_{i k}\right)=\left(p_{i 1}, p_{i 2}, \cdots, p_{i k}, \cdots, p_{i m_{i}}\right)
$$

where $m_{i}$ is the number of attributes for the $i$-th object and $\mathbf{P}_{i}$ is an image of object $a_{i}$. In our case, for example, values of interplanar spaces $d$ and/or intensities $\mathbf{I}$ in a stick diagram serve as parameters $\mathbf{P}$; atomic numbers of elements constituting compound $a_{i}$ can also be added to them.

A set of all the $n$ images $\mathbf{P}=\left\{p_{i}\right\}$ (empirical standards) forms a database that contains information about objects of the set $\mathbf{A}$. The totality of all the possible values $p_{i k}$ forms a space of search parameters $\mathbf{R}$ in which point or vector $\mathbf{P}_{\boldsymbol{i}}$ corresponds to each image $\mathbf{P}_{\boldsymbol{i}}$. The space $\mathbf{R}$ can be defined by means of several well- known methods that I shall not dwell upon. They are considered in works of Fiala (1972; 1976) and in the book (Nakhmanson \& Feklichev, 1990).
Let us consider the identification problem as a first approximation limiting it by the following idealized conditions:

A. All parameters $p_{i k}$ are known absolutely exactly, i.e. there exists one-to-one correspondence between sets $\mathbf{A}$ and $\mathbf{P}$ : one and only one image $\mathbf{P}_{\boldsymbol{i}}$ corresponds to each object $a_{i}$, and vice versa. This means that all the points in $\mathbf{R}$ are absolutely distinguisable.

B. All parameters $p_{i k}$ have different numerical values or, at least, they are distributed in $\mathbf{R}$ uniformly.

C. If an unknown material is a mixture of some objects from the set $\mathbf{A}$, its image $\mathbf{Q}=\left\{q_{s}\right\}$ is an exact linear combination of corresponding images from $\mathbf{P}$ :

$$
\mathbf{Q}=\sum_{j} c_{j} \mathbf{P}_{j}
$$

Summed up in (3) are images of objects forming the unknown mixture. Condition $\mathrm{C}$, in particular, means that when image $\mathbf{Q}$ is obtained in experiment there are no losses of information even if some coefficients $c_{j}$ in equation (3) are very small.

If conditions $\mathrm{A}, \mathrm{B}, \mathrm{C}$ are satisfied, the identification procedure comes to comparison of parameters of each sample $\mathbf{P}_{\boldsymbol{i}}$ with sequence $\left\{g_{s}\right\}$ and selection from the database of those standards whose parameters exactly coincide with some numbers of $\left\{q_{s}\right\}$. In this case there are no such situations as ambiguity of selection (because of conditions A and B) and loss of low-concentration phases (because of condition $\mathrm{C}$ ). There is only one problem left, connected with the speed of the identification process. It can be increased sufficiently if search and comparison are carried out in several steps by means of a number of auxiliary files separated from the main database.

Let us turn to a second approximation, which better corresponds to reality. Let us estimate consequences resulting from violation of conditions $A, B, C$. 
Violation of condition A. Parameters of our standards are not known with absolute accuracy. If we take values $d$ (database images that contain the greater amount of information) as $p_{i k}$, the compounds with similar crystallochemical characteristics have almost the same sets of $d$. Sometimes, because of error in experiment these sets in practice are indistinguishable (e.g. for magnetite $\mathrm{Fe}_{3} \mathrm{O}_{4}$ and magnesioferrite $\mathrm{MgFe}_{2} \mathrm{O}_{4}$ ). Therefore one-to-one correspondence between $\mathbf{A}$ and $\mathbf{P}$ is broken, and some points in the space $\mathbf{R}$ are indistinguishable, which is the first cause of identification ambiguity.

Violation of condition $B$. Many interplanar spaces d belonging to various samples $\mathbf{P}_{\boldsymbol{i}}$ have the same numerical value, and their frequency distribution $U(d)$ is not uniform. The same relates to parameters I and $\mathrm{N}$ and their frequency distributions, $\mathrm{V}(\mathrm{I})$ and $\mathrm{W}(\mathrm{N})$. Distribution $U(d)$ is a unimodal curve with a maximum within $1-2.5 \AA$ that sharply decreases towards small $\mathrm{d}$ and smoothly decreases towards large d; distributions $\mathrm{V}(\mathrm{I})$ and $\mathrm{W}(\mathrm{N})$ are of hyperbolic form. So for $\mathbf{d}$ belonging to $\mathbf{P}$ that are within the range 1 $2.5 \AA$ there exists a high probability of random coincidences (within a certain range of $d$ ) with values $d$ belonging to $\mathbf{Q}$. If in the database $\mathbf{P}$ there are images - X-ray patterns - for which the set of $d$ totally or mostly is within $1-2.5 \AA$, such "short" X-ray patterns will very often coincide with $\mathrm{X}$-ray patterns of objects under study. Similar X-ray patterns are contained in the database, and their amount is at least $3-5 \%$ of the total number of objects $n$ (e.g. 4-783, $\mathrm{Ag} ; 4-784, \mathrm{Au} ; 4-787, \mathrm{Al} ; 4-806, \mathrm{~W} ; 4-836, \mathrm{Cu} ; 6-642$, TiN; 6-675, C; etc.) If by chance two or three of the strongest lines of "short" X-ray patterns from $\mathbf{P}$ coincide with some lines of an unknown pattern they in practice cannot be distinguished from low-concentration phases. This is the second cause of identification ambiguity.

Violation of condition $C$, which always takes place in real experiments (e.g. the loss of weak lines of low-concentration phases), brings about additional uncertainty in identification results. Thus, if two strongest lines of diamond 6-675 with d,I equal to $2.06 \AA, 100$ and $1.26 \AA, 30$ coincide with two lines of the unknown that can be lines of some other phases, one can consider that:

1. This coincidence is accidental, and there is no diamond in the mixture;

2. This coincidence is not accidental, and there is diamond in the mixture as a low-concentration phase, but its two strongest lines overlap lines of basic phases and the other four weak lines were simply not recorded in the experiment.
Violations of conditions A, B or C lead to the result that the identification problem becomes mathematically incorrect (instable by Adamar), and its solution has a probabilistic but not accurate character. In the initial stage of development of automatic identification systems in 1965 - 1985 there were many attempts to create complex algorithms for solution of the problem and to construct complex criteria (FOM) permitting determination of correspondence between the standard and identified X-ray patterns. The credit for development of these methods is shared by J. Hanawalt, L. Frevel, G. G. Johnson, R. Jenkins, R. L. Snyder, J. Fiala and other scientists. However, I would like to emphasize that ambiguity of identification is connected not so much with imperfection of their methods and programs as with objective characteristics of the database: availability in it of similar X-ray patterns as well as of "short" $X$-ray patterns of low information value.

Let us try to lay down ways to decrease identification ambiguity, taking into account the above analysis. Before this we should improve formulation of the goals of qualitative analysis.

Practically all modern systems of automatic identification can determine the basic phases reliably except for the cases when compounds with very similar X-ray patterns (such as magnetite and magnesioferrite) are amongst them. But this is the simplest problem. Much more difficult is the problem of correct differentiation of minor phases without including objects having anomalous short X-ray patterns. I think that this is the main goal of automatic qualitative analysis.

Until now we have usually taken an X-ray pattern, $\mathrm{d}$ and $\mathrm{I}$, to be the parameters $\left\{p_{i k}\right\}$. The traditional method of making the identification results more exact uses an expansion of the search space $\mathbf{R}$, adding information about the chemical compositions of the database standards and the unknown material. However, this method does not always permit getting rid of identification ambiguity. For example, it is impossible to make a choice between $\mathrm{Fe}_{3} \mathrm{O}_{4}$ and $\mathrm{MgFe}_{2} \mathrm{O}_{4}$ if some other phases of the mixture include magnesium (a mixture of magnetite, $\mathrm{Fe}_{3} \mathrm{O}_{4}$, and sellaite, $\mathrm{MgF}_{2}$ is a typical example).

The possibility of expansion of the space $\mathbf{R}$ is limited because only those parameters of substances that obey the additive relationship (3), e.g. infrared spectra, can be included in it. In particular, for multiphase objects, which are our main interest, optical and thermodynamical data are omitted from consideration.

An important step in increasing identification reliability is the use of local methods. By means of an Xray microanalyzer one can obtain data about the el- 
emental composition of each phase; this information is much more valuable then information about chemical composition of the unknown mixture as a whole. In this case there is a partial localization in $\mathbf{R}$ of a number of subspaces corresponding to phases of the mixture, and, as a result, it is possible to solve such problems as choice between $\mathrm{Fe}_{3} \mathrm{O}_{4}$ and $\mathrm{MgFe}_{2} \mathrm{O}_{4}$ or reliable classification of diamond as a false or true phase of low concentration. Actually local methods can eliminate ambiguity of both the first and second type with the same reliability with which they provide information about elemental composition of separate phases of the mixture.

Special preparation of analyzed material, i.e. its division into several samples, each being enriched with one of the constituent phases, is a highly promising method of increasing analysis reliability. In this case several $\mathrm{X}$-ray patterns are entered into the automatic identification system and comparison of their intensities permits determination of minor phases and elimination of ambiguity of the second type. Using the data of quantitative elemental analysis, one can also try to eliminate ambiguity of the first type. This method has been suggested by $\mathrm{J}$. Fiala.

Last in our store of methods we have the increase in accuracy of diffraction measurements and replacement of database standards by more reliable ones. This method corresponds to an approximation to the idealized condition A.

Let us sum up this theoretical discussion of the identification problem to form a clear view of the situation. The article of Johnson (1979) contains a partly humorous but very exact description of a number of requirements that should be met by both the X-ray pattern of material under study (image $\mathbf{Q}$ ) and the skill of the operator who uses the automatic identification program. In particular, Johnson has written the following: "The system is not a cure-all. When the computer prints out the results, it is at that point that the intelligent analyst starts to analyze the results - not just say this is the answer".

I would like to add a pair of additional points to Johnson's words, proceeding from my own experience:

1. There always exist X-ray patterns that can be identified by none of qualitative analysis programs.

2. There always exists a user who thinks that a computer should do all the work for him.

\section{Clusterization}

Let us try to carry out a more profound analysis of the first cause of identification ambiguity. It would be very interesting to clear up "the arrangement" of the database from a crystallochemical point of view: how many groups of similar compounds are in it? what are sizes of these groups? and, last, whether they exist in reality or only in our imagination. The problem formulated in such a way actually comes down to classification of the database, i.e., to separation of similar images $\mathbf{P}_{\boldsymbol{i}}$ in groups (clusters). I, myself, always would like to see these clusters; this, to a great degree, influenced the graphical design of our cluster programs.

First of all, I want to note that classification in space $\mathbf{R}$ is inexpedient. Sets of $d$ spacings for any object of the set $\mathbf{A}$ are totally determined by a crystal system and the values of the cell parameters, so it is considerably simpler to realize clusteriazation in a six-dimensional space of linear and angular parameters of lattice cells. The first program created for this purpose permits one to carry out the following:

1. Selection of crystal system, two parameters (in the general case out of six) and measurement limits for these parameters, e.g. $a_{\min }, a_{\max }$, $c_{\min }, c_{\max }$.

2. Selection from the database of all the compounds in the given crystal system whose parameters are within the given range, e.g. all compounds in the hexagonal system for which $2 \AA<a<20 \AA, 2 \AA<c<20 \AA$.

3. Display on a monitor screen each compound by a point in Cartesian axes $x$ and $y$ corresponding to the first and second of the given crystal parameters (in our example for the hexagonal system $x=a, y=c$ ).

4. An opportunity for the user to outline with a closed line any part of the screen and to obtain the following information about pointscompounds in it:

(a) chemical formulas of compounds;

(b) their names and numbers in the PDF;

(c) stick diagrams (which were displayed on the screen).

A better program that is under development now will provide two more important functions to this list: 
Table 1: Clusters for minerals in the tetragonal system, classified by the $a$ and $c$ lattice parameters.

\begin{tabular}{|llcc|}
\hline No & \multicolumn{1}{c}{ Group } & \multicolumn{2}{c|}{ Ranges of lattice parameters } \\
& & $a, \AA$ & $c, \AA$ \\
\hline 1 & Rutile type & $4.2-5.0$ & $2.6-3.4$ \\
2 & $\mathrm{MgSb}_{2} \mathrm{O}_{6}$ type & $4.6-4.75$ & $9.15-9.25$ \\
3 & $\mathrm{Ag}_{2} \mathrm{FeSnS}_{4}$ type & $5.3-5.75$ & $10.4-11.0$ \\
4 & $\mathrm{CaWO}_{4} \& \mathrm{PbMoO}_{4}$ types & $5.2-5.45$ & $11.4-12.1$ \\
5 & $\mathrm{CaCrO}_{4} \& \mathrm{YVO}_{4}$ types & $7.0-7.25$ & $6.25-6.35$ \\
6 & $\mathrm{Ca}\left(\mathrm{UO}_{2}\right)_{2} \mathrm{AsO}_{4}$ & $6.95-7.2$ & $8.6-9.1$ \\
& type & & $16.8-21.0$ \\
7 & Melilites & $7.5-7.85$ & $5.0-5.1$ \\
& & $10.5-10.6$ & 9.9 \\
8 & $\mathrm{KMn}_{8} \mathrm{O}_{16}$ type & $9.85-10.1$ & $2.85-2.95$ \\
9 & Scapolites & $12.0-12.35$ & $7.35-7.6$ \\
\hline
\end{tabular}

5. The possibility of selecting objects not only according to crystal systems but also according to space groups, chemical composition and a number of other attributes as well.

6. The possibility of selecting as axes $x$ and $y$ not only crystal parameters but any of their functions, such as cell volume, $a / c$ ratio, etc.

These programs permit one to obtain on the computer screen a database section for any parameters; this plane image is very convenient for subsequent interpretation by the user.

Studies have revealed clusters of various shapes and sizes, ranging from strongly localized (within 0.5 $\AA$ for linear crystal parameters) to spread out or extended along one axis (over $1-3 \AA$ ). The main clusters for minerals in the tetragonal crystal system are presented as an example in table 1.

Let us note that in the book by Nakhmanson \& Feklichev (1990) the results of studies that have been carried out are presented in detail. This book is published in Russian but, tables and graphs are intelligible even to English speaking readers. These results lead to the following two conclusions the first of which concerns the topic of this paper, i.e. the identification problem, and the second one is of more general character.

1. Compounds found to be in strongly localized clusters with similar values of crystal parameters have, naturally, very similar X-ray patterns. Although the number of such clusters is not so large, some common or industrially important compounds: spinels, tourmalines, micas, chlorites and a number of others are among them. These compounds are common in the practice of X-ray phase analysis, but operation with them requires special accuracy because of possible identification ambiguity.
2. I can not imagine yet in full measure the capabilities of cluster programs, but it is clear now that they will permit extension of the field of use of the PDF and other similar databases far beyond the limits of the traditional identification problem. In particular, using these programs it is possible to carry out classification of compounds, to find interchangeable materials, and to construct various statistical distributions. However, it seems to me that the most curious question is why some bond lengths between atoms exist in natural and synthetic materials while others do not. We can suppose that this is connected with allowed ionic radii.

\section{References}

Fiala, J. (1972). J. Phys. D: Appl. Phys., 5, 1874-1876. Frala J. (1976). J. Appl. Cryst., 9, 429-432.

Johnson, J. J. (1979). Norelco Reporter, 26, No.1.

NaKhMANSON, M. \& FeKLICHEV V. (1990). Diagnostika Sostava Materialov Rentgenodifrakcionnimi i spectralnymi metodami, Mashinostroenie 


\title{
On the Relative Merits of Cameras and Diffractometers for XRD
}

\author{
P.-E. WERNER \\ Department of Structural Chemistry, Arrhenius Laboratory, \\ S-106 91 Stockholm, Sweden.
}

\begin{abstract}
Obviously there are a large number of studies when Debye- Scherrer and Guinier film techniques are so clearly inferior that no comparison with diffractometers are meaningful. This is not the case for determination of diffraction angles, however. There are only marginal differences in the quality of d-spacings obtainable by modern diffractometers and Guinier systems, but preferences are extremely divergent. However, a film is a detector and a long-time mass storage, whose information might be evaluated any time by a densitometer system or visually inspected. So, the exchange of a film against another recording device requires some good arguments. It will be shown that modern automatic Guinier film scanner systems need not be liable to more subjective influences than automatic diffractometers. The speed and accuracy of a computerized evaluation system for Guinier photographs will be demonstrated. All X-ray diffraction instruments need careful adjustment, but a well adjusted and carefully handled Guinier camera is usually stable for years. Only diffractometers with focussing incident beam monochromators may compete with the resolution of a Guinier photograph, but for profile refinements Guinier and Debye-Scherrer photographs suffer from poorly defined peak shapes. For preliminary studies, including structure determinations and Rietveld refinements, the Guinier technique is a cheap and efficient alternative. It will be argued that a modern laboratory working in the field of X-ray powder diffraction should use diffractometer and camera techniques in parallel.
\end{abstract}

\section{Introduction}

Although not new, the technique of X-ray diffraction is still developing and improving. New X-ray sources and detectors, and new ways of using them in computer-controlled diffractometers, make more advanced experiments possible. During the last few years we have seen time-resolved studies made by electronic position-sensitive detectors, PSDs. Investigations of crystallite size, microstructural imperfections, stacking faults and compositional inhomogenities are, with few exceptions, made with powder diffractometers. Why then should anybody use oldfashioned film methods in powder diffraction work?

Obviously there are a large number of problems where Debye-Scherrer and Guinier film techniques are so clearly inferior that no comparison with diffractometer methods are meaningful. If it were only of interest to judge in general terms how to collect the most accurate powder diffraction data, it should be enough to quote from the preface, by Delhez and Mittemeijer, to the Proceedings of the first European Powder Diffraction Conference held in Munich, Germany, March 1991: "Although this has been the subject of rather recent debate, it is now possible to state that the accuracy achievable with a modern diffractometer in principle surpasses that of the photographic techniques." At first sight this statement seems to be final and sufficient. There are some points in this statement, however, that need to be discussed. What is meant by "in principle," and what is a "modern diffractometer"?

Is a modern diffractometer really the conventional automatic powder diffractometer using a monochromator on the reflected beam side, or is it a diffractometer with an incident-beam focusing monochromator yielding pure $\mathrm{CuK} \alpha_{1}$ radiation? Is it a diffractometer with Bragg-Brentano geometry and a scintillation detector, or is it not regarded as more modern to use a PSD? In principle one cannot use a PSD in the Bragg-Brentano geometry, as the position of the focal circle depends on the angle $\theta_{s}$ between the sample and the primary beam. The diffracted beam is only focused at $2 \theta_{s}, i . e$. at one specific detector position. All other beams reflected from the sample are increasingly defocused with increasing difference from the exact $2 \theta_{s}$ position. Thus, the use of a PSD in Bragg-Brentano mountings is a compro- 
mise between resolution and measuring speed. So far only two questions about the most frequently occurring diffractometer geometry, the Bragg-Brentano mode, have been raised. As will be shown later, however, even the oldest sample mounting, the Debye setting, may be successfully used in a modern powder diffractometer equipped with a focusing incident-beam monochromator. Using a fine-focus $\mathrm{X}$-ray tube, a large focal distance, an internal standard, and a small linear PSD, even this kind of diffractometer may surpass the accuracy in diffraction angles measured by the most accurate Guinier technique. Furthermore, intensity measurements are always more precise with an electronic counter and also more accurate unless the instrument set-up and the corresponding instrument functions are in error. As we shall see, however, this does not mean that the Guinier camera is an historical object that should be relegated to the museum collections.

\section{d-spacings and Bragg intensities.}

$\mathrm{X}$-ray powder diffraction is a well established technique for the phase analysis of solids. Structural studies of polycrystalline samples, not available as single crystals; multiphase analysis, qualitative as well as quantitative, have all benefited from the introduction of powder-pattern characterization. A related application, the measurement of the dimensions and angles of the crystallographic unit cell, has afforded a powerful means for studying and understanding the phenomenon of solid solubility. At a fundamental level these cell parameter measurements serve to convert the atomic parameters, obtained in crystal structure studies, into spatial realities of bond distances and angles for subsequent correlation with physical and chemical properties.

As stated by Mighell \& Stalick (1979), identification of materials by cell parameters is extremely efficient. The NBS Crystal Data File contains unitcell data for many more substances than the total number of patterns, indexed and non-indexed, in the PDF-2 data base. It may also be true that the main reason for a large number of structural investigations not being made is the simple fact that the powder diffraction patterns of the substances have not been indexed.

In general, only the diffraction angles, the Bragg angles, are needed to solve the indexing problem and determine the unit cell parameters. This is fortunate because of the fact that, regardless the method used to collect powder diffraction data, the intensity measurements are often much more dependent on instrument function and sample preparation and thus much less reliable than the observed diffraction angles, especially if an internal standard is used.

The intensity round-robin directed by the JCPDS - International Centre for Diffraction Data, and published by Jenkins \& Schreiner (1989), illustrates this very clearly. From the tests made by twentytwo individual experimentalists using a variety of diffractometer types and a range of configurations of any specific instrument model, a number of interesting conclusions were drawn. It was concluded that although the raw intensity precision for the single person/single laboratory was within a factor of two of the statistical counting limit, systematic intensity variations of $30-60 \%$ with $2 \theta$ angle were found between measured and calculated data. It was stated that systematic errors in powder diffraction data remain abundant, but that, being systematic, these can frequently be addressed by applying external calibration procedures. Naturally all intensity measurements in the round-robin were made with diffractometers, in one case using synchrotron radiation. Usually scintillation detectors, but in two laboratories PSDs, were used. Except for the synchrotron user only one of the two PSD users had an incident-beam monochromator. Although not explicitly stated in the report, it seems clear that all instruments were set up in Bragg-Brentano, or at least in reflection, mode. This was of course done in order to avoid absorption corrections.

There is no reason to believe that any photographic detector is capable of competing successfully with powder diffractometers when it comes to accuracy in intensity measurements. The primary focus of the intensity round-robin was to study measured intensities obtained from modern computercontrolled powder diffractometers and therefore no internal standards were used. The tests, however, were designed to yield information also on the performance of data treatment software packages. Therefore angular accuracy studies were also made. The general conclusion from this part of the study was rather disappointing. Quoting from the report: "Automation of powder diffractometers has not significantly improved d-spacing accuracies of raw data." In many cases very significant $\left(>0.05^{\circ} 2 \theta\right)$ systematic errors were found. For details see the complete report given by Jenkins \& Schreiner (1989).

In the report a special study is made of the $\mathrm{MoO}_{3}$. sample, because the penetration depth in this sample is negligible and the powder packs extremely well, making a smooth surface easy to obtain and a dis- 
placement error less likely. In spite of this, large systematic trends were found in many data sets. Jenkins \& Schreiners made the comment: "One wonders how, with automated systems, such gross errors can go uncorrected!" There may be different ways to explain this. It should be noted, however, that the linear representation of the systematic angular errors (Fig.12 in the report) is a simplified picture of the situation. From the data (Table 27 in the report) it is obvious that the angular error versus $2 \theta$ is often a strongly curved function.

\section{An incident-beam monochromatized focusing powder diffractometer}

The powder diffractometer used in the following tests is a Stoe Stadi/P instrument. It is a computerized high-resolution focusing monochromatic-beam diffractometer. The diffractometer enables the user to choose between transmission measurement using flat samples or capillaries, and reflection measurement (by rotating the sample $90 \mathrm{deg}$ ). In order to provide high resolution, the radius of the recording circle, i. e. the distance between sample and detector, is as large as $220 \mathrm{~mm}$. Thus, a curved germanium monochromator with a focal distance of $440 \mathrm{~mm}$ is used to produce pure $\mathrm{CuK} \alpha_{1}$ radiation.

In a study, by Louër \& Langford (1988), of the use of incident-beam monochromators in conventional Xray powder diffractometry a number of advantages have been summarized:

(a) The removal of $\mathrm{CuK} \alpha_{2}$. reduces the number of peaks, and consequently the resolution of the remaining lines is improved. The peak positions are not biased by any $\mathrm{K} \alpha_{2}$. component. Although the bias can be analytically removed, it may introduce systematic errors unless a precise model for the $\mathrm{K} \alpha_{1}-\mathrm{K} \alpha_{2}$. doublet and its satellite group is used (Parrish, Huang \& Ayers, 1976; Will, Parrish \& Huang, 1983). The $\mathrm{K} \alpha_{1}$ and $\mathrm{K} \alpha_{2}$ lines have different widths and are skewed by different amounts, which is rarely taken into account.

(b) The background has no contribution from filter absorption edges and residual $\mathrm{K} \beta$ lines.

(c) Instrumental line profiles have a simple, well characterized shape whose parameters vary with angle in a straightforward manner.

Louër \& Langford discuss also the advantages of incident-beam monochromators for indexing and determination of cell dimensions, line-profile analysis, structure determination and structure refinement. Although the article deals mainly with a BraggBrentano diffractometer, much of the content is also valid for the Stoe diffractometer discussed above as well as for a conventional Guinier camera.

\section{d-spacings}

Some general comments on intensity and d-spacing measurements have been given above. In the present section we will concentrate on d-spacings alone. The Bragg-Brentano powder diffractometer is generally regarded as the principal source of precision lattice parameters. For several reasons it was also preferred by the National Bureau of Standards (NBS) in the preparation of diffraction patterns for the now defunct NBS monographs Standard X-ray Diffraction Patterns. One reason for the choice of instrument may have been the good possibilities of registering accurate absorption-independent intensities, although this was not the primary aim. Experimental techniques and calculations are well described in the first part of the monographs and will not be repeated here. The description should also be valid for most of the patterns now published in the journal Powder Diffraction. The use of internal standards is recommended, and most of the low-quality patterns in the PDF-2 data base would probably have been much better if this had been a more generally applied procedure.

Several studies of precision measurements of lattice parameters have been published since the article on this subject by Parrish \& Wilson (1959) appeared in the International Tables for Crystallography, Vol.II. It is notable, however, that the main parts of the article deal with Debye-Scherrer cameras and counter-diffractometer methods. Some comments are given about the back-reflection flat-plate camera. The conventional Guinier camera (1937, 1939) is not discussed. This is a pity, since there may be reason to agree with the statement by $G$. Hägg (1986): "In 1937 Professor Guinier, in a different arrangement, ingeniously combined the Johansson element with a focusing powder camera and thus attained what I would call the most perfect instrument existing for powder diffraction." Studies, concerning precision and reproducibility of lattice parameters from Guinier powder patterns have been published by Brown, Edmonds \& Foris (1981) and by Brown \& Foris (1983).

As we are indebted to A.J.C. Wilson for the most extensive mathematical theory of powder diffractometry, I would like to show a quotation from his 
textbook Elements of X-ray Crystallography (1969, p.46): "The general principles of the calculation of lattice parameters from the line positions are the same for counter diffractometers as for powder cameras. However, the greater accuracy resulting from the use of diffractometers makes the results both more accurate and less liable to subjective influences."

It is interesting to compare this statement with the following comment by R. Shirley (1979): "The paramount importance of resolution for indexing work explains the high success rate for focusing camera data, especially from Guinier-Hägg instruments, whose resolution can only be described as superb. It is rather less common (and considerably more expensive) to obtain as good resolution with diffractometer data.", and the conclusion by A. Brown (1986): "Comparison tests, made by members of the Joint Committee for Powder Diffraction Standards, demonstrate that Guinier data are at least equal, and often superior to diffractometer measurements."

As we shall see, these statements by Wilson, Shirley and Brown are not necessarily in contradiction. In order to show this, and also to illustrate the relative merits of diffractometers and focusing cameras, two data sets of the same compound, the solid solution $\left(\mathrm{Zn}_{0.75} \mathrm{Mg}_{0.25}\right)_{3}\left(\mathrm{PO}_{4}\right)_{2}$, were collected. The sample is not a high-symmetry standard compound. It is a normal sample from an ordinary crystallographic study using Rietveld refinements to determine cation distribution in solid solutions (Bénard, Nord, Werner \& Westdahl, 1992). A reasonably complicated powder pattern has been chosen in order to illustrate the importance of good resolution and make it possible to use statistical arguments.

It is the intention to show that a normal routine Guinier film evaluation, one of approximately 2500 per year in our laboratory, gives average deviations between observed and calculated diffraction angles less than $0.01^{\circ}$ in $2 \theta$. Lattice parameters for the compound will be given in order to illustrate the accuracy in d-values. It should be kept in mind that the majority of the patterns obtained in the laboratory are used for identification, indexing, or phase analysis. A few patterns are also collected for structure determinations and refinements. Because of the large number of photographs evaluated per year, it has been found essential to minimize the amount of human interactions and to this end a computerized film scanner system has been designed.

One powder pattern was collected with the Stoe Stadi/P diffractometer described above, using a sam- ple mounted in a $0.5 \mathrm{~mm}$ rotating capillary. The diffraction data were collected with a small linear PSD covering $7.0^{\circ}$ in $2 \theta$. The PSD was moved in steps of $0.2^{\circ}$, thus giving a sum intensity from 35 measurements at each $2 \theta$ position $\left(19^{\circ}<2 \theta<135^{\circ}\right)$. Partly in order to make the following discussion less dependent on the type of diffractometer used, silicon (SRM 640, $a=5.43088 \AA$ ) was added to the sample as an internal standard. The raw data were analysed by a peak-picking program based oll the Morrey fourth derivative algorithm (1968) followed by a program to decompose overlapping peaks and adjust the entire profiles of the lines. The strategy of the program is based on the maximum neighborhood method of Marquardt (1963), which overcomes the problem of highly correlated parameters in nonlinear least-squares refinement. Both programs are available in the Stoe software package. The silicon line positions were then used to derive a second-order polynomial correction function for the $2 \theta$-scale. Finally, 84 uniquely indexed lines of the 128 observed were used in a least-squares refinement of the cell parameters. The cell parameters obtained and some relevant figures of merit are given in table 1 . Table 2 shows a small part of the list of observed and calculated diffraction angles. For comparison, a Guinier photograph of the same compound was produced, also with the addition of silicon as internal standard. The camera used was a Guinier-Hägg camera, XDC $700(\mathrm{R}=50 \mathrm{~mm})$. The photograph was measured and analysed by the automatic Guinier film scanner system. In order to minimize subjective influence (cf. the statement by Wilson above) no manual changes were made in the default input parameters to our Guinier film analysing program, SCANPI. The film scanner, LS18 (Johansson, Palm \& Werner, 1980), is controlled by a PC/AT computer. The SCANPI program evaluates background, performs noise analysis by a procedure similar to the one described by Sonneveld \& Visser (1975), determines peak positions and integrated intensities, identifies the internal-standard lines and calculates sample displacement, zero point error and film-shrinkage factor (see appendix).

Usually some time should have been spent to check the result by visual inspection with a plot module in the program. To rule out any "subjectivity", this check was omitted; and 105 lines, silicon lines automatically excluded, were printed within 6 minutes from the time the Guinier photograph was placed in the film scanner. The cell parameters were indexed and least-squares refined with the same program, PIRUM (Werner, 1969), that was used for the 
Table 1. Unit cell data for $\left(\mathrm{Zn}_{0.75} \mathrm{Mg}_{0.25}\right)_{3}\left(\mathrm{PO}_{4}\right)_{2}$ obtained from different data sets and by different methods.

$\mathrm{N}=$ number of lines. E.s.d.s given in parentheses.

a) Unit cell dimensions from indexed powder diffraction patterns.

\begin{tabular}{|lrrrrr|}
\hline Data & $N$ & $a(\AA)$ & \multicolumn{1}{c}{$b(\AA)$} & $c(\AA)$ & \multicolumn{1}{c|}{$\beta\left(^{\circ}\right)$} \\
\hline Stoe & 128 & 7.55854 & 8.36322 & 5.05213 & 95.0164 \\
& & $(19)$ & $(26)$ & $(13)$ & $(24)$ \\
Guinier & 104 & 7.55831 & 8.36311 & 5.05172 & 95.0128 \\
& & $(39)$ & $(46)$ & $(23)$ & $(38)$ \\
& 50 & 7.55880 & 8.36227 & 5.05156 & 95.0147 \\
& & $(48)$ & $(50)$ & $(43)$ & $(65)$ \\
& 30 & 7.55885 & 8.3628 & 5.0514 & 95.014 \\
& & $(96)$ & $(11)$ & $(10)$ & $(15)$ \\
\hline
\end{tabular}

b) Unit cell dimensions from Rietveld refinements.

\begin{tabular}{|lcccc|}
\hline Data & $a(\AA)$ & $b(\AA)$ & $c(\AA)$ & $\beta^{\circ}$ \\
\hline Stoe & $7.55825(7)$ & $8.36223(7)$ & $5.05195(5)$ & $95.0148(6)$ \\
Guinier & $7.5564(2)$ & $8.3597(3)$ & $5.0502(2)$ & $95.014(2)$ \\
\hline
\end{tabular}

c) Figures of merit.

$M=$ de Wolff figure of merit.(de Wolff, 1968)

Index for the physical plausibility of a unit cell derived solely from powder diffraction data.

$\mathrm{F}=$ Quality index for an indexed powder diffraction pattern. (Smith \& Snyder, 1979)

\begin{tabular}{|lcccc|}
\hline Data & $\mathrm{N}$ & $M_{20}$ & $\mathrm{~F}_{20}$ & $\mathrm{~F}_{N}$ \\
\hline Stoe & 128 & 126 & $205(0.0036,27)$ & $33(0.0070,565)$ \\
Guinier & 104 & 69 & $96(0.0078,27)$ & $53(0.0078,251)$ \\
$"$ & 50 & & & $70(0.0073,98)$ \\
$"$ & 30 & & & $73(0.0084,49)$ \\
\hline
\end{tabular}

Stoe data set. One of the three weakest lines was not indexable, but visual inspection of the photograph revealed that it was a noise contribution. The unit cell parameters obtained are shown in table 1 and observed $2 \theta$ values in table 2 , adjacent to those obtained from the Stoe data.

If the plot program module had been used, it would also have been easy to see that the strongest reflection (210) was overexposed, and its position should normally have been corrected. It has a negligible influence on the cell parameters and STDs shown in table 1, however, and it has therefore been left unadjusted in order to illustrate this kind of problem in photographic methods. It may also be instructive to see how clearly differences between observed and calculated $2 \theta$ values reveal the existence of an error.

The absence of systematic errors is demonstrated in table 1 in two ways. Refinements of unit cell parameters based on different numbers of lines do not show significant differences in the cell dimensions, only STDs increasing with decreasing number of lines. Furthermore, no significant deviations are seen between cell parameters obtained from the Stoe and Guinier data sets.

As can be seen from table 1, Wilson's statement is confirmed, although he had probably a quite different diffractometer geometry in mind. The standard deviations of the unit cell parameters and the average differences between observed and calculated $2 \theta$, especially for the first 20 lines, are smaller for the diffractometer data. This should be no surprise, as the Guinier data have not only a more limited $2 \theta$ range but also rather irregular and asymmetric peak shapes; that makes it more realistic to rely on the 
Table 2. Observed and calculated $2 \theta\left(^{\circ}\right)$ for $\left(\mathrm{Zn}_{0.75} \mathrm{Mg}_{0.25}\right)_{3}\left(\mathrm{PO}_{4}\right)_{2}$.

I-obs Guinier data integrated by SCANPI

I-obs Stoe data from peak program

Systematic absences: $(h 0 l) h+l=2 n+1$ and $(0 k 0) k=2 n+1$.

\begin{tabular}{|rrrrrrr|}
\hline \multicolumn{4}{|c}{ Guinier data } & \multicolumn{3}{c|}{ Stoe data } \\
\hline$(h k l)$ & I-obs & $2 \theta_{\text {obs }}$ & $2 \theta_{\text {calc }}$ & I-obs & $2 \theta_{\text {obs }}$ & $2 \theta_{\text {calc }}$ \\
& & & & & & \\
$\overline{1} 01$ & 65 & 20.315 & 20.334 & 63 & 20.331 & 20.332 \\
011 & 29 & 20.575 & 20.581 & 34 & 20.580 & 20.580 \\
020 & 6 & 21.227 & 20.231 & 10 & 21.228 & 21.230 \\
101 & 28 & 22.059 & 20.069 & 28 & 22.066 & 22.068 \\
$\overline{1} 11$ & 22 & 22.959 & 20.969 & 27 & 22.965 & 22.968 \\
120 & 2 & 24.315 & 24.329 & 4 & 24.334 & 24.328 \\
210 & 100 & 25.903 & 25.934 & 100 & 25.930 & 25.933 \\
021 & 20 & 27.709 & 25.715 & 24 & 27.712 & 27.714 \\
$\overline{1} 21$ & 7 & 29.568 & 29.563 & 11 & 29.561 & 29.562 \\
121 & 2 & 30.812 & 30.811 & 4 & 30.811 & 30.810 \\
220 & 11 & 31.963 & 31.962 & 14 & 31.961 & 31.961 \\
211 & 6 & 32.703 & 32.702 & 8 & 32.699 & 32.701 \\
130 & 11 & 34.277 & 34.273 & 6 & 34.270 & 34.273 \\
002 & 68 & 35.649 & 34.653 & 59 & 35.653 & 35.650 \\
$\overline{2} 21$ & & & 34.660 & & & 35.658 \\
031 & 44 & 36.824 & 36.828 & 37 & 36.825 & 36.827 \\
310 & 18 & 37.366 & 37.379 & 16 & 37.359 & 37.378 \\
221 & 5 & 37.758 & 37.758 & 8 & 37.757 & 37.758 \\
$\overline{1} 12$ & 3 & 38.238 & 38.223 & & & 38.220 \\
$\overline{1} 31$ & & & 38.282 & 4 & 38.261 & 38.280 \\
131 & 7 & 39.281 & 39.283 & 8 & 39.282 & 38.282 \\
$\overline{3} 11$ & 11 & 40.161 & 40.164 & 13 & 40.161 & 40.162 \\
$\vdots$ & $\vdots$ & $\vdots$ & $\vdots$ & $\vdots$ & $\vdots$ & $\vdots$ \\
452 & 2 & 89.441 & 89.446 & 2 & 89.448 & 89.444 \\
315 & & & & 4 & 91.198 & 91.187 \\
044 & & & & & & 91.210 \\
$\vdots$ & $\vdots$ & $\vdots$ & $\vdots$ & $\vdots$ & $\vdots$ & $\vdots$ \\
$\overline{8} 23$ & & & & 2 & 134.255 & 134.246 \\
653 & & & & & & 134.264 \\
\hline & & & & & & \\
\hline
\end{tabular}

peak positions alone. It should be noted, however, that only one of the Stoe data cell parameters deviates marginally more than one STD from the Guinier data cell parameters. One may well ask when such a small improvement is worth the effort, however.

\section{Internal standard and absorption.}

Systematic errors depending on the use of a weakly absorbing material to correct the $2 \theta$ scale for a strongly absorbing material, as found for the BraggBrentano geometry (Brown, 1986), should not occur with a Guinier camera or a diffractometer with transmission geometry and a focusing monochromator. For demonstration, a flat sample of lead nitrate with silicon as internal standard was prepared for data collection in transmission geometry. Since Brown \& Foris (1983) found that the measurement of unit cell dimensions of lead nitrate is sensitive to the specimen preparation, the same flat transmis- 


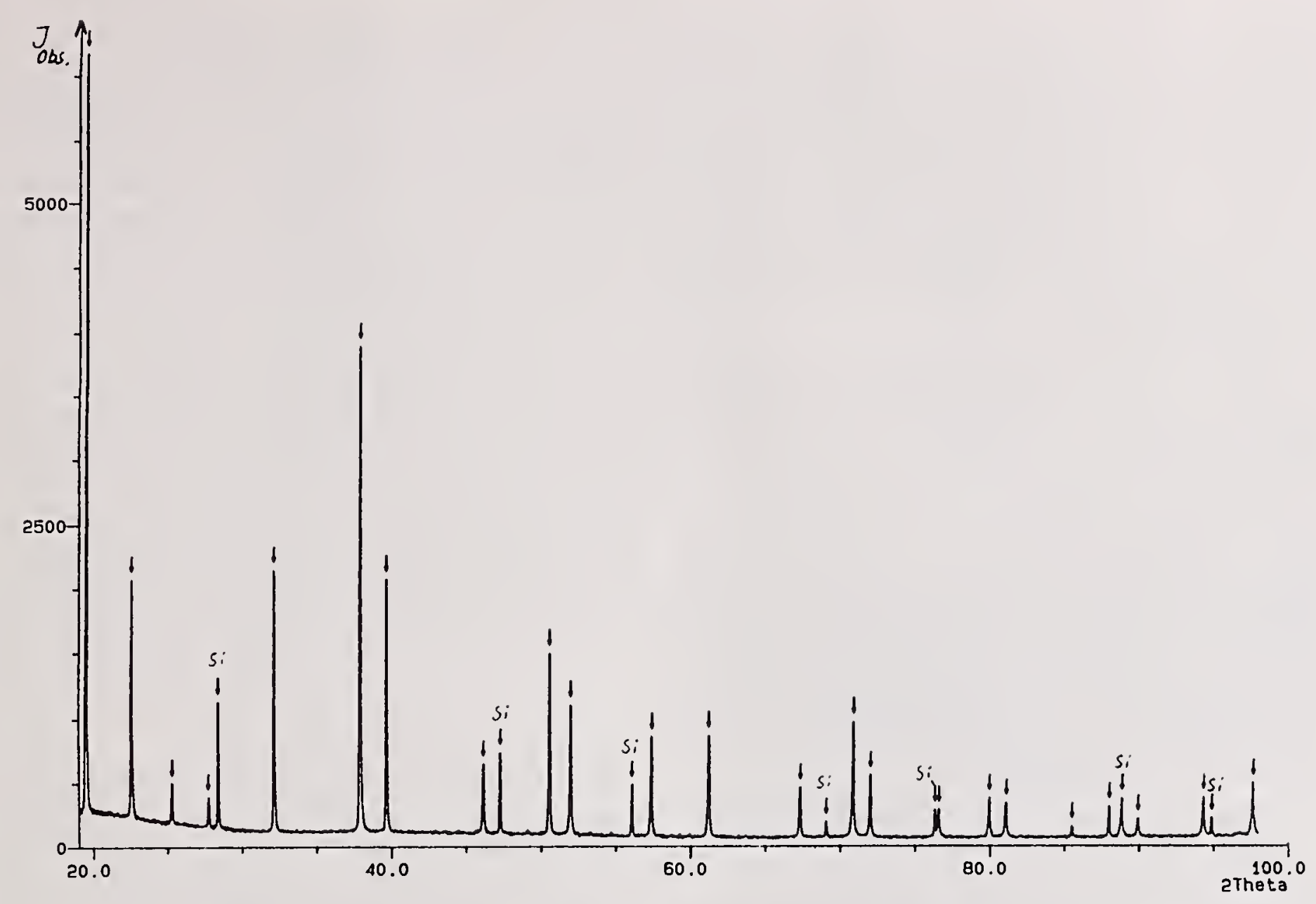

Figure 1: Stadi/P data set of $\mathrm{Pb}\left(\mathrm{NO}_{3}\right)_{2}$ with $\mathrm{Si}$ added as internal standard.

sion sample was used for data collection by the Stoe diffractometer and the automatic Guinier film scanner system. The small linear PSD was used in the same way as described above. For comparison with the Guinier data set, the upper limit in $2 \theta$ was set to $100^{\circ}$. The data sets were treated as described in the previous section. The diffraction pattern obtained with the Stoe diffractometer is shown in figure 1 . It should be noted that all silicon reflections are weak. For a Bragg-Brentano measurement the strongest reflections from the sample and the internal standard should preferably be of equal intensity. Such samples may take some time to prepare.

For the Stoe data the maximum difference between observed and calculated $2 \theta$ was only $0.003^{\circ}$, $\mathrm{F}_{20}=321(0.0012,54)$. and the cubic cell parameter $a=7.85722(3) \AA$. The corresponding figures for the Guinier data were $0.019^{\circ}, \mathrm{F}_{20}=62(0.006,54)$. and 7.8574(2) $\AA$. Thus, no significant systematic error is indicated. It is not possible, however, to know if the accuracy in the Stoe data set equals the precision. It is possible that the accuracy in the internal standard sets the limit.

\section{d-spacing quality needed.}

A recent article by Jenkins \& Nichols (1990) about problems in the derivation of d-values from experimental digital XRD patterns, deals mainly with conventional reflected-beam monochromatized BraggBrentano data. A large number of problems and a number of useful recommendations are described in the article. These will not be repeated here. Only a few points related to the choice of technique will be discussed.

Jenkins \& Nichols (1990) state that, in spite of numerous sources of error in the measurement of $2 \theta$ values and subsequent conversion to $d$-spacings, the derived d's are generally of sufficient quality to allow qualitative phase identification. This was concluded from the result of the round-robin test (Jenkins \& Schreiner, 1989), where it was found that the multiperson/multi-laboratory precision for a binary mixture of $\mathrm{CaCO}_{3} / \mathrm{ZnO}$. was about $0.048^{\circ}$. This may not be good enough for powder indexing, however.

It is a revealing fact that most of the laboratories that have made contributions in the field of powder indexing have also been deeply involved in the prob- 
lem of accurate determination of diffraction angles, whether camera or diffractometer methods have been used. This is not strange; on the contrary, it is a logical part of the indexing work. Although it is difficult to give any quantitative measure of the data quality needed, it is certainly true that there are few fields of endeavour in which careful data preparation is as dramatically rewarded as in computer-based powder indexing (Shirley, 1979).

It should be pointed out, however, that finding the unit cell, i.e. solving the indexing problem, and verifying the physical plausibility of the indexing are different problems. For the latter purpose we may use the criterion that the De Wolff figure of merit $M_{20}$ (De Wolff, 1968) should at least reach a value of 10. Approximate calculations show that for the data sets in the NBS monographs, Standard X-ray Diffraction Patterns, the average $\Delta(2 \theta)$ needed to obtain $M_{20}=10$ is strongly symmetry dependent. Supposing the observed patterns to be equally complete (or incomplete), the following required precision ranges for $2 \theta$ are obtained:

- $0.035^{\circ}-0.06^{\circ}$ for triclinic patterns,

- $0.045^{\circ}-0.07^{\circ}$ for monoclinic patterns, and

- $0.070^{\circ}-0.15^{\circ}$ for orthorhombic patterns.

Solution of the indexing problem usually demands much smaller deviations, however.

The statement by Visser (1969) that the errors in diffraction angles should not exeed $0.03^{\circ}(2 \theta)$ and that the first three lines especially should be as accurate as possible may be regarded as 'rules of thumb'. Programs using different strategies may also be sensitive in different ways to accuracy and completeness. The DICVOL program by Louër and Louër (1972, new program version by Boultif and Louër 1991) is probably less dependent on accuracy in the first loworder lines than for example the TREOR program (Werner, Eriksson \& Westdahl, 1985, new program version 1990), which is more sensitive to accuracy in this range but less dependent on completeness than many other indexing programs.

It is well known that it is possible to obtain accurate d-values also by an external standard technique. From the frequently occurring low data quality in the PDF-2 data base, however, it may be concluded that unless an internal standard technique is used, the experimentalists often have very limited knowledge of the data quality obtained.

\section{Film scanner system.}

It may be concluded that modern powder diffractometers using an incident-beam monochromator can combine the high resolution of a Guinier pattern with mathematically well defined peak shapes. Furthermore, the step intensities are more accurately collected with a diffractometer than by densitometer measurements, which need corrections for nonproportionality between exposure and optical density. However, as long as powder diffractometers not only give much broader peaks, but also peak doublets from $\mathrm{CuK} \alpha_{1}$ and $\alpha_{2}$, Guinier data may be preferable for structure determinations although diffractometer data may be preferred for final Rietveld refinements. This is because high resolution is the most important factor for indexing and for deriving integrated

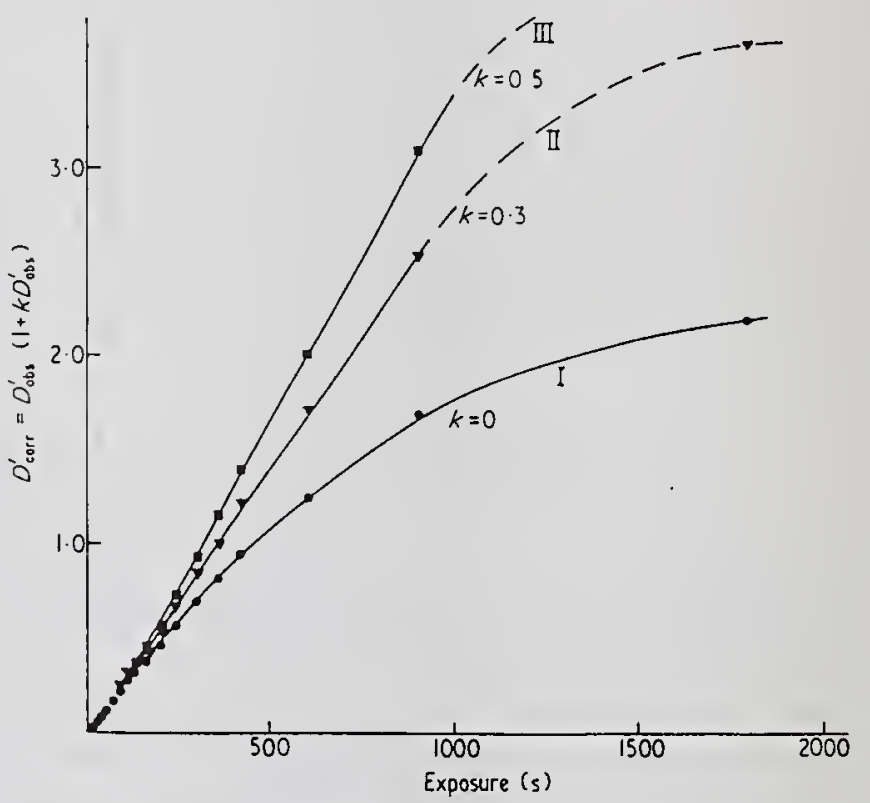

Figure 2: Corrected and uncorrected relative optical density from a density wedge. The relative densities, $\mathrm{D}_{\text {obs }}^{\prime}$ are obtained by subtraction of the local background.

intensities for the Patterson function and for preliminary Fourier calculations using incomplete structure models.

\section{a) Intensities from photographs.}

As stated above, film scanner data need correction for non-proportionality between exposure and optical density. It is well known that it is possible to use different exposure times and to scale the intensities from two or more photographs using only the linear parts of the optical density versus exposure 
Table 3. Data from Rietveld refinements of $\left(\mathrm{Zn}_{0.75} \mathrm{Mg}_{0.25}\right)_{3}\left(\mathrm{PO}_{4}\right)_{2}$.

\begin{tabular}{|c|c|c|}
\hline & Stoe data set & Guinier data set \\
\hline Number of parameters: & 46 & 39 \\
\hline Profile function: & pseudo-Voigt & modified-Lorentzian \\
\hline Background: & refined & not included \\
\hline Step size $(2 \theta)$ : & $0.02^{\circ}$ & $0.0229^{\circ}$ \\
\hline Max. half-width: & $0.137^{\circ}$ & $0.135^{\circ}$ \\
\hline Min. half-width: & $0.099^{\circ}$ & $0.078^{\circ}$ \\
\hline E.s.d. in coordinates: & $0.0001-0.0 .0017$ & $0.0004-0.0027$ \\
\hline $\mathrm{R}_{\mathrm{F}}$ & 0.040 & 0.124 \\
\hline $\mathbf{R}_{\mathrm{B}}$ & 0.042 & 0.081 \\
\hline
\end{tabular}

curve. This is usually not needed, however, if a simple second-order correction procedure is applied to the relative optical densities $D^{\prime}$, i. e. the optical density above the local background.

$$
D_{\text {corr }}^{\prime}=D_{\text {obs }}^{\prime}\left(1+k D_{\text {obs }}^{\prime}\right)
$$

A $k$-value between 0.3 and 0.5 (cf. Fig. 2) may be recommended (Werner, 1971). The local background should be determined with the scanner system. A disadvantage in using intensities derived from densitometer data, however, is that one has to use a weight limit in the Rietveld program, as absolute intensity data are not available.

\section{b) Peak-finding on film data.}

As stated by Jenkins \& Nichols (1990), one major source of confusion is often the recognition of peaks by a given automated system. In the intensity round-robin (Jenkins \& Schreiner, 1989) different users reported from 25 to 53 peaks for $\alpha-\mathrm{Al}_{2} \mathrm{O}_{3}$. over the same $2 \theta$ range, whereas the calculated number is 42 . In order to avoid the extreme sensitivity of the conventional second-order derivative peakhunting method, which often gives too many lines, a different procedure has been used in our film evaluation program.

A peak-finding strategy that works well for photographs with low background and well-defined peak shapes may not be useful for photographs with irregular line broadenings, many overlaps and/or high background levels. Therefore the peak-finding algorithms are not based on any assumption of a general profile function or a smooth variation of the halfwidths with $2 \theta$. In order to increase the range of peak types that can be analysed, three different peak finding algorithms are available.
The first one is, in principle, a normal first-order derivative method with relatively strong constraints. These serve to avoid regions where end-point maxima occur, or where several closely adjacent very small peaks are created by the addition of noise to weak but significant signals. Such regions are analysed in a second pass, which uses maximum deviations from the background for peak definition. These two procedures are normally used.

The third, less frequently used algorithm is a stepwise movement of an integration window of predefined width across all regions where peaks of the first two types are not already defined. The method is similar to that used in some single-crystal diffractometer programs for calculation of integrated intensities.

Two different noise levels are calculated and used by the program. One dynamic noise level (i.e. a function of the local optical density) is defined for the individual step intensities and a second noise level is used for integrated intensities.

\section{Rietveld refinements.}

As stated above, Rietveld refinements using photographic data may be useful in the preliminary stage of a structure determination, although the results are less accurate than refinements involving diffractometer data. There is no need to discuss the well-known Rietveld method in any detail here, but it may be instructive to show why Guinier data should not be underestimated. As an illustration, the two data sets from the solid solution $\left(\mathrm{Zn}_{0.75} \mathrm{Mg}_{0.25}\right)_{3}\left(\mathrm{PO}_{4}\right)_{2}$ discussed above were refined with a local version of the Rietveld program DBW3.2S (Wiles, Sakthivel \& Young, 1988). A pseudo-Voigt profile function was used for the Stoe data. The profile function for 


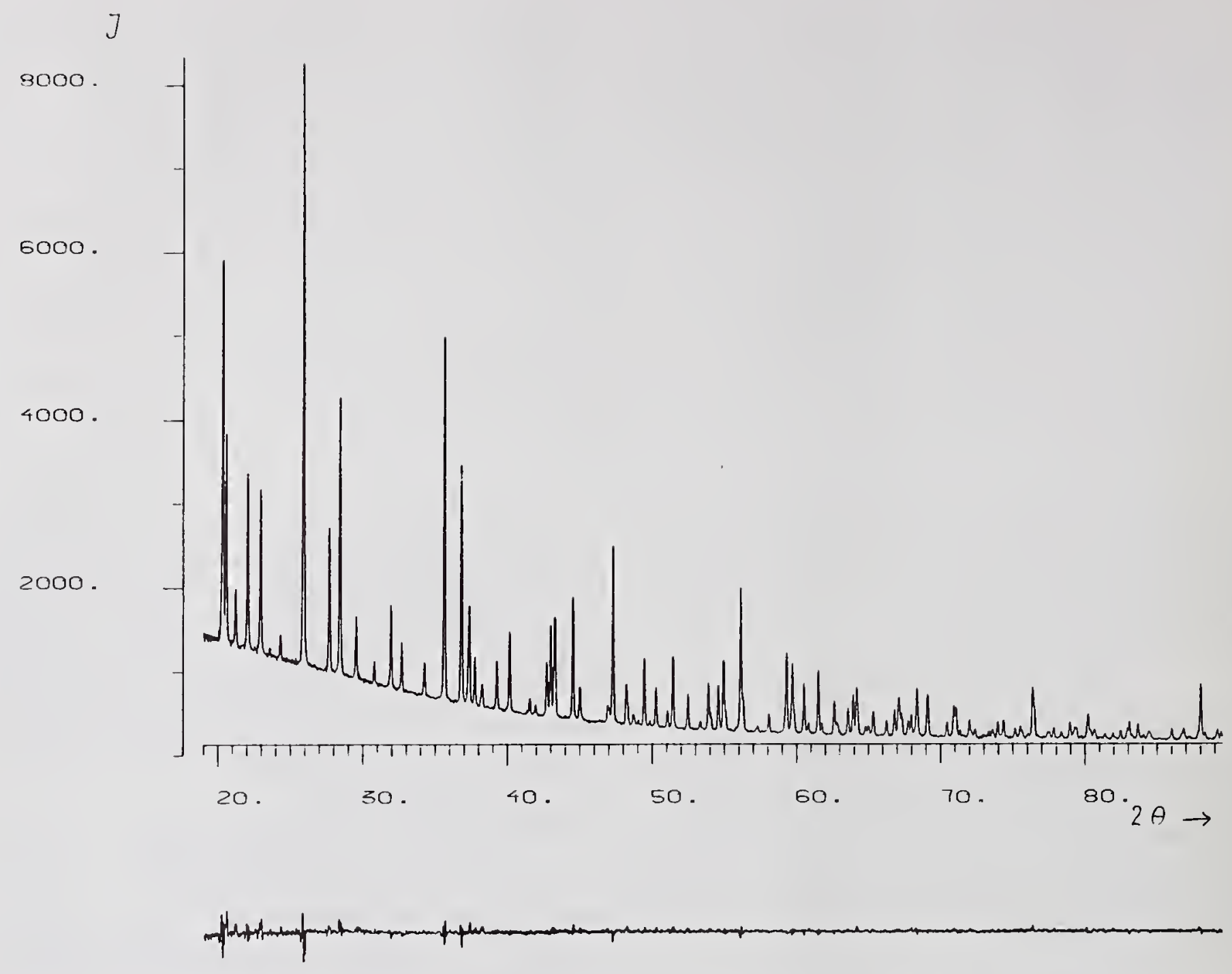

Figure 3: Final Rietveld plot of $\left(\mathrm{Zn}_{0.75} \mathrm{Mg}_{0.25}\right)_{3}\left(\mathrm{PO}_{4}\right)_{2}$ from a Stadi/P data set. The upper curve shows observed data, while the lower curve is the difference between observed and calculated step intensities.

the Guinier refinement was a modified Lorentzian. Some non-structural parameters were naturally different. The amounts of silicon were somewhat different, and normally no internal standard should have been added in a sample used for profile refinement. The number of positional parameters was 18; further information about procedure and results is available in the reference (Bénard, Nord, Werner \& Westdahl, 1992).

The observed intensity curve and the differences between observed and calculated intensities for the Stoe data set are shown in figure 3 . Some information about the refinements is given in table 3 . Of course the diffractometer data set gives a much more accurate final result. Otherwise it would have been difficult to justify an additional, vastly more expensive and time-consuming data collection system. It should be noted, however, that in spite of the fact that a powder diffractometer with exeptionally high resolution has been used, the Guinier lines have smaller half-widths, especially in the low angle range. Although this is of no importance for the final result, it is extremely important for $a b$ initio structure determinations.

The formally refined unit cell dimensions are shown in table $1 \mathrm{~b}$. It should be realised, however, that the cell dimensions obtained by Rietveld refinements are parameters that partly correct the data for non-linear $2 \theta$-scales. The small standard deviations usually obtained for unit cell parameters by Rietveld refinements are therefore often strongly misleading.

Figure 4 shows a small part of the observed and calculated intensity sets from the two Rietveld refinements. 


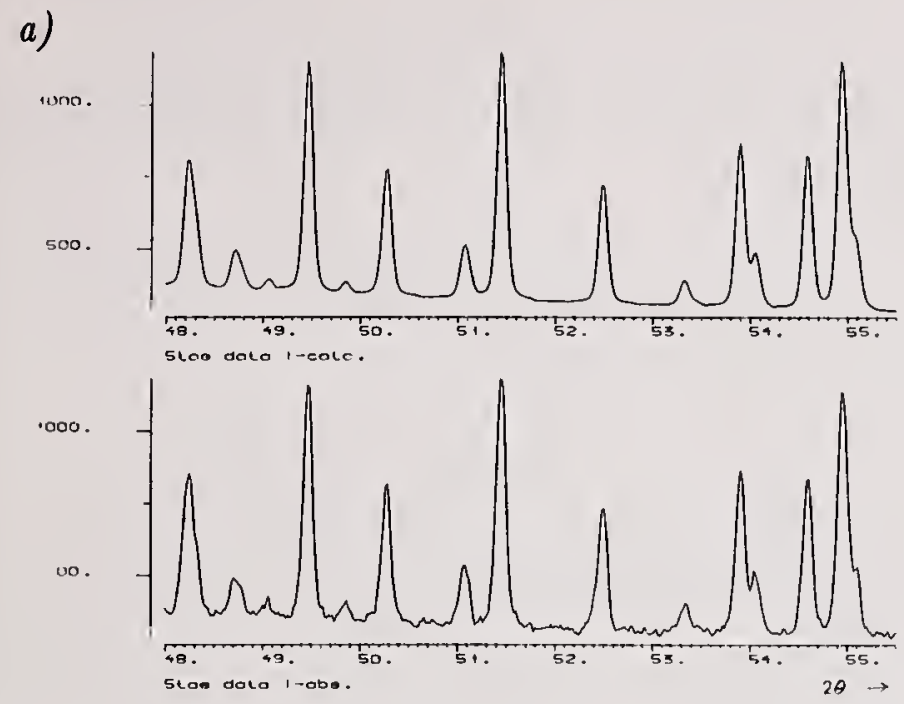

b)
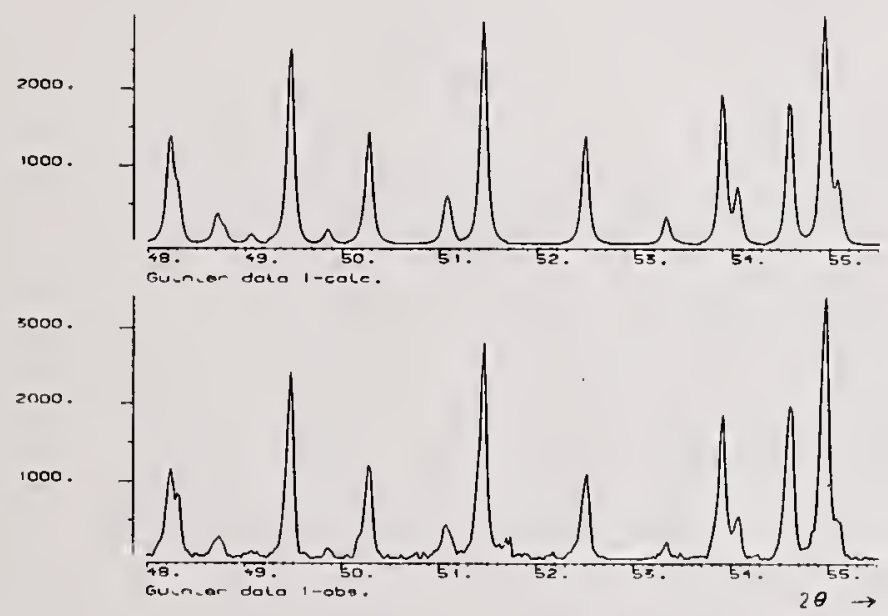

Figure 4: Observed and calculated intensities within the range $48^{\circ}<2 \theta<55.5^{\circ}$ from Rietveld refinements of $\left(\mathrm{Zn}_{0.75} \mathrm{Mg}_{0.25}\right)_{3}\left(\mathrm{PO}_{4}\right)_{2}$.

a) Stadi/P data set. Pseudo-Voigt profile function.

b) Guinier data set. Modified Lorentzian profile function.

\section{Final remarks.}

Different types of equipment for $\mathrm{X}$-ray powder diffraction work have different advantages and disadvantages. A conventional automatic Bragg-Brentano diffractometer with diffracted-beam monochromator usually yields a high peak-to-background ratio, absorption independent intensities, short exposure times and, as most diffractometers, well defined peak shapes. As it cannot compete with the high resolution of a Guinier photograph, however. Another kind of diffractometer has been used in the present work. This diffractometer has a very good resolution at the expense of long measuring times, in spite of the use of a small linear PSD. Operated in the symmetric trans- mission mode it also needs much less sample than a Bragg-Brentano diffractometer. Thus, it is hardly meaningful to discuss powder diffractometers as a homogeneous group of instruments. Diffractometers are also strongly dependent on software systems.

Although, as shown above, it is true that a modern diffractometer may surpass the accuracy of a Guinier camera, it is also true that it is rather difficult, and comparatively expensive to obtain Guinierquality resolution in diffractometer data. All Xray diffraction instruments need careful adjustment, but a well adjusted and carefully handled Guinier camera is usually stable for years. The film is an efficient position-sensitive detector and a long-time mass storage of information that may be visually inspected and evaluated at convenience with a densitometer system. Moreover, it is certainly not true that a computerized Guinier film evaluation system is more liable to subjective influence than an automatic diffractometer.

As illustrated by the present work, both precision and accuracy in $2 \theta$ angles are normally better than $0.01^{\circ}$ in routine measurements of Guinier photographs, if an internal standard and an automated film scanner system are used. This is usually sufficient for phase analysis, indexing and unit cell refinement. Thus, although modern powder diffractometers are indispensable for studies of line broadening, structure refinements, quantitative analyses and time-resolved experiments, it may be strongly recommended to use advanced camera techniques for most of the routine measurements in a laboratory working in the field of powder diffraction.

\section{References}

Bénard, P., Nord, A., Werner, P.-E. \& Westdahl, M. (1992). J. Solid. State Chem. 99, 290-296.

Boultif, A. \& Loü̈r, D. (1991). J. Appl. Cryst. 24, 987-993

Brown, A. (1986). Chemica Scripta 26A, 77-83.

Brown, A., EDMONDS, J. W. \& Foris, C. M. (1981). Adv. X-ray Anal. 24, 111-120.

Brown, A. \& Foris, C. M. (1983). Adv. X-ray Anal. 26, 53-62.

Delhez, R. \& MittemeiJer, E. J. (1991). Materials Science Forum, 79-82, Pt.1

GuINIER, A. (1937). Compt. Rend. 204, 1115-1116.

Guinier, A. (1939). Ann. Phys. 12, 161-237.

Ḧ̈GG, G. (1986). Chemica Scripta 26A, 3-6.

JenKINs, R. \& Nichols, M. (1990). Adv. X-ray Anal. 33, 285-293.

Jenkins, R. \& Schreiner, W. N. (1989). Powd. Diff. 4, 74-100. 
Johansson, K. E., Palm, T. \& Werner, P.-E. (1980). J. Phys. E: Sci. Instrum. 13, 1289-1291.

Louër, D. \& Langford, J. I. (1988). J. Appl. Cryst. 21, 430-437.

LOUËR, D. \& LoUËR, M. (1972). J. Appl. Cryst. 5, 271275.

Loü̈r, D. \& VArgas, R. (1982). J. Appl. Cryst. 15, 542-545.

Marquardt, D. M. (1963). J. Soc. Indust. Appl. Math. 11, 431-441.

Mighell, A. D. \& Stalick, J. K. (1979). Natl. Bur. Stand. (US) Spec. Publ. No.567, 393-403.

Morrey, J. R. (1968). Anal. Chem. 40, 905-914.

Parrish, W., Huang, T. C. \& Ayers, G. L. (1976). Trans. Am. Crystallogr. Assoc. 12, 55-73.

Parrish, W. \& Wilson, A. J. C. (1959). Int. Tables for X-Ray Crystallogr. Vol.II, 216-234

Shtrley, R. (1979). Natl. Bur. Stand. (US) Spec. Publ. No. 567, 361-382.

Smith, G. S. \& Snyder, R. L. (1979). J. Appl. Cryst. 12, 60-65.

Sonneveld, E. J. \& Visser, J. W. (1975). J. Appl. Cryst. 8, 1-7.

VISSER, J. W. (1969). J. Appl. Cryst. 2, 89-95.

WERNER, P.-E. (1969). Arkiv för kemi 31, 513-516.

Werner, P.-E. (1971). J. Phys. E: Sci. Instrum. 4, 351353.

WerNer, P.-E., ERIKSSON, L. \& Westdaht, M. (1985). J. Appl. Cryst. 18, 367-370.

Will, G., Parrish, W. \& Huang, T. C. (1983). J. Appl. Cryst. 16, 611-622.

Wisson, A. J. C. (1970). Elements of X-ray Crystallography, Addison-Wesley Publ. Comp. Inc.

WolfF, P. M. DE (1968). J. Appl. Cryst. 1, 108-113.

\section{Appendix}

Influence of systematic errors in $2 \theta$ on a Guinier film (Brown, 1986).

$\mathbf{S}=$ Measured distance on Guinier film between primary beam line and reflection

$\mathbf{R}=$ Camera radius

$\Psi=$ Angle between primary beam and normal to the specimen

$\varepsilon=$ Sample displacement

$F=$ Film shrinkage factor

$\Delta S_{0} .=$ Zero point error in $\mathrm{S}$

$2 \theta_{\text {corrected }}=A_{0}+A_{1} S+A_{2} \phi$

where

$\phi=\sin (2 \theta) / \cos (\Psi) \cos (2 \theta-\Psi)$,

$A_{1}=90 /(\pi R F)$,

$A_{0}=-\varepsilon A_{1}$,

and

$A_{2}=-\Delta S_{0} A_{1}$. 
National Institute of Standards and Technology Special Publication 846. Proceedings of the international conference Accuracy in Powder Diffraction II, held at NIST, Gaithersburg, MD, May 26-29, 1992. (Issued October 1992)

\title{
Data Optimization and Propagation of Errors in Powder Diffraction
}

\author{
J.-F. BÉRAR \\ Laboratoire de Chimie-Physique du Solide (UA 453 CNRS), Ecole Centrale, 92295 Châtenay, France \\ and LURE, Université Paris-Sud, 92405 Orsay, France.
}

Errors in powder diffraction were widely discussed in the first "Accuracy in Powder Diffraction" meeting (Young, 1979; Albinati, Cooper, Rouse, Thomas \& Willis, 1979; Cooper, Sakata \& Rouse, 1979). In the following years, related papers have been published. The significance of the value of estimated standard deviations (e.s.d.'s) obtained by the Rietveld (1969) method was discussed (Sakata \& Cooper, 1979; Pawley, 1980; Scott, 1983): they are not in accordance with those obtained by intensity refinements, their values being smaller. Still it has been proved that e.s.d.'s are an unbiased estimate of the parameter precision when the assumptions on which the method of least squares is based are satisfied (Prince, 1981; 1985), that is when the model is perfectly suited to the data set. Data collection strategies have been developed to overcome this problem (Hill \& Madsen, $1984 ; 1986 ; 1987)$. Methods have also been proposed to test the validity of the statistical assumptions in Rietveld refinements (Hill \& Flack, 1987). These strategies can be seen as an answer to the question: If we take into account the quality of the results obtained by powder diffraction, is it reasonable to improve the quality of our measurements? Such experiments are time consuming, and wasting time is very expensive in big science.

\section{Data collection strategies}

Since that meeting, in which most of the results come from neutron diffraction, the Rietveld method is now commonly used on data obtained from $\mathrm{X}$ - ray sources, either classical tubes or synchrotron beams. In such cases, or with the new neutron diffractometers, higher resolution can be attained. The number of data collected increases following this resolution improvement. The consequences of this progress in the quality of the measurements have to be considered.

This trend from neutrons to X-ray raises other questions. Can we use the same strategy to optimize data collection with both sources? What do we really want to measure through this diffraction experiment? Are we are really interested in all parameters? Is the same experimental method the right way to get all the parameters that we can physically reach?

If we consider a high resolution synchrotron experiment, the full width at half maximum (FWHM) of lines has a value of some hundredths of a degree, typically $0.03^{\circ} 2 \theta$. The full pattern has to be recorded with a step width of some thousandths of a degree, and the data set will contain more than 30000 values. If we want to perform a full analysis of these data we need a program able to refine not only the structural model (cell parameters, atomic positions, thermal factors) but also other variables related to size, shape, defects and instrumental functions. If we do not know valid and accurate models for all these factors, the refinement will suffer from bias, and it will be difficult to know with accuracy the structural parameters, even if we get very precise values for them. On the other hand, if we wish to know only the cell parameters to study thermal expansion, we need to measure fewer lines, but those measurements should be made with higher angular accuracy. The knowledge of structure depends only on the integrated intensities and can often be obtained using lower resolution and a poor statistical level. For typical structures with fewer than $50 \mathrm{pa}-$ rameters good precision can be achieved with 2500 measurement points.

These relations among resolution, recording step and counting time have been extensively studied by Hill \& Madsen (1984; 1986; 1987). Their conclusions can be roughly summarized as follows: the step width should be between one half and one fifth of the FWHM of the sharpest well resolved line, while the time per step is chosen to obtain some thousand counts at the maximum of the data set. If some evidence of correlation still exists in the data set, a subset should be extracted. This will increase e.s.d.'s, but will reach a point where the statistical assumptions are valid.

The quality of results that can obtained in very short recording time can be surprising $(0.05 \mathrm{~s}$ is given 
as an example by Hill \& Madsen, 1984). Our tendency is to use a visual reference to assess the quality of the data. In figure 1, the highest count is no higher

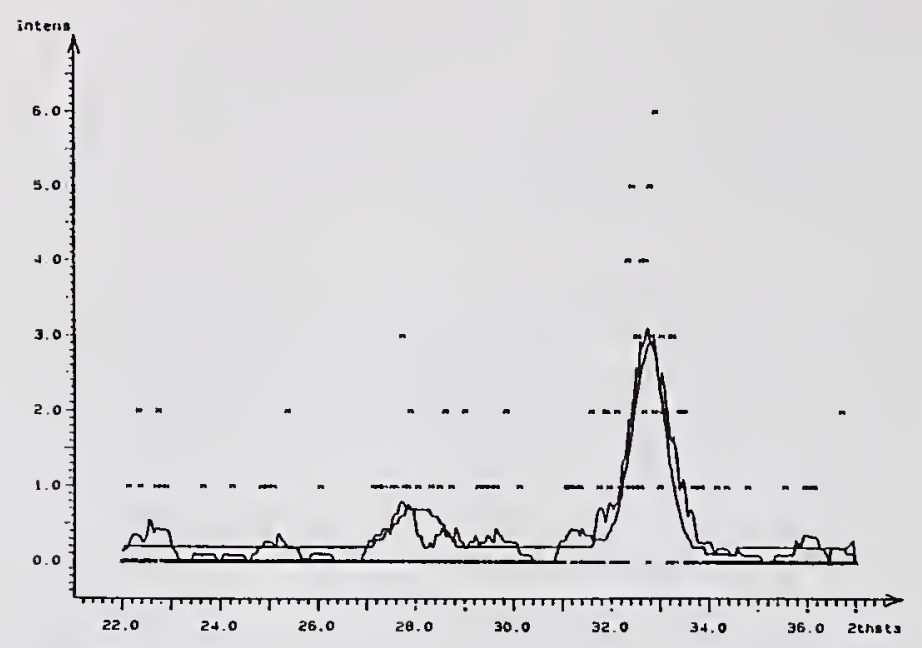

Figure 1: Expected curve $(a)$ in a low counts experiment $(\mathrm{x})$ compared with the result obtained with a digital filter $(b)$.

than 6. Nevertheless the information contained in the data can be extracted even with a simple digital filter adapted to the instrumental function. The resulting curve looks like the one obtained with a recording time a hundred times longer.

As the strategy of collecting data depends on the sample, some remarks can be added to the previous rules. In many problems, we are not only concerned with the structure, but also with its evolution. In such cases it is possible to obtain a very precise knowledge of the parameters if we take into account the dependence of the structure on external factors (time, temperature,...). This can be done either by refining all the collected data simultaneously using a modified program that includes such external dependence (Bérar, 1990), or by fitting the parameter dependence.

In X-ray experiments, data collection is commonly performed by scanning the $2 \theta$ circle with the counter. In such cases, it seems possible to use algorithms that are able to modify the step width and use larger steps in the background. This will allow some time to be spared during measurements, but information contained in the weak lines may be lost.

For instrumental reason, we commonly record each step with a fixed time or fixed monitor counts. Other choices can be made. In X-ray experiments the atomic scattering factors decrease very quickly. If we want to extract valuable information out of data recorded at high angles, we have to increase the quality of these data. It can be useful to record the data using different counting times at low and at high angles. This implies that we use either the same

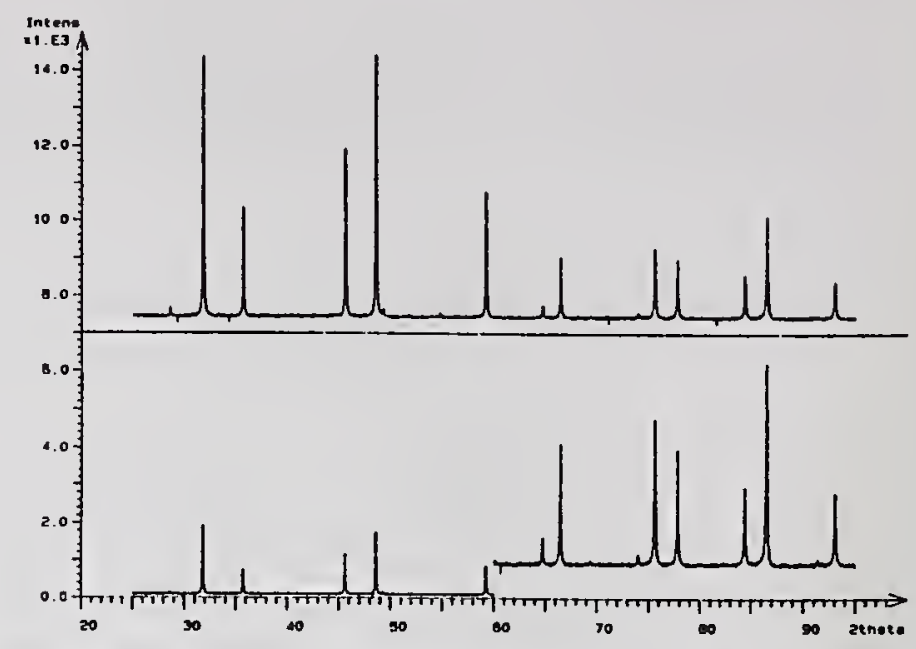

Figure 2: Two possibilities for recording the same $\mathrm{X}$-ray data.

scale factor and adapted weights or observed weights and two related scale factors. Otherwise we will not only waste time, but the data recorded at low angles would be known with unnecessay precision. This compels us to take into account more sophisticated corrections (extinction, ...). Both diagrams in figure 2 need the same recording time. The refinement qualities are similar, but in the case of the diagram recorded with two different step times having a one to eight ratio, the e.s.d.'s on most of the parameters are three times smaller. Commonly with isotropic thermal factors reliability factors $R_{I}$ or $R_{F}$ reach values of a few percent. In order to get a balance between lines, most of the integrated intensities should be chosen in order that the ratios of their e.s.d.'s to their values are in the same range, that is no more than thousand counts, with the peak maxima having fewer counts. Studying real samples, similar but more elaborate strategies should be applied.

\section{E.s.d.'s in the Rietveld method}

In the Rietveld method for structural refinements, the calculated model is directly compared to the observed pattern by a least squares minimisation

$$
S=\sum_{i} w_{i}\left(Y_{o i}-Y_{c i}\right)^{2}=\sum_{i} w_{i} D_{i}^{2}=\sum_{i} a_{i}^{2}
$$

in which $Y_{o i}$ and $Y_{c i}$ stand for the observed and the calculated counts at angle $2 \theta_{i}, w_{i}$ is the associated weight, $D_{i}$ their difference and $a_{i}$ the normalised or weighted one. The summation is carried over the $N$ points in the data set. In this kind of method, there is no requirement for the data to be recorded with fixed step width or counting time. This method does not try to get information out of extracted values of 
integrated intensities. It just samples the diffraction pattern, each data point being an independent measurement. Therefore it is important for the weighting scheme to have a realistic meaning. With the use of multidetectors, the fact that all the data set is often not really covered with the same number of detectors should be taken into account. The use of the Poissonian variance $1 / Y_{o i}$ was unappropriate in old programs in which the background was substracted. However it seems possible to use weights defined using the calculated counts, This can be seen as an approximation to the maximum likehood method, which has been applied with success in fitting experimental data by curves (Antoniadis, Berruyer \& Filhol, 1990).

The calculated intensities $Y_{c i}$ can be expressed by:

$$
Y_{c i}=Y_{b i}+\sum_{j} \sum_{k} K_{j} P_{j k}\left(\theta_{i}-\theta_{k}\right) I_{j k} C_{j k}
$$

$P(\Delta \theta)$ being a normalised profile function whose shape depends on the phase $j$ and the line $k . I_{k}$ is the intensity, which is simply the square of the structure factor for non-magnetic structures. $C_{j k}$ is a correction factor that includes factors like the Lorentz-polarization and, if necessary, orientational factors. $K_{j}$ is the scale factor of the phase. The value $Y_{b i}$ of the background may be represented by polynomials, splines or interpolation functions, which can be refined.

The $S$ sum is minimized using a linear approximation, shifts $X_{p}$ of the $P$ independent parameters are calculated solving the linear system:

$$
\mathbf{M X}=\mathbf{V}
$$

where

$$
\begin{gathered}
M_{p q}=2 \sum_{i} w_{i} \partial Y_{c i} / \partial p \partial Y_{c i} / \partial q \\
V_{p}=2 \sum_{i} w_{i} D_{i} \partial Y_{c i} / \partial p
\end{gathered}
$$

When the minimum of $S$ is reached, the e.s.d. of parameter $q$ is given by

$$
s_{q}^{2}=\left(M^{-1}\right)_{q q} S /(N-P)
$$

It follows that for $\mathrm{N}$ large enough, e.s.d.'s behave like $1 / \sqrt{N}$.

Such a decrease, which is the normal behaviour for parameters obtained by least square analysis, leads to very small values for e.s.d.'s. Sometimes they can be even smaller than values obtained with single crystals. Here was the source of the debate. E.s.d.'s are statistical values, so they cannot tell anything that is not in the model. They are estimates of the precisions of parameters and not of their accuracies. We can obtain very precise but inaccurate (wrong) results (Schwarzenbach, Abrahams, Flack, Gonschorek, Hahn, Huml, Marsh, Prince, Robertson, Rollet \& Wilson, 1989). In the case of the Rietveld method, Prince (1985) has demonstrated that e.s.d.'s are unbiased estimates, but it is assumed that the model is right. Then the remaining differences between the observed and the calculated values are due only to the Poissonian noise. In such case the Goodness of Fit $(G o F)$ has its square distributed like a $\chi^{2}$ with $(N-P)$ degrees of freedom.

$$
G o F=\sqrt{S /(N-P)}
$$

Because the expected value of $G o F$ is 1 , its value in a fit should be smaller than 1.4 if $(N-P)$ is large enough.

In the case of Rietveld refinements, other kinds of discrepancy frequently appear. They are due to serial or local correlations and can be characterized by the Durbin-Watson $d$ distance (Hill, Flack, 1987):

$$
d=\sum_{i=2}^{N}\left(a_{i}-a_{i-1}\right)^{2} / \sum_{i=1}^{N} a_{i}^{2}
$$

For random data, $d$ has an expected value of 2 , and if $(N-P)$ is large enough, $d$ will not deviate from 2 by more than $3 / \sqrt{N}$. Values of the distribution are given in Hill \& Flack (1986) and Theil \& Nagar (1961).

As a consequence of (1), it is normal that the Rietveld e.s.d.'s $s_{q}$ are lower than those calculated by integration or individual fit. The sampling realized by the fit gives a better estimate of the integrated intensity (Table 1). If we add a lot of background data,

Table 1: Precision of intensity measurement for various method.

\begin{tabular}{lrrr} 
& Summation & \multicolumn{2}{c}{ Individual fit } \\
\hline points & 50 & 50 & 200 \\
parameters & 1 & 5 & 5 \\
intensity & $992(33)$ & $975.2(8)$ & $975.2(8)$ \\
background & $19.2(2)$ & $19.200(6)$ & $19.200(3)$
\end{tabular}

the e.s.d.'s on the intensity are close to those previously obtained because we do not modify the $M_{p q}$ matrix elements that are directly related to this estimate. Only the significant points really contribute to the e.s.d.'s. In a Rietveld or a whole pattern fit, most of the parameters are common to all lines. Then the equivalent number of parameter for each line is close to 1 and e.s.d.'s are reduced more than twice in comparison to single line fits. 


\section{Estimated probable error}

With X-ray diffraction, we frequently encounter data that do not fit well. These data exhibit serial or local correlation. Their $d$ values significantly differ from 2 . This was the objection given by Cooper (1982) about the e.s.d.'s, but it was mainly thought that the local correlations where associated with the intensities of lines. This kind of local correlations vanishes when the structural model is right. However, in many data sets there are local correlations that are due to insufficient knowledge of profile functions. It is quite easy

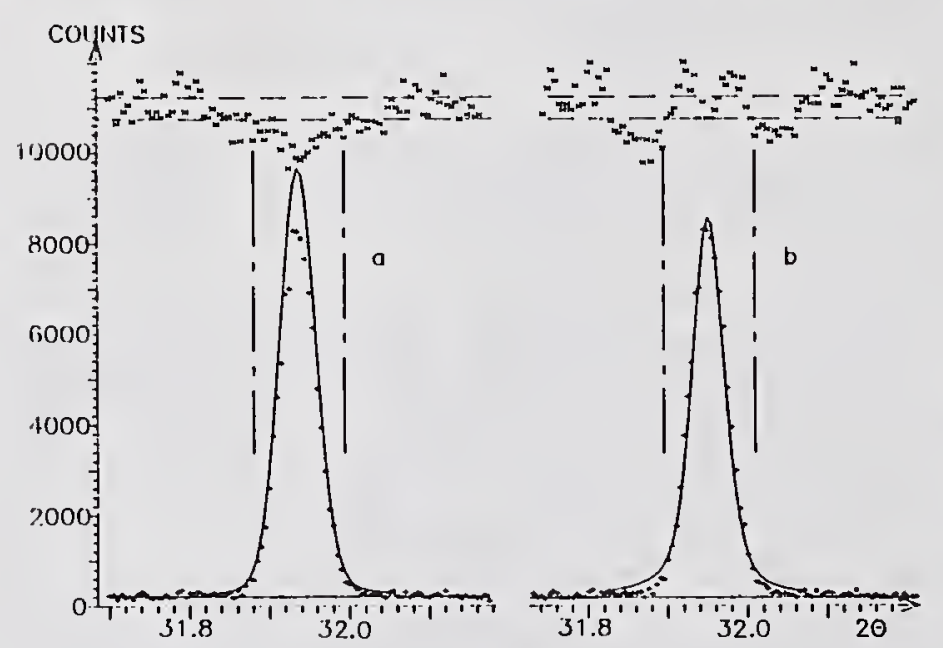

Figure 3: Local correlation associated with profile or intensity misfit.

to characterize the two cases. If the local correlations are due to the profile, they appear on the difference (Fig. 3) as small peaks with opposite sign, whereas if it is an intensity error the correlation length is similar to the line width.

In a pragmatic approach, when this occurs, we can extract subsets out of the recorded data up to a limit where the correlations vanish. Data are then in agreement with the model, and the e.s.d.'s values are meaningful. Following this procedure, mean values of parameters and e.s.d.'s can be directly calculated from equivalent subsets (Table 2).

Table 2: Mean value and e.s.d. obtained with 5 $\alpha \mathrm{PbO}$ simulated data sets $\left(z_{\mathrm{Pb}}=.2344\right)$ including the same profile misfit.

\begin{tabular}{crrr} 
Step width $\left({ }^{\circ} 2 \theta\right)$ & .005 & .020 & .080 \\
\hline$\left\langle z_{P b}\right\rangle$ & .2348 & .2339 & .2339 \\
$s$ & 5 & 7 & 13 \\
$\left\langle s_{k}\right\rangle$ & 3 & 6 & 11 \\
$\left\langle s_{k}^{\prime}\right\rangle$ & 6 & 8 & 12
\end{tabular}

We can obtain similar results assuming that all correlated differences are due to the same cause
(Bérar \& Lelann, 1991). Their contribution are added linearly. The $m$ independent errors are then added quadratically. It is possible to define a level of significance above which differences have to be considered to be correlated. It seem more efficient to split the differences in two terms according to the probability that such differences will be correlated. A modified $S^{\prime}$ can be calculated.

$$
S^{\prime}=\sum_{i}\left(1-z^{2}\right) a_{i}^{2}+\sum_{m}\left(\sum_{m l} z_{i} a_{i}\right)^{2}
$$

In a first try, we have considered that $z$ is pro portional to the probability of $\chi^{2}$ being inferior to $x=a_{i}^{2}+a_{i-1}^{2}$ in the $\chi^{2}$ distribution with two degrees of freedom.

$$
z= \begin{cases}\sqrt{2 \pi x} /(2+\sqrt{2 \pi x}) & \text { if } a_{i} a_{i-1}>0 \\ 0 & \text { otherwise }\end{cases}
$$

But with such a choice, if you look at random data, $S^{\prime}$ will be $20 \%$ higher than $S$ (Ross, 1991). This discrepancy can be reduced to $10 \%$ if we use the ratio of probability of $x$ to taking a value in the $\chi^{2}$ distribution with one or two degrees of freedom. The remaining which could be corrected is due to the probability that $x$ can take a value in random data.

$$
z= \begin{cases}x \sqrt{2 \pi x} /(2+2 x+\sqrt{2 \pi x}) & \text { if } a_{i} a_{i-1}>0 \\ 0 & \text { otherwise }\end{cases}
$$

The probable error $s_{k}^{\prime}$ on the parameter $k$ is in the ratio $\rho=\sqrt{S^{\prime} / S}$ according to the previously calculated e.s.d.'s

$$
s_{k}^{\prime}=\rho s_{k}
$$

that is, the value obtained in the limit where the correlations vanish. In practical cases, the range of $\rho$ is 1.5 to 3 .

As we know in many Rietveld refinements there are some discrepancies. It may seem better to minimise the $S^{\prime}$ sum. Such methods have already been used in other techniques (Mijlhoff, 1991, Alcock, 1991). It corresponds to the use of a non-diagonal weighting scheme (Murata \& Morino, 1966; Beagley, Robiette \& Sheldrick $1968 a ; 1968 b)$. Our first attempts we are not at present sufficient to draw any conclusions.

\section{Precision and accuracy}

We have to remember that e.s.d.'s and the associated probable errors are only precision indicators. They are not concerned with the accuracy of results. The essential problem of accuracy is not satisfactorily resolved, but accuracy is needed when we have 
to compare Rietveld results to others. Accounting for systematic errors as if they are random noise can produce some bias in results, even if statistical assumptions seem to be valid $(G o F \approx 1, d \approx 2)$. If we want to make real progress in accuracy, the only way is to improve the Rietveld model in software or to obtain simpler line shapes, as in the synchrotron case. At present many of the discrepancies can be imputed to profile functions.

Better profile functions have to take into account the real emission spectra, which can include the line commonly referred to as $\alpha_{3}$ (Howard \& Preston, 1989 ), some $W L \alpha$ lines, and part of the continuous spectrum. A better angular dependence of asymmetry and width can be used. In the low angles range instrumental broadening (axial divergency) behaves like $1 / \sin 2 \theta$. The specific problems related to size and shape can be avoided using an $(h k l)$ or an angular dependence of line width parameters; it is easy to expand them using spherical harmonics. A similar expansion allows easier handling of the orientational correction. The software must really take into account the geometrical arrangement in cases of nonstandard geometry (position sensitive detector, ...).

Some other causes of systematic error have to be checked: anomalous dispersion effects; extinction, which has been noticed in powders (Sabine, 1988); and the poor knowledge of background. Thermal diffuse scattering is really nonuniform in space and is a problem in absolute intensity measurements (Suorti, 1977). It will introduce systematic errors into Rietveld refinements. It is mainly this background to signal ratio that promotes single crystal experiments, not the fact that there are overlap problems to solve.

When we want more accurate results, we also need good, well characterized samples.

To conclude, a lot of progress has to be made to obtain more accurate values, but our insufficient knowledge of the profile is not so dramatic. It can be seen with simulated data that the induced bias on structural parameters is very small. Furthermore it seems possible to profit from the remaining local correlations to improve the profile function and then the accuracy of structural parameters. At the present the Rietveld model is not wrong, but it needs to be completed.

\section{References}

Albinati, A., Cooper, M. J., Rouse, K. D., Thomas, M. W. \& Willis, B. T. M. (1979). Natl. Bur. Stand. (US) Spec. Pub. No. 567, pp.203-210.

AlCOCK, N. W. (1991). private communication.

ANToniadis, P., BerRuYer, J. \& Filhol, A. (1990). Acta Cryst. A46, 692-711.

Beagley, B., Robiette, A. G. \& Sheldrick, G. M. (1968a). J. Chem. Soc. A, 3002-3005.

Beagley, B., Robiette, A. G. \& Sheldrick, G. M. (1968b). J. Chem. Soc. A, 3006-3008.

BÉRAR, J.-F. (1990). I. U. Cr. Satel. Meeting "Powder Diffraction", Toulouse.

Bérar, J.-F. \& Lelann, P. (1991). J. Appl. Cryst. 24, 1-5.

Cooper, M. J. (1982). Acta Cryst. A38, 264-269.

Cooper, M. J., Sakata, M. \& Rouse, K. D. (1979). Natl. Bur. Stand. (US) Spec. Pub. No. 567, pp.167-187.

Flack, H. D. \& Vincent, M. G. (1980). Acta Cryst. A36, 495-496.

HILl, R. J. \& FlacK, H. D. (1987). J. Appl. Cryst. 20, 356-361.

Hill, R. J. \& Madsen, I. C. (1984). J. Appl. Cryst. 17, 297-306.

Hill, R. J. \& Madsen, I. C. (1986). J. Appl. Cryst. 19, 10-18.

Hill, R. J. \& Madsen, I. C. (1987). Powd. Diff. 2, 146-162.

Howard, S. A. \& Preston, K. D. (1989). Review in Mineralogy (US) 20, 217-275.

MiJHOLF, F. C. (1991). private communication.

Murata, Y. \& Morino, Y. (1966). Acta Cryst. 20, 605609.

Pawley, G. S. (1980). J. Appl. Cryst. 13, 630-633.

Prince, E. (1981). J. Appl. Cryst. 14, 157-159.

PRINCE, E. (1985). Structure \& Statistics in Crystallography, A. J. C. Wilson, ed. pp 95-103, Guilderland, NY: Adenine Press.

Ross, C. R. (1991). private communication.

Rietveld, H. M. (1969). J. Appl. Cryst. 2, 65-71.

Sabine, T. M. (1988). Aust. J. Phys. 41, 413-422.

Sakata, M. \& Cooper, M. J. (1979). J. Appl. Cryst. 12, 554-563.

SchwarzenbaCh, D., Abrahams, S. C., Flack, H. D., Gonschorek, W., Hahn, Th., Huml, K., Marsh, R. E., Prince, E., Robertson, B. E., Rollet, J. S. \& WIISON, A.J.C. (1989). Acta Cryst. A45, 63-75.

ScotT, H. G. (1983). J. Appl. Cryst. 16, 159-163.

Suorti, P. (1977). Acta Cryst. A33, 1012-1027.

TheIL, H. L., \& Nagar, A. L. (1961). J. Am. Stat. Assoc. 56, 793-800.

Young, R. A. (1979). Natl. Bur. Stand. (US) Spec. Pub. No. 567 , pp.143-165. 
National Institute of Standards and Technology Special Publication 846. Proceedings of the international conference Accuracy in Powder Diffraction II, held at NIST, Gaithersburg, MD, May 26-29, 1992. (Issued October 1992)

\title{
NIST XRD Standard Reference Materials: Their Characterization and Uses
}

\author{
J. P. CLINE \\ Materials Science and Engineering Laboratory, National Institute of Standards and Technology, \\ Gaithersburg, MD 20899 U. S. A.
}

\section{SRM production and certification}

Standard Reference Materials (SRMs) from the National Institute of Standards and Technology (NIST) are designed to increase the accuracy and precision of measurements which are pertinent to science and industry. Powder diffraction SRMs consist of stable materials which have one or more diffraction properties measured and certified. The success of an SRM is dependent on the material and certification measurements being of such quality that the accuracy and precision of the certified values allows for the calibration of subsequent measurements made by the user community. The standardization of such measurements is achieved by the performance of a material rather than the standardization of equipment design and/or measurement procedures.

The certification of a NIST SRM begins with the isolation of a measurement error which may be corrected with the proper use of a standard. A preliminary study is undertaken to determine the feasibility of producing an SRM which will address the problem. In addition, the potential demand for, and impact of, the SRM is evaluated. A proposal for the production of the SRM is then submitted to the Standard Reference Materials Program (SRMP) for review and subsequent funding. The SRMP of NIST operates as a non-profit business with the costs of developing and producing an SRM being fully recovered by the sale of that SRM. Thus the demonstration of the demand for the SRM is critical to a favorable response.

Once the decision to produce the SRM has been reached, an "idealized character" of the SRM material is determined from a review of the literature. The effects of deviations from this "ideal" character exhibited by available materials are the subject of subsequent experiments. The characteristics investigated are those judged as capable of having an effect on the critical property which is at least as large as the smallest conceivable measurement error of that property. The study may include the development of improved measurement techniques which increase the accuracy and precision of the property determinations. Throughout this work the procurement or production of several kilograms of material for the SRM itself is under consideration. The material selected for the SRM must be optimal with respect to the desired property, homogeneous, stable, and of such character as to minimize errors in the measurements made by the end user.

When the required quantity of material has been obtained and verified to be acceptable, it is riffled and bottled by the SRMP. During bottling, a representative sampling of bottles is removed from the stock for certification measurements. The certification process is designed to determine the homogeneity of the material and also determine, via methods traceable to fundamental physical constants, the value of the certified property. However, in the case of certain SRMs, such as the line broadening SRM 660 , the desired property does not lend itself to absolute measurement. In such cases the desired property is determined to be appropriate for the demands placed on the SRM by supplemental research or a round robin study, in which case the certification process is used to determine homogeneity only. Modern statistical procedures are used to design and analyze experiments to insure that the measurements are as accurate as possible and the uncertainties fairly reflect the precision of those measurements.

\section{Line position SRMs}

The primary line position SRM, silicon SRM $640 \mathrm{~b}$, is the most popular of the NIST powder diffraction SRMs. SRM 640b consists of a silicon powder, jet milled to a median particle size of about $5 \mu \mathrm{m}$ from electronic grade single crystal boules, certified with respect to lattice parameter. A second line position SRM 675, fluorophlogopite, is used for low angle measurements. The popularity of SRM $640 \mathrm{~b}$ reflects the widespread adoption of the internal and external standard methods for use in lattice parameter measurements. These procedures involve the determination and application of a delta d curve which represents the end result of the various optical aberrations of the particular diffraction equipment. A polynomial is then fitted to the curve and 
applied either to subsequent measurements in the case of the external standard, or to the line positions of the unknown with which the SRM has been mixed in the case of the internal standard. The external standard method is less reliable because it cannot account for a possible sample displacement error of the unknown. Adoption of the internal standard method has resulted in consistent and routine measurements of lattice parameter to 1 part in $10^{4}$ (Edmonds et al., 1989).

SRM $660, \mathrm{LaB}_{6}$, is certified with respect to lattice parameter. However, its primary virtue is a lack of size- and strain-induced peak broadening. The suitability of this material for the determination of instrumental broadening functions prerequisite for size and strain analysis was determined with a round robin study (Fawcett et al., 1988). The SRM displays evenly spaced, non-overlapping, high intensity diffraction lines whose full-width-half-maximum (FWHM) values measured on fixed slit diffraction equipment closely follow the expected quadratic dependence on $2 \theta$ angle. These properties also render it suitable for use in determining calibration parameters for a Rietveld refinement.

The Rietveld method uses models of the origins of the specific sources of optical aberration to refine the degree to which they contribute to a delta d curve. This technique is applied to data from the unknown only; no standards are used. The delta $\mathrm{d}$ curve derived from an unknown may differ significantly from that of an admixed standard due to variations in character between the unknown and the standard. Therefore, an increase in accuracy could be realized with the use of the Rietveld method. The chief factor preventing the realization of this potential is the lack of fully developed models appropriate for conventional divergent beam $x-$ ray equipment. These models will have to address aberrations which are inherently more complex than those associated with parallel beam equipment. In the case of the software Generalized Structure Analysis System (GSAS) (Larson \& Von Dreele, 1987), the refined delta $d$ curve is obtained with a specimen transparency and sample shift correction. However, examination of actual delta $d$ curves from equipment of various manufacturers indicates that these corrections alone cannot account for the observed aberration curves.

With the application of the pseudo-Voight profile function of GSAS to conventional x-ray diffraction (XRD) data, it is reasonable to assume that the instrumental contribution is primarily Gaussian, while the sample contribution is Lorentzian. Thus refinements of SRM 660 can be used to obtain a set of initial profile parameters; only the Lorentzian terms are released in subsequent refinements. The value of the Lorentzian terms obtained with SRM 660 are then subtracted from those refined for unknowns in size and strain estimations. In this application the models describing optical aberrations affecting peak position can be tested using a material of known lattice parameter. However, in order to perform this function the lattice parameter of the SRM and the wavelength of the $x$-rays must be known to an accuracy and precision greater than the error introduced by improper or incomplete model(s) describing the optical parameters.

The wavelength of $\mathrm{Cu} K \alpha_{1}$ radiation has been determined to be $0.15405945 \mathrm{~nm}$ by combining single crystal lattice spacing measurements (x-ray/optical interferometry) and diffraction angle measurements (Deslattes \& Henins, 1973; Kessler, Deslattes \& Henins, 1979). The quoted value is taken from "The 1986 adjustment of the fundamental constants" and is considered accurate to 0.7 part in $10^{6}$ (Cohen \& Taylor, 1987). The lattice parameters of the silver and tungsten used as internal standards for the certification of SRMs $660,640 \mathrm{~b}$, and 675 were measured in 1966 with a high precision, temperature controlled, back reflection camera (Swanson, Morris \& Evans, 1966). Linkage to the fundamental length standard was through the wavelength of the radiation used.

The procedure by which the lattice parameters of existing SRMs were measured and certified is neither precise nor accurate enough to yield SRMs suitable for development of the Rietveld method as applied to divergent beam $x$-ray diffraction equipment. A project has begun to develop a new high accuracy/precision lattice parameter SRM(s) linked to the fundamental length standard via procedures which are independent of the $\mathrm{x}$-ray wavelength determination. This project involves the lattice parameter determination of a silicon single crystal relative to the iodine stabilized $\mathrm{HeNe}$ laser length standard with an X-Ray Optical Interferometer (XROI). A second experiment will transfer the lattice parameter measurement from the single crystal of silicon to a crystalline powder via $x$-ray diffraction equipment of parallel beam optics. Supplemental studies will include an investigation of the effects of crystallite size on lattice parameters. It is anticipated that this project will result in an SRM in about 2 years.

\section{Instrument sensitivity SRM}

Line position SRMs are used to calibrate equipment with respect to the angular variable of the goniometer. SRM 1976 is a new SRM which is designed to calibrate equipment for the intensity variable as a function of $2 \theta$ angle, i.e., the instrument sensitivity. The need for this SRM was defined by the Jenkins, Schreiner \& Dismore (1992) round robin which was, in turn, prompted by the consideration of a new generation of database by the ICDD. The new database will consist of complete 
digitized diffraction patterns as opposed to reduced $d$ and I values. The removal of any instrumental aberrations from the reference data reported to the ICDD for use in this database will require as yet undeveloped algorithms. The round robin was used to assess the level of variation in instrument performance, which indicates the degree of difficulty to be faced in the building of the new database. Highly uniform, sintered $\alpha$ alumina, corundum structure, plates were circulated among selected respondents with instructions on the collection of a range of intensity data. Among other things, variations in instrument sensitivity of up to $30 \%$ were discovered.

SRM 1976 consists of a sintered $\alpha$ alumina plate originally manufactured as a micro-chip carrier. The plates exhibit considerable, though highly uniform and cylindrically symmetrical, levels of preferred orientation. This cylindrical symmetry allows the use of a sample spinner to reduce the errors of particle counting statistics. The certified values include the lattice parameters, 12 relative intensity values from 25 to 145 degrees $2 \theta$, and absolute variation in intensity. The certification of the last of these parameters allows for comparison of absolute diffraction intensity from equipment at differing locations.

The round robin study indicated only that a problem existed with regards to variation in instrument sensitivity. With the exception of groupings in performance by manufacturer, it indicated little as to its origins. The certified relative intensity data of SRM 1976 were collected on a Philips ${ }^{1}$ diffractometer of conventional optical layout which included a graphite diffracted beam monochromator (Cline, 1992). This "reference" machine was determined to be in "correct" alignment with respect to the results of the Jenkins, Schreiner \& Dismore (1992) round robin. However, due to a lack of understanding of the mechanisms responsible for the effects observed, this machine could not be determined to be any more suited as a reference than any other properly aligned, conventional $x$-ray diffractometer. Early experiments found no dependence of instrument sensitivity on the size of the incident and receiving slits, or the presence or absence of soller slits, either incident and receiving.

The use of SRM 1976 for evaluation of instrument sensitivity entails the collection of data on the test instrument in a manner analogous to that used in the certification. The uniformity of the SRM material with

\footnotetext{
${ }^{1}$ Certain commercial equipment, instruments, or materials are identified in this paper in order to specify the experimental procedure adequately. Such identification is not intended to imply recommendation by NIST, nor is it intended to imply that the materials or equipment identified are necessarily the best available for the purpose.
}

respect to size and strain broadening (minimal) permits the use of peak heights. They were included in the certification. Evaluations performed with the use of peak heights will reflect the lower precision of this type of intensity measurement. Certified integrated intensities were measured using the Siemens version of the NBS*Quant (Hubbard, Robbins \& Snyder, 1983) algorithm. The use of this algorithm is not required, but a credible method of determining a background subtracted integrated intensity measurement should be employed. Graphical evaluation of the performance of the test instrument is accomplished by plotting the ratio of the 12 relative intensities measured from the test instrument to the certified values as a function of $2 \theta$ angle. Such a plot is shown in figure 1.

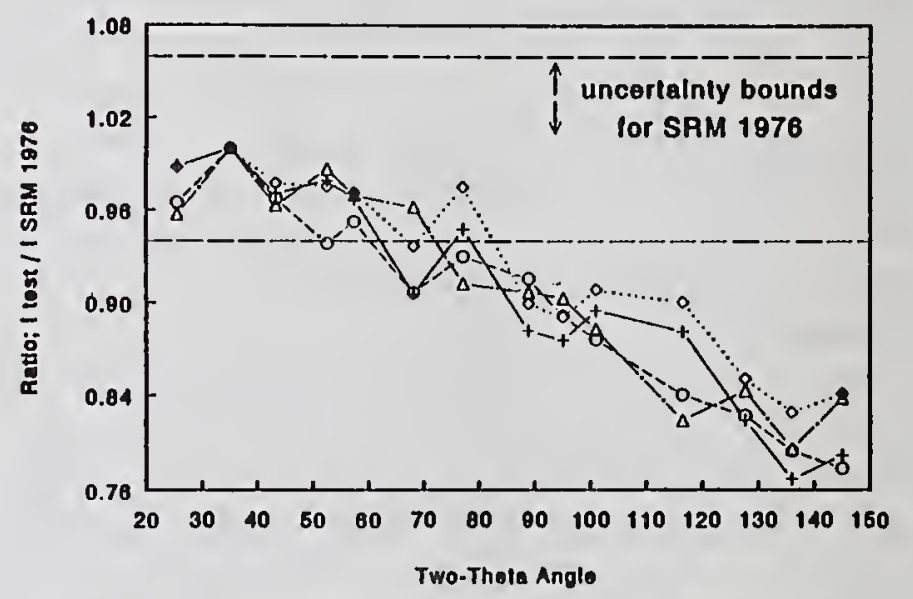

Figure 1. Graphical presentation of test machine results from four samples of SRM 1976.

The upper and lower bounds displayed in figure 1 represent the coverage of $90 \%$ confidence, $95 \%$ coverage tolerance interval for the certified relative intensity data. Tolerance intervals were used due to the expected heterogeneity of the levels of preferred orientation and thus the intensity values measured; no true mean for the population as a whole exists. The preferred orientation is expected to uniformly affect all measurements, thus data analysis assumed one true relative variance (Cline, Schiller \& Jenkins, 1991).

Interpretation of the plot may require some scientific judgement on the part of the operator. Patternless scatter within the error bounds indicate the test machine performs in a manner analogous to the reference machine and needs no correction. If the scatter of the data is such that more than one peak falls outside the error bounds, a lack of precision on the part of the user measurements is indicated. Patterns observed in the data, even within the error bounds, indicate that a correction may be necessary. Procedures to test this hypothesis can be found in the appendix of the SRM 1976 certificate (Cline, 1992). The data of figure 1 clearly indicate that a correction curve should be applied to intensity data collected from this machine. 
During a realignment of the test machine of figure 1 , it was determined that slight changes in the angle of the graphite monochromator crystal would cause changes in instrument sensitivity of up to $20 \%$. The machine was a second Philips diffractometer similar to the reference, with the exception of being equipped with an automatic sample changer as opposed to a sample spinner. The effect occurred through a rocking angle of the graphite crystal within which the intensity of the (104) reflection $\left(35.1^{\circ} 2 \theta\right)$ from an SRM 1976 sample did not vary significantly. The tuning of the monochromator was performed to minimize contributions from the $\mathrm{Cu} K \beta$ and W L lines. Alignment of the crystal to minimize this spectral contamination resulted in the calibration curves of figure 1 . The test machine could be made to perform identically to the reference machine with an appropriate setting of the monochromator crystal. This observation indicates the slight variations in the graphite monochromator angle are responsible for at least part of the effects observed in the Jenkins \& Schreiner round robin. A more exhaustive study of the causes of variation in instrument sensitivity is underway utilizing a survey of selected equipment of different manufacturing origin (Cline, Schiller \& Jenkins, 1992).

\section{Quantitative analysis SRMs}

Quantitative analysis SRMs are powders selected to offer a diffraction intensity as close to the "ideal" as possible. They are of high phase purity, small crystallite size, and of isometric particle morphology. They may be specific to given systems, as in the case of SRMs 1878 and 1879, which are designed for quantification of quartz and cristobalite, respectively, in airborne dusts. Alternatively, SRM 674a consists of a set of five powders of varying absorptivity: $\alpha \mathrm{Al}_{2} \mathrm{O}_{3}$, $\mu=126 \mathrm{~cm}^{-1} ; \mathrm{ZnO}, \mu=279 \mathrm{~cm}^{-1} ; \mathrm{TiO}_{2}, \mu=536 \mathrm{~cm}^{-1}$; $\mathrm{Cr}_{2} \mathrm{O}_{3}, \mu=912^{-1}$; and $\mathrm{CeO}_{2}, \mu=2203 \mathrm{~cm}^{-1}$ (absorptivities are for $\mathrm{Cu} \mathrm{K} \alpha$ radiation). This selection of materials allows for the matching of the absorptivity of the standard to that of the unknown, minimizing the effects of microabsorption.

Recently SRM 676 was certified. It consists of an $\alpha$ alumina powder designed for measurement of $I / I_{c}$ values. The concept of the $I / I_{c}$ as a constant relating a material's diffraction intensity to that of $\alpha$ alumina, corundum, was proposed by Visser \& deWolf (1964). It is a specific case of the more general Reference Intensity Ratio (RIR) later proposed by Chung (1974 $a, b)$. The $I / I_{c}$ concept has been subsequently adopted by the ICDD and is one of the material constants included in the PDF2 database. The RIR of any two phases can be determined from the ratio of their respective $I / L_{c}$ values. Thus the listing of these values in the database allows for quantitative information to be gained in a more routine manner.

The selection of the alumina powder for SRM 676 involved a study of the dependence of diffraction intensity on specific powder characteristics (Cline \& Von Dreele, 1992). The first of these, obviously, is phase purity; a basic problem surfaces immediately for quantitative XRD SRMs based on commercial powders. The ceramics industry requires active powders for use in the manufacture of sintered ware. These powders are generally in the tenths of a micron size range and are of a high surface area. The resultant line broadening is undesirable, but cannot be considered a major factor in selection of quantitative analysis SRMs; a credible intensity measurement will consist of an integrated intensity. The high surface area is detrimental due to the potential increase in the mass fraction of an "amorphous" non-diffracting, surface phase. This surface phase, while possessing some degree of order, does not contribute to Bragg diffraction from the bulk crystal structure. The exact amount of the impurity phase is dependent on the lattice energy of the bulk crystal structure, the specific surface of the material, and its processing history.

The consideration of surface phase contamination is particularly problematic for aluminum oxide powders. Commercial alumina powders are products of the Bayer process wherein, initially, an aluminum hydroxide precursor is chemically generated. This precursor material is subsequently calcined through a series of well known transition aluminas until the high temperature $\alpha$ phase is reached (Wefers \& Bell, 1972). Highly active, sinterable, alumina powders are often incompletely calcined and are phase contaminated by several of the transition aluminas. Complete conversion to the $\alpha$ alumina requires a long duration calcination to at least $1200 \mathrm{C}$. Powders produced by this process can not be considered ideal for XRD purposes either due to their large grains, state of aggregation, and platy crystal habit. Early experiments indicated that a prerequisite for precise $I / I_{c}$ measurements are fully deaggregated powders. Extensive experiments evaluated four highly calcined, deaggregated, phase pure powders of relatively small crystallite (particle) size, for suitability as this SRM material.

The experimental design considered the effects of extinction, the "amorphous" non-diffracting surface layer, and preferred orientation. The first two of these effects will manifest themselves in terms of a reduction in diffraction intensity. The isolation of the material offering the maximum diffraction intensity was accomplished by first spray drying mixtures of the test materials and silicon, SRM 640b, to eliminate the effects of preferred orientation (Cline \& Snyder, 1983). An $1 / I_{c}$ determination of SRM $640 b$ relative to the test 
materials was performed via Rietveld refinements of XRD data collected from these and conventionally prepared specimens. Equipment consisted of a Siemens D500 diffractometer equipped with a focusing $\mathrm{Ge}$ incident beam monochromator, sample spinner, and a position sensitive detector. The conventionally prepared specimens provided data on the levels of preferred orientation via refinements of the March-Dollase parameter (Dollase, 1986). Extinction effects were measured using the Sabine model, (Sabine, 1985; Sabine, Von Dreele \& Jorgensen, 1988) in conjunction with Rietveld analysis of time-of-flight (TOF) neutron measurements. Additional measurements included particle size via laser scattering, specific surface measurements, and examination with a scanning electron microscope.

Results from these four powders as well as the final SRM 676 material are shown in table 1 . A low $I / I_{c}$ value was obtained from Linde $C$ which exhibited no extinction but had the largest surface area of the four test materials. This may indicate that the effect of its presumably higher amorphous content was offset by the extinction effects of the other candidates. However, this conclusion is not supported by the observation that the Sumitomo material exhibited considerable extinction and also displayed a favorable $I / I_{c}$ value. This may indicate an as yet undetermined variable is operative or that the magnitude of the effects is below the measurement precision. The refined March-Dollase parameters, which equals one for a randomly oriented powder, indicated the Linde $\mathrm{C}$ material did not display preferred orientation. The SRM itself was prepared from a second lot of the Linde C product. SRM 676 was certified with respect to lattice parameters and seven relative intensity values from 24 to 78 degrees $2 \theta$. The certification of the relative intensity values allows the user to measure any one of the eight lines for an $\mathrm{I} / \mathrm{I}_{\mathrm{c}}$ determination (use of more than one line is recommended). The machine used for collection of the certified relative intensity data was the "test" machine discussed in the section concerning SRM 1976. In addition to an adjustment of the reported values, the reported uncertainty also had to reflect the measurement uncertainty of SRM 1976 leading to the calibration curve (figure 1). This was done using the statistical technique of bootstrapping. Figure 2 indicates the dramatic increase in the uncertainty from the 30 certification measurements of the (024) reflection of SRM 676 with the incorporation of the uncertainty of test machine measurements of SRM 1976. The three vertical lines represent the size that a $95 \%$ confidence interval for the mean would be when different sources of error are incorporated. The smallest (leftmost) interval includes only the variability in the 30 measurements of SRM 676. In the middle interval, the uncertainty in the calibration curve has been included as well. Finally, the third interval incorporates the uncertainty in the certified values of SRM 1976, as well as the uncertainty in the calibration curve and the variability of the measurements of SRM 676.

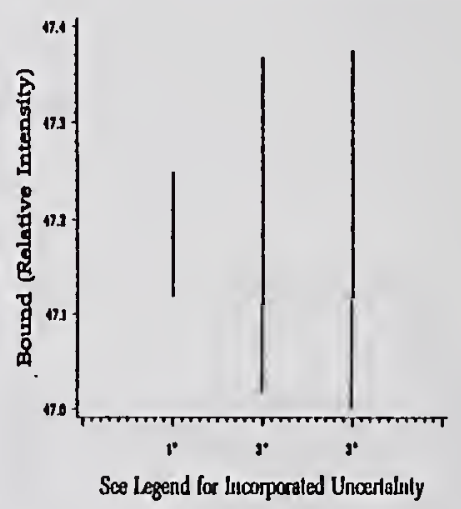

Incopporated Uncertainties

1. Uncorrected Observallons of 676

2. Uncorrecied Observalions of 676 Test Device Catibration

3. Uncorrecled Observallans of 676 Test Devlce Calibration
1976 Cenificalion

Figure 2. Comparison of $95 \%$ confidence intervals of SRM 676 certification data when different sources of error are included.

These data indicate that the uncertainty in relative intensity values, once converted to an interlaboratory scale via SRM 1976, is due as much to the uncertainty in the conversion as to the variability in the uncorrected

Table 1. Results from measurements of SRM 676 candidate materials

\begin{tabular}{|c|c|c|c|c|c|}
\hline $\begin{array}{c}\text { Material or } \\
\text { Supplier }\end{array}$ & $\begin{array}{c}\text { Mean Particle } \\
\text { Size, } \mu \mathrm{m} \\
\text { Laser }\end{array}$ & $\begin{array}{c}\text { Refined } \\
\text { Domain Size, } \\
\mu \mathrm{m} \text { TOF data }\end{array}$ & $\begin{array}{c}\text { BET Surface } \\
\text { Area, m²/gr }\end{array}$ & $\begin{array}{c}\text { I/I Values for } \\
\text { Si, SRM 640b }\end{array}$ & $\begin{array}{c}\text { March - } \\
\text { Dollase coef. }\end{array}$ \\
\hline Linde C & 1.5 & -- & 3.34 & 4.105 & .998 \\
\hline IBM & 4.3 & 1.4 & 1.27 & 4.173 & .818 \\
\hline Sumotomo & 4.3 & 2.5 & .93 & 4.097 & .818 \\
\hline Alcan & 4.0 & 2.7 & 1.07 & 4.176 & .767 \\
\hline SRM 676 & 1.4 & -- & 4.98 & 4.104 & .998 \\
\hline
\end{tabular}


measurements themselves. This suggests that users of SRM 1976 need to incorporate the uncertainty of this correction into the total of their measurements. The bootstrapping approach used here is not practical for user at other laboratories, but a simpler approximation is being developed at NIST and will be described in an upcoming publication (Cline, Schiller \& Jenkins, 1992).

Another new quantitative analysis SRM, which is presently being certified, is specific to analysis of silicon nitride (Cline \& Von Dreele, 1992). The ceramics industry is interested in silicon nitride due to its desirable high temperature properties. Ubiquitous to processing this material is the transformation of the starting low temperature, $\alpha$ phase to the high temperature, $\beta$ phase. Approximately $90 \%$ of typical commercial powder designed for the production of sintered ware will consist of the $\alpha$ phase, with the remainder being amorphous material and the $\beta$ phase. Upon sintering, the $\alpha$ phase transforms into the $\beta$, resulting in an interlocking microstructure of acicular $\beta$ grains. This interlocking microstructure, along with the intrinsic properties of the $\beta$ phase of $\mathrm{Si}_{3} \mathrm{~N}_{4}$, are responsible for the desired high temperature performance and fracture toughness of this material.

The $\alpha$ to $\beta$ ratio of both starting material and the subsequent sintered ware is known to affect properties and is the primary issue addressed by this SRM. However, after discussions with industrial representatives, it became apparent that the ability to analyze for amorphous content was also desired. The SRM itself will consist of two powders, one high in $\alpha$ content, the other high in $\beta$. Each is to be certified with respect to $\alpha / \beta$ ratio and the amorphous content and thus will be suitable for use as a spiking material for user measurements.

The analysis of amorphous content can be performed with the assumption that a spiking phase is $100 \%$ crystalline. Samples of the commercial " $\alpha$ " material selected as the SRM powder were spiked with various concentrations of a phase pure $\beta$ whisker material. XRD samples were spray dried. Rietveld refinements of XRD and TOF data allowed the refinement of a histogram scale factor and the unit cell fraction of the two phases. The use of the Rietveld method circumvents any difficulty caused by the complete interference of the $\beta$ phase's diffraction profiles with those of the $\alpha$. The failure of measured unit cell fractions to follow the expected dependence on the concentration of the spiking phase can be entirely attributed to material in the analyte which is not contributing to Bragg diffraction from either phase, defined in this case as the "amorphous phase." Equations are derived wherein a measured unit cell fraction and the mole fraction of the spikant are related to the mole fractions of the $\alpha$ and $\beta$ phases in the analyte:

$$
S_{\alpha}=\frac{\frac{x_{\alpha}}{Z_{\alpha}} f_{\alpha}}{f_{\alpha}\left(\frac{x_{\alpha}}{Z_{\alpha}}+\frac{x_{\beta}}{Z_{\beta}}\right)+\frac{f_{\beta}}{Z_{\beta}}}
$$

a similar equation may be written for $S_{\beta}$. Also:

$$
\mathrm{x}_{\alpha}+\mathrm{x}_{\beta}+\mathrm{x}_{\mathrm{A}}=1
$$

where:

$\mathrm{f}_{\alpha}=$ mole fraction of "pure" $\alpha$ phase

$f_{\beta}=$ mole fraction of $\beta$ phase

contents of "pure" $\alpha$ phase; unknowns

$\mathrm{x}_{\alpha}=$ mole fraction $\alpha$ phase

$\mathrm{x}_{\beta}=$ mole fraction $\beta$ phase

$\mathrm{x}_{\mathrm{A}}=$ mole fraction amorphous phase

$Z_{\alpha}=4 \quad S_{\alpha}=$ unit cell fraction of $\alpha$ phase

$Z_{\beta}=2 \quad S_{\beta}=$ unit cell fraction of $\beta$ phase

Rearranging the first equation and substituting $1-S_{\alpha}$ for $S_{\beta}$ gives:

$$
\frac{\left(1-f_{\alpha}\right)}{f_{\alpha}}=x_{\alpha} \frac{\left(1-S_{\alpha}\right)}{2 S_{\alpha}}-x_{\beta}
$$

Graphical presentation of this linear relation for the XRD and TOF data is shown in figure 3. The slope of this plot indicates the $\alpha$ content while the $\mathrm{y}$ intercept indicates the $\beta$ content and the difference between the two indicates the amount of the amorphous phase. The XRD data indicate a higher amorphous content than the TOF data which is believed to be caused by a humidity induced atmospheric reaction with one or both phases. This reaction can be assumed to affect the specimens of Gaithersburg, MD more than those of Los Alamos NM. These data indicate this silicon nitride powder consists of $93.8 \% \alpha$ phase, $3.4 \% \beta$ phase, and $2.8 \%$ amorphous phase. This technique can be extended to the measurement of the amorphous or impurity level of any material relative to a second material which can be considered phase pure. The "impurity level" can be considered as the amount of any material which is not contributing to Bragg diffraction from either phase. The extension of this technique to other quantitative SRMs is a topic of current investigation. 


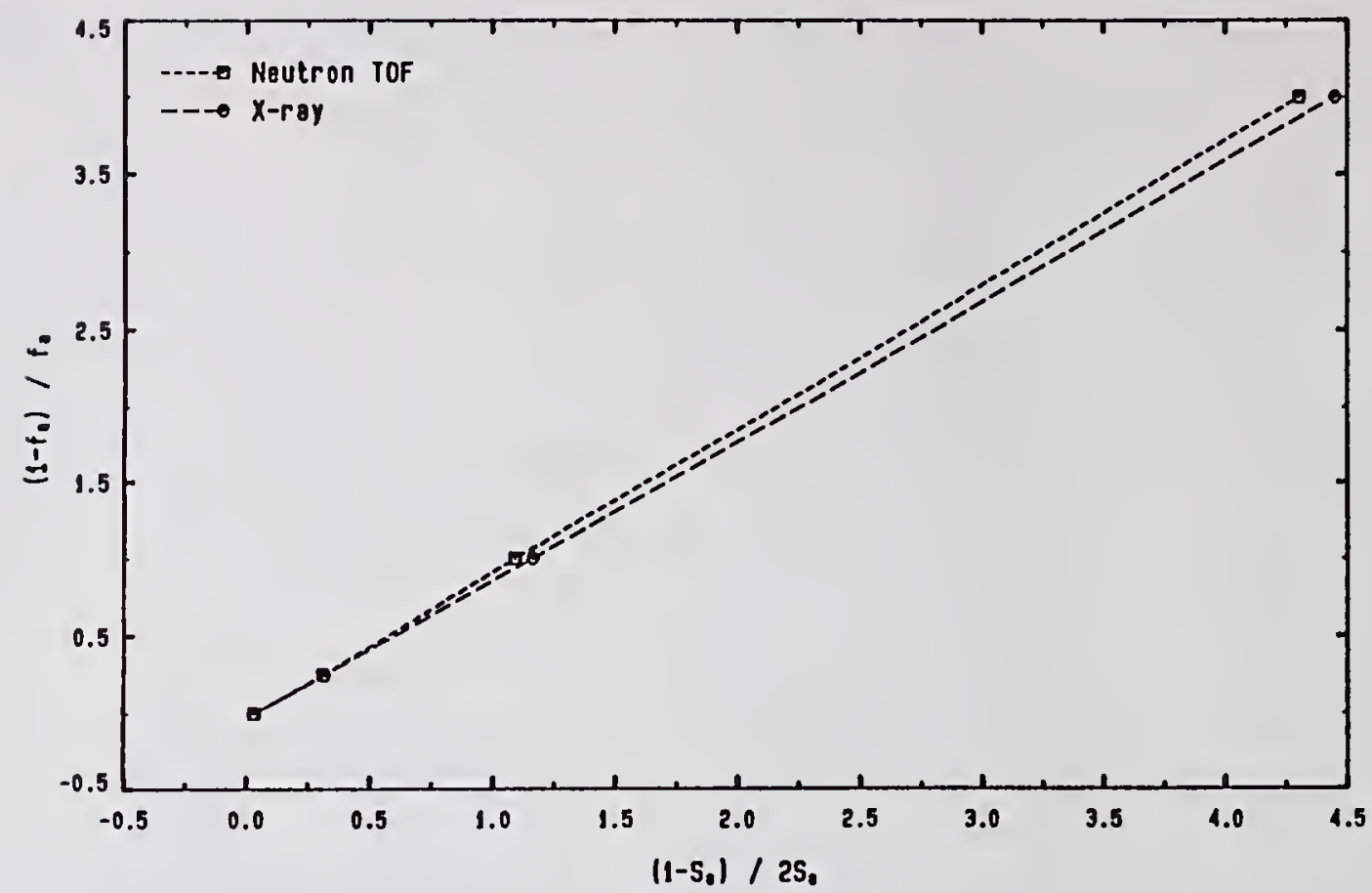

Figure 3. Graphical analysis of TOF and XRD data for the silicon nitride SRM powder of high $\alpha$ phase content.

\section{Acknowledgments}

SRMs $640 \mathrm{~b}, 675,660,1879$, and 1878 were certified under the direction of Camden R. Hubbard.

Collaborators, in order of participation:

Robert B. Von Dreele

Ron Jenkins

Susannah B. Schiller

Hung-Kung Liu

Steve Danforth

Daniel Louër

Bob Newman

Richard D. Deslattes

Ernest G. Kessler, Jr.

SRMP project monitor: Nancy Trahey

SRMP Sales Office: $\quad$ (301) 9756776

FAX (301) 9483730

\section{References}

Chung, F. H. (1974a). J. Appl. Cryst. 7, 525-531.

Chung, F. H. (1974b). J. Appl. Cryst. 7, 519-525.

Cline, J. P. (1992). SRM 1976 Certificate, NIST.

Cline, J. P. \& Snyder, R. L. (1983). Adv. X-ray Anal. 26, 111-116.

Cline, J. P., Schiller, S. B. \& Jenkins, R. (1992). in progress.

Cline, J. P., Schiller, S. B.\& Jenkins, R. (1991). $A d v$. $X$-ray Anal. 35, 341-352.
Cline, J. P. \& Von Dreele, R. B. (1992). in progress. Cohen, E. R. \& Taylor, B. N. (1987). Rev. Mod. Phys. 59, 1121.

Deslattes, R. D. \& Henins, A. (1973). Phys. Rev. Lett. 31, 972.

Dollase, W. A. (1986). J. Appl. Cryst. 19, 267-272.

Edmonds, J. et al. (1989). Methods and Practices in Xray Powder Diffraction, Section 13.1 ICDD.

Fawcett, T. G. et al. (1988). Powder Diffraction, 3, 209-218.

General Structure Analysis System, (Rietveld software package) A. C. Larson \& R. B. Von Dreele, (1987). LANSCE, MS-H805, Los Alamos National Laboratory, Los Alamos, NM 87545.

Hubbard, C. R., Robins, C. R. \& Snyder, R. L. (1983). Adv. X-ray Anal. 26, 149-157.

Jenkins, R., Schreiner, W. \& Dismore, P. (1992) to be submitted to Powder Diffraction.

Kessler, Jr., E. G., Deslattes, R. D. \& Henins, A. (1979). Phys. Rev. A19, 215.

Sabine, T. M. (1985). Aust. J. Phys. 38, 507-18.

Sabine, T. M., Von Dreele, R. B. \& Jørgensen, J. E. (1988). Acta Cryst. A44, 374-79.

Swanson, H. E., Morris, M. C. \& Evans, E. H. (1966). NBS Monograph $\# 25$, sec 4, June 28.

Visser, J. W. \& Wolff, P. M. (1964). de Absolute Intensities. Report 641.109, Technisch Physische, Delft, Netherlands.

Wefers, K. \& Bell, G. M. (1972). Technical Paper \#19, Alcoa Research Laboratories, East St. Louis, Illinois. 
National Institute of Standards and Technology Special Publication 846. Proceedings of the international conference Accuracy in Powder Diffraction II, held at NIST, Gaithersburg, MD, May 26-29, 1992. (Issued October 1992)

\title{
Integrated Software for Structure Solution from Powder Data
}

\author{
LYNNE B. McCusKer
}

Institute of Crystallography, ETH, Zurich, Switzerland

\section{Introduction}

The possibility of applying the extremely welldeveloped and exhaustively-tested single-crystal structure determination techniques to powder data has aroused much interest recently in both crystallographic and materials science circles. Many materials of industrial importance can only be prepared in polycrystalline form, yet knowledge of their structures is essential to the understanding of their properties and to the further development of related materials. In principle, the steps necessary to generate a pseudo-single-crystal data set from powder data are straightforward. In practice, there are many pitfalls awaiting the unwary.

The steps involved in producing a list of $h k l$ 's and their respective integrated intensities are: 1) the collection of the powder diffraction data, 2) the correction of the data (scale, $2 \theta$ zero correction, estimation of the background, etc.), 3) the determination of the peak positions, 4) the indexing of the pattern, 5) the determination of possible and likely space group(s), 6) the extraction of integrated intensities from the powder pattern, and 7) the treatment of severely overlapping reflections. Difficulties can be encountered in any or all of these steps, but experience has shown that the critical points tend to be the data collection itself and steps 5-7. Some practical guidelines for avoiding some of the more common mistakes, and for improving the chances of success are discussed.

Although a number of computer programs are available to perform the necessary calculations for each of these steps (Smith \& Gorter, 1991), no single software package covers the whole procedure. Consequently, data transfer from one system to the next is necessary, and must be done correctly. A concerted effort is presently underway to alleviate this problem by including intensity extraction, at least one treatment for overlapping reflections, and Rietveld refinement in the (until now) single-crystal package $X T A L$. A summary of the plans for and progress with this aspect of XTAL3.2 (Hall \& Stewart, 1992) are presented.

\section{Data collection}

Since data collection is handled in detail elsewhere (e.g. Bish \& Post, 1989 or Hill \& Madsen, 1987), only those aspects critical to structure solution will be mentioned here. If $a b$ initio structure determination is to be attempted, it is essential that the $2 \theta$ values be well calibrated, the measured intensities be accurate, and the resolution of the pattern be optimized.

The calibration of the $2 \theta$ values using an internal standard facilitates the indexing process and allows accurate lattice parameters to be determined. This information can then be used with confidence in the extraction of integrated intensities from data collected without an internal standard using a whole-profile refinement program such as ALLHKL (Pawley, 1981), that of Le Bail, Duroy and Fourquet (1988), EXTRACT (Baerlocher, 1990), or LSQPROF (Jansen, Peschar \& Schenk, 1992a).

Crystallographic methods of structure solution require that the relative intensities of individual reflections be correct. If the crystallites of a powder sample are not randomly oriented during data collection, this requirement is not fulfilled. Such preferred orientation problems should be solved experimentally if at all possible. Putting the sample in a capillary which is then rotated during data collection seems to be the simplest solution, if such a facility is available. If a flat plate sample holder is used, loading the sample either from the back or from the side and avoiding pressing is often sufficient to obtain the necessary random distribution. A further alternative is to prepare the sample using spray drying techniques.

One must also consider the X-ray absorption coefficient of the material under investigation. In general, reflection mode is preferred for strong absorbers, and transmission mode for weak ones. If a flat plate sample is used, care must be also be taken that the sample is deep enough to approximate infinite thickness, and that the X-ray beam remains on the sample (i.e. does not touch the sample holder). This means that a smaller divergence slit is needed at lower angles than at higher angles, and that the data must be corrected accordingly.

To maximize the information content of a powder pattern, the overlap of reflections in $2 \theta$ needs to be minimized. This can be done by taking care that the 
sample itself is highly crystalline, and by optimizing the resolution of the diffractometer used. If the sample broadening is low (i.e. the full width at half maximum (FWHM) of the peaks is determined primarily by the instrumental function), a measurement at a synchrotron radiation facility may be appropriate.

The high intensity and parallel nature of synchrotron radiation allow very high resolution powder diffraction data to be collected. At present, diffractometers with Debye-Scherrer geometry and a simple slit system (Cernik \& Bushnell-Wye, 1991), with Parrish-Hart multiple foils (Parrish, Hart, Huang \& Bellotto, 1987), and with a post-sample analyzer crystal (Cox, Hastings, Cardosa \& Finger, 1986 and Arnold et al., 1989) are available. The first yields simple instrumental peak shapes and good resolution, the second is restricted to flat plate samples but provides high intensity and moderate resolution, and the third results in more complex peak shapes but excellent resolution. Since the last two diffraction geometries are also independent of sample displacement, they provide accurate $2 \theta$ values which are ideal for indexing purposes. The optimal choice of geometry depends upon the quality of the sample and the purpose of the investigation.

\section{Space group determination}

A powder diffraction pattern rarely allows a space group to be determined uniquely. The number of single reflections is limited, so systematic absences are difficult to establish with certainty. Electron diffraction can be very useful in reducing this ambiguity, but, even so, several possible space groups usually emerge. These must then be probed systematically. Certainly for the application of direct methods of structure solution, it is sensible to explore the centrosymmetric possibilities first, but the others should not be forgotten.

Pseudosymmetry can also be exploited in the structure determination process. For example, the calcined form of the aluminophosphate molecular sieve $\mathrm{AlPO}_{4}-18$ can be indexed on an orthorhombic unit cell $(a=13.711, b=12.731$, and $c=18.570 \AA)$ with the possible space groups $C m c m$ or $C m c 2_{1}$, although the structure itself has only monoclinic symmetry (Simmen et al., 1991). The framework topology was established in the space group $\mathrm{Cmcm}$ assuming $\mathrm{Al}=\mathrm{P}$. Only then was the strict alternation of $\mathrm{Al}$ and $\mathrm{P}$ in the tetrahedral sites of the framework taken into account, and the symmetry reduced accordingly to $C 2 / c$. By using this approach, the structure determination problem was reduced from an 18-atom to a 9-atom one. Two similar cases (the aluminophosphates MCS-1 and SAPO-40) are shown in Table 1.

\section{Extraction of reflection intensities}

Individual reflection intensities are extracted from a powder pattern by adjusting lattice parameters, peak shape parameters, $2 \theta$ zero corrections, and $h k l$ intensities to give the best whole-profile fit to the observed diffraction pattern. Many Rietveld refinement (Rietveld, 1969) programs have now been modified to allow whole-profile refinement without a structural model. Originally all parameters were refined using a least-squares algorithm with slack constraints for the intensities of nearly overlapping reflections (Pawley, 1981), but more recently, the simpler and more stable modification first described by Le Bail, Duroy and Fourquet (1988) has been adopted as the favored approach. In this case, the reflection intensities are integrated numerically in an iterative process rather than refined. Other variables are refined as in the Pawley program.

The maximum sensible $2 \theta$ value to use in an extraction procedure depends upon the amount of overlap in the pattern. This is a function of the wavelength used, the FWHM of the peaks, the symmetry, and the lattice constants. At some point, so many reflections overlap that the background is no longer well defined, and the extracted intensities are no longer useful. The determination of this point is not unequivocal, and depends to some extent upon the complexity of the structure under investigation. Several $2 \theta$ limits may have to be tested before an appropriate one is found.

If direct methods are to be used for structure determination, all reflections in the range chosen, whether weak, intermediate, strong or overlapping, should be included in the data set. Weak reflections also contain structural information, and are particularly important for negative quartets. Since many programs set the intensities of missing reflections to zero, the exclusion of overlapping reflections from the data set can result in false information being introduced.

Not only do extraction programs generate a pseudo-single-crystal set of $h \mathrm{kl}$ 's and intensities, they can also be used effectively to evaluate different space group possibilities, to refine lattice parameters, to adjust profile parameters prior to a Rietveld refinement, and to obtain a better estimate of the background.

\section{Treatment of overlapping reflections}

Reflections are considered to overlap in a powder diffraction pattern when their $2 \theta$ values are too close to allow the reflections to be distinguished from one another. This is normally expressed as some fraction of the FWHM, with the fractions varying from 0.1 to 0.5 . When several reflections contribute to what 
Table 1: Some Examples of Structures Solved from Powder Data

\begin{tabular}{|c|c|c|c|c|c|}
\hline Sample & $\mathrm{LaMo}_{5} \mathrm{O}_{8}{ }^{a}$ & Sigma- $2^{b}$ & Cimetidine $e^{c}$ & MCS- $1^{d}$ & SAPO- $40^{e}$ \\
\hline Space Group & $P 2_{1} / a$ & $I 4_{1} / a m d$ & $P 2_{1} / n$ & $P b c n$ & $P m m n$ \\
\hline$a(\AA)$ & 9.91 & 10.24 & 10.21 & 9.65 & 22.04 \\
\hline$b(\AA)$ & 9.09 & 10.24 & 17.97 & 18.03 & 13.70 \\
\hline$c(\AA)$ & 7.57 & 34.38 & 6.52 & 13.91 & 7.12 \\
\hline$\beta\left(^{\circ}\right)$ & 109.05 & & 111.28 & & \\
\hline$V\left(\AA^{3}\right)$ & 645 & 3605 & 1114 & 2420 & 2150 \\
\hline \multicolumn{6}{|l|}{ Reflections (CuK $\left.\alpha_{1}\right)$} \\
\hline Total (up to $75^{\circ} 2 \theta$ ) & 330 & 281 & 568 & 633 & 621 \\
\hline $\begin{array}{l}\text { Overlapping }^{f} \\
\text { (within } 0.5 \cdot F W H M)\end{array}$ & $\begin{array}{c}124 \\
(38 \%)\end{array}$ & $\begin{array}{c}66 \\
(23 \%)\end{array}$ & $\begin{array}{c}217 \\
(38 \%)\end{array}$ & $\begin{array}{c}257 \\
(41 \%)\end{array}$ & $\begin{array}{c}248 \\
(40 \%)\end{array}$ \\
\hline Method & $\begin{array}{l}\text { Direct } \\
\text { Methods }\end{array}$ & $\begin{array}{l}\text { Direct } \\
\text { Methods }\end{array}$ & $\begin{array}{l}\text { Fourier } \\
\text { Cycling }\end{array}$ & $\begin{array}{l}\text { FIPS/Direct } \\
\text { Methods }\end{array}$ & $\begin{array}{l}\text { FIPS/Direct } \\
\text { Methods }\end{array}$ \\
\hline \multicolumn{6}{|l|}{ Atoms/Asym. Unit } \\
\hline Found Initially & $1 \mathrm{La}+5 \mathrm{Mo}$ & $4 \mathrm{Si}+4 \mathrm{O}$ & $1 \mathrm{~S}+3 \mathrm{C}^{g}$ & $6(\mathrm{Al}, \mathrm{P})+9 \mathrm{O}$ & $4(\mathrm{Al}, \mathrm{P})+5 \mathrm{O}$ \\
\hline Subsequent Fouriers & 80 & $3 \mathrm{O}+5 \mathrm{C}+1 \mathrm{~N}$ & $6 \mathrm{~N}+7 \mathrm{C}$ & 30 & $5 \mathrm{O}+6 \mathrm{C}+1 \mathrm{~N}$ \\
\hline Used in Refinement & 14 & 17 & $33^{h}$ & $36^{i}$ & $62^{j}$ \\
\hline 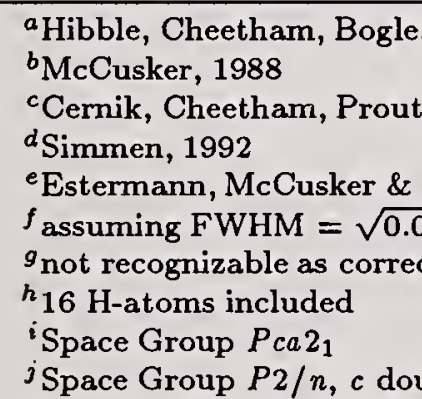 & $\begin{array}{l}\text { Wakerly \& Cox } \\
\text { Watkin, Wilki } \\
\text { aerlocher, } 199 \\
92 \tan \theta+0.00 \\
\text { positions initi }\end{array}$ & $\begin{array}{l}1988 \\
\text { son \& Willis, } 19 \\
\overline{3} \text { (i.e. FWHM r } \\
\text { lly }\end{array}$ & ges from $0.05 t$ & $\left.0.10^{\circ} 2 \theta\right)$ & \\
\hline
\end{tabular}

appears to be a single peak in the diffraction pattern, the total intensity is usually equipartitioned. That is, the intensity ratio of the reflections involved is set to one. If the percentage of overlapping reflections is low, such a data set will usually allow the structure to be solved (see, for example, Sigma-2 in Table 1 ), but as the percentage increases, the chances of success diminish.

Some exciting developments in the treatment of overlapping reflections have been reported in the last year. In their program DOREES, Jansen, Peschar and Schenk (1992b) use a combination of triplet and quartet relationships and a Patterson function calculated using only accurately determined $F_{h k l}$ 's to obtain a more correct estimate of the intensity distribution for overlapping reflections. On the basis of this information, they can judge whether a particular $F_{h k l}$ is likely to be strong, intermediate or weak. The method has been tested on two known structures (one organic and one inorganic), and the data sets were significantly improved.

Cascarano, Favia and Giacovazzo (1992) take advantage of the power of modern computers in their program SIRPOW.91, which is written specifically for structure solution from powder data. They permute the possible intensity ratios of overlapping reflections and apply a multisolution procedure to each data set. The solutions are then analyzed with the help of appropriate figures of merit to identify the correct one. This method has been applied successfully to a number of known structures.

Estermann and Gramlich (1992) have taken a different approach. They generate a Patterson map using equipartitioned data, square the map to emphasize the main features, back-transform this squared map to obtain new Fourier coefficients, and extrapolate these new coefficients to redistribute the relative intensities of overlapping reflections (nonoverlapping ones remain unchanged). This cycle is repeated until the intensity distribution of the overlapping reflections approaches that of the nonoverlapping ones. This Fast Iterative Patterson Squaring (FIPS) method is remarkably fast, and has been applied successfully to many test structures (Estermann, 1991) and to two unknown structures which could not be solved from equipartitioned data (Simmen, 1992 and Estermann, McCusker \& Baerlocher, 1992). 
By producing a data set whose relative intensities are more realistic (i.e. closer to single-crystal values), these methods significantly enhance the success rate for structure solution from powder data. They represent the most important recent advance in the field.

\section{Examples}

Some data for a few of the more complex structures solved $a b$ initio from powder data are given in Table 1. The bottom row, which shows the number of atoms in the asymmetric unit of the final structure, illustrates the structure complexity now within the scope of the powder diffractionist.

The two earlier structures, $\mathrm{LaMo}_{5} \mathrm{O}_{8}$ and Sigma-2, are relatively simple ones, and were solved by direct methods using equipartitioned powder data. However, this approach did not yield recognizable structures for cimetidine, MCS- 1 or SAPO-40.

In the case of cimetidine, only 4 of the 17 highest peaks on the $E$-map were correct, and it was not clear initially which these were. Nonetheless, the positions of all 17 non-hydrogen atoms were determined by an iterative least-squares refinement/Fourier synthesis technique. The procedure was fully automated in the CRYSTALS package (Watkin, Carruthers \& Betteridge, 1985). The inclusion of $16 \mathrm{H}$-atoms in the model then significantly improved subsequent $\mathrm{Ri}$ etveld refinement.

For MCS-1 and SAPO-40, the FIPS method was used to redistribute the intensities of the overlapping reflections more realistically, and then the structures were determined by reapplying direct methods. The redistribution of intensities by the FIPS method appears to allow a larger number of atoms to be found on the initial $E$-map. In both cases, the pseudosymmetry was exploited in the structure solution step. $\mathrm{By}$ assuming that $\mathrm{Al}$ and $\mathrm{P}$ are indistinguishable, the higher space groups could be used to determine the aluminophosphate framework topologies. The strict alternation of $\mathrm{Al}$ and $\mathrm{P}$ in the tetrahedral sites was introduced only at the structure refinement stage.

The number of overlapping reflections given in Table 1 were calculated and included for comparison purposes. In fact, the data collection conditions for the five structures vary significantly. For example, the data for cimetidine were collected at a synchrotron facility (FWHM varying from 0.05 to $0.07^{\circ} 2 \theta$ ), whereas those for SAPO-40 were collected on a laboratory instrument equipped with a position sensitive detector (FWHM varying from 0.10 to $\left.0.14^{\circ} 2 \theta\right)$. The effect of this difference is to reduce the percentage of overlapping reflections for cimetidine to approximately $29 \%$, and to increase that for SAPO-40 to $65 \%$. Consequently, a direct comparison of methods is not possible. Nonetheless, there appears to be a progressive increase in the complexity of structures that have been solved from powder data, and this trend can be expected to continue over the next decade as new methods are developed, improved and combined.

\section{Software}

In figure 1 some of the more commonly used programs for each of the steps required for structure solution using powder data are shown. With the exception of the structure determination program SIMPEL (Peschar, 1990), references for all of these programs have already been cited, or are given in the program list compiled by Smith and Gorter (1991). The purpose of the diagram is to illustrate the variety of programs available without providing an exhaustive list, and to indicate some of the program combinations that have already been used successfully (those connected with arrows). Several laboratories have developed their own inhouse set of software with appropriate bridging programs to transfer information correctly from one system to another, but a completely integrated system is still lacking.

Structure solution from powder data usually involves the use of at least four different program systems. The diffractometer software normally includes data collection, some data correction ( $\alpha_{2}$-stripping, background correction, $2 \theta$ zero correction), and a peakfinding routine. The latter is sometimes coupled to an autoindexing program, but if it is not, the transfer of $2 \theta$ values to stand-alone programs is relatively straightforward. An attractive alternative to using the peakfinding routine in a diffractometer package is the program recently developed by David, Ibberson and Sivia (1992), which is a Bayesian approach to Bragg peak location. In difficult cases, this algorithm may yield peak positions which improve the chances of success in the indexing process.

Most intensity extraction programs are modifications of the Rietveld refinement program used in the last step, but the intermediate steps for the treatment of overlapping reflections and the structure determination itself are separate and require a critical transfer of data. The user must know exactly what form of intensities or structure factors are generated by the extraction program in order to transfer the information correctly. That is, have the intensities been corrected for the Lorentz-polarization factor; have the multiplicities been factored out; is the peak width listed the FWHM, the FWHM/2 or the $\mathrm{FWHM}^{2}$; are the values output $I_{h k l}, F_{h k l}^{2}, F_{h k l}$ or something else? The $h k l$ indices must also correspond to the cell input to the structure determination program. The extraction program may have performed a cell transformation (e.g. changed the 

1) Data Collection
2) Data Correction
3) Peak Positlons
4) Indexing
S) Space Group Determination
6) Intensity Extraction
7) Overlap
8) Structure Determination
9) Rietveld Refinement

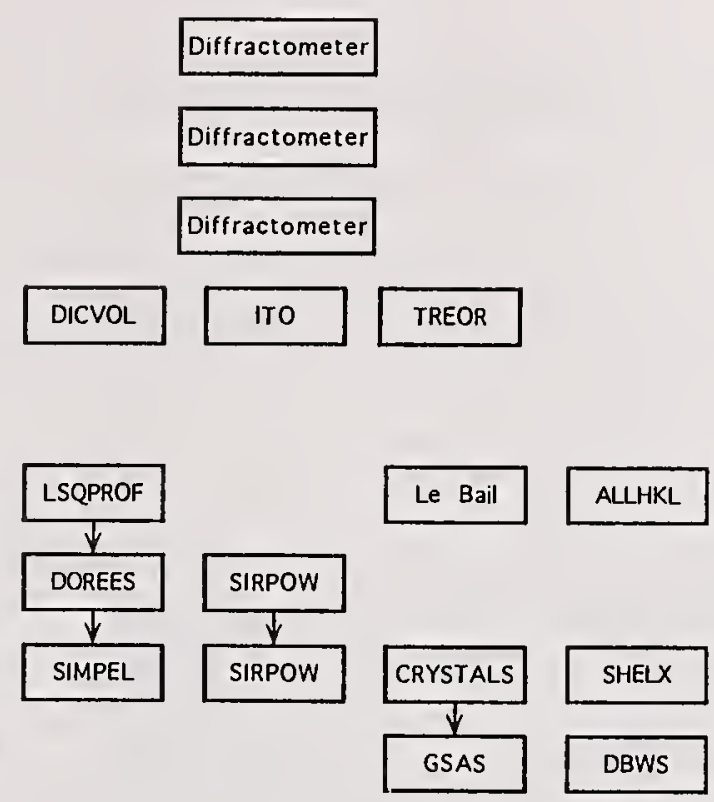

Figure 1: Some Programs Commonly Used in Structure Solution from Powder Data

hexagonal axes of an R-centered cell to the rhombohedral ones) before generating the $h k l$ values. Although these may appear to be trivial and obvious points, they are easily overlooked, and that can lead to long periods of unnecessary frustration.

It is obvious that an integrated system that would perform all the necessary calculations and require no data transfer would be welcome in the powder diffraction community. The editors of the single crystal package $X T A L$ are presently in the process of adding a Rietveld refinement program to the system, and plan to follow that with an extraction program and at least the FIPS method of treating overlapping reflections (the program series shown in the left column of Figure 1). It can be hoped that this trend will continue and that eventually peakfinding and autoindexing routines will also be included. In the meantime, there are plenty of programs available to the crystallographer who is willing to write miniprograms to bridge between the various program systems, and certainly there is no shortage of polycrystalline materials whose structures need to be solved.

\section{References}

A Rnold, H., Bartl, H., Fuess, H., Ihringer, J., Kosten, K., Lö́chner, U., Pennartz, P.U., Prandl, W. \& WroBleWSKI, T. (1989). Rev. Sci. Instrum., 60, 2380-2381. B AERLOCHer, CH. (1990). EXTRACT. A Fortran Program for the Extraction of Integrated Intensities from a Powder Pattern. Institut fuer Kristallographie, ETH, Zurich, Switzerland.

Bish, D.L. \& PosT, J.E. (1989). Editors. Modern Powder Diffraction. Washington: The Mineralogical Society of America.

Cascarano, G., Favia, L. \& Giacovazzo, C. (1992). J. Appl. Cryst. 25, 310-317.

Cernik, R.J. \& Bushnell-Wye, G. (1991). Mater. Sci. Forum, 79-82, 455-462.
Cernik, R.J., Cheetham, A.K., Prout, C.K., Watkin, D.J., Wilkinson, A.P. \& Willis, B.T.M. (1991). J. Appl. Cryst. 24, 222-226.

Cox, D.E., Hastings, J.B., Cardosa, L.P. \& Finger. L.W. (1986). Mater. Sci. Forum, 9, 1-20.

David, W.I.F., Ibberson, R.M. \& Sivia, D.S. (1992). , in preparation.

Estermann, M.A. (1991). Ph.D. Thesis, ETH, Zurich, Switzerland.

ESTERMANN, M.A. \& GRAMLICH, V. (1992). , in preparation. EstermanN, M.A., MCCuSKer, L.B. \& BaERlOCHER, CH. (1992). J. Appl. Cryst. 25, 539-543.

HALL, S.R. \& STEWART, J.M. (1992). Editors. XTAL3.2, in preparation.

Hill, R.J. \& MADSEN, I.C. (1987). Powder Diffraction, 2, 146-162.

Hibble, S.J., Cheetham, A.K., Bogle, A.R.L., Wakerly, H.R. \& Cox, D.E. (1988). J. Am. Chem. Soc. 110, 32953296.

Jansen, J., Peschar, R. \& Schenk, H. (1992a). J. Appl. Cryst. 25, 231-236.

Jansen, J., Peschar, R. \& Schenk, H. (1992b). J. Appl. Cryst. 25, 237-243.

Le Bail, A., Duroy, H. \& Fourquet, J.L. (1988). Mater. Res. Bull. 23,447-452.

MCCusker, L.B. (1988). J. Appl. Cryst. 21, 305-310.

Parrish, W., Hart, M., Huang, T.C. \& Bellotto, M. (1987). Adv. X-ray Anal., 30, 373-381.

PaWley, G.A. (1981). J. Appl. Cryst. 14, 357-361.

PESCHAR, R. (1990). In MolEN, Molecular Structure Solution Procedures, Vol. 3, pp. 59-82. Delft: Enraf-Nonius.

Rietveld, H.M. (1969). J. Appl. Cryst. 2, 65-71.

SIMMEN, A. (1992). Ph.D. Thesis, ETH, Zurich, Switzerland.

Simmen, A., MCCusker, L.B., BAERLOCHER, CH. \& MEIER, W.M. (1991). Zeolites, 11, 654-661.

SMITH, D.K. \& GoRTER, S. (1991). J. Appl. Cryst. 24, 569602.

Watkin, D.J., Carruthers, J.R. \& BetTeridge, P.W. (1985). CRYSTALS User Guide. Chemical Crystallography Laboratory, Univ. of Oxford, England. 
National Institute of Standards and Technology Special Publication 846. Proceedings of the international conference Accuracy in Powder Diffraction II, held at NIST, Gaithersburg, MD, May 26-29, 1992. (Issued October 1992)

\title{
Direct Space Methods of Structure Solution From Powder Diffraction Data
}

\author{
J. M. Newsam, M. W. Deem*and C. M. Freeman \\ Biosym Technologies Inc. 9685 Scranton Road, San Diego, CA 92121-2777, USA
}

\begin{abstract}
Complementing improvements in instrumentation and in more traditional crystallographic approaches, direct space methods are now facilitating the solution of crystal structures based on powder diffraction data. Two such methods are discussed here. In an extension of physical model building methods, unit cell size and symmetry constraints are used to control the combination of particular structural motifs through a graphical interface; model validation or refinement can be achieved by interactively updating simulated analytical data (such as diffraction patterns or NMR spectra) as the developing model is adjusted. Model development can be automated when the reasonableness of a given atomic arrangement is quantified by cost functions or 'energy' terms. These may include first-neighbor distance, bond angle and coordination number constraints etc. Viable models are derived from an initially random distribution of the required number of atoms of each type within the observed unit cell by simulated annealing. The effectiveness of this approach is demonstrated by test applications to the known structures of zeolites and other more condensed oxide phases. It is straightforward to include additional 'energy' terms in this process, such as micropore dimensions in the case of zeolites or, more generally, the degree of match between model and target powder diffraction patterns. Particularly when diffraction pattern matching constraints are included, simulated annealing appears to be an efficient and potentially general route to $a b$ initio structure solutions.
\end{abstract}

\section{Introduction}

How valuable is a knowledge of structure at an atomic level? The answer to this question helps quantify, at one level, the level of resources one is willing to devote to the solution and refinement of a particular crystal structure. Although perhaps difficult to define precisely, particularly in advance of the

* Present address: Department of Chemical Engineering, University of California, Berkeley, CA 94720. availability of the data, the benefits of detailed atomistic structural knowledge are almost universally sufficient to warrant a substantial characterization effort. A large proportion of the interesting new materials being synthesized, however, are only available in polycrystalline form. Even where single crystals can be made, their production at a quality suitable for accurate single crystal diffraction analysis may be time-consuming. As powder diffraction data is usually available immediately, there are often opportunities for ground breaking studies using powder diffraction methods. For example, many of the early studies of $\mathrm{YBa}_{2} \mathrm{Cu}_{3} \mathrm{O}_{7-x}$ used powder diffraction methods (See Johnston, Jacobson, Newsam, Lewandowski, Goshorn et al., (1987) and references cited therein). Improvements in experimentation have helped to improve dramatically the quality of powder diffraction data that are now obtainable. In parallel, computer hardware improvements permit us to exploit computational approaches that even a few years ago would have been perceived as restrictively expensive. In this report we discuss primarily some ways in which modeling or simulation can aid in initial structure solution. Although not addressed here, there have been equally impressive advances in structure refinement methods. Computational tools are also a wellestablished and important means of extracting the maximal value from the atomic structural data that diffraction measurements ultimately provide.

\section{Graphical and geometrical manipulations}

The building of physical models has traditionally been an important means of developing new structural models, particularly of framework structures such as those of zeolites. Physical models capitalize directly on the defined geometrical constraints that the structure class obeys. The selection of those models that might be appropriate for a new material in question is based on the matching of the unit cell sizes, symmetries and computed diffraction patterns with the observed data available. The physical model building approach does not allow the known symmetry or unit cell size to be used directly as a 


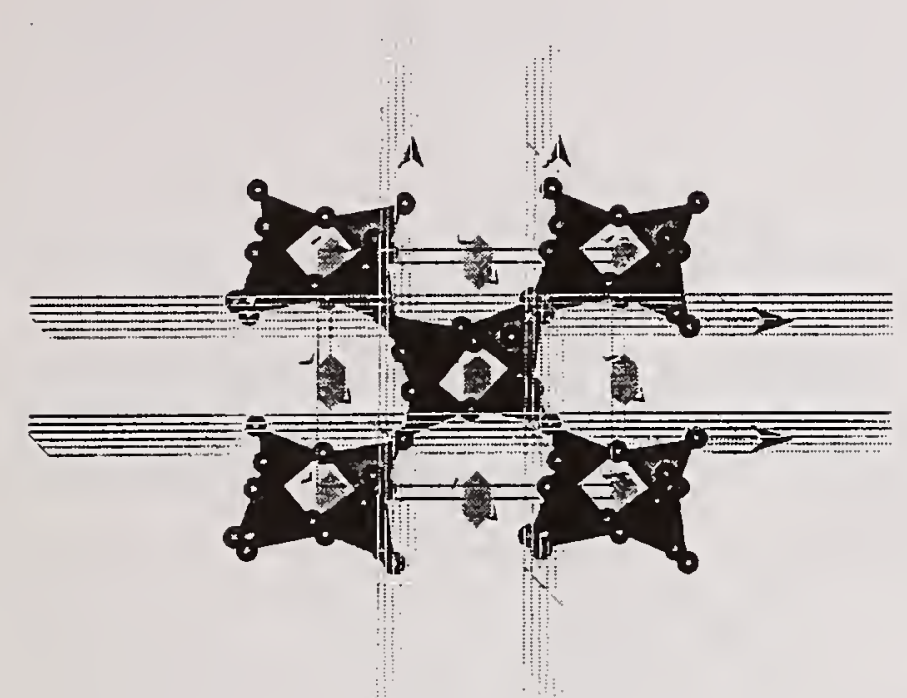

Figure 1: Symmetry elements for space group $P n a 2_{1}$, shown superposed on the framework structure of zeolite Li-A(BW).

constraint. It can be slow and almost inevitably proceeds according to the particular preconceptions of the builder.

Mathematical or computational model building methods can offer substantial advantages. The combination of geometrical constraints and a known unit cell size and space group can, for high symmetry cases, limit greatly the possible atom positions or configurations. Such symmetry arguments (Bragg \& Warren, 1928) have been used often in solving simple inorganic crystal structures. Symmetry constraints can be programmed in a general way so that as an atom or fragment is moved in the structure via a graphical interface, the symmetry-related atoms or fragments are moved in consort. Recognizing the character and location of the symmetry elements that are present in the defined space group (Fig. 1) allows the motion to be constrained so that atoms lying on special positions such as 2 -fold rotation axes or mirror planes remain on those symmetry elements. After molecular fragments or structural motifs such as polyhedra are combined, subject to geometrical rules, unit cell dimensions and space group symmetries can be determined automatically (Fig. 2). Further, model validation or refinement can then be achieved directly by interactively updating simulated analytical data (such as diffraction patterns, EXAFS or NMR spectra) as the developing model is adjusted (Fig. 1) (Newsam, Deem, Freeman, Gorman, Ho et al., 1992).

\section{Automatic model development - Background}

A structure determination from diffraction data involves the development of an initial model, that is the structure solution step, and the completion and refinement of the model. Why are these steps distinct? Today's structure refinement technology can operate effectively only when the starting model is relatively close to the actual model. Classical least squares techniques are unable to locate global minima in parameter spaces that are complex, multi-dimensional and which have many local minima, unless the current model is close to this global minimum. Structure completion tasks, likewise, rely most extensively on interpretations of scattering density maps produced by Fourier inversion of the diffraction data. Such maps must provide at least a recognizable level of definition, placing requirements on both the resolution and the quality of the phasing.

With powder data, as with single crystal data from materials with very large unit cells, the methods that work well for small molecule single crystal work usually cannot be applied directly. Powder diffraction data, even when obtained at high instrumental resolution, necessarily suffer from systematic or accidental peak overlaps, yielding only intensity sums for a significant proportion of the reflections. Although ways are being developed to aid in the proper apportioning of the measured intensity to each of the contributing reflections (David, 1987; Estermann \& Gramlich, 1991), the precision of the decomposed intensities cannot match that obtainable by separate measurements, as is possible with single crystals. The set of reflection intensities obtained by decomposition of the powder diffraction data is, additionally, almost always a 'weak' data set. The precision with which the structure factor amplitudes are defined is modest and the proportion of 'observed' reflections is usually small. This situation, even for single crystal data, often gives rise to problems in the application of Direct Methods. Thus, although significant headway is being made in applying Direct Methods to structure solutions from powder diffraction data (see, e.g., Gilmore, Henderson \& Bricogne, 1991; Morris, Harrison, Nicol, Wilkinson \& Cheetham, 1992), the number of new structures solved this way remains small (McCusker, 1992).

In principle, were our practical description of the interatomic interactions to be sufficiently accurate and were sufficiently powerful numerical optimization tools available, we could truly predict crystal structures from little more than composition data. For some ideal molecular systems such structure prediction, or, more accurately post-diction, has proven possible (Williams, 1967; Gdanitz, 1992). In gen- 
(a)

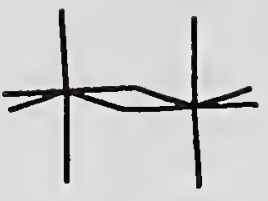

(b)

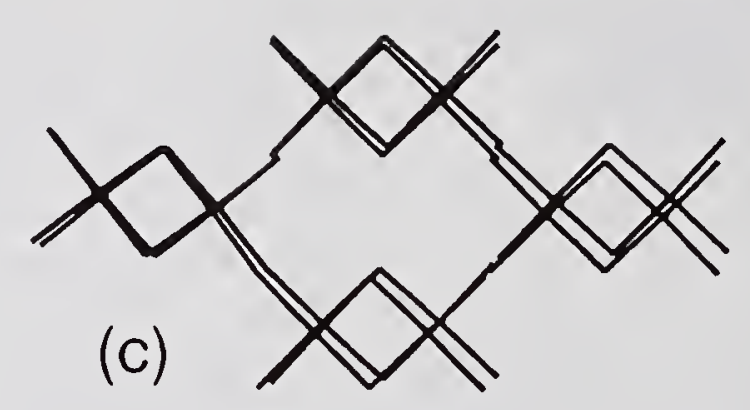

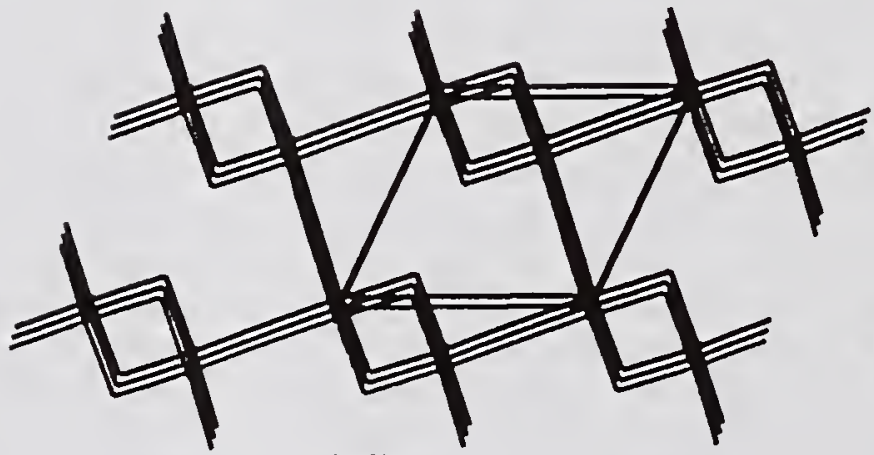

(d)

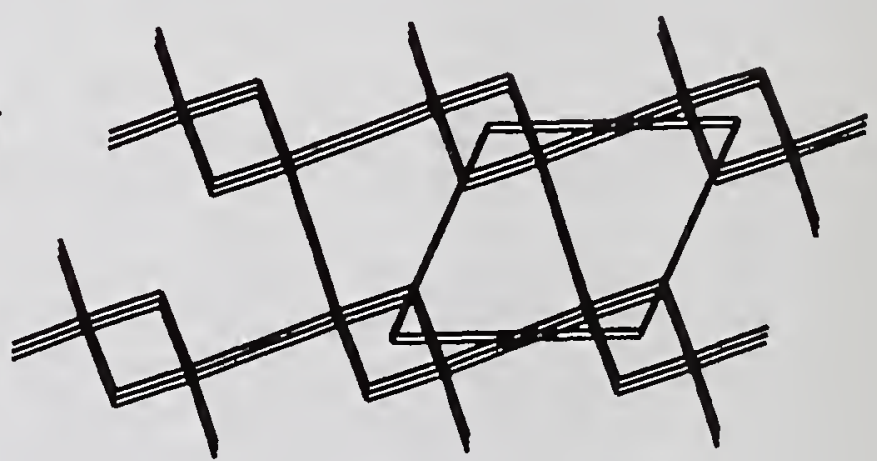

(e)

Figure 2: Edge-shared pairs of octahedra clipped from the corundum structure $(a)$, are optimized (b), and then combined graphically to develop a piece of a hypothetical structural model $(c)$, from which a primitive unit cell $(d)$ and, subsequently, its space group symmetry $(e)$ are automatically determined.

eral, however, we have at hand substantially more information than the chemical composition alone. A successful indexing of the powder diffraction pattern, which can often be done automatically, yields the unit cell dimensions and information on possible space groups. The chemical analysis, combined where necessary with other analytical probes of the unit cell composition, provides the number of atoms or molecules of each type that are contained within this unit cell. The global optimization problem is then that of positioning these species within the unit cell such that the various diffraction data are reproduced. We can also require that the model make reasonable chemical and physical sense and perhaps also match other experimental data. This type of op- timization, the derivation of that set of parameters which will optimally match a set of constraints or observations is truly generic; many scientific problems can be expressed in terms of a function whose minimum value is the solution sought. Any improvements made in algorithms that better address this problem can have immediate impacts in crystallography.

\section{Simulated annealing as an optimization method}

Stimulated in part by the evolution of readily available computer resources, the methodology and application of numerical optimization have progressed 
dramatically. Gradient optimization methods that seek first derivative stationary points were originally developed in the 1950s and 1960s (Powell, 1982; Fletcher \& Powell, 1963; Gill, Murray \& Wright, 1981; Scales, 1985; Press, Flannery, Teukolsky \& Vetterling, 1989; Sedgewick, 1988), and are now quite robust and sophisticated. However, all such methods are, in general, confounded by functions which possess many local minima.

Simulated annealing (Press, Flannery, Teukolsky \& Vetterling, 1989) a comparatively recent development, places little emphasis on the local curvature of the function to be minimized. The resulting procedure is, therefore, less susceptible to simply rolling downhill to the nearest function minimum and provides a higher probability of finding the function's global minimum. The price paid for improved avoidance of local minima is the higher number of function evaluations which must be made to achieve successful minimization. However, if the single point function evaluations do not present a prohibitive computational burden (and the size of burden that is tolerable grows steadily, in consort with hardware performance improvements), the method can show great success (Kirkpatrick, Gelatt \& Vecchi, 1983). Although the present work has uniformly used simulated annealing as the optimization method, combined on occasion with the use of conventional gradient optimizers for final adjustments when viable models have been produced, other interesting approaches to the global optimization problem are also being pursued (Piela, Kostrowicki \& Scheraga, 1989; Somorjai, 1991).

All minimization procedures start from a given proposed solution and iteratively attempt to improve on this starting point. Simulated annealing achieves a smaller degree of local bias than gradient optimizers in its search from the starting point, by accepting with a finite probability configurations which possess higher function values. This property actually permits the optimization to commence from a random starting point. An analogy can be drawn with an ensemble of physical particles at finite temperature. In such a physical system the equilibrium distribution of states accessible at a given temperature characterizes a well defined phase for that system: solid, liquid or gas, for example. Each phase is made up of a collection of 'states' and their associated probabilities. For a physical system the relative probabilities of two states are simply related to the temperature via Boltzman statistics. At high temperature there will be little difference in the probability of any two states, while at low temperature the state with the lowest potential energy will dominate. The 'temperature' of a system may, then, be used as a convenient control of the extent to which states higher in energy than the global minimum are populable.

In the simulated annealing approach to optimization, a 'state' is a possible system configuration; its 'energy' is a measure of the degree to which the configuration matches the target constraints or observations. Thus in fitting a measured diffraction pattern, one configuration of the atoms in the unit cell would be one state, and the conventional weighted residual, $\mathrm{R}_{\mathrm{W}}$, might be taken as its 'energy'. The 'temperature', the amount of thermal 'energy' in the system, determines the relative probabilities of the system adopting two states of different energies.

To locate the sought minimum requires defining a path over the parameter space surface. This path might be a molecular dynamics (MD) trajectory, in which the positions and velocities of the particles in the system are allowed to evolve by solving Newton's equations of motion. The simulation, initially commenced at high temperature, is subjected to an annealing schedule which allows the system to settle into an energy-minimum configuration. Because of the need to ascribe masses to the components of the system molecular dynamics has found greatest use in its application to molecular systems, where the choice of mass is unambiguous, for example in the refinement of protein structures (Brunger, Leahy, Hynes \& Fox, 1991) and the determination of tertiary molecular structure from NMR derived distance constraints (Crippen \& Havel, 1988). Molecular Dynamics, with suitable dummy variables, has also been exploited in combined structural and electronic calculations (Car \& Parinello, 1985; Field, 1991).

In this work, the path in parameter space is driven by the Metropolis importance sampling method (Metropolis, Rosenbluth, Rosenbluth, Teller \& Teller, 1953). A particle is selected at random and displaced; the direction is chosen randomly and the displacement extent is also chosen at random from within defined bounds. The 'energy' of the new trial state, $E_{\text {new }}$, is evaluated and the state is accepted if

$$
\mathrm{E}_{\text {new }} \leq \mathrm{E}_{\text {initial }}
$$

or if

$$
\exp \left\{-\frac{E_{\text {new }}-E_{\text {initial }}}{K T}\right\}<\operatorname{rand}(0,1),
$$

where $E_{\text {initial }}$ is the total 'energy' of the system before the particle displacement, $\mathrm{T}$ is the temperature, $\mathrm{K}$ a constant term and rand $(0,1)$ represents a random number between 0 and 1 . At low values of $T$, the probability of accepting higher energy configurations is small; at high temperature, however, the probability becomes high, allowing substantial tracts of the parameter space to be sampled. When the energy 
function relates to a true thermodynamic energy, the system equilibrium properties at a defined temperature, $\mathrm{T}$, can be evaluated by numerically averaging over the states sampled by the Metropolis scheme, as the method samples the equilibrium states according to the Boltzmann probability distribution.

In simulated annealing, the temperature is raised from an arbitrary point in increments or powers until the system become 'molten'. The 'melting' transition can be recognized by a maximum in the heat capacity of the system as a function of temperature. The heat capacity, $\mathrm{C}_{t}$, is a measure of the degree of energy fluctuation possible in the system at a particular temperature:

$$
\mathrm{C}_{t}=\frac{\left\langle\mathrm{E}_{t}^{2}\right\rangle-\left\langle\mathrm{E}_{t}\right\rangle^{2}}{\mathrm{KT}^{2}}
$$

Alternatively the 'molten' state can be defined as that in which more than, say, $50 \%$ of the attempted moves are accepted (equation 2). From the 'molten' state, the temperature is reduced slowly according to a defined schedule, the particulars of which might depend on the details of the system under investigation (Press, Flannery, Teukolsky \& Vetterling, 1989). Often, for example, the cooling rate is reduced in the vicinity of the change in system heat capacity, $\mathrm{C}_{t}$, that indicates the 'freezing' transition. The annealing procedure corresponds to the physical process of solidification. In the limit of low temperature, only energy reducing moves are accepted (equation 2). Although the terminology of simulated annealing is derived from that of physical simulations, the method can be applied quite generally to functions to be minimized (Kirkpatrick, Gelatt \& Vecchi, 1983). Simulated annealing has, for example, been applied to the traveling salesman problem (Press, Flannery, Teukolsky \& Vetterling, 1989), to integrated circuit design (Kirkpatrick, Gelatt \& Vecchi, 1983), to studying atomic ordering in alloys (Binder, 1987) and aluminosilicates (Soukoulis, 1984; Newsam, 1992), and to curve-fitting.

\section{Cost or 'energy' functions}

\subsection{Geometric and potential functions}

The degree to which a model structure is chemically or physically sensible can be quantified at various levels of sophistication. One of the simplest is a measure of the degree to which unrealistic atomic overlaps are avoided; this criterion can be used in the first stage of model assessment once atoms are introduced at random into the unit cell. At a somewhat more sophisticated level, the measure might capture the reasonableness of the interatomic distances. Bonding criteria require that the distances from a given element in a defined oxidation state to its first and second neighbors will lie within reasonably narrow bounds. Distance targets are therefore a viable and well-used basis for crystal structure optimization. This distance least-squares (DLS) method (Meier \& Villiger, 1969) takes as input a trial or random set of atomic coordinates, the crystal symmetry and possibly atomic site symmetry constraints, and the atomic connectivity or the set of distances to be optimized. It has become a standard method in zeolite structure analysis and has also been successfully employed in studies of various other inorganic crystal structures (Khan, 1976; Tillmanns, Gebert \& Baur, 1973). The function to be minimized is a sum of squares; generally, either the coordinates of the initial model are within the convergence capture radius, or the prescribed distance constraints are sufficiently restricting that the global minimum and the path(s) to it are sufficiently unambiguous that least-squares can be successful. The DLS approach, nevertheless, often has problems with convergence and does not recognize unacceptable atom conflicts unless the corresponding separations are explicitly defined in the initial set of distance targets.

We have been able to use 'energy' functions constructed from simple geometrical terms successfully for structure solutions, particularly for zeolite structures (Fig. 3). These 4-connected framework structures are built from $\mathrm{TO}_{4}$ tetrahedra $(\mathrm{T}=$ Tetrahedral species, $\mathrm{Si}, \mathrm{Al}, \mathrm{Ga}$ etc.) sharing apices. The first phase of framework structure solution requires definition only of the approximate $\mathrm{T}$ - atom positions, for the centers of the T-T vectors are sufficiently close to the actual oxygen atom positions that DLS or refinements against diffraction data can proceed from this starting point. The geometrical constraints on the Tatom configurations obeyed by zeolites and related 4-connected framework structures can be quantified by constructing an 'energy' function that is a sum of terms based on the T-T distances, and the T-T-T angles and average angles observed in known zeolite structures (Fig. 3) (Deem \& Newsam, 1989), and a coordination number term that reflects the requirement of framework 4-connectedness. The degree to which these combined constraints are satisfied by a given arrangement of T-atoms within the unit cell then provides a measure of the reasonableness of that arrangement. In the simulated annealing procedure, the required number of unique T-atoms is introduced at random into the unit cell and the T-atom coordinates are then adjusted so as to optimize the matching with the defined geometrical constraints. Given the openness and flexibility of zeolite structures, we have found it advantageous to limit the number of independent variables to as large a degree as possible. 

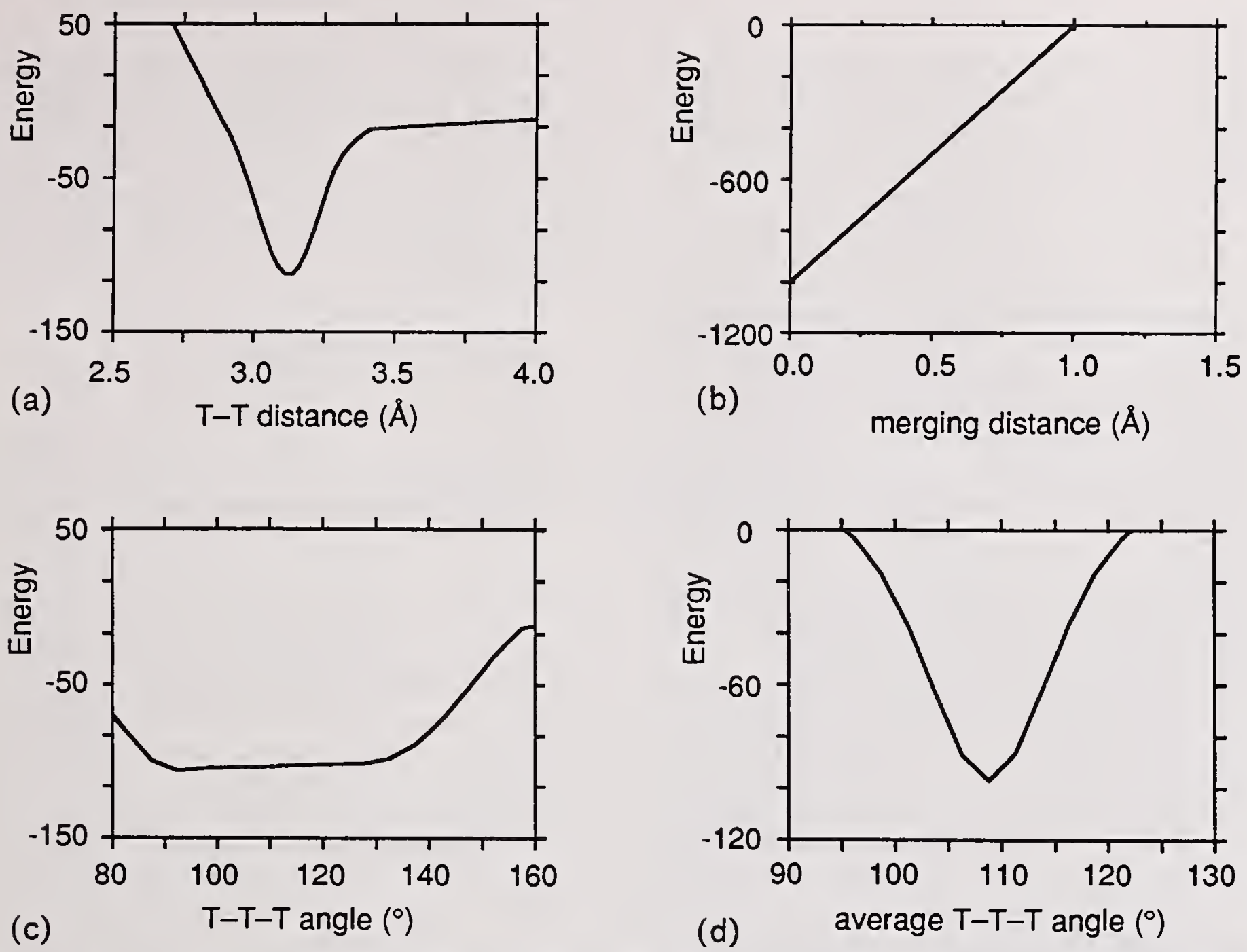

Figure 3: Contributions to the total system 'energy' for a zeolite framework model from each T-T distance $(a)$, individual $(c)$ and average $(d) \mathrm{T}-\mathrm{T}-\mathrm{T}$ angles, and coalescence or merging of symmetry related T-atoms (b) (Deem \& Newsam, 1992).

We therefore impose rigid space group constraints and adjust the positions only of the unique T-atoms; the positions of the other T-atoms that in the coordination shells of the unique $\mathrm{T}$-atoms are generated by the space group symmetry operations.

In many cases, high space group symmetries are involved and there is a tendency for T-atoms to reside on special positions. This possibility is supported by treating all of the T-atoms as being on general positions, but allowing pairs of symmetry related Tatoms to 'merge' when they become separated by less than a defined capture distance during the simulated annealing. In cases where the location of Tatoms on special positions has been inferred from compositional or, perhaps, NMR data, the merging can be promoted by subtracting a contribution to the total 'energy' sum when it occurs (Fig. 3). Other geometrical terms can be included. Projection and pore terms are two that we have found effective. The projection term provides for a bias in favor of structures that, when viewed in projection down a defined crystallographic direction, appear as 3 -connected nets (a property common to many of the known zeolite structures). The pore term is a simple mechanism for favoring structures that have cylindrical or spherical pores; sorption experiments on zeolites often yield data on the character of the pore architecture, particularly the free pore volume and the minimum ring sizes. As noted further below, the full zeolite 'energy' function comprised of these various components, when coupled with the simulated annealing procedure is a very effective means of developing new structural models (Deem \& Newsam, 1989 and 1992).

Beyond the interatomic distances themselves, functions of these distances, that might more realistically approximate the structure energetics can be used as the basis for the 'energy' function. Pannetier et al. (Pannetier, Bassas- Alina, Rodriguez-Carvajal \& Caignaert, 1990) used an 'energy' function com- 
prised of a combination of bond valence and electrostatic terms in determining the structures of $\mathrm{NbF}_{4}$, $\mathrm{K}_{2} \mathrm{NiF}_{4}, \mathrm{BaSiF}_{6}$ and $\mathrm{LiCoF}_{4}$; they have also used simulated annealing as a route to solving magnetic structures (Lacorre \& Pannetier, 1987).

Molecular or crystal mechanics methods, that use effective interatomic potential functions, have achieved wide success in studies of molecular conformation (Burkert \& Allinger, 1982) and molecular crystals (Williams, 1967). Several 'predictions' or refinements of inorganic crystal structures have been reported (Catlow \& Mackrodt, 1982; Maddox, 1988; Hawthorne, 1990; Catlow \& Price, 1990), including several studies of zeolite structures (Bell, Jackson \& Catlow, 1990; Tomlinson, Jackson \& Catlow, 1990). Both DLS and lattice energy minimization (using the METAPOCS code (Parker, Cormack \& Catlow, 1984)) were used in a complementary fashion in determining of the complex structure of zeolite NU-87 (Shannon, Casci, Cox \& Andrews, 1991).

The convergence characteristics of potential energy surfaces based on rigid ion or shell model potentials are poor when the starting model is far from the global minimum; the form of the short range repulsive terms makes it impossible for atoms to interchange positions directly. This problem can be circumvented by staging the application of the potential functions (Freeman \& Catlow, 1991). The starting point is again the generation of random ionic positions within the experimental unit cell. A straightforward distance criterion is used to avoid excessive steric overlap, potentially overlapping positions being rejected and repeated random insertions made until the cell is filled with the required number of atoms or ions of each type. No assumptions of spacegroup symmetry are made throughout the procedure, though translational periodicity is implicit. Having obtained a starting point, simulated annealing, using Metropolis Monte Carlo, is applied to relieve any unreasonably close inter-ionic contacts. The 'energy' function employed in this stage is the computationally convenient $\mathrm{r}^{-12}$ form, with repulsion between ions of similar charge being twice that between pairs of formally dissimilar charges. The effect of this procedure is to evenly disperse the ions within the cell, with locally preferred cation- anion ordering. At the end of the annealing schedule a switch is made to the quadratically convergent conjugate gradient minimization method to optimize the model with respect to the crude 'energy' function. The final stage of the procedure takes the final annealing result and employs full lattice energy minimization using the Born model of the solid, as implemented in the METAPOCS code (Parker, Cormack \& Catlow, 1984; Catalysis 2.0, 1992). The cell dimensions may be maintained throughout at the experimental values. In practice, as in the other simulated annealing procedures, several distinct crystal structure predictions are usually obtained for a given composition and unit cell. The scheme is, therefore, performed repeatedly in order to sample an adequate set of structures possible for the material (Freeman \& Catlow, 1991).

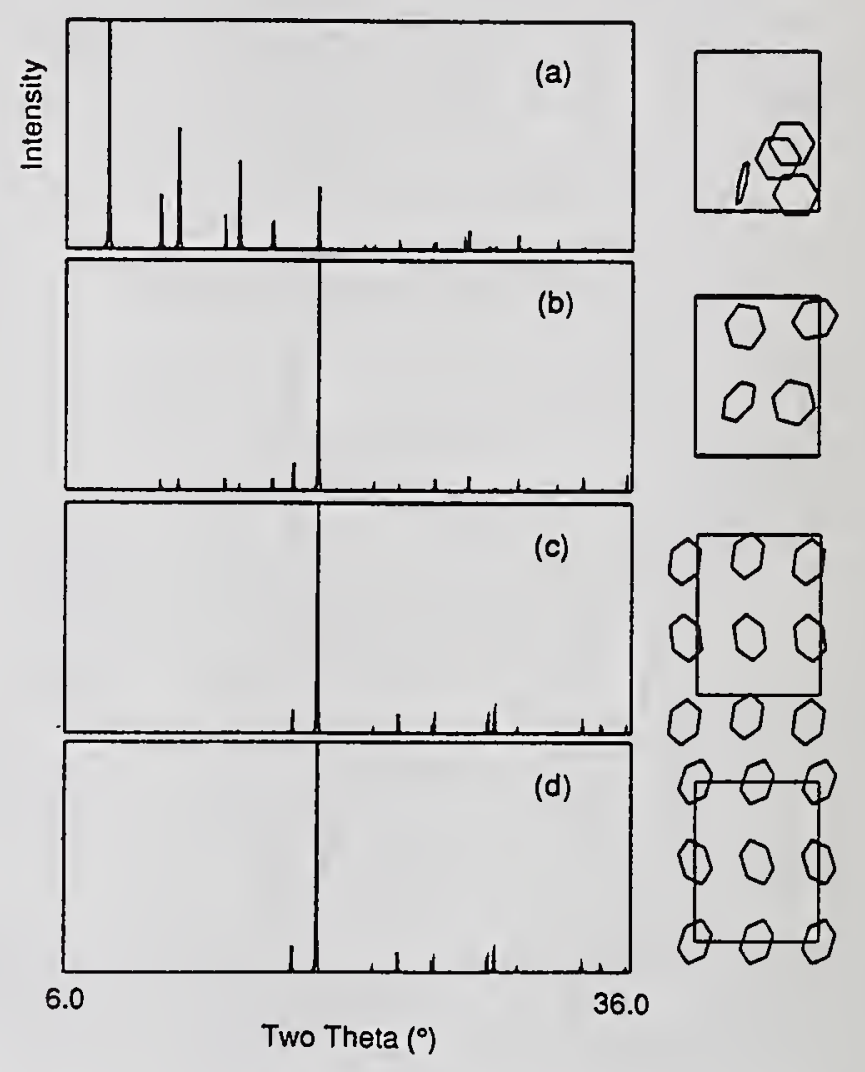

Figure 4: Simulated annealing 'solution' of the crystal structure of benzene using only the fit between target and simulated PXD patterns in the 'energy' function. The rigid benzene molecules are moved and rotated from a random starting configuration (top) using the Metropolis algorithm. The structure obtained by simulated annealing (second from top) was further adjusted using conjugate gradient optimization (third from top). The measured crystal structure is shown at bottom.

\subsection{Matching experimental diffraction or scattering data}

Experiences to date indicate that with chemical composition and unit cell dimensions and, optionally, symmetry as input, simulated annealing using geometrical or potential function terms in the 'energy' function will generate potentially viable structural models. When a number of structures have been produced, the most likely model might be selectable based on the corresponding value of the 'energy' 

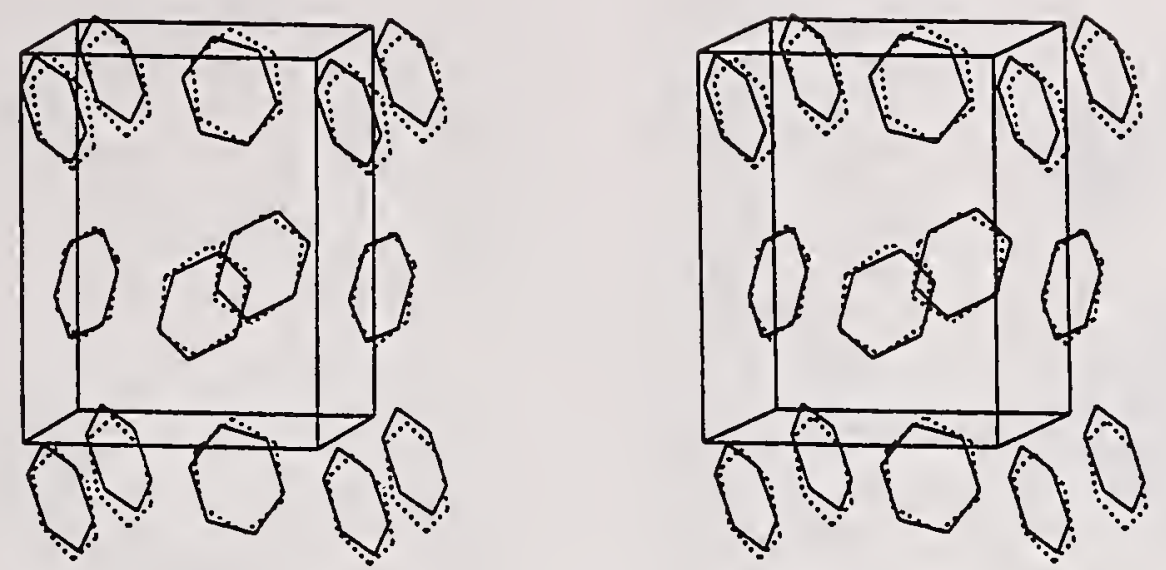

Figure 5: Stereo pair comparing the measured benzene crystal structure (full lines) with the results of simulated annealing using PXD profile matching as the 'energy' function (dashed lines).

function. More typically, this selection must be made based on comparison between model characteristics and experimental data; usually the observed and simulated powder diffraction patterns are compared (Deem \& Newsam, 1989). The degree of matching with experimental data can also be incorporated as an additional term in the 'energy' function expression (Deem \& Newsam, 1992). When the fitting of a target powder X-ray diffraction (PXD) pattern is added to the zeolite cost function, the number of hypothetical but inappropriate structural models is dramatically reduced (Deem \& Newsam, 1992). To test the generality of the methodology, 'solutions' of a large number of known zeolite structures were attempted in which the 'energy' function comprised only the degree of match to the target PXD pattern. Successful results were obtained in a substantial number of cases, even with simulated PXD patterns based only on the T-atom coordinates (Deem \& Newsam, 1992).

This approach is now being extended to attempted solutions of molecular crystal structures, both organic and organometallic. As an illustration of the potential of the method we show in figure 4 results for the benzene crystal structure. The input data were the observed unit cell dimensions, $a=7.44$, $b=9.55, c=6.92 \AA$ and the density (which indicates that the unit cell contains four benzene molecules). Triclinic symmetry, P1, was assumed. The PXD profile $\left(6^{\circ} \leq 2 \theta \leq 36^{\circ}, \lambda=1.5418 \AA\right)$ simulated based on the known crystal structure was used as a target. Simulated annealing was used to adjust the positions and orientations of each of the four independent benzene molecules, treated as rigid molecules, with the profile residual (the normalized sum of the squares of the differences between target and computed PXD profiles point by point across the pattern) being taken as the 'energy' function. The sim- ulation was performed using the Catalysis software (Catalysis 2.0, 1992), augmented with a macro utility. As figure 5 illustrates, the measured crystal structure is reproduced well by this automated structure solution procedure. Applications to other systems are in progress. Simulated annealing has also been applied to the interpretation of experimental scattering data from molten and amorphous materials (McGreevy \& Pusztai, 1990; Keen \& McGreevy, 1990; McGreevy \& Pusztai, 1988).

\section{Some examples of geometric or potential function constraints}

\subsection{Framework structures}

There are currently some 85 known zeolite structure types (Meier \& Olson, 1992; Newsam \& Treacy, 1992). The number of hypothetical 4-connected 3dimensional nets is also growing rapidly (see, e.g., Deem \& Newsam, 1992; Smith, 1988). The simulated annealing method, using only T-T distance, T-T-T angle and average angle, coordination, and merging terms in the zeolite 'energy' function (as described above, figure 3 ), was applied to 84 known zeolite structures, using the crystallographic data given for the representative structure in ZeoFile (Newsam \& Treacy, 1992). The unit cell dimensions and space group, and the numbers of crystallographically distinct T-atoms, $n_{\text {unique, }}$ and the total number of Tatoms in the unit cell, $n_{\mathrm{T}}$, were taken from those of the representative structure. A structure type was considered solved if the coordination sequence for one of the final set of structures generated by the simulated annealing matches that of the representative structure of the known material. (The coordination sequences out to a defined shell, $k$, from the central atoms (Meier \& Moeck, 1979) is defined as the graph of the framework connectivity; for the unit cell 
(a) 34 $-13098$

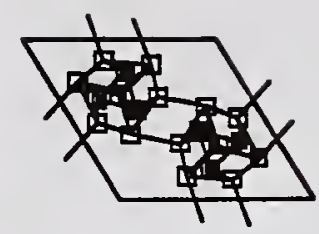

(b) 36

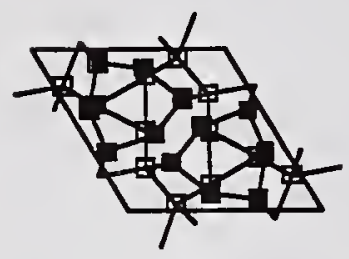

(c) 36

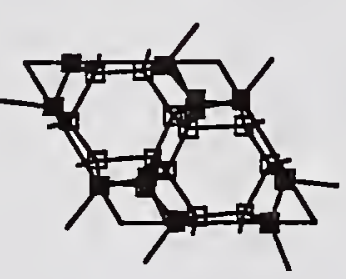

(d) 40

$-4450$

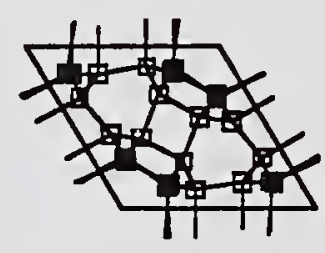

(e) 36

$-10164$

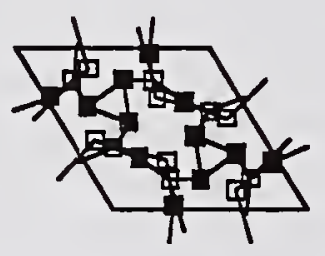

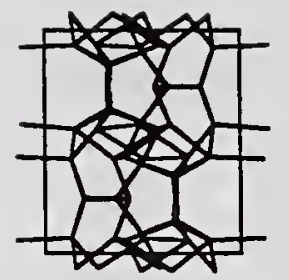
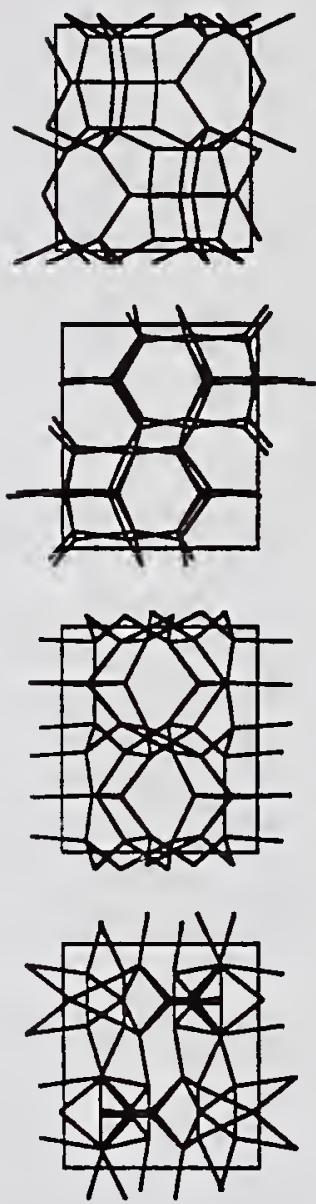

Figure 6: Some of the results of the simulated annealing procedure applied to the known crystallographic data for ZSM-18 $(P 63 / m, a=13.2, c=15.8 \AA$, target number of $T$-atoms per unit cell, $n_{T}=34$ ) (Deem \& Newsam, 1992); the values of $n_{T}$ for each model, and the calculated 'energies' are shown. The actual MEI-framework is (a).

volumes typical of most zeolites, if the coordination sequences out to $k=10$ for two structures are identical, the topologies are identical with a probability that approaches one).

The single first simulated annealing run gives the correct structure in $25(30 \%)$ of the total of 84 cases. Some of the structures that were obtained based on the input data appropriate for the MEI framework of zeolite ZSM-18 are shown in figure 6; an interesting feature of this structure type is the presence of 3 -rings (Newsam \& Treacy, 1992). Of those structures not solved, those having six or fewer unique T-

atoms were selected for further runs, with only the initial random number seed being changed between runs. The method was found to reproduce 57 out of these 65 distinct structures. The full set of calculations consumed about a week of CPU time on a cluster of half-a-dozen $20 \mathrm{MHz}$ Silicon Graphics Personal Irises; a single run consumes typically 10 mins.

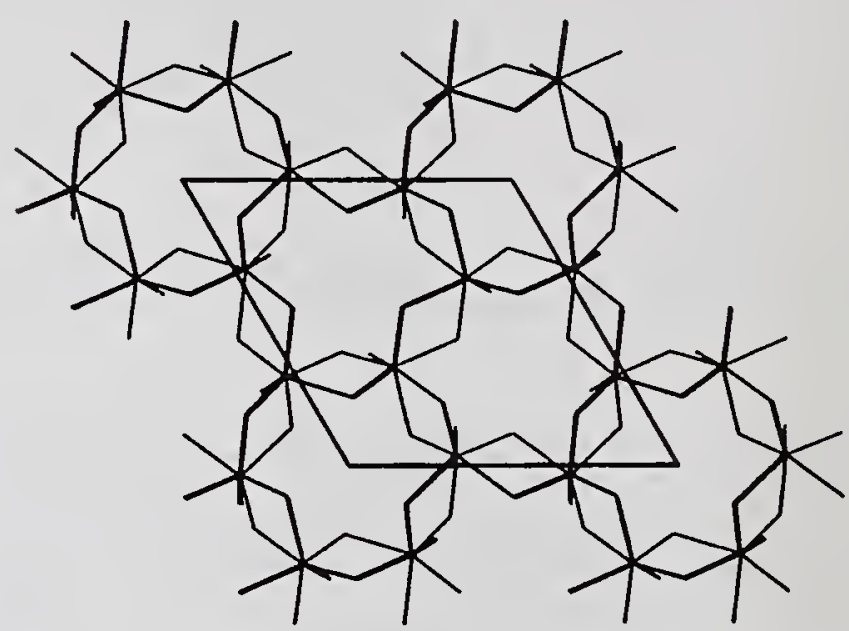

Figure 7: The framework structure of a new sodium zinc arsenate phase solved by simulated annealing using solely geometrical terms (Fig. 3) in the 'energy' function (Nenoff, Harrison, Nicol, Stucky \& Newsam, 1992).

An analogous set of calculations was also performed using, in addition, a PXD diffraction matching term in the zeolite 'energy' function. As experimental PXD data for most of the materials for which structural data are known (Newsam \& Treacy, 1992) are not available, synthetic data were used. The PXD patterns were calculated for $2 \theta \leq 30^{\circ}$ assuming the $\operatorname{CuK} \alpha$ wavelength of $1.5418 \AA$, from the known representative structures assuming, where possible, a chemical composition of $\mathrm{SiO}_{2}$. The maximum intensity in the calculated PXD was scaled to 1000 , and standard deviations of $1,2,3$, and 4 were assigned to intensities greater than $0,40,80$, and 120 in an effort to mimic approximately the uncertainties expected in the experimental data. The PXD pattern measured from a real material will, of course, reflect the scattering from the full unit cell contents, both framework species, non-framework cations, and sorbates or occluded template molecules. However, unless a substantial proportion of heavy elements are present in well-localized positions, the general distribution of intensities will be a reasonable discriminator for possible framework models. Using data carefully measured from real materials we would expect a degree of success similar to that obtained with the synthetic data. The method was again successful in 


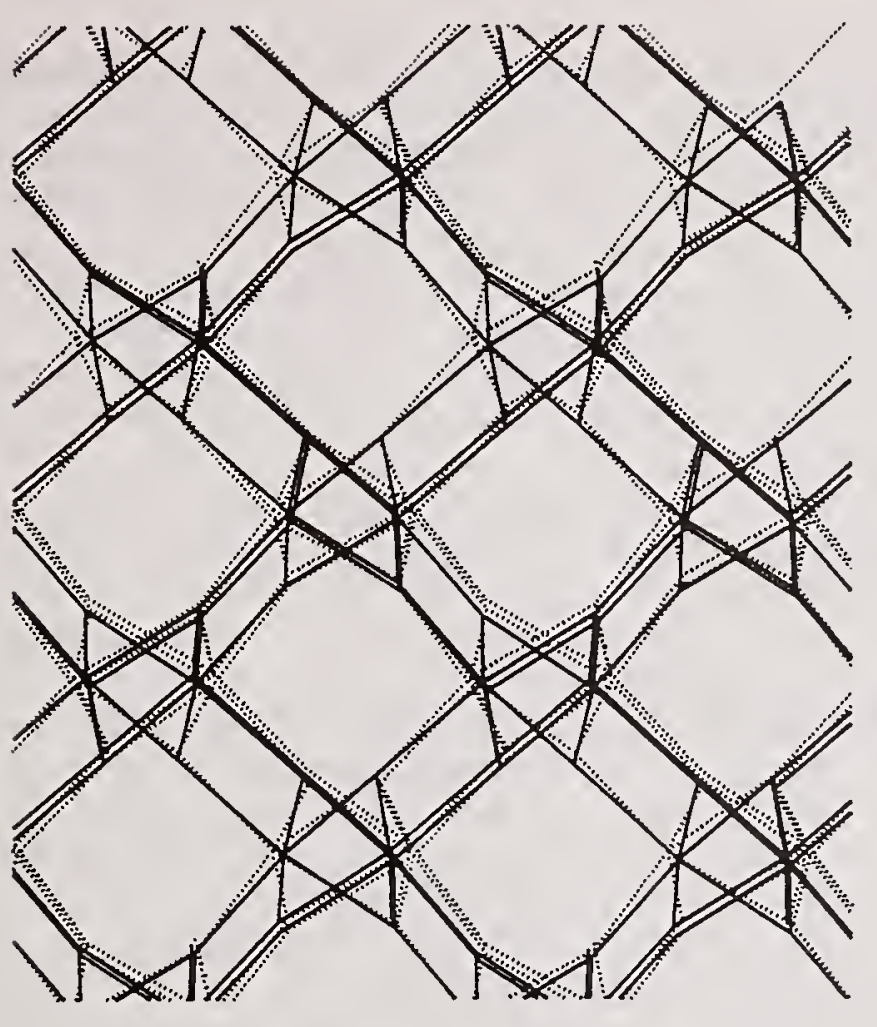

Figure 8: Comparison of the crystal structure of brookite viewed along [001] (full lines) and that generated by simulated annealing using rigid ion model potentials (Freeman, Newsam, Levine \& Catlow, 1992).

the majority of cases, with the PXD pattern matching term acting as a powerful discriminator against inappropriate hypothetical structures.

The simulated annealing method, with the general form of the zeolite 'energy' function described here has been used to solve the structures of some new materials (Deem \& Newsam, 1989), notably the product that results from a framework reconstruction on dehydration of $\mathrm{Na}_{6}\left(\mathrm{ZnAsO}_{4}\right)_{6} \cdot 8 \mathrm{H}_{2} \mathrm{O}$ with the sodalite structure at $190^{\circ} \mathrm{C}$ (Fig. 7) (Nenoff, Harrison, Nicol, Stucky \& Newsam, 1992). Although initially conceived and presented here as an aid to structure solution, the method is also now being used as a true structure design tool.

\subsection{Titania polymorphs}

The use of staged potential functions, culminating in a full lattice energy minimization worked well in 'predicting' the $\mathrm{TiO}_{2}$ rutile structure (Freeman \& Catlow, 1991). As a further test of the procedure we have applied the method to the anatase and brookite polymorphs of $\mathrm{TiO}_{2}$ (Freeman, Newsam, Levine \& Catlow, 1992). The potential model employed in the lattice energy relaxation phase of the calculation was that derived by fitting to the experimental structure

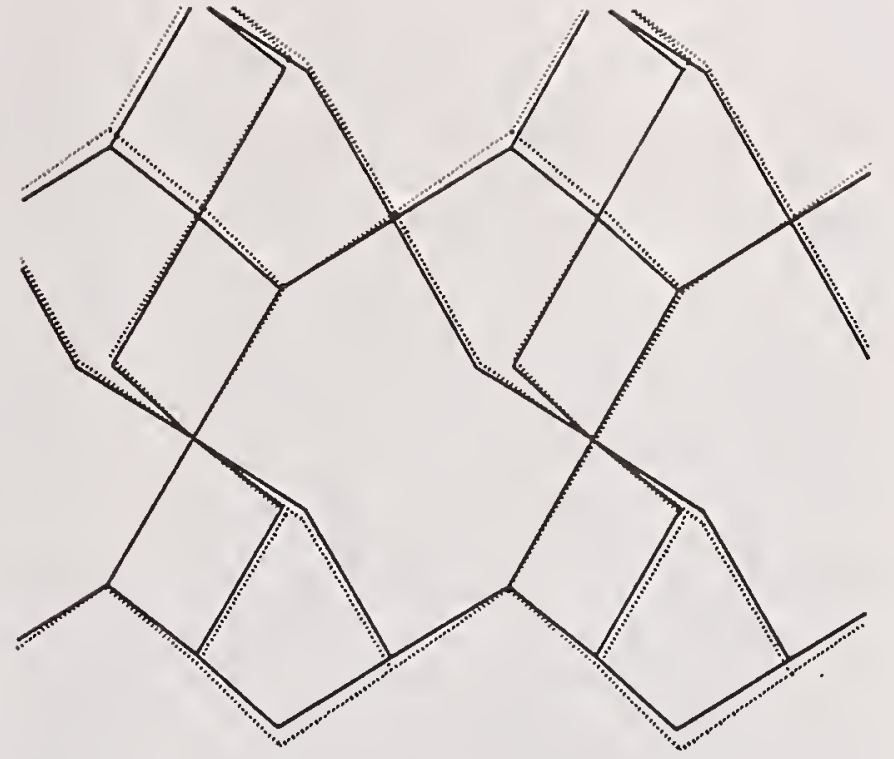

Figure 9: Comparison of the crystal structure of $\mathrm{TiO}_{2}$ (II) (full lines) and that generated by simulated annealing using shell model potentials and the brookite unit cell dimensions (Freeman, Newsam, Levine \& Catlow, 1992) (viewed along [010] in the brookite unit cell).

and properties of rutile. As in the rutile study, 50 trials were performed and the resulting structures were ranked in terms of predicted lattice energy.

For rutile the correct rutile structure is generated in 43 from 50 trials (Freeman \& Catlow, 1991). In the case of anatase the correct structure was generated in 4 of the 50 trial runs, and is the lowest energy structure obtained, whilst for brookite the number of instances of correct structure generation was 3. For brookite it is interesting to note that the correct brookite polymorph was found to be $\sim 0.1 \mathrm{eV}$ less stable than an alternative structure, found in 20 trials. This corresponds to the $\mathrm{TiO}_{2}$ (II) polymorph (Hyde \& Andersson, 1989), but with a doubling of the a lattice vector to match that of brookite's unit cell (Brookite has space group $\mathrm{Pbca}$, with $a=9.116 \AA, b=5.436 \AA, c=5.135 \AA ; \mathrm{TiO}_{2}$ (II) $P b c n$, with $a=4.563 \AA, b=5.469 \AA, c=4.911 \AA)$. Figures 8 and 9 provide a comparison, for representative fragments, of the calculated (dotted line) and the experimental structures (solid) for anatase and brookite respectively. For the higher $\mathrm{TiO}_{2}$ polymorphs the frequency of correct structural prediction was significantly less that in the case of rutile. This is unsurprising given rutile's smaller unit cell and therefore fewer variable parameters to be optimized. Nevertheless the results clearly indicate that, when combined with cell parameters derived from an indexed powder pattern, the method has the potential to assist in the process of structure solution for condensed inorganic materials. 


\section{Conclusion}

When diffraction data from a high quality single crystal are available, conventional structure solution and refinement methods work very effectively. With less precise data, or with data only available from a powder sample, new approaches to structure solutions may have substantial value. Graphical model building methods, coupled with the interactive simulations of diffraction or other spectral data and their comparison against experiment, can be used to develop crystal structure models from discrete molecular components or structural motifs. Unlike physical models, unit cell size and symmetry constraints can be used directly as constraints in the model construction process. By quantifying the reasonableness of a given atomic arrangement by cost or potential functions, viable models can be derived from an initially random distribution of the required number of atoms of each type within the observed unit cell by simulated annealing. The effectiveness of this approach has been demonstrated by test applications to the known structures of zeolites and other more condensed oxide phases. Additional cost functions can be included in this process, such as micropore dimensions in the case of zeolites or, more generally, the degree of match between model and target powder diffraction patterns. Particularly when diffraction pattern matching constraints are included, simulated annealing appears to be an efficient and potentially general route to $a b$ initio structure solutions.

\section{Acknowledgments}

The Biosym Catalysis and Sorption Project is supported by a consortium of industrial and academic institutions; we thank the membership for their guidance, input and for many stimulating discussions.

\section{References}

Bell, R. G., Jackson, R. A. \& Catlow, C. R. A. (1990). J. Chem. Soc. Chem. Comm. 782.

Binder, K., ED. (1987). Applications of the Monte Carlo Method in Statistical Physics, 2nd. Edition (Topics in Current Physics Vol. 36). Berlin: Springer-Verlag. Bragg, W. L. \& WarRen, B. (1928). Zeit. Kristallogr. 69, 168-193.

Brunger, A. T., Leahy, D. J., Hynes, T. R. \& Fox, R. O. (1991). J. Mol. Biol. 221, 239.

BURKERT, U. \& ALLINGER, N. L. (1982). Molecular Mechanics, (ACS monograph No. 177). Washington, DC: American Chemical Society.

Car, R. \& Parinello, M. (1985). Phys. Rev. Lett.55, 2471.
Catalysis 2.0 Software User Manual, San Diego: Biosym Technologies Inc. (1992).

Catlow, C. R. A. \& Mackrodt, W. C. (1982). Computer Simulation of Solids (Lecture Notes in Physics No. 166). Berlin: Springer-Verlag.

Catlow, C. R. A. \& Price, G. D. (1990). Nature 347, 243-248.

Crippen, G. M. \& Havel, T. F. (1988). Distance Geometry and Molecular Conformation, UK: Research Studies Press.

David, W. I. F. (1987). J. Appl. Cryst. 20, 316-319.

Deem, M. W. \& Newsam, J. M. (1989). Nature 342 , 260-262.

Deem, M. W. \& Newsam, J. M. (1992). J. Amer. Chem. Soc. 114, 7189-7198.

Estermann, M. \& Gramilch, V. (1991). Abstracts of 1991 Swiss Crystallographic Society, pp. 22-23.

FIELd, M. (1991). J. Phys. Chem. 95, 5104.

Fletcher, R. \& Powell, M. J. D. (1963). The Computer Journal, 6, 163-168.

Freeman, C. M. \& Catlow, C. R. A. (1991). J. Chem. Soc. Chem. Comm. 89-91.

Freeman, C. M., Newsam, J. M., Levine, S. M. \& Catlow, C. R. A. (1992). J. Mater. Chem. Submitted.

Gdanitz, R. J. (1992). Chem. Phys. Lett. 190, 391-396. Gill, P. E., Murray, W. \& Wright, M. H. (1981). Practical Optimization. London: Academic Press.

Gilmore, C. J., Henderson, K. \& Bricogne, G. (1991). Acta Cryst. A47, 830-841.

HaWthoRne, F. C. (1990). Nature, 345, 297.

Hyde, B. G. \& Andersson, S. (1989). Inorganic Crystal Structures. New York: John Wiley and Sons.

Johnston, D. C., Jacobson, A. J., Newsam, J. M., LEwandowski, J. T., Goshorn, D. P., XIE, D. \& YeLON, W. B. (1987). In Chemistry of High Temperature Superconductors (ACS Symp. Ser. 351), edited by D. L. Nelson, M. S. Whittingham and T. F. George, pp. 136-151. Washington, DC: American Chemical Society.

KeEn, D. A. \& McGreevy, R. L. (1990). Nature, 344, 423-425.

KhaN, A. A. (1976). Acta Cryst. A32, 11-16.

KirkPatrick, S., Gelatt, C. D. \& Vecchi, M. P. (1983). Science 220, 671-680.

Lacorre, P. \& Pannetier, J. (1987). J. Mag. Mat. Mater. 71, 63-82.

MadDox, J. (1988). Nature, 336, 201.

McCusker, L. B. (1992). In Accuracy in Powder Diffraction II (NIST Special Publication), edited by E.

Prince and J. K. Stalick, In press. Bethesda, MD: National Institute of Standards and Technology.

McGreevy, R. L. \& Pusztai, L. (1988). Molecular Simulation, 1, 359-367.

McGreevy, R. L. \& Pusztai, L. (1990). Proc. Roy. Soc. (London) A430, 241-261.

MeIer, W. M. \& Moeck, H. J. (1979). J. Solid State Chem. 27, 349 . 
Merer, W. M. \& Olson, D. H. (1992). Atlas of Zeolite Structure Types (Third Edition). Guildford, UK: Butterworths.

MeIer, W. M. \& Villiger, H. (1969). Zeit. Kristallogr. 129, 411-423.

Metropolis, N., Rosenbluth, A. W., Rosenbluth, M. N., Teller, A. H. \& Teller, G. (1953). J. Chem. Phys. 21, 1087-1092.

MORRIS, R. E., HARrison, W. T. A., Nicol, J. M., Wilkinson, A. P. \& Cheetham, A. K. (1992). Nature. In press.

Nenoff, T. M., Harrison, W. T. A., Nicol, J. M., Stucky, G. D. \& Newsam, J. M. (1992). Zeolites. Submitted.

Newsam, J. M. (1992). In Proc. Ninth Int. Zeolite Conf., edited by R. B. von Ballmoos, J. B. Higgins and M. M. J. Treacy, in press. Amsterdam: ButterworthHeineman.

Newsam, J. M., Deem, M. W., Freeman, C. M., Gorman, A., Ho, A., Koelmel, C. M., Levine, S. M., LI, Y. S., LINDSAY, R. J., NG, J. et al. (1992). In Proc. 2nd. Int. Conf. Comp. Appl. Mater. Molec. Sci. Eng. - CAMSE '92, edited by M. Doyama, in press. Amsterdam: Elsevier.

Newsam, J. M. \& Treacy, M. M. J. (1992). Zeolites. In press.

Pannetier, J., Bassas-Alina, J., Rodriguez-CarvaJAL, J. \& Caignaert, V. (1990). Nature, 346, 242.
Parker, S. C., Cormack, A. N. \& Catlow, C. R. A. (1984). Acta Cryst. B40, 200.

Piela, L., Kostrowicki, J. \& Scheraga, H. A. (1989). J. Phys. Chem. 93, 3339-3346.

Powell, M. J. D., ED. (1982). Nonlinear Optimization. New York: Academic Press.

Press, W. H., Flannery, B. P., Teukolsky, S. A. \& VetTerling, W. T. (1989). Numerical Recipes, The Art of Scientific Computing. Cambridge: Cambridge University Press.

SCAles, L. E. (1985). Introduction to Non-linear Optimization. London: Macmillan.

SEDGEWICK, R. (1988). Algorithms (2nd. Ed.). Reading, MA: Addison-Wesley.

Shannon, M. D., Casci, J. L., Cox, P. A. \& AnDREWS, S. J. (1991). Nature, 353, 417-420.

SMith, J. V. (1988). Chem. Rev. 88, 149-182.

SOMORJaI, R. L. (1991). J. Phys. Chem. 95, 4141-4146.

Soukoulis, C. M. (1984). J. Phys. Chem. 88, 48984901.

Thlmanns, E., Gebert, W. \& Baur, W. H. (1973). J. Solid State Chem. 7, 69-84.

Tomlinson, S. M., Jackson, R. A. \& Catlow, C. R. A. (1990). J. Chem. Soc. Chem. Comm. 813.

Williams, D. E. (1967). J. Chem. Phys. 47, 4680. 
National Institute of Standards and Technology Special Publication 846. Proceedings of the international conference Accuracy in Powder Diffraction II, held at NIST, Gaithersburg, MD, May 26-29, 1992. (Issued October 1992)

\title{
Automatic Indexing: Procedures and Applications
}

\author{
D. LOUËR \\ Laboratoire de Cristallochimie (URA CNRS 1495), Université de Rennes I, \\ Avenue du Général Leclerc, 35042 Rennes cedex, France.
}

\begin{abstract}
Indexing a powder-diffraction pattern by computerbased methods has advanced to the state that it is now possible to determine unit-cell dimensions, regardless of the crystal symmetry, for an unknown material solely from the one-dimensional d-spacing information. It is an essential requirement in a number of applications of the powder diffraction technique; the recent progress in $a b$ initio structure determination from powder data have furthermore demonstrated the fundamental rôle of the indexing of a powder diffraction pattern. A survey of the different approaches used in computer-based methods is given, with emphasis on data accuracy required. The influence of perturbing factors is discussed, e. $g$. random and systematic errors, and some means of reducing the latter are described. The respective merits of modern instruments, operating with monochromatic radiation from conventional or synchrotron $\mathrm{X}$-ray sources, are discussed. Applications are presented, including exceptional examples, such as cases with a dominant zone and unit cells with a large volume.
\end{abstract}

\section{Introduction}

The indexing of a powder pattern is an essential requirement in a number of applications of the powder diffraction technique. Pattern indexing and the determination of symmetry and unit cell parameters allow a unique characterization of the powder material, providing evidence that the sample is a single phase. The recent developments in $a b$ initio structure determination from powder diffraction data have highlighted the fundamental importance of indexing a powder pattern. It is an easy task with accurate data, but the problem is usually complicated by random and systematic errors. Efficient computer-based methods, regardless of symmetry, are currently used; their rate of success is very high, provided that data are accurate. However, their use in routine work re- quires a minimization of systematic errors by knowing perfectly the accuracy attainable by careful alignment of instruments.

The interest in $a b$ initio indexing of a powder diffraction pattern started a few years after the discovery of X-ray diffraction in 1912. Runge (1917) derived some useful specific relations in reciprocal space; later, from similar principles, Ito (1949) and de Wolff (1957) reported a more general method for the geometrical reconstruction of the reciprocal lattice of a crystal from the observed d-spacings. The key rôle of data accuracy for solving the crystallographic problem was clearly expressed by de Wolff (1957), as is shown by this quotation: "It would be quite an easy puzzle if errors of measurement did not exist." In 1968, a simple criterion for selecting correct indexing from false solutions was proposed (de Wolff, 1968); the introduction of this factor was a milestone in the slow progression of powder pattern indexing. At the same time, a computer program, based on the Runge (1917), Ito (1949) and de Wolff $(1957,1968)$ principles, was written by Visser (1969). With the development of computer technology, new approaches using trial-and-error methods were devised: the method based on the permutation of Miller indices by Werner (1964) and the method based on the variation of the lengths of cell edges and interaxial angles by means of a successive dichotomy procedure by Louër \& Louër (1972). The efficiency of these modern computer-based methods was reviewed by Shirley (1978).

The present paper summarizes the basic principles of the indexing of a powder pattern, describes the modern computer-based methods, emphasizes the rôle of data accuracy and presents the features of modern instruments that reduce the influence of perturbing effects. Also, examples, including cases with dominant zones and large unit-cell volumes, are presented. 


\section{Basic principles}

The objective of indexing a powder diffraction pattern is to reconstruct the three-dimensional reciprocal lattice of a solid from the one-dimensional dspacing information available in the pattern. Figure 1 shows how the reciprocal lattices of the randomly oriented crystallites constituting the powder generates, by a rotational projection, a radial distribution of lengths $d^{*}(=1 / d)$ of diffraction vectors. The geometrical restoration of the node positions of

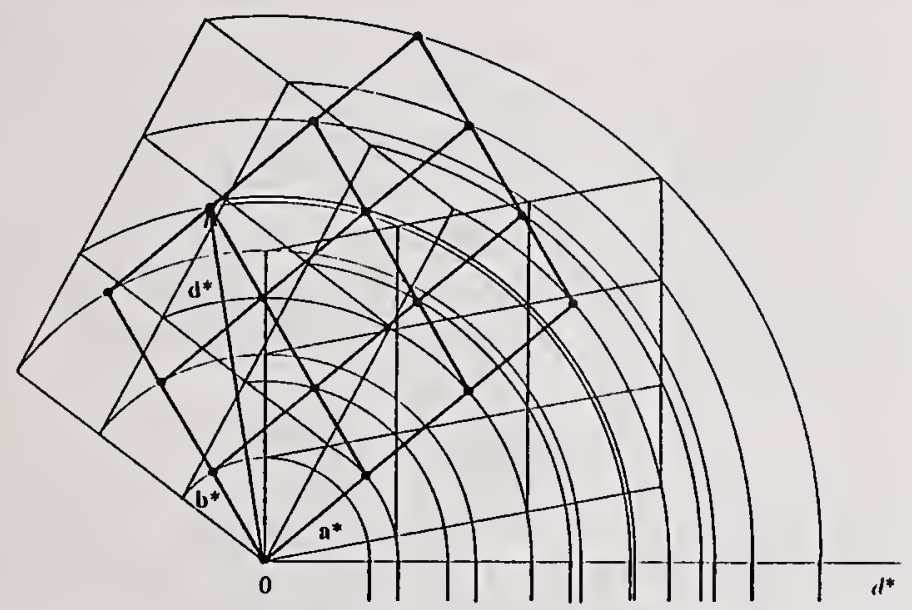

Figure 1: Two-dimensional representation of the distribution of diffraction lines from the projection of randomly directed reciprocal vectors, $\mathrm{d}^{*}$.

the reciprocal lattice from this radial distribution is a crystallographic problem based on algebraic relations; it is also dependent on computing facilities, chemical knowledge of the material, in order to explain (possible) spurious lines due to impurities, and the instrument used for collecting data, since accuracy is required for a successful indexing. The outcome of the indexing will depend on the measurement of d-spacings, derived from $2 \theta$ Bragg angles in angle-dispersive experiments. Consequently, indexing a powder diffraction pattern cannot be dissociated either from the quality of instrument adjustment (or calibration), or from the degree of diffraction line overlap. The latter is related to geometrical coincidences, to the instrument resolution characteristics, and to the microstructure of the material. Indeed, every observed diffraction line ( $h$ profiles) is the convolution of instrumental aberrations ( $g$ profiles), including the wavelength distribution and sampleinduced geometric and physical effects, with contributions that are specific to the microstructure of the sample ( $f$ profiles). These effects reduce the resolution of the pattern, so that some lines cannot be observed.
Each vector $\mathbf{d}_{h k l}^{*}$ is defined in terms of the basis vectors of the reciprocal lattice $\left(\mathbf{a}^{*}, \mathbf{b}^{*}, \mathbf{c}^{*}\right)$ and the Miller indices: $\mathrm{d}_{h k l}^{*}=h \mathbf{a}^{*}+k \mathbf{b}^{*}+l \mathbf{c}^{*}$. The basic relation used for indexing a powder diffraction pattern is obtained by squaring the $d^{*}$ vector:

$$
\begin{aligned}
\mathrm{Q}(h k l)= & \left|\mathrm{d}^{*}\right|^{2} \\
= & h^{2} \mathrm{Q}_{A}+k^{2} \mathrm{Q}_{B}+l^{2} \mathrm{Q}_{C} \\
& +2 k l \mathrm{Q}_{D}+2 h l \mathrm{Q}_{E}+2 h k \mathrm{Q}_{F}
\end{aligned}
$$

where $\mathrm{Q}_{A}=\mathbf{a}^{*} \cdot \mathbf{a}^{*}, \mathrm{Q}_{B}=\mathbf{b}^{*} \cdot \mathbf{b}^{*}, \mathrm{Q}_{C}=\mathbf{c}^{*} \cdot \mathbf{c}^{*}$, $\mathrm{Q}_{D}=\mathbf{b}^{*} \cdot \mathbf{c}^{*}, \mathrm{Q}_{E}=\mathbf{c}^{*} \cdot \mathbf{a}^{*}$ and $\mathrm{Q}_{F}=\mathbf{a}^{*} \cdot \mathbf{b}^{*}$. This quadratic form corresponds to the general triclinic case; it simplifies considerably for the other systems.

The one-dimensional distribution of lengths of reciprocal-lattice vectors is completely defined by the geometry of the unit cell, the lengths of the basis vectors $\left(a^{*}, b^{*}, c^{*}\right)$ and the inter-axial angles of the reciprocal lattice $\left(\alpha^{*}, \beta^{*}, \gamma^{*}\right)$. In practice, some lines are missing, due to symmetry conditions, accidental or exact overlap, and diffraction lines with low intensity. Although the powder diffraction pattern suffers from the collapse of the three-dimensional crystallographic data to the one-dimensional diffraction pattern, some useful mathematical relationships remain:

(i) Algebraic relationships in reciprocal space can be derived from equation (1). Among them are the following general properties:

- Colinear vectors $\mathbf{d}_{h k l}^{*}$ are easily detected by the integer value $n$ of the ratio of their length to the length of the shortest vector $\left(\mathrm{d}_{h_{1} k_{1} l_{1}}^{*}\right)$ in the $[h k l]$ direction: $\mathrm{d}_{h k l}^{*}=$ $n \mathrm{~d}_{h_{1} k_{1} l_{1}}^{*}$. This property can also be used for the detection (and correction) of systematic errors in the pattern.

- Relations for a zone of lattice planes can be derived from the additive properties of $Q_{h k l}$, particularly in the case of triclinic symmetry, e.g. the $\mathrm{Q}(0 k l)$ and $\mathrm{Q}(0 k \bar{l})$ values can be used for the determination of the interaxial angle $\alpha^{*}$. For the detection of $\mathrm{Q}(0 k l)$ and $\mathrm{Q}(0 k \bar{l})$, it can be shown that they are symmetrical with respect to a nonexistent value $\left(\mathrm{Q}_{0 k l}\right)$ calculated for an orthogonal lattice.

Some other general relations have also been derived by de Wolff (1957);

- Additive properties of equation (1) can also be used for finding the $0 k 0$ row in monoclinic cases; an example of a relation that 
serves to locate the 020 reflection is (Smith \& Kahara, 1975):

$$
2 Q_{020}+Q_{h 10}=Q_{h 30} .
$$

(ii) The theoretical number $N$ of diffraction lines in the pattern can be evaluated in the general triclinic case. It is easily demonstrated that the number of radial distances less than $\mathrm{d}_{N}^{*}$ is given by the approximate relation:

$$
N \approx 4 \pi V\left(\mathrm{~d}_{N}^{*}\right)^{3} / 3 m,
$$

where $V$ is the cell volume of the direct lattice. In fact, the actual number of observed lines is much less than $N$. The reduction is caused by the multiplicity factor $m$ of Bragg reflections [ $m$ is given as 2 for triclinic, 4 for monoclinic symmetry, etc. by de Wolff (1961)], the space group extinctions and the non-observed reflections. From this equation, Smith (1977) has derived an empirical relation useful for estimating the unit-cell volume in triclinic cases, based on the $d$ value of the 20th observed line:

$$
V=13.39 \mathrm{~d}_{20}^{3} .
$$

\section{Assessing the reliability of indexing}

The solution to equation 1 is not unique and is also strongly dependent on the accuracy of data. In general, a satisfactory agreement between calculated and observed $Q$ values is not sufficient to be certain of the unit cell. To assess the reliability of an indexed powder diffraction pattern, de Wolff (1968) recommended the use of a criterion based on the ratio of two average quantities: the ratio $\mathrm{Q}_{20} / N_{\text {calc }}$ and the actual discrepancy between observed and calculated $\mathrm{Q}_{i}$ values. This index of reliability is defined as:

$$
M_{20}=\mathrm{Q}_{20} / 2\langle\Delta \mathrm{Q}\rangle N_{\text {calc }},
$$

where $\mathrm{Q}_{20}$ is the value of $\mathrm{Q}$ for the 20th observed line, $\langle\Delta Q\rangle$ is the average absolute discrepancy between $\mathrm{Q}_{i o b s}$ and the nearest $\mathrm{Q}_{\text {calc }}$ values and $N_{\text {calc }}$ is the number of distinct $Q$ values smaller than $Q_{20}$, calculated according to equation (1), excluding any systematic absences, if they are known. $M_{20}$ will be larger if $\langle\Delta \mathrm{Q}\rangle$ is small, and if $N_{\text {calc }}$ is as close as possible to 20. De Wolff stated that incorrect indexing may have a value of $M_{20}$ up to 6, and that correct indexing usually has $M_{20}$ greater than 10. Examples obtained from data collected with different instruments are discussed below in section 7 . They show that values greater than 20 are obtained in routine work, and that they can be considerably greater with high resolution instruments.

The figure of merit $M_{20}$ can be expressed in terms of the cell volume $V$ of the direct lattice, by combining equations 2 and 3 (Werner, 1976):

$$
V=3 m \mathrm{~d}_{20} / 8 \pi\langle\Delta \mathrm{Q}\rangle M_{20} \text {. }
$$

For a given average discrepancy $\langle\Delta \mathrm{Q}\rangle, M_{20}$ and the cell volume are inversely proportional. This result is interesting because it gives a useful argument for searching the smaller cells first in automatic indexing; it is applied in the strategy based on the successive dichotomy method (Louër \& Louër, 1972).

Another figure of merit that is simple to use, $\mathrm{F}_{N}$, has been proposed by Smith and Snyder (1979). Originally intended for rating powder diffraction patterns, it is defined by:

$$
\mathrm{F}_{N}=N /\langle 2 \theta\rangle N_{\text {poss }},
$$

where $N_{\text {poss }}$ is the number of possible diffraction lines up to the $N$ th observed line, and $\langle 2 \theta\rangle$ is the average absolute angular discrepancy between observed and calculated $2 \theta$ values. This index is reported in the form $\mathrm{F}_{N}=\operatorname{value}\left(\langle 2 \theta\rangle, N_{\text {poss }}\right)$. Again, the higher the accuracy of data and the more complete the pattern, the larger is $\mathrm{F}_{N}$. Of special interest is the reported average angular discrepancy $\langle 2 \theta\rangle$, which is another evaluation of the quality of data. The two figures of merit, $M_{20}$ and $\mathrm{F}_{N}$, are usually calculated in indexing programs. The $M_{20}$ factor depends strongly on the crystal symmetry, large $M_{20}$ values being obtained for higher symmetries. Some authors (Shirley, 1980; Werner, 1980) have discussed the relative merits of these factors and argued that $M_{20}$ is to be preferred for testing the reliability of a proposed indexing model; Werner concluded that if $\langle 2 \theta\rangle$ and $N / N_{\text {poss }}$ are identical for a cubic and a triclinic indexing, the cubic indexing is obviously to be preferred.

Recently, Taupin (1988) has proposed another figure of merit based on the information conveyed by the $h k l$ indices assigned to a given diffraction line, namely individual discrepancies between computed and observed line positions, unit-cell volume and systematic extinction rules. This factor has been incorporated in the author's program.

Finally, from the experience of the present author, based on the indexing a great number of powder diffraction patterns, the $M_{20}$ figure of merit has always been found satisfactory in assessing the reliability of a proposed solution. The $F_{20}$ index and the 
reported average angular discrepancy between observed and calculated $2 \theta$ positions constitute other useful indicators of precision. Also, the information contained in equation 4 (a similar equation would be obtained from the $\mathrm{F}_{N}$ index) should be taken into consideration in indexing problems. With accurate data, the solution with the smallest volume is usually the correct one. In some exceptional cases, difficulties can arise from $(i)$ geometrical ambiguities or (ii) when a zone dominates in the twenty first lines. Case $(i)$ has been discussed by Mighell \& Santoro (1975); it can occur if the lattices have high symmetry, e.g. an hexagonal pattern can be indexed by an orthorhombic unit cell having the cell dimensions $a / 2, a \sqrt{3} / 2, c$ and a volume half the hexagonal cell volume. This situation can be detected by analysing separately each crystal system, from high to low symmetries and by the metric relations between parameters of the two cells. In case (ii) solutions with similar unit cell volumes and $M_{20}$ factors can be observed. This case is illustrated by an example in section 9. Nevertheless, whatever the definition of the figure of merit used, the problem of a dominant zone remains unsolved.

\section{Modern methods for the indexing of powder diffraction patterns}

With the availability of computers, automatic programs were devised for indexing powder patterns of solids with an unknown crystal structure. These were reviewed by Shirley several years ago $(1978,1980)$. He also reported an analysis of the success rate of these programs. Since this study, some of the tested programs have been considerably improved. Nowadays, three main important procedures for indexing a powder pattern, regardless the symmetry, are currently used:

(i) The Runge-Ito-de Wolff method is based on algebraic relations in the reciprocal lattice, of which some are given in section 2. A computer program using these principles was written by Visser (1969); it was the first general program, regardless of the symmetry, written for powder pattern indexing. It is well known for its efficiency for low symmetry cases; the most frequent reason for failure is the incompleteness of the input data. The program has frequently been used with success since 1970. It helped to demonstrate that powder pattern indexing can be used with confidence for a unique characterization of powder materials. For example, the Visser program was used to identify four cadmium hydroxide sulphate phases obtained by slow hydrolysis of a neutral cadmium sulphate solution, by indexing the powder diffraction patterns with tetragonal and monoclinic unit cells, with high $M_{20}$ figures of merit (37, 54, 20 and 29) (Walter-Levy, Groult \& Visser, 1974). Later, the correctness of the reported results was demonstrated by single-crystal studies (Labarre, Louër, Louër \& Grandjean, 1976a, 1976b; Louër, Louër \& Grandjean, 1982; Louër, Labarre, Auffrédic \& Louër, 1982).

(ii) The trial-and-error method, based on a permutation of Miller indices for selected base lines, was introduced by Werner (1964), and the principles have since been applied by several authors (Taupin, 1973; Kolhbeck \& Hörl, 1976; Wu, 1989). Among the most popular recent indexing programs, regardless of the symmetry, are TREOR (Werner, Eriksson \& Westdahl, 1985) and the programs by Taupin (1989) and Kolhbech \& Hörl $(1976,1978)$. From its principles, the method has to be sensitive to a dominant zone in the powder data, and provision for such cases can be implemented in programs, as has been done in the program TREOR; this part of the program has been further developed in the program version TREOR90. An objective analysis of the success rate of TREOR has been reported by Werner et al. (1985) on the basis of all powder diffraction data reported in Section 17 of Monograph 25 (Natl. Bur. Stand. (U. S.), 1980). The overall success of the program has been found to be better than $90 \%$, considerably more for orthorhombic and higher symmetries; moreover, it was noted that for a minority of patterns, data were of low quality, mainly due to unstable phases.

(iii) The successive dichotomy method is based on the variation of the lengths of cell edges and interaxial angles by finite ranges, followed by a progressive reduction of intervals by means of a dichotomy procedure. This method, introduced by Louër \& Louër (1972), was first applied to high symmetries and then extended to monoclinic (Louër \& Vargas, 1982). Recently, a considerably improved version, DICVOL91, extended to all crystal systems, has been published (Boultif \& Louër, 1991). The method was described by Shirley (1980) as probably the optimal exhaustive strategy in parameter-space 
within input parameter limits. The property of equation 4 is used for finding the solutions with smaller volumes first. In contrast to other approaches, the calculated patterns for possible solutions are here based on ranges $\left[\mathrm{Q}_{-}(h k l)\right.$, $\left.Q_{+}(h k l)\right]$ for every line, these ranges being progressively reduced by a dichotomy strategy. In the course of the calculation the experimental error of every line, $\Delta Q_{i}$, is rigorously taken into account. Due to the principles of the method, it is not too sensitive to the presence of a dominant zone, unless considerable ambiguity is present in the data used for solving the problem. The strategy for indexing starts from the cubic end of the symmetry sequence and finishes with triclinic symmetry. For an unbiased test of efficiency of the program, data reported in section 17 of Monograph 25 (Natl. Bur. Stand. (U. S.), 1980 ), comprising powder data for all symmetries, were analysed by DICVOL91, as well as all triclinic patterns reported in the complete Monograph 25. All orthorhombic or higher symmetry solutions were found; all solutions with monoclinic symmetry were also found, but for four of them the input absolute error, $\Delta Q_{i}$, of observed peak positions needed to be increased slightly owing to inaccuracy of the reported data; the six powder patterns of triclinic materials in section 17 were also correctly indexed. Powder patterns for triclinic materials, reported in the complete monograph, were indexed, except for three cases; one of them corresponds to a situation (the first possible lines are missing) not implemented in the program, and for the two others the explanation for failure was found to be the low precision of data.

\section{Some considerations on methods and data accuracy}

All authors of indexing programs have emphasized the dramatic effect of data inaccuracy in the search for the correct unit cell and have pointed out that it is the central parameter for a successful outcome of pattern indexing. From the mathematical point of view, equation 1 would be easy to solve if errors did not exist. In fact, taking into account the absolute error, $\Delta Q_{i}$, in every observed $Q_{i}$ value, the indexing of a powder pattern requires that, for a set of parameters $\mathrm{Q}_{A}, \mathrm{Q}_{B}, \mathrm{Q}_{C}, \mathrm{Q}_{D}, \mathrm{Q}_{E}$ and $\mathrm{Q}_{F}$, there exist three $h k l$ integers satisfying the inequality:

$$
\mathrm{Q}_{\mathrm{i}}-\Delta \mathrm{Q}_{\mathrm{i}} \leq \mathrm{Q}(h k l) \leq \mathrm{Q}_{\mathrm{i}}+\Delta \mathrm{Q}_{\mathrm{i}},
$$

where $Q(h k l)$ is given by equation (1). A rigorous analysis of the influence of errors in observed $Q_{i}$ values is difficult. Its disastrous effect is well known and is one of the main reasons, along with incompleteness of data, for failure in indexing powder patterns. By rule of thumb the error in the observed data should be less than $0.03^{\circ}(2 \theta)$ for a wavelength such as copper $K \alpha_{1}$. Before discussing experimental procedures for reducing data inaccuracy, the effect of errors in modern approaches for indexing will be discussed briefly.

In methods of type (ii), solutions are obtained from a set of base lines, against which the remaining lines in the pattern are checked. These solutions are column vectors $\mathbf{P}$ (with elements $\mathrm{Q}_{A}, \mathrm{Q}_{B}, \mathrm{Q}_{C}, \mathrm{Q}_{D}$, $Q_{E}$ and $Q_{F}$ ) derived from a set of linear equations formed by the base lines; in practice, each of them includes implicitly an unknown individual error $\delta Q$; thus, the problem should be formulated by the matrix form:

$$
\mathbf{P}+\delta \mathbf{P}=\mathbf{M}^{-1}(\mathbf{Q}+\delta \mathbf{Q})
$$

where $\mathbf{M}$ is a square matrix whose elements are based on the Miller indices. In this equation, for a reasonable error $\delta \mathbf{P}$, there is no isomorphic relation between the vectors $\mathbf{P}+\delta \mathbf{P}$ and $\mathbf{Q}+\delta Q$, which implies that a small error $(\delta \mathbf{Q})$ in $\mathbf{Q}$ does not necessarily give a small error $(\delta \mathbf{P})$ in $\mathbf{P}$. On the other hand, from a given error vector $\delta \mathbf{P}$ in $\mathbf{P}$, it is possible to calculate exactly the corresponding error $(\delta \mathbf{Q})$ in $\mathbf{Q}$. (This feature of non-bijectivity is at the origin of the successive dichotomy method.) In approach (ii), when the vectors $\mathbf{P}$ have been obtained, they are used to check the second data set formed by the equations describing the remaining diffraction lines. As a consequence of the parameter error $(\delta \mathbf{P})$, expanded error ranges $\Delta Q_{i}^{*}$ must be used, instead of the original expected error $\Delta Q_{i}$. In practice, this problem is solved in current programs and has been discussed by different authors (Werner,1964; Taupin, 1973; Kohlbeck \& Hörl, 1978). It should also be noted that further confidence in a solution is provided if identical solutions are found from different base line sets. In any case, accurate data for the base lines are of special importance for reducing the effect of error propagation. A similar comment is also true for approach (i). The lines used to obtain linear and angular reciprocal lattice parameters must be as accurate as possible in order to have an agreement, within the experimental errors, between observed and calculated $Q_{i}$ values. Also, it is important that there should not be too many lines missing from the pattern. 
The successive dichotomy method

As reported above, the error vector $\delta \mathbf{Q}$ can be calculated from a given vector $\delta \mathrm{P}$, and this calculation can be extended to all possible $h k l$ indices of the input data. This property is the basis of the successive dichotomy method. The parameter axes $P_{\mathbf{j}}$ $\left(P_{\mathrm{j}}=Q_{A}, \ldots, Q_{F}\right.$ or related parameters in direct space) are scanned by linear ranges $\Delta P_{j}\left(=P_{j-}\right.$, $\mathrm{P}_{\mathrm{j}+}$ ), within limits selected by the user. The volume space is thus divided into discrete $\mathrm{j}$-dimensional domains $(j=1, \ldots, 6)$ formed from the ranges $\Delta P_{j}$. Each domain is analysed separately. A calculated pattern is generated, each line being represented in Q-space by an interval [Q- $\left.(h k l), \mathrm{Q}_{+}(h k l)\right]$; the domain is then retained if the observed lines $\mathrm{Q}_{\mathrm{i}}$ lie within the limits

$$
\mathrm{Q}_{-}(h k l)-\Delta \mathrm{Q}_{\mathrm{i}} \leq \mathrm{Q}_{\mathrm{i}} \leq \mathrm{Q}_{+}(h k l)+\Delta \mathrm{Q}_{\mathrm{i}},
$$

which include the maximum expected experimental error, $\Delta Q_{i}$. Otherwise it is discarded. At this stage the correct solution can be included in the domain, which will subsequently be analysed in detail. It can be noted that the $h k l$ indices of the exact solution for every observed line constitute a subset of the set of $h k l$ values that have been found possible at this stage for each line. This is an interesting point, since the introduction of a recursive procedure for the storage of $h k l$ reduces computing times. If all observed $Q_{i}$ values are located within the first calculated limits, each domain is divided into $2 \mathrm{j}$ subdomains by halving the intervals $\left[\mathrm{P}_{\mathbf{j}-}, \mathrm{P}_{\mathbf{j}+}\right]$. Again, calculated lines $\left[\mathrm{Q}_{-}(h k l), \mathrm{Q}_{+}(h k l)\right]$ are generated and compared with the observed data, within the limits of experimental error (relation 6). If a solution still exists, the dichotomy approach is applied successively, up to a maximum of seven times. It is an arborescent procedure that progressively encloses the solution(s). It has been found preferable to apply this technique to direct parameters; the lengths of cell edges and inter-axial angles are scanned by finite ranges $(0.40$ $\AA$ and $5^{\circ}$, respectively), which are progressively reduced. With this stategy, solutions are searched exhaustively in a $\mathrm{j}$-dimensional space. (However, for the triclinic case, $\mathrm{j}=6$, the procedure is applied in $\mathrm{Q}-$ space with some constraints.) The method is simple; it is only necessary to derive the mathematical relations giving the limiting values $\mathrm{Q}_{-}(h k l)$ and $\mathrm{Q}_{+}(h k l)$ for each symmetry. For orthogonal and hexagonal systems this calculation from the intervals $\left[\mathrm{P}_{\mathrm{j}-}, \mathrm{P}_{\mathrm{j}+}\right]$ is trivial (Louër \& Louër, 1972); for low symmetry cases, the calculations are more difficult and have been reported recently (Boultif \& Louër, 1991). Fi- nally, it should also be noted that the method accepts individual experimental error bounds for every observed diffraction line. This feature can be useful in cases where, for some reason, the measurement of a few lines in the pattern is less accurate.

\section{Precision and accuracy of data}

In angle-dispersive powder diffraction, the precision of the position of Bragg reflections is defined by the error $\Delta 2 \theta$. The maximum precision in d-spacing attainable by assuming a constant error $\Delta 2 \theta$ is obtained by differentiating the Bragg equation, which shows that the error in d, proportional to $\cot \theta \Delta \theta$, increases as $\theta$ decreases. Thus, the $d$ values derived from low-angle lines, which play a fundamental rôle in indexing, are most affected by errors. These effects can be illustrated by simulating errors in calculated patterns, as shown in this section. It is beyond the scope of this paper to analyse the origin of random and systematic errors that may occur in powder diffraction; this has been discussed by various authors, e.g. Wilson (1963). In collecting data for indexing a powder pattern, it is strongly recommended that these factors be considered. The effects of all instrumental factors on the quality of data and their influence on the derived interplanar d-spacings must be properly understood to obtain highest accuracy. For example, reproducibility of measurements is an indication of the precision, but does not account for possible systematic errors that can affect the accuracy of data. In practice, it is essential to adjust the instruments correctly and to check the quality of alignment by means of reference materials, which will give an indication of the absolute angular error of data. In what follows the influence of random and systematic errors in input data is described by means of two calculated patterns corresponding to unit cells with a small and a large volume. They correspond to the calculated patterns for a cadmium hydroxide nitrate $(a=3.4206 \AA, b=10.0269 \AA, c=11.0282 \AA$, $\mathrm{V}=378 \AA^{3}$; Auffrédic, Plévert \& Louër, 1990) and barium titanyl oxalate hydrate (BTO) $(a=14.044$ $\AA, b=13.812 \AA, c=13.382 \AA, \beta=91.48^{\circ}, \mathrm{V}=2595$ $\AA^{3}$; Louër, Boultif, Gotor \& Criado, 1990).

The effects of simulated random and systematic errors on solutions can be easily evaluated by means of the successive dichotomy method (program DICVOL91), since the treatment of error is rigorous in this approach. The results are presented until the correct solution is obtained. The default absolute error value for every line (i.e. $0.03^{\circ} 2 \theta$ ) was used for 
the case of maximum random or systematic errors lower than $0.03^{\circ}(2 \theta)$, and was increased for greater simulated errors.

(i) Random errors

Figure 2 shows the effect on the figures of merit (FoM), of a degradation of cadmium-hydroxidenitrate data by a random error. It is remark-

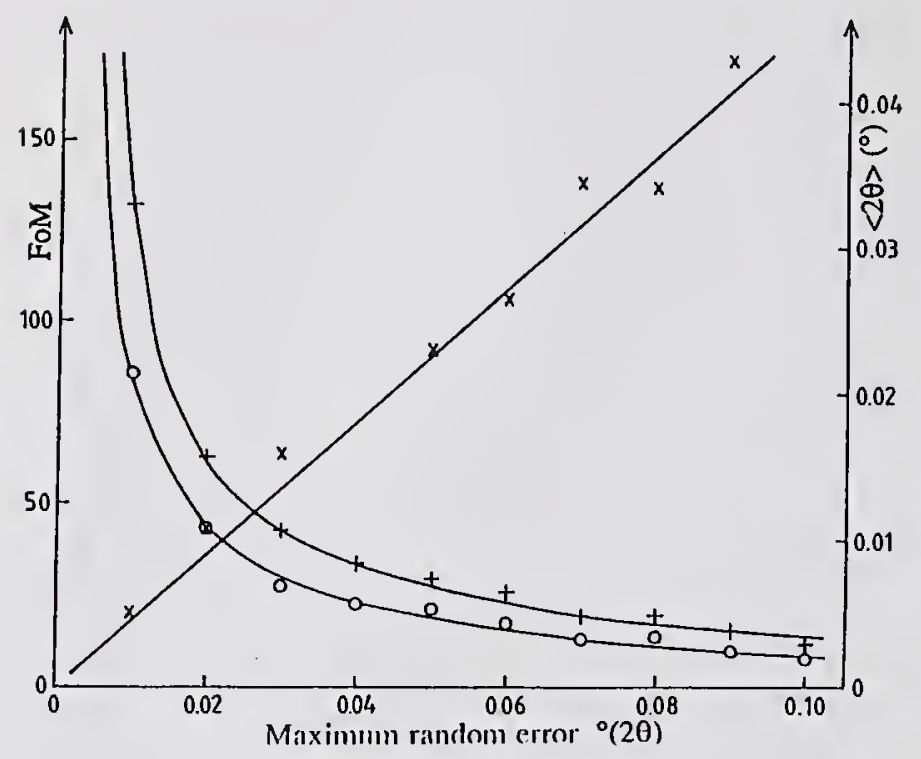

Figure 2: The effect of random error $\left[{ }^{\circ}(2 \theta)\right]$ on the figures of merit (FoM) $M_{20}(\mathrm{o})$ and $\mathrm{F}_{20}(+)$, and on the average angular discrepancy $\langle 2 \theta\rangle\left(^{\circ}\right)(\mathbf{x})$, for cadmium hydroxide nitrate.

able that the correct solution is obtained with large random errors, up to $0.10^{\circ}(2 \theta)$ for the cadmium hydroxide nitrate; it is $0.07^{\circ}(2 \theta)$ for BTO. A similar conclusion was reported by Shirley (1980), who estimated the error limits to be $\pm 0.08^{\circ}$. This result would indicate that the indexing problem is not too sensitive to random errors. The increasing $\Delta \mathrm{d}$ error as $\theta$ decreases, for a constant $\Delta 2 \theta$, explains that, for patterns with low angle lines, like for BTO, the maximum random error acceptable for obtaining the correct solution is less than for the cadmium compound, but it is still large compared with the quality of data that it is possible to obtain by modern powder diffractometry. However, it must be added that this interesting result should not be overemphasized because, with increasing error bounds, the risk of obtaining false solutions is considerably higher. It should be noted that the de Wolff figure of merit is greater than 20 for maximum random errors less than $0.06^{\circ}$ and $0.023^{\circ}(2 \theta)$ for cadmium hydroxide nitrate and barium titanyl oxalate respectively. (ii) Systematic errors

An example of a systematic error can be simulated by a shift of the zero $2 \theta$ position. Data degraded by a zero-point error were used for the pattern of the above materials and again input to DICVOL91. The effect on the figures of merit can be seen in figure 3 for BTO. As for the ran-

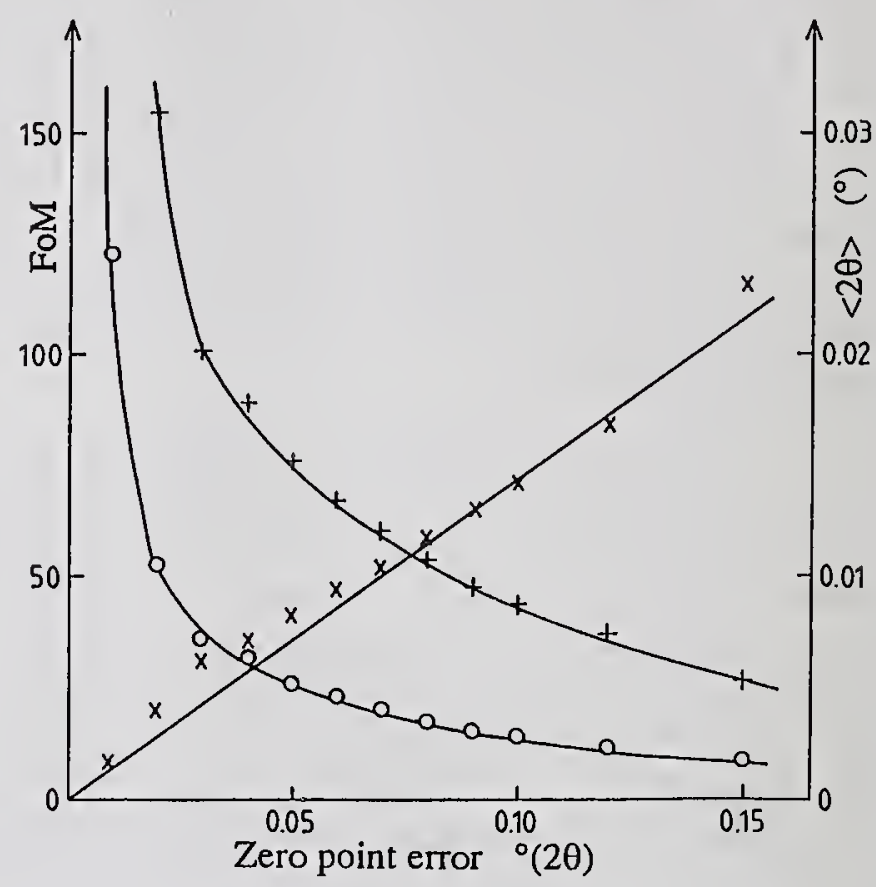

Figure 3: The effect of zero-point error $\left[{ }^{\circ}(2 \theta)\right]$ on the figures of merit (FoM) $M_{20}(\mathrm{o})$ and $\mathrm{F}_{20}(+)$ and on the average angular discrepancy $\langle 2 \theta\rangle\left({ }^{\circ}\right)(\mathrm{x})$, for barium titanyl oxalate hydrate (BTO).

dom error case, the 'correct' solution is obtained for quite large zero-point errors, with the obvious condition than the error bounds in every line tolerated by the program are similar to the angular shift of the zero point. Indeed, the solution is found for shifts as large as $0.15^{\circ}$ and $0.30^{\circ}(2 \theta)$ for $\mathrm{BTO}$ and the cadmium compound, respectively. Again, the successive dichotomy method would not be too sensitive to this systematic error. Figures of merit $M_{20}$ greater than 20 are obtained for zero point errors of $0.12^{\circ}$ and $0.07^{\circ}(2 \theta)$ for the cadmium and BTO compounds respectively. It should be noted that the presence of a zero-point error is easily detected from the refined solution by the angular discrepancies $\Delta 2 \theta_{\mathrm{i}}\left(=2 \theta_{\mathrm{iobs}}-2 \theta_{\mathrm{icalc}}\right)$ displaying a systematic bias as Bragg angles increase.

From these studies, it appears that, for indexing a pattern, the error limit for every diffraction line could be greater than $0.03^{\circ}(2 \theta)$ when the successive 
dichotomy method is employed. However, again this point should not be overemphasized, because it also increases the risk of finding pseudo-solutions and of missing the correct solution. A result with a high figure of merit always inspires confidence, and that is the consequence of a correctly adjusted instrument.

\section{Data collection strategy for indexing}

The most frequent reasons for failure to obtain a correct solution are inaccuracy and incompleteness of data and also the presence of spurious lines due to impurities in the sample. The presence of a second phase in fact implies a mixture of two patterns, with one usually having weaker reflections. The problem of indexing $a b$ initio the two patterns simultaneously seems, in the author's opinion, unsolvable by automatic indexing methods. Some programs will tolerate a few spurious lines; nevertheless, unindexed diffraction lines should always be explained by a chemical reason or by imprecision. In order to ensure a successful outcome of a powder pattern indexing, it is recommended that accurate data from an instrument carefully adjusted or calibrated be used. The user of automatic indexing programs is urged to consider this fact when obtaining the $2 \theta$ positions. As a consequence, a number of critical checks of the instrument adjustment must be made prior to collecting data. High instrumental resolution and accuracy are of paramount importance. Higher resolution allows a less ambiguous decomposition of the powder diffraction pattern, and, although overlapping reflections are not eliminated, the overlap is reduced. High resolution was a common feature of the instruments used by the first workers to practice powder pattern indexing. In particular, monochromatic radiation coupled to a careful calibration of a Guinier camera was used by the Dutch (de Wolff and Visser) and the Swedish (Werner) teams, while the French group (Louër) collected accurate data by means of conventional powder diffractometer with monochromatic X-rays. Because diffractometers, with BraggBrentano geometry, are the instruments commonly employed in powder diffraction, it is of interest to know the precision of peak position that can routinely be achieved. Also, high resolution powder diffractometers with synchrotron radiation have attractive possibilities. Some features of these two currently employed instruments are now presented briefly.

\section{(i) Resolution}

The Instrumental Resolution Functions (IRF), i.e. variation of instrumental line profile FWHM with diffraction angle, have been described by Louër and Langford (1988) for a conventional diffractometer with an incident-beam monochromator and by Langford, Cernik and Louër (1991) for the 9.1 High-Resolution Powder Diffractometer at the SERC Daresbury Laboratory. The IRF curves for flat samples have been discussed for slightly different wavelengths, $1.5405981 \AA$ and $1.4188 \AA$. For the conventional diffractometer, the IRF has a minimum of $0.065^{\circ}(2 \theta)$ at about $30^{\circ}$ and has twice this value at $130^{\circ}(2 \theta)$; a minimum of $0.028^{\circ}(2 \theta)$ is obtained at $30^{\circ}$ and twice this value at $100^{\circ}(2 \theta)$ for the 9.1 HRPD. In both cases, instrumental line profiles can be modelled, to a close approximation, by a single analytical function. The benefit of high resolution can be seen in figure 4 , giving a limited range of patterns registered with the two instruments, for a triclinic zirconium hydroxide nitrate. Partly resolved peaks, frequently vital for successful indexing, are more easily detected with higher resolution, and the separation of close lines, by fitting techniques, becomes more precise. Part of this precision is lost if the $\mathrm{K} \alpha_{1} \alpha_{2}$ doublet is employed.

\section{(ii) Accuracy}

Random errors are minimized by using fitting methods for locating peak positions, but systematic errors need to be reduced by a careful alignment (or calibration) of the instrument. Because data for indexing collected by means of a powder diffractometer are often regarded with suspicion due to zero-point errors, it is of interest to present some results that can be obtained with a Bragg-Brentano geometry and monochromatic radiation, whose features are given elsewhere (Louër, 1991). Before collecting data, two kinds of control are recommended:

- The zero- $2 \theta$ position, which must be close to the true zero, can be established by means of parallel glass-plates located at the centre of the goniometer rotation axis. In general, the value of the zero error can be less than $0.005^{\circ}(2 \theta)$.

- The accuracy of the angular position of the entire goniometer circle must be evaluated. Indeed, the biggest sources of experimental error are due to specimen displacement and, to a considerably less extent with modern diffractometers, misset- 

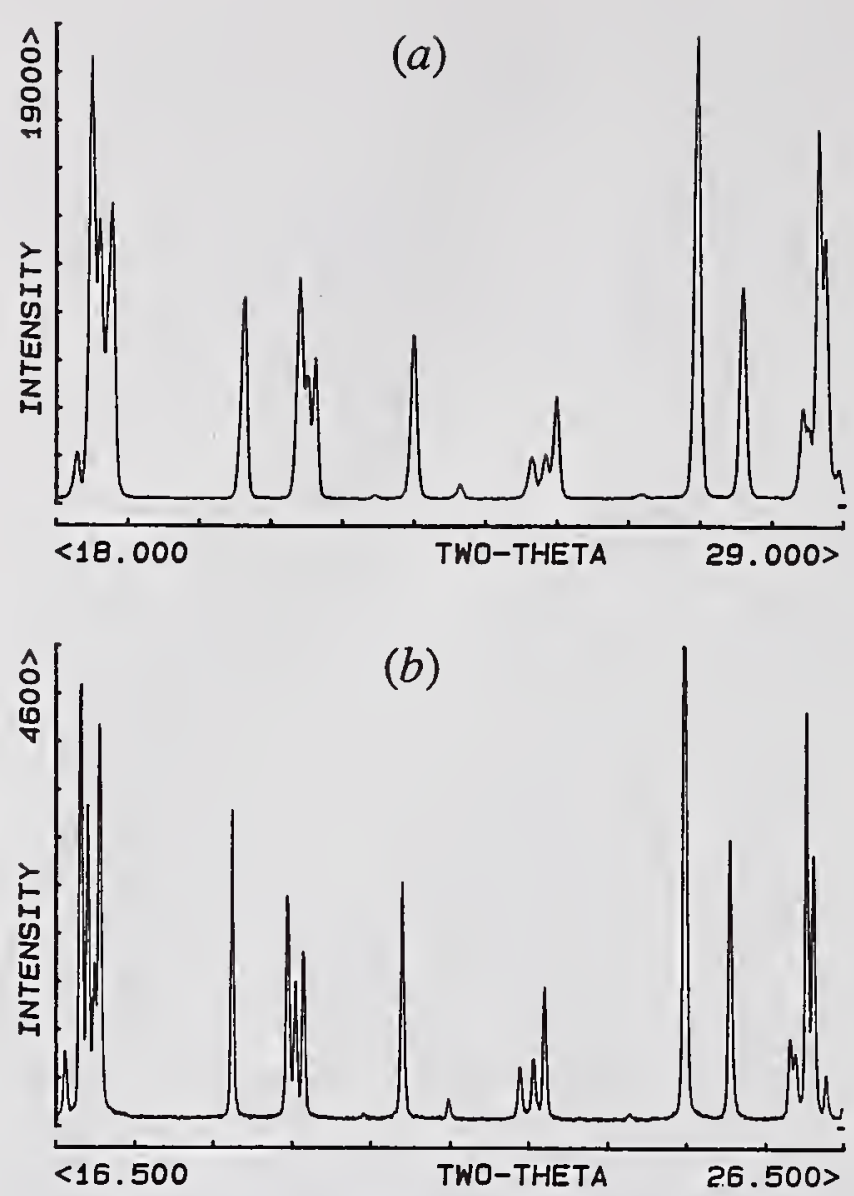

Figure 4: Partial angular ranges of the powder diffraction pattern of zirconium hydroxide nitrate obtained by means of high-resolution powder diffractometers using $(a)$ a conventional X-ray source with monochromatic radiation $(\lambda=1.5405981 \AA)$ and $(b)$ synchrotron radiation $(\lambda=1.4188 \AA)$.

ting of the $\theta / 2 \theta$ adjustment. This can be easily evaluated by using standard reference materials; for this purpose highly oriented crystallites of a layer-type material, deposited in a thin layer on a silicon plate to reduce transparency effects, can be used for checking the adjustment of the diffractometer. For example, the synthetic fluorophlogopite mica (SRM 675: Natl. Bur. Stand., 1982) is suitable for diffractometry by reflection; the basal spacing is $\mathrm{d}(001)=9.98104 \pm 0.00007 \AA$. With a high degree of preferred orientation of crystallites, ten $00 l$ reflections are observed in the angular range $8-135^{\circ} 2 \theta$ for $\lambda=1.54059$ $\AA$. Each diffraction line must produce an identical value of the interlayer spacing $\mathrm{d}(001)=l \times \mathrm{d}(00 l)$. An example of calibration is shown in figure 5 , where the angular discrepancies between observed and calculated $2 \theta$ angles are given for ten diffraction lines. It demonstrates that the angular er-

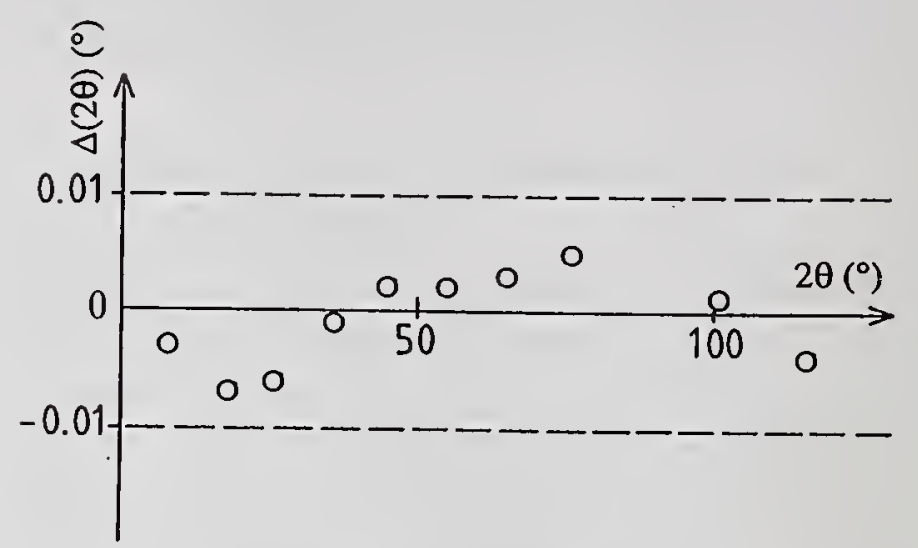

Figure 5: $\Delta 2 \theta\left({ }^{\circ}\right)$ discrepancies between $2 \theta$ angles calculated and observed peak positions for $00 l$ reflections of SRM 675.

ror on peak positions is less than $0.007^{\circ}(2 \theta)$ over the entire goniometer circle. At low angles, axial divergence can produce asymmetry and a shift of lines; this effect is reduced by using Soller slits located in the diffracted beam. The use of high orders of a reflection (if available) can be used for a correction of the positions of low-angle peaks. This practice can be useful when methods are very sensitive to the precision of low-angle lines.

With a correct adjustment of the diffractometer, there is no need to use an internal standard material. Of course, the front surface of the sample has to be very smooth and attention has to be paid to the thickness of the sample in case of materials with only light atoms. In current practice, the absolute error on peak positions can be less than $0.01^{\circ}(2 \theta)$ for well crystallized materials.

(iii) Preparation of data for indexing programs In modern powder diffraction it is a current practice to use fitting techniques to decompose the pattern into Bragg components. Some efficient programs are commercially available and can be used with PCs. In particular, a zoom option is useful to examine low intensity lines. The preparation of data should include the following information for every Bragg component: peak position, relative intensity, FWHM and an 'aspect' indicator (e.g. asymmetry, shoulder, etc.). 
Table 1: $M_{20}$ and $\mathrm{F}_{20}$ figures of merit for indexed powder diffraction patterns, by DICVOL91, from data with different origins : photographic recording $(a)$, conventional diffractometer with monochromatic radiation $(\lambda=1.54059 \AA)(b)$ and $9.1 \operatorname{HRPD}(\lambda=1.40394 \AA)(c)$.

\begin{tabular}{|c|c|c|c|c|c|}
\hline Compound & Reference & Symmetry & Measure type & $M_{20}$ & $\mathrm{~F}_{20}$ \\
\hline$\alpha-\mathrm{Cs}_{2} \mathrm{CdI}_{4}$ & TLL & monoclinic & $a$ & 23 & $39(0.011,46)$ \\
\hline$\beta-\mathrm{Cs}_{2} \mathrm{CdI}_{4}$ & TLL & orthorhombic & $a$ & 20 & $37(0.009,60)$ \\
\hline $\mathrm{Nd}(\mathrm{OH})_{2} \mathrm{NO}_{3} \cdot \mathrm{H}_{2} \mathrm{O}$ & LDHG & monoclinic & $a$ & 34 & $48(0.008,51)$ \\
\hline \multirow{3}{*}{$\mathrm{KCaPO}_{4} \cdot \mathrm{H}_{2} \mathrm{O}$} & LDO & monoclinic & $a$ & 19 & $28(0.012,58)$ \\
\hline & CL & & $b$ & 73 & $107(0.0038,49)$ \\
\hline & CL & & $c$ & 156 & $260(0.0016,47)$ \\
\hline \multirow[t]{2}{*}{$\mathrm{La}(\mathrm{OH})_{2} \mathrm{NO}_{3} \cdot \mathrm{H}_{2} \mathrm{O}$} & LLLG & monoclinic & $b$ & 44 & $67(0.0061,49)$ \\
\hline & CL & & $c$ & 86 & $129(0.0032,49)$ \\
\hline $\mathrm{Cd}_{5}(\mathrm{OH})_{8}\left(\mathrm{NO}_{3}\right)_{2} \cdot 2 \mathrm{H}_{2} \mathrm{O}$ & BL & monoclinic & $b$ & 82 & $151(0.0050,25)$ \\
\hline \multirow[t]{2}{*}{$\mathrm{Ba}(\mathrm{TiO})\left(\mathrm{C}_{2} \mathrm{O}_{4}\right)_{2} \cdot 4 \mathrm{H}_{2} \mathrm{O}$} & LBGC & monoclinic & $b$ & 51 & $173(0.0036,32)$ \\
\hline & CL & & $c$ & 76 & $248(0.0025,32)$ \\
\hline \multirow[t]{2}{*}{$\mathrm{Zr}(\mathrm{OH})_{2}\left(\mathrm{NO}_{3}\right)_{2} \cdot 4 \mathrm{H}_{2} \mathrm{O}$} & BLL & triclinic & $b$ & 54 & $112(0.0059,30)$ \\
\hline & $\mathrm{CL}$ & & $c$ & 295 & $635(0.0012,26)$ \\
\hline \multirow[t]{2}{*}{$\mathrm{PbF}_{2}$} & $\mathrm{CL}$ & orthorhombic & $b$ & 105 & $113(0.0052,34)$ \\
\hline & CL & & $c$ & 699 & $821(0.0006,34)$ \\
\hline
\end{tabular}

TLL: Touchard, Louër \& Louër, (1986); LDHG: Louër, Deneuve, Herviou \& Gourlaouen, (1986); LDO: Louër, Deneuve \& Ouillon, (1987); CL: Cernik \& Louër, (1993); LLLG: Louër, Louër, Lopez Delgado \& Garcia Martinez, (1989); BL: Bénard \& Louër, (1990); LBGC: Louër, Boultif, Gotor \& Criado, (1990); BLL: Bénard, Louër \& Louër, (1991).

In a first indexing attempt, the first twenty lines, not including (less precise) lines with very low intensities, should be used as input data. In a second run the lines with low intensity must be incorporated in input data. If the same solution is obtained, then assurance that the indexing is correct is increased. This strategy can be slightly different if programs tolerate spurious lines.

(iv) Precision of indexing solutions

The fundamental stage for indexing a pattern is the measurement of peak positions. As explained previously, the accuracy is related to the resolution of the instrument, its adjustment, the degree of diffraction line overlap, and the technique used for measuring the peak positions. A few years ago, graphic recording of powder patterns was frequently used, and angular positions were taken as the maximum intensities observed. These measures (type a) are obviously biased in case of line overlap. In modern powder crystallography, step scanning data collection combined with fitting methods gives better estimates of the peak positions. However, the accuracy still depends on the instrument resolu- tion function. In table 1 some indexing results obtained from experimental measures of type $a$ are compared with those obtained by means of a fitting technique applied to data collected with a conventional diffractometer with monochromatic radiation (type $b$ ) and with data collected by means of 9.1 HRPD (type $c$ ). Figures of merit are significantly greater for data obtained by fitting procedures. The mean of $\Delta 2 \theta$ is $0.01^{\circ}$, $0.005^{\circ}$ and $0.0018^{\circ}(2 \theta)$ for measures of type $a$, $b$ and $c$, respectively. These results show the advantage of using synchrotron radiation for $a b$ initio pattern indexing. However, data collected by means of diffractometers with conventional sources and monochromatic radiation lead to excellent results; compared with synchrotron data, the precision of the indexing is lowered by a factor varying from 2 to 7 .

\section{Reliability of indexing solutions}

Although the success rate of computer-based indexing programs is very high, to be accepted with a higher degree of assurance the proposed (mathematical) solutions must satisfy several conditions, which can be summarized as follows: 
- High values of the figures of merit $M_{N}$ and $\mathrm{F}_{N}$ (values of $M_{20}$ greater than 20 are a good indication).

- Since the calculations are usually based on the first twenty lines, all remaining diffraction lines in the pattern must also be indexed, within the error bounds. For this purpose, it is convenient to review the complete observed dataset by means of the computer program NBS*AIDS83 (Mighell, Hubbard \& Stalick, 1981). A benefit of this careful review is an easier derivation of the possible space groups.

- If the density of the material has been measured accurately, an integral number of chemical formula units per unit cell should be obtained.

- If necessary, the use of programs, e. g. CDF$\mathrm{SRCH}$, can be employed to find the normalized unit cell parameters. A subsequent advantage of this analysis is the possibility of interrogating the NIST Crystal Data Base (or another database) to search for apparently isostructural chemically-related materials.

- Finally, due to recent advances in ab initio structure determination, a successful determination of the crystal structure of the material would be an elegant check on the validity of the cell obtained by indexing.

\section{Discussion of particular cases}

The presence of a dominant zone in a pattern introduces a complementary degree of freedom, which can cause problems, with the consequence of possible pseudo-solutions with high figures of merit. Also, the indexing of unit cells with large volumes has always been regarded with suspicion, although there is no mathematical limit for an indexing provided that the information contained in the pattern is sufficiently complete and accurate. A few examples are now discussed.

\section{Cases with a short dimension}

The dominant zone is due to one of the cell edges being significantly shorter than the other two, as can occur for materials with a layer-type structure. The pattern is then characterized by a zero value for one common Miller index for all the first lines of the pattern. This feature can be a problem for methods using base lines for generating solutions and requires
Table 2: Examples of patterns with a dominant zone, indexed by DICVOL91; $N$ : number of successive low-angle diffraction lines having a common zero index.

\begin{tabular}{|lcr|}
\hline Compound & Reference & $N$ \\
\hline $\mathrm{La}(\mathrm{OH})_{2} \mathrm{NO}_{3} \cdot \mathrm{H}_{2} \mathrm{O}$ & LLLG & 6 lines with $k=0$ \\
$\mathrm{Nd}(\mathrm{OH})_{2} \mathrm{NO}_{3} \cdot \mathrm{H}_{2} \mathrm{O}$ & LDHG & 7 lines with $k=0$ \\
$\mathrm{Cd}_{3}(\mathrm{OH})_{5} \mathrm{NO}_{3}$ & $\mathrm{APL}$ & 8 lines with $h=0$ \\
$\mathrm{Zn}(\mathrm{OH}) \mathrm{NO}_{3} \cdot \mathrm{H}_{2} \mathrm{O}$ & $\mathrm{ELW}$ & 13 lines with $k=0$ \\
\hline
\end{tabular}

LLLG : Louër, Louër, Lopez Delgado \& Garcia Martinez, 1989 ; LDHG : Louër, Deneuve, Herviou \& Gourlaouen, 1986 ; APL : Auffrédic, Plévert \& Louër, 1990 ; ELW : Eriksson, Louër \& Werner, 1989.

further consideration; in order to solve this problem effectively, special algorithms for finding possible dominant zones must be incorporated in these approaches (Werner et al., 1985). The successive dichotomy method is, by definition, not sensitive to this situation, unless the number of lines with a common zero-index is exceptionally high. Examples of patterns indexed by DICVOL91 are shown in table 2. The last example, $\mathrm{Zn}(\mathrm{OH})\left(\mathrm{NO}_{3}\right) \cdot \mathrm{H}_{2} \mathrm{O}$ contains 13 first lines with $k=0$, which caused some instability, as is shown by the three similar solutions $\left[694 \AA^{3}\right.$, $\left.M_{20}=17 ; 728 \AA^{3}, M_{20}=16 ; 757 \AA^{3}, M_{20}=18\right]$ obtained from the 20 first lines and an absolute angular error of $0.03^{\circ}(2 \theta)$. To select the correct solution, more lines are necessary, or it is better to review the complete observed dataset by the program NBS*AIDS83, which reveals that only the last is correct. Moreover, since these data, collected by means of a Guinier-Hägg camera, are accurate, the reduction of the error bounds from 0.03 to $0.01^{\circ}(2 \theta)$ leads only to the correct solution. This again demontrates again the importance of accuracy as a stabilising parameter in indexing problems.

\section{Cases with a large unit-cell volume}

If enough crystallographic information is present in the powder diffraction pattern, high instrument resolution, possibly coupled with a judiciously selected wavelength, is required to solve the problem of high line density at low angles. For very high volumes (e.g. greater than $4000 \AA^{3}$ ), synchrotron facilities (resolution, accuracy and tunability of the radiation) are a decided advantage. However, examples using data collected with conventional instruments, have been reported. Among them are the indexing of the patterns of monoclinic halotrycite [V = $3152 \AA^{3}, M_{20}=15, \mathrm{~F}_{20}=35(0.006,98) ;$ Werner, 
1980] and barium titanyl oxalate hydrate (BTO) $\left[\mathrm{V}=2595 \AA^{3}, M_{20}=46, \mathrm{~F}_{20}=107(0.0056,50)\right.$; Louër et al., 1990]. The benefit of using synchrotron radiation for the indexing of patterns of materials with large unit cell volumes can be appreciated with the powder pattern indexing of the zeolitic material Sigma-2 $(\lambda=1.5468 \AA)\left[\mathrm{V}=3604 \AA^{3}, \mathrm{Mc}\right.$ Cusker, (1988)]. For testing the successive dichotomy method, data for two powder patterns, collected on the 9.1 HRPD, were entered in DICVOL91. The first sample was the zeolite ZSM-5 $(\lambda=1.4963 \AA)$, and the solution found is orthorhombic $[a=20.096(6) \AA$, $b=19.955(5) \AA, c=13.433(4) \AA, \mathrm{V}=5384 \AA^{3}$, $\left.M_{20}=24, F_{20}=69(0.0060,48)\right]$, in accordance with a single-crystal study (Olson, Kokotailo, Lawton \& Meier, 1981). The second sample was a synthetic gallophosphate with very large unit cell volume studied by Estermann, McCusker, Baerlocher, Merrouche \& Kessler (1991); data were collected with the wavelength $\lambda=1.70469 \AA$ to have better resolution and accuracy at low angles. From the first 28 reflections input to DICVOL91, a cubic solution was found $\left[a=51.715(2) \AA, \mathrm{V}=138309 \AA^{3}, M_{28}=32\right.$, $\left.\mathrm{F}_{28}=105(0.0013,212)\right]$, in agreement with the reported results.

\section{Conclusion}

Computer-based methods are nowadays powerful for indexing powder diffraction patterns, whatever the crystal symmetry, provided that data are accurate. Recent developments in high-resolution diffractometers using conventional or synchrotron sources offer the possibility of obtaining the required precision routinely. This represents important progress, since the indexing of a powder pattern is required in most applications of powder diffraction. It has become a method for the unique characterization of a single phase, and reported data for a pure material with an unknown structure should always be as precise as possible and analysed by indexing methods, unless a good reason is given; from such a practice the quality of databases would be increased considerably. Recent applications in powder diffraction demonstrate the fundamental rôle of indexing a powder pattern. For example, its power in materials characterization can be seen by the indexing of the pattern of baryum titanyl oxalate hydrate, used as a precursor in the synthesis of high-purity barium titanate. The pattern was long described as complicated and used extensively only for qualitative identification. From accurate data, the pattern was easily indexed with the cell given in Table 1 , showing that this material was in fact a pure phase. Another recent spectacular consequence of powder pattern indexing is the rapid development of $a b$ initio structure determination from powder data. The reconstruction of the reciprocal lattice by means of indexing methods is a crucial and inevitable stage for a subsequent structure analysis, from which the structural chemistry of materials will benefit greatly in the near future.

The author wishes to thank Prof. P.-E. Werner and Dr. J. I. Langford for their many suggestions and critical reading of the manuscript, Dr. C. Baerlocher, who provided the synchrotron powder diffraction data for the two examples with large unit-cell volume, and Dr. R. J. Cernik, who collected synchrotron data for a few samples.

\section{References}

Auffrédic, J. P., Plevert, J. \& Loü̈r, D. (1990). J. Solid State Chem. 84 58-70.

BÉnARd, P. \& Loü̈r, D. (1990). Powder Diffr. 5, 106108.

BÉnARD, P., LouËr, M. \& LouËR, D. (1991). J. Solid State Chem. 94, 27-35.

Boultif, A. \& Loü̈R, D. (1991). J. Appl. Cryst. 24, 987-993.

Cernik, R. J. \& LouËr, D. (1993). J. Appl. Cryst. In press.

Eriksson, L., LouËr, D. \& Werner, P.-E. (1989). J. Solid State Chem. 81, 9-20.

EstermanN, M., McCusker, L. B., BaErlocher, C., Merrouche, A. \& Kessler, H. (1991). Nature (London) 352, 320-323.

Iтo, T. (1949). Nature (London), 164, 755-756.

KoнLвEсK, F. \& HörL, E. M. (1976). J. Appl. Cryst. 9, 28- 33 .

KoHLBEcK, F. \& HörL, E. M. (1978). J. Appl. Cryst. 11, 60-61.

LABARre, J., Loü̈r, D., Loü̈r, M. \& Grandjean, D. (1976a). Acta Cryst. B32, 3250-3253.

LABARre, J., LouËr, D., Loü̈r, M. \& GRANDJEAN, D. $(1976 b)$. Acta Cryst. B32, 3253-3257.

Langford, J. I., CerntK, R. J. \& LouËR, D. (1991). J. Appl. Cryst. 24, 913-919.

Loü̈R, D. (1991). Materials Science Forum, 79-82, 17 26.

Loü̈r, D., Boultif, A., Gotor, F. J. \& Criado, J. M. (1990). Powder Diffr. 5, 162-164.

Loü̈r, D., Deneuve, F., Herviou, C. \& Gourla ouen, C. (1986). Powder Diffr. 1, 263-264.

Loü̈r, D., Deneuve, F. \& Oulllon, N. (1987). Powder Diffr. 2, 253-254.

Loü̈r, D., Labarre, J., Auffrédic, J. P. \& LouËr, M. (1982). Acta Cryst. B28, 1079-1084.

Loü̈r, D. \& Langford, J. I. (1988). J. Appl. Cryst. $21,430-437$. 
LOUËR, D. \& LOUËR, M. (1972). J. Appl. Cryst. 5, 271275.

Loü̈r, D. \& VARGAS, R. (1982). J. Appl. Cryst. 15, $542-545$.

Loü̈r, M., LouËR, D. \& GRANDJEAN, D. (1982). Acta Cryst. B38, 909-912.

LoUËr, M., LoUËr, D., Lopez Delgada, A. \& Garcia Martinez, O. (1989). Eur. J. Solid St. Inorg. Chem. 26, 241-253.

McCusker, L. B. (1988). J. Appl. Cryst. 21, 305-310.

Mighell, A. D., Hubbard, C. R. \& Stalick, J. K. (1981). NBS*AIDS80: a Fortran Program for Crystallographic Data Evaluation. Natl. Bur. Stand. (U. S.). Tech. Note No. 1141. (NBS*AIDS83 is an expanded version of NBS*AIDS80.)

Mighell, A. D. \& Santoro, A. (1975). J. Appl. Cryst. 8, 372-374.

Natl. Bur. Stand. (U. S.) (1980). Monogr. No. 25, §17, pp. 5-86.

Natl. Bur. Stand. (U. S.) (1982). SRM 675, Gaithersburg, MD (USA).

Olson, D. H., Kokotallo, G. T., Lawton, S. L. \& Meier, W. M. (1981). J. Phys. Chem. 85, 2238-2243.

RUNGe, C. (1917). Phys. Z. 18, 509-515.

SHIRLEY, R. (1978). In Computing in Crystallography, edited by H. Schenk, R. Olthof-Hazekamp, H. van Koningsveld \& G. C. Bassi, pp 221-234, Delft Univ. Press.

SHIRLEY, R. (1980). In Accuracy in Powder Diffraction, edited by S. Block \& C. R. Hubbard. Natl. Bur. Stand. (U. S.) Spec. Publ. No 567, pp. 361-382.
SмITH, G. S. (1977). J. Appl. Cryst. 10, 252-255.

Smith, G. S. \& Kahara, E. (1975). J. Appl. Cryst. 8, 681-683.

Smith, G. S. \& SNYder, R. L. (1979). J. Appl. Cryst. $12,60-65$.

TAUPIN, D. (1973). J. Appl. Cryst. 6, 380-385.

TAuPIN, D. (1988). J. Appl. Cryst. 21, 485-489.

TAupIN, D. (1989). J. Appl. Cryst. 22, 455-459.

TOUCHARD, V., LouËr, M. \& LouËr, D. (1986). Powder Diffr. 1, 35-36.

VisSER, J. W. (1969). J. Appl. Cryst. 2, 89-95.

WALter-Levy, L., Groult, D. \& Visser, J. W. (1974). Bull. Soc. Chim. Fr. 1-2, 67-71.

Werner, P.-E. (1964). Z. Krist. 120, 375-387.

Werner, P.-E. (1976). J. Appl. Cryst. 9, 216-219.

Werner, P.-E. (1980). In Accuracy in Powder Diffraction, edited by S. Block \& C. R. Hubbard. Natl. Bur. Stand. (U. S.) Spec. Publ. No 567, pp. 503-509.

Werner, P.-E., ERIKSSON, L. \& WEstdahL, M. (1985). J. Appl. Cryst. 18, 367-370.

Wrson, A. J. C. (1963). Mathematical Theory of X-ray Powder Diffractometry, Eindhoven: Centrex.

WolfF, P. M. DE (1957). Acta Cryst. 10, 590-595.

WolfF, P. M. DE (1961). Acta Cryst. 14, 579-582.

WolfF, P. M. DE (1968). J. Appl. Cryst. 1, 108-113.

WU, E. (1989). J. Appl. Cryst. 22, 506-510. 
National Institute of Standards and Technology Special Publication 846. Proceedings of the international conference Accuracy in Powder Diffraction II, held at NIST, Gaithersburg, MD, May 26-29, 1992. (Issued October 1992)

\title{
Accuracy in Pair Distribution Function Analysis for Crystalline and Non-Crystalline Materials
}

\author{
B. H. TOBY ${ }^{1}$ AND T. EgAMI ${ }^{2}$ \\ ${ }^{1}$ Air Products and Chemicals, Inc., 7201 Hamilton Boulevard, Allentown, PA 18195, U. S. A \\ and \\ ${ }^{2}$ Department of Materials Science and Engineering and \\ Laboratory for Research on the Structure of Matter, \\ University of Pennsylvania, Philadelphia, PA 19104-6272, U. S. A.
}

\begin{abstract}
Pair distribution function (PDF) analysis of neutron and $\mathrm{x}$-ray powder diffraction data is useful for analysis of short-range structure in both amorphous and crystalline materials. The sources for errors in PDF determinations are explored. The significant error sources: termination of the Fourier transform, inability of the instrument to completely resolve the diffraction pattern, inaccurate corrections for experimental artifacts and counting statistics are discussed in detail. Methods for estimating these errors are presented. With care, PDF's can be computed very accurately, as demonstrated by the excellent agreement shown for a standard sample.
\end{abstract}

\section{Introduction}

The atomic Pair Distribution Function (PDF) has been traditionally used for structural studies of noncrystalline materials, where crystallographic analysis cannot be applied (for example see Cargill, 1975, or Wagner, 1972). More recently, analysis of PDF's obtained for crystalline materials has demonstrated features not reflected in the crystal structure (for example see Dmowski et al., 1988, or Toby et al., 1990). While much attention has been applied to understanding the accuracy and precision of the crystallographic results, relatively little attention has been given to understanding and evaluating the precision and accuracy of PDF analysis.

The PDF is determined by direct Fourier transformation of neutron or x-ray powder diffraction data, after application of corrections and shows the probability of finding a pair of atoms at a given interatomic distance (Warren, 1968; Klug and Alexander, 1968). The PDF is similar to a spherically averaged Patterson map. However, for Patterson analysis only Bragg scattering is used, while the PDF also includes diffuse scattering. Thus, the PDF is sensitive to local order while the Patterson map, and indeed all techniques which only utilize Bragg scattering, can only reflect the long-range symmetry in a material.

It is commonly and erroneously thought that the PDF is an approximate technique and the PDF is incapable of observing structure beyond the first few coordination spheres. The PDF of polycrystalline aluminum shown in figure 1 refutes that belief. In this figure, the experimental result is superimposed on the expected result computed from the known crystal structure. It should be noted that the observed and computed PDF's are in excellent agreement and the PDF clearly shows structural features out to at least $40 \AA$. This paper will quantify the factors that limit the accuracy of the PDF and will also show how to obtain the minimum statistical error for a given data collection time by a strategic choice of the scan rate.

\section{Background}

The intensity for scattering of a probe particle usually written as $I(Q, \omega)$ where $\vec{Q}$ is the momentum transfer vector, $\vec{Q}=\vec{k}_{f}-\vec{k}_{i}$, and $\omega$ is the energy transfer, $\omega=\omega_{i}-\omega_{f}$, (subscripts $i$ and $f$ denote the incident and final momentum or energy, respectively). If diffracted intensity is detected without energy discrimination but at fixed $Q, I(Q, \omega)$ will be integrated over all $\omega$. This yields a PDF indicative of the instantaneous correlations of atomic positions.

$$
I(\vec{Q})=\frac{1}{N} \sum_{j, k} b_{j} b_{k}\left\langle\left\langle\exp \left[i \vec{Q} \cdot\left[\vec{r}_{j}(t)-\vec{r}_{k}(t)\right]\right]\right\rangle\right\rangle
$$

where double angle brackets $(\langle\langle\ldots\rangle\rangle)$ indicate a time average, single angle brackets indicate a composi- 
a)

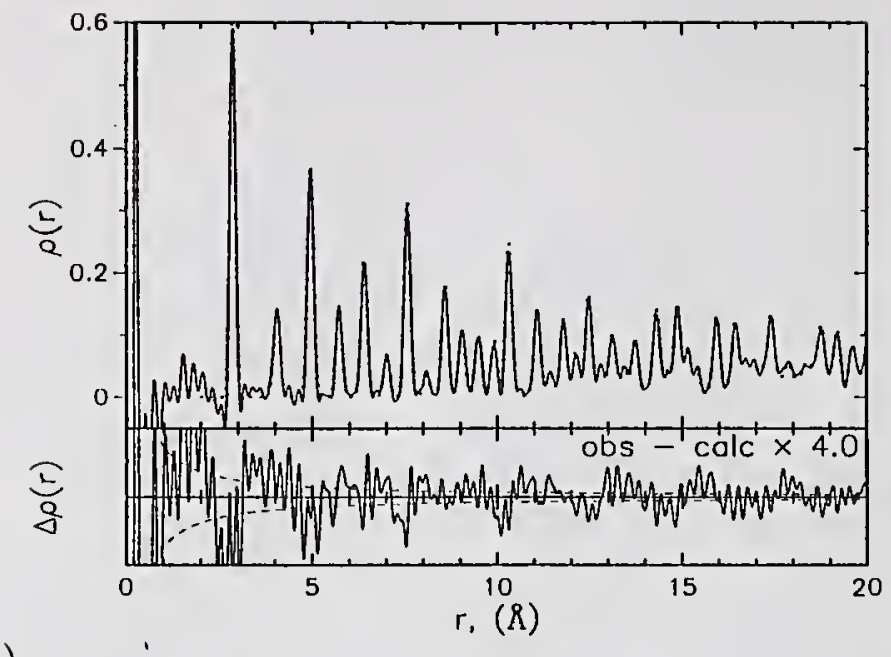

b)

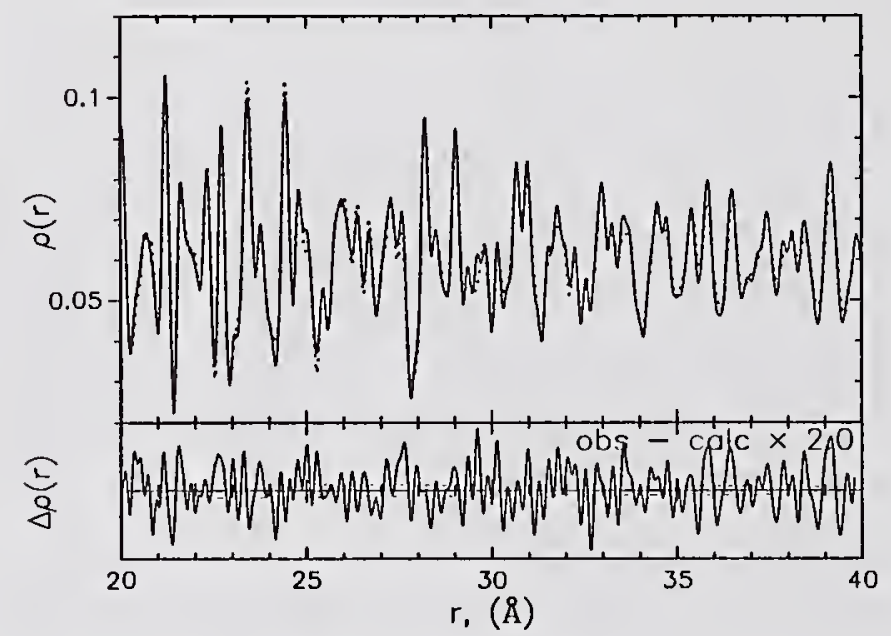

Figure 1: An observed (solid line) and model (dotted line) pair distribution function for polycrystalline $\mathrm{Al}$ powder measured at $50 \mathrm{~K}$ over the range $0-20 \AA$ (a) and 20-40 $\AA(b)$. The lower box shows the deviation between the observed and calculated results (solid line) and plus and minus one estimated standard deviation computed from the counting statistics (dashed line). The lower box contents are magnified $4: 1$ for $(a)$ and $2: 1$ for $(b)$.

tional average, $b_{j}$ is the scattering length for the $j$ th atom, and the summation is performed for all pairs of atoms in the sample (Lovesey, 1984; Warren, 1969; also see Toby and Egami, 1992 for a more complete discussion). It should be noted for detection at fixed $2 \theta, \vec{Q}$ will vary with $\omega$, since Bragg's law, $Q=|\vec{Q}|=4 \pi \sin \theta / \lambda$, is true only for elastic scattering. Thus in practice, data collected without energy discrimination will not directly measure $I(\vec{Q})$ since $Q$ is not constant as $\omega$ changes for constant $\theta$. The Placzek approximation is customarily applied to correct for this shift in $Q$ with $\omega$ (Placzek,
1952). In addition, intensity contributions from multiple scattering and attenuation due to absorption, which depend on the experiment geometry, have been neglected here. Corrections for these factors as well as polarization, background and scaling for the flux incident on the sample must be applied to the observed intensities.

For powder diffraction, a sample composed of a large number of randomly oriented crystallites effectively averages the measurement over all orientations of $\vec{Q}$. The powder-averaged scattering intensity is usually defined as the powder-averaged structure factor, $S(Q)$,

$$
S(Q)=\frac{I(Q)}{\langle b\rangle^{2}}-\frac{\left\langle b^{2}\right\rangle-\langle b\rangle^{2}}{\langle b\rangle^{2}} .
$$

Note that the definition of the structure factor used here differs from the definition for the structure factor, $F_{h k l}$, used in crystallography. For an ideal crystal, $S(Q)$ can be expressed in terms of the crystallographic structure factor, $F_{h k l}$,

$$
S(Q)=\frac{2 \pi^{2}}{v_{c}} \frac{1}{n_{c}\left\langle b^{2}\right\rangle Q^{2} \delta Q} \sum_{Q}^{Q+\delta Q} F_{h k l}^{2}
$$

where the sum is over all reflections with momentum transfer between $Q$ and $Q+\delta Q\left[\vec{Q}=2 \pi\left(h a^{*}+k b^{*}+\right.\right.$ $\left.\left.l c^{*}\right)\right], v_{c}$ is the unit cell volume, $n_{c}$ is the number of atoms in the unit cell.

The PDF, $\rho(r)$, for neutron diffraction is defined for the instantaneous structure as

$$
\rho(\vec{r})=\frac{1}{N} \sum_{j \neq k} \frac{b_{j} b_{k}}{\langle b\rangle^{2}}\left\langle\left\langle\delta\left(\vec{r}-\left[\vec{r}_{j}(t)-\vec{r}_{k}(t)\right]\right)\right\rangle\right\rangle .
$$

The PDF may be obtained from the powder-averaged $S(Q)$ using

$$
\rho(r)-\rho_{0}=\frac{1}{2 \pi^{2} r} \int Q[S(Q)-1] \sin Q r d Q .
$$

The equations given above are strictly accurate for neutron diffraction, but are approximate for x-ray diffraction where the scattering factor, $f_{j}(\theta)$, is substituted for the neutron scattering length, $b_{j}$ (Warren et al., 1936).

The PDF for a collection of atoms may be simulated by counting the interatomic distances between every possible pair of atoms, weighted by $b_{j}$ for each atom. This delta-function PDF is then broadened to simulate thermal motion. For comparison between observed and simulated PDF's, an agreement factor, $A$, is defined in a manner analogous to Rietveld analysis,

$$
A^{2}=\frac{1}{n \rho_{0}^{2}} \sum_{i=1}^{n}\left[\rho_{o b s}\left(r_{i}\right)-\rho_{\text {model }}\left(r_{i}\right)\right]^{2} .
$$


Agreement factors in the range of 0.05 to 0.1 represent very good fits with only minor discrepancies visible to the eye. The agreement between the observed and simulated PDF's given in Fig. 1 are 0.156 and 0.045 over the ranges $2-20 \AA$ and $20-40 \AA$, respectively.

\section{Error sources in the PDF measurement}

All diffraction measurements are subject to random statistical errors. The following section will demonstrate how to estimate these errors. In addition there are three obvious sources for systematic errors in the PDF: (1) termination of the Fourier integration at a finite value of $Q,(2)$ instrumental broadening of the diffractogram, (3) inaccuracies in the experimental corrections applied to the diffraction data. These factors will also be discussed below.

\section{Counting Statistics}

All diffraction measurements either directly or indirectly count quanta and are subject to statistical fluctuations where the estimated standard deviation (e.s.d.) is proportional to the square- root of the number of quanta counted. To determine the e.s.d. for the observed PDF, one can propagate errors from the intensity measurements to the computed PDF values.

The estimated errors for a set of observations may be propagated to estimate the error on a function determined from these observables. In general form, the e.s.d. for a function, $f$, evaluated using a set of independent observations, $O_{1}, O_{2}, \ldots O_{N}$, can be estimated from the e.s.d. of each of the observations using,

$$
\sigma_{f}^{2}=\sum_{j}^{N}\left[\sigma_{O_{j}} \frac{\partial f}{\partial O_{j}}\right]^{2}
$$

(Hamilton, 1964). If the $S(Q)$ values are computed independently, e.g., no observation is used to compute more than one $S(Q)$ value, then the $S(Q)$ values may be treated as an observable. The e.s.d. for the $S(Q)$ values, $\sigma_{S(Q)}$, is then,

$$
\sigma_{S\left(Q_{k}\right)}^{2}=\sum_{j}^{M} \sigma_{O_{j}}^{2}\left(\frac{\partial S\left(Q_{k}\right)}{\partial O_{j}}\right)^{2}
$$

where $M$ independent intensity measurements for sample, background, container scattering, etc., are averaged for each $S(Q)$ value. Note that smoothing removes that independence of $S(Q)$ values and should be avoided. The e.s.d. for $\rho(\mathrm{r})$ may be then computed from

$$
\sigma_{\rho(r)}^{2}=\frac{1}{\left(2 \pi^{2} r\right)^{2}} \sum_{k}\left[\sigma_{S\left(Q_{k}\right)} Q_{k} \Delta Q_{k} \sin Q_{k} r\right]^{2} .
$$

The dashed lines in the lower box of Fig. 1 show the e.s.d. for the observed PDF. These small e.s.d.'s were obtained for only two hours of data collection time for a relatively weak scatterer of neutrons using the Argonne National Laboratory SEPD instrument when the IPNS enriched uranium target was in use.

\section{Systematic Errors}

To quantify the effects of termination and instrumental broadening on the PDF, the ideal total structure factor, $S(Q)$, was computed for a FCC metal and an ideal PDF was then computed from this $S(Q)$. Experimental effects were then simulated by appropriately modifying $S(Q)$ and repeating the PDF computation.

Termination of the Fourier integral at finite $Q$ is equivalent to multiplying $S(Q)$ by a step function. This is equivalent to convoluting the PDF with the Fourier transform of the step function. Indeed, a damping function is commonly applied so that the convolution function appears more Gaussian. Thus, the degree that termination will introduce error into the PDF depends on two factors, the amount of thermal motion (including zero-point vibration) and the intrinsic sharpness of the PDF peaks due to the range of interatomic distances in the material. The effect of termination on the simulated PDF was studied as a function of termination and thermal motion conditions, and the results are shown in figure 2. The smallest values shown for the thermal amplitude, 0.05 and $0.06 \AA$, which are equivalent to isotropic temperature factors, $\mathrm{B}$, of 0.1 and $0.14 \AA^{2}$, respectively, correspond to the estimated zero-point vibration for $\mathrm{Ni}$ and $\mathrm{Cu}$, respectively (International Tables, 1968). The remaining three $\sigma$ values, 0.084 , 0.100 and $0.153 \AA$ ( $\mathrm{B}=0.28,0.39$ and $0.92 \AA^{2}$ ), correspond to the estimated vibration amplitudes for crystalline aluminum at $\mathrm{T}=0,100$ and $295 \mathrm{~K}$ (Ibid.). Except at very low temperatures, most materials exhibit thermal vibration greater than that for $\mathrm{Al}$ at 0 $\mathrm{K}$, so termination at $30 \AA^{-1}$ will be sufficient for accurate determination of the PDF. It should be noted that these values represent upper limits for termination errors. Most crystalline materials and particularly amorphous and quasicrystalline materials tend to have much broader features in their PDF's than FCC metals and their truncation errors are less severe.

The broadening of peaks due to lack of instrumental resolution can seriously limit the usefulness 
Termination Effect on PDF for FCC

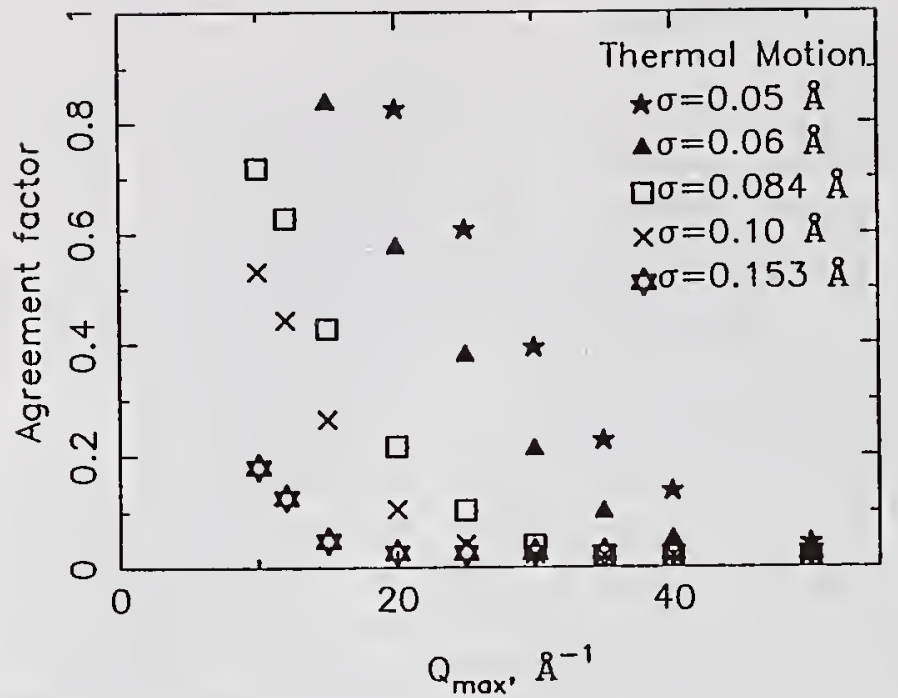

Figure 2: The amount of error introduced into the PDF due to termination of $S(Q)$ with different values of the Debye-Waller temperature factor. Agreement is computed over the range $r=2$ to $20 \AA$ by comparison to a PDF modeled with appropriate broadening.

of diffraction data for conventional crystallographic analysis. In contrast, instrumental resolution has much less impact on PDF computation. In the simplest case, consider an instrument with a Gaussian response function that broadens all peaks by a constant amount in $Q, \Delta Q$. This convolution of $S(Q)$ is equivalent to multiplication of the PDF by a damping function decreasing with $\mathrm{r}$. For moderate broadening values, $\Delta Q$, of 0.05 , the damping of the PDF will be negligible for $r$ below $20 \AA$. For larger values of $\Delta Q$, an exact correction may be applied to the PDF. The only loss due to the instrument response function is a decrease in the signal to noise ratio in the PDF at large $\mathbf{r}$. For instruments where the broadening increases with $Q$, for example pulsed sources, broadening is not a simple convolution of $S(Q)$. The results for a simulation with Gaussian broadening, increasing with $Q$, are shown in figure 3 . For reasonable broadening values, $\Delta Q / Q$ of $5 \%$, again the effect on the PDF is slight. Correction, either by broadening the PDF at large $r$, or by multiplication of the model PDF by a damping function reduces the error further. Consequently, while lack of instrument resolution may limit the range and utility of diffraction data for crystallographic computations, lack of instrumental resolution seldom impedes PDF analysis and can often be compensated by improving statistics and application of corrections to the PDF.

Inaccurate corrections for absorption, multiple
Peak Broadening with $\triangle Q / Q$ Constant

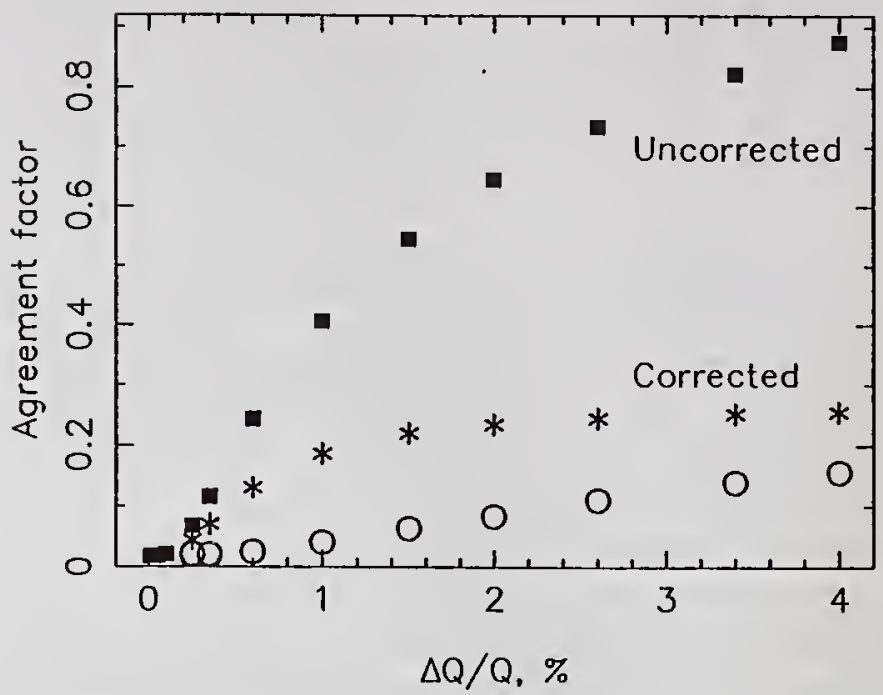

Figure 3: The agreement factor over the range $\mathrm{r}=$ 2 to $20 \AA$ between a model PDF and one computed from a model $S(Q)$ broadened with a instrument response function increasing with $Q$. Data indicated by squares are uncorrected and those shown with asterisks and circles are after correction by an $r$-dependent broadening term and multiplication by a Gaussian envelope function, respectively.

scattering, inelastic or Placzek scattering, sample container and background scattering can also introduce systematic error into the PDF computation. These effects typically vary slowly with $Q$. Thus, any deviations between the computed corrections and the accurate values should also be broad, smoothlyvarying functions of $Q$. Adding such a slowly varying error function to $S(Q)$ will add to $\rho(\mathbf{r})$ an error function with rapid oscillations that will decrease in magnitude as $\mathrm{r}$ increases.

The errors in the PDF for aluminum given in Fig. 1 are approximately three times larger than the expected statistical error, even after compensation for instrumental broadening. Termination errors do not account for more than a third of this error either. This leads to a surprising conclusion that the errors in the PDF due to inaccuracies in the experimental corrections must be of comparable magnitude to termination and statistical errors. Performing PDF computations on well standardized samples is important for characterization of the accuracy of the analysis software.

\section{Optimizing data collection to reduce errors}

In practice, access to synchrotron and neutron 
sources is very limited, and it is very important to obtain the most accurate result for a given total amount of time for a diffraction measurement. Defining a scan rate function as $R(Q)$, where $R(Q)$ is determined by the source intensity, instrument configuration and is proportional to the count time for each point. Thus for a given instrument and a fixed period of time for the complete measurement, the sum of $R(Q)$ must remain constant, but the time for an individual point may be increased or decreased. The number of counts for an individual measurement will be given by

$$
N_{o b s}\left(Q_{k}\right)=R\left(Q_{k}\right) \Delta Q_{k} I\left(Q_{k}\right) .
$$

If the contributions to the e.s.d.'s from experimental corrections such as background subtraction are considered invariant with $Q$, and $S(Q)$ and $R(Q)$ are treated as continuous functions, one obtains

$$
\sigma_{\rho(r)}^{2}=\frac{1}{\left(2 \pi^{2} r\right)^{2}} \int \frac{Q^{2}}{R(Q)<b>^{2}} S(Q) \sin ^{2} Q r d Q .
$$

Further simplification is possible using the approximation that $\sin ^{2} Q r$ averages to $1 / 2$ over a wide range in $Q$ yielding

$$
\sigma_{\rho(r)}^{2} \approx \frac{1}{8 \pi^{4} r^{2}} \int \frac{S(Q) Q^{2}}{R(Q)<b>^{2}} d Q .
$$

The optimum scan rate, $R(Q)$, to yield the smallest e.s.d.'s will be obtained when

$$
R(Q) \propto \frac{Q \sqrt{S(Q)}}{\langle b\rangle}=\frac{Q \sqrt{I(Q)}}{\langle b\rangle^{2}},
$$

because

$$
\sigma_{\rho(r)}^{2} \approx \frac{1}{8 \pi^{4} r^{2}}\left[\int \frac{Q \sqrt{S(Q)}}{\langle b\rangle} d Q\right]^{2} .
$$

In contrast, any other scan rate, such as scanning at a constant rate or waiting for a constant number of counts at each point will yield less accuracy for a given data collection period.

\section{Conclusions}

PDF computation is considered by most crystallographers to be inaccurate, a method of last resort. This conclusion is in error. In fact the PDF may be determined quite accurately with modern instrumentation. The PDF is less sensitive to experimental broadening than crystallographic techniques that fail when diffraction peaks may no longer be resolved.
This work does show that there is room for improvement in the methods used for computation of experimental corrections in $S(Q)$ computations, now that the experimental state-of-the-art has caught up to the software. It should be routine practice to verify the accuracy of software by detemination of the PDF for an appropriate standard. Finally, inclusion of e.s.d.'s with all published PDF's should become routine practice, just as inclusion of e.s.d.'s has become routine for crystallographic computations.

\section{Acknowledgments}

Work at the University of Pennsylvania was supported by the National Science Foundation through Grants DMR90-01704 and DMR88-19885. The Intense Pulsed Neutron Source is operated as a user facility by the U.S. Department of Energy, under Contract W-31-109-Eng-38. We also thank Dr. Diane P. Toby for proofreading this paper.

\section{References}

Cargill, G. S. III (1975). Solid State Physics, 30, 227320.

Dmowski, W., Toby, B. H., Egami, T., Subramanian, M. A., Gopalakrishnan, J. \& Sleight, A. W. (1988). Phys. Rev. Lett. 61, 2608-2611.

Hamilton, W. C. (1964). Statistics in Physical Science, pp. 32-34, New York: Ronald Press.

International Tables for X-Ray Crystallography (1968). Vol. III. Birmingham: Kynoch Press.

Klug, H. P. \& AleXANDeR, L. E. (1968). X-Ray Diffraction Procedures for Polycrystalline and Amorphous Materials, 2nd ed. New York: Wiley.

LOVESEY, S. W. (1984). Theory of Neutron Scattering from Condensed Matter, Volume 1, Oxford: Clarendon Press.

Placzek, G. (1952). Phys. Rev. 86, 377-388.

Toby, B. H., Egami, T., Jorgensen, J. D. \& Subramanian, M. A., (1990). Phys. Rev. Lett. 64, 2414-2417. Toвy, B. H. \& Egami, T., (1992). Acta Cryst. A48, In press.

WAGNER, C. N. J. (1972). Liquid Metals, Chemistry and Physics, edited by S. Z. Beer, pp. 257-329. New York: Marcel Dekker.

Warren, B. E., Krutter, H., \& Morningstar, O. (1936). J. Am. Ceram. Soc. 19, 202-206.

WARrEN, B. E. (1969). X-Ray Diffraction. Reading: Addison-Wesley; also, New York: Dover. 
National Institute of Standards and Technology Special Publication 846. Proceedings of the international conference Accuracy in Powder Diffraction II, held at NIST, Gaithersburg, MD, May 26-29, 1992. (Issued October 1992)

\title{
The Use of the Voigt Function in Determining Microstructural Properties from Diffraction Data by means of Pattern Decomposition
}

\author{
J. I. LANGFORD
}

School of Physics \& Space Research, University of Birmingham, Birmingham B15 2TT, UK

\begin{abstract}
Techniques for studying the microstructural properties of polycrystalline materials by applying pattern decomposition to complete diffraction patterns have advanced substantially since the first conference on Accuracy in Powder Diffraction was held in 1979. In particular, there have been a number of improvements to the method of line-profile analysis based on the Voigt function. The basic concepts of this approach, procedures for applying it and the limitations of the method are reviewed, and developments during the intervening years are illustrated by considering specific applications. The review is self-contained, in that all formulae required to study microstructural properties by means of the Voigt function are included.
\end{abstract}

\section{Introduction}

When the procedure based on the Voigt function for analysing diffraction line broadening in terms of microstructural properties was introduced (Langford, 1978), shortly before the first conference on Accuracy in Powder Diffraction, powder crystallography was enjoying something of a renaissance. This had largely been brought about by the advent, a decade or so earlier, of the Rietveld method for the refinement of crystal structures by means of powder data, but a contributing factor was the introduction of patterndecomposition programs in the mid 1970s, whereby a non-structural model is fitted to a complete diffraction pattern to obtain parameters describing the individual Bragg reflections. However, the method was then only applicable to relatively straightforward cases; nowadays, with more intense sources, instruments with higher resolution, greatly improved software and a better understanding of peak shapes, a wealth of information about the microstructure of polycrystalline materials can be obtained. Even for fairly complex patterns with peak overlap, the mean size and shape of crystallites (coherently diffracting domains) can be determined, various structural 'mistakes' can be studied, and the directional dependence of microstrains and other 'lattice distortions' can be obtained. It is thus timely to review the role of the Voigt function in characterising these quantities and in other applications of powder diffraction.

A brief résumé of the properties of the Voigt function will be given, followed by its use in patterndecomposition methods. A procedure for dealing with instrumental effects will then be considered, and techniques for carrying out line profile analysis based on the Voigt function will be discussed. The last will be illustrated by considering specific cases of sample broadening. It will be demonstrated that, if sufficient reflections are recorded, data are of adequate quality, and a systematic analytical procedure is followed, then meaningful and precise information about sample imperfections can be obtained by the Voigt approach. Examples will be based on data obtained from fixed-wavelength radiation $\left(\mathrm{CuK} \alpha_{1}\right.$, $\lambda=1.540598 \AA$ ), angle-dispersive experiments, but the principles involved apply equally well to energydispersive data.

\section{The Voigt function}

The Voigt function, the convolution of Lorentzian and Gaussian functions, can be represented by (Langford, 1978, equation (18))

$$
I(x)=\left(\beta / \beta_{G}\right) I_{o} \operatorname{Re}\{\omega(z)\},
$$

with

$$
z=\sqrt{\pi} x / \beta_{G}+i y
$$

and

$$
y=\beta_{L} /\left(\sqrt{\pi} \beta_{G}\right),
$$

where $I_{0}$ is the peak intensity, $\beta$ is the integral breadth $\left(=\right.$ area $\left./ I_{o}\right), \beta_{L}$ and $\beta_{G}$ are the integral breadths of the Lorentzian and Gaussian components, and $\operatorname{Re}\{\omega(z)\}$ is the real part of the complex error function.

A Voigt function can be characterised by a shape parameter $\phi$, defined as the ratio of the full width at 
half the peak intensity (FWHM or $2 w$ ) to the integral breadth, or

$$
\phi=\mathrm{FWHM} / \beta \text {. }
$$

From the definition of the Voigtian, it follows that the convolution of two or more such functions is also a Voigt function. The main reason for its introduction as a means of investigating the nature of diffraction line profiles is that $\beta_{L}$ and $\beta_{G}$ can readily be determined from $\beta$ and $\phi$ (vide infra, (11) \& (12)), and that the breadths of the constituent line profiles can then be separated in the usual way; i. e., from the additive property of the breadths of Lorentzian functions [e.g., (14)] and the squares of the breadths of Gaussian functions [e.g., (15)]. The Lorentzian and Gaussian functions are clearly limiting cases of the Voigtian; from Langford (1978), for the former

$$
\phi=2 / \pi=0.6366,
$$

and for the latter

$$
\phi=2 \sqrt{(\ln 2) / \pi}=0.9394 .
$$

\subsection{Applicability of the Voigt approximation}

There is no more theoretical justification for modelling diffraction line profiles by means of the Voigtian than there is for other analytical functions commonly used in line-profile analysis, except perhaps in the case of the instrumental contribution (Louër \& Langford, 1988; Langford, Cernik \& Louër, 1991). However, in practice this function frequently fits symmetric observed line profiles well within the experimental error, or to an adequate degree of ap-

Table 1: Goodness of Fit (GoF) for Voigt and pseudo-Voigt functions fitted to line profiles from tungsten filings.

\begin{tabular}{ccc}
$h k l$ & Voigt & Pseudo-Voigt \\
\hline 110 & 2.3 & 2.5 \\
200 & 1.4 & 1.4 \\
211 & 1.7 & 1.6 \\
220 & 1.2 & 1.2 \\
310 & 1.3 & 1.2 \\
222 & 1.0 & 1.0 \\
312 & 1.5 & 1.4 \\
400 & 1.2 & 1.2 \\
\hline
\end{tabular}

proximation (e.g., figure 1 and table 1; see also Langford \& Wilson, 1978, figures 2 and 3 for fits of the Voigt function to theoretical line profiles for crystallites having various shapes). The quality of the fit, indicated by the Goodness-of-Fit parameter (e.g.,
Hill \& Madsen, 1987) in table 1, depends to some extent on the magnitude of the sample broadening relative to that introduced by the instrument. If the latter is dominant, then the fit tends to be inferior for line profiles at lower angles.

Clearly a single Voigt function cannot be applied if the experimental data are markedly asymmetric. Also, $\phi$ must lie within the Lorentzian and Gaussian limits or, from (5) and (6),

$$
0.6366 \leq \phi \leq 0.9394 \text {. }
$$

Cases have been reported, for data from high resolution instruments, where the intensity in the tails of line profiles decreases more slowly than a Lorentzian ( $\phi<0.6366$; e. g., Plévert \& Louër, 1990), the socalled 'super-Lorentzian' (Wertheim et al., 1974). Such cases have hitherto been rare and, if line-profile analysis is to be applied, then probably the best that can be achieved by using existing techniques is to assume that the reflections are Lorentzian. If $\phi$ is significantly greater than 0.9394 , then it is possible that the fitting procedure is at fault or that the data are suspect.

\section{Experimental considerations and pattern decomposition}

\subsection{Data acquistion}

Line-profile analysis is one of the most exacting applications of powder diffraction, and, for the results to be meaningful, high quality data are essential. Any sample treatment which may affect the properties of interest is clearly to be avoided, but otherwise-standard procedures for sample preparation should be followed. These are described in 'Methods \& Practices in X-ray Powder Diffraction', published by the JCPDS-ICDD. (See also Will, Parrish \& Huang, 1983.)

The data should not be unduly 'noisy', and ideally the standard deviation in the peak intensity of the weakest recorded reflection should not be greater than a few percent. The effects of counting statistics on parameters derived from an analysis based on the Voigt function have been considered by Langford (1980), and these can be appreciable. Since additional systematic errors due to pattern decomposition and assumptions made in the analysis of the data are inevitable, it is desirable to minimise random errors, in so far as is practicable, at the datacollection stage.

The angular increment during data collection is not critical provided that, in addition to the usual criterion based on the aperture of the receiving slit, there are 5 to 10 data points within the FWHM of 
(a) 110

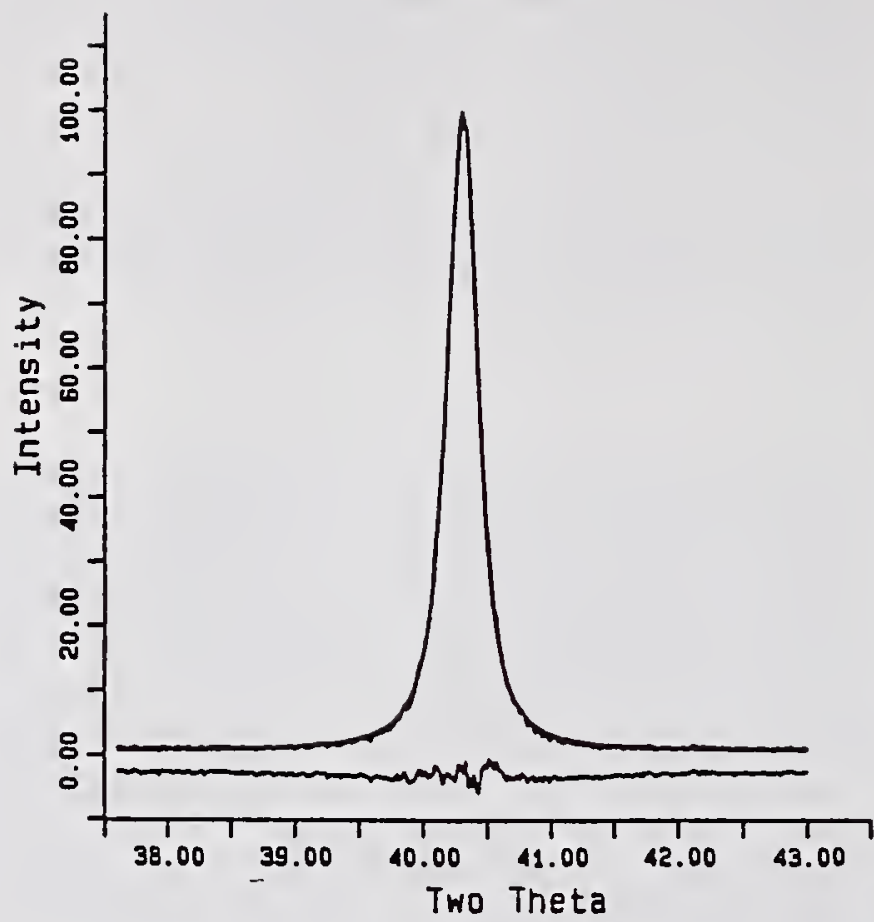

(c) 310

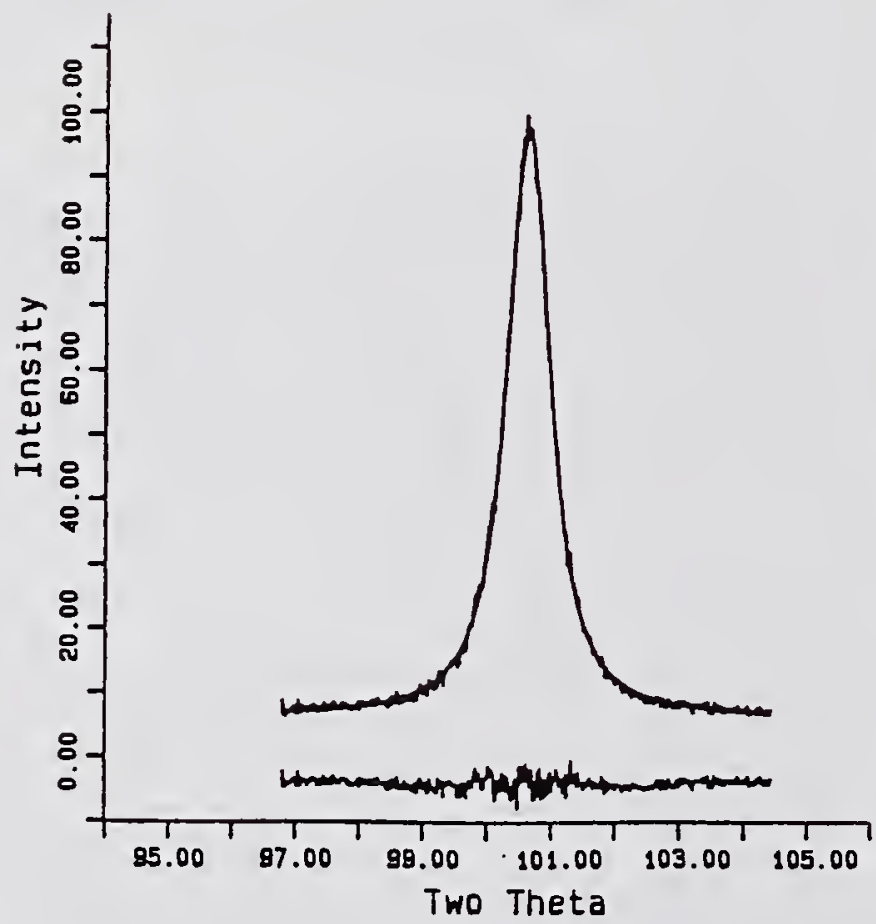

(b) 211

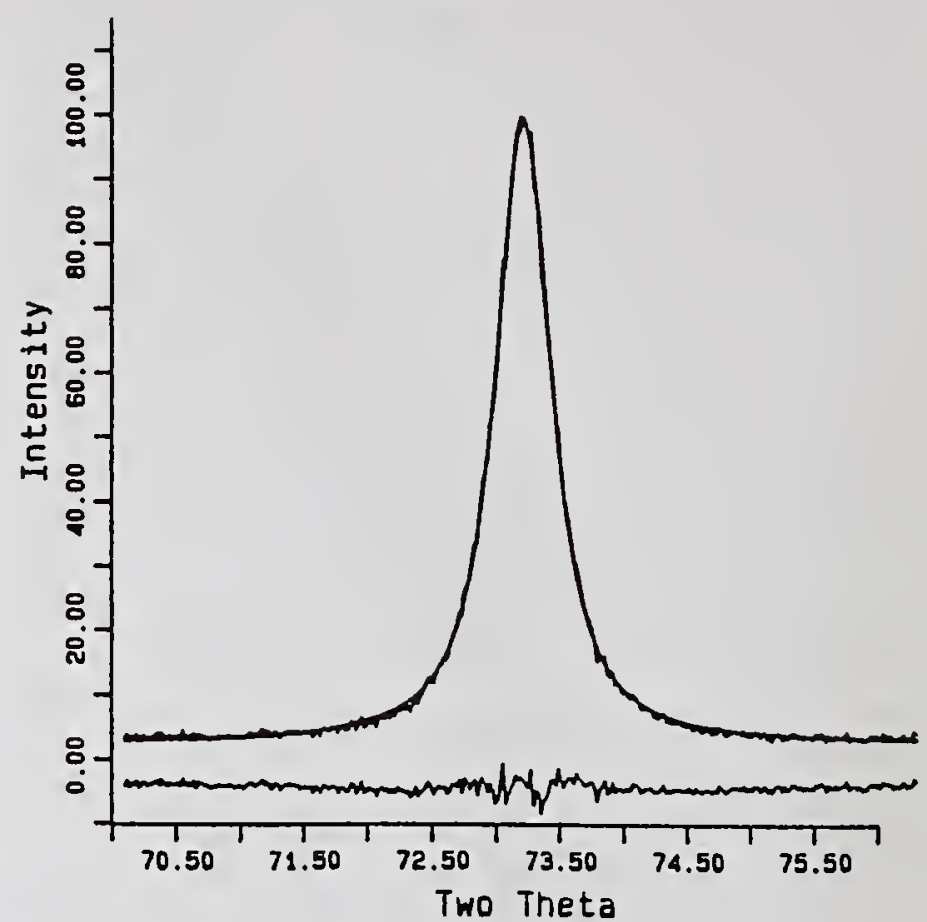

(d) 321

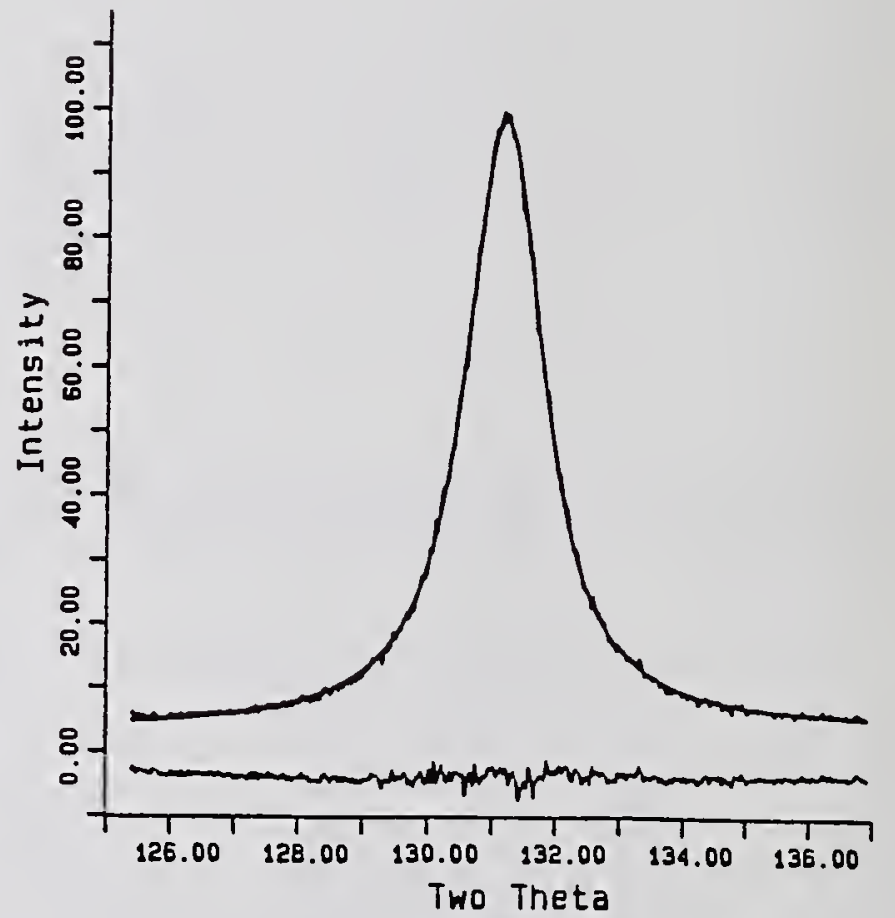

Figure 1: Line profiles for tungsten filings, $\mathrm{CuK} \alpha_{1}$ radiation, with fitted Voigt functions and difference plot.

the narrowest line. As many lines as are feasible must be recorded, up to an angle at which the peak intensity has fallen to a few percent of that of the strongest reflection; if possible, data for at least 20 30 reflections should be obtained if a detailed analysis of sample imperfections is required. It is often convenient to scan the desired angular range in two or more sections, with an increase in the counting time, and perhaps the step length, at higher angles.

It is desirable to use monochromatic radiation 
for line-profile analysis. This can of course be achieved automatically if synchrotron or reactor neutron sources are used, and, in the case of $\mathrm{K} \alpha$ radiation from conventional sealed-tube X-ray sources, the $\mathrm{K} \alpha_{2}$ component can be removed completely by means of an incident-beam focusing monochromator (e.g., Louër \& Langford, 1988). The resulting monochromatic $K \alpha_{1}$ spectrum can then readily be modelled with a simple analytical function, the number of peaks in a diffraction pattern is halved, and the lower background can be assumed to vary linearly over a wide range of angle. Standard analytical procedures for removing the $\mathrm{K} \alpha_{2}$ component, usually based on modifications of the method introduced by Rachinger (1948) (e. g., Delhez \& Mittemeijer, 1975), are adequate for many applications of powder diffraction, but introduce systematic errors in line-profile analysis. These arise because the different widths, shapes and asymmetries of the $K \alpha_{1}$ and $K \alpha_{2}$ components and the $\mathrm{K} \alpha$ satellite lines are not normally taken into account. Such errors are undesirable, but whether or not they are significant depends on the relative magnitudes of the sample and instrumental broadening and the resolution of the instrument.

\subsection{Instrumental effects}

A correction for the contribution from the instrument to the observed data must be made in all studies of sample imperfections by means of line-profile analysis, and inadequate instrumental data can result in severe systematic errors. Instrumental line profiles are normally obtained by means of data from a standard reference material (SRM), and, for samples which are essentially opaque to the radiation used, $\mathrm{LaB}_{6}$ is the SRM marketed by NIST (Fawcett et al., 1988). Alternatively, annealed $\mathrm{BaF}_{2}$ has been found to be eminently suitable for this purpose (Louër \& Langford, 1988). The broadening introduced by sample transparency can be estimated (e.g., Wilson (1963), chapter 3 for flat samples), and, if this is not negligible, an annealed sample of the material of interest should be used. However, in such cases it is important to confirm that line broadening due to residual sample imperfections is inappreciable.

If the breadths of the instrumental line profiles $g(x)$ are obtained by means of an SRM, in general the 'standard' reflections will be at different angles from those of the sample and it is then necessary to interpolate $2 w_{g}$ and $\beta_{g}$ by some function to describe their variation with angle. The nature of the function is unimportant, provided that the fit is adequate, and that frequently used is a quadratic in $\tan \theta$ :

$$
(\text { Breadth })^{2}=U \tan ^{2} \theta+V \tan \theta+W .
$$

$U, V$ and $W$ are then constant for a given instru- mental configuration and can be checked periodically to ensure that alignment has been maintained. A typical instrument resolution function (IRF: FWHM versus $2 \theta$ ), obtained by a least-squares fit of (8) to data from annealed $\mathrm{BaF}_{2}$, is shown in figure 2. An

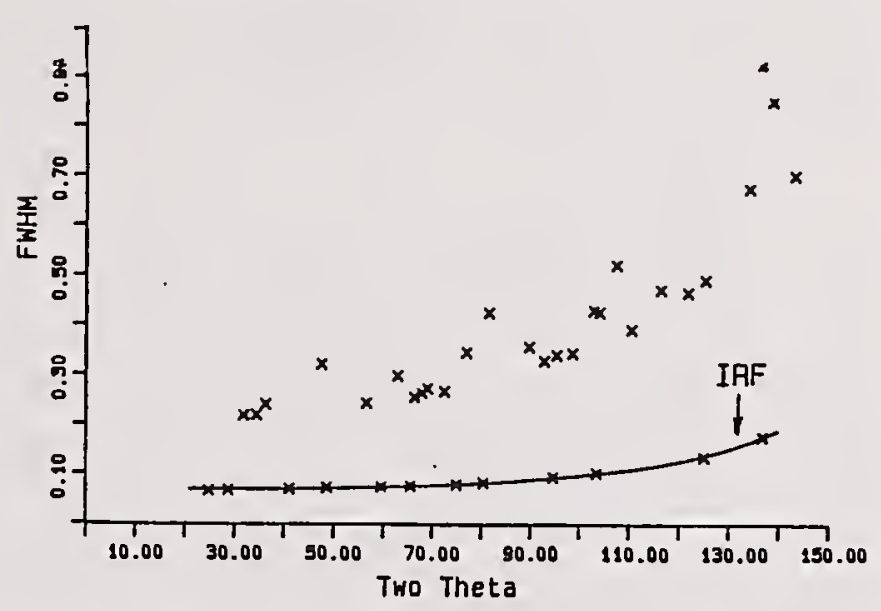

Figure 2: Instrument Resolution Function (IRF) and FWHM for ex-oxalate $\mathrm{ZnO}, \mathrm{CuK} \alpha_{1}$ radiation.

alternative function, with more theoretical justification for X-ray diffractometers, and which may model breadths at low angles more satisfactorily if, for example, axial divergence has a measurable effect, is

$$
(\text { Breadth })^{2}=A \tan ^{2} \theta+B+C \cot ^{2} \theta .
$$

If necessary, a fourth term to account for sample transparency can be added to (9). For a detailed discussion of this function, and a comparison with (8), see Langford (1987), section 2.2. It should be noted that $U, V, W$ or $A, B, C$ are required for both $2 w_{g}$ and $\beta_{g}$ in the Voigt approach to line-profile analysis, in order to obtain $\phi_{g}$ from (4).

\subsection{Pattern decomposition}

In order to investigate the nature of structural imperfections, reliable parameters defining the position, breadth and shape of all accessible Bragg reflections are required. Various programs available for obtaining these quantities by means of pattern decomposition have been reviewed by Smith \& Gorter (1991). The programs employed to obtain data used in the examples given below were PROFIT (Langford et al., 1986) and the Socabim program FIT, available in the PC software package DIFFRAC-AT. The interactive nature of the latter makes it particularly convenient to use, since, with the 'zoom' facility for displaying line-profile tails, erroneous 'fits' are usually apparent. Alternatively, a logarithmic intensity scale can be used.

Ideally, experimental line profiles should be modelled with Voigt functions, as given by (1), and a 
Fortran program for evaluating the complex error function $\omega(z)$ is listed in Appendix 1. $\beta_{f}, \beta_{f L}$, $\beta_{f G}$ and $\phi_{f}$ are then given directly. However, most pattern-decomposition programs employ some other function, notably the pseudo-Voigt and the Pearson VII, when only $\beta_{f}$ and $\phi_{f}$ are obtained. The various functions most commonly used in powder diffraction were defined and reviewed by Wiles \& Young, 1982. The pseudo-Voigt is a close approximation to a Voigtian (Wertheim et al., 1974), and fits obtained by using these functions are frequently identical, within the experimental error (Table 1). In general the Pearson VII is not such a good approximation to a Voigtian. The effects of using parameters obtained from a pseudo-Voigt and Pearson VII, rather than a true Voigtian, and the errors introduced thereby, have been considered by Keijser, Mittemeijer \& Rozendaal (1983). An advantage of these functions is that computational time is usually less than that for the Voigtian.

Ahtee et al., (1984) have shown that, for a Voigt function, $\phi$ is related to $y(3)$ by

$$
\phi=E\left(1+A y+B y^{2}\right) /\left(1+C y+D y^{2}\right),
$$

where $A=0.9039645, B=0.7699548, C=1.364216$, $D=1.136195$ and $E=2 \sqrt{(\ln 2) / \pi}=0.9394372$. (10) is an approximation, but the maximum difference from the exact value is only $0.16 \%$ (at $y=0.15$ ). Thus, if $2 w$ and $\beta$, and hence $\phi$, have been obtained by fitting a pseudo-Voigt or some other function to the observed reflections, then

$$
\begin{aligned}
y= & \left\{(C \phi / E-A)+[C \phi / E-A)^{2}-4(D \phi / E\right. \\
& \left.-B)(\phi / E-1)]^{1 / 2}\right\} /[2(B-D \phi / E)] .
\end{aligned}
$$

$\beta_{G}$ can then be obtained from (Langford, 1978, equation (25))

$$
\beta_{G}=\beta \exp \left(y^{2}\right)[1-\operatorname{erf}(y)],
$$

and $\beta_{L}$ is given by (3).

\section{Preliminary analysis of line breadths and shapes}

\subsection{Preliminary assessment}

Before one embarks on a detailed analysis of line breadths, the nature of any microstructural properties in the sample must first be ascertained. The first stage in this procedure is to compare the FWHM $\left(2 w_{h}\right)$ of the observed line profiles $\mathrm{h}(x)$ with the IRF. $2 w_{h}$ for a sample of $\mathrm{ZnO}$ obtained by thermal decomposition of the oxalate (Langford et al., 1992) is plotted in figure 2, and it is clear that $2 w_{h} \gg 2 w_{g}$ for all reflections, and that there is thus appreciable sample broadening. Also, the scatter for the $2 w_{h}$ data is considerably greater than would be expected from random effects ( $c f$ the data for the IRF), and the broadening due to the sample thus exhibits a marked 'anisotropy' or lattice-direction dependence. Plots such as figure 2 serve to indicate whether or not the data merit further analysis in terms of structural imperfections. As part of the general assessment of the data, the diffraction pattern can be reviewed by using the observed peak positions with the program NBS*AIDS83 (Mighell, Hubbard \& Stalick, 1983), or some other program which obtains refined unit-cell dimensions. This will indicate if there are any significant sample-induced peak displacements, such as can arise from some structural imperfections. The magnitude and direction of systematic peak shifts can be of importance in analysing and interpreting sample line broadening, particularly if there is evidence of a contribution from structural 'mistakes'.

\subsection{Instrumental correction}

The integral breadths $\beta_{f}$ of the line profiles $\mathrm{f}(x)$ due to sample imperfections are required in order to characterise microstructural properties. If all line profiles are assumed to be Voigtian, since $\mathrm{h}(x)$ is the convolution of $\mathrm{f}(x)$ and $\mathrm{g}(x)$, i.e.,

$$
\mathrm{h}(x)=\mathrm{f}(x) * \mathrm{~g}(x)
$$

then

$$
\beta_{f L}=\beta_{h L}-\beta_{g L},
$$

$$
\beta_{f G}^{2}=\beta_{h G}^{2}-\beta_{g G}^{2} .
$$

If Voigt functions have been used in the pattern decomposition, then (14) and (15) can be applied directly to obtain $\beta_{f L}$ and $\beta_{f G}$. If some other function is used to model reflections, then $y_{h}$ and $y_{g}$ are given by (11) with $\phi=\phi_{h}$ and $\phi=\phi_{g}$, respectively. $\beta_{h G}$ and $\beta_{g G}$ are then obtained by inserting $y_{h}, \beta_{h}$ or $y_{g}$, $\beta_{g}$ in (12), and the corresponding Lorentzian components are given by (3). This procedure is shown schematically in Langford et al., (1988) figure 3.

Depending on the nature of any imperfections present, $\beta_{f L}$ and $\beta_{f G}$ may be needed in a subsequent analysis of line broadening, but the Voigtian breadth $\beta_{f}$ is required in the preliminary evaluation of the data. This is obtained from (12), with $\beta_{G}=\beta_{f G}$ and $y=y_{f}=\beta_{f L} /\left(\sqrt{\pi} \beta_{f G}\right)$. Additionally, $\phi_{f}$ is given by (10), again with $y=y_{f}$. Hitherto, breadths have been expressed in angular units $(2 \theta)$, but the subsequent analysis is simplifed if $\beta_{f}$ is given in terms of reciprocal units, where

$$
\beta_{f}^{*}=\beta_{f} \cos \theta / \lambda
$$


In what follows, reciprocal units are implied throughout, but for convenience $\beta_{f}^{*}$ will be denoted by $\beta_{f}$.

\subsection{Williamson-Hall plot}

The next stage in the analysis is to ascertain if the breadths depend on $d^{*}(=2 \sin \theta / \lambda)$ and to investigate the nature of any $[h k l]$ (i.e., lattice-direction) dependence. For this purpose the Williamson-Hall plot (Williamson \& Hall, 1953) is used, in which $\beta_{f}$ (in reciprocal units) is plotted as a function of $d^{*}$, with the addition of the Miller indices $h k l$ for each point. It should be noted that the Williamson-Hall plot should not be used quantitatively, other than as a first approximation, if $\beta_{f}$ has both $d^{*}$-independent and $d^{*}$-dependent contributions, since the implicit assumption is that the corresponding line profiles are Lorentzian, which is unlikely to occur in practice. Nevertheless, such a plot gives a valuable insight into the nature of any structural imperfections present and the procedure to be adopted in subsequent analyses. The use of the Williamson-Hall plot can best be demonstrated by considering various specific cases of sample broadening.

\section{(a) Ex-hydroxide nitrate $\mathrm{ZnO}$, sample $A$ [Fig. 3(a)]}

From the Williamson-Hall plot for a sample of $\mathrm{ZnO}$ obtained by the thermal decomposition of the hydroxide nitrate (Louër et al., 1983), $\beta_{f}$ is the same, to within the experimental error, for different orders of reflections $(h 00, h h 0,00 l)$, indicating that there is not a measurable contribution from microstrains or other 'lattice distortions', which introduce an order dependence. Furthermore, the intercept for the $00 l$ reflections is much less than for the $h k 0$, suggesting that on average the crystallites or domains have a prismatic form, with the axis in the $c$ direction and an axial dimension greater than the base diameter. Since there is not a significant difference in $\beta_{f}$ for the $h 00$ and $h k 0$ reflections, the prisms may be regarded as having a circular section. A subsequent analysis of the breadths for this sample would thus be based on strain-free crystallites whose shape, on average, is a cylinder. The data for the Williamson-Hall plot are taken from Langford et al., (1986).

\section{(b) Tungsten filings [Fig. 3(b)]}

The breadths for a sample of tungsten filings (Table 2) increase linearly with $d^{*}$, and there is a non-zero intercept, indicating that microstrains are appreciable, and that there is a measurable 'size' effect. The points do not display a significant scatter, so the strain is isotropic, as would be expected for coldworked tungsten. There is also a grain or sub-grain structure within the filings, and this may represent regions bounded by dislocations.

\section{(c) Ex-oxalate $\mathrm{ZnO}$ [Fig. 3(c)]}

For the ex-oxalate $\mathrm{ZnO}$ data (Langford et al., 1992, Table 3), it is immediately apparent from the Williamson-Hall plot that the values of $\beta_{f}$ are distributed among three groups, according to the values of $h \mathrm{kl}$ :

Group $1\left(\beta_{f 1}\right) h k 0$ and $h-k=3 n$. Group $2\left(\beta_{f 2}\right) h-k=3 n \pm 1, l$ odd. Group $3\left(\beta_{f 3}\right) h-k=3 n \pm 1, l$ even.

Furthermore, $\beta_{f 1}<\beta_{f 2}<\beta_{f 3}$ for almost all the data. This behaviour is a classic case of broadening due to stacking faults in materials belonging to the hexagonal system, which had not been been reported previously for $\mathrm{ZnO}$. From the theory of stacking-fault broadening (e.g., Wilson, 1962, chapter 6), group 1 reflections are unaffected by 'mistakes'. For these lines, $\beta_{f}$ is independent of $d^{*}$, again indicating that microstrains are negligible. Since the plot for group 1 reflections has a non-zero intercept, the crystallites or domains are of relatively small size. Also, there is little scatter for these data and the lack of 'anisotropy' suggests that on average the crystallites may be regarded as spherical, or as prismatic with equal apparent dimensions in the axial and basal directions. (See section 5.3.) The ex-oxalate $\mathrm{ZnO}$ thus represents a case of combined 'size' and 'mistake' broadening, but with no measurable strain contribution.

(d) Ex-hydroxide nitrate $\mathrm{ZnO}$, sample $B$ [Fig. 3(d)]

This sample has been considered by Langford et al. (1986) and by Sonneveld et al. (1986). From the Williamson-Hall plot, $\beta_{f}$ lines for $h 00$ and $00 l$ reflections have non-zero and slightly different slopes, indicative of the presence of microstrains which display a degree of anisotropy. There is also a 'size' effect (non-zero intercepts) and the crystallites are prismatic ( $00 l$ intercept $<h 00$ intercept). $\beta_{f}$ for the 110 reflection is close to the $h 00$ line, again suggesting that on average the crystallite shape is cylindrical. A curious feature for this sample is that $\beta_{f}$ for all other $h k l$ lies on, or close to, the $h k 0$ line. This may be due to an inverse correlation between domain size and strain. There is, however, no evidence of measurable broadening due to stacking faults. For $\mathrm{ZnO}$ sample $B$, a subsequent analysis would thus be based on combined 'size' and strain broadening, with anisotropic strain and possibly a cylindrical model for the crystallite shape. 

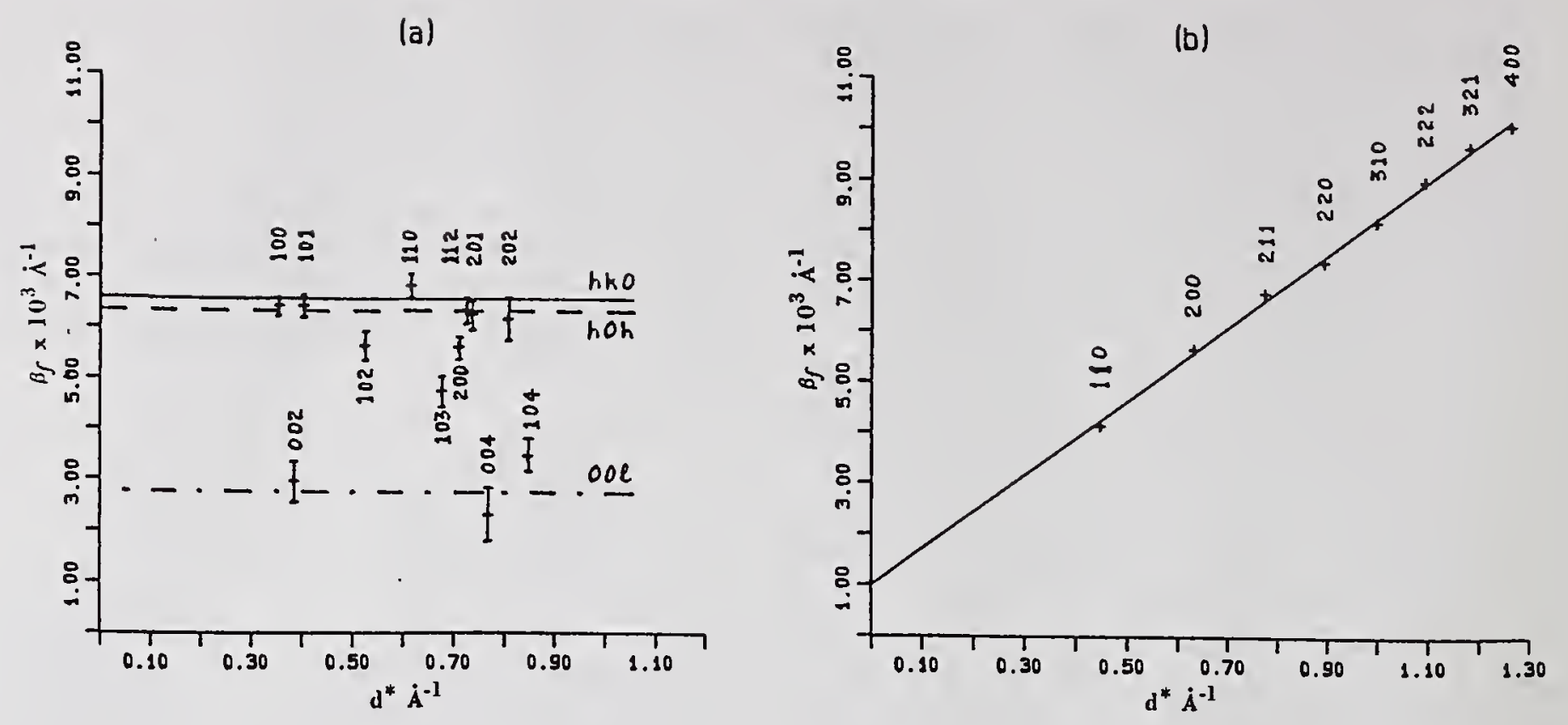

(c)

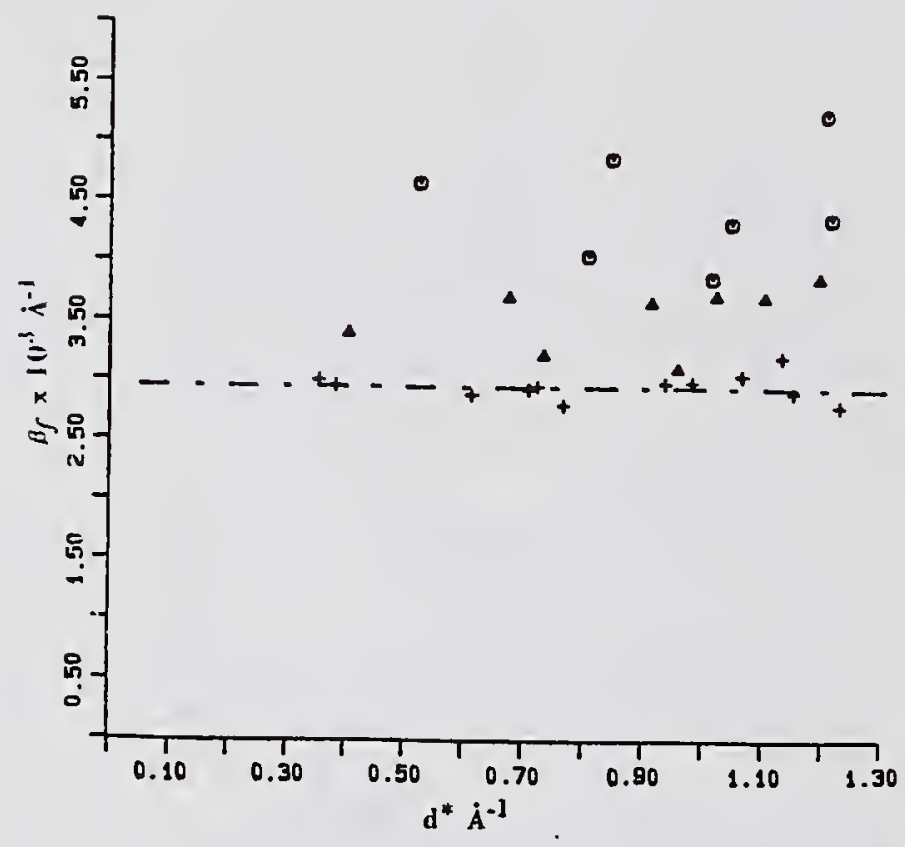

(d)

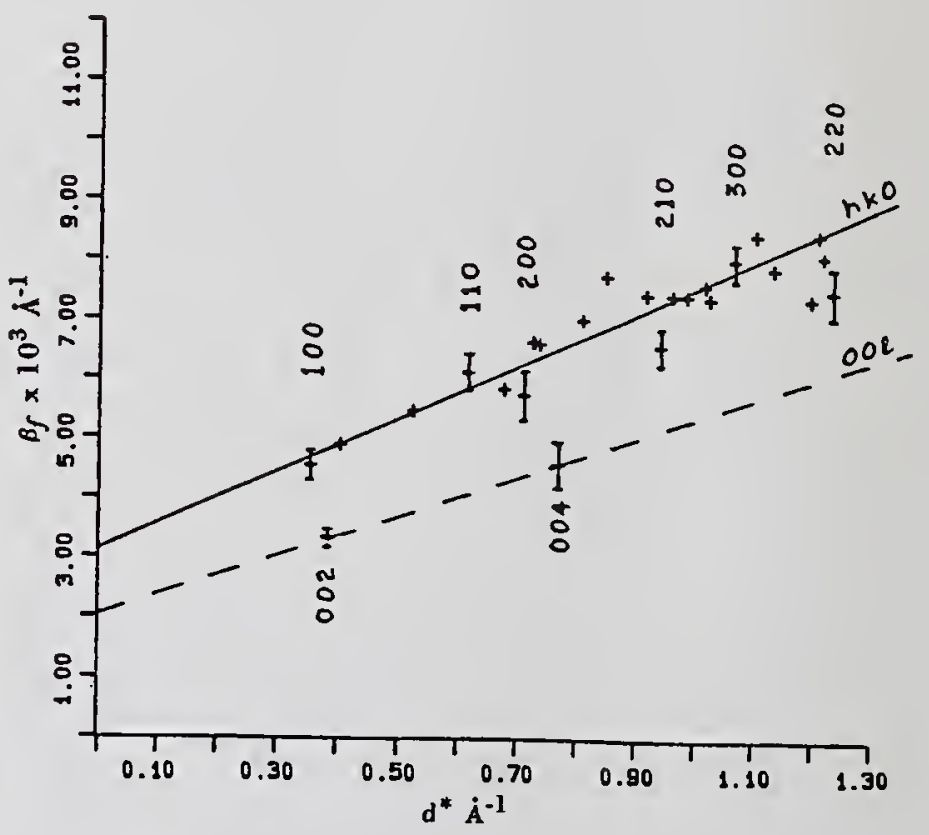

Figure 3: Williamson-Hall plots. ( $a$ ) ex-hydroxide nitrate $\mathrm{ZnO}$, sample $A$. (b) tungsten filings. (c) ex-oxalate $\mathrm{ZnO} ;+h k 0$ and $h-k=3 n, \Delta h-k=3 n \pm 1, l$ odd, $\odot h-k=3 n \pm 1, l$ even. $(d)$ Ex-hydroxide nitrate $\mathrm{ZnO}$, sample $B$.

\subsection{Shape of sample line profiles}

To conclude the preliminary assessment of the data, the nature of the shape parameter is considered, i.e., whether the profiles are predominantly Lorentzian or Gaussian, and if there is any dependence of $\phi_{f}$ on $d^{*}$ or lattice direction. The line profile shape for the $\mathrm{ZnO}$ sample $A$ data is closely Lorentzian (Langford et al., 1986), as is that for the tungsten filings [from table $\left.2,\left\langle\phi_{f}\right\rangle=0.673(11)\right]$, and there is no evidence of any $d^{*}$ or lattice-direction dependence for either of these samples. For the ex-oxalate $\mathrm{ZnO}$, on the other hand, $\left\langle\phi_{f}\right\rangle=0.778(19)$ for group 1 reflections and $0.714(25)$ for groups 2 and 3 . There is thus a significant Gaussian component in this instance which is greater for the group 1 ('size only') reflections than for groups 2 and 3. $\phi_{f}$ increases steadily for $\mathrm{ZnO}$ 
Table 2: Line breadths and shape parameter for tungsten filings; apparent size and strain from single-line method.

\begin{tabular}{crrrrrrr}
$\begin{array}{c}d^{*} \\
\AA^{-1}\end{array}$ & $\begin{array}{c}\beta_{f} \\
\times 10^{3} \AA^{-1}\end{array}$ & $\begin{array}{c}\beta_{f L} \\
\times 10^{3} \AA^{-1}\end{array}$ & $\begin{array}{c}\beta_{f G} \\
\times 10^{3} \AA^{-1}\end{array}$ & $\phi_{f}$ & $\begin{array}{c}\varepsilon \\
\AA\end{array}$ & $\begin{array}{c}\eta \\
\times 10^{3}\end{array}$ & $\begin{array}{c}\tilde{\mathrm{e}} \\
\times 10^{3}\end{array}$ \\
\hline 0.447 & 4.153 & 3.407 & 1.503 & 0.697 & 294 & 6.70 & 1.34 \\
0.632 & 5.664 & 5.046 & 1.561 & 0.673 & 198 & 4.95 & 0.99 \\
0.774 & 6.774 & 6.109 & 1.763 & 0.670 & 164 & 4.55 & 0.91 \\
0.894 & 7.407 & 6.448 & 2.239 & 0.680 & 155 & 5.00 & 1.00 \\
0.999 & 8.203 & 7.455 & 2.054 & 0.667 & 134 & 4.10 & 0.82 \\
1.094 & 9.010 & 8.088 & 2.398 & 0.671 & 124 & 4.40 & 0.88 \\
1.182 & 9.679 & 9.020 & 2.074 & 0.659 & 111 & 3.50 & 0.70 \\
1.264 & 10.107 & 9.273 & 2.399 & 0.664 & 108 & 3.80 & 0.76 \\
\hline
\end{tabular}

sample $B$ (Langford et al., 1986, Fig.4), which is consistent with a markedly Gaussian character of the strain component of $\mathrm{f}(x)$.

\section{Microstructural properties from line breadths}

5.1 Ex-hydroxide nitrate $\mathrm{ZnO}$, sample A: crystallite size only

The analysis of the data for $\mathrm{ZnO}$ sample $A$ is representative of an important application of line-profile analysis, since strain-free materials occur frequently in practice, particularly ceramics and other compounds which have been produced at a high temperature. In such cases, if there is no evidence of 'mistake' broadening (section 5.3), $\beta_{f}$ can be attributed solely to the effects of crystallite or domain size and shape, and

$$
\beta_{f}=1 / \varepsilon
$$

where $\varepsilon$ is an apparent size, equivalent to the volume average of the thickness, measured perpendicular to the reflecting planes (Appendix 2 and Wilson, 1962). It should be noted that an analysis of line breadths based on Fourier coefficients (e.g., Warren, 1969) gives a different measure of the apparent size, namely an area average of the crystallites, projected on to the reflecting planes (Wilson, 1963, chapter 9). In general this will be less than the apparent size obtained from the integral breadth. An indication of the mean dimensions of the crystallites or domains can be obtained by fitting a suitable model to the values of $\varepsilon$ (Appendix 2).

In the case of $\mathrm{ZnO}$ sample $A$, the Williamson-Hall plot suggests that the morphology of the crystallites approximates to a cylinder, with its axis parallel to $c$, which is not unreasonable for a hexagonal material. (A case for which a hexagonal prism is a better model, again for a sample of $\mathrm{ZnO}$, has been considered by Vargas, Louër \& Langford, 1983.) $\varepsilon$ is related to the mean diameter $D$ and the height $H$ of a cylinder, and the angle $\phi_{z}$ defining the direction of the diffraction vector, by (A4) and (A5) of Appendix 2. These equations are fitted to the observed values of $\varepsilon$ by the method of non-linear least squares, and the result of this procedure is shown in figure 4, which depicts a cross-section through the 'average' cylinder. From this analysis, $\langle D\rangle=180(10) \AA$, and $\langle H\rangle=270(60) \AA$. From figure 4 , it can be seen that a cylinder is a close approximation to the 'average' morphology of the crystallites. The results of TEM measurements are in broad agreement with this result and demonstrate that the particles of the powder are in fact single crystals (Louër et al., 1983).

\subsection{Tungsten filings: isotropic size and strain broad- ening}

If order-independent and order-dependent broadening occur simultaneously, then the analysis is less straightforward than in the 'size only' case. The two contributions to $\beta_{f}$ are normally regarded as 'size' and 'strain' broadening, though the former can include a contribution from structural 'mistakes' and the mean separation of dislocations, and the latter can arise from a compositional gradient within the sample. $\mathrm{f}(x)$ is then the convolution of $\mathrm{S}(x)$, the line profile due to all 'size' effects, and $\mathrm{D}(x)$, that due to strain, actual or apparent, or

$$
f(x)=S(x) * D(x)
$$

There are various approaches to separating $\beta_{S}$ and $\beta_{D}$, the integral breadths of $\mathrm{S}(x)$ and $\mathrm{D}(x)$, all based on assumptions regarding the nature of the line profiles due to 'size' and 'strain', and these are considered below. 


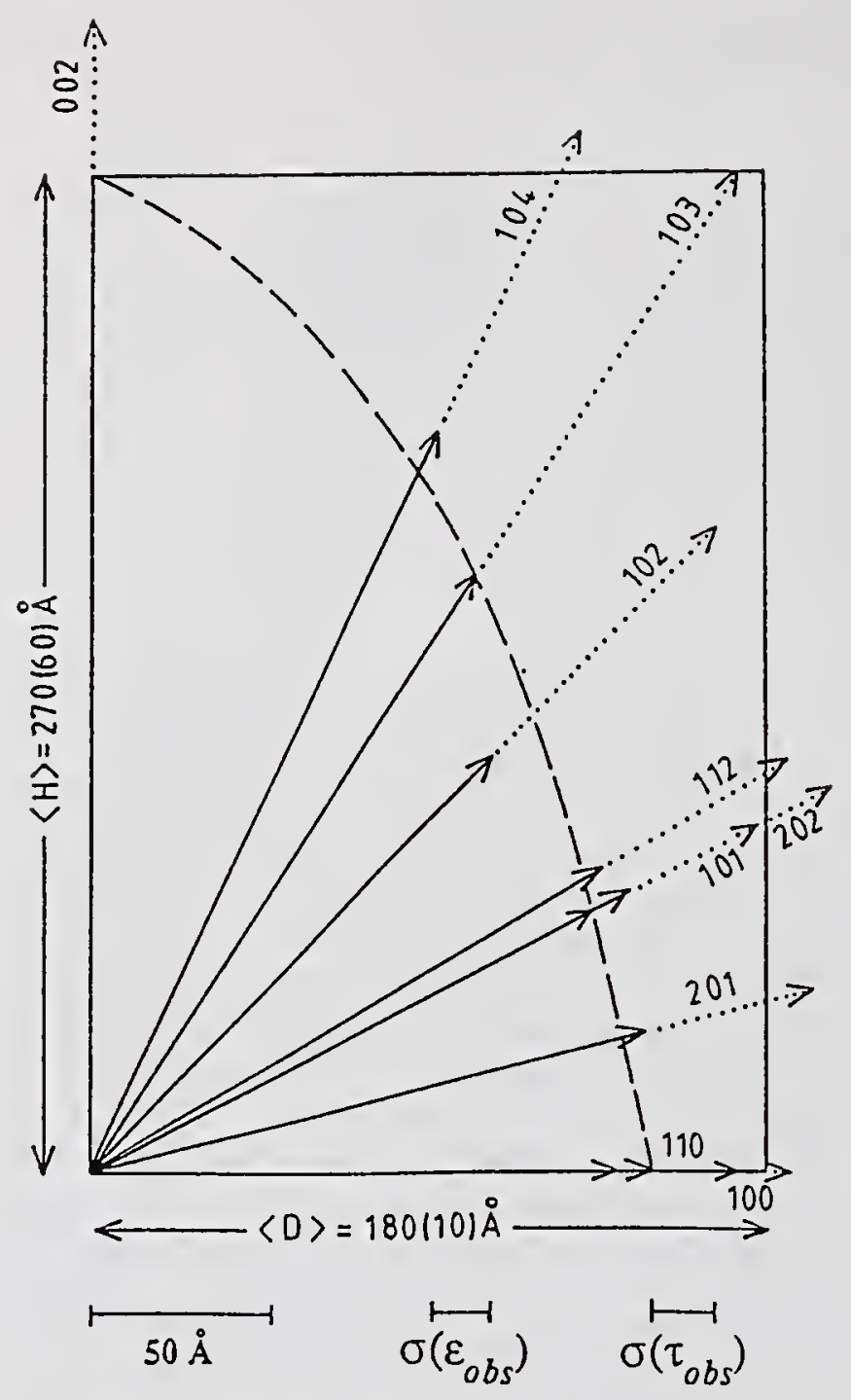

Figure 4: Least-squares fit of (A4) and (A5) (cylindrical model) to line breadths from ex-hydroxide nitrate, sample $A$ (Langford et al., 1992); - $\varepsilon_{o b s}$, - - - $\varepsilon_{c a l}, \cdots \cdots \cdots \tau_{\text {obs }}$.

(a) 'Average' Size-Strain Plot

If the Williamson-Hall plot does not indicate a marked anisotropy of size or strain broadening, as is the case for the tungsten sample, the 'average' sizestrain plot may be employed to obtain $\left\langle\beta_{S}\right\rangle$ and $\left\langle\beta_{D}\right\rangle$. Halder \& Wagner (1966) have shown that an approximation to the integral breadth of a Voigt function is given by

$$
\beta^{2}=\beta_{L} \beta+\beta_{G}^{2},
$$

which is accurate to within about $5 \%, \beta$ being less than the true value for a given $y$ (Langford, 1978). If it is assumed that $\beta_{f}$ is Voigtian, that the Lorentzian component is due solely to size effects, or

$$
\beta_{L}=\beta_{S}=1 / \varepsilon
$$

and that $\beta_{G}$ arises from strain, or

$$
\beta_{G}=\beta_{D}=\eta d^{*} / 2,
$$

where $\eta$ is a measure of the strain, then (19) may be written as

$$
\left(\beta / d^{*}\right)^{2}=\varepsilon^{-1} \beta /\left(d^{*}\right)^{2}+(\eta / 2)^{2} .
$$

The implications of assuming that the 'size' and 'strain' profiles are Lorentzian and Gaussian respectively have been discussed by Delhez et al., (1993, Appendix B). From (22), the intercept of the plot of $\left(\beta / d^{*}\right)^{2}$ versus $\beta /\left(d^{*}\right)^{2}$ gives the mean value of $\eta$, and the slope gives the mean apparent size $\langle\varepsilon\rangle$. Despite the approximation and assumptions made, this plot has the advantage that data for reflections at low and intermediate angles are given more weight than those at higher angles, which are often less reliable.

From the 'average' size-strain plot for the tungsten filings (Fig. 5), $\langle\varepsilon\rangle=640(70) \AA$, and $\langle\eta\rangle=$

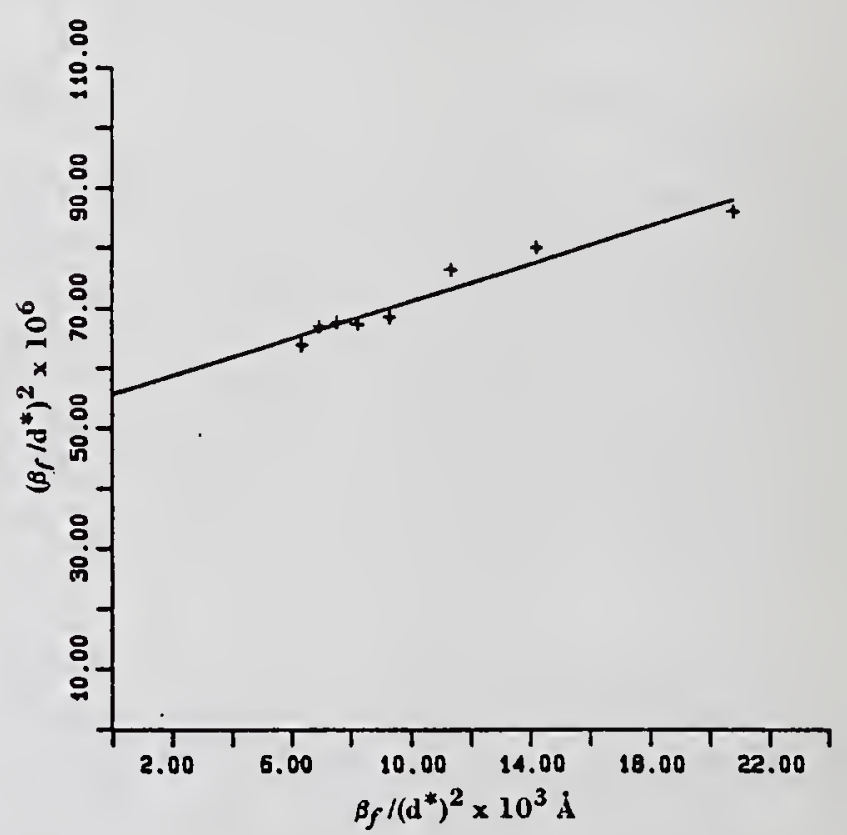

Figure 5: 'Average' size-strain plot for tungsten filings.

$14.9(3) \times 10^{-3}$. Since the line breadths show no $[h k l]$ dependence, the domains may be regarded as having spherical form, on average, with a diameter $\langle\mathrm{D}\rangle=860(90) \AA$ [Appendix 2, (A2)]. The value reported for the strain depends on the stress model invoked (Stokes \& Wilson, 1944; see also Langford et al., 1988). If the rms strain $\tilde{\mathrm{e}}\left(\equiv\left\langle e^{2}\right\rangle^{1 / 2}\right)$ is considered to be appropriate, then

$$
\tilde{\mathrm{e}}=\eta /(2 \sqrt{(2 \pi)} \cong \eta / 5,
$$

and, for the tungsten filings, $\langle\tilde{e}\rangle=2.98(5) \times 10^{-3}$. 

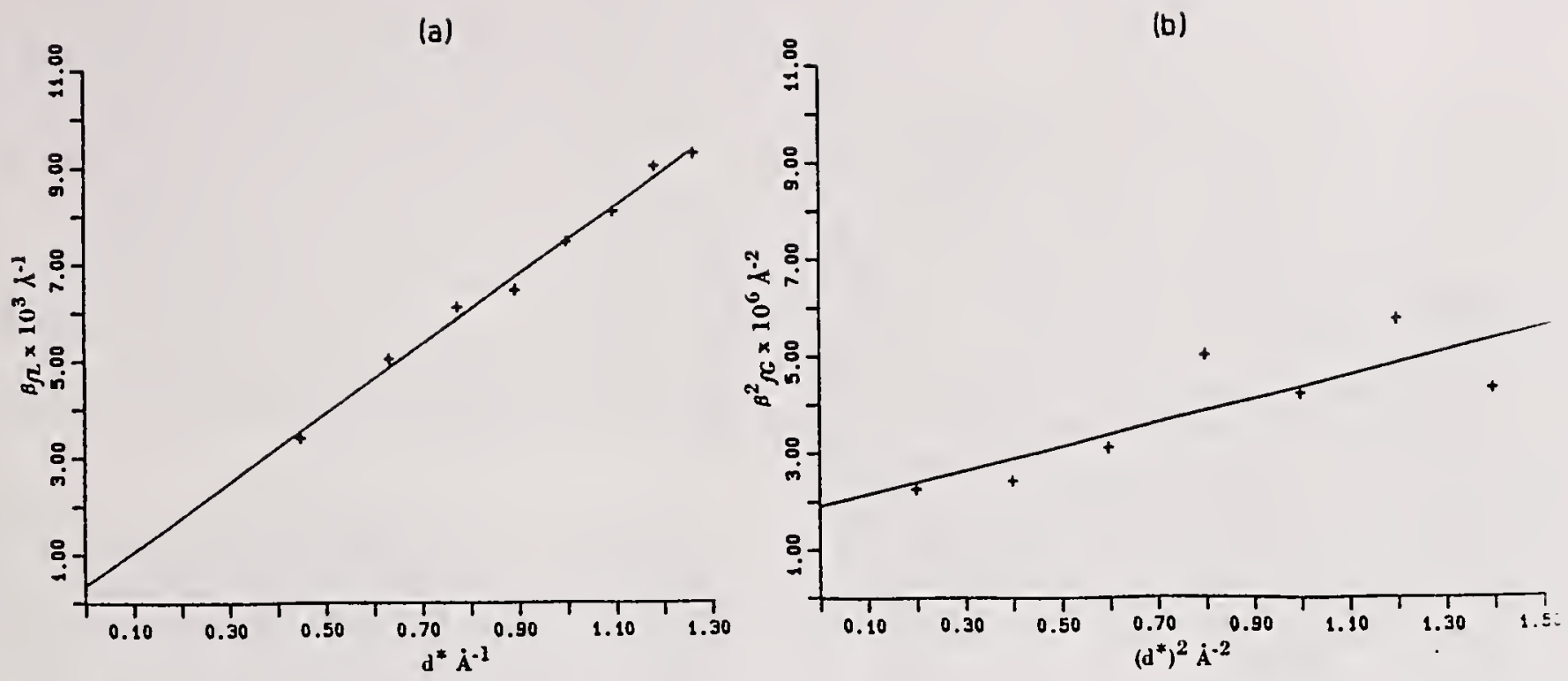

Figure 6: Separation of $d^{*}$-independent and $d^{*}$-dependent contributions to line breadths for tungsten filings (multiple-line analysis). (a) Lorentzian components. (b) Gaussian components.

\section{(b) Multiple Line Analysis}

If the values of $\beta_{f}$ show little or no [ $\left.h k l\right]$ dependence, as is the case for the tungsten sample, $\beta_{S}$ and $\beta_{D}$ may be separated by assuming that both are the breadths of Voigtian functions, with components $\beta_{S L}, \beta_{S G}$ and $\beta_{D L}, \beta_{D G}$ respectively. The average values of these quantities can be obtained from plots of $\beta_{f L}$ versus $d^{*}$ [Fig. 6(a)] and $\beta_{f G}^{2}$ versus $\left(d^{*}\right)^{2}$ [Fig. 6(b)]. If such plots are linear, as are those of figure 6 , then the intercept of figure $6(\mathrm{a})$ gives $\beta_{L}=0.36(3) \times 10^{-3} \AA^{-1}$, and, from figure $6(\mathrm{~b}), \beta_{S G}=1.38(21) \times 10^{-3} \AA^{-1}$. $\beta_{S L}$ and $\beta_{S G}$ can then be combined by means of (12) and (3), with $y=\beta_{S L} /\left(\sqrt{\pi} \beta_{S G}\right)$, to give $\beta_{S}=1.43(25) \times 10^{-3} \AA^{-1}$, with $\langle\varepsilon\rangle=620(100) \AA$. Implicit in the above assumption is that $\eta$ also has Lorentzian and Gaussian components $\eta_{L}$ and $\eta_{G}$. The slope of figure $6(\mathrm{a})$ is then $\eta_{L} / 2$, and that of figure $6(\mathrm{~b})$ is $\left(\eta_{G} / 2\right)^{2}$. By again using (12) and (3), with $y=\eta_{L} /\left(\sqrt{\pi} \eta_{G}\right)$, $\langle\eta\rangle=15.2(6) \times 10^{-3}$, and $\tilde{\mathrm{e}}=3.04(12) \times 10^{-3}$. It should be noted that, as with approach (a), the multiple-line analysis gives average values of crystallite size and microstrain and does not furnish information on any $[h k l]$ dependence of these quantities.

It is also of interest to examine the Voigt shape parameter $\phi$ for the size and strain broadening. From (10), $\left\langle\phi_{S}\right\rangle=0.884(38)$, and $\left\langle\phi_{D}\right\rangle=0.660(5)$. Thus, if $\mathrm{f}(x)$ is in fact a Voigt function, then its size component is strongly Gaussian and the strain component is almost pure Lorentzian. However, from table 2 $\left\langle\phi_{f}\right\rangle(=0.673(12))$ is close to the Lorentzian limit, as would be expected, since the line broadening is dominated by the microstrain component.

\section{(c) Multiple-Order Analysis}

If data for two or more orders of a reflection are available, then a Voigt approach which yields information on crystallite shape and anisotropy of microstrains can be employed. However, this depends on the assumption that, for a given series of reflections, $\beta_{f L}$ and $\beta_{f G}$ vary linearly with $d^{*}$ and $\left(d^{*}\right)^{2}$ respectively. While this is clearly the case for all reflections for the tungsten sample (Fig. 6), in general at least three orders of a reflection are required to confirm that this is so. By using

$$
\beta_{f L}=\beta_{S L}+\left(\eta_{L} / 2\right) d^{*}
$$

and

$$
\beta_{f G}^{2}=\beta_{S G}^{2}+\left(\eta_{G} / 2\right)^{2}\left(d^{*}\right)^{2}
$$

and the procedure outlined in (b) for combining $\beta_{S L}, \beta_{S G}$ and $\beta_{D L}, \beta_{D G}, \varepsilon=720(110) \AA$, and $\tilde{\mathrm{e}}=$ $2.90(9) \times 10^{-3}$ for the 200 and 400 . These values confirm the lack of an $[h k l]$-dependent broadening for the tungsten sample, as before.

\section{(d) Single-Line Analysis}

In the single-line method of Keijser et al. (1982), it is normally assumed that $\beta_{f L}$ is due only to size effects, and $\beta_{f G}$ is attributed to strain. Since this approach is applied to data for each line separately, information on crystallite shape and anisotropy of strain can in principle be extracted, but the results will clearly be influenced markedly by this assumption. In the single-line method

$$
\beta_{f L}=1 / \varepsilon
$$


and

$$
\beta_{f G}=\eta d^{*} / 2 .
$$

Values of $\varepsilon, \eta$ and ẽ obtained from (25) and (26) are given in table 2, from which $\langle\varepsilon\rangle=160(60) \AA$ and $\tilde{\mathrm{e}}$ $=0.93(2) \times 10^{-3}$, which are significantly less than the values obtained previously. Also, from table $2, \varepsilon$ and $\tilde{e}$ decrease steadily, whereas both quantities should be constant, aside from variations due to crystallite shape or elastic anisotropy, for all values of $d^{*}$. However, $\phi_{f}$ is close to the Lorentzian limit for all lines (section 4.4), and the WilliamsonHall plot [Fig. 3(b)] indicates that $\beta_{f}$ is dominated by the effects of microstrains. This suggests that the line profile due to the latter may be predominantly Lorentzian in character. If $\beta_{f L}$ and $\beta_{f G}$ in (25) and (26) are interchanged, then $\langle\varepsilon\rangle=515(100) \AA$, and $\langle\tilde{\mathrm{e}}\rangle=3.00(11) \times 10^{-3}$.

The average values of $\varepsilon$ and $\tilde{e}$ obtained by applying the four approaches to the tungsten data are summarised in table 3 . There is good agreement between the results of $(a)$ to $(c)$; strain is determined with fairly high precision, though the standard deviation in the size estimates is somewhat larger. From the customary procedure for $(d), \varepsilon$ and $\tilde{\mathrm{e}}$ are considerably less than for other methods, but this is to be expected, since it has been shown that Lorentziansize and Gaussian-strain line profiles are not close approximations for the tungsten data. However, this represents a special case which is not necessarily applicable generally. It should be noted that, if data can only be obtained for a few lines and there are no multiple orders, then a single-line analysis is the only method available.

\subsection{Ex-oxalate $\mathrm{ZnO}$ : crystallite-size and 'mistake' broadening}

The microstructure of this sample has been investigated by Langford et al. (1992). The WilliamsonHall plot revealed that there are three catagories of reflections, and those of group 1 ( $h k 0$ and $h-k=$ $3 n$ ), which are unaffected by stacking faults, are used to obtain the mean crystallite size and shape. By assuming a spherical morphology, $\langle D\rangle=453(17) \AA$, and, if the form is assumed to be cylindrical, $\langle D\rangle=$ $404(7) \AA$, and $\langle H\rangle=351(9) \AA$. Although there is little to choose between the two models from statistical considerations, the latter is probably more realistic on physical grounds. As noted previously (section 4.4), $\mathrm{f}(x)$ for group 1 reflections has approximately the same shape for all $h k l$ and has a significant Gaussian component. The ex-oxalate $\mathrm{ZnO}$ is strain-free, but if measurable microstrains were present, then the analysis of 5.2 would be applied to the group 1 reflections.

There are no measurable peak displacements for this sample, which suggests that the lattice 'mistakes' are stacking faults, since other forms of fault can introduce a peak shift. The next stage in the analysis is to separate the breadth contribution $\beta_{m}$ due to stacking faults from that arising from size effects ( $\beta_{S}$ for groups 2 and 3 reflections). For this purpose, $\beta_{S}$ is calculated from (A2) or (A4) and (A5), according to whether the spherical or cylindrical model is used. There are then two approaches for obtaining $\beta_{m}$. The simpler method is to assume that the 'size' and 'fault' profiles are both Lorentzian, when

$$
\beta_{m}=\beta_{f}-\beta_{S} .
$$

The theoretical line profile for stacking-fault broadening is approximately Lorentzian (Wilson, 1962, chapter 6), but it has been demonstrated that the 'size' profiles are not. Thus, an approach based on the Voigt function can again be applied, where

$$
\beta_{m}=\beta_{f L}-\beta_{S L}
$$

and

$$
\beta_{S G}=\beta_{f G} .
$$

Since $\beta_{f G}$ and $\phi_{S}$ are known from the group 1 reflections, $\beta_{S L}$ can be obtained by solving (11) for $y$ (Langford et al., 1992, Appendix I). $\beta_{m}$ is then given by (28).

The final stage is to obtain the stacking-fault probability $\alpha$ from $\beta_{m}$, and for hexagonal materials (from Wilson, 1962, chapter 6)

$$
\beta_{m}=\alpha \cos \phi_{z} / 2 c, h-k=3 n \pm 1, l \text { odd }
$$

and

$$
\beta_{m}=3 \alpha \cos \phi_{z} / 2 c, h-k=3 n \pm 1, l \text { even. }
$$

From the Lorentzian approximation, cylindrical model, $\langle\alpha\rangle=0.0069(11)$ for group 2 reflections and $\langle\alpha\rangle=0.0068(3)$ for group 3 . These values correspond to a mean separation between stacking faults $(=c / 2 \alpha)$ of $380(30) \AA$, or about one fault per crystallite, on average; $i$. e., according to this approximation, the crystallites are in fact domains bounded by fault planes.

By assuming that the 'size' profiles are Voigtian, $\langle\alpha\rangle=0.014(3)$ for the group 2 reflections and $\langle\alpha\rangle=$ $0.009(1)$ for group 3 , with about two faults per crystallite, on average. The difference between the two estimates of $\alpha$ suggests that the line profiles due to stacking faults are not strictly Lorentzian. An analysis based on Lorentzian functions gives highly consistent results, though there are clearly systematic errors. That based on the Voigt function is probably more accurate, though less precise. For a more 
Table 3: Summary of size-strain analysis for tungsten filings

\begin{tabular}{|c|c|c|c|c|c|}
\hline Method & $\begin{array}{l}\langle\varepsilon\rangle \\
\AA\end{array}$ & $\left\langle\phi_{\varepsilon}\right\rangle$ & $\begin{array}{c}\langle\eta\rangle \\
\times 10^{3} \\
\end{array}$ & $\left\langle\phi_{\eta}\right\rangle$ & $\begin{array}{r} \\
\tilde{\mathrm{e}} \\
\times 10^{3} \\
\end{array}$ \\
\hline 'Average' s-s plot & $640(70)$ & - & $14.9(3)$ & - & $2.98(5)$ \\
\hline Multiple line & $620(100)$ & $0.884(38)$ & $15.2(6)$ & $0.660(5)$ & $3.04(12)$ \\
\hline $\begin{array}{ll}\text { Multiple order } & \text { (a) } h h 0 \\
& \text { (b) } h 00\end{array}$ & $\begin{array}{l}720(110) \\
580(90)\end{array}$ & $\begin{array}{l}0.872 \\
0.808\end{array}$ & $\begin{array}{l}15.4(6) \\
14.5(5)\end{array}$ & $\begin{array}{l}0.675 \\
0.663\end{array}$ & $\begin{array}{l}3.07(11) \\
2.90(9)\end{array}$ \\
\hline $\begin{array}{l}\text { Single line (average) } \\
\text { (a) L size G strain } \\
\text { (b) G size L strain }\end{array}$ & $\begin{array}{l}160(60) \\
520(100)\end{array}$ & - & $\begin{array}{l}4.70(10) \\
15.1(5)\end{array}$ & - & $\begin{array}{l}0.93(2) \\
3.00(11)\end{array}$ \\
\hline
\end{tabular}

detailed discussion of the analysis of the ex-oxalate $\mathrm{ZnO}$ data, see Langford et al., 1992.

Other cases of 'mistake' broadening are treated similarly. For example, twinning in the orthorhombic phase of the high $\mathrm{T}_{c}$ ceramic $\mathrm{YBa}_{2} \mathrm{Cu}_{3} \mathrm{O}_{7-\delta}$ does not affect the $h h l$. planes. In this case a parallelepiped, given by (A8), can be used to model the domain shape (Rand, Langford \& Abell, 1992). A theoretical treatment of the line broadening and peak displacement due to various types of mistake is given in Wilson, 1962. (See also Warren, 1969, chapter 13).

\subsection{Ex-hydroxide nitrate $Z n O$, sample $B$ : size and strain broadening}

The Williamson-Hall plot indicates that, at least to a first approximation, there are two main groups of reflections for $\mathrm{ZnO}$ sample $B$, namely $h k l$ with $h, k \neq 0$ and $00 l$. The 'average' size-strain plot and multiple-line analysis can be applied to the former group, since the [hkl] dependence of the breadths of these reflections is not large. From the intercept of the former [(22) and Fig. 7] $\langle\eta\rangle=9.8(5) \times 10^{-3}$, and $\langle\tilde{e}\rangle=1.96(11) \times 10^{-3}$. From the slope, $\langle\varepsilon\rangle=$ $240(12) \AA$, and, if spherical domains are assumed, $\langle D\rangle=320(16) \AA$. However, from a multiple-order analysis (Sonneveld et al., 1986), there appears to be a small $[h k l]$ dependence of both size and strain broadening, and, from the former, the domain shape can be modelled by a cylinder of diameter about $375 \AA$ and height $420 \AA$. The strain estimated by this method was found to be $2.9 \times 10^{-3}$ in the basal plane of the cylinder, with $2.0 \times 10^{-3}$ in the direction of its axis. The results of a single-line analysis are also given by Sonneveld $e t$ al. and, excluding $00 l$ data, $\langle\varepsilon\rangle=270(50) \AA$ and $\langle\tilde{e}\rangle=2.4(5) \times 10^{-3}$ from this approach, and, for a spherical model, $\langle D\rangle=360(70) \AA$. The agreement between the values estimated from

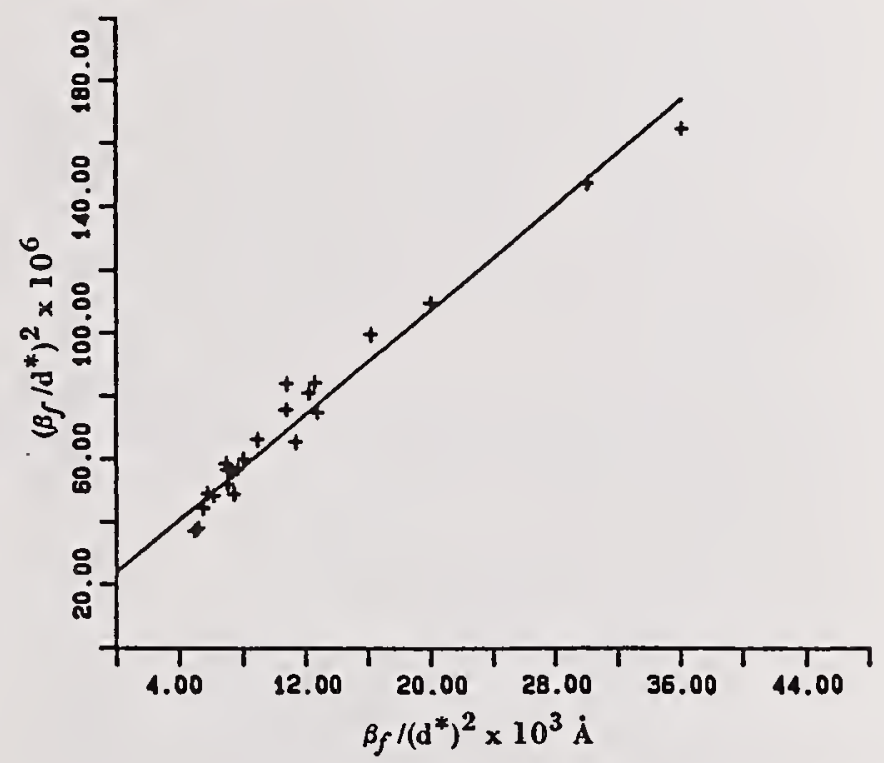

Figure 7: 'Average' size-strain plot for ex-hydroxide nitrate $\mathrm{ZnO}$, sample $B$.

the single-line approach and those from other methods is thus better than for the tungsten filings. This is to be expected, since it has been shown that the line profiles due to microstrains are more Gaussian in character for $\mathrm{ZnO}$ sample $B$ (section 4.4).

\section{Summary}

After obtaining data of adequate quality for the sample of interest and for an instrumental standard, followed by pattern decomposition, the procedure for carrying out line profile analysis by means of the Voigt function is as follows:

1. Compare the angular variation of the FWHM with the IRF to ascertain if there is measur- 
able sample broadening, and if there is any [hkl] (i.e., lattice-direction) dependence (section 4.1). If an annealed version of the sample is used as an instrumental standard, then the FWHM for this should also be compared with the IRF. Use NBS*AIDS83 or an equivalent program to ascertain if there are any measurable peak shifts.

2. Check that the Voigt function is appropriate for analysing line breadths (criterion (7) and inappreciable asymmetry: section 2.1)

3. Correct data for instrumental effects to obtain breadths $\beta_{f}$ and $2 w_{f}$ for the sample line profiles $[(14) \&(15)]$ and hence the shape factors $\phi_{f}(4)$. Obtain $\beta_{f L}$ and $\beta_{f G}$ from (11), (12) and (3) and convert all integral breadths to reciprocal units (section 4.2).

4. Make Williamson-Hall plot $\left(\beta_{f}\right.$ versus $\left.d^{*}\right)$ and add Miller indices. Ascertain the nature of the sample broadening and note any $[h k l]$ dependence of line breadths (section 4.3). Examine $\phi_{f}$ to determine the Lorentzian/Gaussian character of $\mathrm{f}(x)$, and whether the profile shape has any $d^{*}$ or $[h k l]$ dependence (section 4.4 ).

5. Carry out a detailed analysis of line breadths in accordance with the information obtained from step 4. This should be based on one or more of the procedures given in section 5 :

\section{1: Size-only broadening}

5.2: 'Isotropic' size and strain broadening

5.3: Size and 'mistake' broadening

5.4: 'Anisotropic' size and strain broadening

\section{Conclusions}

The Voigt function can be used in the majority of cases to study microcrystalline properties by means of diffraction data. It is only inapplicable when line profiles are markedly asymmetric, and when the shape factor $\phi_{h}$ is outside of the Voigtian range. The success of the method depends critically on the quality of the data, the number of lines recorded (except for high symmetry materials) and the calibre of the pattern-decomposition program used. The last can be a limitation at present, and pattern decomposition is an area where further development is needed. From the examples given in section 5 , it is evident that a considerable amount of information on structural imperfections can be obtained from an approach based on the Voigt function, and that, particularly for the ex-oxalate $\mathrm{ZnO}$ and tungsten samples, a relatively high degree of precision can be achieved. However, the assumptions made in the analysis introduce systematic errors, particularly when 'size' and 'strain' broadening occurs simultaneously, and the results are not necessarily accurate. Nevertheless, if meaningful data are available for a large number of lines, the degree of self-consistency usually gives an indication of the validity of the results. The foregoing has been concerned with studies of microstructural properties by using data from observable reflections. However, after obtaining parameters defining structural imperfections, it is a relatively simple matter to reverse the procedure given above to model all line profiles of a diffraction pattern, as is required in the Rietveld method for the refinement of crystal structures. This technique has been discussed further by Delhez et al. (1993).

Much of this work originated from collaboration over a period of many years with Dr. D. Louër, of the University of Rennes, France. I am indebted to him for providing the $\mathrm{ZnO}$ data and also to $\mathrm{Dr}$. J. P. Auffrédic, who prepared the samples. I am also grateful to Professor E. J. Mittemeijer and his colleagues of TU Delft, the Netherlands, for many helpful discussions and assistance in developing the method of line profile analysis based on the Voigt function. 
Appendix 1. Evaluation of complex error function.

FUNCTION CDWERF(Z) 01

C Evaluation of complex error function $\omega(x+i y)=\exp \left(-z^{2}\right)(1-\operatorname{erf}(-i z)) \quad 02$

C J.I. Langford, School of Physics \& Space Research, University of 03

C Birmingham, Birmingham B15 2TT, UK. 04

C 15.iii.82; based on a CERN Computer Centre algorithm 05

C 06

IMPLICIT DOUBLE PRECISION (R-Y),COMPLEX*16 (D,Z) 07

DOUBLE PRECISION VCONST $\quad 08$

COMPLEX*16 CDWERF $\quad 09$

REAL LAMDA 10

LOGICAL B 11

VCONST $=1.0 / \mathrm{DSQRT}(\operatorname{DATAN}(0.1 \mathrm{D} 01)) \quad 12$

$\mathrm{XX}=\mathrm{DREAL}(\mathrm{Z}) \quad 13$

$\mathrm{YY}=\mathrm{DIMAG}(\mathrm{Z}) \quad 14$

$\begin{array}{ll}\mathrm{X}=\mathrm{DABS}(\mathrm{XX}) & 15\end{array}$

$\mathrm{Y}=\mathrm{DABS}(\mathrm{YY}) \quad 16$

IF (Y.LT.4.29.AND.X.LT.5.33) GO TO 1

$\mathrm{W}=0 . \quad 18$

$\mathrm{NC}=0 \quad 19$

$\mathrm{NU}=8 \quad 20$

LAMDA $=0 . \quad 21$

$\mathrm{B}=$.TRUE. $\quad 22$

GO TO 2

$1 \mathrm{~S}=(1.0-\mathrm{Y} / 4.29)^{*} \operatorname{SQRT}\left(1.0-(\mathrm{X} / 5.33)^{* *} 2\right)$

$\mathrm{W}=1.6^{*} \mathrm{~S} \quad 25$

$\mathrm{W} 2=2.0^{*} \mathrm{~W} \quad 26$

$\mathrm{NC}=6+\operatorname{INT}\left(23.0^{*} \mathrm{~S}\right) \quad 27$

$\mathrm{NU}=9+\mathrm{INT}\left(21.0^{*} \mathrm{~S}\right) \quad 28$

LAMDA $=\mathrm{W} 22^{* *} \mathrm{NC} \quad 29$

$\mathrm{B}=$ LAMDA.EQ.0.

$2 \quad \mathrm{R} 1=0 . \quad 31$

$\mathrm{R} 2=0 . \quad 32$

$\mathrm{S} 1=0 . \quad 33$

$\mathrm{S} 2=0 . \quad 34$

$\mathrm{N}=\mathrm{NU}+1$

$3 \quad \mathrm{~N}=\mathrm{N}-1$

$\mathrm{VN}=\mathrm{N}+1 \quad 37$

$\mathrm{T} 1=\mathrm{Y}+\mathrm{W}+\mathrm{VN} * \mathrm{R} 1$

$\mathrm{T} 2=\mathrm{X}-\mathrm{VN} * \mathrm{R} 2 \quad 39$

$\mathrm{U}=0.5 /(\mathrm{T} 1 * \mathrm{~T} 1+\mathrm{T} 2 * \mathrm{~T} 2)$

$\mathrm{R} 1=\mathrm{U}^{* \mathrm{~T} 1}$

$\mathrm{R} 2=\mathrm{U}^{*} \mathrm{~T} 2$

IF (W.LE.0.0.OR.N.GT.NC) GO TO 4

$\mathrm{T} 1=\mathrm{LAMDA}+\mathrm{S} 1$

$\mathrm{S} 1=\mathrm{R} 1{ }^{*} \mathrm{~T} 1-\mathrm{R} 2{ }^{*} \mathrm{~S} 2$

$\mathrm{S} 2=\mathrm{R} 2 * \mathrm{~T} 1+\mathrm{R} 1{ }^{*} \mathrm{~S} 2 \quad 46$

LAMDA = LAMDA/W2 $\quad 47$

4 IF (N.GT.0) GO TO $3 \quad 48$

$\mathrm{C}^{* * * * * * * * * * * * * * * * * * * * * * * * * * * * * * * * * * * * * * * * * * * * * * * * * * * * * * * * * * * * * * * * * * * * * * * * * * * * * *}$ 


\begin{tabular}{|c|c|c|}
\hline & IF (B) GO TO 5 & 49 \\
\hline & $\mathrm{RS} 1=\mathrm{S} 1$ & 50 \\
\hline & $\mathrm{RS} 2=\mathrm{S} 2$ & 51 \\
\hline & GO TO 6 & 52 \\
\hline \multirow[t]{2}{*}{5} & $\mathrm{RS} 1=\mathrm{R} 1$ & 53 \\
\hline & $\mathrm{RS} 2=\mathrm{R} 2$ & 54 \\
\hline \multirow[t]{6}{*}{6} & $\mathrm{RS} 1=\mathrm{VCONST} * \mathrm{RS} 1$ & 55 \\
\hline & $\mathrm{IF}(\mathrm{Y} . \mathrm{EQ} .0 .0) \mathrm{RS} 1=\operatorname{DEXP}(-\mathrm{X} * \mathrm{X})$ & 56 \\
\hline & CDWERF = DCMPLX (RS1, VCONST $*$ RS2) & 57 \\
\hline & IF (YY.LT.0.0) GO TO 7 & 58 \\
\hline & IF (XX.LT.0.0) CDWERF = DCONJG(CDWERF) & 59 \\
\hline & RETURN & 60 \\
\hline \multirow[t]{4}{*}{7} & CDWERF $=2.0^{*} \operatorname{CDEXP}\left(-\mathrm{DCMPLX}(\mathrm{X}, \mathrm{Y})^{* *} 2\right)-\mathrm{CDWERF}$ & 61 \\
\hline & IF (XX.GT.0.0) CDWERF = DCONJG(CDWERF) & 62 \\
\hline & RETURN & 63 \\
\hline & END & 64 \\
\hline
\end{tabular}

Appendix 2. Apparent and 'true' size of crystallites or domains

The reciprocal of the integral breadth of a diffraction line profile due to the finite size of crystallites or domains is the volume-weighted average thickness $\varepsilon$, measured in the direction of the diffraction vector. $\varepsilon$ is thus an apparent size and, if $t$ is a distance measured in this direction, then (Wilson, 1962, chapter 4)

$$
\varepsilon=\frac{1}{V(0)} \int_{V} t \mathrm{~d} v
$$

where $V(0)$ is the volume of the crystallite. By taking into account the $h k l$ dependence of $\varepsilon$ and assuming that on average the crystallites have a particular form, the mean dimensions can be obtained by a non-linear least-squares procedure, in which $\varepsilon_{o b s}$ is compared with $\varepsilon_{c a l}$, the value calculated from parameters describing the selected shape and its orientation with respect to the crystallographic axes. A useful construction for providing a visual indication of the validity of the model is given by a comparison of the observed and calculated thickness, $\tau_{o b s}$ and $\tau_{c a l}$, measured from a suitable origin in the direction of the diffraction vector. Equations for $\varepsilon$ and $\tau$ are given below for crystallites having the form of spheres, cylinders and parallelepipeds.

\section{Spheres:}

For spherical crystallites of diameter $D$ [Wilson (1962), chapter 4, (12)]

$$
\varepsilon=3 D / 4
$$

and

$$
\tau=D
$$

for all $h k l$.

Cylinders:

$\varepsilon$ for cylindrical crystallites of diameter $D$ and height $H$ for a reflection whose normal makes an angle $\phi_{z}$ with the cylinder axis may be obtained from Langford and Louër (1982), (10), (17) and (18):

$$
\begin{aligned}
\varepsilon= & \frac{D}{\pi} \csc \phi_{z}\left[\frac{8}{3}+2 q \cos ^{-1} q\right. \\
& -\frac{1}{2 q} \sin ^{-1} q-\frac{5}{2} \sqrt{\left(1-q^{2}\right)} \\
& \left.+\frac{1}{3} \sqrt{\left(1-q^{2}\right)^{3}}\right], 0 \leq \phi_{z} \leq \Phi
\end{aligned}
$$

or

$$
\varepsilon=D \csc \phi_{z}\left[\frac{8}{3 \pi}-\frac{1}{4 q}\right], \Phi \leq \phi_{z} \leq \frac{\pi}{2},
$$

where $q=H \tan \phi_{z} / D$ and $\Phi=\tan ^{-1}(D / H)$. In this instance it is convenient to take as origin the intersection of a diameter of the base and a generator of the cylinder, when

$$
\tau=H \sec \phi_{z}, 0 \leq \phi_{z} \leq \Phi,
$$

and

$$
\tau=D \csc \phi_{z}, \Phi \leq \phi_{z} \leq \frac{\pi}{2} .
$$

While crystallites are unlikely to be cylindrical in reality, a hexagonal prism may be approximated by this form.

Parallelepipeds: The procedure introduced by Wilson (1962, chapter 4) can be used to obtain $\varepsilon$ for 
a parallelepiped. Consider a parallelepiped defined by the vectors $\mathbf{A}, \mathbf{B}$, and $\mathbf{C}$, with angles $\alpha=\mathbf{B}^{-} \mathbf{C}$, $\beta=\mathbf{C}^{\wedge} \mathbf{A}$, and $\gamma=\mathbf{A} \wedge \mathbf{B}$, and with a vertex as the origin. If $\mathbf{A}$ is taken along the $x$ axis, $\mathbf{B}$ is in the $x-y$ plane, and $\phi_{x}, \phi_{y}, \phi_{z}$ are the angles between the direction of the diffraction vector and $x, y$ and $z$ respectively, then

$$
\begin{aligned}
\varepsilon= & 2 \tau\left[1-\frac{1}{2}(p+q+r) \tau\right. \\
& \left.+\frac{1}{3}(p q+q r+r p) \tau^{2}-\frac{1}{4} p q r \tau^{3}\right]
\end{aligned}
$$

where

$$
\begin{aligned}
p & =\cos \phi_{x} / A \\
q & =\cos \phi_{y} \csc \gamma / B \\
r & =\cos \phi_{z} \sin \gamma / C D
\end{aligned}
$$

with

$D=\left(1-\cos ^{2} \alpha-\cos ^{2} \beta-\cos ^{2} \gamma+2 \cos \alpha \cos \beta \cos \gamma\right)^{1 / 2}$.

The value of $\tau$ to be used in (A8) depends on which face is intersected by a vector through the origin and parallel to the diffraction vector. If face $A$ is defined as the face parallel to the plane containing $\mathbf{B}$ and $\mathbf{C}$, at a distance $A$ from it, with similar definitions for faces $B$ and $C$, then,

if $\tau$ terminates in face $A$,

$$
\tau=\frac{A \sin \gamma}{\sin \gamma \cos \phi_{x}-\cos \gamma \cos \phi_{y}-F \cos \phi_{z} / D}
$$

if $\tau$ terminates in face $B$,

$$
\tau=\frac{B \sin \gamma}{\cos \phi_{y}-E \cos \phi_{z} / D}
$$

and if $\tau$ terminates in face $\mathrm{C}$,

$$
\tau=\frac{C D \csc \gamma}{\cos \phi_{z}}
$$

where $E=\cos \alpha-\cos \beta \cos \gamma$ and $F=\cos \beta-$ $\cos \gamma \cos \alpha$.

The above equations simplify considerably for a rectangular parallelepiped, which can be used to model a 'tetragonal' form and a cube. The orientation of the parallelepiped relative to the orthogonal axes $x, y, z$ was chosen arbitrarily in the above. In any particular application it may be necessary to rotate $\mathbf{A}, \mathbf{B}, \mathbf{C}$ by means of the Euler transformation.

\section{References}

Ahtee, M., Unonius, L., Nurmela, M., \& Suortti, P. (1984). J. Appl. Cryst. 17, 352-357.

Delhez, R., KeiJSer, Th. H. De, Langford, J. I., LouËr, D., MitTemeiJer, E. J. \& SonNeveld, E. J. (1993). In The Rietveld Method. R. A. Young, ed. Oxford: Oxford University Press, pp.132-166, in press.

Delhez, R. \& MittemeiJer, E. J. (1975). J. Appl. Cryst. 8, 609-611.

Fawcett, T. G., Crowder, C. E., Brownell, S. J., Zhang, Y., Hubbard, C. R., Schreiner, W., Hamill, G. P., Huang, T. C., Sabino, E., LangFORD, J. I., HAMILTON, R. \& LOUËR, D. (1988). Powder Diff. 3, 209-218.

HALDER, N. C. \& WAGNER, C. N. J. (1966). Adv. X-ray Anal. 9, 91-102.

Hill, R. J. \& Madsen, I. C. (1987). Powder Diff. 2, 146-162.

JCPDS/ICDD (1989). Methods \& Practices in $X$ Ray Powder Diffraction, R. Jenkins, ed. Swarthmore, PA:ICDD.

Keijser, Th. H. DE, Langford, J. I., Mittemeijer, E. J. \& Vogels, A. P. B. (1982). J. Appl. Cryst. 15, 308-314.

Keijser, Th. H. DE, Mittemeijer, E. J. \& RozenDAAL, H. C. F. (1983). J. Appl. Cryst. 16, 309-316.

LangFord, J. I. (1978). J. Appl. Cryst. 11, 10-14.

Langford, J. I. (1980). In Accuracy in Powder Diffraction, NBS Spec. Pub. No. 567, S. Block and C. R. Hubbard, eds. pp 255-269. Gaithersburg, MD: U.S. Department of Commerce.

Langford, J. I. (1987). Prog. Cryst. Growth $\&$ Charact. $14,185-211$.

LangFord, J. I., Boultif, A., Auffrédic, J. P. \& LOUËR, D. (1992). J. Appl. Cryst. 25, In press.

LANGFord, J. I., CERNIK, R. J. \& LOUËR, D. (1991). J. Appl. Cryst. 24, 913-919.

Langford, J. I., Delhez, R., Keijser, Th. H. De \& MittemeiJer, E. J. (1988). Aust. J. Phys. 41, 173187.

Langford, J. I. \& LouËr, D. (1982). J. Appl. Cryst. 15, 20-26.

LANGFORD, J. I., LouËR, D., SONNEveld, E. J. \& Visser, J. W. (1986). Powder Diff. 1, 211-221.

Louër, D., Auffrédic, J. P., Ciosmak, D. \& NiePce, J. C. (1983). J. Appl. Cryst. 16, 183-191.

Louër, D. \& LangFord, J. I. (1988). J. Appl. Cryst. 21, 430-437.

Mighell, A. D., Hubbard, C. R. \& Stalick, J. K. (1981). NBS Technical Note No.1141. Gaithersburg, MD: U.S. Department of Commerce.

Plévert, J. \& LouËr, D. (1990). J. Chim. Phys. 87, 1427-1440.

RACHINGer, W. A. (1948). J. Sci. Instrum. 25, 254-255.

RAND, M., LANGFord, J. I. \& Abell, J. S. (1992). J. Cryogenics, submitted for publication. 
Smith, D. K. \& Gorter, S. (1991). J. Appl. Cryst. 24, 369-401.

Sonneveld, E. J., Delhez, R., KeIJSER, Th. H. DE, LANGFord, J. I., MitTEMEIJER, E. J., VisSer, J. W. \& LoUËR, D. (1986). In Proceedings of 12 th Conference on Applied Crystallography, Cieszyn, Poland. Katowice: Silesian University.

Stokes, A. R. \& Wilson, A. J. C. (1944). Proc. Phys. Soc. Lond. 56, 174-181.

VARGAS, R., LOUËR, D. \& LANGFoRD, J. I. (1983). $J$. Appl. Cryst. 16, 512-518.

WARREN, B. E. (1969). $X$-ray Diffraction. Reading MA: Addison- Wesley.
Wertheim, G. K., Butler, M. A., West, K. W. \& Buchanan, D. N. E. (1974). Rev. Sci. Instrum. 45, 1369-1371.

WILES, D. B. \& Young, R. A. (1981). J. Appl. Cryst. 14, 149-151.

Will, G., Parrish, W. \& Huang, T. C. (1983). J. Appl. Cryst. 16, 611-622.

Williamson, G. K. \& Hall, W. H. (1953). Acta Met. 1, 22-31.

WILSoN, A. J. C. (1962). X-ray Optics, 2nd edition. London: Methuen.

WILSON, A. J. C. (1963). Mathematical Theory of X-ray Powder Diffractometry. Eindhoven: Centrex. 
National Institute of Standards and Technology Special Publication 846. Proceedings of the international conference Accuracy in Powder Diffraction II, held at NIST, Gaithersburg, MD, May 26-29, 1992. (Issued October 1992)

\title{
Preferred Orientation Effects
}

\author{
V. VALVODA \\ Charles University, Faculty of Mathematics and Physics, \\ 12116 Prague 2, Czechoslovakia
}

\begin{abstract}
Methods of texture correction in samples with axial symmetry introduced by spinning the sample during measurements are reviewed and experimentally tested. The most accurate methods are based on a representation of the orientation distribution of crystallites by a set of symmetrized harmonics with coefficients that can be determined from the orientation distribution of a small number of crystallographic planes (as measured on a texture goniometer) or from the relative intensities of all reflections (as measured on a powder diffractometer) when the structure of the sample is known, at least approximately. The latter case is met in quantitative phase analysis and in structure refinement procedures. In special cases of fiber textures simpler analytical functions (like exponential functions) are sufficient for texture description and handling. The Harris texture index can also be used for texture characterization and correction when the texture is not too strong.
\end{abstract}

\section{Introduction}

Non-random orientation distribution of crystallites in powder specimens or compact polycrystalline samples can influence almost all accurate diffraction measurements. This regards mainly quantitative phase analysis and structure determination because both these methods are based on intensities of reflections from a randomly oriented assembly of particles. However, a strong preferred orientation (texture) also causes problems in phase identification, stress measurements and profile analysis, especially in thin films. On the other hand, as has been pointed out by Bunge, Dahms \& Brokmeier (1989), texture can also help to separate completely overlapped reflections, like 330 and 411 reflections in the cubic $\gamma$-brass structure, if the sample has an approriate preferred orientation.

The effect of preferred orientation in powder specimens can be reduced by special preparation tech- niques (see, e.g., Smith \& Barrett, 1979). However, such techniques cannot be used in the case of compact polycrystalline samples and, as has been shown by Suortti \& Jennings (1977), the inhomogeneity of the packing of the crystallites in a loosely pressed specimen will cause an uncontrollable variation in the intensity distribution, making accurate intensity measurements impossible. Another experimental method of texture suppression is a measurement that integrates diffracted intensities over all (or almost all) sample orientations (Will, 1991). This approach is time consuming, and accuracy is usually limited by incomplete pole figure integration together with systematic errors appearing at large inclinations of flat specimens.

The present paper is mainly focused on methods of correction of integrated intensities for the effect of preferred orientation in samples with axial symmetry introduced by spinning the sample during measurements. These methods have found application in accurate powder diffractometry directed to structure refinement and phase analysis or to studies of charge distribution and thermal vibrations of atoms.

\section{Theoretical background and corrections based on texture goniometer measurements}

The sample statistics in powder diffraction are improved by spinning the sample. The rotation of the sample introduces in every crystallite a special direction $\mathbf{P}$ called the polar axis (in the case of the Bragg-Brentano diffractometer this polar axis coincides with the normal to the flat sample surface). The polar axis density, $W(\theta, \varphi)$, is defined as proportional to the volume fraction, $d V / V$, of the diffracting crystallites whose polar axis falls within the solid angle $d \Omega$ about the direction $(\theta, \varphi)$ in the crystallographic coordinate system (Fig. 1),

$$
W(\vartheta, \varphi)=\frac{d V}{V} \frac{d \Omega}{4 \pi}
$$

In a randomly oriented sample $W(\theta, \varphi)=W_{\text {ran }}=1$. 

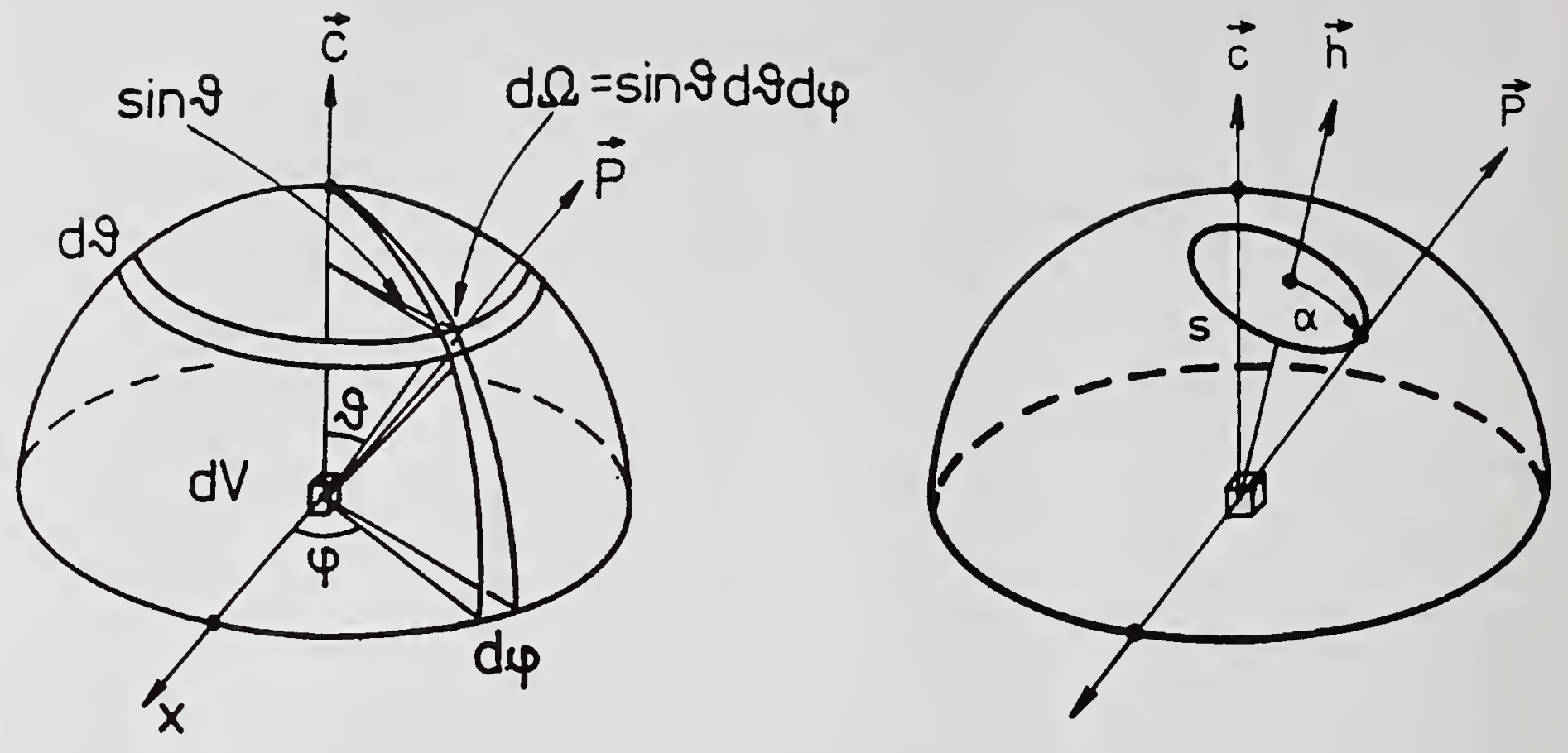

Figure 1: Left: Polar axis $\mathbf{P}$ in a solid angle $d \Omega$ in the direction of $(\theta, \varphi)$ in a crystallographic coordinate system. Right: During integration of reflection $\mathbf{h} \equiv h k l$ the polar axis $\mathbf{P}$ is circulating along the circle $s$ whose radius corresponds to the angle $\alpha$ of sample inclination.

When measuring the intensity of reflection $h \mathrm{kl}$ with the sample inclined by angle $\alpha$ (which is the angle between the reciprocal lattice vector $\mathbf{h} \equiv[h k l]$ and the polar axis $\mathbf{P}$ ) only those crystallites whose polar axes $\mathbf{P}$ are pointing in a direction that forms angle $\alpha$ with the direction $\mathbf{h}$ can contribute to the measured intensity (Fig. 1, right). When the sample is spinning, the direction $\mathbf{P}$ turns uniformly along the circle $s$ on the unit sphere, and the integrated intensity of reflection $\mathbf{h}$ is

$$
I^{h}(\alpha)=\frac{I_{r a n}^{h}}{2 \pi \sin \alpha} \oint W(\vartheta, \varphi) d s,
$$

where $I_{\text {ran }}^{h}$ is the intensity corresponding to a randomly oriented sample. Because the integral on the right side of equation 2 depends solely on the value of angle $\alpha$ we can rewrite this equation in the form

$$
I^{h}(\alpha)=I_{\text {ran }}^{h} W^{h}(\alpha),
$$

where $W^{h}(\alpha)$ represents an average polar axis density along the circle of integration. The intensity $I_{r a n}^{h}$ of the random sample can be thus obtained from the intensity $I^{h}(0)$ measured in ordinary diffraction geometry (i.e. with $\alpha=0$ ) if we know the value $W^{h}(0)=W\left(\theta_{h}, \varphi_{h}\right):$

$$
I^{h}(0)=I_{r a n}^{h} W^{h}(0) .
$$

In principle, $W\left(\theta_{h}, \varphi_{h}\right)$, as well as the pole density at any other location, could be determined from complete texture analysis by measuring pole density distributions of a number of diffracting planes followed by inversion of the data to yield pole densities for all other, unmeasured, planes (see, for example, Roe \& Kriegbaum, 1964 or Bunge, 1985). Such measurements require a multiaxis pole-figure goniometer and correction for such effects as absorption, defocusing and surface roughness.

Method A. A variant of this approach, specific for a spinning sample, has been applied by Järvinen, Merisalo, Pesonen \& Inkinen (1970), Pesonen, Järvinen \& Kurki-Suonio (1973) and Pesonen (1979). This method involves an analytical description of the polar axis density $W$ by means of the expansion in terms of a set of properly symmetrized harmonics $Y_{i j}$ :

$$
W(\vartheta, \varphi)=\sum_{i j} C_{i j} Y_{i j}(\vartheta, \varphi) .
$$

Performing the integration (2) results in the equation

$$
W^{h}(\alpha)=\sum_{i j} C_{i j} Y_{i j}\left(\vartheta_{h}, \varphi_{h}\right) P_{i}(\cos \alpha),
$$

where $P_{i}(\cos \alpha)$ is a Legendre polynomial and $\left(\theta_{h}, \varphi_{h}\right)$ are the spherical coordinates of the reciprocal lattice vector $\mathbf{h}$ with respect to crystallographic 
Table 1: Picking rules for indices of symmetrized harmonics

$$
Y_{i j \pm}=N_{i j} P_{i}^{j}(\cos \vartheta) \begin{array}{ll}
\cos j \varphi & (\text { for }+) \\
\sin j \varphi & (\text { for }-)
\end{array}
$$

\begin{tabular}{|c|c|c|}
\hline Laue class & $\begin{array}{l}\text { Choice of } \\
\text { coordinate } \\
\text { axes }\end{array}$ & $\begin{array}{l}\text { Indices of } \\
\text { symmetrized } \\
Y_{i, j, \pm}\end{array}$ \\
\hline$\overline{1}$ & any & $(2 \lambda, \mu, \pm)$ \\
\hline $2 / m$ & $z \| 2, z \perp m$ & $(2 \lambda, 2 \mu, \pm)$ \\
\hline$m m m$ & $z\|m, y \perp m, z\| m$ & $(2 \lambda, 2 \mu,+)$ \\
\hline $4 / m$ & $z \| 4, z \perp m$ & $(2 \lambda, 4 \mu, \pm)$ \\
\hline $4 / m m m$ & $z \| 4, y \perp m$ & $(2 \lambda, 4 \mu,+)$ \\
\hline$\overline{3}$ & $z \| \overline{3}$ & $(2 \lambda, 3 \mu, \pm)$ \\
\hline$\overline{3} m$ & $z \| \overline{3}, y \perp m$ & $(2 \lambda, 3 \mu,+)$ \\
\hline & $x \perp m$ & $\begin{array}{l}(2 \lambda, 3 \mu,+ \\
\text { for even } \mu, \\
- \text { for odd } \mu)\end{array}$ \\
\hline $6 / m$ & $z \| 6, z \perp m$ & $(2 \lambda, 6 \mu, \pm)$ \\
\hline $6 / \mathrm{mmm}$ & $z \| 6, z \perp m, y \perp m$ & $(2 \lambda, 6 \mu,+)$ \\
\hline
\end{tabular}

In the cubic classes $m \overline{3}$ and $m \overline{3} m$ the symmetrized functions up to the 8th order are as follows:

\begin{tabular}{llllcc} 
Laue class & \multicolumn{6}{l}{ Cubic Harmonics } \\
$m \overline{3}$ & $K_{0}$ & $K_{4}$ & $K_{61}$ & $K_{62}$ & $K_{8}$ \\
$m \overline{3} m$ & $K_{0}$ & $K_{4}$ & $K_{6}$ & - & $K_{8}$
\end{tabular}

axes $\mathbf{a}, \mathbf{b}, \mathbf{c}$. The needed value of $W^{h}(0)$ is then given by the expression

$$
W^{h}(0)=\sum_{i j} C_{i j} Y_{i j}\left(\vartheta_{h}, \varphi_{h}\right)
$$

because $P_{i \geq 1}=1$ for $\alpha=0$.

Practical application of method $A$ consists of three steps:

(i) Determination of indices of non-vanishing symmetrized harmonics for given Laue class according to table 1 and their calculation according to tables 7 and 8 (see Appendix).

(ii) Determination of unknown coefficients $C_{i j}$ by simultaneously fitting functions $W^{h}(\alpha) / W^{h}(0)$ to the ratios $I^{h}(\alpha) / I^{h}(0)$ measured in steps (say $10^{\circ}$ ) of angle $\alpha$ for a few reflections (usually 2 to 4 ). The standard linear least-squares method is sufficient in these calculations.

(iii) Calculation of the values $W^{h}(0)$ according to equation 7 for all reflections measured in normal Bragg-Brentano geometry. Dividing the observed $I^{h}(0)$ values by $W^{h}(0)$ gives the required intensities $I_{\text {ran }}^{h}$ of the sample with random orientation of crys- tallites (See equation 4).

\section{Corrections based on analysis of intensities of all measured reflections}

It has been shown (Valvoda, 1987) that the coefficients $C_{i j}$ determining the polar density distribution can also be obtained from the whole set of integrated intensities $I^{h}(0)$ as measured in ordinary Bragg-Brentano arrangement. The following three methods, $B, C$ and $D$, assume that we know the structure of the sample under study exactly, or we are at the stage of structure refinement.

Methods $B$ and $C$. If we know the relative intensities $R_{h}$ of the sample with random orientation of crystallites then

$$
I_{\text {ran }}^{h}=k R^{h},
$$

where $k$ is an unknown scale factor. The combination of (4), (7) and (8) for two reflections $h_{1}$ and $h_{2}$ leads to the relation

$$
\sum_{i j}\left\{\left[I^{h_{1}}(0) R^{h_{2}} / I^{h_{2}}(0) R^{h_{1}}\right] Y_{i j}^{h_{2}}-Y_{i j}^{h_{1}}\right\} C_{i j}=0 .
$$

The system of linear equations (9) for different pairs $h_{1}$ and $h_{2}$ can be solved with respect to $C_{i j}$ either directly (when the number of selected independent equations is equal to the number of coefficients required) or by linear least-squares (when the number of independent equations exceeds the number of coefficients demanded). These two computational procedures will henceforth be designated as methods $B$ and $C$, respectively.

Method $D$. In structure refinement with an overall Debye parameter $B$ and relative intensities $R_{o}^{h}$ of the static structure,

$$
I^{h}(0)=k \cdot R_{o}^{h} \exp \left(-2 B \lambda^{-2} \sin ^{2} \theta\right) \sum_{i j} C_{i j} Y_{i j}^{h} .
$$

The system of equations (10) for all measured reflections contains the following unknown parameters: a scale factor $k$, structural parameters appearing in $R_{o}^{h}$, a Debye parameter $B$ and texture coefficients $C_{i j}$. All these parameters can be refined by a nonlinear least-squares method starting with estimates of the texture coefficients derived by method $B$. In the example shown below the program POWLS (Will, Frazer \& Cox, 1965) was used. On the other hand, equation (10) is also the basic equation for structure refinement by means of a Rietveld-type program with simultaneous texture refinement. In 
such a case the list of unknown parameters is extended by lattice parameters, line profile parameters and instrumental parameters (zero shift, angular dependence of line breadths, etc.). A Rietveld refinement program with texture correction according to equation (10) was introduced by Ahtee, Nurmela, Suortti \& Järvinen (1989).

\section{Experimental tests of texture corrections based on the description of polar density in terms of symmetrized harmonics}

Method $A$ was tested by Järvinen, Merisalo, Pesonen \& Inkinen (1970), by Pesonen, Järvinen \& KurkiSuonio (1973), and by Pesonen (1979), on samples of crystals with cubic, hexagonal and tetragonal symmetry, using a texture goniometer in reflection geometry. The results have shown that measurements of $I(\alpha) / I(0)$ for three reflections are sufficient for the determination of accurate texture corrections using only four terms in the expansion for the polar axis distribution $W$. The corrected intensity values were in agreement, within $1 \%$ or less, with those of a specimen in which the orientation distribution was random within the limits of experimental error.

Paakari, Blomberg, Serimaa \& Järvinen (1988) applied this method in the case of monoclinic crystals of cellulose using only two measurements of five reflections, one in symmetrical reflection $\left(\alpha=0^{\circ}\right)$ and one in symmetrical transmission $\left(\alpha=90^{\circ}\right)$. It has been found that the effect of texture can be reduced by an order of magnitude, which is sufficient accuracy for applying the method to quantitative determination of the crystallinity of cellulose.

A comparative test of methods $A-D$ on a powder sample of titanium carbide with the $\mathrm{NaCl}$ type of structure (Valvoda, 1987) verified the equivalence of both basic approaches in this case. The results of this test are summarized in tables 2-4 and in figures 2 and 3.

The integrated intensities, measured with a specimen spinner and $\mathrm{Cu} \mathrm{K}_{\alpha}$ radiation on a Philips X-ray powder diffractometer, are presented in table 2 . The texture coefficients derived from these intensity values by methods $B, C$ and $D$ are given in the last three columns of table 4 . The relative intensities $R^{h}$ of a random sample used in methods $B$ and $C$ were calculated from the known structure of the sample with the use of a Debye-parameter value $B=0.34 \AA^{2}$. This value was estimated from the pairs of reflections $111 / 222$ and $200 / 400$. Only the first three texture coefficients were determined by method $B$ with the reflection 111 as $\mathbf{h}_{1}$ and the reflections 200,220 and 311 as $\mathbf{h}_{2}$ in (9). The parameters refined in method $D$
Table 2: Comparison of intensities $\left(I_{o b s} / W\right)$ corrected for the effect of texture from the data of Table 4 ( $I_{\text {ran }}$ are the intensities calculated for a random specimen with $k=7.49$ and $B=0.33 \AA^{2}$ )

\begin{tabular}{|c|c|c|c|c|c|c|}
\hline$h k l$ & $I_{\text {obs }}$ & $I_{\text {ran }}$ & $A$ & $B$ & $C$ & $D$ \\
\hline 111 & 133.8 & 139.9 & $140 \cdot 6$ & 140.2 & 139.9 & $140 \cdot 8$ \\
\hline 200 & 190.8 & $146 \cdot 1$ & 146.5 & $146 \cdot 1$ & 145.8 & 146.9 \\
\hline 220 & 79.7 & 85.9 & 86.5 & 85.9 & 86.7 & 86.3 \\
\hline 311 & $47 \cdot 1$ & 46.8 & 46.5 & 46.7 & $46 \cdot 6$ & 46.5 \\
\hline 222 & 24.7 & $25 \cdot 9$ & 25.9 & 25.8 & $25 \cdot 8$ & 25.9 \\
\hline 400 & 15.9 & $12 \cdot 1$ & 12.2 & 12.1 & $12 \cdot 1$ & 12.2 \\
\hline 331 & 18.9 & $20 \cdot 6$ & $20 \cdot 2$ & $20 \cdot 3$ & $20 \cdot 2$ & $20 \cdot 1$ \\
\hline 420 & 38.8 & 39.8 & $39 \cdot 2$ & 38.9 & 39.4 & $39 \cdot 6$ \\
\hline 422 & 39.9 & $41 \cdot 7$ & 41.9 & 42.2 & 41.8 & 41.6 \\
\hline 333 & $36 \cdot 1$ & 33.9 & $34 \cdot 5$ & 34.4 & 34.4 & 34.7 \\
\hline
\end{tabular}

\begin{tabular}{|c|c|c|c|c|c|c|}
\hline & $C_{4}$ & $C_{6}$ & $C_{8}$ & $C_{10}$ & $k$ & $B$ \\
\hline$C_{4}$ & 1.00 & -0.27 & -0.27 & 0.78 & 0.07 & 0.09 \\
\hline$C_{6}$ & -0.27 & 1.00 & 0.15 & -0.41 & -0.36 & -0.28 \\
\hline$C_{8}$ & -0.27 & 0.15 & 1.00 & -0.02 & -0.77 & -0.65 \\
\hline$C_{10}$ & 0.78 & -0.41 & -0.02 & 1.00 & $-0 \cdot 10$ & -0.06 \\
\hline$k$ & 0.07 & -0.36 & -0.77 & -0.10 & 1.00 & 0.88 \\
\hline$B$ & 0.09 & -0.28 & -0.65 & -0.06 & 0.88 & 1.00 \\
\hline
\end{tabular}

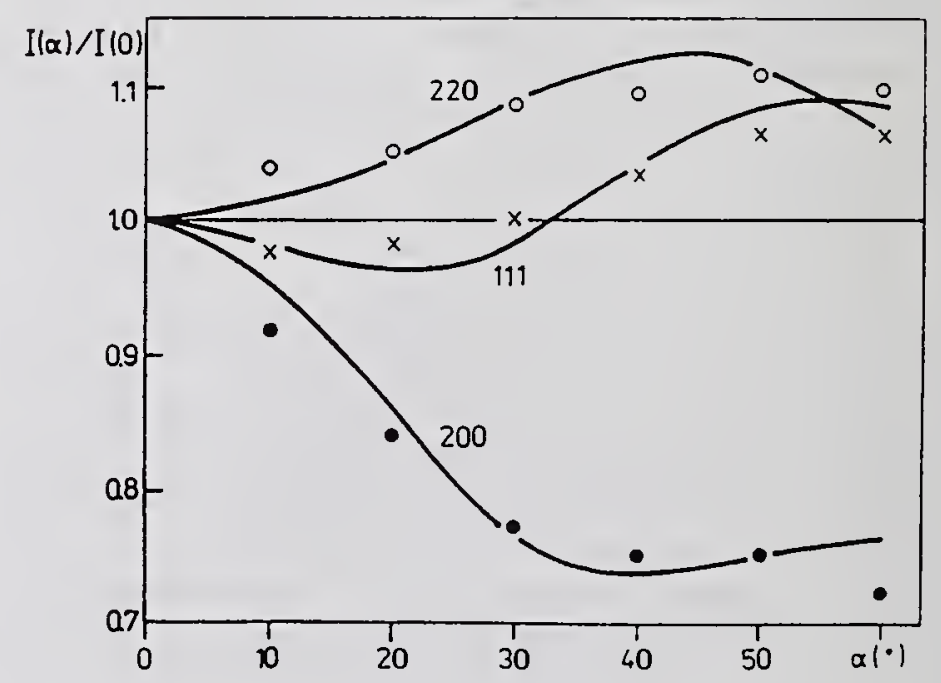

Figure 2: Plane-normal orientation distribution of the first three reflections of the specimen $T_{i} C_{0.96}$ as measured on the Schulz texture goniometer. Solid curves were obtained by fitting cubic harmonics expansions to all experimental values simultaneously (method A). - Reflections 200, × 111, ○ 220 . 
Table 4: Texture coefficients determined from the powder diffraction data of $\mathrm{TiC}_{0.96}$ (methods B-D) and their comparison with the values obtained from texture measurements (method $A$ )

$k$ is a scale factor, $B$ the overall Debye parameter, $r$ the correlation coefficient and $R_{w I}$ is the residual index.

\begin{tabular}{|c|c|c|c|c|}
\hline \multirow[t]{2}{*}{ Method } & $A$ & $B$ & $C$ & \multirow{2}{*}{$\begin{array}{c}D \\
\text { Non-linear } \\
\text { least squares } \\
\text { All }\end{array}$} \\
\hline & $\begin{array}{c}\text { Texture } \\
\text { goniometer }\end{array}$ & \multicolumn{2}{|c|}{$\begin{array}{l}\text { Linear least squares } \\
\left(B_{\text {estim. }}=0 \cdot 34 \AA^{2}\right)\end{array}$} & \\
\hline$C_{4}$ & 0.192 & $0 \cdot 199$ & $0 \cdot 190$ & $0.181(8)$ \\
\hline$C_{6}$ & 0.072 & 0.067 & 0.077 & $0.073(5)$ \\
\hline$C_{8}$ & $0 \cdot 063$ & 0.069 & 0.067 & $0.065(8)$ \\
\hline$C_{10}$ & -0.011 & - & -0.014 & $-0.020(7)$ \\
\hline$k$ & $7 \cdot 49(5)$ & $7 \cdot 46(6)$ & $7 \cdot 47(5)$ & $7 \cdot 50(6)$ \\
\hline$B\left(\stackrel{\circ}{A}^{2}\right)$ & $0.33(2)$ & $0.32(2)$ & $0.33(1)$ & $0.33(2)$ \\
\hline$r$ & 0.988 & 0.985 & 0.989 & 0.984 \\
\hline$R_{w I} \times 100$ & 0.83 & 0.95 & 0.83 & 0.88 \\
\hline
\end{tabular}

Estimated standard deviations corresponding to the last digits are given in parentheses.

by the program POWLS (Will, Frazer \& Cox, 1965) using (10) are given in the last column of table 4; the correlation matrix of these parameters is given in table 3 .

Table 2 compares intensities corrected for the texture effect with the observed values $I_{o b s}$ and values $I_{\text {ran }}$ corresponding to a random specimen (calculated according to equation (8) with scale factor and Debye parameter values corresponding to method $A$ ). These last two parameters were determined by linear regression of the data presented in Wilson plots, i. e. as $\ln \left[I^{h}(0) / R_{o}^{h} W^{h}(0)\right]$ versus $\sin ^{2} \theta$, and their values are given, together with the values of the correlation coefficients, in the lower part of table 4. An example of such a Wilson plot is given in figure 3 . The goodness of the fit of the corrected and calculated intensities is also expressed in table 4 by means of the residual index $R_{w I}$ based on weighted intensities.

Inspection of table 2 shows that methods $B, C$ and $D$, which are based on the powder diffractometer data and on the known relative intensities of a random specimen, lead to corrected intensities deviating from those obtained by method $A$ by less than $1 \%$.

Method $D$ has also been tested in a Rietveld refinement program by Ahtee, Nurmela, Suortti \& Järvinen (1989) on samples of $\mathrm{Ni}, \mathrm{Mg}$ and $\mathrm{NaNO}_{3}$. The results demonstrated clearly that the wholepattern fit was substantially improved when the texture correction was included in the refinement, and that the model is able to handle correction factors that range between 0.5 and 2.3 .

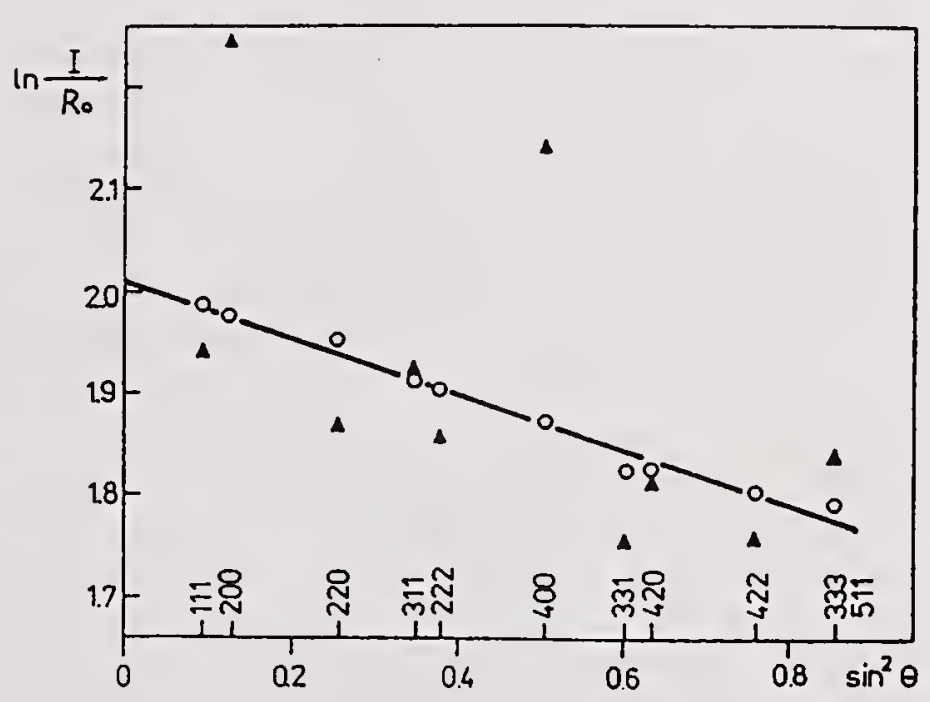

Figure 3: Wilson plots for the specimen $T i C_{0.96}$. $\Delta$ Before texture correction $[I=I(0)]$; $\circ$ after texture correction $[I=I(0) / W(0)]$, based on the coefficients determined by method $C$. 


\section{Less exact methods and special cases}

Method E: Texture Index. A texture index concept was originally introduced by Harris (1952). It is applied in quantitative phase analysis for correcting the effect of texture, or in thin film studies as a measure of degree of preferred orientation. The texture index $T$ of the reflection $h_{i}=h_{i} k_{i} l_{i}$ is defined as

$$
T\left(h_{i}\right)=\frac{I\left(h_{i}\right) / R\left(h_{i}\right)}{\frac{1}{n} \sum_{j=1}^{n} I\left(h_{j}\right) / R\left(h_{j}\right)} .
$$

Using equations (4) and (8), we can reformulate (11) in terms of polar densities $W$ as follows:

$$
T\left(h_{i}\right)=W\left(h_{i}\right) / \frac{1}{n} \sum_{j=1}^{n} W\left(h_{j}\right) .
$$

In the case of random orientation, $W\left(h_{i}\right)=1$ for all $h_{i}$, and the value of texture index is one. Harris assumes that, for a large set of reflections, the value of the denominator in (12) is near one. In such a case $T\left(h_{i}\right)=W\left(h_{i}\right)$, and the texture index can be used as a texture correction instead of $W^{h}(0)$ in equation (4). It has been shown by Valvoda \& Järvinen (1990) that this is true in cases of a weak texture, whereas in cases of stronger textures this assumption is invalid. This is because of incorrect averaging of pole densities in the form of an arithmetic mean in the denominator of equation (12) instead of an appropriate integral.

Method F: Single Pole-Density Profile. This method can be used for texture correction of intensities diffracted from cylindrically symmetric flat-plate or capillary specimens, composed of effectively rodor disc-shaped crystallites. Several analytical functions used to approximate a single pole-density profile $W^{h}(\alpha)$ are presented in table 5.

Dollase (1986) has tested these functions on two kinds of trigonal samples. In the case of $\mathrm{CaCO}_{3}$ the texture was too weak to demonstrate a preference for any of the tested functions. In the case of stronger preferred orientation of (1011) planes in huntite, $\mathrm{CaMg}_{3}\left(\mathrm{CO}_{3}\right)_{4}$, an $R$ factor of $29 \%$ and unreasonably high temperature factors were obtained without a texture correction. Correction based on functions 1 and 2 resulted in $R$ factors of $10 \%$ while the remaining functions gave $R$ factors of 6 to $7 \%$ in the structure refinement procedure. In these refinements functions from table 5 replace the sum on the right side of equation (10), and the adjustable coefficients $G, b$ or $r$ of these functions, which reflect the strength of the preferred orientation, are refined together with structural parameters. None of the functions (other than 6) is normalized to unit
Table 5: Mathematical forms of proposed pole-figure profiles

No. Function

Reference

$\begin{array}{ll}1 & \exp \left[-G \alpha^{2}\right] \\ 2 & \exp \left[G(\pi / 2-\alpha)^{2}\right] \\ 3 & \exp \left[-G \sin ^{2} \alpha\right] \\ 4 & \exp \left[-G\left(1-\cos ^{3} \alpha\right)\right] \\ 5 & b+(1-b) \exp \left[-G \alpha^{2}\right] \\ 6 & \left(r^{2} \cos ^{2} \alpha+r^{-1} \sin ^{2} \alpha\right)^{-3 / 2}\end{array}$

(e)

(e)

(a)- Uda (1967), (b)- Rietveld (1969), (c)- Parrish \& Huang (198 3), (d)- Will, Parrish \& Huang (1983), (e)- Capková \& Valvoda (1974), (f)- Sasa \& Uda (1976), (g)- Toraya \& Marumo (1981), (h)- March (1932)

integral, which means that a change in an adjustable coefficient must be counterbalanced by a change in the overall scale factor $k$. The angle $\alpha$ represents angular separation of a reciprocal lattice vector $\mathbf{h}$ of a measured reflection and the reciprocal lattice vector $h_{0}$ corresponding to the direction of preferred orientation.

Functions 3 and 4 were also tested by Čapková \& Valvoda (1974) on samples of $\mathrm{Mg}$ and $\mathrm{Mg}_{3} \mathrm{Cd}$ prepared in different ways by measurements on a texture goniometer. The single pole-density profiles can also be constructed from the complete set of $I^{h}(0)$ values measured on an ordinary powder diffractometer because

$$
\frac{W^{h_{\circ}}(\alpha)}{W^{h_{o}}(0)}=\frac{I^{h}(0)}{I^{h_{o}}(0)} \frac{R^{h_{\circ}}}{R^{h}}=g(\alpha),
$$

as follows from combination of equations (3), (4) and (8) (See Valvoda, 1986). The results of both approaches are compared in figure 4.

Regardless of the analytical form of $g(\alpha)$, the pole densities $W^{h_{\circ}}(0)$ for correction of the intensity of reflection $h_{o}$ can be obtained from the normalization condition

$$
\begin{aligned}
\frac{1}{4 \pi} \int_{4 \pi} W^{h_{\circ}}(\alpha) d \Omega & =W^{h_{\circ}}(0) \int_{0}^{\pi / 2} g(\alpha) \sin \alpha d \alpha \\
& =1
\end{aligned}
$$

and the pole densities $W^{h}(0)$ for other reflections can be derived from equation (13) because $W^{h}(0)=$ $W^{h_{o}}(\alpha)$. Figure 5 shows the integrands of equation (14) for the case of magnesium and magnesium alloys with [002] preferred orientation and table 6 compares the pole densities $W^{002}(0)$ obtained by a graphical integration of profiles in figure 5 with those obtained by method $A$. 


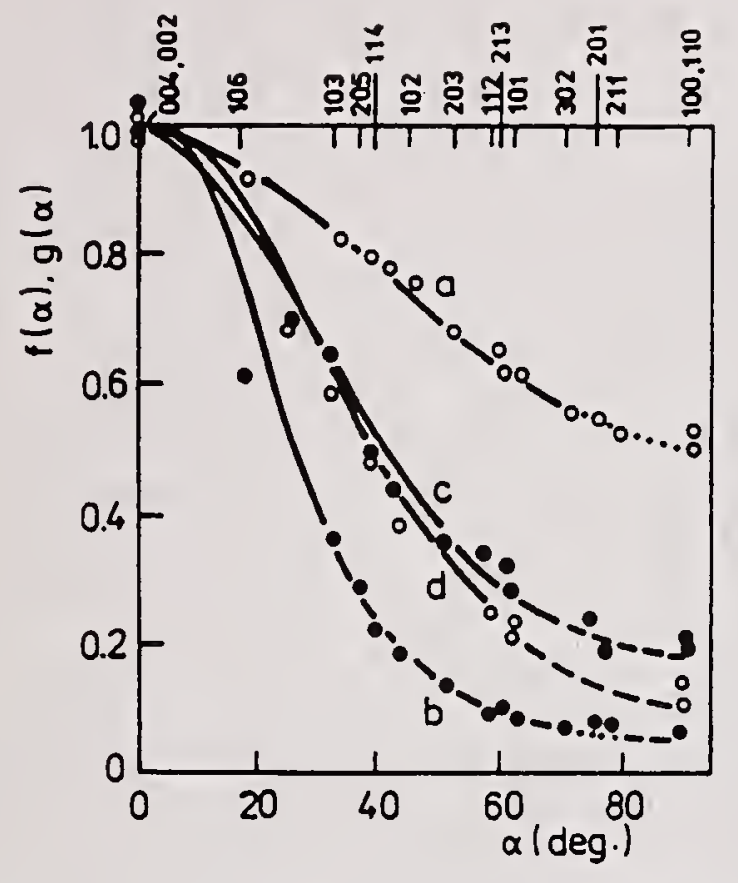

Figure 4: Approximation of the normalized pole distribution $f(\alpha)=I^{002}(\alpha) / I^{002}(0)$ (given by full lines) by the set of discrete $g(\alpha)$ values (given by full and open circles) calculated according to (13) from intensities measured at zero inclination angle of the specimen. (a) $\mathrm{Mg}$-specimen $\mathrm{B}$, (b) $\mathrm{Mg}$-specimen $\mathrm{C}$, (c) $\mathrm{Mg}_{3} \mathrm{Cd}$-specimen $\mathrm{D}$ and (d) $\mathrm{Mg}_{3} \mathrm{Cd}$-specimen $\mathrm{E}$.

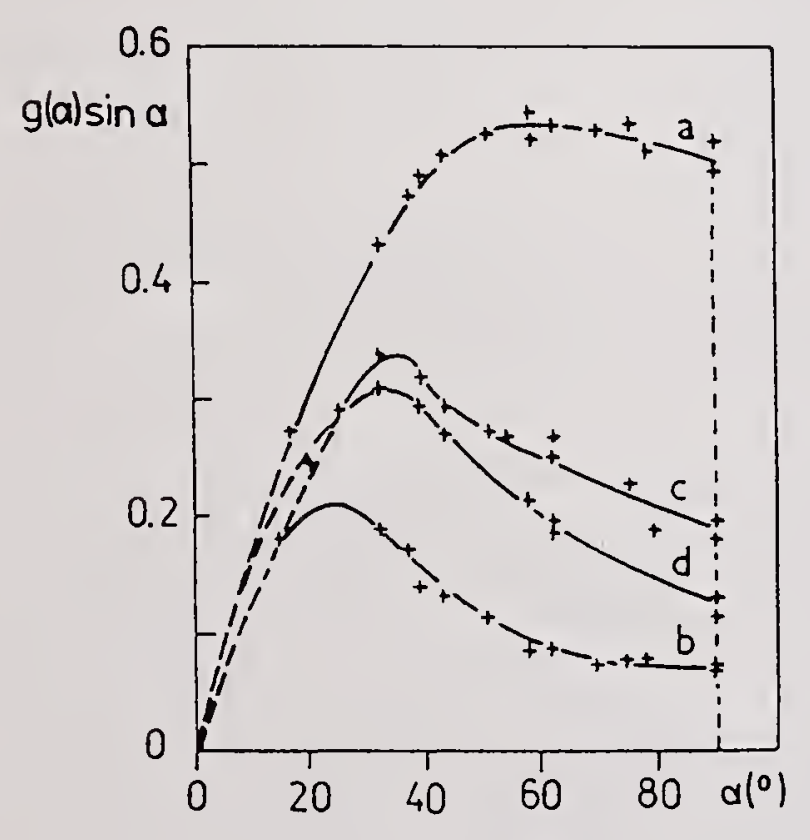

Figure 5: The functions $g(\alpha) \sin \alpha$ obtained from Figure 4 for specimens B, C, D and $\mathrm{E}$ of magnesium and $\mathrm{Mg}_{3} \mathrm{Cd}$ alloys.
Table 6: Comparison of pole densities $W^{002}(0)$ of magnesium and $\mathrm{Mg}_{3} \mathrm{Cd}$ alloys as obtained from Equations (13) and (14) with the results of method A (after Valvoda, 1986)

\begin{tabular}{lcccc}
\multicolumn{1}{c}{ Sample } & $B$ & $C$ & $D$ & $E$ \\
Equation (13) and (14) & 1.51 & 5.26 & 2.70 & 3.03 \\
Method A & 1.62 & 4.97 & 2.64 & 3.02
\end{tabular}

\section{Thin films}

Medium and strong textures appear in thin films as a rule. Strong textures complicate phase identification and profile analysis of weak reflections. In some cases an asymmetric reflection geometry can reveal more well defined peaks. Texture effects can also be partially eliminated by using DOSOPHATEX (doseur oscillant de phase texturé) diffraction system introduced by Terrat (1987) which uses sample rotation of $360^{\circ}$ around its polar axis and $\alpha$-inclinations from $-60^{\circ}$ to $+60^{\circ}$ in each step of a $\theta-2 \theta$ scan. Strong textures also complicate stress analysis by the appearance of so-called 'snake tails' in $\sin ^{2} \psi$ plots of lattice spacings (Marion \& Cohen, 1987).

Apart from a complete texture analysis by means of a texture goniometer, there also exist less accurate ways of texture characterization:

(i) $\omega$-scan of the reflection $h_{\circ}$ from the preferentially oriented planes gives a profile that can serve as a first approximation to the true single pole-density profile (see example shown in figure 6). Its full width at half maximum is inversely proportional to the degree of texture.

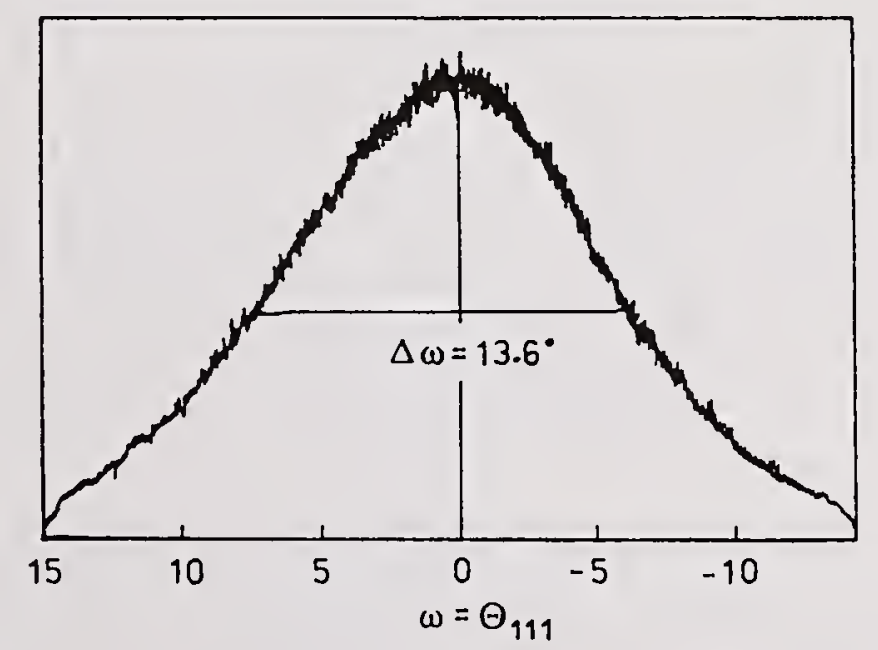

Figure 6: Characterization of normal texture by the breadth of an $\boldsymbol{\omega}$-scan profile. Reflection 111 of thin film of TiN. 
(ii) Texture index (Equation (11)) is another quantity that can also be used for an approximate characterization of preferred orientation in thin films. The values of texture indices of individual reflections divided by their sum should be in some cases regarded as a measure of the volume fraction of crystallites grown with given planes parallel to the substrate surface rather than a measure of deviation of orientation distribution of crystallites from the random orientation. This point of view seems to be realistic, especially in films composed of columnar grains. However, great care has to be given to the effect of absorption, which is no longer independent of angle in thin films, even in the case of a symmetrical reflection geometry. The accuracy of the method can be estimated by checking whether the sum of texture indices of all reflections divided by their number is actually equal to one, as it should be according to equation (12). In cases of strong textures this condition can be strongly violated due to the reasons discussed in section 5 .

\section{Conclusions}

The flexibility of the expansion in symmetrized harmonics as a description of the orientation distributions of crystallites and the axial symmetry introduced by specimen spinning, which has reduced the problem to only one variable (angle $\alpha$ ), are two basic tools of the currently most accurate methods of texture correction.

These corrections can be calculated from the complete set of measured intensities of reflections if the structure of the sample is known, at least approximately. In the opposite case, when the structure is unknown, the orientation distributions of three crystallographic planes, as determined by a texture goniometer measurement, is usually sufficient for calculation of corrections for all reflections. The accuracy of these methods is comparable with the accuracy of intensity measurements. The most promising application is probably in structure determination and refinement from powder data, using programs based either on fitting of integrated intensities or on fitting of the whole diffraction profile (as in the Rietveld method).

Even the less accurate methods based on a particular choice of function type in describing single pole-density profiles or on the whole set of measured reflections, are applicable in cases when preferentially oriented systems of planes have no other symmetry equivalent members. This is usually the case for samples composed of disc- or rod-shaped crystallites. When properly understood, the Harris texture index can also serve as a first approximation in texture characterization and correction.

\section{References}

Ahtee, M., Nurmela, M., Suortti, P., \& Järvinen, M. (1989). J. Appl. Cryst. 22, 261-268.

Bunge, H. J. (1985). Preferred Orientation in Deformed Metals and Rocks. Orlando: Academic Press.

Bunge, H. J., DaHMS, M., \& BROKMEIER, H. G. (1989). Collected Abstracts of the XII. Hungarian Diffraction Conference. Budapest: Roland Eötvös Physical Society.

Čapková, P., \& Valvoda, V. (1974). Czech. J. Phys. B24, 891-900.

Dollase, W. A. (1986). J. Appl. Cryst. 19, 267-272.

Harris, G. B. (1952). Phil. Mag. 43, 113-123.

Järvinen, M., Merisalo, M., Pesonen, A.,\& INKINEN, O. (1970). J. Appl. Cryst. 3, 313-318.

MarCh, A. (1932). Z. Kristallogr. 81, 285-297.

Paakari, T., Blomberg, M., SerimaA, R., \& Järvinen, M. (1988). J. Appl. Cryst. 21, 393-397.

Parrish, W., \& Huang, T. C. (1983). Adv. X-ray Anal. 26, 35-44.

Pesonen, A. (1972). J. Appl. Cryst. 12, 460-463.

Pesonen, A., JärVinen, M., \& KurKi-Suonio, K. (1973). Phys. Fenn. 8, 81-91.

Rietveld, H. M. (1969). J. Appl. Cryst. 2, 65-71.

Roe, R. J., \& Kriegbaum, W. R. (1964). J. Chem. Phys. 40, 2608-2615.

SASA, Y., \& UdA, M. (1976). J. Solid State Chem. 18, 63-68.

Smith, D. K., \& BarretT, C. S. (1979). Adv. X-ray Anal. 22, 1-12.

Suortti, P., \& Jennings, L. D. (1977). Acta Cryst. A33, 1012-1027.

Terrat, J. P. (1987). 6th International Conference on Ion and Plasma Assisted Techniques, Brighton. Edinburg: CEP Consultants Ltg., p.411.

Toraya, H., \& Marumo, F. (1981). Mineral. J. 10, 211-221.

UdA, M. (1967). Z. Anorg. Allg. Chem. 350, 105-109.

Valvoda, V. (1986). Powder Diffraction 1 (No 2), 28-32.

Valvoda, V. (1987). J. Appl. Cryst. 20, 453-456.

Valvoda, V., \& JÄrvinen, M. (1990). Powder Diffraction 5 (No 4), 200-203.

Will, G., Frazer, B. C., \& Cox, D. E. (1965). Acta Cryst 19, 854-857.

Will, G., Parrish, W., \& Huang, T. C. (1983). J. Appl. Cryst. 16, 611-622.

WILL, G. (1991). Materials Science Forum 79-82, 207220. 


\section{Appendix}

Table 7: Real spherical harmonic functions $Y_{i j \pm}(\vartheta, \varphi)$. The norm is chosen so that $\max |Y \||=1$

$\begin{array}{ll}i j \pm & Y_{i j \pm}(\vartheta, \varphi) \\ 00 & 1 \\ 20 & 0.5\left(3 \cos ^{2} \vartheta-1\right) \\ 21+ & 2 \cos \vartheta \sin \vartheta \cos \varphi \\ 21- & 2 \cos \vartheta \sin \vartheta \sin \varphi \\ 22+ & \sin ^{2} \vartheta \cos 2 \varphi \\ 22- & \sin ^{2} \vartheta \sin 2 \varphi \\ 40 & 0.12500\left(35 \cos ^{4} \vartheta-3 \cos ^{2} \vartheta+3\right) \\ 41+ & 0.94695\left(7 \cos ^{2} \vartheta-3\right) \cos \vartheta \sin \vartheta \cos \varphi \\ 41- & 0.94695\left(7 \cos ^{2} \vartheta-3\right) \cos \vartheta \sin \vartheta \sin \varphi \\ 42+ & 0.77778\left(7 \cos ^{2} \vartheta-1\right) \sin { }^{2} \vartheta \cos \varphi \\ 42- & 0.77778\left(7 \cos ^{2} \vartheta-1\right) \sin \vartheta \sin \varphi \\ 43+ & 3.07920 \cos ^{2} \sin ^{3} \vartheta \cos 3 \varphi \\ 43- & 3.07920 \cos \vartheta \sin ^{3} \vartheta \sin 3 \varphi \\ 44+ & \sin ^{4} \vartheta \cos 4 \varphi \\ 44- & \sin \vartheta \sin 4 \varphi \\ 60 & 0.06250\left(231 \cos ^{6} \vartheta-315 \cos ^{4} \vartheta+105 \cos \vartheta-5\right) \\ 61+ & 0.69140\left(33 \cos ^{4} \vartheta-30 \cos ^{2} \vartheta-5\right) \cos \vartheta \sin \vartheta \cos \varphi \\ 61- & 0.69140\left(33 \cos ^{4} \vartheta-30 \cos ^{2} \vartheta-5\right) \cos \vartheta \sin \vartheta \sin \varphi \\ 63+ & 1.41685\left(11 \cos ^{2} \vartheta-3\right) \cos \vartheta \sin { }^{3} \vartheta \cos 3 \varphi \\ 63- & 1.41685\left(11 \cos ^{2} \vartheta-3\right) \cos \vartheta \sin 3 \vartheta \sin 3 \varphi \\ 64+ & 0.81675\left(11 \cos ^{2} \vartheta-1\right) \sin ^{4} \vartheta \cos 4 \varphi \\ 64- & 0.81675\left(11 \cos ^{2} \vartheta-1\right) \sin ^{4} \vartheta \sin 4 \varphi \\ 65+ & 3.86393 \cos ^{2} \sin ^{5} \vartheta \cos 5 \varphi \\ 65- & 3.86393 \cos \vartheta \sin ^{5} \vartheta \sin 5 \varphi \\ 66+ & \sin ^{6} \vartheta \cos 6 \varphi \\ 66- & \sin ^{6} \vartheta \sin 6 \varphi \\ & \end{array}$

Table 8: Cubic harmonics $K_{i j}(\vartheta, \varphi)$

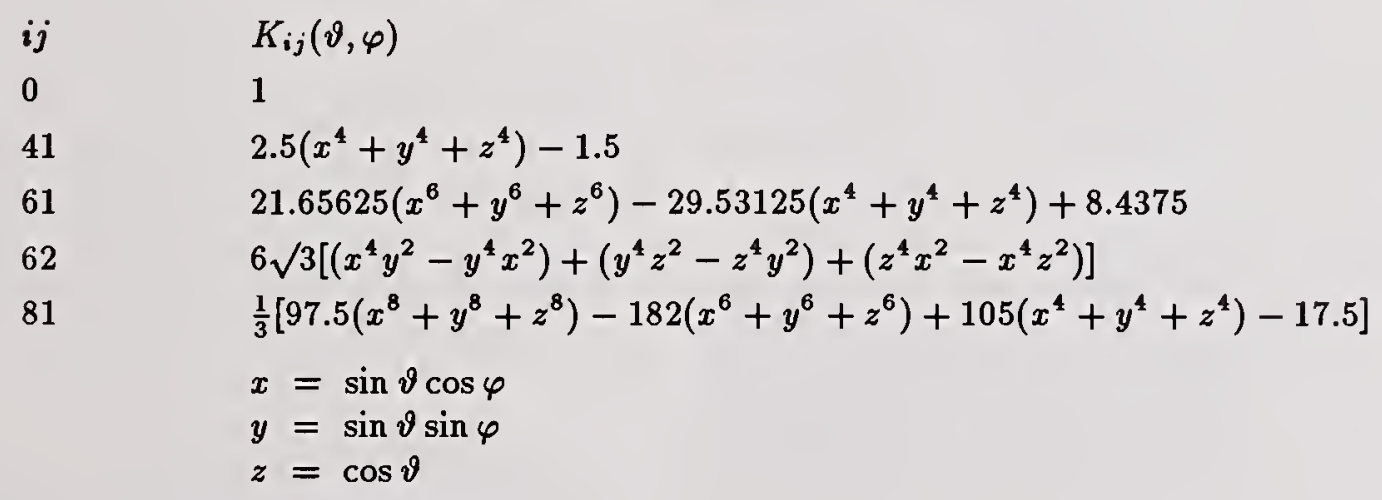


National Institute of Standards and Technology Special Publication 846. Proceedings of the international conference Accuracy in Powder Diffraction II, held at NIST, Gaithersburg, MD, May 26-29, 1992. (Issued October 1992)

\title{
Residual Stress Measurements with Neutrons
}

\author{
T. M. Holden AND J. H. RooT \\ AECL Research, Chalk River Laboratories, \\ Chalk River, Ontario, Canada, KOJ 1 Jo
}

\begin{abstract}
Mechanical methods of residual stress measurement currently claim an accuracy of $\pm(10-20) \mathrm{MPa}$ for a given stress component. This corresponds to an accuracy of $\pm 0.3 \times 10^{-4}$ in each of three principal strain components. For example, the changes in the (110) plane spacing of iron, $2.02 \AA$, have to be obtained with an accuracy of $\pm 0.00006 \AA$, which is a worthy goal in any high resolution neutron diffraction measurement and not too far from being achievable.

The determination of residual strain fields involves a difference measurement, under identical diffractometer conditions, between a Bragg peak position in the sample displaying the strain field and a reference sample which is determined to be strain free. For confidence in the strain results we measure interplanar spacings, not just shifts in spacings, and this requires calibration of the neutron wavelength with a standard powder sample under identical diffractometer conditions. This permits intercomparisons to be made among different spectrometers at different times and between conventional and time-offlight diffractometer measurements. Presently the typical accuracy achieved is $\pm(0.5-1.0) \times 10^{-2} \%$ in calibrated plane spacing measurements.

The need to measure strain tensors associated with intergranular or type II stresses, for example in $\mathrm{Zr} 2.5 \% \mathrm{Nb}$ alloys, poses more severe requirements. In this case $\mathrm{d}$-spacings are measured as a function of orientation on a sample completely bathed in the neutron beam. Strains are derived by comparison with reference d-spacings calculated from the literature, or from high temperature measurements, or from single crystals. For this situation, a high resolution configuration with Soller collimators is required. The crucial requirement is accurate centering of the sample on an Eulerian cradle and careful checks on the centering by measuring first and second orders of several reflections at different sample orientations. The accuracy in repeat measurements of separately mounted specimens each properly cal-
\end{abstract}

ibrated against a standard powder is again in the range $\pm(0.5-1.0) \times 10^{-2} \%$.

\section{Introduction}

The accuracy of residual stress measurements by neutron diffraction methods must eventually be able to match the current accuracy, $\pm(10-20) \mathrm{MPa}$, claimed for each stress component by mechanical methods. This corresponds to an accuracy of $\pm 0.3 \times 10^{-4}$ in each of the strain components, and this is close to being achieved.

The accuracy of elastic residual strain measurements depends on the factors that govern the accuracy of any neutron diffraction experiment. These include: optimization of the angular resolution by appropriate choices of collimation, monochromator mosaic spread and take-off angle; adequate knowledge of the peak shape; and a good signal-to-noise ratio in the diffraction peaks. However, in measurements of lattice strain, additional factors must be considered.

Studies of both macroscopic residual strain fields in engineering components, as well as intergranular strains, often referred to as microstrains, are made at Chalk River. Macroscopic strains occur when the manufacture of an object entails an inhomogeneous distribution of plastic deformation. An example is provided by a weldment, where plastic deformation occurs on heating and cooling in and near the fusion zone, but elastic deformation occurs further away. The strain field has dimensions of order tens of $\mathrm{mm}$ and may extend over the whole sample. Microstrains occur because individual grains in a polycrystal may be plastically deformed in manufacture while others are not because of the anisotropy of deformation properties in the crystal lattice. Microstrains vary on the length scale of the grain size. 


\section{Macroscopic strain fields}

The measurements of macroscopic strain fields at depth below the surface of engineering components are possible because neutrons readily penetrate most materials of interest. Narrow beams of neutrons, defined by slits in absorbing cadmium in both the incident and scattered beams, are arranged to intersect over the centre of rotation of the sample table. The region of overlap is termed the gauge volume by analogy with strain gauges. The sample, mounted on an XYZ translator table, can then be moved so that any desired location lies in the gauge volume. The diffraction peak measured at this location then gives the average interplanar spacing, $\mathrm{d}_{h k l}$, over the gauge volume for grains whose plane normals $(h, k, l)$ lie along the scattering vector. High accuracy is achievable because a set of difference measurements are made under identical diffractometer conditions between a Bragg peak position in the sample displaying the strain field and a reference sample which is determined to be strain free. The following sections deal with the principal sources of error, systematic and random.

\subsection{Beam centering}

The gauge volume should be centered over the rotational axis of the sample table so the same location in the sample is examined for any choice of scattering vector direction. A fine polyethylene pin is centered on the table by checking its precession on rotation of the table through $360^{\circ}$ with a theodolite (Smith et $a l ., 1988)$, which also provides a reference line of site. A plumb-bob lies over the pin as a useful secondary reference. Since neutrons scatter incoherently from hydrogenous materials, the detector may be set at any angle and the incident slit is traversed across the neutron beam to find the slit position at which the count rate is maximized. The process is repeated with the slit in the scattered beam.

\subsection{Wavelength calibration}

The average wavelength passing through the slit system is calibrated with a standard Si powder sample obtained from the National Institute of Standards and Technology (NIST). A calibration with three or more peaks yields the effective zero-angle offset, $\theta_{0}$, of the mechanical scale as well as the mean wavelength from Bragg's law,

$$
\lambda=2 \mathrm{~d} \sin \left(\theta-\theta_{0}\right) .
$$

Without careful centering of the gauge volume, the calibration measurements made over a wide angular range will yield an incorrect wavelength and zeroangle offset. Intercomparison between the results for the same sample at different spectrometers is then possible. For example, measurements at Chalk River by conventional diffraction on a diametrally compressed ring in 1986 agreed within the quoted errors of $\pm 0.0003 \AA$ and $\pm 0.0002 \AA$ for the (111) and (002) reflections, with measurements by time-offlight diffraction at Los Alamos in 1989 (Holden et al., 1990) with errors of $\pm 0.00015 \AA$ and $\pm 0.00016 \AA$ for the same reflections. A similar slit system had been set up at the time-of-flight diffractometer at Los Alamos, using the $2 \theta=90^{\circ}$ banks, and the slit system had been calibrated with a standard powder. Assurance of the interplanar spacings generates confidence in the correctness of the strains.

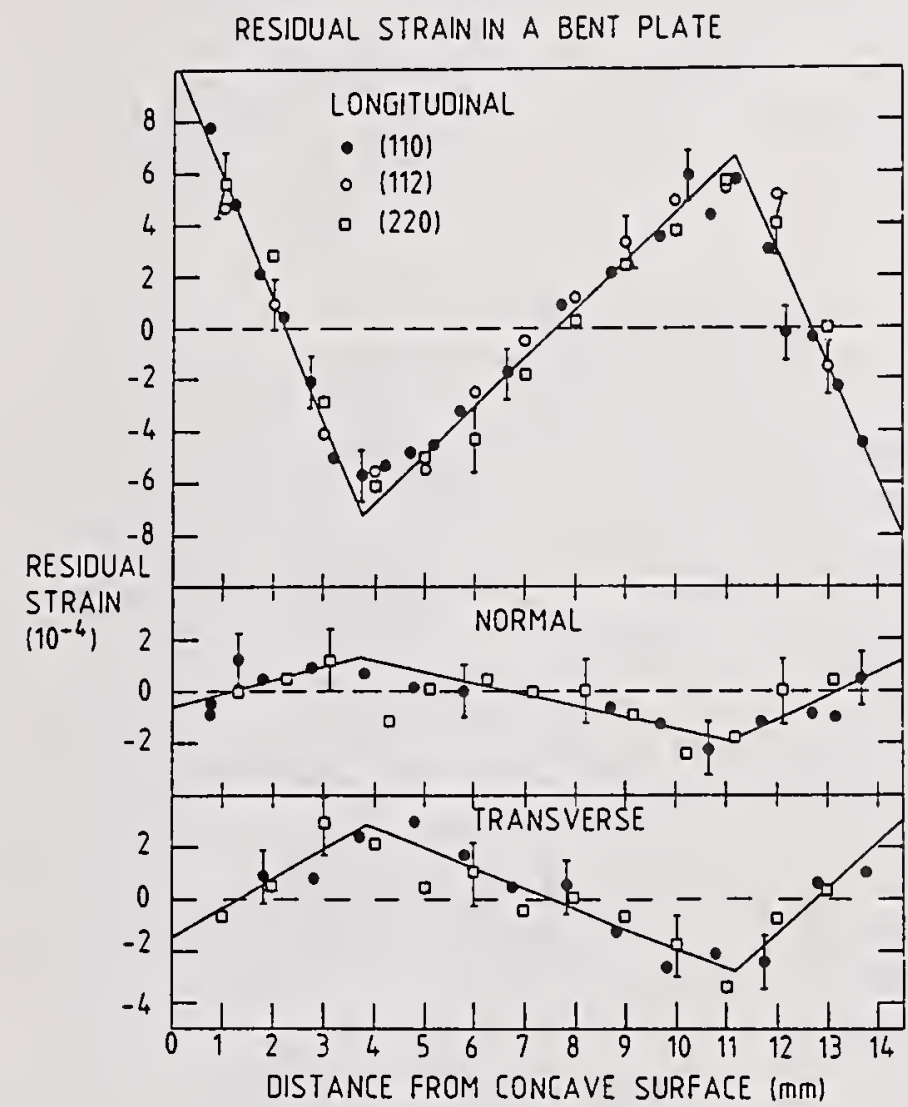

Figure 1: Residual elastic strain components in a bent plate, determined with the aid of the (110), (112) and (220) reflections of the body-centered cubic steel alloy.

Figure 1 shows the results of experiments on a 14.5 mm thick plate of HY80 steel that had been bent so that the regions furthest from the neutral axis had yielded plastically. On removing the load, the plate had retained a permanent set. Measurements are shown of strains measured in the longitudinal, transverse and normal directions of the plate with the 
aid of the 110,112 and 220 reflections of the bodycentered structure. Two separate experiments were carried out at two neutron wavelengths to collect this data. Typical errors in individual d-spacing measurements were $1.01373 \pm 0.00008 \AA, 1.17100 \pm 0.00008 \AA$ and $2.02990 \pm 0.00012 \AA$ for the 220,112 and 110 reflections. The independent sets of measurements, when converted to strain, agree within the quoted experimental errors.

\subsection{Peak Fitting, reproducibility and setting accuracy}

In conventional diffractometry, the angular instrumental resolution is close to Gaussian. To obtain the peak position, width and intensity, a Gaussian peak shape with a sloping background is fitted to the experimental data. The precision of peak fitting depends on the peak width as well as the signal strength and is $\pm 0.003^{\circ}$ at best. This figure is also typical of the reproducibility when measurements are repeatedly made at the same spatial locations with the same set-up. The precision of setting the detector angle is also in this range for our diffractometer.

\subsection{Reference lattice spacings}

The identification of a strain-free reference, with respect to which the strains are calculated from interplanar spacings, is potentially a source of systematic error. The basis of mechanical methods of measuring stress fields is that cutting a small section of material from the workpiece removes the field in that section. In many cases, a small sample is cut from the workpiece. Measurements are made on this reference sample under exactly the same conditions as the sample of interest, in different orientations and in several slightly different locations. This provides an average, $\mathrm{d}_{h k l}^{0}$, and a standard error with which to compute the strains and their errors from $\mathrm{d}_{h k l}$ measured at locations within the object, through the formula,

$$
\epsilon_{h k l}=\left(\mathrm{d}_{h k l}-\mathrm{d}_{h k l}^{0}\right) / \mathrm{d}_{h k l}^{0} .
$$

If the reference sample is annealed to relieve the stress thermally, great care must be taken not to change the microstructure or the state of solution of minor alloying additions.

Sometimes a localized region of the sample will have been subjected to thermal or plastic distortion, and then it is possible to take as reference the spacings in the sample well removed from the localized region.

The reference lattice spacing has also been identified by computing the equilibrium of forces in the section and finding the value which most closely satisfies the condition of no net force (Prask \& Choi, 1987). Since these conditions are for the behaviour of the ensemble of all orientations of grains, and since different crystal orientations do display different stresses in the same orientation, this criterion must be used judiciously and would ideally be computed from the average behaviour of all the different planes.

Brand (1991) has pointed out that the curvature of the diffraction cones can be significant when the slit defining the scattered beam is close to the gauge volume for scattering angles not equal to $90^{\circ}$, and that this can introduce effective peak shifts. This source of systematic error can be minimized by ensuring that the slit arrangement is not changed between measurements on the object and the reference specimen.

\subsection{Grain size effects}

The accuracy of lattice spacing measurements is strongly affected by grain size. The reproducibility at precisely the same spatial location is as stated in section 2.3 , but the scatter among the results from location to location in the sample may be two or three times worse than this for grain sizes of order 100-150 $\mu \mathrm{m}$, which are common for austenitic stainless steel (Holden, Root, Fidleris, Holt \& Roy, 1988). To ensure that the same grains contribute at every angle under a Bragg peak in these conditions, it is very important to do $\theta-2 \theta$ scans, otherwise the peaks will be ragged and shifted randomly due to the statistics of grain selection. However, even if the sample is half-angled, there can be a marked scatter in strain. The exact strain in each grain is determined by the boundary conditions with its juxtaposed neighbours. For small grain sizes, the ensemble of all grain environments is sampled adequately. For large grains, only 10-20 grains may contribute to the average in a $10 \mathrm{~mm}^{3}$ gauge volume. Thus, a poor sampling of the environment and subsequent scatter occur. The grain size may actually be deduced from the r.m.s. variation of intensity in the Bragg peaks as we traverse through the sample. It is often possible to use the symmetry of the sample to make measurements at many equivalent points and thereby increase the number of grains contributing to the average result (Holden, Root, Fidleris, Holt \& Roy, 1988).

\subsection{Near surface effects}

When the gauge volume is only partly within a component surface, such as in attempts to measure near-surface strains, there are possibilities for large systematic errors. These originate from two causes: 
the offset of the effective "centre of gravity" of the scattering volume from the centre of the spectrometer table and the selection of only part of the wavelength distribution across the neutron beam. The latter error can be several times larger than the former if special care is not taken to minimize it. To control the wavelength distribution across the beam, there must be good $\left(\sim 0.3^{\circ}\right)$ collimation between the source and the monochromator, as can be seen from considerations of the incident resolution (Cooper \& Nathans, 1967). In the work of Ezeilo et al. (1990), the effect of the long guide before the monochromator at the D1A station at the ILL reactor may have had the same effect as a collimator before the monochromator.

\subsection{Multidetectors}

In order to increase the rate of data acquisition, a position-sensitive detector [see for example, Krawitz, Rudnick, Butler \& Cohen (1986)] or a multidetector such as the one used at Chalk River with, for example, 15- 25 closely spaced wires or counters are employed. A complete diffraction peak can then be collected with a small number of settings of the counter position. Collimation is achieved by the distance of the counting element to a slit placed within 20-30 $\mathrm{mm}$ of the sample. Parallax occurs for wires offset from the central wire, which not only spreads the gauge volume along the incident beam, but also corresponds to a different counter angle than the nominal setting. Parallax is minimized by placing the exit slit as close as possible to the gauge volume, although this is sometimes prohibited by the desired movement of the sample. The precise angle of the counter element can be computed from the offset of the exit slit from the centre of the gauge volume. It is important to rock the sample angle, $\theta$, during the measurement so that a common subset of grains contributes to the scattering in each element of the counter.

Near-surface measurements should not be made with a multidetector because the parallax will certainly introduce errors.

\section{Grain interaction strains}

Grain interaction strains arise because of the difference in plasticity of grains of different crystal orientations juxtaposed in the material. For $\mathrm{Zr}$ and $\mathrm{Ti}$, the [0002] direction is elastically and plastically stiff while directions in the basal plane such as [1010] and equivalent directions are relatively soft (MacEwen,
Table 1: Comparison of lattice spacing measurements derived from two experiments on the same Zircaloy-2 sample

$\begin{array}{ccc}\text { Direction } & \text { Experiment \#1 } & \begin{array}{c}\text { Experiment \#2 } \\ \AA\end{array} \\ & & \\ {[0002]} & c=5.1500 \pm 0.0004 & 5.1489 \pm 0.0002 \\ {[10 \overline{1} 0]} & a=3.2306 \pm 0.0002 & 3.2306 \pm 0.0002 \\ {[\overline{1} 2 \overline{1} 0]} & a=3.2322 \pm 0.0001 & 3.2320 \pm 0.0001 \\ {[10 \overline{1} 1]} & d=2.4584 \pm 0.0001 & 2.4583 \pm 0.0001 \\ {[10 \overline{1} 2]} & d=1.8948 \pm 0.0001 & 1.8947 \pm 0.0001\end{array}$

Faber \& Turner, 1983). On imposing and removing a load that exceeds the yield point for some grains, the (0002) orientations will tend to be left in a state of tensile residual strain, and the $\langle 10 \overline{1} 0\rangle$ in residual elastic compression. Strong crystallographic texture, which usually occurs for $\mathrm{Zr}$, can generate macroscopic differences in strain relaxation, even though the strains are on the scale of the grains. This, in turn, affects engineering properties such as in-reactor growth (Fidleris, Tucker \& Adamson, 1987).

The very presence of strong crystallographic texture, however, opens up the possibility of measuring the complete strain tensor for an assembly of grains that have nearly the same orientations, termed a family of grains in this article, because there is a one-to-one correspondence between direction in the sample and crystallographic direction. Grain interactions are intensive in the sense that a sample may be cut in two halves and still contain the same information. A composite sample may thus be assembled from small pieces, free of macroscopic stress. The composite sample may have a volume of order $10^{3} \mathrm{~mm}^{3}$, completely bathed by the neutron beam at all orientations.

A prerequisite is the determination of the texture of the sample and calculation of the orientation distribution function. This permits identification of the various "families" of grain orientation of technological interest and subsequent calculation of the sample orientation to pick up particular reflections, say, for example, the [12 $\overline{1} 0]$ reflection, from each family of grains.

\subsection{Sample centering}

The goal is to measure the strain tensor, $\epsilon_{i j}$, for a given family of grains from measurements of strains in perhaps 20 orientations. The goal in accuracy is the measurement of $\mathrm{d}_{h k i l}$ to $\pm 0.0001 \AA$, which cor- 
Table 2: Comparison of strain tensors derived by fitting equation 2 to 25 data points measured in each of two experiments on a Zircaloy-2 sample.

$$
\begin{aligned}
& \# 1 \epsilon_{i j}\left(10^{-4}\right) \\
& \left(\begin{array}{ccc}
-0.3 \pm 0.4 & -0.5 \pm 0.4 & 0.4 \pm 0.5 \\
-0.5 \pm 0.4 & 3.9 \pm 0.4 & -0.9 \pm 0.5 \\
0.4 \pm 0.5 & -0.9 \pm 0.5 & -4.6 \pm 0.9
\end{array}\right) \\
& \begin{array}{c}
\# 2 \epsilon_{i j}\left(10^{-4}\right) \\
\left(\begin{array}{ccc}
-1.1 \pm 0.3 & 0.3 \pm 0.2 & 0.6 \pm 0.4 \\
0.3 \pm 0.2 & 4.2 \pm 0.3 & -2.2 \pm 0.7 \\
0.6 \pm 0.4 & -2.2 \pm 0.7 & -3.3 \pm 0.6
\end{array}\right)
\end{array}
\end{aligned}
$$

responds to an accuracy in strain of better than $\pm 1 \times 10^{-4}$. A typical high-resolution diffractometer set-up is required with the sample mounted on an Eulerian cradle. The measurements at Chalk River (Holden, Root, Fidleris, Holt \& Roy, 1988) were made with a $\mathrm{Ge}(331)$ monochromator, of mosaic $0.2^{\circ}$, at a take-off angle of $100^{\circ}$ and collimation of $0.3^{\circ}$, before and after the sample. The wavelength was chosen to allow measurements of the $\{10 \overline{1} 0\}$, $\{10 \overline{1} 1\},\{0002\},\{10 \overline{1} 2\},\{20 \overline{2} 0\},\{20 \overline{2} 2\}$ and $\{0004\}$ reflections. Crucial to the accuracy is the careful centering of the sample at the centre of rotation of the spectrometer table and the centre of the Eulerian cradle. This is done optically and checked by computing the effective offset angle, $\theta_{0}^{\prime}$, in the Bragg equation, (1), from the first and second orders of at least a dozen reflections at different orientations of the cylindrical sample in the beam. A typical average value of $\theta_{0}^{\prime}$ for an individual sample is $-0.003 \pm 0.005^{\circ}$, where the error is the r.m.s. spread for all the different reflections. Systematic variations in $\theta_{0}^{\prime}$ must be absent. Since very high intensities can be achieved with large volume samples and marked texture, errors of $\pm 0.003^{\circ}$ in the fitted Gaussians are usual.

\subsection{Wavelength calibration}

The wavelength and scale offset are measured with the standard Si powder sample. The offsets mentioned in the previous paragraph will be deviations from the Si powder, $\theta_{0}$, for each sample. The calibration is vital since absolute d-spacing measurements are being sought here. The reference values for lattice spacing are obtained in a separate experiment, or from the literature. The repeatability of the measurements, taking the sample off the spectrometer and measuring it again subsequently on the same or a different spectrometer, but calibrated with the same standard, was within the goal set in section 3.1. Results for a Zircaloy-2 sample are shown in table 1 .

\subsection{Reference d-spacings}

The major source of error in strain measurements of this kind is the uncertainty in the strain-free reference, $d_{h k i l}^{0}$. Because of the coefficient of thermal expansion in the $c$ direction of the hcp lattice, $\alpha_{c}$, is twice that in the $a$ direction, $\alpha_{a}$, thermal strains are always generated on cooling from some elevated annealing temperature, where strains might have been eliminated. To circumvent this problem, the temperature of an alloy was raised in steps, and the plane spacing measured in different directions at each step, until all directions gave the same spacing to within the experimental errors. This is a strain-free spacing at the annealing temperature. Then the single crystal thermal expansion between the strain-free temperature and room temperature was used to find the unconstrained alloy parameters at room temperature. The reference spacings for alloys were also found by taking the concentration derivative of the interplanar spacing change, $\partial / \partial c\left(\mathrm{~d}_{h k i l}\right)$, from sets of polycrystal measurements and then applying this change to single crystal, i.e., unconstrained, plane spacings. The two methods agree within an uncertainty of about $\pm 1 \times 10^{-4}$ for the $a$ lattice parameter for both $\mathrm{Zr}-2.5 \mathrm{Nb}$ and Zircaloy-2 but less well for the c-parameter $\pm(2-3) \times 10^{-4}$.

\subsection{Strain tensors}

The strain tensor, $\epsilon_{i j}$, determined with respect to Cartesian crystallographic coordinates, $(X, Y, Z)$, [1010], $(X, Y, Z),[12 \overline{1} 0]$ and $(X, Y, Z),[0002]$, is obtained by fitting the measured strains to the expression,

$$
\frac{\Delta \mathrm{d}}{\mathrm{d}}=\sum_{i j} \epsilon_{i j} l_{i} l_{j},
$$

where $l_{i}$ and $l_{j}$ are direction cosines for the direction of measurement. Six values with zero uncertainty are required, in principle, to determine a strain tensor. For the uncertainties characteristic of neutron strain measurements, $\pm(10-30) \%$, we require 
many measurements to be made, and, in our case, at least twenty strain measurements are fitted to obtain the tensor elements with a precision approaching $\pm 1 \times 10^{-4}$. Table 2 shows values of the strain tensor determined on two separate occasions on the same sample which agree within the errors.

\section{Acknowledgments}

We would like to acknowledge discussions of the accuracy of strain measurements with R. A. Holt, P. J. Webster and J. Schröder.

\section{References}

Brand, P. C. (1991). Thesis, University of Twente, ECN-R-91-006.

CoOper, M. J. \& Nathans, R. (1967). Acta Cryst. 23, $357-67$.

Ezemo, A. N., Webster, P. S., Webster, G. A. \& WeBster, P. J. (1991). Proceedings of the NATO Advanced Research Workshop on Measurement of Residual and Applied Stress using Neutron Diffraction. Edited by M. T. Hutchings and A. D. Krawitz. Amsterdam: Kluwer Academic Publishers, B. V.
FIDleris, V., Tucker, R. P. \& Adamson, R. B. (1987). ASTM STP 939, 49- 85

Holden, T. M., Root, J. H., Fidleris, V., Holt, R. A. \& RoY, G. (1988). Materials Science Forum $27 / 28,359-370$.

Holden, T. M., Hosbons, R. R., MacEwen, S. R., Flower, E. C., BourKe, M. A. \& Goldstone, J. A. (1991). Proceedings of the NATO Advanced Research Workshop on Measurement of Residual and Applied Stress using Neutron Diffraction. Edited by M. T. Hutchings and A. D. Krawitz. Amsterdam: Kluwer Academic Publishers, B. V.

Krawitz, A. D., Rudnick, P. J., Butler, B. D. \& CoHEN, J. B. (1986). Advances in X-ray Analysis. Edited by C. S. Barrett, J. B. Cohen, J. Faber, Jr., R. Jenkins, D. E. Leyden, J. C. Russ and P. K. Predecki, 29, 163-71.

MacEwen, S. R., Faber, J., JR. \& Turner, A. P. L. (1983). Acta. Metall. 31, 657-76.

Prask, H. J. \& ChoI, C. S. (1987). In Residual Stress in Design, Process and Materials Selection. Edited by W. B. Young, p. 21-26, ASM International.

Smith, D. J., Leggatt, R. H., Webster, G. A., MacGillivray, H. J., Webster, P. J. \& Mills, G. (1988). J. Strain Anal. 23, 201-11. 
National Institute of Standards and Technology Special Publication 846. Proceedings of the international conference Accuracy in Powder Diffraction II, held at NIST, Gaithersburg, MD, May 26-29, 1992. (Issued October 1992)

\title{
Modelling Anisotropic Crystallite Size/Microstrain in Rietveld Analysis
}

\author{
A. Le BAIL \\ Laboratoire des Fluorures, Université du Maine, 72017 Le Mans Cedex, France
}

\begin{abstract}
Studies aiming at modelling crystallite size/microstrain (particularly anisotropic) by a direct approach in Rietveld analysis are reviewed. Possible evolutions are suggested.
\end{abstract}

\section{Introduction}

The Rietveld method (Rietveld, 1967, 1969) offers a powerful approach for characterizing crystallized powdered materials (for a review concerning mainly neutron diffraction: Hewat, 1986). Application of this method has dramatically increased in recent years, partly due to the efforts to find new high $\mathrm{T}_{c}$ superconductors. Problems in solid state chemistry frequently involve powders, which may be easily verified in any recent issue of a specialized journal like the Journal of Solid State Chemistry, where it is not rare that more than half the papers make use of modern powder methodology. Organometallic and organic chemistry may be behind in this domain, but this could change in the future. An increasing number of dedicated ultra-high resolution powder instruments is available using synchroton sources (Cox, 1991); their development will probably have some impact on pussible amelioration of in-laboratory diffractometers. Repeated demonstration of the feasability of $a b$ initio structure determination from powder data is certainly another cause of increasing numbers of applications (Cheetham \& Wilkinson, 1991). There is a new golden age for the powder method, so more problems delaying the routine use of pattern decomposition and profile analysis in structure determination are now coming to light. Anisotropic line broadening intrinsic to the sample is one such problem, particularly noticeable when using ultrahigh resolution.

Real materials may be affected by a variety of defects (concentration variation, macrostrain, microstrain, size, stacking fault, antiphase domains ...), all able to influence the shape and width of the powder diffraction pattern reflections. Moreover, the three-dimensional order may be highly perturbed, as in polymers and liquid crystals, with the most extreme situation corresponding to amorphous materials. The subject of this paper is mainly restricted to the attempts to interpret line broadening in terms of anisotropic crystallite size/microstrain (S/M) effects in Rietveld refinement (RR). Reviews on this subject (at least partly) may be found in Louër (1988), Young \& Desai (1989), Delhez, de Keijser, Langford, Louër, Mittemeijer \& Sonneveld (1989). Without the structure constraint, an alternative is the determination of microstructural properties by means of pattern decomposition (for a review, see Langford, 1987 and in this volume; Toraya, 1989). Some definitions about S/M will be given. Then earlier works taking account of isotropic effects in RR will be briefly discussed before showing how the general anisotropic case has been treated by various authors. Special attention will be given to experimental applications. A test case will be considered, and the results from different methods will be compared. Finally, a projection for the future will be attempted.

\section{Statistical approach to size/microstrain}

There is an enormous specialized bibliography about effects of imperfections of all kinds on diffraction phenomena, and some controversy. Because theories are generally not directly applicable, quite a large number of numerical methods were derived to approach experimental cases. They cannot be detailed here; the reader will be generally directed towards recent review articles. Several models have been proposed to quantitatively estimate lattice distortions. For instance, different formulae have been given for the experimentally observable integral breadth. According to the work of Warren \& Averbach (1950) (WA), the integral breadth, $\beta_{\mathrm{d}}$ for "microstrain" broadening is a function of the mean-square lattice distortion $\left\langle\varepsilon^{2}\right\rangle$; $\beta_{\mathrm{d}}$ is related to an index of the "paracrystalline" distortion in the work of Hosemann \& Bagchi (1962); $\beta_{d}$ is related to the "maximum distortion" $e$ defined by 
Wilson (1949); a direct relationship exists between $e$ and $\left\langle\varepsilon^{2}\right\rangle$ (Buchanan, McCullough \& Miller, 1966). In the more recent controversy about the $S / M$ view of WA for cold-worked metals, one can find a theory developed for symmetrical X-ray diffraction lines broadened by dislocations (for a review, see Wilkens, 1979). Ergun (1970) stated that highly defective lattices must be characterized by a "mean defectfree distance" rather than a domain size. Nevertheless, the method most applied (when possible, i.e. if harmonics are isolated, see below) remains the WA method. Its formulation was based on the work of Bertaut (1949) for the definition of the size distribution. It is important to note that the WA theory is general and must not be confused with the so-called WA multiple line method for S/M separation, which uses some assumptions.

According to the formalism of Warren (1959, 1969), the experimentally observable power per unit length $P^{\prime}(2 \theta)$ of the intersection of the diffraction cone with a receiving surface at a distance $R$ from the sample (equation 13.24 in Warren, 1969) may be expressed as a Fourier series, whose coefficients are $A(n)=\left\langle\cos 2 \pi l Z_{n}\right\rangle N_{n} / N_{3}$ and $B(n)=$ $\left\langle\sin 2 \pi l Z_{n}\right\rangle N_{n} / N_{3}$ for a family of $00 l$ reflections. $N_{n}=\sum_{i=\ln l}^{\infty}(i-\ln l) \mathrm{P}(i)$ is the average number of $n$ pairs of cells per column; $\mathrm{P}(i)$ is the fraction of the columns of length $i$ cells (i.e. the surfaceweighted size distribution) and $N_{3}$ is its mean (a related "volume-weighted" size distribution function may be defined); $Z_{n}$ is the component (along the normal to the diffracting plane) of the displacement vectors for a pair of $n$ apart cells. In fact, this Fourier series represents the "true profile" $f$, which, by convolution with the "instrumental profile" $g$, gives the experimental profile $h=f^{*} g$. At this stage, no assumption about the distribution $\mathrm{P}_{n}(Z)$ of local strain is made. Thus asymmetry of a reflection is possible in this rather general formulation (asymmetry cannot be due to size effect but to strain according to the sine term). This formulation clearly shows that strain effect on broadening is dependent on $l$, whereas size effect is not; the possibility of separating the two contributions originates from this different behaviour in $2 \theta$ for the same $h \mathrm{kl}$ family of reflections. However, some simplifications are necessary. Concerning microstrain, the assumption that $\left\langle\cos 2 \pi l Z_{n}\right\rangle=\exp \left(-2 \pi^{2} l^{2}\left\langle Z_{n}^{2}\right\rangle\right)$ is exact if local strain has a Gaussian distribution $\mathrm{P}_{n}(Z)=(a / \sqrt{\pi}) \exp \left(-a^{2} Z_{n}^{2}\right)$, whatever the value of $n$. This assumption (or expanding $\left(\cos 2 \pi l Z_{n}\right)$ as a series approximated by a few of the first terms) pro- vides a way to separate size and microstrain effects if at least two orders of a reflection family are available. As this is rare, methods were developed to separate $\mathrm{S} / \mathrm{M}$ effects from one reflection, called "single line" methods (SL). Assumptions used in SL methods come from the fact that the profile shape is governed by some particular variations of $\left\langle Z_{n}^{2}\right\rangle$ versus $n$. If the local strain distribution $\mathrm{P}_{n}(Z)$ is Gaussian, the associated reflection profile shape is Cauchy-like if $\left\langle Z_{n}^{2}\right\rangle=n\left\langle Z_{1}^{2}\right\rangle$; it is Gaussian if $\left\langle Z_{n}^{2}\right\rangle=n^{2}\left\langle Z_{1}^{2}\right\rangle$. Concerning the size effect, Cauchy-like profile has been widely adopted, though this corresponds to a very particular size distribution function. To be clear, the same profile shape associated with size and microstrain effects renders the separation impossible with SL methods but possible with whole pattern fitting like RR because of the different $2 \theta$ dependency. Recent reviews on SL methods may be found in Delhez, de Keijser \& Mittemeijer (1982); Nandi, Kuo, Schlosberg, Wissler, Cohen \& Crist (1984).

Because of these approximations, the "size" and "microstrain" parameters as obtained from the WA or SL methods need to be used cautiously. "Sense and nonsense" of these parameters were discussed recently (Delhez, de Keijser, Mittemeijer \& Langford, 1988); the question of accuracy of $S / M$ values from X-ray diffraction line profiles using Fourier series was treated by Delhez, de Keijser \& Mittemeijer (1980). It is a paradox that, as will be apparent below, current methods aiming at extracting $S / M$ by direct approach in RR use mainly the basic principles of SL methods, while the Rietveld method was developed to minimize overlapping effects.

\section{Profile shapes in Rietveld analysis}

Many analytical profile shapes are routinely used in RR. Some are fortunately compatible with oversimplifications associated with the SL methods. They were mainly introduced for RR applications to Xray powder data (for a review: Young \& Wiles, 1982). For constant wavelength powder data, the list of such profile shapes includes Gaussian (G), Lorentzian (L) (or Cauchyan (C)), intermediate Lorentzian (IL), Voigtian (V) (but see below) and its approximations the pseudo-Voigtian (pV) and Pearson VII (PVII). Other descriptions than analytical have been used in RR: learned profile shape by numerical fitting (Hepp \& Baerlocher, 1988) or by Fourier coefficient representation (Le Bail, 1984); analytical Fourier coefficients were also used (Le Bail, Duroy \& Fourquet, 1988). 
Modelling isotropic crystallite size/microstrain in Rietveld analysis

Formulae enabling extraction of S/M parameters after a RR using V, pV and PVII were listed by de Keijser, Mittemeijer \& Rozendaal (1983). The possibility of anisotropic effect was not considered, and no application was given. The cases of PVII and pV were treated by giving equivalence of breadth with $\mathrm{V}$ (the Voigt function was introduced in RR later).

Extraction of isotropic particle-size and microstrain broadening have been taken into account in a modified Rietveld program by Cox (1984), introducing the constraint of the different $2 \theta$ dependency for size and microstrain using $\mathrm{pV}$.

Considered as the best approach to profile-shape for $S / M$ interpretation in a simplified view, the Voigt function was used much earlier in SL methods (Langford, 1978; de Keijser, Langford, Mittemeijer \& Vogels, 1982) than in the Rietveld method (Ahtee, Unonius, Nurmela \& Suortti, 1984; David \& Matthewman, 1985). The latter took advantage of the possible physical meaning of $\mathrm{V}$ by extraction of a mean crystallite size for a de-intercalated $\mathrm{Li}_{1-x} \mathrm{Mn}_{2} \mathrm{O}_{4}$ spinel. The $\mathrm{L}$ part of $\mathrm{V}$, with a $1 / \cos \theta$ dependency, was assumed to come from the size effect.

A neutron powder diffraction study of materials presenting various peak shapes and crystallite sizes was realized by Hill \& Howard (1985), showing that at least a part of isotropic S/M effects is handled empirically by standard Rietveld procedures with $\mathrm{pV}$ or PVII. Convolute profiles were obtained numerically by Howard \& Snyder $(1985,1989)$. The algorithm was implemented in a modified Rietveld program able to extract isotropic S/M parameters; applications were made on La-substituted $\mathrm{SrTiO}_{3}$ and Al specimens (Yau \& Howard, 1989; Howard, Yau \& Anderson, 1989).

Young \& Desai (1989) reviewed the literature (including anisotropic) and developed an approach allowing both the size and the microstrain broadening to have both $\mathrm{L}$ and $\mathrm{G}$ components, using the pseudoVoigt function of Thompson, Cox \& Hastings (1987). Preliminary results with $\mathrm{MgO}$ (no anisotropy modelled) suggested that the assignment of the $L$ component to size and the $G$ component to microstrain broadenings cannot be a general procedure.

The most amazing applications of the Rietveld method including isotropic microstrain effects were for modelling the structures of some amorphous materials. The simultaneous fit of three neutron patterns for $\mathrm{Pb}_{2} \mathrm{M}_{2} \mathrm{~F}_{9}$ fluoride glasses ( $\mathrm{M}=\mathrm{Zn}, \mathrm{Fe}$;
$\mathrm{Mn}, \mathrm{Fe} ; \mathrm{Mn}, \mathrm{V})$ was made by using different crystalline models, supposing isomorphous substitution (Le Bail \& Jacoboni, 1984; Le Bail, $1985 a$; Le Bail, Jacoboni \& De Pape, 1985). The simultaneous fit of the neutron and X-ray patterns for a $\mathrm{BaZ}_{\mathrm{r}_{2}} \mathrm{~F}_{10}$ glass was also done (Le Bail, Boulard \& Jacoboni, 1987). Moreover, the structure of liquid alloys $\mathrm{Al}_{1-x} \mathrm{M}_{x}$ (M $=\mathrm{Mn}$ or $\mathrm{FeCr}$ ) was simulated in this way (Maret, Chieux, Dubois \& Pasturel, 1991). Instrumental effects were neglected in these studies. Atomic coordinates and cell parameters of the crystalline models were refined (thermal motion effects were considered as included in a refined disorder parameter having the $\left\langle Z_{1}^{2}\right\rangle$ meaning) using $G$ profile shapes (their widths were so large that more than 1000 reflections could overlap, depending on the structuremodel complexity).

Rietveld application to amorphous materials may be used to eliminate wrong structural models. However, it is insufficient to establish the validity of a model, even if the fit is quite "good." Very good fits $\left(\mathrm{R}_{p}<4 \%\right)$ were generally obtained with unrealistic individual distances between pairs of atoms in the final refined model. However, the mean distances for particular types of pairs were generally credible. After all, this holds also for other methods of simulating amorphous materials structures, such as molecular dynamics or model-building with relaxation.

\section{Modelling anisotropic S/M in Rietveld analysis: chronology of attempts}

Attempts to model anisotropic S/M effects in RR began in almost the same years that attempts to model isotropic effects began. The exhaustivity of the list of studies presented below, although they are not so numerous, cannot be guaranteed; not all were in the Journal of Applied Crystallography. Many of them were found in congress proceedings.

1985- Greaves (1985) encountered a problem of anisotropy in the neutron study of $\mathrm{Ni}(\mathrm{OD})_{2}$. The $\mathrm{RR}$ was unsatisfactory with $\mathrm{R}_{w p}=14.1 \%$ (using G). Individual fits of some reflections indicated that the crystallites were plate-like with mean size $400 \AA$ and $1800 \AA$ along [001] and [110], respectively. The variation law of the FWHM was modified by adding a term depending on the angle between $[\mathrm{hkl}]$ and [001]. This way, $R_{w p}$ decreased to $8.5 \%$. This may be considered to be the first empirical approach to anisotropic broadening ( $\mathrm{G}$ functions correspond to unphysical $\mathrm{P}(n)<0$ for low $n$ values). 
The idea of approaching anisotropy for both size and microstrain by rank 2 symmetrical tensors was soon tested (Le Bail, 1985a,b) using a modified Rietveld-type program specially conceived to treat such effects. A three-dimensional mean apparent coherency domain and a three-dimensional mean microstrain were defined, in which the mean size $M_{h k l}$ and $\left\langle Z_{1}^{2}\right\rangle_{h k l}$ were both approximated by using an equation giving the distance from the origin of an ellipsoid to its surface. This way, some self-consistency in the anisotropy is forced that would not be obtained by simple independent fits of widths. Applications were to $\mathrm{ZnO}, \mathrm{NiF}_{2}$, and $\mathrm{W}$ samples (the latter being isotropic) that were highly deformed by grinding. The instrumental $g$ contribution to the $h$ profile was obtained from well crystallized samples (with same formula). The process uses a description of profiles by Fourier coefficients. This choice was made in order to simplify the convolution of size and microstrain associated profiles between themselves and with the $g$ profile (multiplication in Fourier space). This method will be denoted the "Rietveld Fourier" method (RF). Some oversimplifications were used: $A_{n}^{s}(h k l)$ was modelled to correspond to $\mathrm{L}$ profiles and $A_{n}^{d}(h k l)$ to profiles continuously varying between $\mathrm{L}$ and $\mathrm{G}$ by using the hypothesis $\left\langle Z_{n}^{2}\right\rangle_{h k l}=\ln l^{K}\left\langle Z_{1}^{2}\right\rangle_{h k l}$ and refining K (Adler \& Houska, 1979). A quasi size-only sample was also studied: $\mathrm{Ni}(\mathrm{OH})_{2}$ prepared by precipitation. Profile shapes associated with the $K$ value for microstrain were found intermediary between $\mathrm{G}(\mathrm{K}=2)$ and $\mathrm{L}(\mathrm{K}$ $=1$ ); $\mathrm{K}$ took the value 1.4 for both cold-worked $\mathrm{ZnO}$ and W. However, the shape was a "super-Lorentzian" for $\mathrm{NiF}_{2}(\mathrm{~K}=0.8)$.

1986- An application of the RF method to the neutron powder diffraction patterns $(50 \mathrm{~K}$ and $4.2 \mathrm{~K})$ of $\mathrm{MnNbF}_{6}$ (Bizot, Chassaing, Pannetier, Leblanc, Le Bail \& Férey, 1986) greatly improved the fit, decreasing $R_{p}$ values by more than $4 \%$. The anisotropy effect was complex (reflections with $l$ indices larger than $h$ or $k$ are sharp, whereas the others are significantly broadened) and no attempt was really made to further understand its physical origin.

Using the same RF method, Gibaud, Le Bail \& Bulou (1986) presented a study of antiphase domain size effects for $\mathrm{KAlF}_{4}$ (easily produced by simple handpulverizing, with no strain effect). The broadened reflections were due only to some $\mathrm{F}$ atoms $(\mathrm{K}, \mathrm{Al}$ and a part of the $\mathrm{F}$ atoms did not contribute to these reflections because of their particular positions, leading to special extinction conditions). For such a problem, there are at least two sets of reflections to be treated differently in line shape and width; the $g$ contribution was obtained from the sample itself in a refinement excluding the broadened reflections.

Uniaxial anisotropy of strain together with the differently constrained $\mathrm{S} / \mathrm{M} 2 \theta$ dependence were used with neutron time-of-flight (TOF) data for $\mathrm{Pb}_{0.76} \mathrm{Ca}_{0.24} \mathrm{TiO}_{3}$ (Rotella \& Richardson, 1986, 1987).

Thompson, Reilly, Corliss, Hastings \& Hempelmann (1986) studied a deuterided $\mathrm{LaNi}_{5}$ sample according to a two stage process. First the profile parameters for S/M effects were deduced from a Williamson-Hall (1953) plot; second they were imposed in the Rietveld refinement made with $\mathrm{pV}$, using $\mathrm{G}$ (with imposed $2 \theta$ dependence) for the $g$ function (from a $\mathrm{ZrNiAl}$ reference sample), $\mathrm{L}$ for the size effect (isotropic) and $\mathrm{G}$ for the distortion (anisotropic with no effect along [001]).

1987- Thompson, Reilly \& Hastings (1987) reported a study of de-deuterided $\mathrm{LaNi}_{5}$, assuming an isotropic size effect (L shape); the microstrain was allowed to vary anisotropically ( $G$ shape) through the elasticity equations, reducing to 4 unknowns in the hexagonal system (21 components in the most general case). The $g$ profile was fixed in the refinement and the $f^{*} g$ convolution was realized through the possibilities of $\mathrm{pV}$.

Lartigue, Le Bail \& Percheron-Guegan (1987) published another application of the RF method to $\mathrm{LaNi}_{5} \mathrm{D}_{6.7}$. This material is strongly affected by repeated operations of deuterium (or hydrogen) absorption and desorption. The distortion along [ $h k 0]$ directions of this trigonal phase was found to be much larger than along the [00l] direction, and this was consistent with the size effect (the mean apparent domain sizes for the volume distribution were estimated to be 160 and $>5000 \AA$, respectively, in these directions). Both size and strain anisotropy were modelled by ellipsoids of revolution along the $c$ axis. The major interest of this study was to propose a repartition of deuterium atoms from the simultaneous structure refinement.

TOF neutron diffraction study on $\mathrm{Pd}_{2} \mathrm{MnSn}$ was reported by Kamiyama, Shinohara, Tomiyoshi, Asano, Yamamoto \& Watanabe (1987). The simultaneous effects of crystallite size, microstrain and antiphase domain boundaries were taken into the standard Rietveld method; full details were given later (1990; see below).

1989- Delhez, de Keijser, Langford, Louër, Mittemeijer \& Sonneveld (1989) reviewed the litera- 
ture about crystal imperfection broadening and peak shape in the Rietveld method, and proposed a two stage approach. In stage I, pattern decomposition is used to obtain line-shape parameters for a number of more or less resolved reflections in the pattern; with the help of these data, the $h k l$ dependence of the line-shape parameters is modelled. In stage II, crystal-structure refinement by the Rietveld method is performed using the line-shape model obtained in stage $I$. This is not exactly a modelling of $S / M$ in $\mathrm{RR}$ since the approach is not direct. Application was made to $\mathrm{ZnO}$-B data, (Langford, Louër, Sonneveld \& Visser, 1986) for which stage I allowed the conclusion that two different laws could fit the angular width variation of $00 l$ reflections apart from all the others (with PVII) well. These laws, once established, were kept fixed in stage II. However, the authors tried ultimately to refine the set of line shape parameters corresponding to the non- $00 l$ reflections.

1990- The RF method was also applied to improve the fit during the $a b$ initio structure determination of $\mathrm{Cu}_{3} \mathrm{~V}_{2} \mathrm{O}_{7}(\mathrm{OH})_{2} \cdot 2 \mathrm{H}_{2} \mathrm{O}$ (Lafontaine, Le Bail \& Férey, 1990). The anisotropic broadening was found to be mainly due to size effect. This was not unexpected, because of the method of synthesis (precipitation) and the layer structure adopted by this material.

The full details of the TOF neutron diffraction study on $\mathrm{Pd}_{2} \mathrm{MnSn}$ were reported by Kamiyama, Shinohara, Tomiyoshi, Minonishi, Yamamoto, Asano \& Watanabe (1990). All the contributions of defects (size: Lorentzian profile, anisotropic; microstrain: Voigtian profile, anisotropic; antiphase domain boundary with particular $h k l$ dependency) and the $g$ profile were expressed in terms of Fourier coefficients.

Simultaneous structure and S/M refinement by the Rietveld method was also proposed by Lutterotti \& Scardi (1990). They used pV functions; the anisotropy of both the crystallite size (corresponding to $L$ profile shape) and microstrain were modelled by using two tensors in a procedure similar to that used for the temperature factors. Applications were made to tetragonal $\mathrm{ZrO}_{2}$, tetragonal $\mathrm{Zr}_{0.82} \mathrm{Ce}_{0.18} \mathrm{O}_{2}$ and $\alpha-\mathrm{Al}_{2} \mathrm{O}_{3}$.

1991- A study of $\mathrm{La}_{2} \mathrm{NiO}_{4}$ presenting an anisotropic microstrain effect (no size effect) was made by Rodriguez-Carjaval, Fernandez-Diaz \& Martinez (1991), using the FULLPROF program (RodriguezCarjaval, 1990). The particular hkl dependence of the contribution to the FWHM of reflections was described with only one adjustable parameter. FULL-
PROF is one of the most versatile Rietveld programs for fixed wavelength, based on the code of the Wiles \& Young (DBW) program (1981). In its 2.1 version, FULLPROF proposes no less than nine possible pre-established models for domain size and eight models for microstrain effects. The general formulation of the anisotropic size effect assumes that the average diameter can be expressed as an ellipsoid with refinable parameters (six maximum), with $\mathrm{L}$ profile shape. Up to ten parameters (corresponding to the fluctuations and correlations of direct cell parameters) may be refined to describe the general anisotropic strain broadening case, with G profile shape.

Scardi, Lutterotti, Di Maggio \& Maistrelli (1991) improved the method proposed by Lutterotti et al. (1990) by considering a more general expression for the crystallite size distribution leading to both $G$ and $\mathrm{L}$ components of profiles. Applications were to $\mathrm{Zr}_{0.85} \mathrm{Ce}_{0.15} \mathrm{O}_{2}, \mathrm{Ni}(\mathrm{OH})_{2}$ prepared by precipitation and a two-phase sample $\mathrm{Ni}(\mathrm{OH})_{2}-\mathrm{NiO}$ obtained by thermal treatment of $\mathrm{Ni}(\mathrm{OH})_{2}$.

Millini, Perego \& Brückner (1991) implemented in a RR program a theoretical approach relating the FWHM (L profile) of each $h k l$ reflection to the average crystal lengths parallel to the crystallographic axes. The instrumental part was treated with $\mathrm{G}$ profiles (X-ray powder data), and the convolution $f^{*} g$ was taken into account by $\mathrm{pV}$ profiles. Applications were made to layered zirconium phosphites and zirconium phosphate-phosphite derivatives with large improvements in agreement factors when compared to RR without crystallite size optimization.

\section{Modelling anisotropic S/M in Rietveld analysis: comments}

The evolution of methods in the above list of applications to real cases, since 1985 , is to increase complications from the most simple approaches for which both $\mathrm{S} / \mathrm{M}$ effects were each assigned to have a quite precise profile shape ( $\mathrm{L}$ and/or $\mathrm{G}$ ) to the most complex for which both effects would be described as having both $\mathrm{L}$ and $\mathrm{G}$ components to profile shapes. Two very distinct types of profile-shape representations were used: analytical or by Fourier coefficients. However, up to now, these two approaches use similar approximations to model the size and microstrain effects. This could change in the future: all that is done with analytical profiles may be done easily with Fourier representation, the reverse being far from true (see below). 
No example of application of modelling anisotropic $\mathrm{S} / \mathrm{M}$ in $\mathrm{RR}$ for ultra-high resolution synchrotron powder data was found in the list above. Of course, there is a degree of line broadening associated with $\mathrm{S} / \mathrm{M}$ effects rendering such ultra-high resolution useless. However, below such a limit very fine effects are expected to be observed from synchrotron data, and they will probably be very difficult to model.

Extraction of S/M effects from whole-profile fitting procedures has advantages relative to the WA multiple line method. The series expansion of the cosine term is not truncated; as it is a fit, there is no profile truncation effect in the usual sense of the WA analysis (there is no Fourier inversion). However the location of the background has no exact solution. Also, there is no need to deconvolute to obtain $f$; instead, profiles are convoluted. Nevertheless, convolution of analytical profiles may prove to be difficult when the $g$ (and also $f$ ) profiles are asymmetric. The limitations are those of the oversimplifications retained: if the sample shows effects not exactly described by the formulae used (this would generally be the case), the kind of error is not predictable; large discrepancies in results are expected when applying different approaches to the same data set. Actually, there is no example where an asymmetry effect intrinsic to the sample has been taken into account, with extraction of a physical meaning, in RR.

The simplest and most efficient way to model anisotropic effects is certainly to describe the variation in real space (in directions normal to the reflecting planes) of mean size and microstrain by ellipsoids. The nature of powder diffraction, which is to superpose the equivalent $h k l$ reflections with a multiplicity $p$, leads to limitations of such a procedure. Lutterotti \& Scardi (1990) noticed that it was necessary to apply some restrictions similar to those used for the anisotropic temperature factors in order to respect the symmetry properties of the crystal, so they used $M_{11}=M_{22} \neq M_{33}$ and $M_{13}=M_{23} \neq M_{12}$ (four unknowns) for a tetragonal case belonging to the $4 / \mathrm{mmm}$ Laue class. Restrictions could in fact be more drastic, and only two unknowns should be refined in this case unless certain conditions are fulfilled, or unless one is not trying to obtain an ellipsoid. As the anisotropic thermal parameters cannot, in general, be used with the factored form of the structure factor expression (the contribution of each atom to the structure factor must be calculated separately and added), the six parameters corresponding to the general ellipsoid description cannot be refined unless the $h k l$ contributions are considered sep- arately and added (with the exception of Friedel's pairs): i.e., the classical multiplicity factor cannot be used if the symmetry is not triclinic. Anisotropic temperature factors have the same effect on equivalent reflections, anisotropic S/M effects do not. To illustrate this, imagine an orthorhombic phase with a needle-like shape elongated in the $a$ direction showing a topotactic phase transition to a tetragonal phase with correspondence of cell parameters. In such a case, the inevitable superposition of $h 00$ and $0 k 0$ reflections on the powder pattern would probably lead to the conclusion that the size distribution function is bimodal (Young \& Sakthivel, 1988), with profiles quite difficult to reproduce. Can we expect to treat such a problem with an ellipsoid that would not be of revolution along the $c$ axis? To try that, it would at least be necessary to calculate separately the shape and width of the $h 00$ and $0 k 0$ reflections. Instability of such a calculation is probable; it would be necessary to start refinement by giving different values to $M_{11}$ and $M_{22}$. Now if the refinement is performed without separating the equivalent reflections (Laue class $4 / \mathrm{mmm}$ ) it is a priori nonsense to refine more than two parameters $\left(\mathrm{M}_{11}=\mathrm{M}_{22} \neq \mathrm{M}_{33}\right)$, i.e. an ellipsoid of revolution along the $c$ axis. In this sense it is not possible to detect any anisotropy for a cubic compound (special effects on some $h k l$ classes, corresponding to stacking faults, antiphase domain boundaries and so on, are of course not concerned in this discussion of a generalized anisotropic effect).

So, what does one do with the 21 parameters of the elasticity equations in the procedure of Thompson et al. (1987) or the ten parameters of FULLPROF in the case of microstrain effects? Restrictions were not given so the user must be careful. I recognize not having respected these necessary restrictions in one case, when applying the RF method to $\mathrm{NiF}_{2}$ (Le Bail, $1985 a, b)$. This material has the rutile structure belonging to the $4 / \mathrm{mmm}$ Laue class; I tried to refine independently three unknowns for both $\mathrm{S} / \mathrm{M}$ effects, the $11=22$, the 33 and the 12 terms of the symmetrical rank two tensors. Since I did not separate the $\bar{h} k l$ from the $h k l$ during refinements, I must now conclude that, for being self-consistent, the broadening effect should be the same for the $\bar{h} k l$ and $h k l$ reflections, and thus the cross term in my formula ought to be $|h k| \mathrm{S}_{12}$ and not $h k \mathrm{~S}_{12}$. Thus, considering the refined values I had obtained, it was not an ellipsoid that defined the mean domain size; it was a mathematical object of which the section in the $00 l$ plane was not an ellipse but a sort of four-branched star, a quite curious shape for the "mean crystallite." 
Quite excellent results have been obtained in most of the experimental cases cited above (i.e. low $\mathrm{R}_{p}$, indicating some quality of the fit, comparable to those obtained when no $\mathrm{S} / \mathrm{M}$ effect is present). The excellence of these results is in fact questionable, and it must be emphasized that it concerns generally "school cases": materials with simple structures (having few reflection overlapping problems) sometimes specially prepared to show the feasibility of such studies. The cold-worked materials, like $\mathrm{ZnO}$ for instance, probably really present nearly centered and symmetrical Gaussian local distributions of microstrain (corresponding to the approximation made), or materials with size-effect only may have size distributions well approaching that corresponding to Lorentzian profile shape (three independent workers studied anisotropic size effect by RR of $\mathrm{Ni}(\mathrm{OH})_{2}$ or $\mathrm{Ni}(\mathrm{OD})_{2}$; several teams studied $\mathrm{ZnO}$ as a test case). In spite of these "excellent" results, one must realize that a common point to all proposed methods is an extreme oversimplification unfavorable to any accuracy in the deduced crystallite size and microstrain parameters. However, the accuracy in structure parameters certainly benefited from the account of anisotropic effects. In most of these studies, the comparison of results with and without $\mathrm{S} / \mathrm{M}$ modelling evidenced a considerable decrease in $\mathrm{R}_{p}$ and $\mathrm{R}_{\mathrm{B}}$ (up to $10 \%$ in some cases).

The first version of the RF method (Le Bail, $1985 a, b)$ had the inconvenience of modelling $g$ from a reference reflection, which must be chosen isolated on the pattern of a well-crystallized sample. The whole-pattern fit was realized (Le Bail, 1984) by convoluting the reference profile with an analytical one having a pseudo-Caglioti $2 \theta$ dependence (Caglioti, Paoletti \& Ricci, 1958). This was a problem when no reference reflection could be isolated. This inconvenience was suppressed in a further version in which the $g$ function could be modelled more easily from analytical Fourier coefficients (Le Bail, Duroy $\&$ Fourquet, 1988). However, the first version may sometimes work better for modelling $g$ (particularly when asymmetry is present at low diffracting angle).

The two stage process of Thompson et al. (1986) is very similar to that proposed later by Delhez et al. (1989). The first stage would be hardly practicable in case of important overlapping. On the other hand, it is evidence that interpretation of $S / M$ in $R R$ is not realizable in one stage for any sample. There cannot be any routine in such a kind of study; each case is a special case. In fact, the first stage may be: one observes that standard RR gives poor results; stage two: one tries to identify what kind of problem it is; stage three ..., but why not refine the profile parameters in the last stage? Remember also that doing a RR supposes that the structure is known, at least for a large part.

\section{Some selected RR cases without S/M modelling}

Problems of anisotropic line broadening were encountered in many published papers using standard RR in which some efforts merit to be emphasized. A very pronounced anisotropic broadening effect considerably limited the quality of the Rietveld refinement in the case of $\mathrm{PbC}_{2} \mathrm{O}_{4}$ (Christensen, Cox \& Lehmann, 1989), although it did not make impossible the $a b$ initio structure determination. The structure being triclinic, $\mathrm{PbC}_{2} \mathrm{O}_{4}$ would be a very interesting example for testing the general anisotropic case with ellipsoids. An approach to S/M was presented from individual peak fits of the synchrotron data that revealed all of the reflections to be broadened appreciably by comparison with the instrumental resolution. Those with scattering vectors close to the [001] axis showed an additional systematic broadening consistent with stacking faults in this direction. $\mathrm{R}_{\mathrm{B}}$ and $\mathrm{R}_{w_{p}}(\%)$ were respectively 10.0 and 21.3 for synchrotron and 10.1 and 17.9 for neutron data. This shows how the problem was not anecdotal.

Some authors have sometimes detected problems of stacking fault, antiphase domain and so on (effects on some classes of reflections), and have tried to fit two independent sets of linewidths (or more) in order to improve the structure parameters. This has been done for two $a b$ initio structure determinations from powder data with qualitative interpretation of faulting. For instance, reflections $h k l, l$ odd were fitted with different profile parameters than those with $l$ even in the case of $\mathrm{Rb}\left(\mathrm{VO}_{2}\right)\left(\mathrm{HPO}_{4}\right)$ and $\mathrm{Cs}\left(\mathrm{VO}_{2}\right)\left(\mathrm{HPO}_{4}\right)$ (Amoros, Beltran-Porter, Le Bail, Férey \& Villeneuve, 1988). Reflections $h k l$, $h$ odd were treated separately from the others in the case of $\mathrm{Cd}_{3}(\mathrm{OH})_{5}\left(\mathrm{NO}_{3}\right)$ (Plévert, Louër \& Louër, 1989). Some other works where a significant amount of anisotropic broadening was noticed are: the study of $\mathrm{La}_{2} \mathrm{CuO}_{4+\delta}$ at $297 \mathrm{~K}$ (Zolliker, Cox, Parise, McCarron III \& Farneth, 1990); the study of the $\mathrm{VOPO}_{4} \cdot 2 \mathrm{D}_{2} \mathrm{O}$ structure (Tachez, Theobald, Bernard \& Hewat, 1982). 
Table 1: Size and microstrain parameters for $\mathrm{ZnO}-\mathrm{B}$

\begin{tabular}{|c|c|c|c|c|c|c|c|c|c|c|c|}
\hline \multirow[b]{2}{*}{ lines } & & \multicolumn{5}{|c|}{$\langle\mathrm{D}\rangle \mathrm{v}(\AA)$} & \multicolumn{5}{|c|}{$e \times 10^{3}$} \\
\hline & & $\bar{A}$ & $\overline{\mathrm{B}}$ & $\mathrm{C}$ & $\overline{\mathrm{D}}$ & $\bar{E}$ & $\bar{A}$ & $\bar{B}$ & $\mathrm{C}$ & $\mathrm{D}$ & $\bar{E}$ \\
\hline$(h 00)$ & $\begin{array}{l}(100) \\
(200) \\
(300)\end{array}$ & $\begin{array}{l}392 \\
352 \\
186\end{array}$ & 318 & 287 & 260 & 288 & $\begin{array}{l}3.8 \\
2.7 \\
1.9\end{array}$ & 2.9 & 2.3 & 2.0 & $7.1 ; 2.5$ \\
\hline$(h 0 h)$ & $\begin{array}{l}(101) \\
(202)\end{array}$ & $\begin{array}{l}327 \\
238\end{array}$ & 305 & 313 & 337 & 316 & $\begin{array}{l}3.3 \\
2.5\end{array}$ & 3.2 & 2.7 & 2.9 & $7.3 ; 2.5$ \\
\hline$(00 l)$ & $\begin{array}{l}(002) \\
(004)\end{array}$ & $\begin{array}{l}484 \\
391\end{array}$ & 417 & 408 & 490 & 598 & $\begin{array}{l}2.3 \\
1.7\end{array}$ & 2.0 & 1.6 & 2.0 & $7.8 ; 2.2$ \\
\hline
\end{tabular}

A : PVII, single line (Sonneveld et al., 1986)

B : PVII, multiple line (Sonneveld et al., 1986)

C : PVII, multiple line (Langford et al., 1986)

$\mathrm{D}: \mathrm{pV}$, multiple line (Langford et al., 1986)

$\mathrm{E}: \mathrm{RF}$ method (Two approximate " $e$ " values are given at distances $7.36 \AA$ and at $\langle\mathrm{D}\rangle \mathrm{v}$.)

Application of the RF method to $\mathrm{ZnO}-\mathrm{B}$ and comparison with previous results

A rather well-documented "size-strain" test case is $\mathrm{ZnO}$-B. The conventional X-ray powder pattern has been studied by means of Total Pattern Analysis (Langford et al., 1986; Sonneveld, Delhez, de Keijser, Langford, Mittemeijer, Visser \& Louër, 1986). Results have been extensively discussed by Langford (1987), Langford, Delhez, de Keijser \& Mittemeijer (1988) and Delhez et al. (1989). The latter made a $\mathrm{RR}$ on the $\mathrm{ZnO}$ - $\mathrm{B}$ data (see above), with final residuals $R_{B}=0.028$ and $R_{p}=0.039$ (background (probably) not subtracted (BNS); to understand the interest of giving "background subtracted" (BS) $\mathrm{R}_{p}$, see Hill \& Fisher, 1990). The RF method was thus applied in its original form (Le Bail, $1985 a, b$ ) to the same data set for comparison purposes. The $g$ profile component of $h$ was modelled from the $\mathrm{ZnO}$ standard (Langford et al., 1986) in a preliminary step by a RR (Le Bail, 1984) leading to the residuals $R_{B}=0.022$ and $\mathrm{R}_{p}=0.089$ (BS). Results on $g$ would probably have been better without the presence of a small $\alpha_{2}$ component residue $(<3 \%)$. Good results are really essential on the $g$ part in order to model correctly $h=f^{*} g$ in the next step. It is noteworthy that the best $\mathrm{RR}$ results on this standard $\mathrm{ZnO}$ data set obtained by using the FULLPROF program were with PVII, leading to residuals $R_{B}=0.031 R_{p}=0.124$ (BS) or $\mathrm{R}_{p}=0.108$ (BNS). Then, the ZnO-B pattern was fitted keeping the $g$ part fixed, using ellipsoids of revolution along the $c$ axis for both the $S / M$ effects, with $L$ shape for the size and intermediate $\mathrm{G} / \mathrm{L}$ shape for the microstrain. Final reliabilities were $R_{B}=0.017$ and $R_{p}=0.063(B S)$, with quite comparable structure parameters as those quoted by Delhez et al. (1989).

The $S / M$ values are compared in Table 1 with those from previous studies. As the RF method gives the mean of the area-weighted size distribution function $\langle D\rangle$ a, it was multiplied by two to be compared with $\langle D\rangle v$ (volume weighted), the relation being exact for $\mathrm{L}$ profiles. On the other hand, the RF method gives $\left\langle Z_{n}^{2}\right\rangle=\ln l^{K}\left\langle Z_{1}^{2}\right\rangle$, and $\mathrm{K}$ was refined to $1.43(6)$; thus the comparison with $e$ given in previous studies is not straigthforward, since $e=(1 / 2) \sqrt{2 \pi}\left\langle e^{2}\right\rangle^{1 / 2}$ (de Keijser et al., 1983) only if $\left\langle e_{n}^{2}\right\rangle=\left\langle e^{2}\right\rangle$ (note that $\left.\left\langle e_{n}^{2}\right\rangle=\left\langle Z_{n}^{2}\right\rangle / n^{2}\right)$, i. e. if the microstrain profiles are 
$\mathrm{G}$ (corresponding to $\mathrm{K}=2$ ). Thus, two values calculated as $(1 / 2) \sqrt{2 \pi}\left(\left\langle Z_{1}^{2}\right\rangle \ln l^{K-2}\right)^{1 / 2}$ were given as an approximation for $e$ (at $n=1$, corresponding to the choice of a $7.359 \AA$ reference length, associated with the interval of definition of the reflections, and at the $n$ value corresponding to $\langle D\rangle \mathrm{v})$. The discrepancies in Table 1 give an idea of the poor accuracy now attainable on S/M parameters. All methods use large assumptions, and it is not possible to find out whether one result is better than another. One cannot expect to obtain more than an order of magnitude for the extracted S/M parameters (in this sense, the results of Table 1 are quite similar).

\section{In the future}

It is not yet possible to recommend a particular choice of a procedure for analysis of anisotropic line broadening effects by $R R$ from the jungle of possibilities listed above. What will be useful in the future is somewhat unpredictable. However, any progress in line broadening understanding will certainly come from the fact that whole pattern fitting, with or without a structure model, will allow the suppression of some oversimplifications, since overlapping problems are partly overcome in these methods. Some directions for doing that may be suggested that could be taken from the recent literature or by a complication of formulae used currently, going crescendo towards the general 13.24 equation in Warren (1969).

There still exist activities in the $\mathrm{S} / \mathrm{M}$ domain which have been said to be in infancy. Some selected works are listed in the following. An "Information Theory" approach was devised in order to obtain crystallite-size distributions from X-ray line broadening by Guérin, Alvarez, Rebollo Neira, Plastino $\&$ Bonetto (1986). In very recent works, some approaches to flexible size distribution functions were made. It has been shown that the Voigt functions may be considered to be a good approximation to some particular size distribution functions $\mathrm{P}(n)$ (Selivanov \& Smyslov, 1991). Moreover, exactly the same formulation was proposed for the strain effect (Yuming, Shansan \& Yenchin, 1982), so the description of both $\mathrm{S} / \mathrm{M}$ effects by $\mathrm{V}$ functions may have physical justifications. Other recent work that could serve as a basis for modelling size effects quite different from "Cauchy-like" is that of Rao \& Houska (1986). They showed (for a normal distribution of diameters of statistically spherical subgrains) that the diffraction profile is not very sensitive to the fine details of the size distribution, but is rather sensitive to the mean column height $N_{3}$ and the column-height variation coefficient, $V_{c}$, the latter giving a measure of the spread of the distribution. Two parameters may thus describe $A_{n}^{s}(h k l)$. The question is how to insure some self consistency if subgrains are statistically ellipsoidal instead of spherical? Asymmetry problems may have a solution: random-layer line profiles may be synthesized by Fourier transform methods, as shown by Ruland (1967). A theory has been developed for asymmetric line broadening of plastically deformed crystals (Groma, Ungar \& Wilkens, 1988). A new method of synthesizing the reflection profile, based on the calculation of Fourier coefficients of instrumental functions, was recently presented (Kogan \& Kupriyanov, 1992). Ideas may be found in the domain of modelling powder patterns; for instance, a general recursion method for calculating diffracted intensities from crystals containing planar faults has been proposed recently (Treacy, Newsam \& Deem, 1991).

Improvements could be obtained by the suppression of some practiced approximations having catastrophic effects when reflections are strongly broadened. Actually, the Lorentz-polarization factor variation over one peak, which may be quite large at low angle, is not taken into account. Instead, the value of LP at the $2 \theta_{0}$ position is applied to the entire reflection.

Now, the possibilities of going more closely to the 13.24 equation of Warren (1969) will be considered. Profile shape description by analytical functions, such as $\mathrm{L}$ or $\mathrm{G}$ or convolution of these two (Voigtian), seems to be in a "golden age," but may have no future in the sense that evolution is hardly possible because these shapes correspond to very special cases of S/M. There are not many other analytical profile shapes corresponding to physically realistic S/M effects, and the inevitable convolution $h=f^{*} g$, which may have a simple analytical solution for $L, G$ and $V$, could cause some problems with other ones, although convolute profiles can be obtained numerically (Howard \& Snyder, 1985, 1989). Quite good fits, according to actual criterion of what is a good fit may be $R_{p}<12 \%$ (BS), are obtained with V, PV and/or PVII even for cases with strong anisotropy. A possible limit to the future evolution with analytical profiles appears to be consideration of $S$ and $M$ as having both $L$ and $G$ components, using ellipsoids or something else to describe anisotropy. However, this affirmation may be wrong.

The alternative to analytical profile shapes, i.e. the description of profile shapes in terms of Fourier 
coefficients, has large possibilities of evolution for two main reasons: the mathematical convolution is obtained from a simple multiplication, and a great variety of $\mathrm{S} / \mathrm{M}$ effects may be modelled through flexible expressions for $A_{n}^{s}(h k l)$ and $A_{n}^{d}(h k l)$. Towards the 13.24 equation (Warren,1969), one can imagine quite a lot, starting from the current state of the RF method. The variation law of $\left\langle Z_{n}^{2}\right\rangle$ with $n$ could be, in a first step, made dependent on $h k l$, providing up to 6 more parameters in the general case with $\mathrm{K}(h k l)$. More flexible size distributions allowing definition of $A_{n}^{s}(h k l)$ with a few parameters could be introduced (see Rao et al., 1986; Scardi et al., 1991). The $\left\langle\sin 2 \pi l Z_{n}\right\rangle$ term could be expanded as a series to try to handle asymmetry and could have $h k l$ dependence. Currently, two ellipsoids are used to simulate the mean $\mathrm{N}_{3}(h k l)$ of the size distribution and $\left\langle Z_{1}^{2}\right\rangle(h k l)$. We could go further and imagine that it would be possible to approximate the size and microstrain distributions $\left(\mathrm{P}(n)_{h k l}\right.$ and $\left.\mathrm{P}_{n}(\mathrm{Z})_{h k l}\right)$ themselves by ellipsoids for each $n$ and $Z$ value. With Fourier series, the number of steps that may be expected in order to describe the $\mathrm{Z}$ variable of the microstrain distribution depends on the number of harmonics in each $h \mathrm{kl}$ family, whereas the number of steps to describe $n$ depends on the number of points defining the reflection profiles: typically one could have 50 to $100 n$ values (defining each reflection on 100 to 200 points) and maybe 5 to $10 \mathrm{Z}$ values (if the pattern is recorded with low wavelength up to large diffracting angle) corresponding to a total of 300 to 1100 unknowns to be multiplied by 1 to 6 depending on the restrictions on the ellipsoids. Before claiming that this is impossible, remember that Fourier transforms have a role to play here (moreover with physical meaning), and that one profile defined by 201 steps (for instance) may be transformed into a Fourier series having 101 harmonic numbers (if symmetrical), all being significant if the profile is a "sizeonly" $f$ profile. Since the problem depends on the profile shape, all data points contribute ( 15000 points for a pattern with $0.01^{\circ} 2 \theta$ between 10 and $160^{\circ} 2 \theta$ ), so, is it really impossible? One can imagine considerably reducing the number of unknowns if each microstrain distribution is assumed to be Gaussian, so only the width and centroid position would have to be found for each $n$ and $h k l$ value. The number of unknowns reduces to 150 to 300 (or 100 to 200 if the Gaussian distributions of microstrain are centered on the mean cell parameters), to be multiplied by 1 to 6 . The problem has to be correctly formulated with constraints to force $\mathrm{P}(n)$ to be positive or zero. Never- theless, the three-dimensional self consistency would be perhaps difficult to ensure. Being a chemist, I am afraid that $I$ have not enough mathematical qualifications to try to do it! On the other hand, as stated by Langford (1987), for many applications it is often not necessary to obtain microstructural parameters with a high absolute accuracy. One may be mainly interested in improving the structure parameters and not wish to give a meaningful physical sense to functions used for modelling profile shapes (to model empirically, but perfectly, powder patterns for structure refinement purposes appears not so useless, particularly for ultra-high resolution data from synchrotron X-rays). To do that, one can imagine using direction-related ellipsoids for both $\mathrm{L}$ and $\mathrm{G}$ parts, both having different $2 \theta$ dependence, and not taking into account the instrumental part $g$. This would probably provide a "good" fit favorable to the structure accuracy.

\section{Conclusion}

A question is: does simultaneous structure-S/M refinement increase the credibility of all parameters? Moreover, why do it when more or less perfect fits may be obtained without structure constraints, without space group constraint, and even without cell constraints? It is clear that the fewer the constraints, the more the fit may be perfect. It may even be argued that profile decomposition techniques are free from the structural model errors and the preferred orientation correction, unlike the Rietveld method; I believe in turn that the structure constraint is a "garde-fou" in case of serious overlapping of peaks. Some Rietveld programs now offer the possiblity to switch off the structure constraint when desired, and to perform iterations of the Rietveld decomposition formula leading to a cell and space-group constrained full-pattern profile fitting (Le Bail, 1992). Nevertheless, the high quality of the fit obtained for $\mathrm{CuO}$ by pattern fitting, without structure, using PVII (Langford \& Louër, 1991) is not attainable now by any $\mathrm{S} / \mathrm{M}$ modelling in Rietveld analysis. In this case, residual effects after annealing at $1273 \mathrm{~K}$ were an $\mathrm{hkl}$ dependent skewing and line broadening due to a distribution of $d$ spacing.

When I have sample effects, unless there is a special interest in studying these effects, I do my best to obtain a well-crystallized sample by some chemical or physical means, but this is not always possible. The state of the art has to progress, and real accuracy concerning S/M will certainly not be obtained unless 
$\mathrm{R}_{p}<4$ or maybe $2 \%$ (BS). We are far from routinely obtaining such low values. It would be necessary to improve instrumentation, sample alignment, theories of sample effects, etc. Modelling $g$ in the Rietveld method must be at the same level of accuracy as modelling $h$ (Langford, Cernik \& Louër, 1991). However, if such low $\mathrm{R}_{p}$ are obtained by adding say 200 profile parameters to be refined, I am not sure that this would be highly credible. On the other hand, a rigorous treatment of the problem theoretically involves much more (by Fourier transform), and the results would be credible without the overlapping problem. The study of defective single microcrystals using synchrotron radiation is also a possibility of the present decade. A full account (three-dimensional) of the $f$ profiles of all reflections would be of the highest interest. I am afraid for the next generation of users (solid state chemists or physicists, for instance) that the level of sophistication will seriously increase in the future.

\section{Acknowledgments}

The author is grateful to J. I. Langford, D. Louër, E. J. Sonneveld and J. W. Visser for kindly supplying the patterns of $\mathrm{ZnO}-\mathrm{B}$ and well-crystallized $\mathrm{ZnO}$ studied in their 1986 paper.

\section{References}

Adler, T. \& Houska, C. R. (1979). J. Appl. Phys. 50, 3282-3287.

Ahtee, M., Unonius, L., Nurmela, M. \& Suortti, P. (1984). J. Appl. Cryst. 17, 352-357.

Amoros, P., Beltran-Porter, D., Le Ball, A., FÉREY, G. \& Villeneuve, G. (1988). Eur. J. Solid State Inorg. Chem. 25, 599-607.

Bertaut, E. F. (1949). C. R. Acad. Sci. 228, 492-494. Bizot, D., Chassaing, J., Pannetier, J., Leblanc, M., Le BaIl, A. \& Férey, G. (1986). Solid State Commun. 58, 71-74.

Buchanan, D. R., McCullough, R. L. \& MLler, R. L. (1966). Acta Cryst. 20, 922-924.

Caglioti, G., Paoletti, A. \& Ricci, F. P. (1958). Nucl. Instrum. 3, 223-228.

Cheetham, A. K. \& Wilkinson, A. P. (1991). J. Phys. Chem. Solids 52, 1199-1208.

Christensen, A. N., Cox, D. E. \& Lehmann, M. S. (1989). Acta Chem. Scand. 43, 19-25.

Cox, D. E. (1984). Acta Cryst. A40, Suppl. C369.

Cox, D. E. (1991). Handbook on Synchrotron Radiation, Elsevier Science Publishers, Brown, G. \& Moncton, D. E., eds. Vol. 3, 155-200.
David, W. I. F. \& Matthewman, J. C. (1985). J. Appl. Cryst. 18, 461-466.

Delhez, R., KeiJser, Th. H. DE, Langford, J. I., LOUËr, D., MitTemeiJer, E. J. \& SONNEveld, E. J. (1989). The Rietveld Method, R. A. Young, ed. Oxford: Oxford University Press

Delhez, R., KeiJser, Th. H. DE \& MittemeiJer, E. J. (1980). Accuracy in Powder Diffraction, S. Block \& C. R. Hubbard, eds. Natl. Bur. Stand. (U. S.) Spec. Publ. 567, pp. 213-253.

Delhez, R., Keijser, Th. H. DE \& MittemeiJer, E. J. (1982). Fresenius Z. Anal. Chem. 312, 1-16.

Delhez, R., KeiJser, Th. H. DE, MittemeiJer, E. J. \& Langford, J. I. (1988). Aust. J. Phys. 41, 213-227. Ergun, S. (1970). Phys. Rev. B 1, 3371-3380.

Groma, I., Ungar, T. \& WLikens, M. (1988). J. Appl. Cryst. 21, 47-53.

Gibaud, A., Le Ball, A. \& Bulou, A. (1986). J. Phys. C. 19, 4623-4633.

Greaves, C. (1985). J. Appl. Cryst. 18, 48-50.

Gú́rin, D. M. A., Alvarez, A. G., Rebollo Neira, L. E., Plastino, A. \& Bonetto, R. D. (1986). Acta Cryst. A42, 30-35.

Hepp, A. \& Baerlocher, Ch. (1988). Aust. J. Phys. 41, 229-236.

Hewat, A. W. (1986). Chem. Scripta 26A, 119-130.

Hill, R. J. \& Howard, C. J. (1985). J. Appl. Cryst. $18,173-180$.

HLL, R. J. \& Fisher, R. X. (1990). J. Appl. Cryst. 23, 462-468.

HosemanN, R. \& Bagchi, S. N. (1962). Direct Analysis of Diffraction By Matter, Amsterdam: North-Holland.

HoWARD, S. A. \& SNYDER, R. L. (1985). Materials Science Research; Symposium on Advances in Materials Research 19, 57-71.

Howard, S. A. \& SNYDER, R. L. (1989). J. Appl. Cryst. 22, 238-243.

Howard, S. A., YaU, J. K. \& ANderson, H. A. (1989). J. Appl. Phys. 65, 1492-1498.

Kamiyama, T., Shinohara, T., Tomiyoshi, S., Asano, H., Yamamoto, H. \& Watanabe, N. (1987). Proc. Int. Symp. on Physics of Magnetic Materials, Sendai, April 1987, M. Takahasa, S. Maekawa, Y. Gondo \& H. Nose, eds. Singapore: World Scientific, pp 579-582.

Kamiyama, T., Shinohara, T., Tomiyoshi, S., MInonishi, Y., Yamamoto, H., Asano, H. \& WatanABE, N. (1990). J. Appl. Phys. 68, 4741-4750.

Keijser, Th. H. DE, LangFord, J. I., Mittemeijer, E. J. \& Vogels, A. B. P. (1982). J. Appl. Cryst. 15, 308-314.

Keijser, Th. H. DE, Mittemeijer, E. J. \& RozenDAAL, H. C. F. (1983). J. Appl. Cryst. 16, 309-316.

Kogan, V. A. \& KupriYanov, M. F. (1992). J. Appl. Cryst. 25, 16-25.

Lafontaine, M. A., Le Bail, A. \& Férey, G. (1990). J. Solid State Chem. 85, 220-227. 
LangFord, J. I. (1978). J. Appl. Cryst. 11, 10-14

Langford, J. I. (1987). Prog. Crystal Growth and Charact. 14, 185-211.

LANGFord, J. I., CERNIK, R. J. \& Loü̈r, D. (1991). J. Appl. Cryst. 24, 913-919.

Langford, J. I., Delhez, R., KeIJSER, Th. H. \& MiTTEMEIJER, E. J. (1988). Aust. J. Phys. 41, 173-187.

Langford, J. I. \& Loü̈r, D. (1991). J. Appl. Cryst. 24, 149-155.

LANGFORD, J. I., Loü̈r, D., SONNEVELD, E. J. \& Visser, J. W. (1986). Powd. Diffr. 1, 211-221.

Lartigue, C., Le BaIl, A. \& Percheron-Guegan, A. (1987). J. Less Common Met. 129, 65-76.

Le BaIl, A. (1984). Acta Cryst. A40, Suppl. C369.

LE BAIl, A. (1985a). PhD Thesis, Le Mans.

LE BAIL, A. (1985b). Proc. 10th Collogue Rayons $X$, Siemens, Grenoble, 45-58.

LE BAIl, A. (1992). In this volume.

LE BaIL, A., Boulard, B. \& Jacoboni, C. (1987). Mater. Sci. Forum, 19-20, 127-136.

Le BaIl, A., Duroy, H. \& Fourquet, J. L. (1988). Mater. Res. Bull. 23, 447-452.

Le BaIl, A. \& Jacoboni, C. (1984). Acta Cryst. A40, Suppl. C477.

Le BaIL, A., Jacoboni, C. \& De Pape, R. (1985). J. de Physique, Colloque C8, 46, 163-168.

LOUËR, D. (1988). Chemica Scripta 28, 89-95.

Lutterotti, L. \& Scardi, P. (1990). J. Appl. Cryst. 23, 246-252.

Maret, M., Chieux, P., Dubois, J. M. \& Pasturel, A. (1991). J. Phys.: Condens. Matter 3, 2801-2817.

Millini, R., Perego, G. \& Brǘckner, S. (1991). Mater. Sci. Forum 79-82, 239-244.

NANDI, R. K., KUO, H. K., ScholsBerG, W., Wissler, G., Cohen, J. B. \& Crist, B. JR. (1984). J. Appl. Cryst. 17, 22-26.

Plévert, J., LouËr, M. \& Loü̈r, D. (1989). J. Appl. Cryst. 22, 470-475.

Rao, S. \& Houska, C. R. (1986). Acta Cryst. A42, 613.

RIETVELD, H. M. (1967). Acta Cryst. 22, 151-152.

Rietveld, H. M. (1969). J. Appl. Cryst. 2, 65-71.

Rodriguez-Carjaval, J. (1990). Abst. Satellite Meeting on Powder Diffraction of the XVth IUCr Congress (Toulouse), 127-128.

Rodriguez-Carjaval, J., Fernandez-Diaz, M. T. \& Martinez, J. L. (1991). J. Phys.: Condens. Matter 3, 3215-3234.

Rotella, F. J. \& Richardson, J. W. JR. (1986). Inst. Phys. Conf. Ser. 81, 27-36.
Rotella, F. J. \& Richardson, J. W. JR. (1987).

Trans. Amer. Cryst. Assoc. 23, 83-86.

Ruland, W. (1967). Acta Cryst. 22, 615-623.

Scardi, P. Lutterotti, L., Di Maggio, R. \& Maistrelli, P. (1991). Mat. Sci. Forum 79-82, 233238.

Selivanov, V. N. \& Smyslov, E. F. (1991). Zavod. Lab. 57, 28-29; Engl. Transl.: (1991) Industrial Lab. 57, 709-710.

Sonneveld, R. J., Delhez, R., Keijser, TH. H. DE, LANGFord, J. I., MitTEMEiJer, E. J., VisSER, J. W. \& LoÜ̈R, D. (1986). Proc. 12th Conf. on Applied Crystallography Katowice:Silesian University, 26-31.

Tachez, M., Theobald, F., Bernard, J. \& Hewat, A. W. (1982). Rev. Chim. Miner. 19, 291-300.

Thompson, P., Reilly, J. J., Corliss, L. M., HastIngs, J. M. \& Hempelmann, R. (1986). J. Phys. F. 16, 675-685.

Thompson, P., Reilly, J. J. \& Hastings, J. M. (1987). J. Less Common Met. 129, 105-114.

Thompson, P., Cox, D. E. \& Hastings, J. B. (1987). J. Appl. Cryst. 20, 79-83.

Toraya, H. (1989). Powd. Diffr. 4, 130-136.

Treacy, M. M. J., Newsam, J. M. \& DeEM, M. W. (1991). Proc. Roy. Soc. Lond. A433, 499-520.

Warren, B. E. (1959). Prog. Met. Phys. 8, 147-202.

WARREN, B. E. (1969). In X-ray Diffraction New York: Addison-Wesley.

Warren, B. E. \& Averbach, B. L. (1950). J. Appl. Phys. 21, 595-599.

WILKENS, M. (1979). J. Appl. Cryst. 12, 119-125.

Wiles, D. B. \& Young, R. A. (1981). J. Appl. Cryst. 14, 149-151.

Williamson, G. K. \& Hall, W. M. (1953). Acta Met. 1, 22-31.

WILson, A. J. C. (1949). X-Ray Optics, London: Methuen.

YaU, J. K. \& Howard, S. A. (1989). J. Appl. Cryst. 22, 244-251.

Young, R. A. \& Wiles, D. B. (1982). J. Appl. Cryst. $15,430-438$

Young, R. A. \& Desai, P. (1989). Archiwum Nauki o Materialach 10, 71-90.

Young, R. A. \& SaKthivel, A. (1988). J. Appl. Cryst. 21, 416-425.

Yuming, W., Shansan, L. \& Yenchin, L. (1982). $J$. Appl. Cryst., 15, 35-38.

Zolliker, P., Cox, D. E., Parise, J. B. McCarron, E. M. III \& Farneth, W. E. (1990). Phys. Rev. B 42, 6332-6341. 
National Institute of Standards and Technology Special Publication 846. Proceedings of the international conference Accuracy in Powder Diffraction II, held at NIST, Gaithersburg, MD, May 26-29, 1992. (Issued October 1992)

\title{
Structure Building with Rietveld Analysis
}

\author{
D. L. BISH \\ MS D469, Los Alamos National Laboratory \\ Los Alamos, NM 87545, U. S. A.
}

\section{Introduction}

The Rietveld method was originally conceived (Rietveld, $1967,1969)$ to refine crystal structures using neutron powder diffraction data. By its very nature, structure refinement relies on the existence of a reasonably complete structure model. Diffraction data are then used to improve, or to refine, the model so that optimum agreement between observed and calculated observations is obtained. Such refinement methods have been critically important over the past few decades in expanding our knowledge of the details of thousands of crystal structures. The Rietveld method has been adapted over the years to be used with X-ray powder diffraction data, in addition to neutron diffraction data, thereby greatly expanding the application of the method. However, the user of the method is still faced with the necessity of having a general knowledge of the crystal structure of the phase (or phases) in question before beginning refinement.

The Rietveld method has been used in recent years with other ancillary techniques to expand the number of crystalline phases that can be analyzed and the amount of information obtainable. High-resolution transmission electron microscopy (HRTEM) images (e.g., Post and Bish, 1988; Richardson and Vogt, 1992) have been used in some cases to provide starting models for structure refinement, and distance least-squares (DLS) methods (e.g., McCusker and Baerlocher, 1984) are very commonly used. The Rietveld method was used in these cases to evaluate and to refine proposed structure models. In several other applications, observed and calculated structure factors extracted from Rietveld refinements have been used as input to Fourier syntheses (e.g., Adams and Hewat, 1981; Lager et al., 1987). Difference-Fourier $(\Delta F)$ syntheses have been used very effectively to locate the positions of extraframework atoms or to investigate the possibility of multiple or split atom positions in a variety of materials, including zeolites and clay minerals. Thus, although the Rietveld method was originally designed for structure refinement, it is very well suited to structure solution. This paper will review the results of partial and complete structure solution using the Rietveld method, emphasizing both the strengths and weaknesses of the combined methods. In addition, it will review the benefits and difficulties of using several types of observations, e.g., neutron and X-ray powder diffraction data, in Rietveld refinements. It will not review $a b$ initio structure determinations that use a combination of profile fitting to obtain individual reflection intensities, direct or Patterson methods to obtain an approximate structure, and final Rietveld refinement (e.g., Clearfield et al., 1984) as this combination of methods warrants a separate paper.

\section{Starting models}

Because any crystal structure refinement relies on a reasonable beginning crystal structure, determination of the starting model is a crucial part of any Rietveld refinement. Geometrical constraints and crystal chemical intuition have played a part in solving structures since the first crystal structure determinations in the early part of this century. However, as the complexity of structures increases, additional information is usually required. In recent years, further data have come in the form of HRTEM images; these images have been used to provide structure models. In several cases, the structure models derived from HRTEM images were then further optimized using DLS modeling to provide a useful starting model for Rietveld refinement.

\subsection{High-resolution TEM data}

Within the past 20 years, transmission electron microscopy has advanced to the point where it can provide images of the atomic arrangement in a crystal, often easily yielding insights into general structural schemes and, sometimes, solving apparently intractable problems (e.g., Veblen, 1985; Buseck and Veblen, 1988). It is important to note, however, that HRTEM images must be used with caution. Minor changes in focus in the microscope can cause large changes in the high-resolution image, greatly changing the images (e.g., Guthrie and Veblen, 1990). To interpret the images unambiguously, it is necessary to couple 
HRTEM observations with modeling of the images using a multi-slice calculation; such calculations require at least an approximate knowledge of the structure (e.g., O'Keefe, 1984).

In an example of the use of HRTEM images to elucidate the structure of an unknown material, Turner and Buseck (1981) and Post and Bish (1988) recognized similarities between the chain structures of hollandite $\left(\mathrm{BaMn}_{8} \mathrm{O}_{16}\right)$ and romanechite $\left(\mathrm{BaMn}_{5} \mathrm{O}_{10} \bullet \mathrm{H}_{2} \mathrm{O}\right)$ and HRTEM images of todorokite. The hollandite structure consists of double chains of $\mathrm{Mn}-\mathrm{O}$ octahedra forming $2 \times 2$ tunnels, and romanechite has both double and triple chains, forming $2 \times 3$ tunnels (Figure 1). HRTEM images (Turner and Buseck, 1981) suggested that todorokite has a $3 \times 3$ tunnel structure, although other authors had suggested a layer structure. Post and Bish derived a starting model for todorokite using DLS modeling, assuming a $3 \times 3$ tunnel structure inferred from HRTEM images and observed $\mathrm{Mn}-\mathrm{O}$ and $\mathrm{O}-\mathrm{O}$ bond distances from hollandite and romanechite. A reasonable refinement was obtained, in spite of the very poor quality of the X-ray powder diffraction pattern due to structural disorder; the refinement confirmed the $3 \times 3$ tunnel structure. In a similar situation, Richardson and Vogt (1992) used HRTEM images, symmetry information, DLS modeling, and comparisons with the structure of the zeolite VPI-5 to obtain a reasonable starting model for $\mathrm{AlPO}_{4}-8$. They successfully refined their model structure using neutron powder diffraction data. It is important to note, however, that just as with the first structure solutions in the early part of this century, crystal chemical intuition was important with both structure determinations, as was informed interpretation of the HRTEM images.

The structure determination of $\mathrm{TiO}_{2}(\mathrm{~B})$ by Banfield et al. (1991) using HRTEM and DLS modeling illustrates the utility of this combination of methods and is an example of the importance of HRTEM image simulations. A model structure was obtained from HRTEM images of semicoherently intergrown lamellae of the B phase in anatase (also $\mathrm{TiO}_{2}$ ). The authors confirmed the validity of the model structure by comparing computercalculated images with their observed images. They then refined their model structure using DLS methods, assuming bond lengths taken from the well-known polymorphs of $\mathrm{TiO}_{2}$, anatase and rutile. Image simulations for their model structure agreed closely with the observed images, and it is noteworthy that their final model structure reproduced the polyhedral distortions predicted for the ideal $\mathrm{TiO}_{2}(\mathrm{~B})$ structure using electrostatic minimization procedures. Although Banfield et al. did not proceed with a Rietveld structure refinement due to a lack of material, their procedure could be applied in detail to virtually any unknown structure.
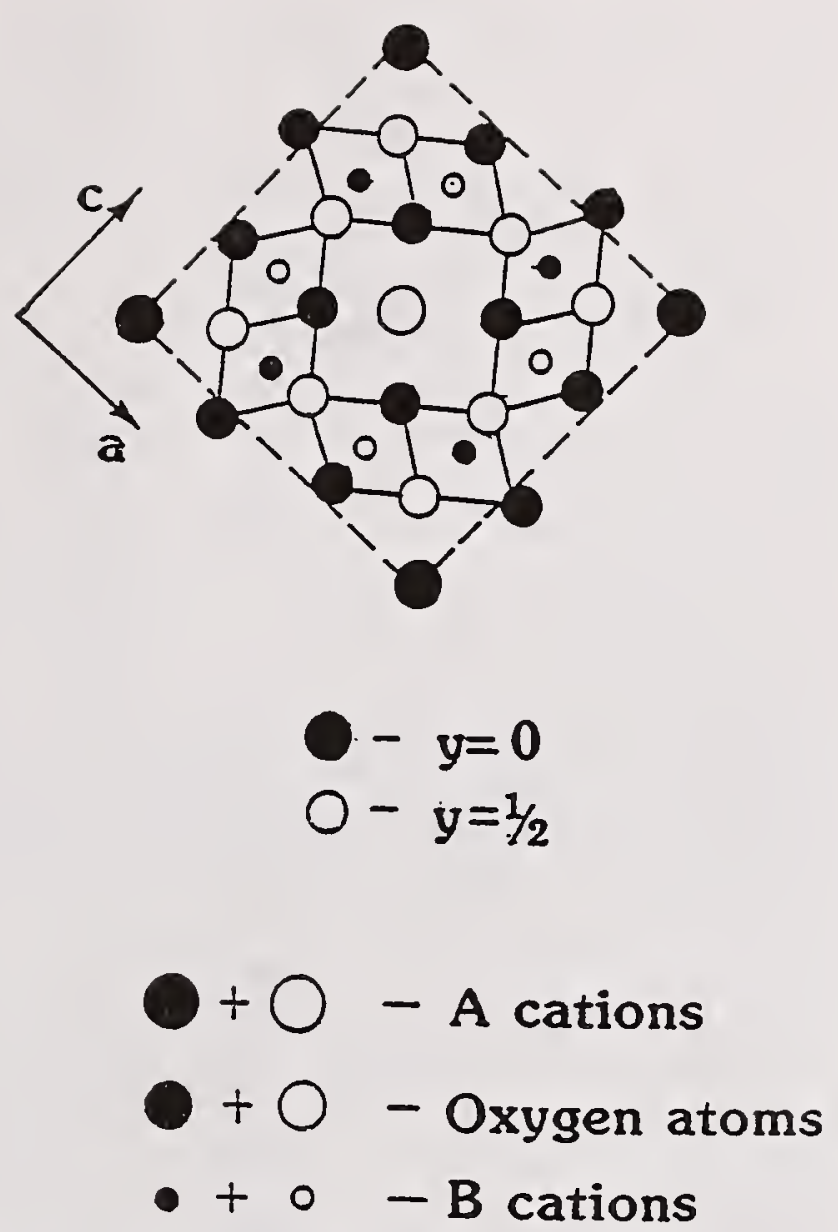

Figure 1. Projection of the hollandite structure down the monoclinic $b$ axis, illustrating the $2 \times 2 \mathrm{Mn}-\mathrm{O}$ octahedral structure (from Post, 1981).

\subsection{Distance least-squares modeling}

DLS modeling (Meier and Villiger, 1969) is a powerful method for determining whether a particular model is geometrically possible. The method is similar in many respects to conventional crystal structure refinement, particularly in that it requires a reasonable starting model. These models can be obtained through analogy with other structures or by crystal chemical reasoning (or from HRTEM images). Fortunately, the DLS method is very robust and will usually converge, even given very poor starting models. An optimum distance model is obtained by varying atomic coordinates and lattice parameters to minimize discrepancies between interatomic distances calculated for the model structure and prescribed (input) distances that comprise the observations. Input distances are usually obtained from published, well-refined analogous structures and can often be estimated to within several hundredths of an Ångstrom. Weights applied to distances are usually either related to Pauling bond strengths or to some empirical measurement, such as stretching force constants; weights based on the latter weight shorter distances and those involving highly charged ions 
much more heavily than the former method. Known lattice parameters can be used often to constrain the refinements further, resulting in more reasonable structures. When beginning with unknown or poorly known structures, lattice parameters can usually be obtained by indexing the powder or electron diffraction pattern using well-established techniques.

DLS modeling of a completely unknown structure such as todorokite requires some imagination and adjunct information (such as HRTEM images), but modeling a variant of a known material can be quite easy. For example, if an intercalation reaction in a layered material such as kaolinite, $\mathrm{Al}_{2} \mathrm{Si}_{2} \mathrm{O}_{5}(\mathrm{OH})_{4}$, gives rise to a larger unit repeat in the direction perpendicular to the layers, DLS modeling can use the parent structure with the new lattice parameters to represent the new structure. Of course, one must either provide lattice parameters or use distance observations sufficient to constrain the structure in three dimensions. After any DLS modeling, calculated diffraction patterns usually provide a quick idea of how reasonable the structure model is, easily revealing whether the symmetry and cell are correct and how closely the model structure approximates the real one. This procedure is similar to that used by Banfield et al. (1991) with HRTEM images, namely obtaining a model structure and determining how well observations agree with those calculated based on the model. In some cases, a DLS model structure can minimize the problems of falseminima in a Rietveld refinement, as appears to be the case for kaolinite (Bish and Von Dreele, 1989). Rietveld refinement of kaolinite using a previously published structure as a starting model consistently resulted in a similar structure with unusual bond distances. Refinement of the kaolinite structure using a DLS structure as the starting model resulted in convergence to a reasonable structure with normal bond distances. It is noteworthy in this case that application of heavily-weighted soft constraints during refinement led to the same false-minimum structure when starting with the published structure.

\section{Partial structure solution using $\Delta \mathrm{F}$ maps}

Difference-Fourier synthesis is a method used for refining crystal structures and is particularly well suited for locating scattering density missing from the structure model. A $\Delta \mathrm{F}$ synthesis is unaffected by series termination errors and uses structure factors $\left(F_{o b s}\right)$ obtained from observed intensities and those calculated using the model structure $\left(\mathrm{F}_{\text {call }}\right)$. It involves calculating the function

$$
\begin{aligned}
& \mathrm{D}(\mathrm{x}, \mathrm{y}, \mathrm{z})=1 / \mathrm{V} \Sigma_{\mathrm{h}} \Sigma_{\mathrm{k}} \Sigma_{1}\left(\mathrm{~F}_{\mathrm{o}} \mathrm{b}_{\mathrm{s}}^{\mathrm{k} 1}-\right. \\
& \left.\mathrm{F}_{\mathrm{ca} \text { a }}^{\mathrm{h} k 1}\right) \cos 2 \pi(\mathrm{hx}+\mathrm{ky}+\mathrm{lz}) \text {. }
\end{aligned}
$$

There are many factors to consider when calculating $\Delta \mathrm{F}$ maps, whether from powder or single-crystal diffraction data. First, in this calculation, the sign (or phase) of $F_{\text {obs }}$ is assumed to be the same as $F_{\text {calc }}$. Therefore, if a significant amount of scattering density is missing or misplaced, the calculated signs (or phases) may be incorrect. For example, Rietveld refinements and $\Delta F$ calculations of synthetic Cs A and Y zeolites (Bish, unpublished) were fraught with difficulties because the omitted Cs atoms resulted in many incorrect structure factors (magnitudes and phases). Further, during the initial stages of refinement when some atoms are omitted, it is likely that temperature factors and scale factors will be incorrect, often by a large amount. An additional important consideration when calculating $\Delta \mathrm{F}$ maps during Rietveld refinement is that decomposition of overlapping peaks is performed assuming the ratios of the calculated intensities of the contributing reflections. Thus, results from a set of Rietveld refinements and $\Delta \mathrm{F}$-map calculations may be very different from those that would be obtained from profile refinement, where observed intensities are generally obtained independent of any model. In general, electron-density (or neutron-density) maxima are lower for Rietveld-derived $\Delta \mathrm{F}$ maps than for those obtained from single-crystal data.

Because of these complications, location of missing atoms is always an iterative process, where atoms are found, added to the model if reasonable (in scattering power and position), and another $\Delta \mathrm{F}$ map is calculated after extraction of observed F's. As more of the correct scattering material is added to the model, the calculated F's (magnitudes and phases) and scale factors become more correct, and the decomposition of overlapping peaks is improved. These complications also dictate how a Rietveld refinement must be performed to obtain reasonable calculated F's. Typically, a minimum of parameters is varied, and structural parameters are usually not refined. If structural parameters are varied, they may adjust in an attempt to compensate for the missing scattering power, yielding an unreasonable structure and complicating the interpretation of the $\Delta \mathrm{F}$ map. In a Rietveld refinement $/ \Delta \mathrm{F}$ map calculation, the scale factor(s) and background parameters are usually varied first, followed by the lattice parameters. In some cases it is preferable to obtain the scale factor from high-angle data, fix it, and then calculate a $\Delta F$ map. Some profile parameters may be refined, although heavily overlapped patterns may require either fixing or manual adjustment of these parameters if observed and calculated patterns are significantly different. In practice, it appears that $\Delta \mathrm{F}$ maps are relatively insensitive to a preferred orientation correction, although it is preferable to use data unaffected by orientation. 
A test case using the known structure of the zeolite analcime, $\mathrm{NaAlSi}_{2} \mathrm{O}_{6} \bullet \mathrm{H}_{2} \mathrm{O}$, illustrates some common features of partial structure solution during Rietveld refinement. Rietveld refinement of analcime was conducted using the tetragonal structure of Mazzi and Galli (1978) and X-ray powder diffraction data. For the test case, both the $\mathrm{Na}$ and $\mathrm{H}_{2} \mathrm{O}$ sites were purposely left vacant, and initial cycles varied only the scale, background, and lattice parameters. Five large positive peaks appeared on the first $\Delta F$ map (Figure 2) calculated; the two tetrahedral Al,Si sites (which were fully occupied in the model), the two $\mathrm{Na}$ sites, and the $\mathrm{H}_{2} \mathrm{O}$ site. The refined scale factor was 239.2. Interestingly, the $\mathrm{Na}$ positions in Figure 2 reveal the same anisotropy observed by Mazzi and Galli (1978) using single-crystal $\mathrm{X}$-ray diffraction data. After insertion of the two $\mathrm{Na}$ atoms, refinement was continued and another $\Delta \mathrm{F}$ map was calculated. Three large positive peaks appeared on the resultant map; the two tetrahedral $\mathrm{Al}, \mathrm{Si}$ sites and the $\mathrm{H}_{2} \mathrm{O}$ site, but little evidence of the $\mathrm{Na}$ sites. The refined scale factor was 291.3. At this stage, the $\mathrm{H}_{2} \mathrm{O}$ molecule (represented by an $\mathrm{O}$ atom) was inserted and occupancies of the $\mathrm{Na}$ and $\mathrm{H}_{2} \mathrm{O}$ sites and temperature factors of all atoms were refined. A $\triangle F$ map calculated after this stage revealed no large peaks, and the scale factor was 456.4 . Removing the $\mathrm{H}_{2} \mathrm{O}$ from this refined structure yielded a $\Delta \mathrm{F}$ map that had only a single peak due to $\mathrm{H}_{2} \mathrm{O}$ (Figure 3). The appearance of density at the tetrahedral atom positions during the initial stages of refinement and $\Delta F$ map calculation was probably due to a combination of an incorrect scale factor and incorrect temperature factors. Determination of an initial scale factor using only high-angle data may have partially compensated for these discrepancies. Results of this simple test refinement should be considered when performing Rietveld refinement coupled with calculation of $\Delta \mathrm{F}$ maps on a poorly known structure. Several further examples will illustrate the potential of these methods when applied to incompletely known structures.

\subsection{Todorokite}

The structure of todorokite, $(\mathrm{Na}, \mathrm{Ca}, \mathrm{K})_{0.55}$ $\left(\mathrm{Mn}^{4+}, \mathrm{Mn}^{3+}\right)_{5.6} \mathrm{Mg}_{0.4} \mathrm{O}_{12} \bullet 4 \mathrm{H}_{2} \mathrm{O}$, was first solved and refined by Post and Bish (1988) as outlined above. After determination of the $\mathrm{Mn}-\mathrm{O}$ framework structure using a DLS starting model and Rietveld refinement, a remaining question was the positions of the included $\mathrm{Na}$, $\mathrm{Ca}, \mathrm{K}$, and $\mathrm{H}_{2} \mathrm{O}$. Post and Bish used Rietveld-derived observed intensities and the DLS model structure to calculate $\Delta \mathrm{F}$ maps for two different todorokites. All of the electron density on the maps was located in the tunnel region (Figure 4) and was similar for both

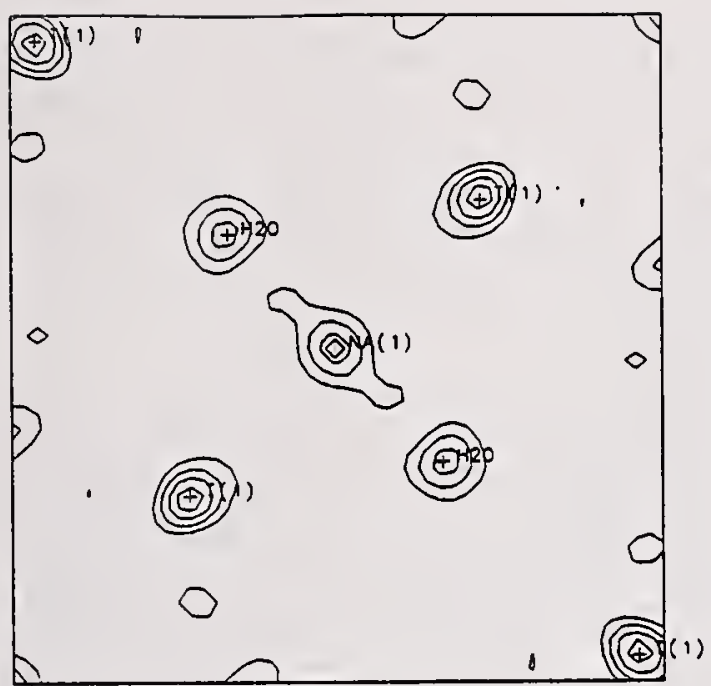

Figure 2. Difference-Fourier map of analcime at $z=0$, calculated without $\mathrm{Na}$ or $\mathrm{H}_{2} \mathrm{O}$ in the model. Contours are drawn at 2.0, 4.0, 6.0, and 8.0 electrons.

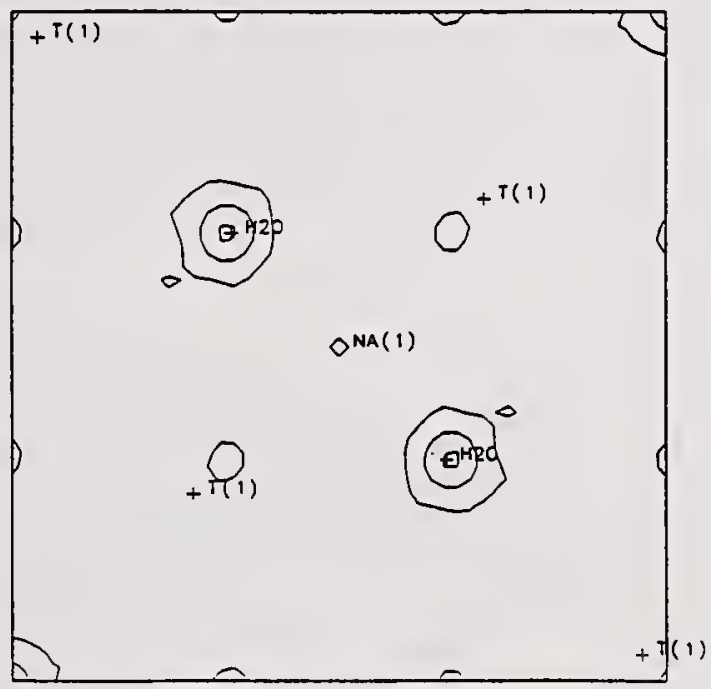

Figure 3. Difference-Fourier map of analcime at $z=0$, calculated after full refinement and subsequent removal of $\mathrm{H}_{2} \mathrm{O}$. Contours are drawn at 1.0, 3.0, and 5.0 electrons.

samples, suggesting that the information was not an artifact. Since most of the non-framework material in todorokite is $\mathrm{H}_{2} \mathrm{O}$, the three largest peaks in the $\Delta \mathrm{F}$ maps were assigned to $\mathrm{H}_{2} \mathrm{O}$, and Rietveld refinement was continued. The positions and occupancies of the three tunnel sites were refined successfully, yielding a significant reduction in the $R_{\mathrm{wp}}$ values. Although it is often difficult to distinguish between contributions from tunnel cations and $\mathrm{H}_{2} \mathrm{O}$ molecules, Post and Bish conducted electrostatic minimizations strongly suggesting that the two largest regions of electron density correspond to $\mathrm{H}_{2} \mathrm{O}$ whereas the density near 
$(1 / 2,1 / 2,1 / 2)$ is due to tunnel $\mathrm{Ca}$ and $\mathrm{Na}$. Given the disordered nature of todorokite and the resultant poor quality of the diffraction data, it is remarkable that such a cohesive picture of the todorokite structure emerged, attesting to the power of the DLS-Rietveld-energy minimization combination. The final refined structure is shown in Figure 5.

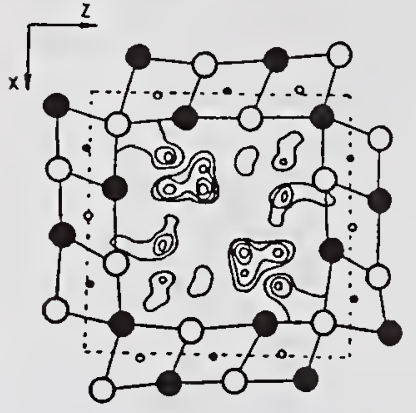

a

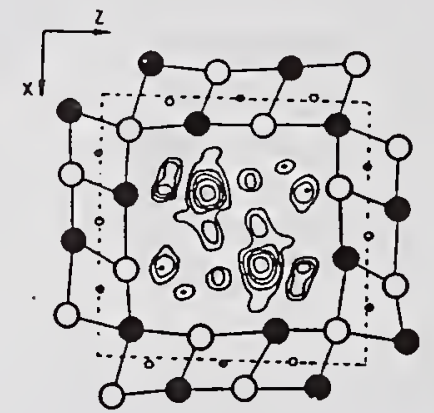

b
Figure 4. Difference-Fourier maps at $y=0$ superimposed onto projections of the $3 \times 3$ tunnel structure of todorokite, looking down $b$, for samples from (a) South Africa and (b) Cuba. Filled circles are atoms at $y=0$ and open circles are those at $y=1 / 2$. Large circles are $\mathrm{O}$ and small circles indicate $\mathrm{Mn}$. Contour interval is $0.4 \mathrm{e}^{-} / \AA^{3}$. The todorokite unit cell is indicated by the dotted lines (from Post and Bish, 1988).

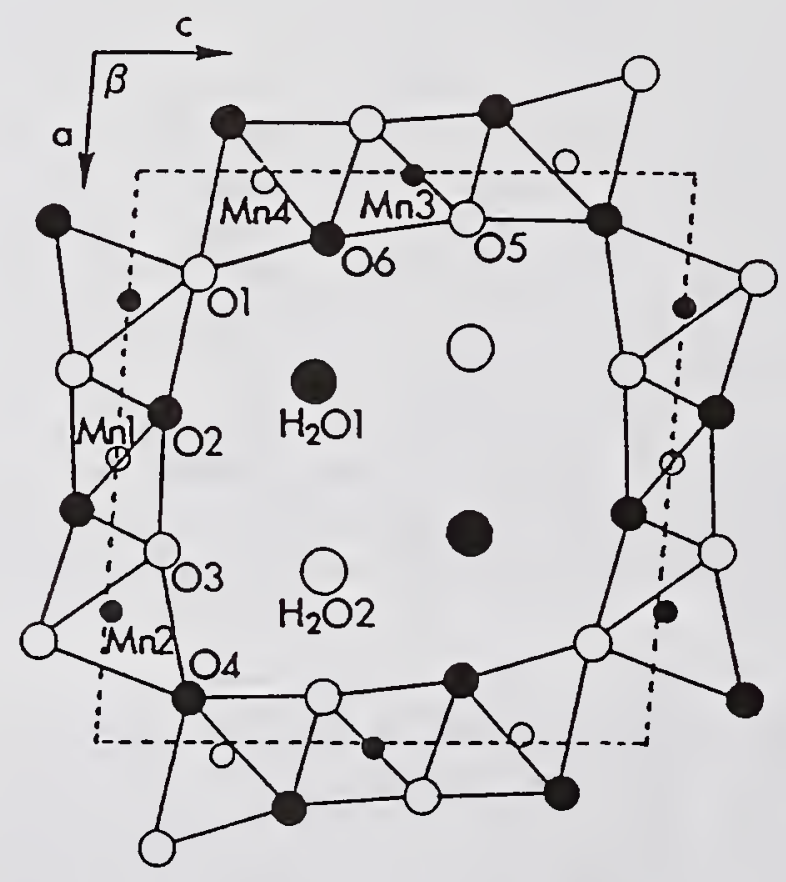

Figure 5. Projection of the final refined structure of todorokite down $b$. Filled circles represent atoms at $\mathrm{y}=0$ and open circles are atoms at $\mathrm{y}=1 / 2$ (from Post and Bish, 1988).

\subsection{Location of hydrogen atoms in kaolinite and dickite}

One of the more interesting aspects of structural studies of layer silicates and clay minerals is the location of the $\mathrm{H}$ atoms that so significantly affect their properties. The structure of kaolinite, $\mathrm{Al}_{2} \mathrm{Si}_{2} \mathrm{O}_{5}(\mathrm{OH})_{4}$, was outlined as early as 1930, but it was not until 1983 that determinations of the locations of the $\mathrm{H}$ positions were first made (Adams, 1983; Suitch and Young, 1983). Before that date, Giese and Datta (1973) and Giese (1982) had determined $H$ positions using electrostatic modeling methods. Both Adams (1983) and Suitch and Young (1983) used published structures as starting models. Adams determined the $\mathrm{H}$ positions using $\Delta \mathrm{F}$ maps, but his results appear to have been affected by disorder and two-dimensional diffraction effects in the sample. Suitch and Young assumed $\mathrm{H}$ positions for their starting model and they assumed a lower space group symmetry $(P 1)$ than accepted for kaolinite $(C 1)$, resulting in an apparent false-minimum structure. More recently, Young and Hewat (1988) re-refined the kaolinite structure, again assuming a complete structural starting model and the lower space group symmetry and obtaining a structure similar to that obtained by Suitch and Young. Bish and Von Dreele (1989) refined the structure of kaolinite using $\mathrm{X}$-ray powder diffraction data, but they used a DLS model in $C 1$ as the starting structure. Their final refined structure appeared accurate, with reasonable bond lengths.

In 1990, Bish and Von Dreele re-examined the kaolinite structure using constant-wavelength neutron powder diffraction data and the non-hydrogen structure of Bish and Von Dreele (1989) as a starting model to rectify the differences between the earlier refinements. They calculated $\Delta \mathrm{F}$ maps, and the four largest negative regions of neutron density appeared reasonable for the four $\mathrm{H}$ positions (Figure 6). They then refined the complete structure, using $\Delta \mathrm{F}$ maps and anisotropic refinements to examine the nature of the $\mathrm{H}$ sites. There was no significant anisotropy in any of the four $\mathrm{H}$ positions, although the inner-hydroxyl $\mathrm{H}$ was slightly smeared along the $z$ direction and the three inner-surface hydroxyl $\mathrm{H}$ atoms had larger displacement parameters within the plane of the layers. These results do not agree with those of Adams (1983), Suitch and Young (1983), or Young and Hewat (1988), probably because of the difficulties with those refinements mentioned above. It is noteworthy that Guthrie and Bish (1991) reproduced the refined $H$ positions using an electrostatic minimization procedure. Calculated energy contours also showed that the inner-surface hydroxyl $\mathrm{H}$ atoms are situated in disk-shaped minima approximately parallel to (001) and the inner-hydroxyl $\mathrm{H}$ minimum is approximately perpendicular to (001), in agreement with the results of the Rietveld refinement. 
a)

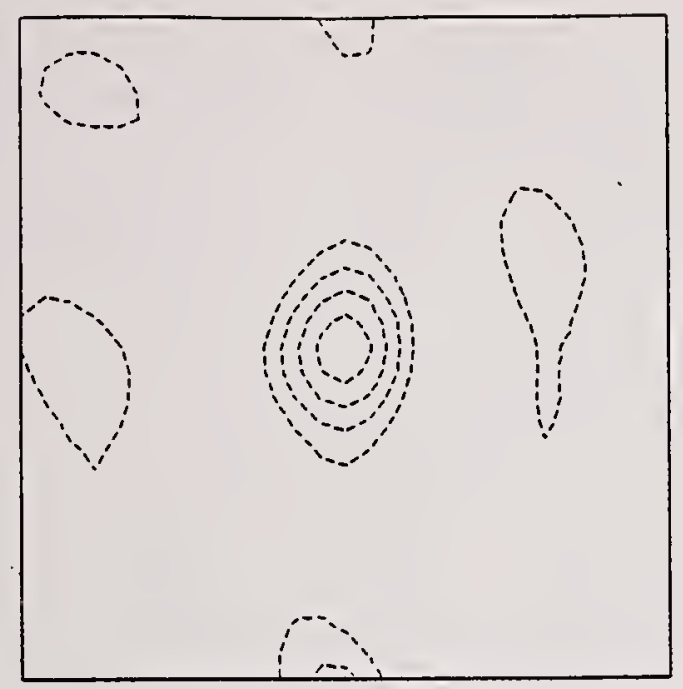

b)

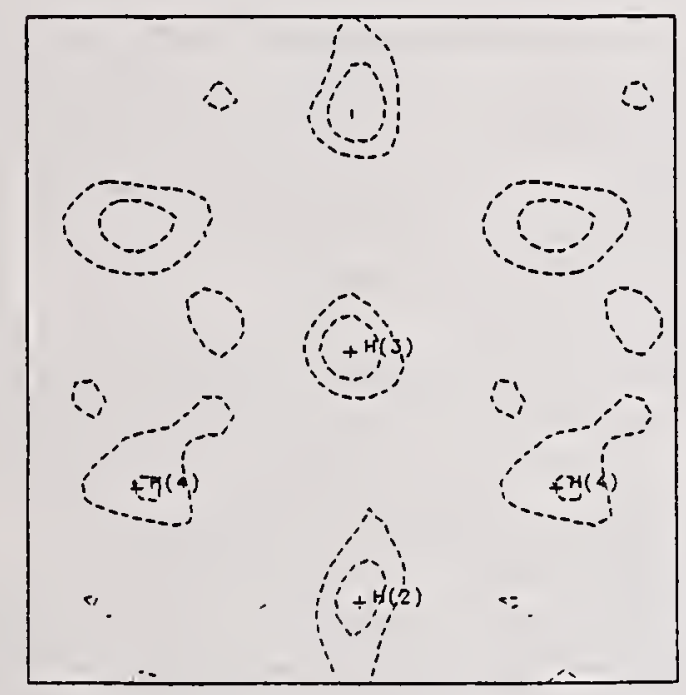

Figure 6. Difference-Fourier maps for kaolinite illustrating the positions of a) $\mathrm{H} 1$ within the kaolinite layer at $y=0.329$, contours drawn at $-0.20,-0.15$, and -0.10 , and -0.05 ; and b) $\mathrm{H} 2, \mathrm{H} 3$, and $\mathrm{H} 4$ in the kaolinite interlayer; contours drawn at $-0.3,-0.2$, and -0.1 . The $a$ and $b$ axes are approximately horizontal and vertical, respectively, in both figures.

Until recently, there was a similar lack of information on $\mathrm{H}$ positions in the related mineral, dickite, a polymorph of $\mathrm{Al}_{2} \mathrm{Si}_{2} \mathrm{O}_{5}(\mathrm{OH})_{4}$. In probably the first application of the Rietveld method to a layer silicate, Adams and Hewat (1981) examined the dickite structure using Fourier methods and neutron powder diffraction data. Their $\mathrm{H}$ positions, obtained on a sample exhibiting some two-dimensional diffraction effects, do not agree well with subsequent single-crystal X-ray determinations. Therefore, Bish and Johnston (1992) re-investigated the structure of dickite using lowtemperature time-of-flight neutron powder diffraction data, the Rietveld method, and Fourier syntheses, in addition to variable-temperature Fourier-transform infrared (FTIR) spectroscopy. Using a recent X-ray structure as a starting model, with no $\mathrm{H}$ atoms included, the four $\mathrm{H}$ atoms were unambiguously located as the four largest negative regions of density on $\Delta F$ maps (Figure 7). The minor anisotropy noted for the $\mathrm{H}$ atoms in kaolinite was not observed for dickite. The observed $\mathrm{H}$ positions were most similar to the recent singlecrystal X-ray refinement of Joswig and Drits (1986), although the $\mathrm{O}-\mathrm{H}$ bond distances found by Bish and Johnston appear to be more accurate. $\mathrm{OH}$ geometries, and the changes occurring from room temperature to 12 $\mathrm{K}$, are consistent with the FTIR spectra as a function of temperature and lend credence to the Rietveld-refined results. The relative ease with which the $H$ positions were obtained in a powdered clay mineral such as dickite is remarkable when one considers that barely 25 years ago such information would have been out of reach.

a)

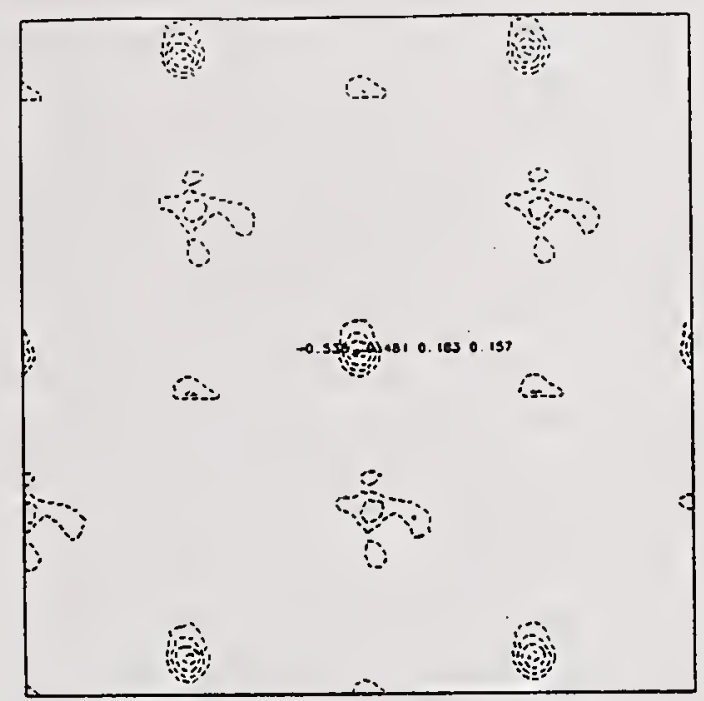

b)

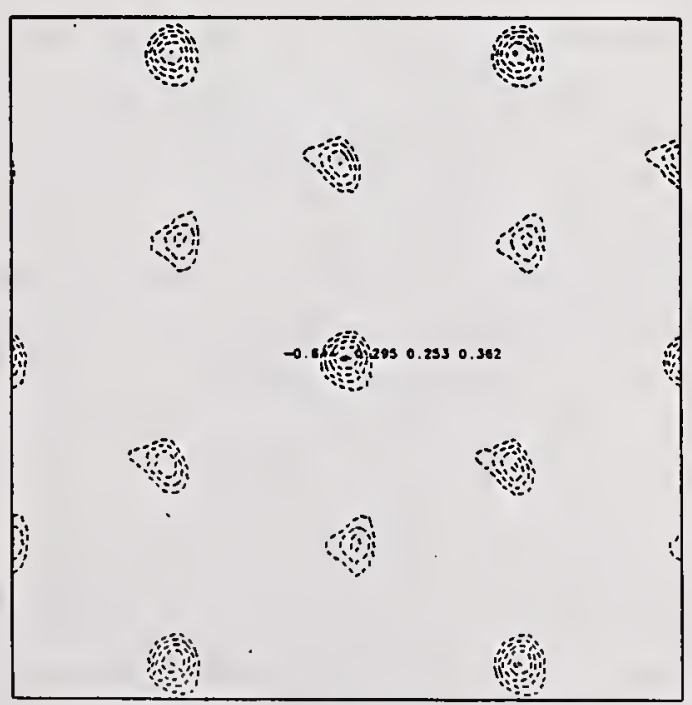

Figure 7. Difference-Fourier maps for dickite illustrating the positions of a) $\mathrm{H} 1$ within the dickite layer; contours drawn at $-0.5,-0.4,-0.3$, and -0.2 ; and b) $\mathrm{H} 2$, $\mathrm{H} 3$, and $\mathrm{H} 4$, in the dickite interlayer; contours drawn at $-0.6,-0.5,-0.4,-0.3$, and -0.2 . The $a$ and $b$ axes are approximately horizontal and vertical, respectively, in both figures. 
It is important to make one final comment regarding the use of $\Delta F$ methods to locate $H$ (or $D$ ) using neutron powder diffraction data. It is common practice in such experiments to attempt to replace $H$ with $D$ to reduce incoherent scattering. If the replacement can be made to go to completion, it should facilitate improved analysis due to a lowered background and improved signal-to-noise ratio. However, one can easily envision a situation in which only partial substitution of $\mathrm{D}$ for $\mathrm{H}$ is accomplished, on a statistical basis. Such a result could produce $\Delta \mathrm{F}$ maps revealing little or no information about the positions of $\mathrm{H}$ or $\mathrm{D}$ due to averaging of the negative neutron scattering length of $\mathrm{H}$ and the positive neutron scattering length of $D$. One could create a material with an average neutron scattering length of zero in the $\mathrm{H}$ sites.

\subsection{The interlayer structure of kaolinite intercalates}

The determination of the interlayer structure of the kaolinite-hydrazine intercalate is a good example of the utility of combined DLS modeling, $\Delta \mathrm{F}$ methods, and Rietveld refinement. Although kaolinite, a layer silicate consisting of sheets extending in two dimensions but with weak bonding between the sheets, does not normally expand, it interacts strongly with several liquids to create expanded intercalates. When kaolinite is exposed to anhydrous hydrazine, $\mathrm{N}_{2} \mathrm{H}_{4}$, it expands to a material with either a 9.5 or a $10.4 \AA$ basal (001) spacing, depending on the vapor pressure of water. Although the starting kaolinite may not possess good three-dimensional order, the $9.5 \dot{A}$ complex usually exhibits three-dimensional order sufficient to apply the Rietveld method. As a first step, the structure of the $9.5 \dot{A}$ intercalate was modeled using DLS, assuming the structure and $a, b, \alpha$, and $\gamma$ lattice parameters of a $7 \dot{A}$, non-expanded kaolinite, and a value of $\beta$ of $114^{\circ}$, yielding a value for $c$ of $10.5 \dot{A}$ obtained from a measured $d_{001}$ of $9.52 \dot{A}$. It was assumed in the DLS model that the stacking of layers in the intercalate was the same as in unexpanded kaolinite. The DLS structure was used as the starting model for Rietveld refinement, with the $\mathrm{N}_{2} \mathrm{H}_{4}$ molecule omitted. The two largest peaks in a $\Delta \mathrm{F}$ map were in the interlayer region and were $1.40 \dot{\mathrm{A}}$ apart, consistent with an $\mathrm{N}_{2} \mathrm{H}_{4}$ molecule. The molecule is canted within the interlayer, and there is evidence from the $\Delta \mathrm{F}$ maps for positional disorder of the $\mathrm{N}$ atom closest to the hydroxyl interlayer surface (Figure 8). Inclusion of these two $\mathrm{N}$ atoms in the structure model and continued isotropic refinement yielded an $\mathrm{R}_{\mathrm{up}}$ of $25 \%$, compared with $36 \%$ without the $\mathrm{N}$ atoms, although the refinement is still preliminary. This structure determination/refinement resolved the question of the exact orientation of the $\mathrm{N}_{2} \mathrm{H}_{4}$ molecule within the kaolinite interlayer, and the refinement result

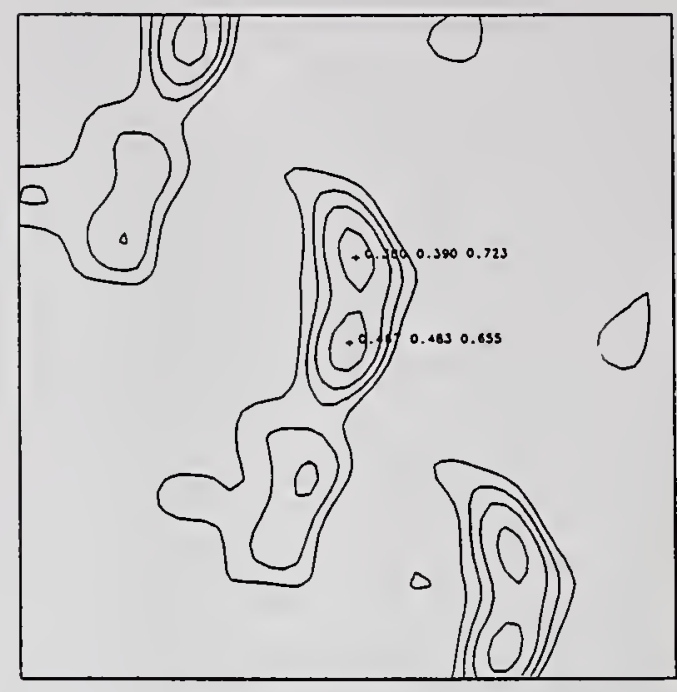

Figure 8. Difference-Fourier map for kaolinitehydrazine showing the $\mathrm{N}_{2} \mathrm{H}_{4}$ molecule represented by the two largest peaks at $(0.38,0.39,0.723)$ and $(0.467$, $0.483,0.655)$ in the interlayer space. Contours are drawn at $0.15,0.30,0.45$, and 0.65 electrons. The $c$ axis is approximately vertical.

suggests that the intercalate has the same layer stacking as kaolinite. Given the low atomic number of the $\mathrm{N}$ and the comparatively poor quality of the material, it is remarkable that such a result was obtained.

Comparable results were obtained using this methodology with a similar kaolinite intercalate. Costanzo et al. (1980) created a stable $8.4 \AA$ hydrate of kaolinite by expanding the material with dimethylsulfoxide and then reacting the expanded material in a solution containing $\mathrm{NH}_{4} \mathrm{~F}$, thereby partially or completely fluorinating the interlayer hydroxyl groups. The resultant material has an enlarged $c$ lattice dimension and three-dimensional order sufficient to apply the Rietveld method. A starting structure model was obtained using DLS methods, and the location of interlayer water molecules was determined from Rietveld refinement and $\Delta \mathrm{F}$ maps (Bish et al., 1992). The largest peak on the $\Delta F$ maps was midway between the layers, with only two other minor peaks above zero density. Rietveld refinement, incorporating an $O$ atom at the position of the largest peak, yielded an occupancy of $\mathrm{H}_{2} \mathrm{O}$ of 0.75 , positioned almost exactly midway between the silicate layers. Subsequent $\Delta F$ maps revealed no significant areas of electron density.

\subsection{Exchangeable cations and water in sepiolite}

The study of sepiolite by Bish and Post (1987) is an example of the use of Rietveld $/ \Delta F$ methods for elucidating the nature of the occupants of cavities in open-structure materials for which large single crystals cannot be obtained. A schematic structure of sepiolite, $\mathrm{Mg}_{4} \mathrm{Si}_{6} \mathrm{O}_{15}(\mathrm{OH})_{2} \cdot 6 \mathrm{H}_{2} \mathrm{O}$, was proposed by Nagy and 
Bradley (1955) and Brauner and Preisinger (1956) using so-called fiber diagrams (two-dimensional data). However, the details of the atom distribution in the structural tunnels between the silicate chains remained poorly understood. Bish and Post used X-ray data, with the Brauner and Preisinger framework as a starting model, to locate the tunnel occupants. Several iterations of Rietveld refinement and $\Delta \mathrm{F}$ map calculation revealed the presence of several $\mathrm{H}_{2} \mathrm{O}$ sites and several $\mathrm{Mg}$ sites (as judged by interatomic distances). The greatest difficulty faced in interpreting the $\Delta F$ maps was deciding which peaks were real; all of the tunnel occupants are low-atomic number atoms, and most or all of the sites are probably partially occupied. In cases such as this, one must rely on crystal chemical reasoning, using information such as bond distances and observed stoichiometry. Refinements of Bish and Post confirmed the presence of all water molecules postulated by Brauner and Preisinger (1956) but did not support several split-atom sites determined by Rautureau and Tchoubar (1974) using two-dimensional electron diffraction data. The Rietveld refinement of sepiolite constituted essentially the first three-dimensional structural study of sepiolite.

\section{Other structure refinement methods}

In addition to the use of the above model-building and structure-solution methods in connection with Rietveld refinement, there are several other techniques that can be used profitably during or after a refinement to improve and/or to supplement the information gained. These techniques include the use of so-called soft and hard distance constraints during refinement (see Baerlocher, 1986), use of multiple observations such as both X-ray and neutron diffraction data, analysis of the sample by other methods to obtain supporting information, and modeling of the refined crystal structure using energy minimization methods. These different methods have the effects of stabilizing difficult refinements, minimizing the problems of false-minimum structures, and supporting and rationalizing the final refined structure.

\subsection{Use of distance constraints}

Soft distance constraints, in the form of prescribed interatomic distances and angles obtained from wellrefined similar structures, can be used to supplement diffraction observations, but they do not reduce the number of variables. Their weight is typically chosen manually, often higher during beginning cycles and lower (or even zero) during the later stages of refinement. Such geometric restrictions have been used to great advantage in refinements of high-symmetry materials with numerous atoms, particularly those with poor ratios of observations/variables (e.g., Baerlocher, 1984). This procedure is exactly analogous to performing a combined DLS/Rietveld refinement. The use of soft constraints often improves the behavior of a refinement in the initial stages, but the constraints cannot always be completely removed (Baerlocher, 1986). Although the use of incorrect soft constraints can lead to false-minima structures, Baerlocher (1986) argued that such structures can be detected. Arguments have also been advanced that the use of soft constraints will unreasonably bias the outcome of a refinement, but the additional observations are usually known with high precision and one is simply exploiting the wealth of accurate crystal chemical and structural information available. In spite of this, however, it is important to state that the use of soft distances constraints in the final stages of a Rietveld refinement will affect the derived bond lengths to varying degrees, depending upon the quality of the refinement and the weighting of the soft constraints. A poor refinement with poor data can still yield bond distances that appear acceptable if numerous soft distance constraints are used with high weights in the final stages of a refinement.

Hard constraints, known as rigid-body constraints in single-crystal refinements (e.g., Finger and Prince, 1975), differ from soft constraints in that they reduce the number of variables. These constraints are usually used with molecular groups, such as $\mathrm{CO}_{3}{ }^{2}$, in which the molecular conformation (distances and angles) is fixed and only the position and orientation of the group are refined. This method is very effective when studying the structures of complex materials containing essentially isolated molecular groups, such as an adsorbate in a zeolite cavity. As with soft constraints, the intra-molecular distances and angles can often be predicted with high precision and accuracy, and these constraints should not unduly bias a refinement. However, also as with soft constraints, highly weighted hard constraints can unduly influence the results of a refinement.

\subsection{Joint X-ray and neutron refinements}

A very effective method that has seen little application in Rietveld analysis is the simultaneous use of both Xray and neutron powder data. The use of combined data generally provides a more complete structure determination, in part because of the very different variation of scattering length (scattering factor) with atomic number for $\mathrm{X}$-rays compared with neutrons. For example, there is no $\sin \theta / \lambda$ fall off of scattering factor for neutrons as is seen for all atoms with $\mathrm{X}$-rays, yielding lower intensities at high angles. In addition, whereas adjacent atoms in the periodic chart have 
virtually identical X-ray scattering factors, they may have very different neutron scattering lengths. Of course, the large variation of neutron scattering length even among isotopes of the same element is often exploited in structural studies that would be impossible with X-ray diffraction data. The strengths of neutron diffraction are illustrated by attempts to differentiate between $\mathrm{Fe}$ and $\mathrm{Mn}$ on different sites. Although their $\mathrm{X}$-ray scattering factors are almost identical, the neutron scattering length for $\mathrm{Mn}$ is $-0.373 \times 10^{-12} \mathrm{~cm}$ compared with that for $\mathrm{Fe}$ of $0.954 \times 10^{-12}$, providing for very easy discrimination between the two species. Sometimes, the reverse is true, where atoms widely separated in the periodic chart have very similar neutron scattering lengths, but very different $\mathrm{X}$-ray scattering factors. The ultimate result of using combinations of $\mathrm{X}$-ray and neutron observations is that refinement of individual anisotropic thermal parameters and site occupancies can be accomplished for complex structures. This method also minimizes problems with false-minima refined structures since a model must agree with very different types of data.

Williams et al. (1988) illustrated the utility of this combination in their study of superconducting $\mathrm{YBa}_{2} \mathrm{Cu}_{3} \mathrm{O}_{7 \times \mathrm{x}}$. Although the structure of this material had been studied several times and was well known before their study, most of the structural studies utilized neutron diffraction data. Because the neutron scattering lengths of $\mathrm{Y}\left(0.775 \times 10^{-12}\right)$ and $\mathrm{Cu}\left(0.7718 \times 10^{-12}\right)$ are virtually identical, the earlier neutron studies were unable to identify uniquely the distribution and ordering of these two cations. The structure refined using both $\mathrm{X}$-ray and neutron powder diffraction data was much more precise than previous determinations, and the refinement showed definitively that there was no disorder between the $\mathrm{Y}$ and $\mathrm{Cu}$ sites. In addition, the refinement provided a complete picture of the anisotropic thermal motion of all the atoms (Figure 9). Clearly, the combined use of multiple types of observations makes the Rietveld method much more powerful than originally conceived, potentially allowing for determination of intricate details of site occupancy and thermal motion of powdered materials. The only caveat that must be recognized is that all sets of data must be obtained under identical conditions, so that the lattice parameters and occupancies of any environmentally sensitive sites are the same.

\subsection{Supporting observations}

A few final comments should be made concerning observations that can (and often should) be made in support of a structural determination by Rietveld methods. For example, it has become routine to determine cation distributions in Fe-bearing materials using Mossbauer spectroscopy, and Nord and Ericsson (1982, 1985) and Nord (1986) used this combination very effectively. These authors gave several examples illustrating the benefits of using the Rietveld/Mossbauer combination, in particular elucidating Fe-Mn ordering. This combination allows an understanding of complex structures that could not be unraveled with only one technique. Another useful combination that has not been exploited is the use of magic-angle spinning nuclear magnetic resonance (MAS-NMR) spectroscopy with Rietveld refinement. ${ }^{29}$ Si MAS-NMR spectroscopy has proven particularly valuable in elucidating Al-Si ordering in silicates and should be used more often in conjunction with structure determinations and Rietveld refinements.

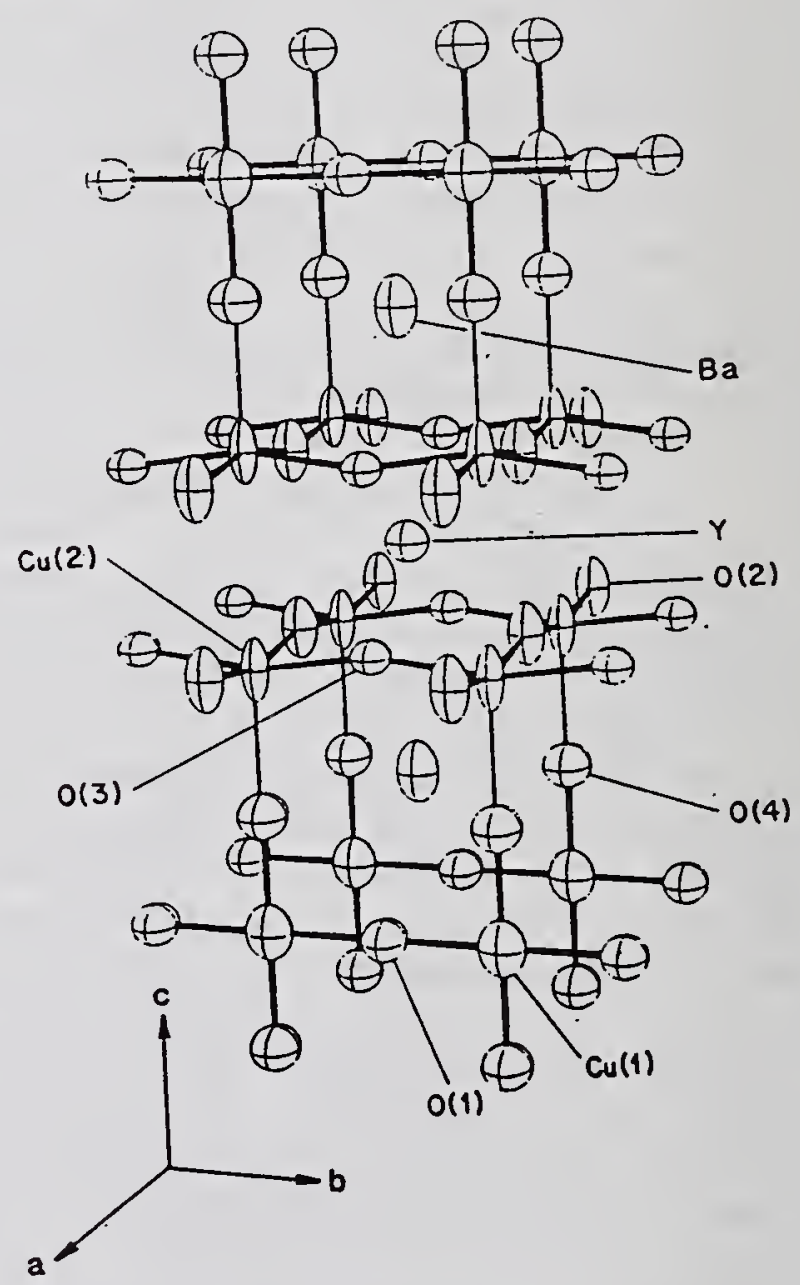

Figure 9. Structure of orthorhombic $\mathrm{YBa}_{2} \mathrm{Cu}_{3} \mathrm{O}_{7-\mathrm{x}}$, with thermal ellipsoids drawn at $99 \%$ probability surfaces (from Williams et al., 1988).

A final method that is proving particularly useful in supporting determinations of $\mathrm{H}$-atom orientations and extra-framework species positions is electrostatic energy minimization. This method combines calculation of the Coulomb portion of the lattice energy with some formulation of the short-range repulsive energies to determine the minimum-energy configuration of a particular arrangement of atoms. Guthrie and Bish 
(1991) applied this method to a study of the $\mathrm{H}$ orientations in kaolinite, for which $\mathrm{H}$ positions had just recently been determined using Rietveld refinement $/ \Delta F$ methods. It is extremely interesting that the results ofthe electrostatic minimizations reproduced both the $\mathrm{H}$ positions and the general shape of the potential wells within which the $\mathrm{H}$ atoms resided. Thus, this method explained and supported the anisotropic thermal motion of the $\mathrm{H}$ atoms observed in the Rietveld refinement. Giese (1982) and Guthrie and Bish (1991) also used these methods to predict the $\mathrm{H}$ positions and orientations in layer silicates for which complete structure refinements have not been performed. Catlow et al. (1986) described numerous uses of electrostatic methods, including studying cation distributions in zeolites, and they commented on the possibilities of coupling energy minimizations with structure refinements using diffraction data.

\section{Summary}

With careful and imaginative application of several ancillary techniques, the Rietveld method can provide structural information for powdered materials that was, until recently, out of reach. Structure building with HRTEM images and DLS methods can provide useful starting models for refinement and can allow solution of previously unknown structures. In addition, the use of DLS models can minimize the problems of falseminimum refined structures. One of the most powerful combinations for structure determination is the use of Rietveld refinement with difference-Fourier syntheses. A variety of important structural problems can be solved with this combination, including location of missing atoms, including $\mathrm{H}$ (D) atoms and extraframework atoms in open framework or layer-structure materials.

Supplementing diffraction observations with soft distance constraints can stabilize the initial stages of a refinement and can sometimes allow refinement of structures that otherwise could not be refined. However, their indiscriminate use can significantly affect derived bond distances. A potentially very beneficial enhancement of the Rietveld method is the use of both neutron and X-ray powder diffraction data with a single refinement. Such a combination can provide superior structural details, including useful anisotropic temperature factors and high-precision site occupancies. All structural results can benefit from support from other observations, including spectroscopic data and electrostatic energy minimizations that should allow a large amount of structural information to be extracted from powdered materials.

\section{Acknowledgment}

It is a pleasure to acknowledge the never-failing assistance of Dr. R. B. Von Dreele throughout my studies, and Dr. G. D. Guthrie for helpful comments on the manuscript. In addition, I have also benefitted from ongoing discussions with Dr. J. E. Post concerning Rietveld refinements of mineral structures.

\section{References}

Adams, J. M., (1983). Clays \& Clay Minerals 31, 352356.

Adams, J. M. and Hewat, A. W. (1981). Clays \& Clay Min. 29, 316-319.

Baerlocher, C. (1984). Proc. 6th Int. Zeolite Conf., Reno, USA, 823-833.

Baerlocher, C. (1986). Zeolites 6, 325-333.

Banfield, J. F., Veblen, D. R., and Smith, D. J. (1991). Amer. Mineral. 76, 343-353.

Bish, D. L. and Johnston, C. T. (1992). Proc. 29th Annual Clay Minerals Soc. Meeting.

Bish, D. L. and Post, J. E. (1987). Proc. 24th Annual Clay Minerals Soc. Meeting, 33.

Bish, D. L. and Von Dreele, R. B. (1989). Clays \& Clay Minerals 37, 289-296.

Bish, D. L. and Von Dreele, R. B. (1990). Progr. \& Abstracts, 27th Annual Clay Minerals Soc. Meeting, 25.

Bish, D. L., Giese, R. F., Jr., and Costanzo, P. M. (1992). Proc. 29th Annual Clay Minerals Soc. Meeting.

Brauner, K. and Preisinger, A. (1956). Miner. Petrogr. Mitt. 6, 120-140 (1956).

Buseck, P. R. and Veblen, D. R. (1988). Mineralogy, chapter 9, Oxford Univ. Press, New York, 308-377.

Catlow, C. R. A., Doherty, M., Price, G. D., Sanders, M. J., and Parker, S. C. (1986). Materials Sci. Forum 7, 163-176.

Clearfield, A., McCusker, L. B., and Rudolf, P. R. (1984). Inorg. Chem. 23, 4679-4682.

Costanzo, P. M., Clemency, C. V., and Giese, R. F., Jr. (1980). Clays \& Clay Minerals 28, 155-156.

Finger, L. W. and Prince, E. (1975). Natl. Bur. Stand. (U.S.) Tech. Note 854, $133 \mathrm{pp}$.

Giese, R. F., Jr. (1982). Bull. Minéral. 105, 417-424. Giese, R. F. and Datta, P. (1973). Amer. Mineral. 58, 471-479.

Guthrie, G. D. and Bish, D. L. (1991). Progr. \& Abstracts, 28th Annual Clay Minerals Soc. Meeting, 63.

Guthrie, G. D. and Veblen, D. R. (1990). Amer. Mineral. 75, 276-288. 
Joswig, W. and Drits, V. A. (1986). N. Jb. Miner. Mh. 19-22.

Lager, G. A., Armbruster, Th., and Faber, J. (1987). Amer. Mineral. 72, 756-765.

Mazzi, F. and Galli, E. (1978). Amer. Mineral. 63, 448-460.

McCusker, L. B. and Baerlocher, C. (1984). Proc. 6th Int. Zeolite Conf., Reno, Nevada, 812-822.

Meier, W. M. and Villiger, H. (1969). Zeit. Krist. 129, 411-423.

Nagy, B. and Bradley, W. F. (1955). Amer. Mineral. 40, 885-892 (1955).

Nord, A. G. and Ericsson, T. (1982). Amer. Mineral. 67, 826-832.

Nord, A. G. and Ericsson, T. (1985). Amer. Mineral. 70, 624-629.

Nord, A. G. (1986). Chemica Scripta 26A, 115-118.

O'Keefe, M. A. (1984). Electron Optical Systems, J. J. Hren, F. A. Lenz, E. Munro, and P. B. Sewell, eds., SEM Inc., AMF O'Hare, Chicago, IL, 209220.

Post, J. E. (1981). Ph.D. thesis, Arizona State University, $321 \mathrm{pp}$.

Post, J. E. and Bish, D. L. (1988). Amer. Mineral. 73, 861-869.

Rautureau, M. and Tchoubar, C. (1974). C. R. Acad. Sc. Paris 278B, 25-28.

Richardson, J. W., Jr. and Vogt, E. T. C. (1992). Zeolites 12, 13-19.

Rietveld, H. M. (1967). Acta Cryst 22, 151-152.

Rietveld, H. M. (1969). J. Appl. Cryst. 2, 65-71.

Suitch, P. R. and Young, R. A. (1983). Clays and Clay Minerals 31, 357-366.

Turner, S. and Buseck, P. R. (1981). Science 212, 1024-1027.

Veblen, D. R. (1985). Mineralogical Assoc. Canada, 63-90.

Williams, A., Kwei, G. H., Von Dreele, R. B., Larson, A. C., Raistrick, I. D., and Bish, D. L. (1988). Phys. Rev. B 37, 7960-7962.

Young, R. A. and Hewat, A. W. (1988). Clays \& Clay Minerals 36, 225-232. 
National Institute of Standards and Technology Special Publication 846. Proceedings of the international conference Accuracy in Powder Diffraction II, held at NIST, Gaithersburg, MD, May 26-29, 1992. (Issued October 1992)

\title{
Anharmonic Thermal Parameters, Disorder and Phase Transitions
}

\author{
H. BOYSEN
}

Institut für Kristallographie, Universität München, 8000 München 2, Theresienstr. 41, Germany

\begin{abstract}
Anharmonic atomic displacement parameters may become quite important in structure refinements from powder data in many practical situations, especially at high temperatures. Valuable information is obtained for materials like fast ionic conductors, polymorphic substances close to the phase transition and statically disordered crystals. A standard Rietveld program has accordingly been modified using a Gram-Charlier series expansion of the DebyeWaller factor up to the fourth order. The basic theory, the refinement procedures, several examples and possible future developments are discussed.
\end{abstract}

\section{Introduction}

In conventional crystal structure analysis from Bragg diffraction data the effect of thermal motion of the atoms is taken care of by the harmonic Debye-Waller factor (HDWF), which corresponds to a Gaussian probability distribution of the atoms in real space. Anharmonic thermal vibrations, although evidenced by many physical properties like thermal expansion, heat conductivity, specific heat anomalies, interaction of phonons etc., are usually considered to have a negligible influence on the Bragg intensities. If the amplitudes of the thermal motion of the atoms become large, however, e. g. at high temperatures, this is no longer true, and anharmonic Debye-Waller factors (ADWF) have to be considered. Clearly, for an atom in a non-centrosymmetric site symmetry a description by a Gaussian distribution is not adequate. More generally, the local environment, e.g. the directions of weak and hard bonds, will determine the form of the distribution. Examples of materials exhibiting large amplitude motions are ionic conductors and substances undergoing phase transitions close to the transition point. On the other hand, static displacements of the atoms in disordered crystals can also often be successfully described by ADWF's. Therefore, to emphasize the applicability of DWF's to both static and dynamic disorder the term "atomic" (ADP) instead of "thermal" displacement parameters should be used (Dunitz cited in Kuhs, 1992).

In the past most of the work concerning ADWF's has been done with single crystals, although single line powder intensities have also occasionally been analyzed (e.g. Matsubara, 1975). With the high intensity and resolution of powder diffractometers available nowadays, however, such anharmonicities may become important and should be considered in the refinement. In particular, the increasing interest in high temperature crystallography and the related physical properties, together with the availability of good diffraction furnaces, clearly demands the inclusion of ADWF's in the refinement programs.

In section 2 of this paper a brief outline of the theory of ADWF's will be given, following an entirely phenomenological approach. For a more detailed discussion, and also the physical background, see the recent review by Kuhs (1992) and the references therein. Refinement procedures, evaluation of the results, and a comparison with the alternative split atom model, are discussed in section 3. The application of ADWF's is illustrated in section 4 by several examples including fast ionic conductors, (order/disorder) phase transitions and static disorder. Finally, the conclusions in section 5 also give an outlook on what can be expected from future developments.

\section{Theory}

In a scattering experiment (X-ray or neutron) the amplitude of the scattered wave $A(\mathbf{Q})$ (where $\mathbf{Q}$ is the scattering vector, $Q=4 \pi \sin (\Theta) / \lambda, 2 \Theta$ the scattering angle and $\lambda$ the wavelength) is given by the Fourier transform of the electron density or scattering length weighted nuclear density, respectively, $\rho(\mathbf{r})$. As long as an averaged structure can be defined, $\rho(\mathbf{r})$ can be divided into

$$
\rho(\mathbf{r})=\langle\rho(\mathbf{r})\rangle+\Delta \rho(\mathbf{r}),
$$

where the definition means $\langle\Delta \rho(\mathbf{r})\rangle=0 .\langle\rho\rangle$ is the projection of all unit cells into a single one (divided 
by the number of unit cells) repeated strictly periodically in space. Then the intensity of the scattered beam $I=A A^{*}$ is given by the Fourier transform of

$$
\rho(\mathbf{r}) * \rho(-\mathbf{r})=\langle\rho\rangle *\langle\rho\rangle+\Delta \rho * \Delta \rho,
$$

where $*$ denotes convolution. Thus it can be divided into a Bragg $\left(I_{B}\right)$ and a diffuse $\left(I_{D}\right)$ part

$$
I=I_{B}+I_{D} .
$$

The average structure may be written

$$
\langle\rho(\mathbf{r})\rangle=\left[\sum_{k} n_{k} \delta\left(\mathbf{r}-\mathbf{r}_{k}\right) * \rho_{k}(\mathbf{r}) * p d f_{k}(\mathbf{u})\right] * l(\mathbf{r}),
$$

where the contents of the unit cell ( $k$ runs over all atoms in the unit cell) are convoluted with the lattice function $l(\mathbf{r})=\sum_{l} \delta\left(\mathbf{r}-\mathbf{r}_{l}\right)$, where $l$ runs over all unit cells. $n_{k}$ takes care of the occupation of a specific site by different atom species (including vacancies), $\rho_{k}$ is the atomic electron distribution for $\mathrm{X}$-rays or $b_{k} \delta\left(\mathbf{r}^{\prime}\right)$ for neutrons $\left(b_{k}\right.$ is the coherent nuclear scattering length) and $p d f_{k}$ the probability density function that describes the space and time averaged distribution of the atoms around the equilibrium position $\mathbf{r}_{k}\left(\mathbf{u}=\mathbf{r}-\mathbf{r}_{k}\right)$. From the definition $\int p d f(\mathbf{r}) d \mathbf{r}=1$. Fourier transformation gives

$$
A(\mathbf{Q})=\left[\sum_{k} n_{k} e^{i \mathbf{Q} \mathbf{r}_{k}} f_{k}(\mathbf{Q}) T_{k}(\mathbf{Q})\right] L(\mathbf{Q}) .
$$

$f_{k}(\mathbf{Q})$, the $\mathrm{X}$-ray scattering amplitude has to be replaced by $b_{k}$ for neutrons. $L(\mathrm{Q})$ is the lattice function in reciprocal space and $T_{k}(\mathrm{Q})$ the temperature factor. The term in square brackets represents the usual structure factor, $F(\mathrm{Q})$. The main result is that the temperature factor is the Fourier transform of the $p d f$ in a very general sense, i. e. for any distribution, static or dynamic

$$
T(\mathbf{Q})=\int p d f(\mathbf{u}) e^{i \mathbf{Q} \mathbf{u}} d^{3} \mathbf{u} .
$$

For a trivariate Gaussian $p d f$ the temperature factor is also Gaussian and we obtain the usual HDWF. In the isotropic case

$$
p d f(\mathbf{u})=\left(2 \pi\left\langle u^{2}\right\rangle\right)^{-3 / 2} \exp \left(-\frac{u^{2}}{2\left\langle u^{2}\right\rangle}\right)
$$

and

$$
T(\mathbf{Q})=\exp \left(-\frac{1}{2} Q^{2}\left\langle u^{2}\right\rangle\right)=\exp \left(-B \frac{\sin ^{2} \Theta}{\lambda^{2}}\right)
$$

where $\left\langle u^{2}\right\rangle$ is the mean square displacement, and $B=$ $8 \pi^{2}\left\langle u^{2}\right\rangle$ is the usual isotropic atomic displacement parameter.

In lattice dynamics (applicable only for dynamic displacements) this result is derived in the "harmonic" approximation by truncating the crystal potential

$$
\begin{gathered}
V=V_{0}+\frac{1}{2} \sum_{\alpha k l} \sum_{\alpha^{\prime} k^{\prime} l^{\prime}} \Phi_{\alpha k l \alpha^{\prime} k^{\prime} l^{\prime} u_{\alpha}}(k l) u_{\alpha^{\prime}}\left(k^{\prime} l^{\prime}\right) \\
+V_{3}+V_{4}+\ldots
\end{gathered}
$$

after the quadratic term. Inclusion of higher order terms to account for the anharmonicity is extremely complicated and has only been carried out for a few simple cases. Instead, at high temperatures, one can make a drastic simplification and neglect all interactions between the atoms, i.e. we regard each atom as an Einstein oscillator performing independent vibrations in a one particle potential (OPP)

$$
V=V_{0}+\frac{1}{2} \alpha|\mathbf{u}|^{2}+\beta f_{3}(\mathbf{u})+\gamma f_{4}(\mathbf{u})+\ldots,
$$

where $f_{3}$ and $f_{4}$ denote homogeneous functions of order 3 and 4 , respectively. This approximation can be further justified as we are only interested in the average distribution of the atoms. Moreover, the $p d f$ can be calculated by applying Boltzmann statistics

$$
p d f(\mathbf{u})=e^{-V(\mathbf{u}) / k T} / Z=p d f(\mathbf{0}) e^{-V(\mathbf{u}) / k T},
$$

where $Z$ is the partition function and the second equality is obtained by setting $V(0)=0$.

In summary, equations (6) and (10) define the relations among the potential, the $p d f$ and the temperature factor. Hence, each of these quantities can be used, and is used in the literature, as the starting point for approximation of ADWF's. After Dawson's generalized structure factor formalism (Dawson, 1967) introducing imaginary parts to account for anti-symmetry, the OPP expansion (Willis, 1969; Tanaka \& Marumo, 1983), which starts from equation (9), the expansion of the $p d f$ adapted to a specific problem [e.g. spherical harmonics for orientationally disordered crystals (Press \& Hüller, 1973; Prandl, 1981)], and the moment or cumulant expansions (Johnson, 1969; Johnson \& Levy, 1974), which are based on statistics, have been employed. Here, for purely mathematical reasons (analytical calculation of $p d f$ and OPP, applicability to all symmetries, easy inclusion into refinement programs), the quasimoment expansion has been chosen. This is given by 


$$
\begin{aligned}
T(\mathbf{Q}) & =T^{\text {harm }}(\mathbf{Q})\left[1+\frac{i^{3}}{3 !} C^{j k l} Q_{j} Q_{k} Q_{l}\right. \\
& \left.+\frac{i^{4}}{4 !} D^{j k l m} Q_{j} Q_{k} Q_{l} Q_{m}+\ldots\right]
\end{aligned}
$$

(summation convention), which is known as the Gram-Charlier series. After Fourier transformation

$$
\begin{aligned}
p d f(\mathbf{u}) & =p d f^{h a r m}(\mathbf{u})\left[1+\frac{1}{3 !} C^{j k l} H_{j k l}(\mathbf{u})\right. \\
& \left.+\frac{1}{4 !} D^{j k l m} H_{j k l m}(\mathbf{u})+\ldots\right],
\end{aligned}
$$

where $H_{j k}$.. are the so-called Hermite polynomials. Up to the fourth order there are 10 coefficients $C^{j k l}$ and 15 coefficients $D^{j \mathrm{klm}}$. These coefficients are (covariant) components of tensors and are subject to restrictions imposed by the local site symmetry of the atom under consideration, i. e., their number is reduced in many practical cases. The Hermite polynomials and the symmetry constraints for tensors up to the fourth order may be found in Johnson \& Levy (1974). It should be emphasized that the refinable parameters $C^{j k l}$ and $D^{j k l m}$ have no direct physical meaning, and, moreover, there are often correlations with the second order (harmonic) coefficients and even the positional parameters. The refined positional parameter does not necessarily coincide with the center of gravity of the distribution. Therefore, to interprete the results, one always has to calculate the $p d f$ and/or the potential.

Since $\langle\rho\rangle$ is a space and time average one can in general not distinguish between static and dynamic disorder. Some hints may, however, be obtained by analyzing the temperature dependence of the DWF. In the harmonic case with purely dynamic disorder the mean square displacements $\left\langle u^{2}\right\rangle_{d y n}$ should be proportional to the temperature at high enough temperatures (classical regime), as may be derived easily from equations (9) and (10). Therefore, a plot of $\left\langle u^{2}\right\rangle$ versus $T$ should extrapolate to zero at $0 \mathrm{~K}$. Assuming that any static disorder is independent of temperature, there will be a constant additional $\left\langle u^{2}\right\rangle_{\text {stat }}$, which can be read off the ordinate at $0 \mathrm{~K}$ by the same extrapolation procedure. Care has, however, to be taken with this widely used method, since (1) the static disorder may also in fact be temperature dependent; (2) there may be couplings between the static and dynamic components; (3) at too low temperatures $\left(T \leq \Theta_{D}\right.$, where $\Theta_{D}$ is the Debye temperature) quantum mechanical effects cause deviations from linearity (zero point motion!); and (4) at too high temperatures anharmonicities again cause deviations from linearity. The latter may be simply seen from the quasi-harmonic approximation, which accounts for thermal expansion by replacing the normal mode frequencies $\omega$ through $\omega_{q h}^{-2}=\omega^{-2}\left(1+2 \gamma_{G} \chi T\right)$, where $\gamma_{G}$ is the Grüneisen contstant, and $\chi$ is the volume expansion coefficient. Then

$$
B^{q h}=B^{h a r m}\left(1+2 \gamma_{G} \chi T\right),
$$

i. e., proportional to $T+$ const. $\times T^{2}$.

If ADWF's have been refined, similar extrapolations hold for $C^{j k l}$ and $D^{j k l m}$, which should be proportional to $T^{2}$ and $T^{3}$, respectively, but should again be used only with care as discussed above.

A better method seems to be the consideration of the temperature dependence of the potentials (Bachmann \& Schulz, 1984). Clearly, in the purely dynamic case this should be constant, whereas in the presence of static contributions it makes no sense at all and consequently will change with temperature (becoming virtually steeper with increasing temperature, since the relative contribution of thermal motion increases).

\section{Data evaluation}

The Gram-Charlier series expansion (equation (11a)) up to the fourth order has been included in the multiphase Rietveld program of Thomas \& Bendall (1978). The restriction to four orders seems to be sufficient in the wide majority of cases (also in single crystal work). The program consists of two parts, the preparation program PREP and the refinement program PROF. At present only Gaussian line profiles are available for neutrons. This has been found to be adequate in most cases. In fact, the deviations at the tails of the reflexions (calculated too low) which are observed in the presence of strong thermal diffuse scattering (TDS) are considered to be even advantageous, since a (partial) correction for this effect is made by this procedure. This has been confirmed by test refinements on lead. On the other hand, simple, modified and intermediate Lorentzians are supplied for X-rays to account for the intrinsic different line shape (intermediate Lorentzians gave the best fits for the local diffractometer). Problems may arise, however, in both cases, if strong particle size and/or strain broadening is present in the sample.

Further analysis of the results is done with the single crystal system PROMETHEUS (Zucker et al., 1983), which allows the calculation of $p d f$ maps, potentials and Fourier maps. Higher orders (up to six) and other expansions (Edgeworth) may be tried also. For this purpose an output file is provided which contains the "observed" integrated (Bragg) intensities

$$
I_{o b s}(h)=\sum_{i} y_{i o b s} \frac{y_{i c a l c}(h)}{y_{i c a l c}}
$$

( $h$ stands for the reflexion indices $h k l, y_{i o b s / c a l c}$ are the observed and calculated profile intensities, and 
the summation runs over all $y_{i}$ that can contribute to a specific reflexion $h$ ) and the corresponding variances

$$
\sigma\left(I_{o b s}(h)\right)=\sqrt{\sum_{i}\left[\sigma\left(y_{i o b s}\right) \frac{y_{i c a l c}(h)}{y_{i c a l c}}\right]^{2}} .
$$

Further input files for PROMETHEUS are prepared from the refined parameters of PROF by a special program RIPR. Note that $p d f$ maps and potentials only need the final refined parameters of PROF, while Fourier maps and other expansions require the list of (pseudo) Bragg intensities. The latter are of course biased by the weighting scheme in equation (13) (ambiguous for many overlapping reflexions), so that care has to be taken in interpreting these results. Difference Fourier maps have, however, been used successfully, e.g., to locate interstitial atoms, as long as the reflection overlap is not too large. Misleading results have sometimes been obtained with strongly overlapping diagrams.

To estimate the significance of the results one should distinguish two situations:

(1) For low symmetry space groups and/or low atomic site symmetries the number of structural parameters $p_{s}$ (including the scale factor) is usually much larger than the number of "instrumental" parameters $p_{i}$ (half width parameters, lattice constants, etc.). During the refinement the number of anharmonic coefficients should be increased stepwise fixing non-significant ones to zero at each step. The conventional agreement factors

$$
R_{w p}=\sqrt{\sum_{i} w_{i}\left(y_{i o b s}-y_{i c a l c}\right)^{2} / \sum_{i} w_{i} y_{i o b s}^{2}}
$$

and the corresponding goodness of fit $\left(=R_{w p} / R_{\text {exp }}\right)$

$$
g o f_{p}=\sqrt{\sum_{i} w_{i}\left(y_{i o b s}-y_{i c a l c}\right)^{2} /\left(m_{y}-p\right)}
$$

are adequate in this case. Here $m_{y}$ is the number of profile intensities, $p\left(=p_{s}+p_{i}\right)$ the total number of refinable parameters and $w_{i}=1 / \sigma^{2}\left(y_{i}\right)$ the weight.

(2) For high symmetries (both space group and site point group) $p_{s}$ is small and about the same as $p_{i}$. Then the consideration of anharmonicity requires only a few additional parameters. Consequently, the effect on the conventional $g o f_{p}$ is small, since the denominator in equation (14b) is only slightly changed. Instead, a much larger effect may be obtained, if one uses a weighted Bragg R-factor

$$
R_{w B}=\sqrt{\sum_{h} w_{I}\left(I_{o b s}(h)-I_{c a l c}(h)\right)^{2} / \sum_{h} w_{I} I_{o b s}^{2}(h)}
$$

and

$$
g \circ f_{B}=\sqrt{\sum_{h} w_{I}\left(I_{o b s}(h)-I_{c a l c}(h)\right)^{2} /\left(m_{I}-p_{s}\right)},
$$

where $w_{I}=1 / \sigma^{2}\left(I_{o b s}(h)\right)$ and $m_{I}$ is the number of reflections. Clearly, this procedure is statistically not fully correct, since it neglects the correlations with the instrumental parameters and those between the "observed" intensities. In general, however, the correlations between structural and instrumental paramaters are small (although this has to be checked in each application) and, for high symmetries, the reflection overlap is not too severe. The latter limits, however, the applicability of equations (15) for low symmetries, and therefore equations (14) are recommended in this case. It may be added that a weighted Bragg R-factor is generally preferred to the usually quoted unweighted $R_{B}$, since it also avoids unrealistically large values encountered sometimes, when near the end of the dataset or near excluded regions some reflections contribute to the fit only with their tails. In any case, to avoid spurious results, one should keep only those terms in the refinement that are very clearly significant (more than, say, four or five estimated standard deviations (e.s.d.)).

Of course one always starts a refinement with HDWF's. Apart from the natural expectation that allowance should be made for anharmonicity for diagrams taken at very high temperatures or for weakly bonded crystals, other criteria might also indicate its necessity, e.g. very large $B$ values and a temperature dependence following equation (12). A very strong indication has been found to be occupation numbers $n$ coming out considerably less than one (unless this is due to nonstoichiometry etc.). Another strong hint is a large and modulated diffuse background, which always indicates strong disorder (compare equations (2) and (3)).

As has been shown by Bachmann \& Schulz (1984), a mathematically equivalent description of nonGaussian $p d f$ 's is the use of split atoms. Strictly this is true only if an infinite number of split positions is used, and the anharmonic expansion is carried to infinity. In practice both models must be truncated, so similar but slightly different distributions are obtained. Depending on the actual problem one or the other can provide a better description. For example, for a disorder type where two atoms are distributed randomly over two closely spaced equilibrium positions, the split model is superior. Very often, however, one encounters severe convergence problems, due to very strong correlations in the split model, unless the starting values are already close to the final ones. Such correlation problems are usually less severe in the anharmonic model. The latter finds the 
correct distribution simply by refining the higher order coefficients, whereas in the split model a number of possible displaced positions (magnitude and direction) have to be tried as long as nothing is known from the beginning. Hence, even if the split model turns out to be superior at the end, first hints may often be obtainend more easily by refining anharmonic terms first. Moreover, quite often the number of free parameters is less in the anharmonic model.

A final remark concerns the use of Fourier maps calculated from the "observed" structure factors, which in principle can give the same information. Apart from the ambiguity in the "observed" intensities (see above), they always suffer from truncation effects, which are avoided in the $p d f$ approach.

\section{Examples}

In order to demonstrate the necessity to employ ADWF's in the refinements from powder diffraction patterns even for "normal" substances at elevated temperatures, an experiment has been carried out on zincblende $(\mathrm{ZnS})$ up to $1223 \mathrm{~K}$ (Boysen, Steger, Hewat \& Buevoz, 1981). Both atoms lie in tetrahedral site symmetry $(\overline{4} 3 \mathrm{~m})$. The appropiate single third order anharmonic coefficient becomes significant (larger than one e.s.d.) above $\approx 900 \mathrm{~K}$. It is in good agreement (within one e.s.d.) with a thorough single crystal investigation at room temperature (Cooper, Rouse \& Fuess, 1973) and is proportional to $T^{2}$, as expected (although the e.s.d.'s were too large to really confirm this without doubt). The corresponding $p d f$ has lobes pointing towards the corners of a tetrahedron opposite to the $\mathrm{Zn}-\mathrm{S}$ bonds.

This example clearly shows the importance of third order anharmonic terms for atoms lying in noncentrosymmetric site symmetries. In this investigation the OPP expansion (which is equivalent to the Gram-Charlier formalism in this special case), as well as single line fits, were employed. The anharmonicity could be traced back to intensity changes (as compared to the harmonic case) of individual reflections that amounted to as much as about $6 \%$. This provides an estimate of the required statistical precision of the measurement.

Much larger anharmonic effects were found for the special materials in the following examples. Most of the experiments discussed below were carried out with neutrons, since they usually allow more reliable access to temperature factors. With X-rays one also has to keep in mind that asphericities of the atomic electron density can cause similar effects and can in general not be easily distinguished from thermal motion.

\section{(a) Fast ionic conductors}

Particularly interesting materials exhibiting large atomic motions are superionic conductors. The $p d f$ 's of the mobile ions will indicate the diffusion pathways. The maximum potential along these paths may be compared with activation energies obtained e.g. from conductivity measurements: they should be equal, if no additional energy is required for the formation of defects. Note that the potential barrier derived from the scattering experiment only contains the activation energy for migration.
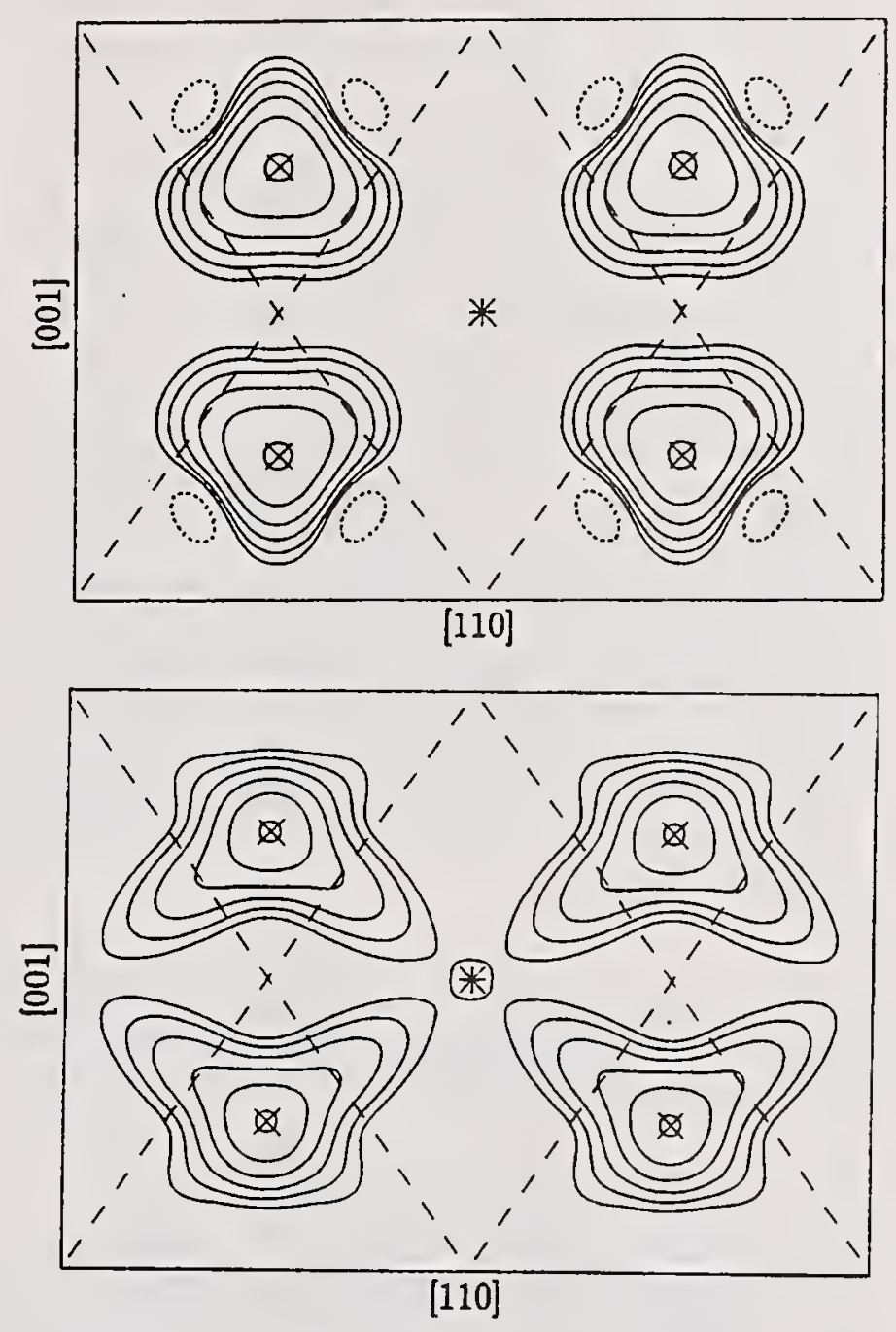

Figure 1: pdf map of $\mathrm{Cu}$ in $\mathrm{CuI}$ at $698 \mathrm{~K}$ for anharmonic (top) and split (bottom) models. Contour lines refer to $3,6,12,24,48$ and $96 \%$ of maximum. Negative values are dashed. Tetrahedral and octahedral positions are marked by a cross and a star, respectively. Straight dashed lines indicate the traces of the faces of the surrounding I-tetrahedron.

CuI (Yu, Boysen \& Schulz, 1990) has the zincblende structure at room temperature $(\gamma$-phase, 
space group $F \overline{4} 3 m$ ) and transforms to the $\alpha$-phase (disregarding an intermediate hexagonal $\beta$-phase) at high temperatures. Here the anions keep their arrangement as in the $\gamma$-phase, while the cations are randomly distributed over all tetrahedral sites. The space group is $F m \overline{3} m$. In figure 1 the $p d f$ maps of the $\mathrm{Cu}$ cations at $698 \mathrm{~K}$ are compared for a split and an anharmonic model. Although they look quite different at first sight, they both indicate a similar diffusion path, first along [111] through the face of the surrounding I-tetrahedron into the octahedral cavity, where it turns to [001] and reenters the neighbouring tetrahedral site along the equivalent [ $\overline{1} \overline{1} 1]$ direction. At this temperature both models yielded practically the same R-values. Hence the real $p d f$ is probably in between. On the other hand, a distinction could be made in favour of the split model at $633 \mathrm{~K}$ and the anharmonic model at $763 \mathrm{~K}$. Altogether these findings may be understood by a transition from a jump to a diffusion like behaviour (shorter and longer residence times on interstitial positions). At the highest temperature the diffusion path has moved a little more inwards towards the direct connection between the tetrahedral sites (through the edges). The potential barrier along these diffusion paths turns out to be independent of temperature and is in excellent agreement with determinations of the activation energy from conductivity and NMR measurements. Very similar results have recently been obtained for the $\gamma$ phase of $\mathrm{CuCl}$ (Graneli et al., 1992) and $\mathrm{CuBr}$ (Altorfer \& Bührer, 1992). Measurements at a number of temperatures nicely revealed the expected proportionality between the single third order anharmonic coefficient $C 123$ and $T^{2}$.

Table 1: Agreement factors of different models for $\mathrm{CuI}$ at $763 \mathrm{~K}\left(\mathrm{~h}=\right.$ harmonic, $\mathrm{s}=$ split, $3=3^{\text {rd }}$ order, $4=4^{\text {th }}$ order anharmonic)

$\begin{array}{ccccc} & h & s & 3 & 4 \\ \mathrm{R}_{w p}(\%) & 9.40 & 8.76 & 8.63 & 8.62 \\ g \circ f_{p} & 1.30 & 1.21 & 1.19 & 1.19 \\ \mathrm{R}_{w B}(\%) & 7.04 & 5.26 & 4.50 & 4.60 \\ g \circ f_{B} & 2.33 & 1.87 & 1.54 & 1.69 \\ p_{s} & 3 & 5 & 4 & 6 \\ p_{i} & 6 & 6 & 6 & 6\end{array}$

Table 1 summarizes the refinement statistics for different models at $763 \mathrm{~K}$. As anticipated before, the preference for the (third order) anharmonic model is hardly seen in the profile $\mathrm{R}$-factors, whereas it is quite obvious in the weighted Bragg R-factors: the lower $R_{w B}$ is obtained with fewer structural parameters! Fourth order terms are clearly not significant: while $R_{w p}$ is slightly reduced, $R_{w B}$ becomes even larger. This is, of course, a consequence of the fact that the fit is still done on the profile intensities. It thus demonstrates the statistical shortcomings of this procedure. Finally, table 1 shows that the harmonic model (isotropic by symmetry) is definitely not adequate even with powder data of moderate quality: this investigation was carried out on a low flux reactor (instrument MAN I at the FRM in Garching). The errors in the $p d f$ maps are about $3 \%$ of the maximum (the lowest contour line in figure 1 ; i.e., the negative regions are not significant) as estimated by calculating $p d f$ maps with the parameters changed by one e.s.d. This procedure for estimating the errors can, however, no longer be applied, if the number of parameters becomes larger. Statistically relevant error maps may be calculated with the help of the Monte-Carlo method and have recently been incorporated in the program package PROMETHEUS by Kuhs (1992).

Doped zirconia $\left(\mathrm{ZrO}_{2}\right)$ and ceria $\left(\mathrm{CeO}_{2}\right)$ are examples of high temperature anionic conductors. They may serve here to illustrate the temperature dependence of the harmonic and anharmonic coefficients and the discrimination of static and dynamic contributions (Martin et al., 1992). Figure 2(a) shows the harmonic $B$ values of oxygen in CSZ $(15 \% \mathrm{CaO})$ derived from powder and single crystal data. Extrapolations to $0 \mathrm{~K}$ indicate the presence of static contributions, which become larger at lower temperatures. This is consistent with an analysis of the diffuse scattering in single crystals (Neder, Frey \& Schulz, 1990), which revealed correlated clusters the number of which decreases strongly around $900 \mathrm{~K}$ (Proffen et al., 1992). As anticipated before, the presence of static disorder may also be inferred from the temperature dependence of the potentials, [See Fig.2(b)]. They are not, of course, real potentials in this case. Care has to be taken in interpreting potentials unless the absence of static disorder has been proven. The deviation from a linear temperature dependence of the third order anharmonic coefficient $C 123$ when plotted versus $T^{2}$, shown in figure $2(c)$, again reflects the presence of static disorder. On the other hand, the expected behaviour for (almost) purely dynamic disorder was found for Y-TZP (tetragonal zirconia polycrystal, $3 \% \mathrm{Y}_{2} \mathrm{O}_{3}$ ) and ceria $\left(3 \% \mathrm{Y}_{2} \mathrm{O}_{3}\right)$, as is also shown in Fig.2(c). It may be worth mentioning that the $p d f$ maps of TZP (which can only be prepared in polycrystalline form!) did not indicate any enhanced ionic conductivity at low temperatures as compared to CSZ, although this was found in conductivity measurements. Hence, one has to conclude either that the conduction takes place predominantly along grain boundaries or intergrown glassy regions of the sintered material rather than in the bulk, or, more probably, that the larger static 
(a)

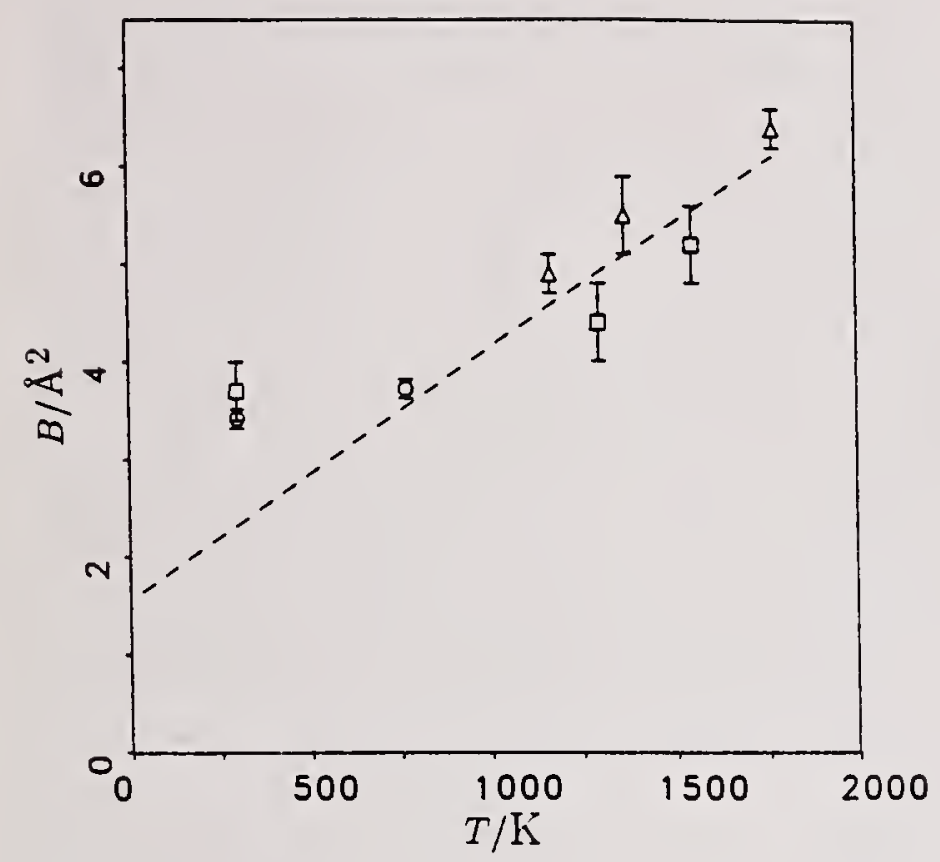

(b)

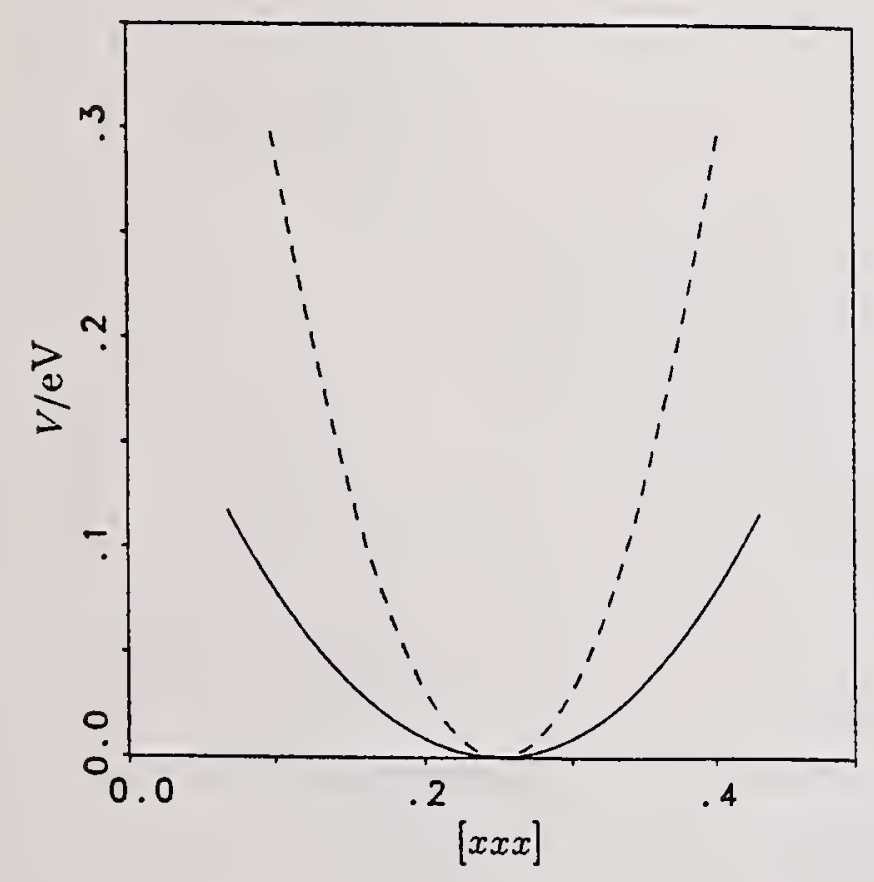

short range order found in CSZ is responsible for the reduced conductivity in that material.

A comparative study of tetragonal zirconia with $\mathrm{X}$-rays and neutrons (Martin, 1992) yielded rather good agreement between the two methods provided a careful absorption correction was made for the $\mathrm{X}$-ray data (by using two diffraction geometries, Debye-Scherrer and Bragg-Brentano, and adjusting the absorption coefficient). Non-perfect corrections resulted in wrong isotropic $B$ values. Nevertheless, the anharmonicity and therefore the form of the $p d f$ was described correctly. (c)

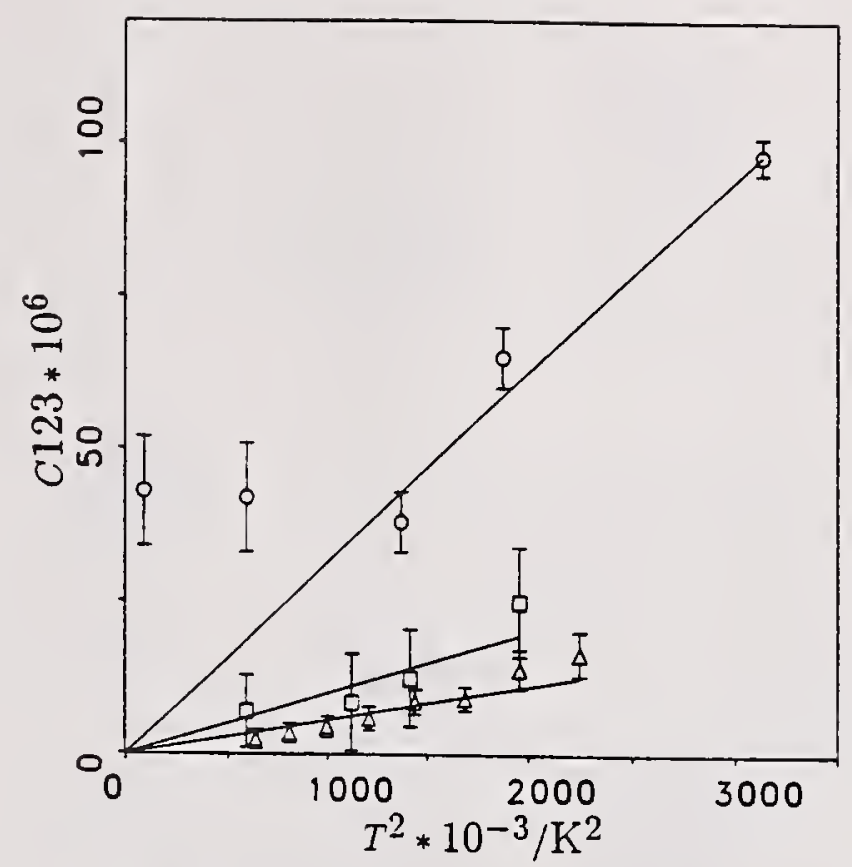

Figure 2: Temperature dependence of various parameters in zirconia. (a) $B(\mathrm{O})$ of $\mathrm{CSZ}$ from different powder (circles and squares) and single crystal (triangles) measurements. The dashed line indicates the extrapolation of the high temperature values to $0 \mathrm{~K}$. (b) Section through the potential at room temperature (solid line) and $770 \mathrm{~K}$ (dashed line) for CSZ. (c) Anharmonic third order coefficient plotted versus $T^{2}$ for CSZ (circles), Y-TZP (squares) and ceria (triangles).

\section{(b) Phase transitions}

Anharmonicity is a prerequisite for any structural phase transition. Large amplitude atomic motions may be appreciated from the fluctuation-dissipation theorem

$$
\chi=\langle A A\rangle / k T,
$$

where $\chi$ is the susceptibility (which should diverge at a second order phase transition) and $A$ is the amplitude of the critical normal mode (or spin normal mode in the case of order-disorder transitions). Note, however, that the ADP's represent an average over all normal modes, i.e. the additional contribution of the critical mode may be relatively small. For a general discussion of DWF theories near structural phase transitions see Bruce \& Cowley (1981). A cusp-like increase of the HDWF near $T_{c}$ is predicted and has been observed $e . g$. by Ivanov et al. (1979) in ferroelectric perovskite-type structures and by Kihara (1990) at the $\alpha-\beta$ transition in quartz. Again, more information about the critical mode could be obtained from "anharmonic" refinements. 


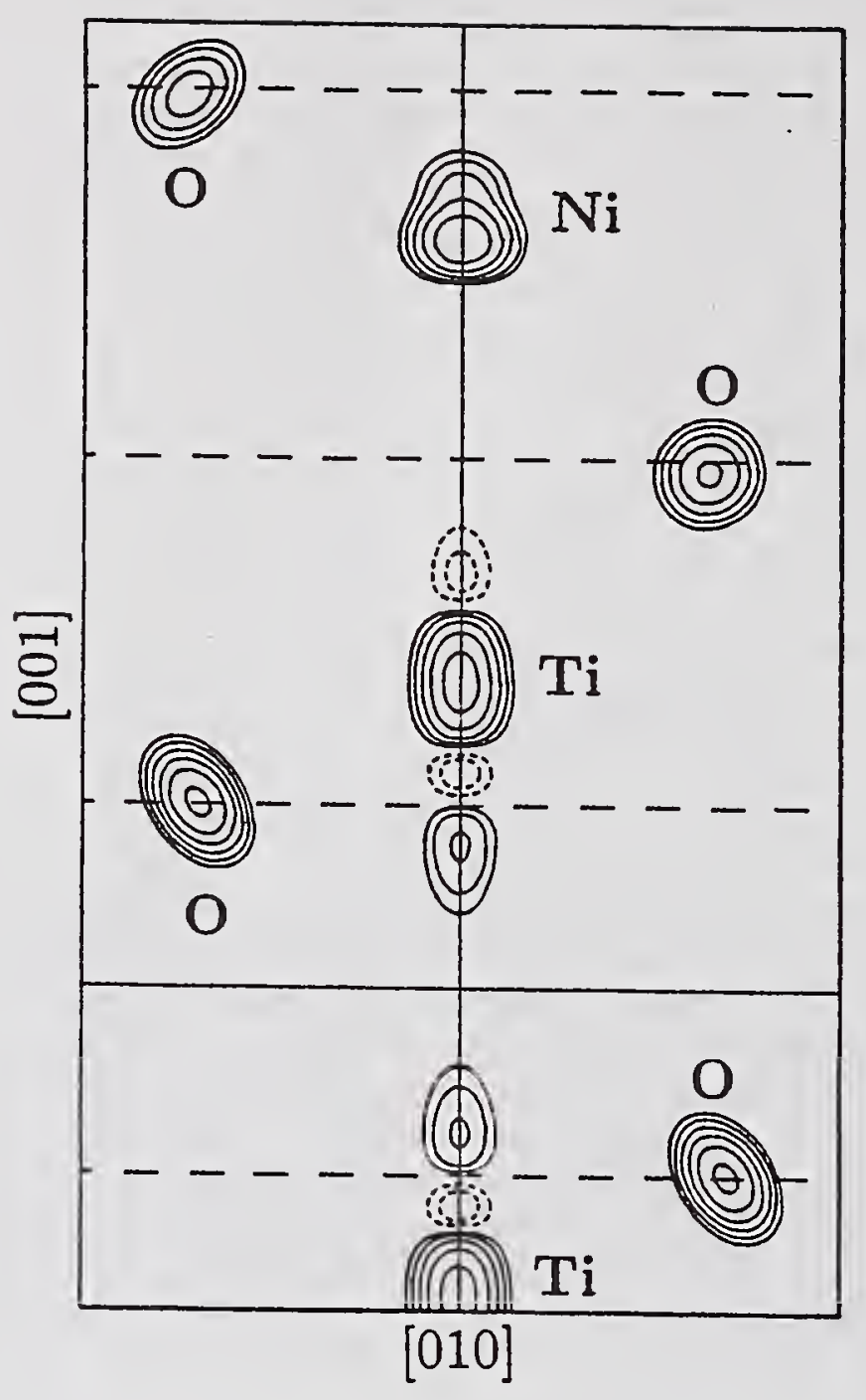

Figure 3: pdf-map of $\mathrm{NiTiO}_{3}$ at $1520 \mathrm{~K}$. Ni and Ti are on the threefold axis, while oxygens are not all exactly on the section shown. The planes of oxygens are indicated by the dashed lines. Contour lines as in figure 1.

At room temperature $\mathrm{NiTiO}_{3}$ possesses the ordered ilmenite structure $(R \overline{3})$ which may be envisaged as a nearly hexagonal close packing of the oxygens with the cations occupying $2 / 3$ of the octahedral voids in an ordered sequence along the threefold axis. At $T_{c}(\approx 1560 \mathrm{~K})$ it undergoes a phase transition to a disordered corundum structure $(R \overline{3} c)$ with a random occupation of the same cation sites (Lerch et al., 1992). Besides the order parameter describing the cation exchange there are further displacive degrees of freedom belonging to the same symmetry species. A neutron powder investigation of the structural changes with temperature (Lerch et al., 1992) revealed not only a critical increase of the HDWF as $T \rightarrow T_{c}$, but also unexpected coupling relations between the different order parameters. The reason for this could be found from "anharmonic" refinements. As shown in the $p d f$ map at about $40 \mathrm{~K}$ below $T_{c}$ (Fig. 3) the cation densities are deformed and, most important, there is some additional density even in the normally (i.e., far below and above $T_{c}$ ) empty octahedron. Hence, a description in terms of a pure site exchange process is by far too simple. In more accurate phase transition theories this (probably dynamic) delocalization of the atoms has to be taken into account. A rather similar situation has also been found in the ferroelectric-paraelectric transition in $\mathrm{LiNbO}_{3}$ (Boysen \& Altorfer, 1992), i. e., it seems to be a more general phenomenon. In both cases trials using split models failed due to very strong correlations.

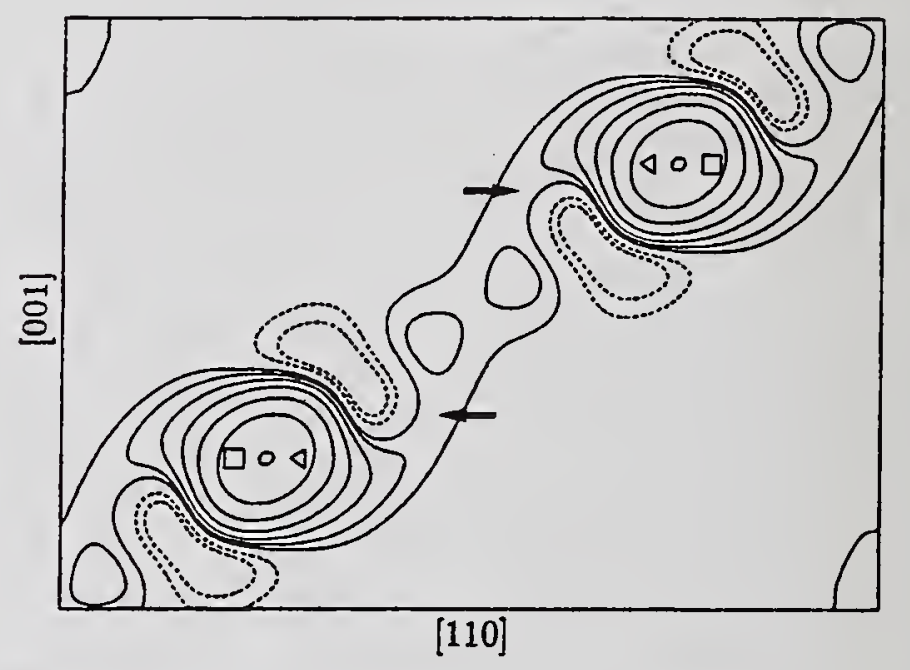

Figure 4: $p d f$ map of $\mathrm{K}$ in leucite at 1073K. Arrows point to the positions of the potential barriers along the diffusion path. Two equivalent $K$ positions resulting from the tetragonal refinement are marked by squares and triangles, respectively. Contour lines as in figure 1 , but starting at $1.5 \%$.

\section{(c) Static disorder}

Static displacement distributions in disordered crystals, (e.g. solid solutions) are almost never truly Gaussian (Krivoglaz, 1969) and require the inclusion of higher order terms. One example (CSZ) has already been mentioned. Another example is the framework silicate leucite, $\mathrm{K}\left(\mathrm{Al}, \mathrm{Si}_{2}\right) \mathrm{O}_{6}$, although it also exhibits aspects of ionic conductivity and phase transitions. The structure consists of corner-linked $(\mathrm{Al}, \mathrm{Si}) \mathrm{O}_{4}$-tetrahedra forming 4-, 6- and 8-fold rings perpendicular to $\langle 100\rangle,\langle 111\rangle$ and $\langle 110\rangle$, respectively. $\mathrm{K}$ atoms are located in non-intersecting tunnels along 
$\langle 111\rangle$ created by the 6 -fold rings. The structure is cubic $(I a 3 d)$ above $T_{c} \approx 940 \mathrm{~K}$ and tetragonal below $\left(I 4_{1} /\right.$ acd and $\left.I 4_{1} / a\right)$. In the cubic phase the space group symmetry requires statistical occupation of $\mathrm{Al}$ and $\mathrm{Si}$ on the tetrahedral sites, while partial ordering is allowed (and is realised) in the tetragonal phases (Boysen, 1990). A conventional harmonic refinement yielded extremely large $B(\mathrm{~K}) \approx 29 \AA^{2}$, which could be reduced to $B(\mathrm{~K}) \approx 16 \AA^{2}$ if the occupancy of $\mathrm{K}$ was also refined: $n(\mathrm{~K}) \approx 0.84$. As mentioned, both findings are strong indications of anharmonicity. After introducing the appropiate anharmonic terms $n(\mathrm{~K})$ returned to full occupancy. The corresponding $p d f$ of $\mathrm{K}$ at $1073 \mathrm{~K}$ is shown in figure 4. Two features may be discerned: (1) there is a continuous probability density all along the tunnel, and, (2) there are lobes along $\langle 110\rangle$. (1) may be related to ionic conductivity, which has also been found from dielectric measurements (Rüscher et al., 1987), again with a remarkable agreement of the activation energy with the potential barriers at the positions marked by the arrows in figure 4. (2) may be interpreted as static short range order: if the $\mathrm{Al} / \mathrm{Si}$ distribution is not completely random (local tetragonal symmetry) the $\mathrm{K}$ ions are displaced towards the potential Al-sites. In fact, an equally good fit, again with $n(K) \approx 1$, could be obtained in space group $I 4_{1} /$ acd with a strictly cubic metric. The resulting positions of the $\mathrm{K}$ atoms are marked in figure 4 for two equivalent distortions. It may be concluded that both models are approximations to the real one: tetragonal distortions on a very fine scale within a strictly cubic framework. It should be added that (of course unphysical) negative $p d f$ 's like those in figure 4 (and figure 3 ) are frequently encountered near very steep features in the true $p d f$, a consequence of the truncation of the series.

\section{Conclusions}

The above examples should have demonstrated that valuable information can be obtained from an analysis of anharmonic temperature factors also in the case of powder diffraction. For a proper understanding of the physical and chemical properties of matter a detailed knowledge of the structure is essential, and this includes not only average positions of the atoms, but also their static and dynamic displacements and disorder. All of the examples represent relatively simple structures, with the consequence that the reflection overlap is not too severe. In more complicated structures, however, the strong overlap and the large number of refinable parameters cause problems that eventually lead to unphysical results. Such a situation was, e.g., encountered in the tetragonal leucite phases and in $\left(\mathrm{Pb}_{0.92} \mathrm{Ba}_{0.08}\right)_{3}\left(\mathrm{PO}_{4}\right)_{2}$ (Hensler et al., 1992), where an anharmonic refinement was possible in the high temperature trigonal phase but not in the low temperature monoclinic one. It may therefore be hoped that with even higher quality data (resolution and intensity) such problems may be tackled in the future. Furthermore, the extension of the scattering diagram to large $Q$-values (small wavelenghts and large scattering angles) is also generally helpful, since the influence of the anharmonic terms increases with $Q$ (see equation (11)).

Although the Gram-Charlier expansion is sufficient in a wide range of applications, there are limitations in some special cases. Note that the truncation of the series restricts its application to relatively small anharmonicities. In an anharmonic refinement of non-stoichiometric $\mathrm{Li}_{x} \mathrm{Al}(0.8 \leq x \leq 1)$ (Tadin et al., 1991) lobes in the pdf of Li indicated a diffusion along certain zig-zag-chains. The fit could then be considerably improved by placing interstitial $\mathrm{Li}$ atoms all along this path, leading to a nearly uniform density distribution. However, this could only be achieved by fixing the positions, occupancies and thermal parameters. Extreme correlations between these parameters prevented a refinement. Therefore, a problem adapted expansion of the $p d f$ that uses only a small number of parameters (e.g. cylinder functions) would be helpful in this case. Another example, where problem adapted expansions might be superior is rotational motions of rigid groups in molecular crystals. Such a treatment based on spherical harmonics (Press \& Hüller, 1973; Prandl, 1981) has been used, e.g., by Hohlwein et al. (1987) for the orientational disorder in $\mathrm{CsNO}_{2}$.

A particularly interesting field of future applications of ADWF's seems to be the critical behaviour near phase transitions (in a wide sense, i. e., including melting, decomposition and chemical reactions). Current theories are based on anharmonicity (mainly in the quasiharmonic approximation) and disorder. Very little has been done so far even with single crystals. More experimental evidence could challenge further theoretical studies. Powder diffraction can be advantageous because it is less susceptible to systematic errors (e.g. the change of mosaicity/extinction) and the ease of data collection, allowing structure determinations at many temperatures in a reasonable time. High quality furnaces with very good temperature homogeneity and stability (a few hundredths of a degree) are necessary for such investigations.

More work is needed for the correction of thermal diffuse scattering (TDS) and multiple scattering in strongly anharmonic systems. Another point concerns the relation of anharmonicities to line broadening effects like microstrains and particle sizes.

In all of the examples given in section 4 a strong 
and modulated diffuse background immediately signaled the presence of disorder. This background by itself contains valuable information about the correlations of the deviations from the average structure (compare equations (2) and (3)). Attempts to calculate this diffuse background include the use of the Warren-Cowley short range order formalism (Warren, 1969; for an example (NaTl) see Schneider, 1988), Fourier-filtering (Richardson \& Faber, 1986) and low dimensional disorder phenomena (corresponding to diffuse streaks and planes; Boysen, 1985). The final goal will be to refine this background simultaneously with the sharp Bragg peaks. In any case, an accurate determination of the background is important to define a reliable baseline underneath the Bragg peaks, especially in the presence of sharper features (TDS peaks, low dimensional disorder). It has been found, however, that varying the background mainly affected the harmonic part of the DWF and to a lesser extent the anharmonic one.

Although, as shown before, a distinction between static and dynamic disorder can often be obtained from an analysis of the temperature dependence of the harmonic and anharmonic ADP's, more definite conclusions could be drawn by using both elastic (with an analyzer in the diffracted beam) and integral (i.e. energy integrated) neutron diffraction patterns (Boysen \& Schneider, 1986). Not only can the static or dynamic nature of the diffuse background and therefore the origin of the disorder be determined, but also an estimate of the TDS contribution to the Bragg intensities can be made.

This work was supported by the BMFT under project No. 03-SC2LMU3.

\section{References}

Altorfer, F. \& BüHrER, W. (1992). Progress-Report $L N S-161$, ETH Zürich, 114-115.

BaCHManN, R. \& Schulz, H. (1984). Acta Cryst. A40, 668- 675 .

BOYSEN, H. (1985). J. Appl. Cryst. 18, 320-325.

BOYsEN, H. (1990). in Phase transitions in ferroelastic and co-elastic crystals, edited by E. K. H. Salje, pp. 334-349. Cambridge: Cambridge University Press.

BOYSEN, H. \& SCHNEIDER, J. (1986). Z. Kristallogr. 174, 26-27.

Boysen, H., Steger, G., Hewat, A. W. \& Buevoz, J. L. (1982). Neutron Scattering-1981. AIP Conference Proceedings, Vol. 89, 202-204. New York: American Institute of Physics.

BOYSEN, H. \& Altorfer, F. (1992). in preparation.

Bruce, A. D. \& Cowley, R. A. (1981). Structural Phase Transitions. London: Taylor \& Francis Ltd.

Cooper, M. J., Rouse, K. D. \& Fuess, H. (1973). Acta Cryst. A29, 49-56.
Dawson, B. (1967). Proc. Roy. Soc. A298, 255-288.

Graneli, B., Fischer, P. \& Dahlborg, U. (1992). Progress-Report LNS-161, ETH Zürich, 112-113.

Hensler, J., Boysen, H., Bismayer, U., Vogt, T., Francke, W. \& Heituans, P. (1992). Z. Kristallogr., submitted.

Hohlwein, D., Hoser, A. \& Prande, W. (1987). $Z$. Kristallogr. 177, 93-102.

IVANOV, S. A., TChorneI, S. A., Michaichenko, B. P. \& Benevtsev, J. A. (1979). Ukrainian Physical Journal 24, 1574-1576.

JoHNSON, C. K. (1969). Acta Cryst. A25, 187-194.

JoHNson, C. K. \& LEVy, H. A. (1974). International Tables for X-ray Crystallography, Vol. IV, 311-336. Birmingham: Kynoch Press.

KiHARA, K. (1992). Europ. J. Mineral. 2, 63-77.

Krivoglaz, M. A. (1969). The Theory of X-ray and Thermal Neutron Scattering by Real Crystals. London: Plenum Press.

Kuns, W. (1992). Acta Cryst. A48, 80-98.

Lerch, M., Boysen, H., Neder, R., Frey, F. \& Laqua, W. (1992). J. Phys. Chem. Solids 53, 11531156.

Lerch, M., Boysen, H., Frey, F. \& Vogt, T. (1992). in preparation.

Martin, U., Frey, F. \& Boysen, H. (1992). Acta Cryst., submitted.

MARTIN, U. (1992). Thesis. Universität München.

Matsubara, T. (1975). J. Phys. Soc. Jpn. 38, 10761079.

Neder, R. B., Frey, F. \& Schulz, H. (1990). Acta Cryst. A46, 792-809.

PrandL, W. (1981). Acta Cryst. A37, 811-818.

Press, W. \& Hüller, A. (1973). Acta Cryst. A29, 252256.

Proffen, T., Neder, R., Frey, F., Keen, D. A. \& ZEYEN, C. (1992). Acta Cryst., submitted.

Richardson JR., J. W. \& FABer JR., J. (1986). $A d v$. in X-ray Anal. 29, 143-152.

Rüscher, C., PAPENdick, M., Boysen, H., Putnis, A. \& SALJE, E. (1987). Z. Kristallogr. 178, 195-196.

SCHNEIDER, J. (1988). Materials Science Forum 27-28, 63-68.

TAdin, M., Schneider, J., Boysen, H. \& Frey, F. (1991). Materials Science Forum 79-82, 635-641.

Tanaka, K. \& Marumo, F. (1983). Acta Cryst. A39, 631-641.

Thomas, M. W. \& Bendall, P. J. (1978). Acta Cryst. A34, 351.

WARREN, B. E. (1969). X-Ray Diffraction. AddisonWesley.

WILLIS, B. T. M. (1969). Acta Cryst. A25, 227-300.

Yu, Y., Boysen, H. \& Schulz, H. (1990). Z. Kristallogr. 191, 79-91.

Zucker, U. H., Perenthaler, E., Kuhs, W., BachMANN, R. \& SchUlz, H. (1983). J. Appl. Crystallogr. 16,358 . 
National Institute of Standards and Technology Special Publication 846. Proceedings of the international conference Accuracy in Powder Diffraction II, held at NIST, Gaithersburg, MD, May 26-29, 1992. (Issued October 1992)

\title{
Recent Advances in the Use of Anomalous Dispersion Effects
}

\author{
J. P. AtTField
}

\author{
Department of Chemistry, University of Cambridge, \\ Lensfield Road, Cambridge CB2 1EW, \\ and Interdisciplinary Research Centre in Superconductivity, \\ Madingley Road, Cambridge, CBз OHE, U.K.
}

\begin{abstract}
Recent advances have centred upon the use of synchrotron X-ray wavelengths that are very close to (usually within $50 \mathrm{eV}$ of) an elemental absorption edge, giving rise to very large anomalous dispersion effects. Rietveld refinements using such resonant $\mathrm{X}$ ray diffraction data enable the distribution of the absorbing element over crystallographically distinct sites within a structure to be determined precisely, even when other elements with very similar atomic numbers are present e.g. $\mathrm{Fe}$ in $\mathrm{FeNi}_{2}\left(\mathrm{BO}_{3}\right) \mathrm{O}_{2}$ using data collected at the $\mathrm{Fe} \mathrm{K}$ edge. Distributions of more than two cations can be found by simultaneously fitting a structural model to resonant profiles collected at several absorption edges. Resonant diffraction data may also be used to study different electronic states of the absorbing element if these are ordered within a crystal structure, e.g. $\mathrm{Eu}^{2+}$ and $\mathrm{Eu}^{3+}$ in $\mathrm{Eu}_{3} \mathrm{O}_{4}$, due to differences in the absorption edges of the two states. This leads to the possibility of obtaining site-resolved XANES spectra. The accuracy of the results depends upon the accuracy with which the anomalous dispersion coefficients $\mathrm{f}^{\prime}$ and $\mathrm{f}^{\prime \prime}$ are known; they can be calculated theoretically or determined from X-ray absorption spectra or crystallographic refinements. Strategies for collecting resonant diffraction data will also be discussed.
\end{abstract}

\section{Introduction}

The intense white $\mathrm{X}$-ray beams produced by electron synchrotrons have recently been used in anomalous scattering powder diffraction experiments. These studies are typically performed close to elemental absorption edges and so are termed 'resonant' X-ray diffraction experiments. Resonant diffraction experiments tend to fall into three classes:

(i) Fundamental studies of simple materials in which reference values of the anomalous dispersion coefficients are determined and strategies for powder methods are explored. (ii) Elemental contrast experiments to determine the distribution of the resonant element within a crystal structure in the presence of other elements with similar atomic numbers.

(iii) Electronic contrast experiments in which the differences between inequivalent states of the resonant element, usually different valence states, are studied through differences in their anomalous dispersion coefficients.

In this review, the origin of anomalous scattering, experimental considerations, and examples of the above experiment types will be discussed, especially in the latter two areas where anomalous dispersion gives information about elemental or electronic distributions that is often not available from other techniques.

\section{Anomalous scattering and $\mathrm{X}$-ray absorption}

The atomic scattering factor for $\mathrm{X}$-rays, $\mathrm{f}$, contains both a normal (Thomson) term, $\mathrm{f}^{n}$, and an anomalous dispersion contribution consisting of real, $\mathrm{f}^{\prime}$, and imaginary, $\mathrm{f}^{\prime \prime}$, terms.

$$
f=f^{n}+f^{\prime}(E)+i f^{\prime \prime}(E)
$$

The normal scattering depends upon the electron distribution and varies only with $\sin \theta / \lambda$, whereas the anomalous contribution results from spectroscopic processes, and so is highly energy dependent. The curves for $f^{\prime}(E)$ and $f^{\prime \prime}(E)$ are directly related to the linear photoelectric absorption coefficient $\mu(\mathrm{E})$ through the optical theorem:

$$
\mathrm{f}^{\prime \prime}(\mathrm{E})=\left(m c / 2 h e^{2} N\right) \mathrm{E} \mu(\mathrm{E}),
$$

where $m=$ electron mass, $c=$ velocity of light, $h=$ Planck's constant, $e=$ electron charge, $N=$ number density of atoms; and the Kramers-Kronig dispersion relationships:

$$
f^{\prime}(E)=(2 / \pi) \int_{0}^{\infty}\left[E^{*} f^{\prime \prime}\left(E^{*}\right) /\left(E^{2}-E^{* 2}\right)\right] d E^{*}
$$



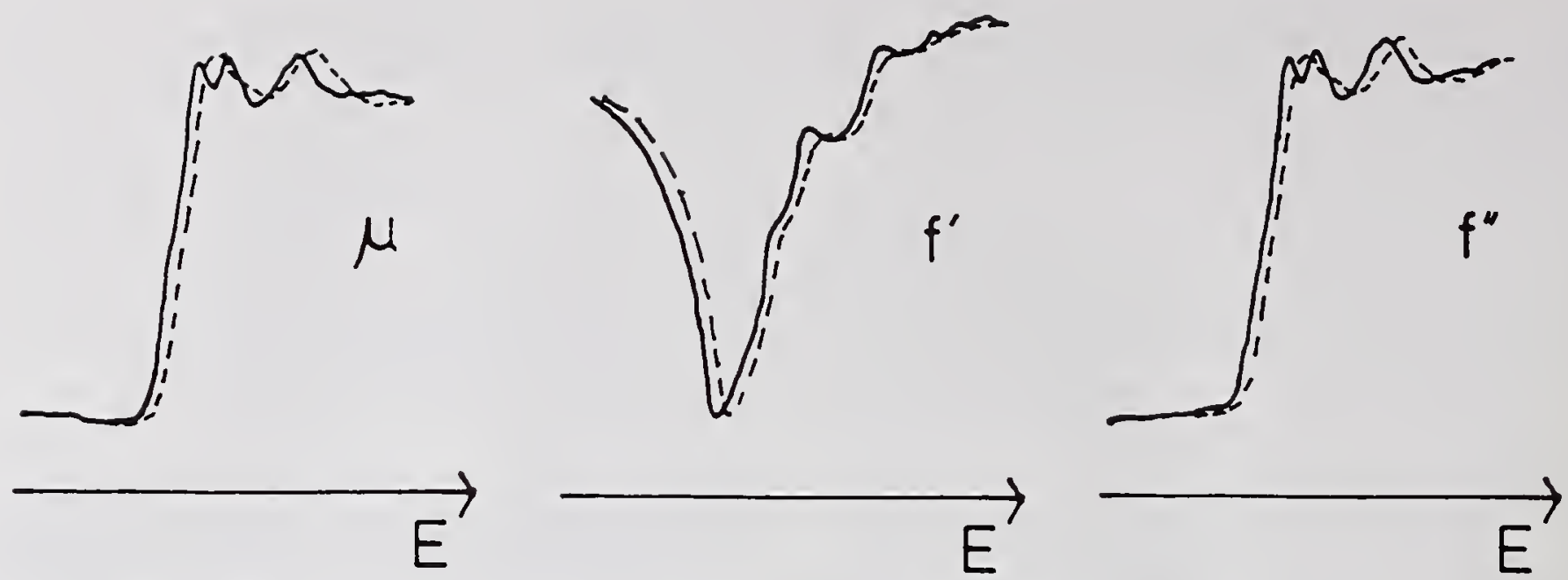

Figure 1: Schematic energy variations of the photoelectric absorption coefficient, $\mu$, and the anomalous dispersion terms $\mathrm{f}^{\prime}$ and $\mathrm{f}^{\prime \prime}$ (continuous lines). The same quantities are also shown for a higher oxidation state of the same element (broken lines).

$$
f^{\prime \prime}(E)=(2 E / \pi) \int_{0}^{\infty}\left[f^{\prime}\left(E^{*}\right) /\left(E^{2}-E^{* 2}\right)\right] d E
$$

by taking the Cauchy principal values of the integrals in (3) and (4).

X-ray absorption spectra of solids contain discontinuities ('edges') at the threshold energies for electronic transitions from core levels to excited states, e.g. $1 s \rightarrow n p$ for the $\mathrm{K}$ edge. Accessible X-ray wavelengths are available for elements with $Z>20$ at K-edges from $\mathrm{Ca}(3.07 \AA)$ to $\mathrm{Ag}(0.49 \AA)$ and $\mathrm{L}$ and $\mathrm{M}$ edges beyond $\mathrm{Cd}\left(\mathrm{L}_{\mathrm{I}}=3.09 \AA\right)$. XANES (X-ray Absorption near-Edge Structure) and EXAFS (Extended X-ray Absorption Fine Structure) effects are also apparent in such spectra. XANES features around the edge arise from electronic transitions to localised excited states, which may result in a very intense 'white line' absorption peak (Brown, Peierls \& Stern, 1977). The EXAFS modulations result from the backscattering of the emitted photoelectron by surrounding atoms and persist for several hundred $\mathrm{eV}$ above the edge.

Through the above equations, the features in $\mu(\mathrm{E})$ are present in the $\mathrm{f}^{\prime \prime}(\mathrm{E})$ curve and, less straightforwardly, in $f^{\prime}(E)$, as shown in figure 1 . This diagram also illustrates the use of resonant $\mathrm{X}$-ray energies for elemental and electronic contrast studies. The former studies are made possible by the large negative values of $\mathrm{f}^{\prime}$ around the edge, which significantly reduce the overall atomic scattering factor of the resonant element. The edge position tends to vary with the electronic state of the resonant element, and increases by several $\mathrm{eV}$ for each unit increase in oxidation number, and so discernible differences between the $\mathbf{f}^{\prime}$ or $\mathrm{f}^{\prime \prime}$ curves for different electronic states may be found from resonant $\mathrm{X}$-ray diffraction experi- ments. The anomalous terms account for an increasing proportion of the total scattering as $2 \theta$ increases, as $\mathrm{f}^{\prime}$ and $\mathbf{f}^{\prime \prime}$ show virtually no fall-off with $\sin \theta / \lambda$ (Templeton, Templeton, Phizackerley \& Hodgson, 1982).

Anomalous dispersion curves can be determined experimentally from X-ray absorption spectra using equations (2) and (3) (Bonse \& Hartmann-Lotsch, 1984; Hoyt, De Fontaine \& Warburton, 1984; Dreier, Rabe, Malzfeldt, \& Neimann, 1984). Good agreement has been found between such spectroscopic results and values refined from single crystal data (Templeton \& Templeton, 1982, 1985, 1988, 1990; Templeton, Templeton, Phillips \& Hodgson, 1980; Templeton, Templeton, Phizackerley \& Hodgson, 1982). Anomalous coefficients can also be determined from powder diffraction data, as described below, or by X-ray interferometry Begum, Hart, Lea, \& Siddons, 1986). Theoretical methods (Creagh, 1988) such as the relativistic dipole calculations of Cromer \& Liberman $(1970,1981)$ are commonly used to compute $\mathrm{f}^{\prime}$ and $\mathrm{f}^{\prime \prime}$ (Cromer, 1983), but these do not take account of the valence shift and local edge features described above, and so are of limited accuracy at resonant wavelengths. Only crystallographic methods allow anomalous coefficients to be measured independently at different sites within the same structure, giving rise to the new developments described here.

\section{Experimental considerations}

The use of existing synchrotron diffractometers for angle-dispersive resonant powder $\mathrm{X}$-ray experiments has been discussed by Wilkinson, Cox \& Cheetham 
(1991). Specific considerations for resonant studies are the wavelength and sample absorption. The energy spread and mean wavelength must be known accurately, and it is desirable to be able to select the wavelength accurately and to vary it in relation to the absorption edge position. If data are collected above the edge, then large sample absorption and background fluorescence make the choice of sample geometry and detection system important.

The energy spread within the X-ray beam is determined by the monochromator band-pass, given by:

$$
\Delta \mathrm{E} / \mathrm{E}=\sqrt{\left(\Delta \phi \cot \theta_{\mathrm{B}}\right)^{2}+(\Delta \mathrm{d} / \mathrm{d})^{2}},
$$

where $\Delta \phi$ is the angular divergence of the beam in the scattering plane, $\theta_{\mathrm{B}}$ is the monochromator Bragg angle, and $\Delta d / d$ is the divergence of the monochromator, which for a perfect crystal is related to the intrinsic Darwin width of the reflection plane. Typical values for the $\Delta d / d$ contribution at $1.5418 \AA$ $(8.04 \mathrm{keV})$ are $\Delta \mathrm{E}=1.1,0.5,2.6$, and $1.2 \mathrm{eV}$ for $\mathrm{Si}(111), \mathrm{Si}(220), \mathrm{Ge}(111)$, and $\mathrm{Ge}(220)$ perfect crystals, respectively (Freund, 1987). The wavelength thus needs to be determined with an accuracy of $\Delta \lambda / \lambda \sim 10^{-4}$ at such energies, usually by fitting several peaks from a $\mathrm{Si}$ standard. Recalibration is typically performed each time the wavelength is changed. This can be done conveniently from the positions of the sample peaks.

For many experiments the wavelength is chosen relative to the positions of features in the absorption edge spectrum. The widths of absorption edge features tend to increase with energy, from $\mathrm{Cu}=1.5$ to $\mathrm{Sn} \sim 8 \mathrm{eV}$ for $\mathrm{K}$ edges (Hamalainen, Manninen, Suortti, Collins, Cooper \& Laundy, 1989; Bambynek, Craseman, Fink, Freund, Mark, Swift, Price \& Rao, 1972), placing a limit upon the necessary experimental energy resolution. As the experimentally observable absorption curve is a convolution of the true curve with the monochromator band-pass, it is highly desirable to be able to record the spectrum in situ. rather than relying on spectra recorded on other instruments. This can be done by a direct transmission experiment, but a more convenient alternative is to monitor the sample fluorescence at an angle away from the Bragg peaks. With a detector of poor energy resolution, the variation of background intensity with energy gives a measure of the fluorescence, and an approximate $\mu(\mathrm{E})$ spectrum can be derived (Attfield, 1991). Alternatively, solid state detectors offering good energy resolution can be used to measure the fluorescence spectrum directly (Wilkinson, Cox \& Cheetham, 1991).

When resonant diffraction is performed above the threshold of the absorption edge then sample absorption becomes severe. Geometry-dependent absorption corrections must be applied to the diffraction data, except for flat plate samples in reflection mode, which are therefore preferable for most experiments. However, even in this geometry, surface roughness can provide a small angle dependent correction, and at very absorbing wavelengths the penetration depth may be so small that incomplete powder averaging or surface texture may result in systematic errors in the diffraction intensities. The high fluorescent background at such wavelengths can be reduced by using Soller slits and a solid state detector with a high energy resolution (200-400 eV) or a crystal analyser with a gas proportional or scintillation detector. The latter combination gives a very high peak to background ratio, but the low acceptance angle at the detector may exacerbate the systematic errors at absorbing wavelengths.

\section{Results of resonant powder diffraction experiments}

\section{i) Fundamental Studies}

The sensitivity of powder refinements to $\mathrm{f}^{\prime}$ and $\mathrm{f}^{\prime \prime}$ in comparison to single crystal studies can be demonstrated by considering the structure factors $\mathrm{F}_{ \pm}$for a Friedel pair of reflections $\pm h k l$ with only one anomalously scattering element in the unit cell:

$$
\begin{aligned}
\mathbf{F}_{ \pm}= & A+a\left(\mathrm{f}^{\prime}+i \mathrm{f}^{\prime \prime}\right) \\
& \pm i\left[B+b\left(\mathbf{f}^{\prime}+i \mathbf{f}^{\prime \prime}\right)\right]
\end{aligned}
$$

where $A / B$ and $a / b$ are the summed cos / sin terms for the normal and anomalous contributions, respectively. Hence,

$$
\begin{aligned}
\mathrm{F}_{ \pm}^{2}= & A^{2}+B^{2}+\left(a^{2}+b^{2}\right)\left(\mathrm{f}^{2}+\mathrm{f}^{\prime 2}\right) \\
& +2(A a+B b) \mathrm{f}^{\prime} \pm 2(B a+A b) \mathrm{f}^{\prime \prime}
\end{aligned}
$$

In the centrosymmetric case this reduces to:

$$
\mathrm{F}_{ \pm}^{2}=A^{2}+a^{2}\left(\mathrm{f}^{2}+\mathrm{f}^{\prime 2}\right)+2 A a \mathrm{f}^{\prime}
$$

In a powder pattern $\mathrm{F}_{+}^{2}$ and $\mathrm{F}_{-}^{2}$ are overlapped, whereas they can be measured independently in single crystal studies. For a centrosymmetric structure $\mathrm{F}_{+}^{2}=\mathrm{F}_{-}^{2}$ so there is no loss of information in the powder pattern compared to the single crystal case, beyond the usual compression of three-dimensional data into a radial pattern. For small anomalous coefficients, the refinements are sensitive to $\mathrm{f}^{\prime}$ through the cross term $A a f^{\prime}$ but are relatively insensitive to $\mathrm{f}^{\prime \prime}$.

Single crystal studies of non-centrosymmetric materials are able to determine $\mathrm{f}^{\prime \prime}$ from the difference between $\mathrm{F}_{+}^{2}$ and $\mathrm{F}_{-}^{2}$ (equation (7)), whereas in powder diffraction only their sum is measured:

$$
\begin{aligned}
\mathrm{F}_{+}^{2}+\mathrm{F}_{-}^{2}= & 2 A^{2}+2 B^{2} \\
& +2\left(a^{2}+b^{2}\right)\left(\mathrm{f}^{2}+\mathrm{f}^{\prime 2}\right) \\
& +4(A a+B b) \mathrm{f}^{\prime} .
\end{aligned}
$$


Hence the Bijvoet effect information that is used to solve complex non-centrosymmetric structures (Karle, 1989) and determine absolute configurations (Flack, 1983) is lost in a powder pattern. Again, for small anomalous coefficients the powder data are much more sensitive to variations in $\mathrm{f}^{\prime}$ than in $\mathrm{f}^{\prime \prime}$.

Strategies for determining anomalous dispersion coefficients from powder patterns have been discussed for Ni (Suortti, Hastings \& Cox, 1985) and $\mathrm{Yb}_{2} \mathrm{O}_{3}$ (Will, Masciocchi, Hart \& Parrish, 1987). A variety of reference materials have also been used to obtain $\mathrm{f}^{\prime}$ values for a given geometry or valence state as part of the elemental and electronic contrast experiments described below, e.g. $\quad \mathrm{Fe}_{2} \mathrm{O}_{3}$ (Perkins \& Attfield, 1991) and $\mathrm{Fe}_{3}\left(\mathrm{PO}_{4}\right)_{2}$ (Warner, Wilkinson, Cheetham \& Cox, 1991) for studies of iron oxo-salts and $\mathrm{Cu}_{2} \mathrm{O}, \mathrm{CuO}$ and $\mathrm{KCuO}_{2}$ (Kwei, Von Dreele, Williams, Goldstone, Lawson \& Warburton, 1990) for the investigation of copper valences in $\mathrm{YBa}_{2} \mathrm{Cu}_{3} \mathrm{O}_{6+x}$. In all cases, data were collected below the absorption edge and used to refine $\mathrm{f}^{\prime}$ by conventional integrated intensity or Rietveld (1969) methods with $\mathrm{f}^{\prime \prime}$ fixed at a calculated value. For example, in the study of $\mathrm{Yb}_{2} \mathrm{O}_{3}$ by Will, Masciocchi, Hart \& Parrish (1987), $f^{\prime}$ values up to -21.2 e/atom at $8 \mathrm{eV}$ below the $\mathrm{L}_{\mathrm{III}}$ edge were obtained, due to a white line at the absorption edge.

Although high precision in $\mathrm{f}^{\prime}$ may be obtained, accuracy may be limited by correlations with temperature and scale factors. The former problem may be minimised by collecting data over as wide a $\sin \theta / \lambda$ range as possible, or by determining temperature factors from another off-edge or neutron diffraction profile. When the resonant element is the dominant $\mathrm{X}$-ray scatterer, then $\mathrm{f}^{\prime}$ tends to correlate with the scale factor, requiring an intensity standard or measurements of absolute diffraction peak intensities (Suortti, Hastings \& Cox, 1985).

Resonant powder diffraction data can also be used to study the anisotropy of the anomalous scattering coefficients that results from anisotropy in $\mu(\mathrm{E})$. This can lead to the breakdown of extinction rules such as for the $n$-glide planes in $\mathrm{Cu}_{2} \mathrm{O}$ with $P n 3 m$ symmetry (Kirfel, Eichhorn \& Wroblewski, 1988).

\section{(ii) Elemental contrast}

These experiments provide the most straightforward and useful application of resonant X-ray diffraction. When two or more elements of similar atomic number are disordered within a structure, then resonant data collected at one of the elemental absorption edges can be used to refine the individual distributions. Examples are given in table 1 . In all cases contrast is achieved by using a wavelength just below the absorption edge, where sample absorption is low and so $\mathrm{f}^{\prime \prime}$ is small and calculable, but large negative values of $\mathrm{f}^{\prime}$ can be achieved, giving marked changes in

Table 1: Materials studied by elemental contrast resonant powder X-ray diffraction, with the resonant element(s) underlined. ' $N$ ' indicates a simultaneous refinement using resonant $\mathrm{X}$-ray and neutron profiles.

$\mathrm{EuSm}_{2} \mathrm{O}_{4}$
$\mathrm{Fe}_{0} .50 \mathrm{Co}_{0.48} \underline{\mathrm{V}}_{0.02}, \mathrm{~N}$
$\mathrm{FeCo}_{2}\left(\mathrm{PO}_{4}\right)_{3}$
$\underline{\mathrm{Fe}}_{2} \mathrm{BO}_{3} \mathrm{O}_{2}$
$\underline{\mathrm{La}}_{0.9} \underline{\mathrm{Gd}}_{0.9} \underline{\mathrm{S}}_{0.2} \mathrm{CuO}_{4}$

$\underline{\mathrm{Y}}_{0.19} \underline{\mathrm{Zr}}_{0.81} \mathrm{O}_{1.90}$ $\mathrm{YBa}_{2}(\underline{\mathrm{Cu}}, \underline{\mathrm{Ga}})_{3} \mathrm{O}_{7-x}, \mathrm{~N}$

$\mathrm{YBa}_{2}(\mathrm{Cu}, \underline{\mathrm{Zn}})_{3} \mathrm{O}_{7-x}$ $\mathrm{YBa}_{2}(\underline{\mathrm{Cu}}, \underline{\mathrm{Zn}})_{3} \mathrm{O}_{7-x}, \mathrm{~N}$
Attfield (1990).

Williams et al. (1990).

Warner et al. (1991).

Perkins \& Attfield (1991).

Kwei, Von Dreele, Cheong et al. (1990).

Moroney et al. (1988).

Kwei, Von Dreele, Williams et al. (1990).

Howland et al. (1989).

Kwei, Von Dreele, Williams et al. (1990).

the peak intensities.

An illustrative example is the study of $\mathrm{FeNi}_{2}\left(\mathrm{BO}_{3}\right) \mathrm{O}_{2}$ (Perkins \& Attfield, 1991) in which a single resonant pattern collected $16 \mathrm{eV}$ below the $\mathrm{Fe} \mathrm{K}$ edge was used to refine the $\mathrm{Fe} / \mathrm{Ni}$ occupancies of four crystallographically distinct octahedral sites (Fig. 2). The profile fit is shown in figure 3 , and the results are summarised in table 2 . The refinement gives typical precision for the fractional coordinates and other structural parameters, and also $\mathrm{Fe} / \mathrm{Ni}$ occupancies precise to $0.6 \%$ for the extra effort of selecting a resonant wavelength. The refined value of $\mathrm{f}^{\prime}$ from an $\mathrm{Fe}_{2} \mathrm{O}_{3}$ standard was $-6.94(3)$ (calculated $\mathrm{f}^{\prime}=$ $-6.61 \mathrm{e} /$ atom), which greatly reduced the Fe scattering factor $\left(\mathrm{f}^{n}(0)=26\right.$ e/atom). A variation on this type of study was carried out by Moroney, Thompson \& Cox (1988) on yttrium-stabilised cubic zirconia, $\mathrm{Y}_{0.19} \mathrm{Zr}_{0.81} \mathrm{O}_{1.90}$. In this case the $\mathrm{Y} / \mathrm{Zr}$ occupancy of the single cation site was fixed, and resonant data were used to find how each species was disordered around the ideal position by determining its temperature factor independently. The isotropic B-factors were found to be $\mathrm{Y}=0.64(15) \AA^{2}, \mathrm{Zr}=1.18(4) \AA^{2}$.

Contrasting elements with similar atomic numbers by resonant diffraction compares favourably with the traditional neutron diffraction method. Resonant diffraction is general for elements with $\mathrm{Z}>20$ and can be used for highly neutron absorbing materials, e.g. $\operatorname{EuSm}_{2} \mathrm{O}_{4}$ (Attfield, 1990) or for elements with similar neutron scattering lengths, e.g. $\mathrm{Fe}$ and $\mathrm{Ni}$ in $\mathrm{FeNi}_{2}\left(\mathrm{BO}_{3}\right) \mathrm{O}_{2}$ above. Warner, Wilkinson, Cheetham \& Cox (1991) have shown that independent Rietveld refinements of the $\mathrm{Fe} / \mathrm{Co}$ distribution in $\mathrm{FeCo}_{2}\left(\mathrm{PO}_{4}\right)_{3}$ with resonant $\mathrm{X}$-ray and neutron diffraction data give comparable results. The values of $x$ for the distribution 
Table 2: Results of the resonant X-ray refinement of orthorhombic $\mathrm{FeNi}_{2}\left(\mathrm{BO}_{3}\right) \mathrm{O}_{2}($ Perkins \& Attfield, 1991). The cation occupancies were constrained to give the correct overall stoichiometry.

\begin{tabular}{|c|c|c|c|c|c|c|c|}
\hline \multicolumn{3}{|c|}{ Number of points $=9062$} & \multicolumn{5}{|c|}{ Number of reflections $=189$} \\
\hline \multicolumn{2}{|c|}{ R-Factors (\%): } & $\mathrm{R}_{w p}=12.6$ & \multicolumn{2}{|c|}{$\mathrm{R}_{p}=9.5$} & \multirow{2}{*}{$\begin{array}{c}\mathrm{R}_{I}=4.8 \\
2.23794(9)\end{array}$} & \multirow{2}{*}{\multicolumn{2}{|c|}{$\begin{array}{l}\mathrm{R}_{E x}=8.3 \\
c=3.00620(2)\end{array}$}} \\
\hline Cell P & rameters $(\AA)$ & $a=9$. & $21048(7)$ & & & & \\
\hline \multicolumn{8}{|c|}{ Atomic Parameters (space group Pbam): } \\
\hline Site & Multiplicity & $\mathrm{x}$ & $\mathrm{y}$ & $\mathrm{z}$ & $\mathrm{B}_{i s o}\left(\AA^{2}\right)$ & $\% \mathrm{Fe}$ & $\% \mathrm{Ni}$ \\
\hline$M(1)$ & 2 & 0 & 0 & 0 & $0.13(3)$ & $8.8(6)$ & $91.2(6)$ \\
\hline$M(2)$ & 2 & $1 / 2$ & 0 & $1 / 2$ & 0.13 & $38.5(6)$ & $61.5(6)$ \\
\hline$M(3)$ & 4 & $-0.0001(2)$ & $0.2814(4)$ & 0 & 0.13 & $14.4(5)$ & $85.6(5)$ \\
\hline $\mathrm{M}(4)$ & 4 & $0.7378(2)$ & $0.3848(1)$ & $1 / 2$ & 0.13 & $61.9(4)$ & $38.1(4)$ \\
\hline $\mathrm{B}(1)$ & 4 & $0.274(1)$ & $0.3602(9)$ & $1 / 2$ & $0.3(2)$ & & \\
\hline $\mathrm{O}(1)$ & 4 & $0.8516(5)$ & $0.0438(4)$ & $1 / 2$ & $0.13(4)$ & & \\
\hline$O(2)$ & 4 & $0.3910(5)$ & $0.0763(4)$ & 0 & 0.13 & & \\
\hline $\mathrm{O}(3)$ & 4 & $0.6276(5)$ & $0.1394(5)$ & $1 / 2$ & 0.13 & & \\
\hline$O(4)$ & 4 & $0.8497(6)$ & $0.1444(5)$ & 0 & 0.13 & & \\
\hline $\mathrm{O}(5)$ & 4 & $0.8497(6)$ & $0.2395(4)$ & $1 / 2$ & 0.13 & & \\
\hline
\end{tabular}

${ }^{\mathrm{VI}}\left(\mathrm{Co}_{x} \mathrm{Fe}_{1-x}\right)^{\mathrm{V}}\left(\mathrm{Co}_{2-x} \mathrm{Fe}_{x}\right)\left(\mathrm{PO}_{4}\right)_{3}$ over the five and six-coordinate cation sites were found to be $0.502(8)$ and $0.486(10)$ for time-of-flight neutron and resonant $\mathrm{X}$-ray diffraction, respectively. The contrast gained by inducing $\mathrm{f}^{\prime}(\mathrm{Fe})=-7.8 \mathrm{e} /$ atom, $9 \mathrm{eV}$ below the Fe K edge, was approximately equivalent to that between the neutron scattering lengths of $\mathrm{b}(\mathrm{Fe})=0.954$ and $\mathrm{b}(\mathrm{Co})=0.253 \times 10^{-14} \mathrm{~m}$.

A further advantage of resonant X-ray diffraction for elemental contrast studies is the ability to collect data at the edges of several elements to solve complex disorder problems involving more than two elements. Simultaneous refinements of a combination of resonant synchrotron X-ray diffraction profiles, laboratory X-ray data, and neutron patterns have been used by Kwei, Von Dreele, Williams, Goldstone, Lawson \& Warburton (1990) to obtain precise structural models for several compounds listed in table 2. For example, the distribution of cations over the eight and nine-coordinate sites in the $\mathrm{T}^{*}$ type superconductor $\left(\mathrm{La}_{0.9} \mathrm{Gd}_{0.9} \mathrm{Sr}_{0.2}\right) \mathrm{CuO}_{4}$ was found to be ${ }^{I X}\left(\mathrm{La}_{0.8} \mathrm{Sr}_{0.2}\right)^{\mathrm{VIII}}\left(\mathrm{La}_{0.1} \mathrm{Gd}_{0.9}\right) \mathrm{CuO}_{4}$ (Kwei, Von Dreele, Cheong, Fisk \& Thompson, 1990).

(iii) Electronic contrast

Studies of differences in anomalous scattering from a resonant element at two or more crystallographically distinct sites are shown in table 3 , and have recently been reviewed (Attfield, 1991). The ability of resonant X-ray diffraction to contrast ordered valence states was illustrated by a study of $\mathrm{Eu}_{3} \mathrm{O}_{4}$ using a single powder diffraction pattern collected at the Eu $\mathrm{L}_{\text {III }}$ edge with $\lambda=1.7771(1) \AA(6976.9 \mathrm{eV})$ (Attfield, 1990). $\mathrm{Eu}^{2+}\left(\mathrm{Eu}^{3+}\right)_{2} \mathrm{O}_{4}$ is a localised valence compound in which $\mathrm{Eu}^{2+}$ occupies one of three crys-
Table 3: Compounds studied by electronic contrast resonant powder X-ray diffraction, with the different states of the resonant element(s) underlined.

$$
\begin{aligned}
& \alpha \text {-Ce, valence fluctuations Kwei, Von Dreele, } \\
& \text { Williams et al. } \\
& \text { (1990). } \\
& \underline{\mathrm{Eu}}^{2+}+\underline{\mathrm{Eu}}_{2}^{3+} \mathrm{O}_{4} \\
& \mathrm{YBa}_{2} \mathrm{Cu}(1) \mathrm{Cu}(2)_{2} \mathrm{O}_{6+x} \\
& x=0.09,0.38,0.72,0.95 \\
& \mathrm{YBa}_{2} \mathrm{Cu}(1) \mathrm{Cu}(2)_{2} \mathrm{O}_{6+x}, \quad \text { Attfield (1991). } \\
& x=0.27 \\
& \mathrm{Y}_{3}\left({ }^{\mathrm{IV}} \underline{\mathrm{Ga}}^{3+}\right)_{3}\left({ }^{\mathrm{VI}} \underline{\mathrm{Ga}}^{3+}\right)_{2} \mathrm{O}_{12} \quad \text { Wilkinson, Cox } \\
& \text { \& Cheetham } \\
& \text { (1991). }
\end{aligned}
$$

tallographically distinct $\mathrm{Eu}$ sites and $\mathrm{Eu}^{3+}$ fills the other two. In order to determine the valence distribution from the anomalous scattering alone, Rietveld fits were performed for the three possible ways of ordering the valences over the three sites by refining both $\mathrm{f}^{\prime}$ and $\mathrm{f}^{\prime \prime}$ for $\mathrm{Eu}^{2+}$ and $\mathrm{Eu}^{3+}$ independently. The value of the integrated peak intensity residual, $R_{I}$, was significantly lower for the correct model (11.8\%) than for the other two (18.5 and $17.8 \%)$. The refined anomalous coefficients were compared to published curves for $f^{\prime}(E)$ and $f^{\prime \prime}(E)$ around lanthanide $\mathrm{L}_{\text {III }}$ edges (Templeton, Templeton, Phizackerley \& Hodgson, 1982), enabling the energy shifts of the $L_{\text {III }}$ 


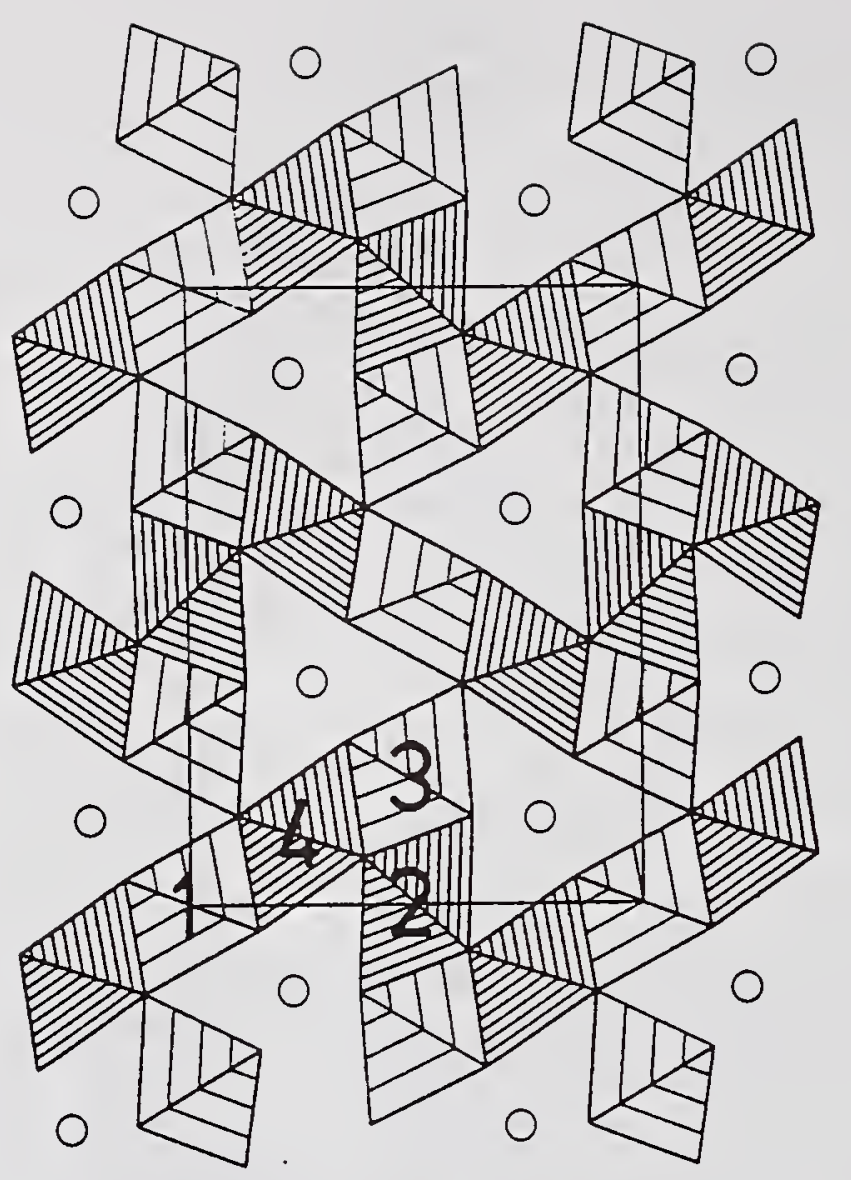

Figure 2: (001) polyhedral view of $\mathrm{FeNi}_{2}\left(\mathrm{BO}_{3}\right) \mathrm{O}_{2}$ with the unit cell marked ( $a$ horizontal, $b$ vertical) and the boron atoms shown as circles. The four crystallographically distinct octahedra are labelled, and those centred in the $z=0 / 1 / 2$ planes are lightly / heavily hatched.

edges of $\mathrm{Eu}^{2+}$ and $\mathrm{Eu}^{3+}$ relative to the beam energy to be estimated as $-2.3(10)$ and $4.2(8) \mathrm{eV}$ respectively (Fig. 4). The valence shift of $6.5(13) \mathrm{eV}$ is in excellent agreement with the difference of $6.4(3) \mathrm{eV}$ from the X-ray absorption spectra of $\mathrm{EuO}$ and $\mathrm{Eu}_{2} \mathrm{O}_{3}$.

In the study of $\mathrm{Eu}_{3} \mathrm{O}_{4}$, large differences between the anomalous scattering coefficients of different oxidation states were observed as the chosen wavelength lay between the edge energies of the two oxidation states. However, the high sample absorption resulted in low counting statistics and poor precision in the refined anomalous coefficients. To avoid these problems, other electronic contrast experiments have been performed at pre-edge energies to obtain good counting statistics, from which significant differences in refined $\mathrm{f}^{\prime}$ values have been obtained. For example, a study of $\mathrm{Ga}^{+} \mathrm{Ga}^{3+} \mathrm{Cl}_{4}$ (Wilkinson, Cheetham $\&$ Cox, 1991) revealed differences up to $3.2 \mathrm{e} /$ atom in $\mathrm{f}^{\prime}$ between the $\mathrm{Ga}^{+}$and $\mathrm{Ga}^{3+}$ sites. The studies in table 3 show that resonant powder $\mathrm{X}$-ray diffraction

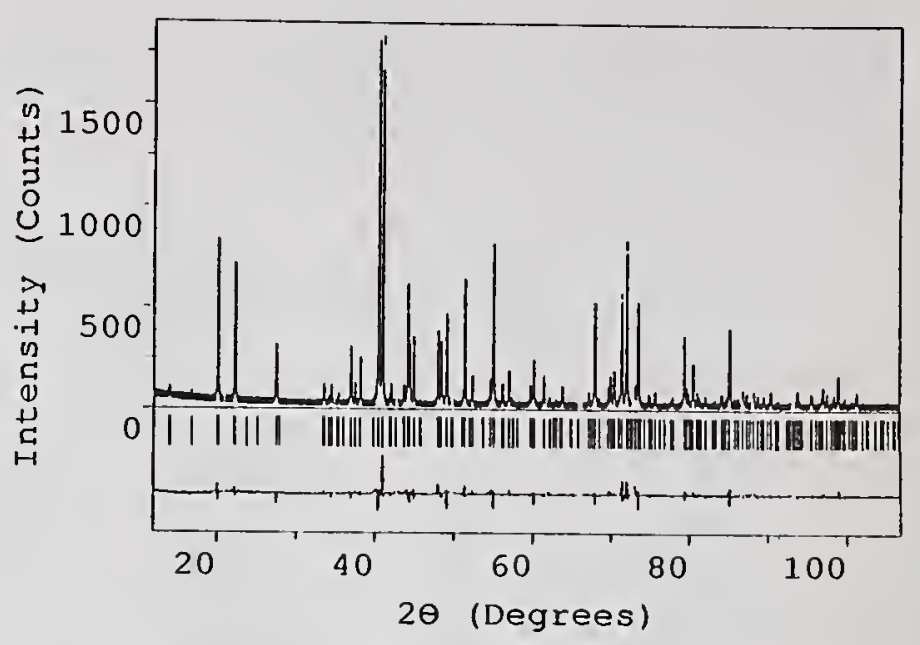

Figure 3: Observed (dashes), calculated (full line), and difference resonant $\mathrm{X}$-ray diffraction profiles for $\mathrm{FeNi}_{2}\left(\mathrm{BO}_{3}\right) \mathrm{O}_{2}$ with the reflection positions marked (Perkins \& Attfield, 1991).

can be used to demonstrate that different valence states, and perhaps differently coordinated sites, of the same element present in the same structure may have significantly different anomalous scattering coefficients. However, the interpretation of such results is not always straightforward. In the case of $\mathrm{Eu}_{3} \mathrm{O}_{4}$, a single pattern was used to refine four anomalous terms, which could be related to the published $\mathrm{f}^{\prime}(\mathrm{E})$ and $f^{\prime \prime}(E)$ curves only by assuming that the edge shifts but does not change shape in going from $\mathrm{Eu}^{2+}$ to $\mathrm{Eu}^{3+}$. A better approach is to use $\mathrm{f}^{\prime}(\mathrm{E})$ or $\mathrm{f}^{\prime \prime}(\mathrm{E})$ curves derived from appropriate standards, for example $\mathrm{Cu}+1,+2$, and +3 oxides to interpret results from $\mathrm{YBa}_{2} \mathrm{Cu}_{3} \mathrm{O}_{6+x}$ (Kwei, Von Dreele, Williams, Goldstone, Lawson \& Warburton, 1990), but even this involves a certain level of approximation, as the XANES features are sensitive to local electronic structure as well as oxidation state (Alp, Goodman, Soderholm, Mini, Ramanathan, Shenoy, \& Bommannavar, 1989). The influence of site geometry has been investigated in the garnet $\mathrm{Y}_{3} \mathrm{Ga}_{5} \mathrm{O}_{12}$ (Wilkinson, Cox, \& Cheetham, 1991). A small difference in $\mathrm{f}^{\prime}$ between octahedrally and tetrahedrally coordinated $\mathrm{Ga}^{3+}$ was found, but the range of observations was too limited to determine the origin of the difference.

In view of the problems in interpreting electronic contrast results, the ultimate aim of such experiments might be to produce resolved X-ray absorption edge spectra for each crystallographically independent site. In principle, this could be achieved by collecting data at energies across the edge, refining $f^{\prime}$ and $f^{\prime \prime}$ for each site, and scaling the $f^{\prime \prime}(E)$ curves to $\mu(\mathrm{E})$ via equation (2). This is not yet practicable, as sample absorption necessitates very long count- 


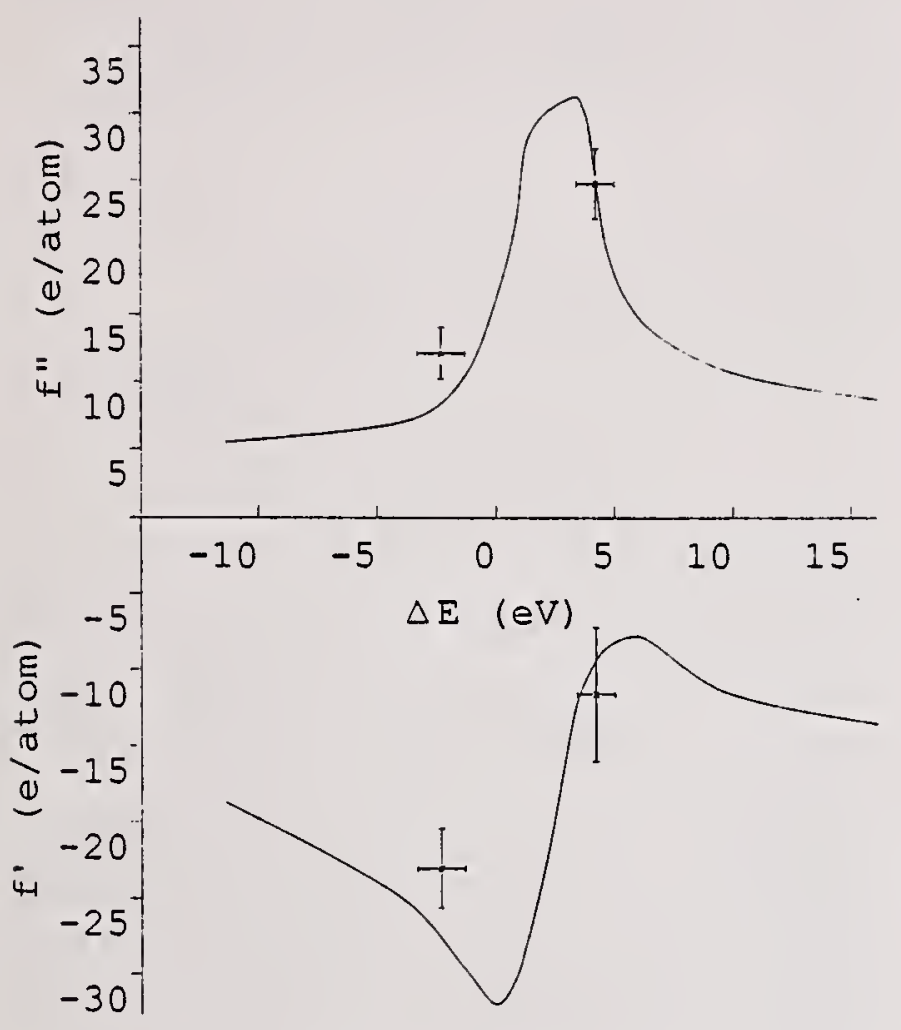

Figure 4: Curves for the variation of $\mathrm{f}^{\prime}$ and $\mathrm{f}^{\prime \prime}$ with $\Delta \mathrm{E}\left(=\mathrm{E}_{\text {Beam }}-\mathrm{E}_{\mathrm{Edge}}\right)$ around the Eu LIII absorption edge. The points show the refined values for $\mathrm{Eu}^{2+}$ $(\Delta \mathrm{E}=-2.3(10) \mathrm{eV})$ and $\mathrm{Eu}^{3+}(\Delta \mathrm{E}=4.2(8) \mathrm{eV})$ in $\mathrm{Eu}_{3} \mathrm{O}_{4}$ (Attfield, 1990).

ing times at highly absorbing energies, and correlations between the refined $f^{\prime}$ and $f^{\prime \prime}$ values may be severe. However, a simple experiment using limited crystallographic data to resolve an X-ray absorption spectrum has been demonstrated for $\mathrm{YBa}_{2} \mathrm{Cu}_{3} \mathrm{O}_{6.27}$ (Attfield, 1991). The variations of $\left|F_{001}\right|$ and $\left|F_{111}\right|$ in the near-edge region were measured (Fig. 5) and used to divide the bulk $\mathrm{Cu} \mathrm{K}$ edge spectrum into curves for the two $\mathrm{Cu}$ sites (Fig. 6). Although the results are of very limited precision, they do reveal that the $\mathrm{Cu}(1)$ edge is broader, with unresolved pre-edge features, and at a lower energy than that of $\mathrm{Cu}(2)$. This is consistent with the accepted view that both $\mathrm{Cu}^{+}$and $\mathrm{Cu}^{2+}$ are present on the $\mathrm{Cu}(1)$ (chain) sites, but only $\mathrm{Cu}^{2+}$ is on the $\mathrm{Cu}(2)$ (plane) sites for this oxygen content.

Resonant X-ray diffraction may also be able to investigate systems in which valence fluctuations are too rapid to give rise to a structural distortion. Kwei, Von Dreele, Williams, Goldstone, Lawson \& Warburton (1990) report a study of the low temperature $\alpha$ form of cerium metal. If the previously determined average valence of +3.6 results from fluctuations between +3 and +4 states that are ordered for a sufficiently long time to give coherent Bragg diffraction a)

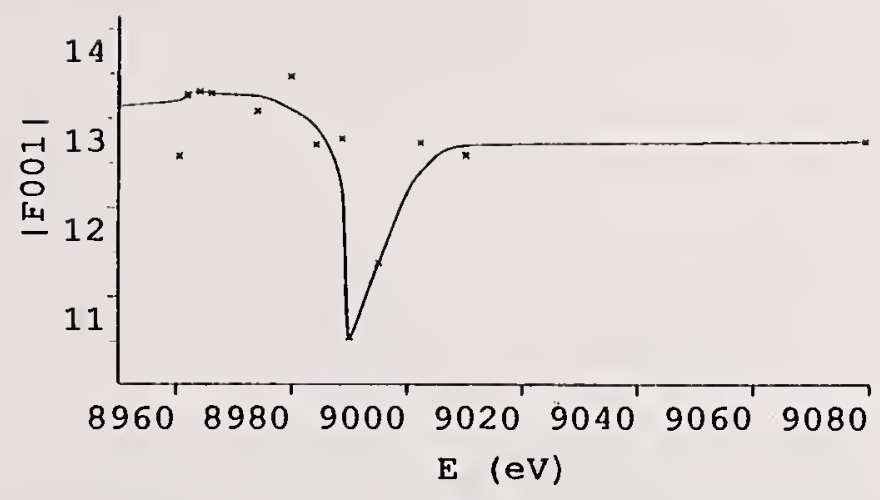

b)

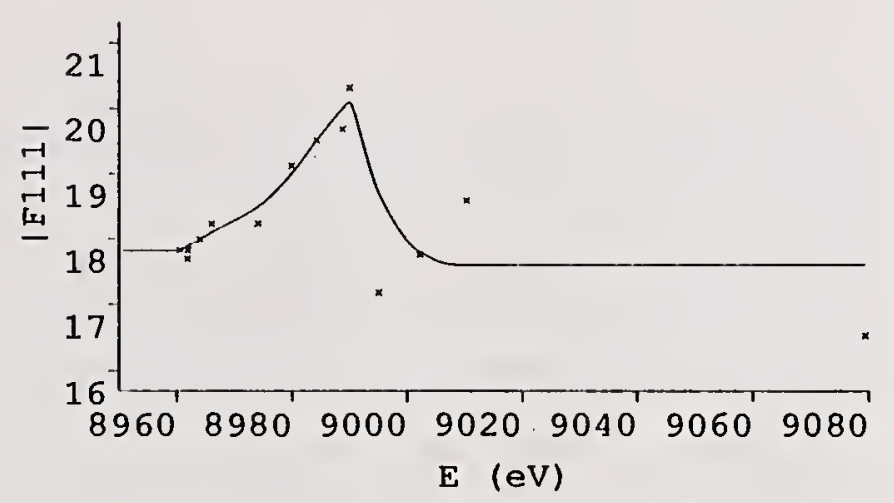

Figure 5: The energy variation of $|\mathrm{F}(001)|$ and $|\mathrm{F}(111)|$ for $\mathrm{YBa}_{2} \mathrm{Cu}_{3} \mathrm{O}_{6.27}$ around the $\mathrm{Cu} \mathrm{K}$ edge from Attfield (1991). The smooth curves are a fit to the data by eye.

$\left(\sim 10^{-15}-10^{-16} \mathrm{~s}\right)$ then superlattice peaks should be observable at resonant wavelengths. A long scan $7 \mathrm{eV}$ below the Ce $\mathrm{L}_{\mathrm{III}}$ edge, revealed a weak peak at the position expected for the otherwise forbidden 100 reflection, but this result awaits further confirmation.

\section{Conclusions}

Recent resonant X-ray diffraction studies have shown that large anomalous scattering effects may be observed close to absorption edges using high resolution powder synchrotron instruments. Accurate $\mathrm{f}^{\prime}$ values may be determined from pre-edge data, but powder refinements are less well suited for studying $f^{\prime}$ and $f^{\prime \prime}$ at absorbing energies within the edge. The large $f^{\prime}$ values obtained in the pre-edge region enable the resonant element to be distinguished from neighbouring elements, and such elemental contrast refinements give site occupancies of comparable precision to those typically obtained by powder neutron diffraction. 


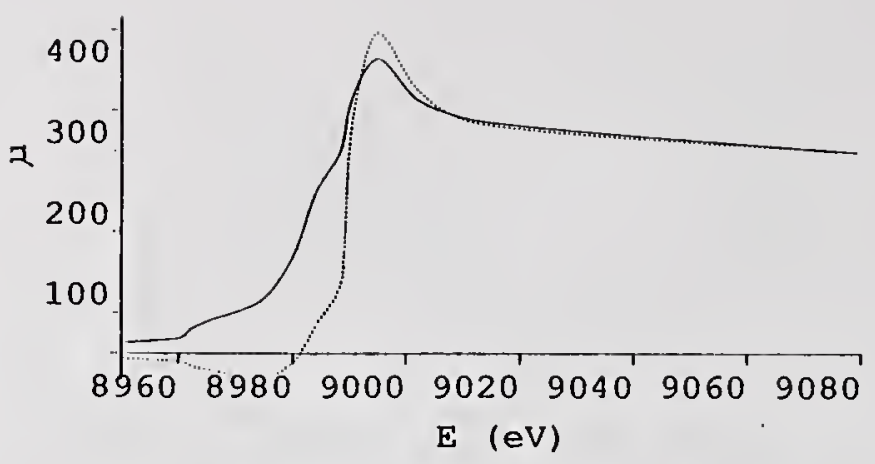

Figure 6: Site-resolved Cu $\mathrm{K}$ edge $\mathrm{X}$-ray absorption spectra for $\mathrm{YBa}_{2} \mathrm{Cu}_{3} \mathrm{O}_{6.27} ; \mathrm{Cu}(1)=$ solid line; $\mathrm{Cu}(2)$ $=$ dashed line (Attfield, 1991).

Electronic contrast experiments show that significant differences in the anomalous scattering from different oxidation states in the same crystal structure can be measured experimentally. In many cases, these differences can be related to valence effects in the X-ray absorption edge and suggest the possibility of deriving site-resolved X-ray absorption edge spectra.

These results establish resonant powder X-ray diffraction as a tool for studying elemental distributions and the valence states of atoms at different sites within crystalline materials. Further improvements, particularly in electronic contrast experiments, will result from the future use of very intense synchrotron sources.

\section{References}

Alp, E. E., Goodman, G. L., Soderholm, L., Mini, S. M., Ramanathan, M., Shenoy, G. K. \& Bommannavar, A. S. (1989). J. Phys.: Condens. Matter 1, 6463-6468.

ATTFIELD, J. P. (1990). Nature (London) 343, 46-49.

AttField, J. P. (1991). J. Phys. Chem. Solids 53, 1243-1249.

Bambynek, W., Craseman, B., Fink, R. W., FreUND, H. -U., Mark, H., Swift, C. D., Price, R. E. \& RAO, P. V. (1972). Rev. Mod. Phys. 44, 716-813.

Begum, R., Hart, M., Lea, K. R. \& Siddons, D. P. (1986). Acta Cryst. A42, 456-464.

Bonse, U. \& Hartmann-Lotsch, I. (1984). Nucl. Instrum. Methods 222, 185-188.

Brown, M., Peierls R.E. \& Stern, E.A. (1977).

Phys. Rev. B 15, 738-744.

Creagh, D. C. (1988). Austr. J. Phys. 41, 487-501.

Cromer, D. T. (1983). J. Appl. Cryst. 16, 437.

Cromer, D. T. \& Liberman, D. (1970). J. Chem. Phys. 53, 1891-1898.

Cromer, D. T. \& Liberman, D. (1981). Acta Cryst. A37, 267-268.
Dreier, P., Rabe, P., Malzfeldt, W. \& NeimanN, W. (1984). J. Phys. C 17, 3123-3136.

FlaCK, H. D. (1983). Acta Cryst. A39, 876-881.

FreUND, A. K. (1987). $X$-ray Optics ESRF internal report.

Hamalainen, K., Manninen, S., Suortti, P., Collins, S. P., CoOPER, M. J. \& LAUNDY, D. (1989). J. Phys. Condens. Mat. 1, 5955-5964.

Howland, R. S., Geballe, T. H., Laderman, S. S., Fischer-Colbrie, A., Scott, M., TARascon, J. M. \& Barboux, P. (1989). Phys. Rev. B 39, 9017-9027.

Hoyt, J. J., De Fontaine, D. \& Warburton, W. K. (1984). J. Appl. Cryst. 17, 344-351.

Karle, J. (1989). Physics Today 42, No. 6, 22-29.

KIRFEL, A., EICHHORN K. \& WROBLEWSKI, T. (1988). HASYLAB Jahresbericht.

Kwei, G. H., Von Dreele, R. B., Cheong, S-. W., FISK, Z. \& ThOMPSON, J. D. (1990). Phys. Rev. B 41, 1889-1893.

Kwei, G. H., Von Dreele, R. B., Williams, A., Goldstone, J. A., LaWson II, A. C. \& WarburTON, W. K. (1990). J. Mol. Structure 223, 383-406.

Moroney, L. M., Thompson, P. \& Cox, D. E. (1988). J. Appl. Cryst. 21, 206-208.

Perkins, D. A. \& AttField, J. P. (1991). J. Chem. Soc., Chem. Comm. 229-231.

RIETVELd, H. M. (1969). J. Appl. Cryst. 2, 65-71.

Suortti, P., Hastings, J. B. \& Cox, D. E. (1985). Acta Cryst. A41, 413-420.

Templeton, D. H. \& Templeton, L. K. (1982). Acta Cryst. A38, 62-67.

Templeton, D. H. \& Templeton, L. K. (1985). Acta Cryst. A41, 133-142.

Templeton, D. H., Templeton, L. K., Phillips, J. C. \& Hodgson, K. O. (1980). Acta Cryst. A36, 436442.

Templeton, L. K. \& Templeton, D. H. (1988). J. Appl. Cryst. 21, 558-561.

Templeton, L. K. \& Templeton, D. H. (1990). J. Appl. Cryst. 23, 18-20.

Templeton, L. K., Templeton, D. H., PhizackerLEY, R.P. \& Hodgson, K.O. (1982). Acta Cryst. A38, 74-78.

Warner, J. K., Wilkinson, A. P., Cheetham, A. K. \& Cox, D. E. (1991). J. Phys. Chem. Solids B 53, 1251-1256.

Wilkinson, A. P., Cheetham, A. K. \& Cox, D. E. (1991). Acta Cryst. B47, 155-161.

Wilkinson, A. P., Cox, D. E. \& Cheetham, A. K. (1991). J. Phys. Chem. Solids B 53, 1257-1266.

WILl, G., Masciocchi, N., Hart, M. \& Parrish, W. (1987). Acta Cryst. A43, 677-683.

Williams, A., Kwei, G.H., Ortiz, A. T., Karnowski, M. \& Warburton, W. K. (1990). J. Mater. Res. 5, 1197-1200. 
National Institute of Standards and Technology Special Publication 846. Proceedings of the international conference Accuracy in Powder Diffraction II, held at NIST, Gaithersburg, MD, May 26-29, 1992. (Issued October 1992)

\title{
Instrumentation for Studies at the Powder/Single-crystal Boundary
}

\author{
L. W. FINGER
}

\author{
Geophysical Laboratory and Center for High Pressure Research, \\ Carnegie Institution of Washington, Washington DC 20015-1305, U. S. A.
}

\begin{abstract}
Crystal structures have been refined for single crystals that have volumes on the order of $1 \mu \mathrm{m}^{3}$ and unknown structures have been solved for crystals with volumes of the order of $10^{3} \mu \mathrm{m}^{3}$. Study of microcrystals gives rise to a number of special problems, such as maximization of signal to noise by reduction of background, development of an incident beam that is homogeneous on the scale of $\mu \mathrm{m}$, and reduction of data collection time by use of parallel recording techniques. Solutions to these problems have been attempted at a number of beam lines at various synchrotrons, using both monochromatic and polychromatic sources. On the latter type of beam, the flux tends to be more homogeneous; however, the analysis is more complicated. For monochromatic radiation, the experiment is similar to that performed in the laboratory, but requires considerable beam time for the data collection. With current sources, the scattering power required for structural refinement has been reduced by approximately six orders of magnitude relative to the laboratory. The next generation of machines should be bright enough to reduce the scattering power by another three orders of magnitude, if means are found to handle the crystals.
\end{abstract}

\section{Introduction}

It is now possible to obtain accurate intensities from crystals that are the size of an individual grain in a powdered sample. This method is the only single-crystal technique available for materials whose single crystals are smaller than the $50-100 \mu \mathrm{m}$ size needed for conventional diffractometry. Microcrystal diffraction studies that have used monochromatic synchrotron beams are typified by Eisenberger et al. (1984), Bachmann et al. (1985), Rieck et al. (1988), and King et al. (1991a,b). Despite significant advances in deconvolution of a complex powder pattern, and solution of the crystal structure with standard structure solution techniques such as direct methods (McCusker, 1988), or by the Patterson method (Attfield et al., 1988), there are still structures for which single-crystal intensities are needed. Small crystals are also needed to obtain accurate intensity data that are as free as possible from the effects of extinction and absorption. Such data are needed if accurate electron densities are to be obtained.

Ability to study small volumes has also been important in the field of high-pressure investigations, where the maximum pressure attainable is, in part, determined by the area ratio between the external load device and the sample chamber. Because the thickness of the sample is also constrained, sample chamber volumes on the order of $1000 \mu \mathrm{m}^{3}$ are common. Although the bulk of high-pressure, X-ray studies have been performed with powdered samples, a number of recent investigations have involved single crystals (Mao et al., 1988; Hemley et al., 1990; LeToullec et al., 1992a,b).

As the crystal being studied in a single-crystal experiment approaches a size appropriate for an individual grain in a powdered sample, i.e. on the order of $1 \mu \mathrm{m}$, a number of experimental requirements become quite severe. For example, the process of selecting and mounting a microcrystal is not trivial - a micromanipulator is essential. In addition, the amount of extraneous material such as fiber and glue in the beam must be carefully controlled; otherwise the amorphous scattering will swamp the Bragg scattering from the crystal. To control background from air scattering, the beam size must be greatly reduced. If possible, beam paths should be filled with $\mathrm{He}$ or evacuated. The crystal must be centered extremely carefully; otherwise it may precess out of the beam as the diffractometer axes are moved. Harder to control, the so-called "sphere of confusion", which is the volume of space swept out by a perfectly-centered, point sample must also be very small. One is also confronted with the "normal" problems of synchrotron 
experimentation, i.e. an unsteady source intensity and too little beam time available for the experiment.

This contribution reviews the progress made in the study of microcrystals and presents some of the details of apparatus under development at present sources and planned for the next generation of synchrotrons. Particular attention will be directed toward the effectiveness of the design in solving the problems outlined above.

\section{Effective crystal size}

In any discussion of what constitutes a "small" crystal, the fact that materials differ in their ability to scatter X-rays, primarily due to effects such as atomic number and density, must be taken into account. For a single crystal, monochromatic experiment, Warren $(1969$, p. 46) shows that the total diffracted energy for a reflection $h k l$ is proportional to $\left(F_{h k l} / V_{U}\right)^{2} V_{C} \lambda^{3}$, where $F_{h k l}$ is the structure factor for $h k l, V_{U}$ is the unit-cell volume, $V_{C}$ is the volume of the crystal, and $\lambda$ is the wavelength of the radiation. Lorentz-polarization factors and absorption are ignored. The maximum for this power would be obtained at a scattering angle of zero, where all atoms would scatter in phase and thermal effects would be negated. We can, therefore, use $F_{000}$ instead to yield the scattering power, $S$, defined as

$$
S=\left(\frac{F_{000}}{V_{U}}\right)^{2} V_{C} \lambda^{3}
$$

which is a dimensionless measure of how well a crystal scatters radiation. For experiments that use polychromatic radiation, the equation needs to be modified; however, it still serves as an effective metric for the scattering expected for a given crystal. Note that all factors, except $V_{C}$, are fixed by the crystallography of the material.

With a conventional source, $S$ must be no smaller than $10^{16}-10^{17}$ for a successful diffraction experiment to be performed. In a study of solid hydrogen at $5.4 \mathrm{GPa}$ pressure and room temperature by Hazen et al. $(1987)\left(S=7 \times 10^{15}\right.$, Table 1$)$, the intensity of the strongest diffraction maximum was only 68 counts per second for an Mo, fine-focus, X-ray tube operated at a power of $1.4 \mathrm{~kW}$. Signal to noise was less than 7 . Although the intensity data were sufficient to demonstrate that $\mathrm{H}_{2}$ has a hexagonal, not cubic, structure under these conditions, it is clear that the experiment would be impossible if the scattering power were reduced by another order of magnitude. At $6 \mathrm{GPa}$, the crystal either fragmented or became broadened
Table 1: Crystal Volume and Scattering Power.

\begin{tabular}{cccc}
\hline \hline Year & $\begin{array}{c}\text { Volume, } \\
\left(\mu \mathrm{m}^{3}\right)\end{array}$ & $\mathrm{S}$ & Ref \\
& & & \\
1987 & $9 \times 10^{5}$ & $7 \times 10^{15}$ & $(1)$ \\
1984 & 800 & $3 \times 10^{14}$ & $(2)$ \\
1985 & 200 & $1.3 \times 10^{14}$ & $(3)$ \\
1988 & 2.2 & $7.1 \times 10^{12}$ & $(4)$ \\
1991 & 0.02 & $?$ & $(5)$ \\
1991 & 0.03 & $1.8 \times 10^{10}$ & $(6)$
\end{tabular}

(1) Hazen et al. (1987) $-\mathrm{H}_{2}-$ Sealed Source.

(2) Eisenberger et al. (1984) $-\mathrm{Na}_{6} \mathrm{Al}_{6} \mathrm{Si}_{6} \mathrm{O}_{24}$ - Monochromatic Synchrotron

(3) Bachmann et al. (1985) $-\mathrm{CaF}_{2}$ - Monochromatic Synchrotron

(4) Rieck et al. (1988) $-\mathrm{CaF}_{2}$ - Monochromatic Synchrotron

(5) Ohsumi et al. (1991) - Mo - Polychromatic Synchrotron

(6) Skelton et al. (1991) - Bi - Polychromatic Synchrotron

by strain, and the diffraction could no longer be detected with the laboratory source. Note that the integrated intensity is proportional to $S$; however, the detectibility of a given $h k l$ is more dependent upon the peak intensity, which is inversely proportional to the peak width. The optimum peak to background is obtained if the beam divergence matches the mosaic spread of the crystal (Rieck \& Schulz, 1991); however, most real crystals have a much broader spread than synchrotron beams.

With synchrotron sources, the increases in both total flux and, more importantly, brightness, have resulted in a decrease in the minimum size of crystals and scattering power for crystals that can be studied. Table 1 lists some of the notable experiments and the date published.

\section{Monochromatic synchrotron sources}

As noted in Table 1 (references $2-5$ ), there has been a considerable decrease in crystal volume and $S$ for studies using monochromatic synchrotron radiation. In the first of these investigations, Eisenberger 


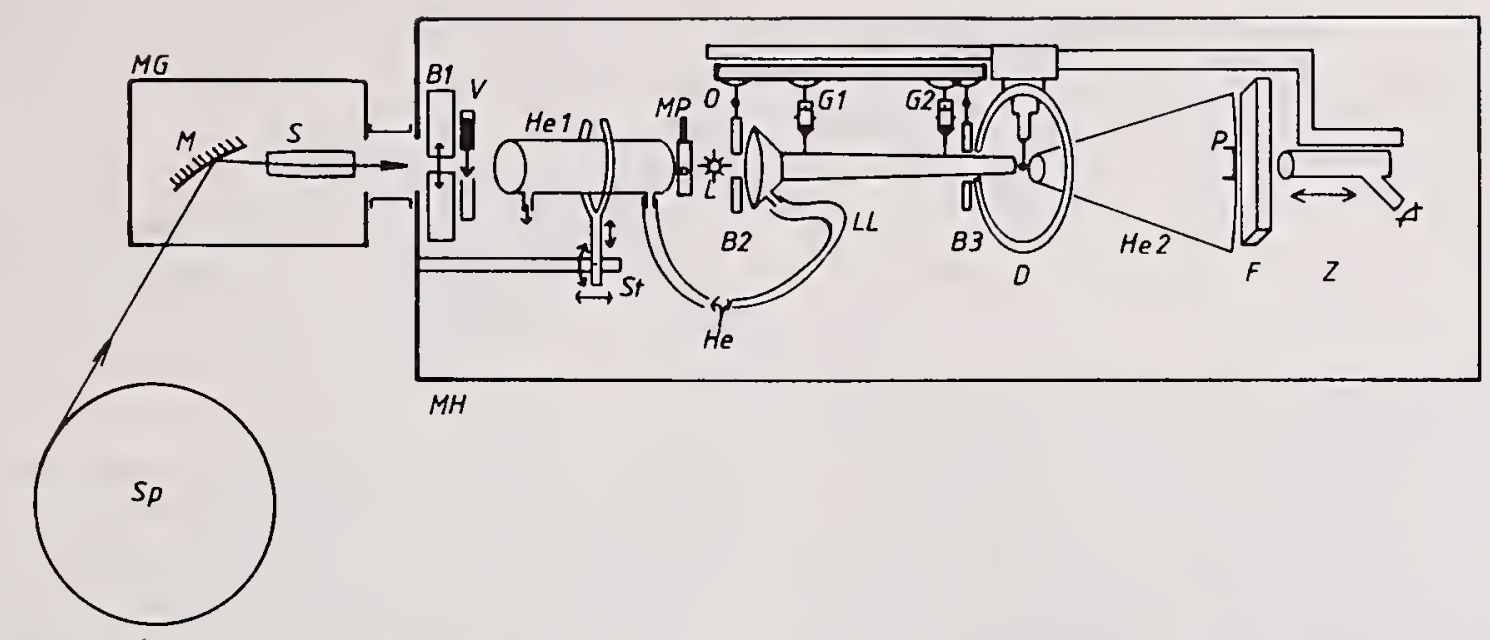

Figure 1: A possible experimental station for microcrystal examinations: Sp: storage ring; MG: monochromator housing; M: curved monochromator; S: focusing mirror; MH: experimental hutch; B1, B2, B3: pinholes or diaphragms; V: beam shutter; He1, He2: helium or vacuum paths; MP: beam monitor; He: helium or vacuum source; St: stage; LL: collimator or X-ray guide tube; G1, G2: collimator positioning goniometers; O: optical bench; D: diffractometer; P: beamstop; F: area detector; Z: telescope. Adapted from Rieck \& Schulz (1991).

et al. (1984) did not measure a complete set of intensities for structural analysis, but used two $h k l$ 's at two wavelengths each to estimate the minimum size of crystals that could be studied. Their prediction that "complete data sets could be measured from crystals having volumes less that $1 \mu \mathrm{m}^{3 "}$ has already been realized. In a review of the literature for X-ray studies of microcrystals using monochromatic synchrotron sources, Rieck \& Schulz (1991) present a diagram of a diffractometer suitable for microcrystal studies, reproduced here as Figure 1 . Such an instrument would provide the high flux and brightness required for examination of small crystals, particularly if mounted on a wiggler or undulator beam line. In addition, the use of small pinholes and helium or vacuum beam paths will reduce background due to general scatter; thereby increasing signal to noise. The use of an area detector will also facilitate rapid data collection, which is necessary given the difficulty of obtaining long periods of time on such beam lines. In addition, this detector will also help in the determination of crystal orientation. If the unit-cell constants are unknown, this step can be rather time consuming. Note that for the smallest crystals, it is not possible, in general, to perform alignment in an off-line experiment.

Variations in the flux density of the beam on a $\mu \mathrm{m}$ scale is a matter of concern. Although the beam incident upon the monochromator is usually very ho- mogeneous, the scale of imperfections in the bent crystal and in the mirror make it difficult to ensure that the flux density incident upon the sample is not changing as it moves within the sphere of confusion of the diffractometer. King et al. (1991a,b) have used slits several meters apart to define an X-ray beam with a narrow angle of acceptance, which reduces fluctuations arising from small changes in the electron orbit. The resulting beam is relatively uniform over a $250 \mu \mathrm{m}$ diameter. Variations in the total flux, after beam monitor corrections, are on the order of $5 \%$ throughout the ring current decay and refill cycle (King, personal communication 1992). This variation is definitely not random, but has a minimum just after the fill, followed by a steady increase as the beam decays. A maximum is reached in midcycle, followed by a slight decay near the end of the fill. Such behavior is tentatively ascribed to thermal variations in the mirror between the ring and the monochromator. As shown by King et al. (1991a,b), such a system may be used to measure accurate intensities for crystals with dimensions on the order of $10 \mu \mathrm{m}$.

The data of Rieck et al. (1988), as presented by Rieck \& Schulz (1991) are quite different. For a wide variety of refinements, the temperature factors for the $\mathrm{Ca}$ and $\mathrm{F}$ ions in the $\mathrm{CaF}_{2}$ structure are nearly identical with the results of lattice dynamics calculations (Elcombe \& Pryor, 1970). For the $2.2 \mu \mathrm{m}^{3}$ crys- 


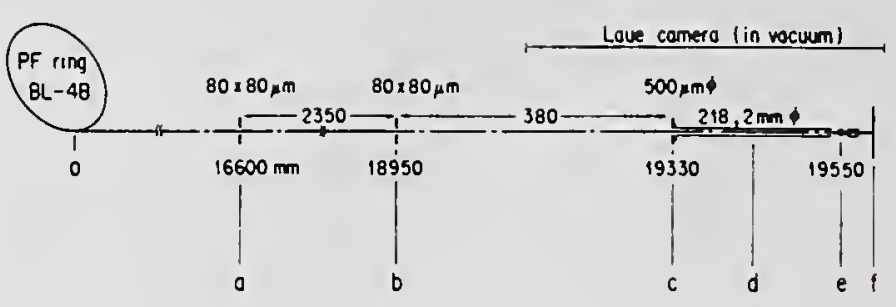

Figure 2: The optical system employed in the Laue diffractometer at Photon Factory. Letters $a, b, c$ indicate the first and second slit, and a pinhole, respectively. A beam tube is indicated by $d$, and letters $e$ and $f$ indicate the sample and image plate detector. The numbers below the horizontal line are the distances from the source. Adapted from Ohsumi (1992).

tal, however, the factors are suddenly much larger, indicating some uncorrected systematic error, which may be caused by crystal imperfections noted below. It is also possible that microvariations in the flux density of the beam are the cause of the systematic error. King (personal communication, 1992) suggests that the present generation of high-brightness synchrotrons may be more susceptible to this problem than the first generation of machines, because their electron bunch size is smaller making it easier to detect the effects of variations in the electron orbit.

\section{Polychromatic sources}

Because it is impossible to ensure that any beam arising from a monochromator and/or focusing mirror is homogeneous on a very small scale, other investigators have chosen an alternative approach that eliminates all active optical elements. This is accomplished by using the "white" radiation from either a bending magnet or a wiggler to illuminate the sample directly. The only elements between the storage ring and the sample are filters, windows, and pinholes; none of which should introduce appreciable beam inhomogeneity. Ohsumi et al. (1990) describe a Laue diffractometer that uses an image plate as a detector (Fig. 2). This instrument has exceedingly low background as the slit system has been designed to eliminate the scattered radiation arising from the interaction of the polychromatic beam with the beam defining aperatures. This diffractometer has been used to study spheres of Mo, $0.8 \mu \mathrm{m}$ in diameter. One such sphere was found to be twinned and the

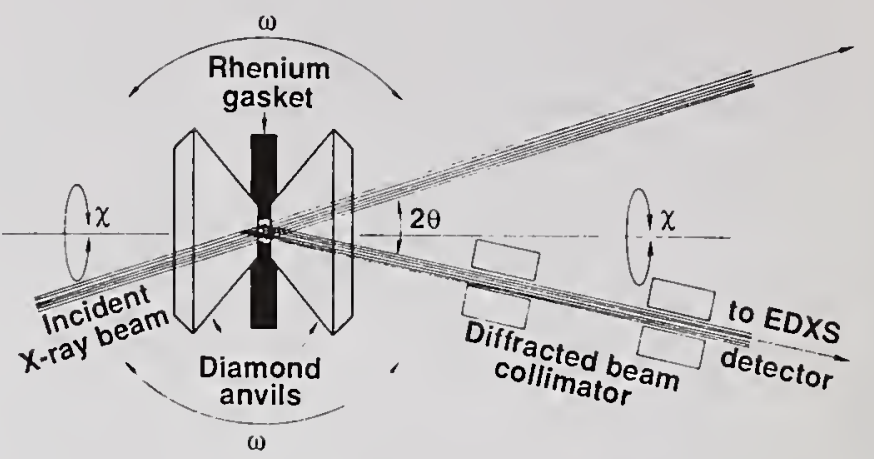

Figure 3: Schematic diagram of Laue two-circle, Xray diffractometer used for single-crystal diffraction studies of crystals with small values of scattering power. The motions on $\chi$ and $\omega$ are used to position a crystal into diffracting condition. Approximately $30 \%$ of the allowable diffraction data is accessible. For samples that are not contained within a diamond anvil cell, a modified $\phi$-axis, which allows complete access to reciprocal space is added. Figure adapted from Mao et al. (1988)

volume fraction of the twin individuals were varied by least-squares analysis. The volume of the lesser part refined to a value of $0.02 \mu \mathrm{m}^{3}$, too small to be seen in an optical microscope. Despite this small volume, they were able to refine the temperature factor of Mo to a value of $0.08(6) \AA^{2}$, a value compatible with that obtained with powder diffraction on the same material.

For a Laue diffractometer that does not have any means of energy resolution, it can be difficult to index the pattern. In addition, only axial ratios, not absolute axial lengths, may be determined. For this reason, Ohsumi et al. (1992) have added a solid-state detector. An additional benefit is that the X-ray fluorescence effect may be used to measure the heavy element composition of the sample.

A different type of single-crystal diffractometer (Figure 3) for a polychromatic incident beam has been developed at NSLS, initially on beamline X7a, and more recently, on beamline $\mathrm{X} 17 \mathrm{C}$, which has a superconducting wiggler source. This instrument, initially used to study the crystallized form of normally gaseous materials such as hydrogen, deuterium, etc. (Mao et al., 1988; Hemley et al., 1990; LeToullec et al., 1992a, 1992b; Vos et al., 1992), has been extended to study the crystallography of a series of Taylor wires (Taylor, 1924), which consist of small metallic filaments with submicrometer diameters contained within glass capillaries (Skelton et al., 1991). Such samples are particularly difficult because 
the glass container has a diameter of approximately $10 \mu \mathrm{m}$, i.e. a volume that is 2 to 3 orders of magnitude larger than the crystal. The background level from this amorphous material is very large, however, this instrument has been able to detect diffraction from a Bi wire only $670 \AA$ in diameter, and to determine the lattice constants of a previously unknown form of Bi (Finger et al., in preparation). A severe difficulty of this instrument is that very large amounts of time are required to locate the diffraction maxima needed to determine the orientation and lattice of the crystal, particularly, when the scattering power of the sample is very small. To overcome this problem, an imaging plate is being added, which will be used to locate the position of diffraction maxima, and to calculate changes in angles $\omega$ and $\chi$ necessary to bring the diffraction into the detector. Additional efforts are still needed to reduce background and improve signal to noise.

D. Häusermann (personal communication, 1992) has begun work on a Laue diffractometer of a new design for ESRF. This instrument uses a solid-state detector with a coaxial design in which the active crystal has a hollow center. This design permits the use of an active region large enough for a scattering angle of roughly $7^{\circ}$ while still maintaining acceptible energy resolution. The collimator assembly consists of inner and outer tungsten carbide cones separacted by a few $\mu \mathrm{m}$. For powdered samples, the entire Debye-Scherrer ring is collected at once, greatly increasing the data collection rate and reducing some of the effects of preferred orientation. With single crystals, the search process is reduced to a single scan on $\omega$. If an additional thin slit is available to mask all but a narrow region of the cone, single crystal reflections can be located and indexed.

\section{Crystal perfection}

Although it might seem likely that small crystals are more likely to be free of many effects such as twinning and low-angle grain boundaries, the limited experience suggests that microcrystals are no better than larger ones. For example, Ohsumi et al. (1990) found that one of their two Mo spheres was twinned. Similarly, the $2.2 \mu \mathrm{m}^{3}$ crystal of $\mathrm{CaF}_{2}$ studied by Rieck et al. (1988) showed the presence of extra crystallites is some scans (Fig. 4).

\section{Future sources}

Although the developments in microcrystal diffraction have been spectacular on first-generation syn-

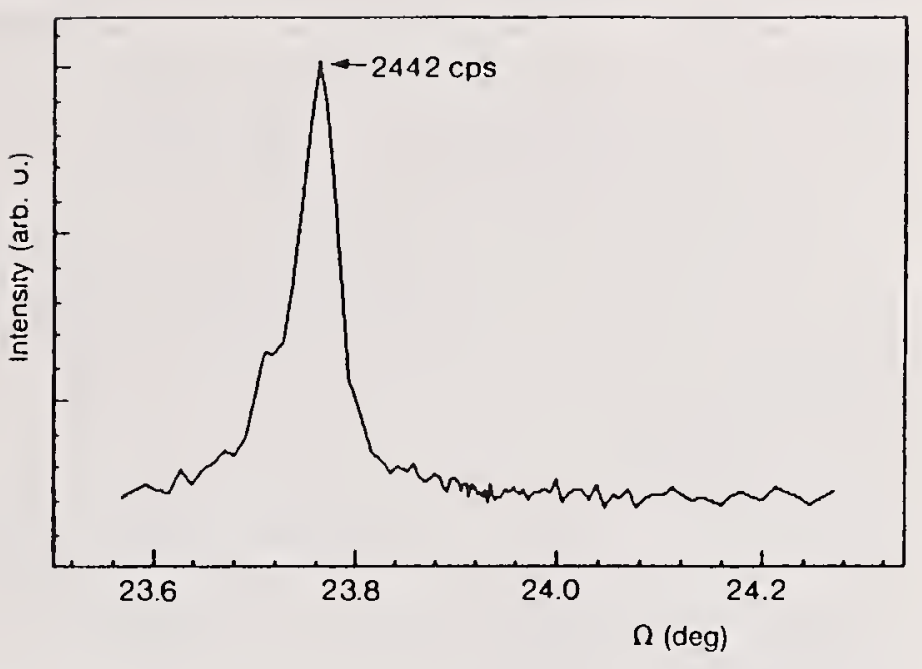

Figure 4: Omega scan (rocking curve) of the 202 reflection of a $\mathrm{CaF}_{2}$ crystal with volume of $2.2 \mu \mathrm{m}^{3}$. Note the left-side shoulder indicating a second crystallite. Figure from Rieck \& Schulz (1991).

chrotrons, and significant results are now obtained on the second-generation machines, one can expect even more advances with the increased brightness expected for the third-generation machines now being built at Grenoble (ESRF - $6 \mathrm{GeV}$ ), Argonne National Laboratory (APS - $7 \mathrm{GeV}$ ), and Japan (Spring8 $8 \mathrm{GeV})$. These machines are optimized for insertion devices such as wigglers, which produce a polychromatic beam, and undulators, which have peaks in the energy spectrum and are used with monochromators. Once the problems of increased heating of optical components have been solved so that a uniform flux is achieved at the sample position, it should be possible to reduce the scattering power by another three orders of magnitude. At this point, linear dimensions of the crystals will be approaching the limit at which Scherrer broadening (Warren, 1969, p. 253) will make it no longer possible to detect the diffracted intensity. At this size, it will be impossible to resolve the crystals with an optical microscope; therefore, it will be necessary to mount the crystals in a scanning electron microscope.

\section{Conclusions}

As shown by Bachmann et al. (1985) and King et al. $(1991 \mathrm{a}, \mathrm{b})$, it is possible to measure accurate intensity information for crystals that have scattering powers as large as $10^{14}$ on monochromatic beamlines at both first- and second-generation synchrotrons. As the scattering power of the crystal is reduced into 
the $10^{12}$ range, diffracted intensity can be observed; however crystal structure refinements on such small crystals have not yielded satisfactory results to date. One possibility is that the incident beam may not be sufficiently homogeneous at the $1 \mu \mathrm{m}$ scale.

Although correction of measured intensities for instrumental effects are not trivial for Laue geometry, it has been shown that such calculations are sufficiently accurate to yield meaningful structural parameters, even for crystals with scattering powers in the $10^{12}$ range. In addition, the simple X-ray optics should lead to a more homogeneous beam, even at the submicron scale. Although either a solidstate detector, or an image plate would be sufficient to measure complete intensities at a polychromatic beamline, there are considerable advantages in speed and flexibility if both are available.

\section{References}

Attfield, J. P., Cheetham, A. K., Cox, D. E. \& Sleight, A. W. (1988). J. Applied Crystallography, 21, 452-457.

BachmanN, R., Kohler, H., Schulz, H. \& Weber, H-P. (1985). Acta Crystallographica, A41, 35-40.

Eisenberger, P., Newsam, J. M., Leonowicz, M. E. \& Vaughan, D. E. W. (1984). Nature, 309, 45-47.

Hazen, R. M., MaO, H. K., Finger, L. W. \& Hemley, R. J. (1987). Physical Review B, 36, 3944-3947.

Hemley, R. J., MaO, H. K., Finger, L. W., JephCOAT, A. P., Hazen, R. M. \& Zha, C. S. (1990). Physical Review B, 6458-6470.
King, H. E. Jr., Mundi, L. A., Strohmaier, K. G. \& Haushalter, R. C. (1991a). J. Solid State Chem., 92, 1-7.

King, H. E. JR., Mundi, L. A., Strohmaier, K. G. \& Haushalter, R. C. (1991b). J. Solid State Chem., 92, 154-158.

LeToullec. R., Loubeyre, P., Pinceaux, J. P., Mao, H. K. \& Hu, J. (1992a). High Pressure Research, 6, 379-388.

LeToullec. R., Loubeyre, P., Pinceaux, J. P., Mao, H. K. \& Hu, J. (1992b). High Pressure Research, 8, 691-696.

Mao, H. K., Jephcoat, A. P., Hemley, R. J., Finger, L. W., Zha, C. S., HAZEN, R. M. \& Cox, D. E. (1988). Science, 239, 1131-1134.

MCCUSKer, L. B. (1988). J. Applied Crystallography, 21, 305-310.

Ohsumi, K., Hagiya, K. \& Ohmasa, M. (1990). Acta Crystallographica, A46, C-26.

RIECK, W., Euler, H., Schulz, H. \& SchlldCAMP, W. (1988). Acta Crystallographica, A44, 1099-1101.

RECK, W. \& Schulz, H. (1991). Handbook on Synchrotron Radiation, Vol. 3, G. Brown, D.E. Moncton, Eds., 267-290.

Skelton, E. F., Ayers, J. D., QAdri, S. B., Moulton, N. E., Cooper, K. P., Finger, L. W., MaO, H. K. \& Hu, Z. (1991). Science, 253, 1123-1125.

TAYLOR, G. F. (1924). Physical Review, 23, 655-660.

Vos, W., Finger, L. W., Hemley, R. J., Hu, J. Z., MaO, H. K. \& Schouten, J. A. (1992). Nature, in press.

WARREN, B. E. (1969). X-ray Diffraction. Reading, Mass: Addison-Wesley. 
National Institute of Standards and Technology Special Publication 846. Proceedings of the international conference Accuracy in Powder Diffraction II, held at NIST, Gaithersburg, MD, May 26-29, 1992. (Issued October 1992)

\title{
Time-resolved X-ray Diffraction Studies of Crystallization in Metallic Glasses
}

\author{
M. Sutton, S. Brauer, Y. S. Yang, H. E. Fischer and J. O. Ström-Olsen \\ Centre for the Physics of Materials and the Department of Physics, McGill University, \\ 3600 University Street, Montréal, Québec, Canada H3A 2 T8
}

\section{G. B. STEPHENSON}

\author{
IBM Research Division, Thomas J. Watson Center, \\ Yorktown Heights, NY, U. S. A. 10598
}

\begin{abstract}
The IBM/MIT beamline X-20C at the National Synchrotron Light Source has been optimized for time-resolved scattering measurements. Using a multilayer monochromator and a fast linear positionsensitive detector system, scattering patterns from a transforming material can be acquired with $3 \mathrm{~ms}$ time-resolution. In conjunction with a fast pyrometric temperature controller which has been developed to change and regulate the sample temperature with microsecond response, we have been studying the kinetics of crystallization of metallic glasses on time-scales varying from minutes to milliseconds. In a typical measurement, two synchronized positionsensitive detectors provide complementary in situ information about the transformation. The first is used for large-angle scattering and the second detector measures small-angle scattering. At relatively slow transformation rates, quantitative measurements of crystal volume fractions as small as $10^{-4}$ are possible. In many systems, as the transformation rate is increased, the crystallization mechanism changes such that new metastable crystal phases are formed en route to the equilibrium structure.
\end{abstract}

\section{Introduction}

X-ray diffraction has played a key role in our understanding of the structure of materials. However, one would often like to have structural information for phenomena in systems whose structures evolve with time. To acquire this information, the time required to measure a diffraction pattern must be of suitable duration.

One way to study these systems is to compare standard X-ray measurements made before and after any changes. Also, it is often possible to arrest the development in mid-process and thus obtain information on intermediate structures. Classic examples of such ex situ measurements are diffraction measurements made after heat treatments in which the samples have been annealed at high temperatures for varying amounts of time. For fast processes, such studies are often not possible or are fraught with systematic errors in which the structure has evolved in unexpected ways during the processing used to arrest the time evolution. In spite of these limitations this technique has been and is still very useful. Provided that the processing is fast enough, this method allows for measurements on time scales shorter than the time to measure a diffraction pattern.

Another possible technique relies on the ability to cycle a system repeatedly through its time evolution sequence. This allows measurements to average over many cycles and build up a diffraction pattern slowly over time. This powerful technique is not always applicable, since not all processing can be cycled or cycled reproducibly enough. When applicable, this technique will also give time resolutions which are not limited by the time to accumulate a complete diffraction pattern.

The method we will emphasize in this article is to make in situ measurements by recording a sequence of diffraction patterns in real time. This often sets the most severe experimental requirements, but it has the advantage that the analysis of the data is more straight forward and systematic errors are greatly reduced. The time resolution obtainable in this way directly depends on the time to accumulate each diffraction pattern. With conventional X-ray sources, as found in most laboratories, the time to obtain a diffraction pattern ranges from minutes to days. With the advent of the new synchrotron X-ray sources, this time can be reduced to milliseconds. 


\section{Metallic glasses}

In this article, we will use crystallization studies of metallic glasses as examples in which the time evolution of structure is important. Metallic glasses are formed by cooling molten metal alloys rapidly enough to inhibit the formation of any crystalline order. The absence of lattice periodicity leads to several characteristics which make glasses interesting both from the point of view of our fundamental understanding of materials and for materials applications (Luborsky, 1983). In the simplist picture, metallic glasses are in a thermally arrested metastable state. When heated, a rich assortment of non-equilibrium phenomena occur as they transform into their equilibrium phases by nucleation and growth.

A single transformation of an amorphous material into a crystalline phase can be classified into three types depending upon the phases involved in the transformation. The least complicated transformation is called a polymorphic crystallization. This involves the transformation of a metallic glass into a crystal phase with the same composition and does not require the diffusion of material over large distances. A more complicated type, called primary crystallization, occurs when the composition of the crystal phase is different from the starting composition. As primary crystallization proceeds, the composition of the remaining amorphous material changes with time and it eventually crystallizes into another phase or phases with different compositions. This type of crystallization is thus inherently a multistep process. The third type is called eutectic crystallization and involves the simultaneous formation of two crystal phases with the correct compositions so as to leave the remaining amorphous material at its original composition. Such a transformation involves diffusion on length scales comparable to the separation of the two crystal phases and often results in a lamellar microstructure.

For a particular alloy and composition, crystallization may not proceed directly to the equilibrium structure on the phase diagram and may involve many competing transformations, metastable phases and the formation of phases which must relax to reach equilibrium. In situ time-resolved diffraction provides an ideal tool for investigating these irreversible processes. It allows one to monitor the appearance and disappearance of phases to give a qualitative understanding of the transformation processes. Furthermore, it is possible to measure the amounts and morphologies of the various phases. This allows quantitative comparison to theoretical models of the kinetics. In this paper we will give several examples to demonstrate the different types of information that can be obtained.

\section{Experimental setup}

The IBM/MIT beamline X-20C at the National Synchrotron Light Source (NSLS) has been optimized for time-resolved scattering measurements. The details of the experimental configuration is discussed elsewhere(Stephenson, 1988, Stephenson et al., 1989 and Brauer et al., 1992). This beam line is equipped with a $\mathrm{Pt}$ coated silicon 1:1 focusing mirror, which collects up to $4 \mathrm{mrad}$ of bending-magnet synchrotron radiation and focuses it into a $1 \mathrm{~mm} \times 1 \mathrm{~mm}$ spot $22 \mathrm{~m}$ from the source. The X-ray wavelength is selected by a double crystal monochromator, typically in the range 1.5 to $2.0 \AA$. The beam line vacuum is separated from ring vacuum by a $B$ window at one end and the $\mathrm{X}$-rays exit the beam pipe from another $\mathrm{Be}$ window near the sample position. Three aspects of this setup are emphasized here.

The first aspect is the use of a $1 \%$ bandpass monochromator based on synthetic multilayers of tungsten and silicon. This greatly increases the incident X-ray intensity although at some loss of diffraction resolution. The resulting increase in intensity for diffuse scattering is much greater than the corresponding increase for sharp Bragg peaks, and so this loss of diffraction resolution actually dramatically improves measurements of diffuse scattering.

The second aspect is the use of fast linear detector arrays to collect many diffraction angles in parallel at count rates approaching a million counts per second per angle. In our recent measurements we are using two such detectors to simultaneously collect small-angle and large-angle X-ray scattering. The two measurements give complementary information on crystal structure, the phases present and on the microstructure. With this setup, powder diffraction profiles covering ten to twenty degrees of $2 \theta$ can be collected with a time resolution of a few milliseconds.

The third aspect is the development of in situ techniques to rapidly measure and control the sample environment on a time scale fast enough to take advantage of the millisecond time resolution(Stephenson et al., 1989). Since one is comparing diffraction profiles taken under almost identical conditions, one of the primary advantages of in situ measurements is that quite small structural changes can be detected. Furthermore, one can observe and often understand the nature of the temporal evolution in the structure even though a complete structural determination is not possible.

Our metallic glasses are made by melt spinning. This produces a ribbon of material which is 10 to 30 $\mu \mathrm{m}$ thick and 1 to $2 \mathrm{~mm}$ wide. For our experiments a 


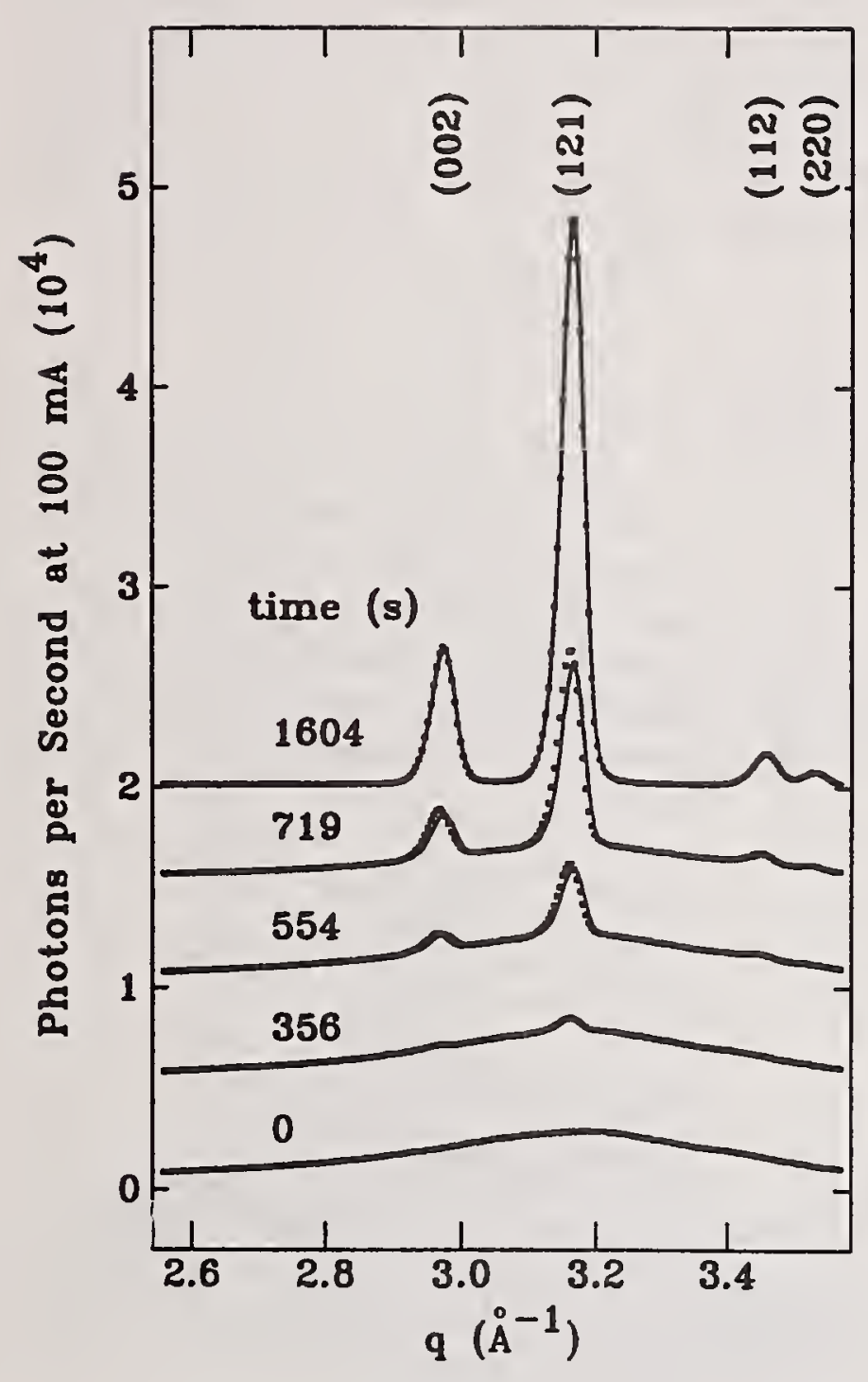

Figure 1: Time-resolved diffraction of $\mathrm{Co}_{2} \mathrm{~B}$ at $T_{a}=675 \mathrm{~K}$. Each successive pattern is offset by 5000 counts/second for clarity. The time per diffraction pattern is $6.6 \mathrm{~s}$. The crystal structure of $\mathrm{Co}_{2} \mathrm{~B}$ is $I 4 / \mathrm{mcm}\left(D_{4 h}^{18}\right)$ with $\mathrm{a}=5.015 \AA$ and $\mathrm{c}=4.22 \AA$. The indexing of the peaks is shown in the figure. The solid lines are fits to the data as discussed in the text.
$30 \mathrm{~mm}$ length is suspended with one end held under slight tension to compensate for thermal expansion. The ribbons are heated directly by passing current through them and their temperature is controlled using an infrared pyrometer. This thermal geometry with its small thermal mass allows us to increase the temperature of our samples by $1000{ }^{\circ} \mathrm{C}$ in under a millisecond (Brauer et. al, 1990) and then hold the temperature constant at the anneal temperature $T_{a}$. By surrounding the sample with $\mathrm{He}$, cooling rates of over $1000{ }^{\circ} \mathrm{C} / \mathrm{s}$ can be obtained. A typical measurement involves acquiring 100 to 500 consecutive diffraction patterns. The temperature step is timed to occur after several low temperature patterns are taken and to end so that the last few patterns can be taken at room temperature. This enables us to monitor what happens during the heating and cooling of our samples. We define $t=0$ as the time at which $T_{a}$ is reached. In our current setup, up to four voltage signals are digitized at a rate 10 times faster than the time per diffraction pattern. Two of these are used to measure incident beam intensity and the temperature. Measurements for large-angle scattering can be made in reflection or in transmission. Using reflection tends to give counting rates 2 to 5 times higher than transmission.

\section{$\mathrm{Co}_{2} \mathrm{~B}$}

Figure 1 shows a sequence of diffraction patterns for different times during the polymorphic crystallization of $\mathrm{Co}_{2} \mathrm{~B}$ for $T_{a}=675 \mathrm{~K}$. The measurements were taken in a transmission geometry. The time $t=0 \mathrm{~s}$ profile shows the characteristic scattering of a metallic glass. As the material crystallizes this scattering reduces in intensity and is replaced by the characteristic diffraction pattern of a polycrystalline material shown by the $t=1604 \mathrm{~s}$ profile. Since the intensity of X-rays from a synchrotron changes with time, all intensities have all been normalized to a beam current of $100 \mathrm{~mA}$. The X-ray ring at NSLS typically operates between 150 and $250 \mathrm{~mA}$. Figure 1 shows that we routinely obtain count rates of 40,000 to $60,000 \mathrm{cts} / \mathrm{sec} /$ angle for transmission. For reflection this becomes 100,000 to $250,000 \mathrm{cts} / \mathrm{sec} /$ angle. The minimum time to read out a scan from the linear detector is under $3 \mathrm{~ms}$. From these numbers one can see that diffraction patterns with maxima of several hundred counts can be taken with a time resolution of $3 \mathrm{~ms}$.

To determine the crystalline volume fraction for this data, each diffraction profile is fit by least squares to a simple linear combination of the initial amorphous scattering $I_{a}(q)$, and the final crystalline scattering $I_{c}(q)$. Explicitly, the scattering intensity 


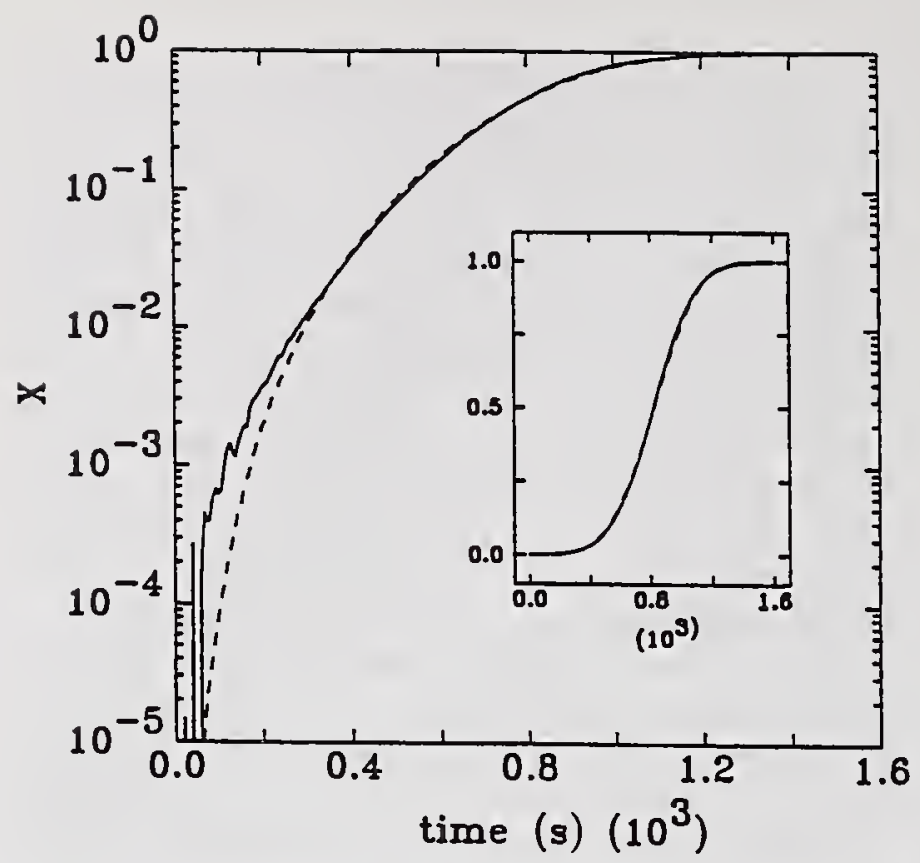

Figure 2: Crystallized volume fraction of $\mathrm{Co}_{2} \mathrm{~B}$ versus time. The solid line is a fit to $x(t)=1-e^{-a t^{4}}$, with $a=1.5 \times 10^{-12} \mathrm{~s}^{-4}$. Inset is same data with linear scales.

for a given wave vector $q$ and time $t$, is

$$
I(q, t)=[1-x(t)] I_{a}(q)+x(t) I_{c}(q)
$$

where $x(t)$ is a measure of the volume fraction. Providing that the scattering profile of each phase is independent of time, the most sensitive way to extract $x$ is to use the measured initial and final scattering patterns, i.e. $I_{a}(q)=I\left(q, t_{\text {initial }}\right)$ and $I_{c}(q)=I\left(q, t_{\text {final }}\right)$. This procedure implicitly tests whether the increase of crystalline scattering is compensated for by a corresponding loss of amorphous scattering. Furthermore, any systematic deviations of the model from the data directly indicate when the model breaks down. The results of such fits are plotted in figure 2. Note that volume fractions smaller than 1 part per $10^{4}$ are easily seen for data which have a large number of counts per diffraction angle. A fit to a simple model of nucleation and growth (Luborsky, 1983) is also plotted in the figure to show how kinetic parameters can be obtained from the data.

As with all powder X-ray diffraction measurements care must taken to avoid insufficient powder averaging. In our scattering geometry, large crystallites result in peaks which are sharper than resolution the intensities of which are unusually sensitive to sample and beam motion. The use of melt spun metallic ribbons seems to be particularly advantageous in giving small grain sizes and avoiding these problems.

\section{$\mathrm{FeZr}_{2}$ and $\mathrm{CoZr}_{2}$}

Figure 3 shows the polymorphic crystallization of $\mathrm{FeZr}_{2}$. Again, the initial amorphous pattern disappears with time to be replaced by a polycrystalline powder pattern. This time, however, the crystal scattering continues to evolve in time; the peak heights grow and the peak widths decrease as the sample continues to anneal. The crystal structure of this

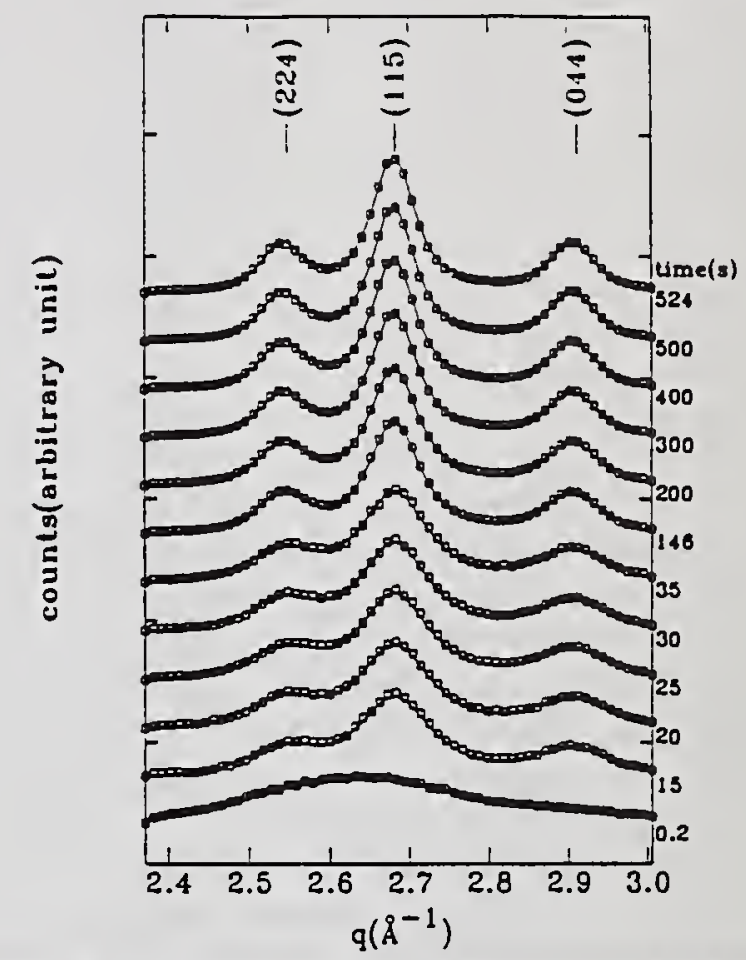

Figure 3: Time-resolved diffraction of $\mathrm{FeZr}_{2}$ at $T_{a}=690 \mathrm{~K}$. The time per diffraction pattern is 0.2 $\mathrm{s}$ up until $40 \mathrm{~s}$ and $2.0 \mathrm{~s}$ thereafter. Solid lines are fits to a model discussed in the text and the crystal Bragg peaks are labeled.

phase of $\mathrm{FeZr}_{2}$ is known to be cubic $\left(F d \overline{3} m\left(O_{h}^{7}\right)\right)$ with a lattice constant of $12.2 \AA$. Because the crystalline scattering evolves with time, the simple linear decomposition of line shapes used in equation 1 must be modified. The solid lines in the figure are based on fitting the crystal profiles as the sum of three Gaussian peaks. The positions and the integrated intensities of these peaks are obtained by fitting the final scattering pattern. Intermediate patterns are then fit to equation 1 with the $I_{c}(q)$ replaced by this functional form, $I_{c}(q, w(t))$ where $w(t)$ is a common peak width. A least squares fitting procedure is used to determine $x(t)$ and $w(t)$ and none of the other parameters are varied. In general, one could imagine the need for models of the scattering which cover the spectrum from simple ones, as those used here, to ones as complicated as those required for a complete Rietveld analysis.

From the fits, it is found that for this run, $x(t)=$ 


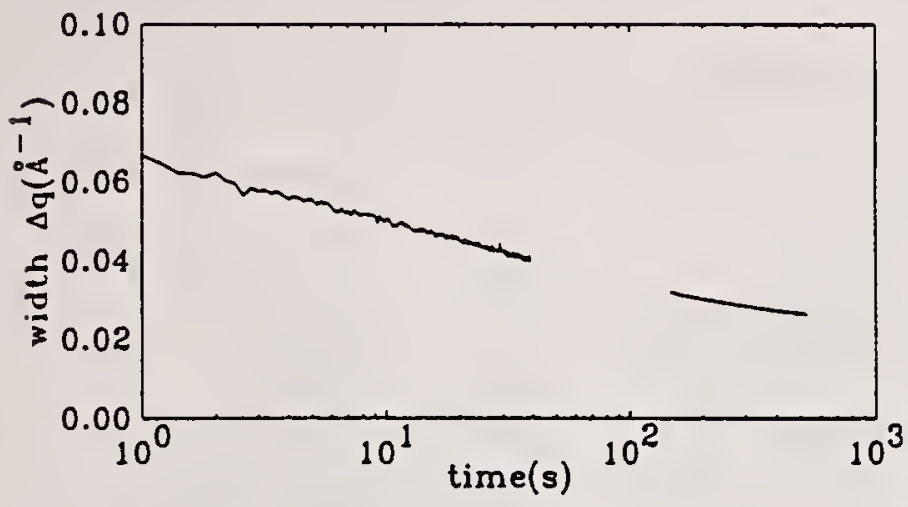

Figure 4: Peak width (half width half maximum) as a function of time for $\mathrm{FeZr}_{2}$. Gap in data results from the transfer of the data of the first part of the scan and starting the second part.

1.0 is reached within the first 20 seconds, and the peak width continues to evolve for the duration of the measurement. The measured width is plotted in figure 4. This phase of $\mathrm{FeZr}_{2}$ is known to have

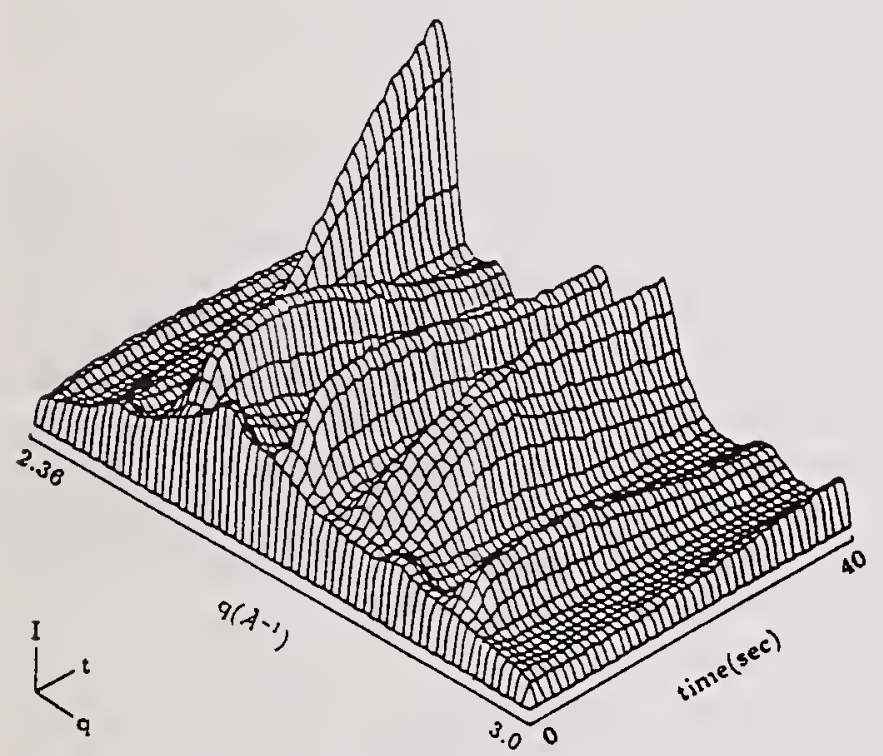

Figure 5: A three dimensional representation of the scattering as a function of time for $\mathrm{CoZr}_{2}$ annealed at $T_{a}=720 \mathrm{~K}$. The time per diffraction pattern is 0.1 s. At this temperature crystallization occurs in well under $0.1 \mathrm{~s}$ and the first pattern is due to the cubic structure.

small crystallite sizes when prepared from melt spun ribbons (Altounian, Volkert and Ström-Olsen 1985). Assuming the width is only due to finite sized crystallites and using the Scherrer equation, particles are $\approx 50 \AA$ at $t=1 s$ and grow to over $125 \AA$ at 500 s.

The crystallization of $\mathrm{CoZr}_{2}$ is shown in figure 5. The first hot diffraction pattern indicates that the amorphous phase has already transformed. This crystal phase has the same cubic structure as de-

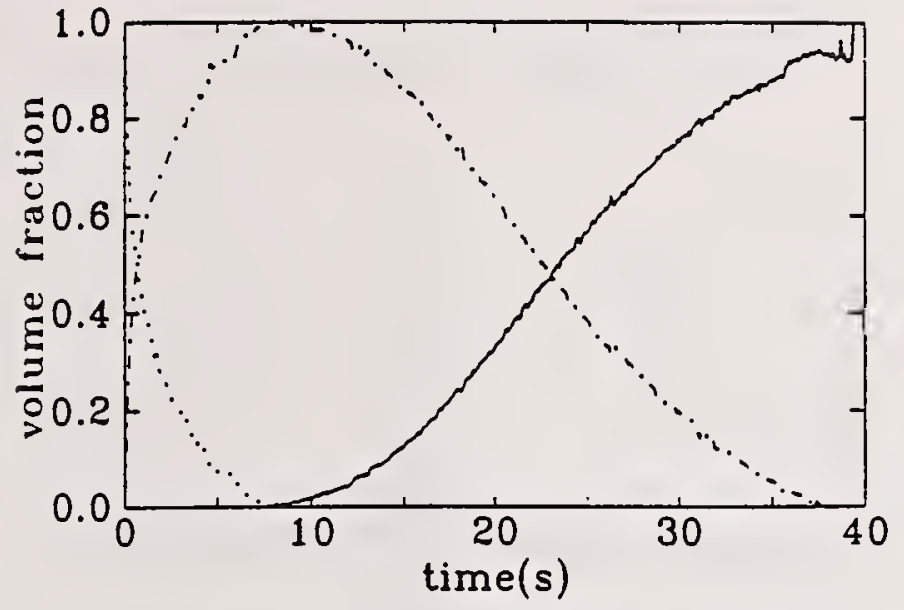

Figure 6: The time dependence of the volume fractions for the data in figure 5 . The dotted, dashed and solid lines represent the volume fractions for the cubic, the unknown and the tetragonal phases respectively.

scribed above for $\mathrm{FeZr}_{2}$. From figure 5 it is immediately obvious that two other phases are involved in the crystallization. One can see that the cubic phase lasts for less than 10 seconds. A second, previously undiscovered, metastable phase appears for $\approx$ 40 seconds. This phase does not appear in crystallization at low temperatures. The final phase which appears is the known equilibrium tetragonal phase of $\mathrm{CoZr}_{2}, I 4 / \mathrm{mcm}\left(D_{4 h}^{18}\right)$. Extending equation 1 to include more phases is straight forward and produces volume fractions versus time for each of the phases, as shown in figure 6 . With our ability to rapidly quench samples, we have been able to obtain specimens with large volume fractions of this new unknown phase. It is hoped that with more complete powder diffraction profiles, TEM and selected area diffraction more information can be obtained to uniquely determine this crystal structure.

\section{Eutectic $\mathrm{Co}_{89} \mathrm{Zr}_{11}$}

Figure 7 shows the crystallization of $\mathrm{Co}_{89} \mathrm{Zr}_{11}$. These data look very similar to those in figures 1,3 and 5 . In this crystallization both $\beta$-Co $\left(F m \overline{3} m\left(O_{h}^{5}\right)\right)$ and $\mathrm{Co}_{5} \mathrm{Zr}\left(F \overline{4} 3 m\left(T_{d}^{2}\right)\right)$ appear. The occurrence of the (200) of $\beta$-Co and the (220) of $\mathrm{Co}_{5} \mathrm{Zr}$ in isolated regions of the profile enable us to monitor the appearance of each of the phases independently. The extra structure in the final scattering pattern is due to the formation of a small amount of $\alpha-$ Co.

Figure 8 shows the time evolution of the smallangle scattering for the same composition ribbon at a slightly lower temperature but for a much longer annealing time. A well defined small-angle peak near 


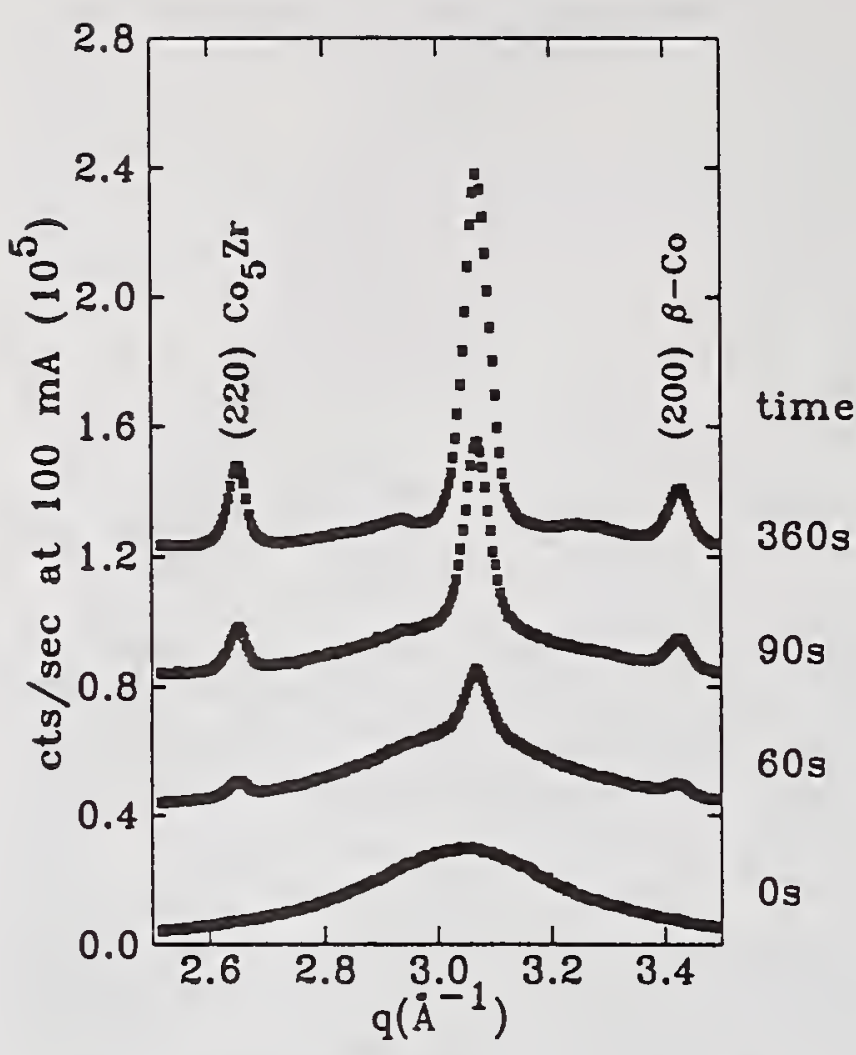

Figure 7: Crystallization of $\mathrm{Co}_{89} \mathrm{Z}_{\mathrm{r}_{11}}$ at $T \approx 830 \mathrm{~K}$, large- angle scattering. The peak at $3.05 \AA^{-1}$ is the superposition of the (311) of $\mathrm{Co}_{5} \mathrm{Zr}$ and the (111) of $\beta-$ Co.

$q=.07 \AA^{-1}$ is clearly seen. This peak corresponds to the eutectic formation of $\mathrm{Co}$ and $\mathrm{Co}_{5} \mathrm{Z}_{\mathrm{r}}$ with an interlamellar spacing of just under $100 \AA$. From the high angle data we can measure the order of appearance of the phases, and the relative volume fractions. From the small-angle data we can study the evolution of the eutectic structures. By making these measurements at several compositions around the eutectic composition and at several temperatures, we will not only be able to test theories for the time evolution of eutectic crystallization, but we will also be able to measure the cross-over from eutectic crystallization to primary crystallization. The ability to measure both small and large-angle scattering is important for these studies.

\section{Conclusions}

Typically, further measurements are needed to fully understand the structures seen in our in situ work. We often perform ex situ measurements using $\mathrm{X}$ ray diffraction and electron microscopy to help identify phases. From our time-resolved data we can determine the appropriate times for quenching our samples. Furthermore, in situ quenching gives us confidence that the quenched-in structure has not

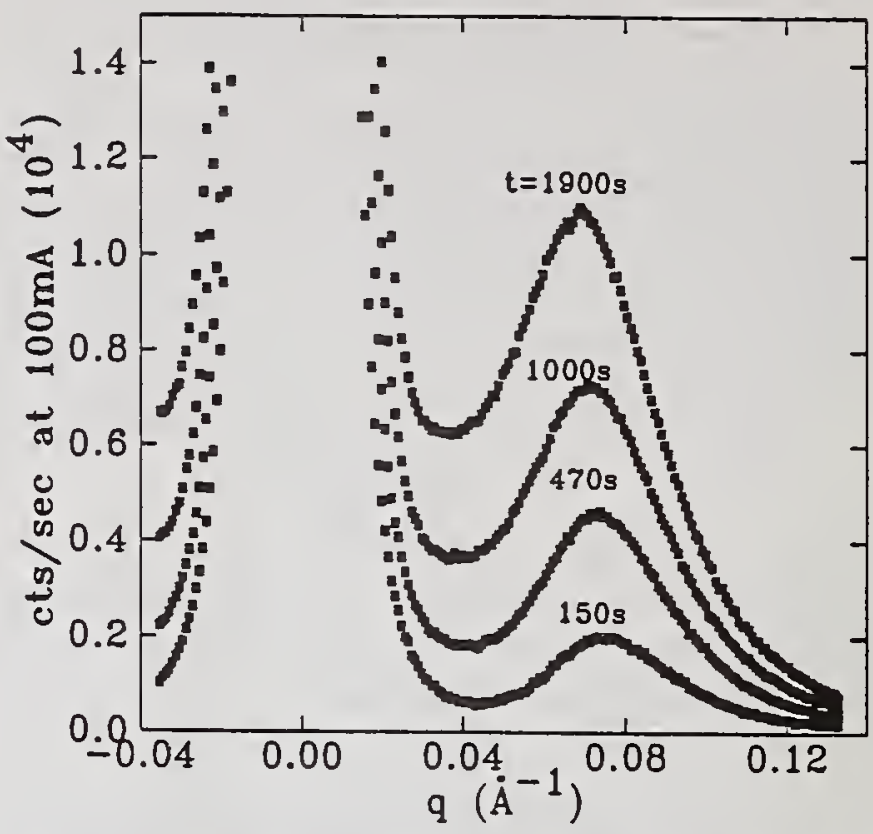

Figure 8: Small-angle scattering from $\mathrm{Co}_{89} \mathrm{Z}_{\mathrm{r}_{11}}$ crystallizing at $T \approx 820 \mathrm{~K}$.

changed so that further ex situ measurements do indeed represent the transient structures of the transformation under study.

In this paper we have tried to emphasize the wide range of structural information that can be measured by time-resolved X-ray diffraction. Metallic glasses provide a particularly rich set of structural transformations and clearly demonstrate both the power of time-resolved diffraction in unraveling these transformations and the necessity for using it. We have also emphasized in situ measurements since these allow one to use the continuity in the time evolution of the scattering to help ensure that some aspect of the transformations is not missed.

\section{References}

Altounian, Z., Volkert, C. A. \& Ström-Olsen, J. O. (1985). J. Appl. Phys. 17, 1777-1782.

Brauer, S., Ryan, D. H., Ström-Olsen, J. O., Sutton, M. \& Stephenson, G. B. (1990). Rev. Sci. Instrum. 61, 2214-2219.

Brauer, S., Ström-Olsen, J. O., Sutton, M., YANG, Y. S., Zaluska, A., Stephenson, G. B. \& Köster, U. (1992). Phys. Rev B, 45, 7704-7715.

LuBorsky, F. E. (1983). Amorphous Metallic Alloys, London: Butterworths \& Co. Ltd.

StEPHENSON, G. B. (1988). Nucl. Inst. and Meth. A266, 447-451.

STEPHENSON, G. B., Ludwig JR., K.F., JoRdanSweet, J. L., Brauer, S., Mainville, J., Yang, Y. S. \& SutTon, M. (1989). Rev. Sci. Inst., 60, 15371540. 
National Institute of Standards and Technology Special Publication 846. Proceedings of the international conference Accuracy in Powder Diffraction II, held at NIST, Gaithersburg, MD, May 26-29, 1992. (Issued October 1992)

\title{
New Developments in High-Pressure Neutron Powder Diffraction
}

\author{
R. J. Nelmes*and J. S. Loveday \\ Department of Physics, The University of Edinburgh, Edinburgh, EH9 3JZ, U.K.
}

AND J. M. BESSON

Physique des Milieux Condensés, Université P. et M. Curie, Jussieu, Paris cedex 05, France

For high-pressure neutron powder diffraction studies, time-of-flight methods have considerable advantages. The fixed scattering geometry greatly simplifies the construction of the cell in reducing the the size of the windows required for incident and diffracted beams. Diffraction at a fixed scattering angle also allows the construction of simple collimation to screen out background scattering from the material around the sample.

Amongst the earliest work to exploit this technique is that of Brugger and co-workers (Brugger, Bennion $\&$ Hall, 1965), who developed an alumina-insert cell capable of reaching $3 \mathrm{GPa}$ and an opposed-anvil cell that achieved $7 \mathrm{GPa}$ (Brugger, Bennion, Worlton \& Myers, 1969). A number of successful studies were performed, including an investigation of Bi II (Brugger, Bennion \& Worlton, 1967).

This project, and particularly the work on the opposed-anvil cell was curtailed by the closure of the Idaho Falls reactor, but a programme based around the alumina-insert cell was continued at Argonne National Laboratory. Many notable high-pressure studies have been carried out there including work on $\mathrm{ReO}_{3}$ and deuterated ice (Jorgensen \& Worlton, 1984; Jørgensen, Jorgensen, Batlogg, Remeika \& Axe, 1986). Over the past few years the Argonne group has also been responsible for much of the successful high-pressure diffraction work on high$\mathrm{T}_{c}$ superconductors (Pei et al., 1989; Jorgensen et al., 1990; Yamada et al., 1990). This recent work, however, has generally made use of a large sample volume in a gas cell (Yamada et al., 1990), which gives higher precision on the initial (small) rates of change of bondlengths with pressure.

Work using angle-dispersive diffraction on reactor sources has all been largely confined to pressures

\footnotetext{
*To whom correspondence should be addressed.
}
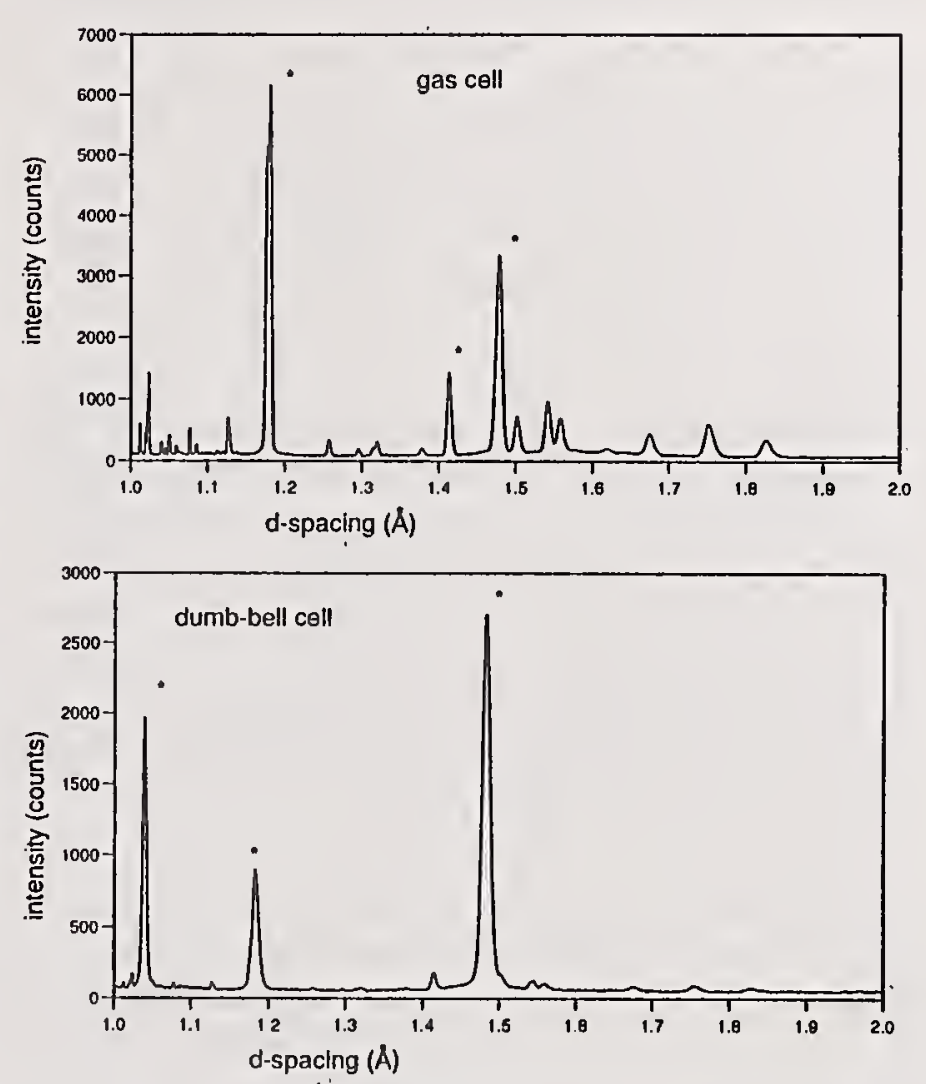

Figure 1. Powder patterns collected from samples of $\mathrm{La}_{2-x} \mathrm{Sr}_{x} \mathrm{CuO}_{4}$ at ILL, Grenoble. The upper pattern was collected in an aluminium gas-cell with a sample volume of $3000 \mathrm{~mm}^{3}$ on the $\mathrm{D} 1 \mathrm{~A}$ instrument. The lower pattern was collected using a steel "dumb-bell" cell on D1A with a sample volume of approximately $500 \mathrm{~mm}^{3}$. The asterisks mark non-sample peaks.

below $1 \mathrm{GPa}$, where larger sample volumes allow the background scattering from the cell materials to be tolerated. The problems of background scattering are illustrated by the patterns shown in figure 1 , which are dominated by this scattering even with the large sample volume available with the gas cell. 
One of the successful studies using a reactor source at pressures above $1 \mathrm{GPa}$ is the series of experiments described in Kuhs, Finney, Vettier \& Bliss (1984) on deuterated ice. Long count times were employed to obtain high quality data, and values of the O-D bondlength were obtained with a precision of $0.007 \AA$. It was also possible to observe indications of multisite disorder of the oxygen site in ice VII.

Thus we see that until recently, with the exception of the Brugger opposed-anvil cell, which was not pursued, neutron diffraction has been limited to the 3 $\mathrm{GPa}$ achievable with clamp cells and often much less. However there are now two independent attempts in progress to extend this pressure range significantly.

One development is based around a reactor source in Russia and employs cells giving a small sample volume of only $1 \mathrm{~mm}^{3}$ or less (Glazkov, Naumov, Somenkov \& Shil'stein,1988; Goncharenko, Glazkov, Irodova \& Somenkov, 1991). With such small samples, the pressure cell has to be almost completely surrounded by detectors in the (horizontal) scattering plane. Each individual detector is carefully collimated to view just the region of the sample and is shielded to minimise contaminant and background scattering. Successful structure refinements of $\mathrm{AlD}_{3}$ up to $7 \mathrm{GPa}$ have recently been reported (Goncharenko, Glazkov, Irodova \& Somenkov, 1991). However the very small sample volume used in this technique means that it is not expected to yield full structure refinements other than for the most strongly scattering materials.

The other new development is our own, using timeof-flight techniques on the UK pulsed neutron source, ISIS, at the Rutherford Appleton Laboratory. This project is based around a novel opposed-anvil cell specifically designed to achieve pressures of $10 \mathrm{GPa}$ and above with a sample volume of the order of 100 $\mathrm{mm}^{3}$.

The cell is shown in figure 2 and consists of a sample mounted between two tungsten carbide or sintered-diamond anvils and surrounded by a gasket. Pressure is applied by a compact 200 tonne hydraulic-ram (HR), which allows in situ variation of the pressure. The complex anvil profile is important to the operation of the cell. The quasi-spherical sample volume $(\mathrm{S})$ has the role of increasing the sample volume (compared with flat anvils), and its shape also ensures a more homogeneous pressure distribution. The key feature is the outer toroidal groove (T), which inhibits side extrusion of the gasket and hence allows higher pressures to be reached. Details of the cell and ram design may be found in papers

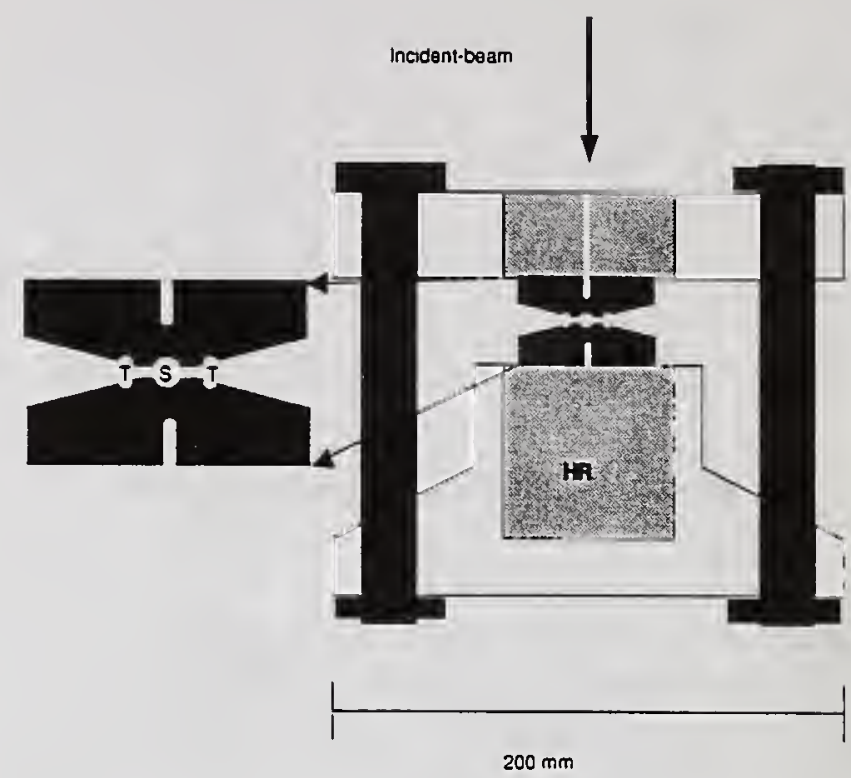

Figure 2: A schematic cross section through the Paris-Edinburgh cell showing the hydraulic ram (HR). The enlargement shows the toroidal gasket groove $(\mathrm{T})$ and the sample space $(\mathrm{S})$.

by Besson et al. (1992a, b). The performance of the equipment has been tested and proved in making an accurate study of the equation of state of LiD (Besson et al., 1992b).

The cell is designed for use on the POLARIS powder diffractometer at ISIS. It is mounted with its axis horizontal so that the the incident beam enters through the hole in the front of the cell. To ensure that the beam illuminates the minimum amount of non-sample material, the diameter of the beam is reduced to slightly less than that of the sample by a collimator fixed to the front of the cell. The diffraction pattern is collected by detectors placed at $2 \theta=90^{\circ}$, and background scattering from the illuminated parts of the anvils is screened out by coating the inner (opposing) faces of the anvils with a neutron absorbing material (either $\mathrm{Gd}_{2} \mathrm{O}_{3}$ or $\mathrm{Cd}$ ). Further shielding is placed around the cell and the scattered-beam flight paths to reduce the background due to other sources. After careful optimisation of the shielding it is now possible to achieve signal:background ratios of up to 100:1 with no detectable diffraction peaks due to the anvil materials, as shown in figure 3 .

Once good quality data are obtained, any systematic effects on the relative intensities must be corrected. In the case of the Paris-Edinburgh cell the principal systematic error is that due to attenuation by the cell materials. Finite-element calculations and 


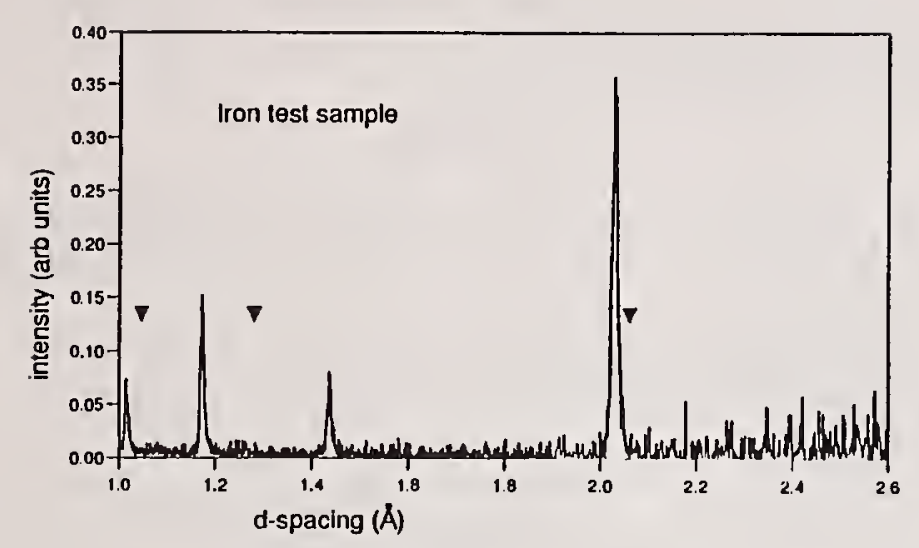

Figure 3. A time-of-flight spectrum collected from a sample of iron in the Paris-Edinburgh cell. The marks show the expected positions of the diffraction peaks due to the anvils.

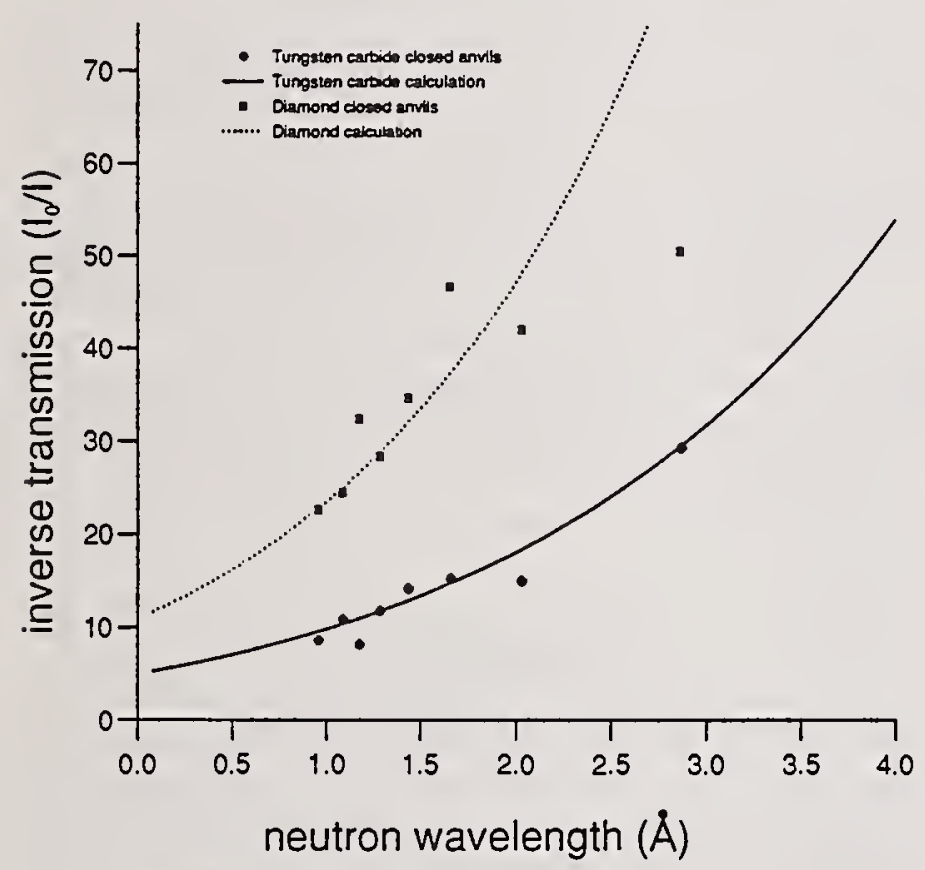

Figure 4. The inverse transmission factor (defined as $\mathrm{I}_{0} / \mathrm{I}$ ) as a function of neutron wavelength for tungsten carbide anvils (circles,solid line), and sintereddiamond anvils (squares,dotted line). The symbols show measurements made using an iron pellet sample, with the anvils fully closed together, while the lines are the results of the corresponding finiteelement calculations.

measurements show that this can vary by as much as a factor of 20 (Fig. 4) over the wavelength range of interest $(0.7-3 \AA)$. If left uncorrected, such severe attenuation significantly biases the refined values of the positional parameters. The form of attenuation curve is a function of the anvil separation, and hence pressure, so attenuation may introduce spurious pressure dependences to the refined values of structural parameters. Accurate corrections using the finite-element calculations are thus essential.

The experience gained on the neutron scattering aspects of the cell has been used in a high-pressure study of the structure of deuterated ice VIII. This was chosen as it is a problem of suitable difficulty and interest that is appropriate to study by neutron diffraction. In addition, previous neutron diffraction studies in the 2-3 GPa range (Kuhs, Finney, Vettier \& Bliss, 1984; Jorgensen \& Worlton, 1984) provide a check on the accuracy of data in the low-pressure region. Ice VIII is stable only below $0^{\circ} \mathrm{C}$, and so the sample and cell were cooled by a stream of cold nitrogen gas.

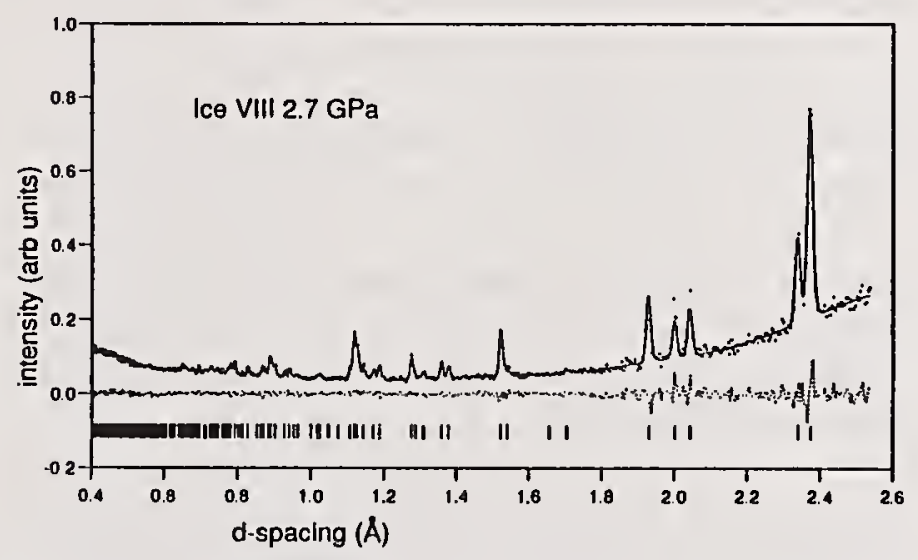

Figure 5: The measured spectrum of $\mathrm{D}_{2} \mathrm{O}$ ice VIII at $2.7 \mathrm{GPa}$ and $-5{ }^{\circ} \mathrm{C}$ (dots) and the results of a Rietveld profile refinement (solid line).

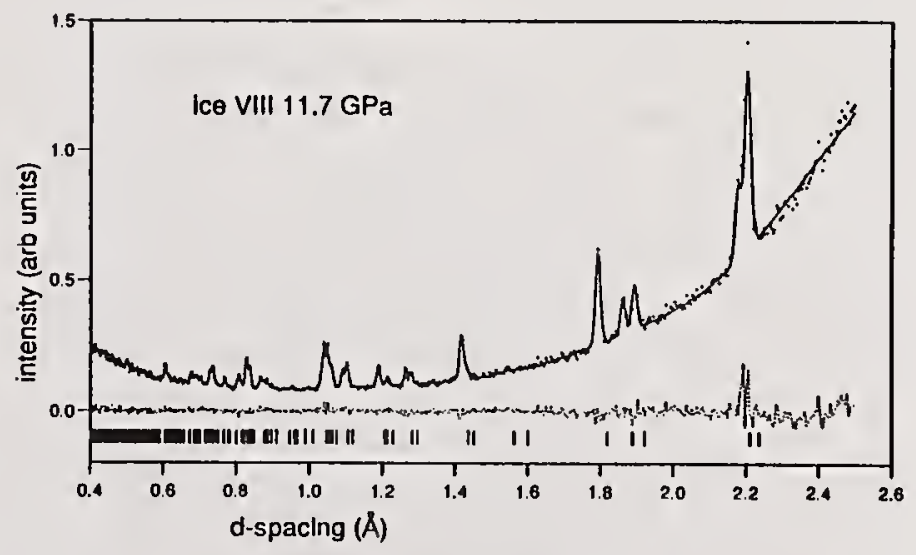

Figure 6: The measured spectrum of $\mathrm{D}_{2} \mathrm{O}$ ice VIII at $11.7 \mathrm{GPa}$ and $-5^{\circ} \mathrm{C}$ (dots) and the results of a Rietveld profile refinement (solid line).

Figures 5 and 6 show the measured and calculated spectra obtained from a profile refinement of corrected data collected at pressures of 2.7 and 11.7 
GPa. To illustrate the precision achieved, the values obtained for the O-D bondlength in preliminary profile refinements are shown in figure 7 , along with

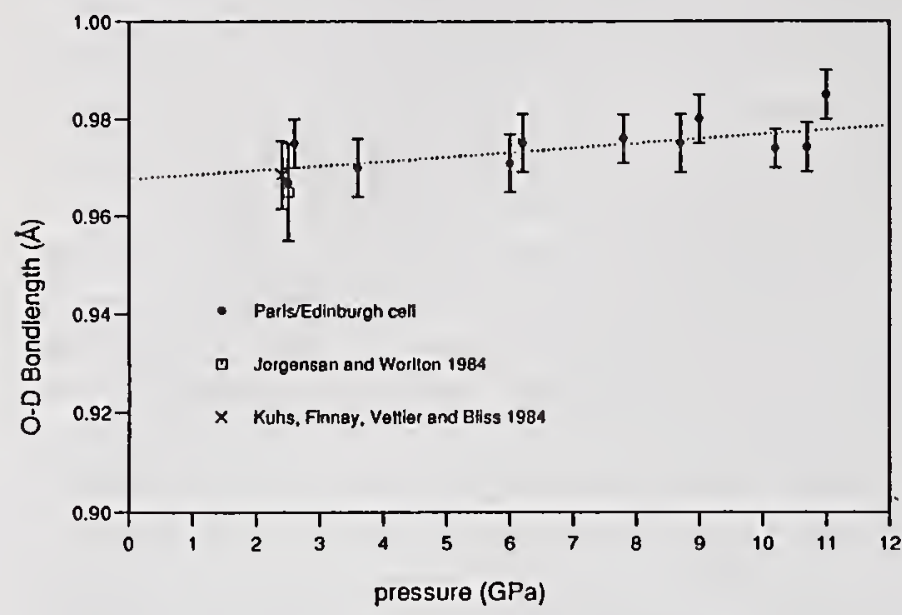

Figure 7: The pressure dependence of the intramolecular O-D bondlength in ice VIII. The values shown as circles were measured in the ParisEdinburgh cell while those shown as a cross and an open-square were taken from previous work (Kuhs, Finney, Vettier \& Bliss, 1984; Jorgensen \& Worlton, 1984). The dotted line is a fit to our values.

the results of previous studies (Jorgensen \& Worlton, 1984; Kuhs, Finney, Vettier \& Bliss, 1984). The pressures were derived from the measured unit-cell volume and the equation-of-state of ice VII (Hemley et al., 1987), which differs very little in density from ice VIII. The level of random variation of the values suggests that the true e.s.d.'s are approximately $0.005 \AA$, as shown. The overall internal consistency of the values, which were obtained from three separate loadings, along with the good agreement with previous measurements, suggests that the contributions from non-random errors are rather small. The rate of change of $\mathrm{O}-\mathrm{D}$ with pressure appears to be around $0.001 \AA / \mathrm{GPa}$, which compares with a predicted variation of $0.0018 \AA / G P a$ (Klug \& Whalley, 1987). The very small size of these changes illustrates the need to obtain precise data over a wide range of pressures in order to draw meaningful conclusions about such structural variations.

The results achieved to date give considerable cause for optimism concerning the feasibility of performing structural studies routinely to $20 \mathrm{GPa}$. Further work is needed to reduce the background to the levels necessary for work with smaller sample volumes (to attain higher pressures) or to study weaker scatterers. The factor of 10 improvement in count rate that will become available with a new, larger de- tector bank, currently being constructed, will make such studies feasible.

\section{References}

Besson, J. M., Nelmes, R. J., Hamel, G., Loveday, J. S., WeIll, G. \& Hull, S. (1992a). Physica B, 180, in press.

Besson, J. M., Hamel, G., WeLl, G. , Nelmes, R. J., Loveday, J. S. \& Hull, S. (1992b). Phys. Rev. B, $45,2613$.

Brugger, R. M., Bennion, R. B. \& Hall, H. T. (1965). Bull. Am. Phys. Soc. Series II, 10, 172.

Brugger, R. M., Bennion, R. B. \& Worlton, T. G. (1967). Phys. Lett. A, 24, 714.

Brugger, R. M., Bennion, R. B., Worlton, T. G. \& MYERs, W. R. (1969). Proceedings of the Symposium on Crystal Structures at High Pressure 141. Seattle: American Crystallographic Assoc.

Glazkov, V. P., Naumov, I. V., Somenkov, V. A. \& SHIL'steIN, S. SH. (1988). Nucl.Inst and Meth., A264, 367.

Goncharenko, I. N., Glazkov, V. P, Irodova, A. V. \& Somenkov, V. A. (1991). Physica B, A264, 117.

Hemley, R. J., JephcoAt, A. P., MaO, H. K., ZhU, C. S., Finger, L. W. \& Cox, D. E. (1987). Nature, 330, 737.

Jørgensen, J. E., Jorgensen, J. D., Batlogg, B., Remeika, J. P. \& Axe, J. D. (1983). Phys. Rev. B, 33, 4793 .

Jorgenson, J. D. \& Worlton, T. G. (1984). J. Chem. Phys. 81, 3211.

Jorgensen, J. D., Pei, S., Lightfoot, P., Hinks, D. G., Veal, B. W., Dabrowski, B., Paulikas, A. P., Kleb, K. \& Brown, I. D. (1990). Physica C, 171, 93. Klug, D. D. \& Whalley, E. (1984). J.Chem.Phys., 81, 1220.

Kuhs, W. F., Finney, J. L., Vettier, C. \& Bliss, D. V. (1984). J. Chem. Phys., 81, 3612.

Pei, S., Jorgensen, J. D., Hinks, D. G., Dabrowski, B., Lightfoot, P. \& Richards, D. R. (1990). Physica $C, 171,175$.

Yamada, Y., Jorgensen, J. D., Pei, S., Lightfoot, P., Kodama, Y., Masumoto, T. \& Izumi, T. (1991). Physica C, 173, 101. 
National Institute of Standards and Technology Special Publication 846. Proceedings of the international conference Accuracy in Powder Diffraction II, held at NIST, Gaithersburg, MD, May 26-29, 1992. (Issued October 1992)

\title{
Crystal Structure Analysis by Fitting of Powder Diffraction Data on Energy Dispersion
}

\author{
T. YAMANAKA \\ College of General Education, Osaka University, \\ Machikaneyama Toyonaka Osaka 560, Japan
}

\begin{abstract}
A profile fitting method based on energy dispersive (ED) spectra has been established. The ED measurement has a large advantage for diffraction studies under various physical conditions, such as at high temperature and pressure, because of the fixed Xray diffraction system. However, ED spectra present numerous difficulties in respect to diffraction intensities. The diffraction intensities must be corrected for source intensity distribution, absorption, escape efficiency and background as a function of energy. Energy resolution and accuracy of the structure refinement have also been tested. The structure refinements were performed on ED spectra taken with synchrotron radiation at the Photon Factory using a diamond anvil pressure cell and multi-anvil pressure apparatus.
\end{abstract}

\section{Introduction}

Crystal structure analysis based on the powder diffraction method has been advanced by the progress in profile fitting methods (Rietveld, 1969; Langford, 1981). Numerous discussions have been presented on the analysis of not only neutron but also X-ray diffraction profiles (Parrish, Huang \& Ayers, 1976; Young, Mackie \& Von Dreele, 1977; Malmros \& Thomas, 1977; McNelles \& Cambell, 1975). In recent years, synchrotron radiation (SR) sources have induced extensive powder diffraction studies because of their excellent beam characteristics. Thus the field of application of powder diffraction has been greatly broadened.

Energy dispersive (ED) diffraction experiments using a white X-ray source, as well as angle dispersive diffraction, have become more popular. ED experiments can be made under fixed X-ray optical conditions, where $\mathrm{X}$-ray source, sample and detector are set in place. The method has, therefore, a great advantage for in situ diffraction studies under variable physical conditions such as high pressure and high or low temperature.

Discussions on the ED diffraction intensity were presented by Buras, Olsen, Gerward, Selsmark \& Andersen (1975). Wilson (1973), Gerward, Morup \& Topsoe (1976) and Fukamachi, Hosoya \& Terasaki (1973) have discussed the profile shape. The application of profile analysis of the ED spectrum to structure refinement was discussed by Glazer, Hidaka \& Bordas (1978).

Various difficulties encountered in the profile fitting of ED spectra have been overcome by empirical corrections to the diffraction intensities. Yamanaka \& Ogata (1991) detailed correction of the intensity for source intensity distribution, absorption, escape efficiency and background as a function of energy. The profile fitting is performed by the profile decomposition method, using the whole ED pattern (Pawley, 1981).

\section{Intensity measurement of ED spectra}

Diffraction intensity measurements based on ED spectra require a white X-ray source having uniform and large intensity. Synchrotron radiation and tungsten or silver rotated anode targets in laboratory sources are extremely well-suited for ED diffraction studies. Radiation emitted from the positron storage ring at the Photon Factory (PF) with $2.5 \mathrm{GeV}$ and $300 \mathrm{~mA}$ and from the Accumulator Ring (AR) of TRISTAN with $6.5 \mathrm{GeV}$ and $50 \mathrm{~mA}$ provides $\mathrm{X}$ ray sources having maximum energies of $40 \mathrm{keV}$ and $120 \mathrm{keV}$, respectively. In the latter case, it is possible to detect diffraction peaks falling in the range $0.7113 \AA<d_{H}<14.227 \AA$ with the detector set at $2 \theta=10^{\circ}$. By changing the detector angular position, we can shift the detectable energy region. Because of the nature of the synchrotron radiation beam, a parallel-beam mode can be used instead of a focusedbeam mode. Therefore a very small divergence of the incident source gives a sharp diffraction profile. 


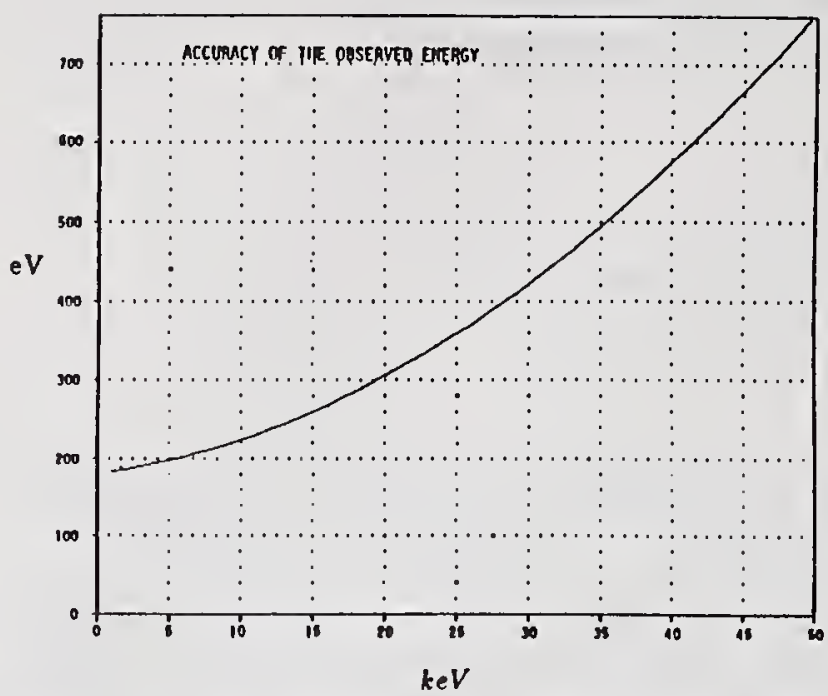

Figure 1: Energy resolution of Ge (intrinsic) detector with a resolution of $150 \mathrm{eV}$.

In the present experiment intensities were measured using a $\mathrm{Ge}$ (intrinsic) solid state detector (SSD). In cases where a sample contains elements with larger atomic number than $\mathrm{Ge}(\mathrm{Z}=32)$, X-ray fluorescence interferes with the diffraction pattern. The fluorescence increases the photon count error due to pulse pileup and a large dead time. For the elimination of the fluorescence, a Zn foil absorption filter was used.

The energy-resolution of the SSD was tested in advance. The full width half maximum (FWHM) of the fluorescent peak of $\mathrm{MnK} \alpha$ indicated a resolution of about $150 \mathrm{eV}$. Figure 1 presents the energy resolution of the Ge SSD. This resolution is related to the accuracy of the interplanar distance $d_{h k l}(\AA)$. That the precision of $d_{h k l}$ varies with the angular position $(\theta)$ of the detector can be inferred from the formula

$$
d_{h k l}=\frac{12.398}{2(E+\Delta E) \sin \theta}
$$

A higher angular position $\theta$ of the detector ensures a more precise determination of $d$. Figure 2 shows the systematic error of $\Delta d$ depending on the energy of diffraction peaks for a SSD with an energy resolution of $200 \mathrm{eV}$. It ensures a precision higher than \pm 0.005 $\AA$, but indicates $\pm 0.001 \AA$ at best. The value of $\frac{\Delta d}{d}$ is less than 0.001 . It is necessary to take into account the energy resolution for the determination of the lattice constants.

An intensity measurement was made using a multichannel analyzer (MCA) and computer aided measurement and control (CAMAC) module. A linear relationship between the channel and energy was confirmed by the known fluorescent X-ray energies of

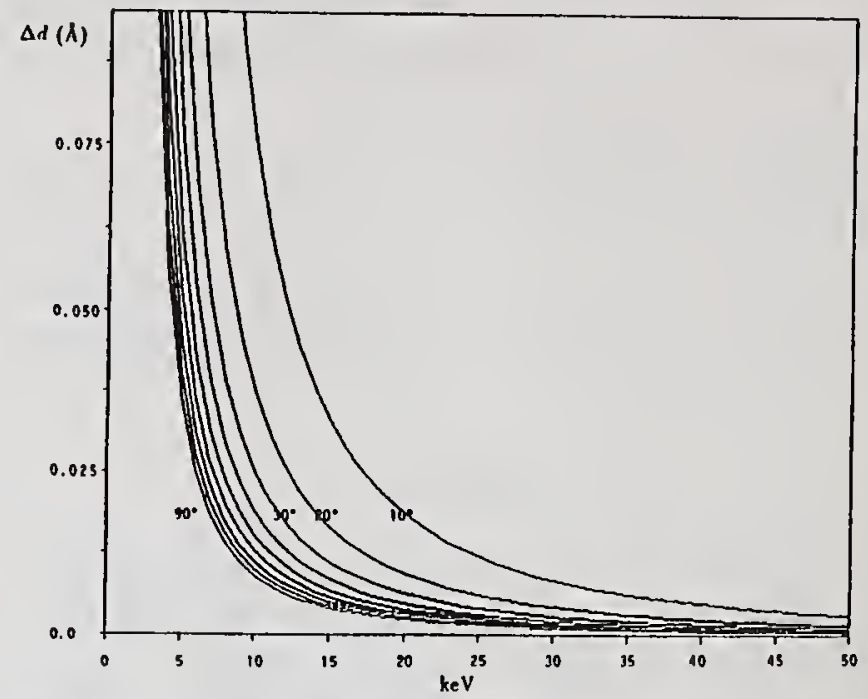

Figure 2: $\Delta d$ accuracy with respect to photon energy. Energy resolution of the SSD is $200 \mathrm{eV}$ at $5.89 \mathrm{keV}$.

several elements. The zero position and the channel/energy relationship were tested by the leastsquares method, using as standards the fluorescence of the elements Ti, Ni, Y, Mo, Mn, Zn, Se and Zr. The linearity of the quantum efficiency of the SSD at each channel was also confirmed using an Am radioactive source. The photon counting efficiency of the SSD was tested in the energy region of the measured spectrum. In this test several parameters of MCA, AD converter and amplifier have to be optimized. Since the frequency of AD converter clock pulses was $100 \mathrm{MHz}$, the shaping time was $10 \mu \mathrm{s}$. The X-ray source intensity was adjusted in order to prevent the number of photons from exceeding 1.5 kcps per channel throughout all 2048 or 4096 channels. The dead time was kept within $5 \%$.

The photon counting efficiency is discontinuous at the $\mathrm{K}$ absorption edge ( $11.103 \mathrm{keV})$ of Ge. Therefore, great care should be taken as to the angular position of the SSD in order to keep the diffraction peaks from occupying the above-mentioned position.

\section{Correction of the diffraction intensity}

(a) Intensity distribution of the white X-ray source

For ED experiments the energy distribution of the SR X-ray source ideally should be continuous, without any characteristic X-ray lines such as are found in Bremsstrahlung X-ray sources. The source, however, does not have a uniform intensity throughout the whole energy region. An experimental investigation of the intensity distribution of the source was made by varying the energy positions of the reflections, using a fixed-time intensity measurement on a 


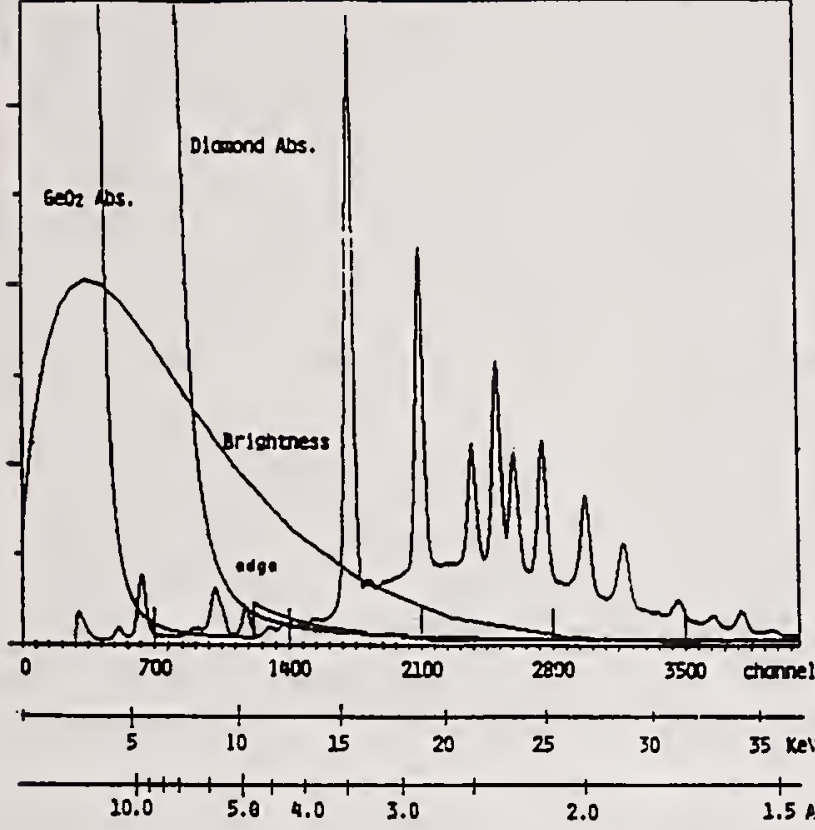

Figure 3: Intensity correction of the photon count distribution of the source and the absorption of $\exp (-\mu r)$. The SR source is emitted from the storage ring of electrons with the energy of $2.4 \mathrm{GeV}$ and $300 \mathrm{~mA}$. The absorption corrections are calculated for the diamond anvil cell and $\mathrm{GeO}_{2}$ sample.

Si crystal plate cut parallel to (111). An approximation to the SR source intensity distribution was also made using a Bessel function. An example of the SR source from a bending magnet with $2.5 \mathrm{GeV}$ and 300 $\mathrm{mA}$ is shown in figure 3 . The maximum brightness of the source is located at $2.9 \mathrm{keV}$.

\section{(b) Correction of the escape peak intensity}

When photons have larger energies than that of the $\mathrm{K}$ absorption edge of $\mathrm{Ge}$, energy escape arises. Then the accompanying escape peaks appear beside Bragg peaks at positions $E_{\mathrm{Bragg}}-9.87 \mathrm{keV}$. The escape yield $\varepsilon(E)$ depends on energy. The observed intensity, $y_{o b s}(E)$, is related to the true intensity, $y_{o}(E)$, of the diffraction peak by:

$$
y_{o b s}(E)=y_{o}(E)[1-\varepsilon(E)]
$$

The intensity of the escape peak is given as

$$
y_{o b s}\left(E-E_{o}\right)=\varepsilon\left(E-E_{o}\right) y_{o}\left(E-E_{o}\right)
$$

where $E_{o}$ is the energy of the characteristic X-ray of Ge-K.

It is necessary to determine the correction curve for the energy dependence of $\varepsilon(E)$. The integrated intensity of reflection $h h h$ from a Si single crystal plate was measured using a $\mathrm{Ge}$ (intrinsic) SSD. The correction curve for $\varepsilon(E)$ was previously shown in
Yamanaka \& Ogata (1991). Measurement was carried out by varying the angular position of the SSD. An empirical equation of the correction curve for the escape efficiency was determined by least-squares calculation, as expressed below:

$$
\varepsilon\left(E_{o}\right)=\exp (-0.455 \times E(k e V)-2.2236)
$$

\section{(c) Background intensity}

Profile analysis demands an accurate measurement of the true Bragg reflection intensity, excluding the background intensity which is caused by X-ray scattering other than Bragg reflection. An attempt has been made in the present program to express the background intensities in the whole energy range by several different functions in order to ensure highly precise structure determination. Among many trials, the best function for fitting the background shape was found to be a sum of a linear function and a modified pseudo-Voigt function:

$$
\begin{array}{r}
y\left(E_{i}\right)=a E_{i}+b+P\left[\eta \exp \left(\frac{E-E_{i}}{\Gamma}\right)^{2}\right. \\
\left.+(1-\eta)\left\{1+4 \frac{E-E_{i}}{\Gamma}\right\}^{2}\right]
\end{array}
$$

where $a, b, P, E, \Gamma$ and $\eta$ are variable parameters.

A part of photon energy is lost by the release of recoil electrons, and it produces Compton scattering. The incoherent scattering due to the Compton effect generates an aperiodic intensity. Thus the ED spectra include the Compton scattered spectra.

\section{(d) X-ray absorption correction}

An incident X-ray having an energy greater than the ionization energy induces photon absorption by liberation of an atomic electron. The observed intensity $I$ is derived from $I=I_{0} \exp (-\mu r)$, where $I_{0}$ is the incident intensity, $\mu\left(\mathrm{cm}^{-1}\right)$ is the linear absorption coefficient, and $r(\mathrm{~cm})$ is the X-ray path length in the material. The linear absorption coefficient as a function of energy can be calculated from the mass absorption coefficient $\frac{\mu}{\rho}\left(\mathrm{cm}^{2} \mathrm{~g}^{-1}\right)$, because the $\mathrm{X}$ ray absorption varies with the photon energy of the wavelength. The mass absorption coefficient of an element is calculated by

$$
\frac{\mu}{\rho}=\frac{\rho_{p}}{A}+\frac{\rho_{s} Z}{A}
$$

where $A$ is the atomic mass and $\sigma_{p}$ and $\sigma_{s}$ are defined by the photoelectric cross-sections for individual electron orbits derived from quantum mechanics. The mass absorption coefficient of each atom has been given as a function of energy of the primary X-ray wave (Sasaki, 1990). 
In a previous paper (Yamanaka \& Ogata, 1991), the mass absorption coefficient derived from the empirical equation of Victoreen $(1948,1949)$ was used:

$$
\frac{\mu}{\rho}=C_{1} \lambda^{3}-D_{1} \lambda^{4}+\frac{\sigma N Z}{A}
$$

The coefficients $C_{1}, D_{1}$, and $\sigma$ were taken from the International Tables for $X$-ray Crystallography, Vol. III. In equation (7), $N$ denotes Avogadro's Number, $Z$ indicates an atomic number, and $A$ represents atomic mass. When a sample contains an element with its absorption edge in the measured energy range, $C_{z}$ and $D_{z}$ should also be taken into account.

\section{ED diffraction profile}

\section{(a) Integrated intensity of diffraction peaks}

Several functions for describing the powder diffraction profile have been proposed for angular dispersion spectra. The profile decomposition method was applied to the ED spectra. Then each integrated intensity in the superposed diffraction peaks was assigned separately.

For the least-squares profile fitting of the ED spectra, the initial parameters of the crystal structure were required. The fitting procedure optimized the structure parameters by the least-squares method so as to ensure a good agreement with the ED diffraction intensity.

The energy positions of the ED diffraction peaks $\left(E_{i}\right)$ can be determined from $E_{H}=12.398 / 2 d_{H} \sin \theta$, where $\theta$ denotes the angular position of the detector. The integrated intensity of reflection $H$ can be derived from the following equation:

$$
\begin{aligned}
I_{H}= & I_{o}\left(E_{H}\right)-p\left\{\frac{1+\cos ^{2} 2 \theta}{\sin ^{2} 2 \theta \cdot \cos \theta}\right\}\left|F_{H}\right|^{2} \\
& \times \exp \left(-\frac{2 B \cdot E_{H}^{2} \sin ^{2} \theta}{h^{2} c^{2}}\right) A\left(E_{H}\right)
\end{aligned}
$$

$I_{o}\left(E_{H}\right)$ : source intensity at' the energy $E_{H}$ $p$ : multiplicity parameter

$F_{H}$ : structure factor of reflection $H$

$B$ : overall temperature factor

$A\left(E_{H}\right):$ X-ray absorption term at the energy $E_{H}$

SR X-rays have an approximately horizontal polarization and thus the polarization factor, $(1+\cos 2 \theta)$, is neglected.

\section{(b) Profile function}

The full width at half maximum (FWHM) of ED diffraction peaks is generally larger than is found in angular dispersion data. This is particularly noticeable at high energies. The diffraction profile varies not only with the crystallized state but also such optical and instrumental conditions as $\mathrm{X}$-ray source, slit system and SSD resolution. Therefore, an optimum function had to be selected for the description of profile shapes.

In the case of X-ray angular dispersion diffraction, functions such as Gaussian, Lorentzian, Pearson VII and Voigt have been attempted to ensure accord with the observed profile. Glazer et al. (1978) applied the Gaussian function in profile fitting to the ED pattern. However, the present program uses the pseudo-Voigt function, which satisfactorily models the profiles.

$$
\begin{gathered}
y\left(2 \Delta E_{i H}\right)=C\left\{\eta \exp \left(-X^{2}\right)+(1-\eta) \frac{1}{1+Y^{2}}\right\} \\
\Delta E_{i H}=E_{i}-E_{H} \\
X=2 \frac{\Delta E_{i H}}{\Gamma_{H}(\text { Gaussian })} \\
Y=2 \frac{\Delta E_{i H}}{\Gamma_{H}(\text { Lorentzian })} \\
E_{H}: \text { energy of the reflection } H \\
E_{i}: \text { variable energy } \\
\Gamma_{H} \text { (Gauss), } \Gamma_{H} \text { (Lorentz) : variable energy }
\end{gathered}
$$

The FWHM of the diffraction profile is not constant, but increases with energy $E_{H}$. The energy dependency of $\Gamma$ was expressed by the empirical formula

$$
\Gamma=U E_{H}^{2}+V E_{H}+W
$$

using the observed data presented in figure 4. This procedure is in conformity with the procedure of Glazer et al. (1978). U, V and W are variable parameters in the least-squares fitting.

Unlike the angular dispersion diffraction profiles, the profile of the ED spectrum remains asymmetric, and consequently the following correction term has been introduced:

$$
a\left(\Delta E_{i H}\right)=1-A\left\{4 E_{H}^{2}-\left(\frac{12.398}{d_{H}}\right)^{2}\right\}^{\frac{1}{2}} \Delta E_{i H}
$$

The profile decomposition divides the superposed diffraction patterns into the respective diffraction profiles in accordance with the corresponding profile functions, thereby determining the integrated intensity, peak position, profile pattern parameters (FWHM, peak height, Gaussian fraction) and background intensity. 


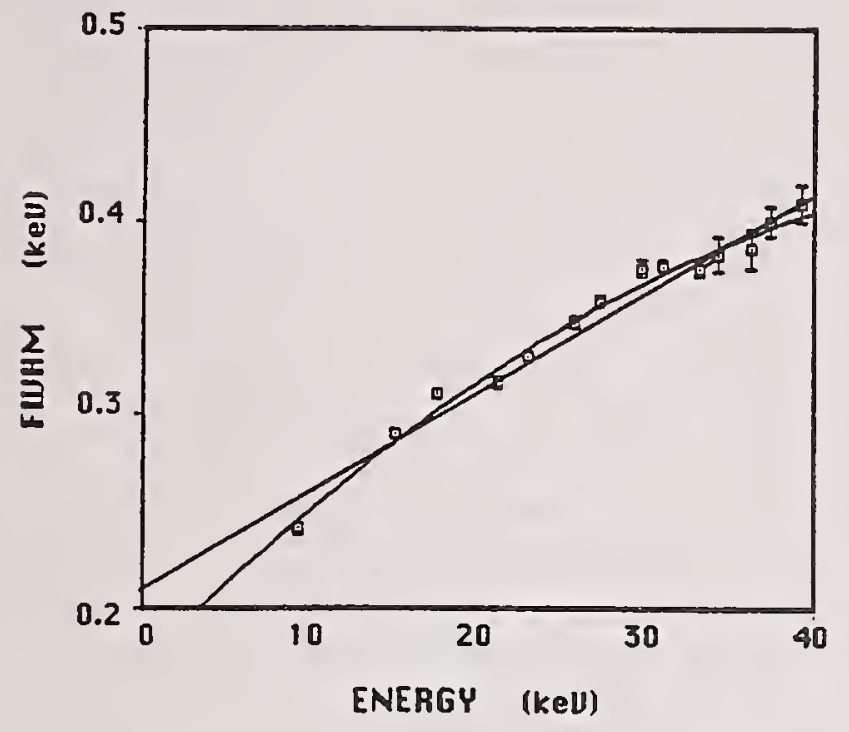

$\begin{array}{ll}F W H M=0.21311+0.00508 E & R^{2}=0.970 \\ F W H M=0.17194+0.00888 E-0.00007 E^{2} & R^{2}=0.986\end{array}$

Figure 4: Variation of FWHM with energy. Linear and quadratic functions have been fitted to the observed variation in the Si ED spectrum.

\section{(c) Program}

The integrated intensity $I_{H}$ derived from equation (8) is converted into the diffraction intensity $y_{c}\left(E_{i}\right)$ of the respective energies in accordance with the following formula:

$$
y_{c}\left(E_{i}\right)=B(E i)+\sum y\left(2 \Delta E_{i H}\right) I_{H}
$$

where

$B\left(E_{i}\right)$ : background

$y\left(2 \Delta E_{i H}\right):$ profile function defined by eq. (9).

The structure factors $F_{o}$ are derived from the observed diffraction intensities $y_{o}\left(E_{i}\right)$ of the respective energies in the 4096 individual channels of the MCA. Structural parameters, including lattice constants, atomic coordinates and temperature factors, are optimized by the least squares method so as to minimize the standard residual:

$$
\sigma=\sum w_{i}\left\{y_{o}\left(E_{i}-y_{c}\left(E_{i}\right)\right\}^{2}\right.
$$

The scale factor, profile parameters and background are also optimized by the refinement. Figure 5 indicates the flow chart of the computer program. The Gauss-Newton method is applied as the nonlinear minimum square method for the parameter refinement.
Three $\mathrm{R}$ factors are calculated for the determination of the results of refinement.

$$
\begin{aligned}
R_{w D} & =\left[\frac{\sum w_{i}\left\{y_{o}\left(E_{i}-E_{c}\right)\right\}^{2}}{\sum w_{i}\left\{y_{o}\left(E_{i}\right)\right\}^{2}}\right]^{\frac{1}{2}} \\
R_{D} & =\frac{\sum\left|y_{o}\left(E_{i}\right)-y_{c}\left(E_{i}\right)\right|}{\sum y_{o}\left(E_{i}\right)} \\
R_{s} & =\frac{\sum\left|I_{o}(H)-I_{c}(H)\right|}{\sum I_{o}(H)}
\end{aligned}
$$

\section{Structure analysis and kinetic study of the transition at high pressures}

Profile fitting of ED spectra of two $\mathrm{GeO}_{2}$ polymorphs, hexagonal $\left(\mathrm{P}_{2} 21, Z=3\right)$ and tetragonal $\left(\mathrm{P} 4_{2} / \mathrm{mmm}, Z=2\right)$, were performed under various high pressures and temperatures using a diamond anvil pressure cell with a platinum ring heater (Yamanaka \& Ogata, 1991). Synchrotron radiation with $2.5 \mathrm{GeV}$ and maximum current of $300 \mathrm{~mA}$ at the Photon Factory in Tsukuba was used as the X-ray source. The diamond anvil cell was set on the spinner, which was rotated at the rate of $1 / 3 \mathrm{rpm}$ around the $\phi$ axis and simultaneously oscillated in the range of $\pm 5^{\circ}$ around the $\omega$ axis for a randomization of the sample orientation and for reducing the effect of grain growth. A white X-ray beam of $50 \mu m \phi$ was applied. Figure 6 shows the profile fitting of the ED spectrum of hexagonal $\mathrm{GeO}_{2}$ at $2.9 \sim 3.0 \mathrm{GPa}$ and $493 \mathrm{~K}$.

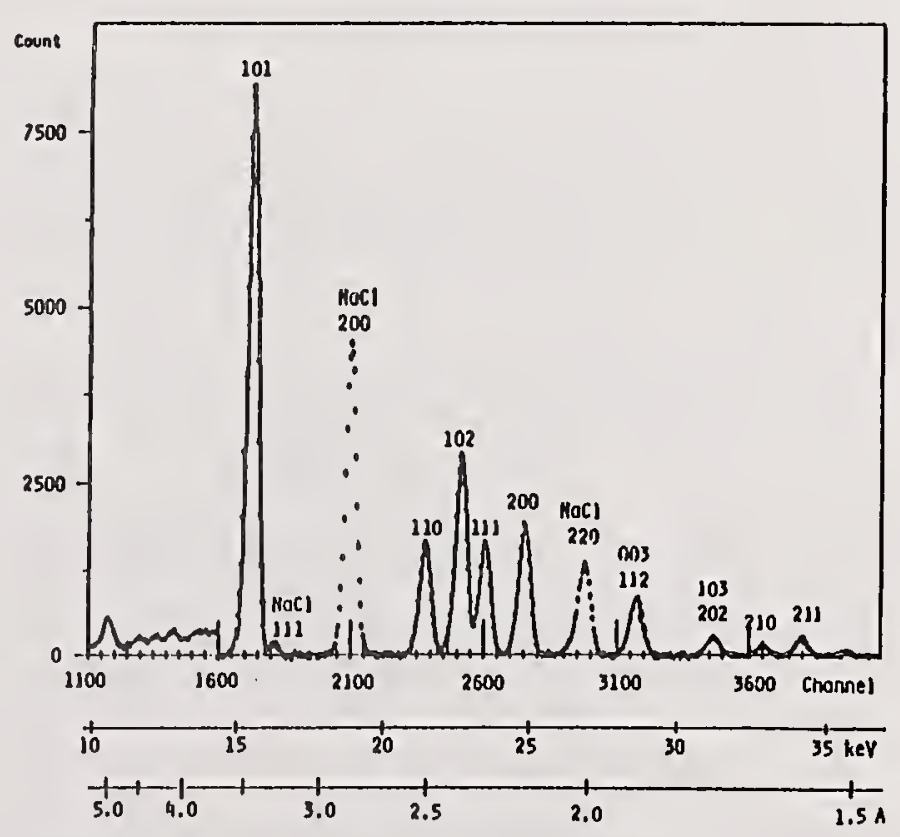

Figure 6: Profile fitting of the ED spectrum of hexagonal $\mathrm{GeO}_{2}$ at $2.9 \mathrm{GPa}$ and $493 \mathrm{~K}$.

Structure refinements by profile fitting were made after the many intensity corrections mentioned 


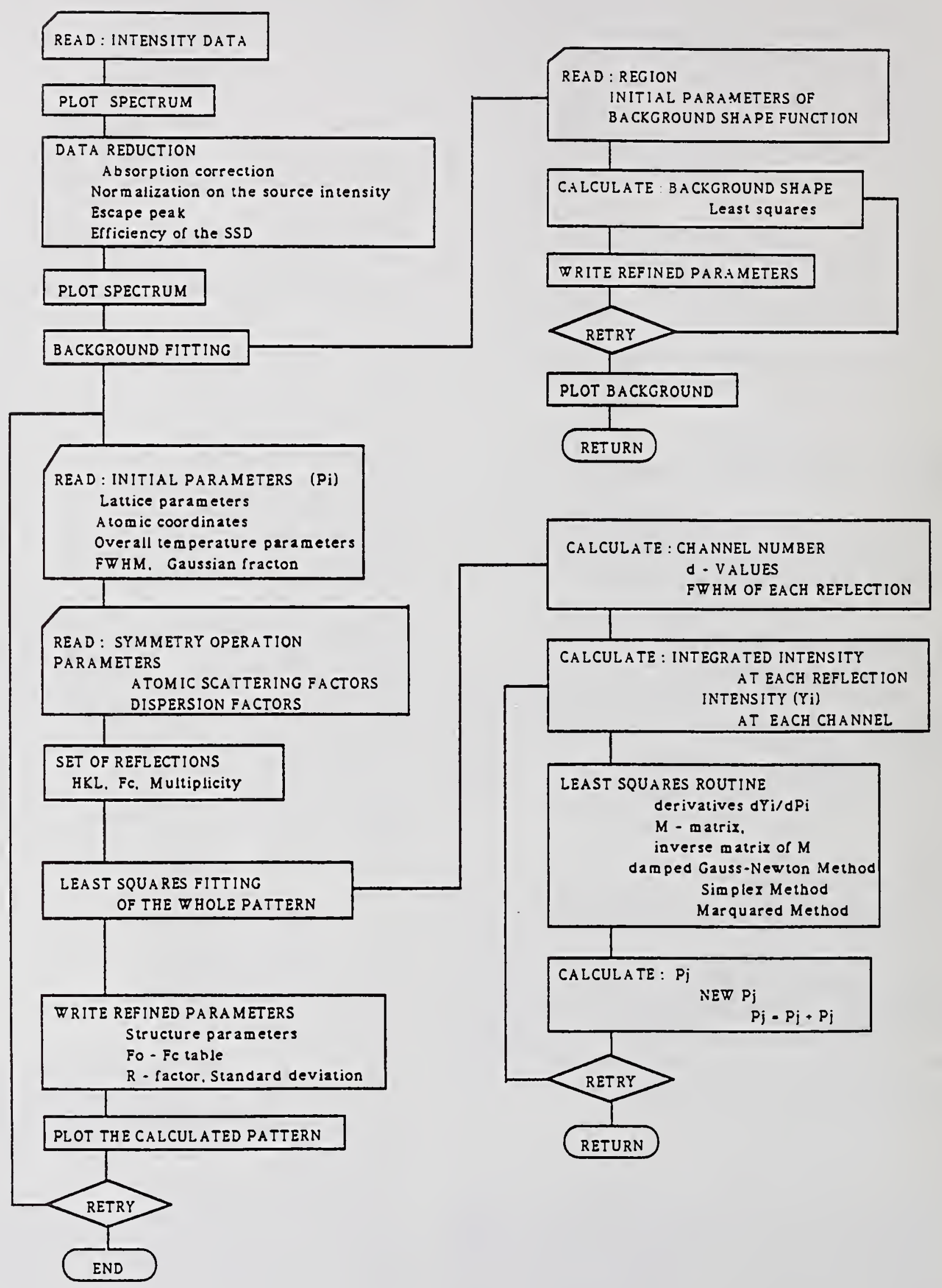

Figure 5: Flow chart of the program of the ED spectrum profile fitting. 
above. The final residual factor of the least-squares calculation was $R_{D}=0.088$. From the lattice parameters as a function of pressure determined by the profile fitting, volumetric compressibility, equation of state and isothermal bulk modulus were calculated (Yamanaka \& Ogata, 1991). Profile fitting was applied to the kinetic study of the structure transition from hexagonal to tetragonal structure (Yamanaka, Sugiyama \& Ogata, 1992). The transition rate was calculated from the mass fractions that were obtained from the precise measurement of the diffraction intensities of both phases in the time resolved ED spectra at several fixed pressures and temperatures.

The above structure study had a drawback due to the small effective energy region, within $40 \mathrm{keV}$. The small number of photon counts at higher energy emitted from the bending magnet at the Photon Factory storage ring could not provide large enough intensities to use in the refinement. However, a multianvil pressure apparatus MAX80 (Shimomura et al., 1989) set on the beam line of the Accumulator Ring of TRISTAN with $6.5 \mathrm{GeV}$ and $50 \mathrm{~mA}$ overcomes the difficulty for the structure refinements. The ED spectrum with an energy range of $10 \sim 150 \mathrm{keV}$ is available for profile fitting at high pressures. The pressure apparatus, with anvils composed of sintered diamond instead of tungsten carbide, can give a maximum pressure of $13 \mathrm{GPa}$ and temperature of 1700 ${ }^{\circ} \mathrm{C}$; the sample volume in the anvil is $2 \mathrm{~mm}$ in diameter and $3 \mathrm{~mm}$ high.

Structure refinements of Mo ( $\operatorname{Im} 3 m, Z=2)$ were examined by profile fitting of the ED spectrum (figure 7 ) falling in the range of $5.0 \sim 0.6 \AA$ observed at $5.9 \mathrm{GPa}$ and $800^{\circ} \mathrm{C}$ using the high pressure apparatus (Kikegawa et al., personal communication). The measurement duration was $1171 \mathrm{sec}$ with the ring current $8 \mathrm{~mA}$. The observed peaks in the spectrum are much more intense than those from the normal bending magnet with $2.5 \mathrm{GeV}$ and $300 \mathrm{~mA}$, and higher accuracy of the profile fitting can be expected than that obtained from the ordinary ED measurement.

\section{References}

Buras, B., Olsen, J. S., Gerward, L., Selsmark, B. \& ANDERSEN, A. L. (1975). Acta Cryst. A 31, 327$333 \mathrm{C}$.

Fukamachi, T., Hosoya, S. \& Terasaki, O. (1973). J. Appl. Cryst. 6, 117-122.

Gerward, L., Morup, S. \& Topsoe, H. (1976). J. Appl. Phys. 47, 822-825.

Glazer, A. H., Hidaka, M. \& Bordas, J. (1978). J. Appl. Cryst. 11, 165-172.

LangFord, J. I. (1981). Acta Cryst. A 37 Supplement. sample: Mo powder

$X$-rny source: SR (6. SGeY 30mA at AR)

$P$ and $T$ condition: $P=5.9 \mathrm{GPa} \quad T=300 \mathrm{~K}$ by MAX80

Derector: Ge-SSD. $2 \theta=8^{\circ}$, time $=1171 \mathrm{sec}$

E(keV)=0.0736 $\times \mathrm{N}(\mathrm{ch})+1.0746 \mathrm{mux}$ chso $=2048$
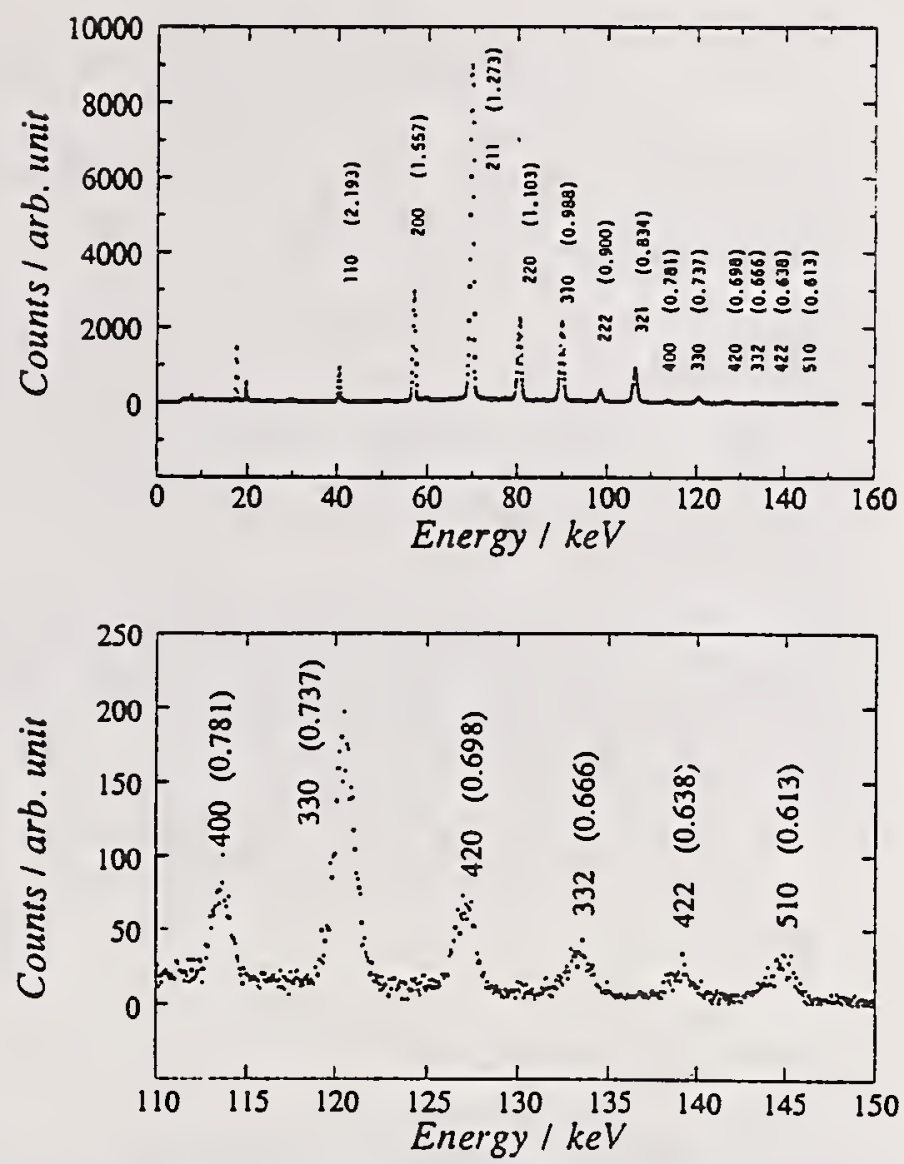

Figure 7: ED spectrum of Mo.

Malmros, G. \& Thomas, J. O. (1977). J. Appl. Cryst. 10, 7-11.

McNelles, L. A. \& Cambell, J. L. (1975). Nucl. Instr. Meth. 127, 73-81.

Parrish, W., Huang, T. C. \& Ayers, G. L. (1976). Trans. Am. Cryst. Assoc. 12, 55-73.

PaWley, G. S. (1981). J.Appl. Cryst. 14, 357.

RIETVeld, H. M. (1969). J. Appl. Cryst. 2, 65-71.

SASAKI, S. (1990). X-ray Absorption coefficients of the elements, KEK Rep. 90-16.

Shimomura, O., Taniguchi, T., Kanda, H., Akaishi, M., Yamaoka, S., Nagashima, T., Kikegawa, T., Kato, T. \& Yaoita, K. (1990). PF Activity, Rep. 290.

Victoreen, J. A. (1948). J. Appl. Phys. 19, 855-860.

Victoreen, J. A. (1949). J. Appl. Phys. 20, 1141-1147.

WiLson, A. J. C. (1973). J. Appl. Cryst. 6, 230-237.

Yamanaka, T. \& Ogata, K. (1991). J. Appl. Cryst. 24, 111-118.

Yamanaka, T., Sugiyama, K. \& Ogata, K. (1992). $J$. Appl. Cryst. 25, 11-15.

Young, R. A., Mackie, P. E. \& Von Dreele, R. B. (1977). J. Appl. Cryst. 10, 55-73. 
O1.1 THE USER-ASSISTED AUTO CALIBRATION PROCESS IN THE $\mu$ PDSM MICRO POWER DIFFRACTION SEARCH/MATCH. R. Gary Marquart, Fein-Marquart Associates, lnc., 7215 York Road, Baltimore, Maryland 21212, USA

$\mu$ PDSM (micro Power Diffraction Search/Match) continues to be improved and augmented. The latest addition is the ability to use multiple identified phases as internal standards. Even with well-aligned instruments and carefully prepared and mounted samples, some amount of systematic error is usually noted. The JCPDS database size is large and ever increasing; reducing systematic error permits the use of tighter match parameters, thereby enhancing the search/match specificity.

However, it is possible that large systematic errors will preclude automatic assignment of lines from the proposed internal standards to the correct corresponding problem pattern lines. For those cases, $\mu$ PDSM provides for user interaction, both numerically and graphically, in the correction/ calibration process, with the user identifying the fiduciary lines and assigning their correct values, both directly and by reference to the lines of selected PDF patterns.

The final internal standard calibration is iterative and uses all matched lines from the user-selected standards, weighing the contribution of each by its relative significance as defined by the relationship of its intensity to the user-specified standard error at that intensity.

O1.2 THE LIMITING EFFECT OF CRYSTALLITE SIZE ON ACCURACY IN QUANTITATIVE POWDER DIFFRACTION ANALYSIS. Deane K. Smith, Department of Geosciences. University Park, PA 16802, USA.

The effective number of diffacting crystallites in a sample is usually the limiting sample parameter on the potential accuracy obtainahle in quantitative powder diffraction analysis. A detailed analysis of the interactions hetween the number and orientations of the crystallites and the optics of the diffractometer indicate that the typical quantitative limit in present practices is of the order of 1 weight percent. Reduction of crystallite sizes to an average of 1 micrometer is critical to achieving this accuracy. This limit applies to whole-pattern matching and to the Rietveld method.

Detailed analysis of the ray paths of the diffracting crystallites reveals the primary angular limit of crystallite diffraction. The rocking range of the individual crystallites and the image size of the focal spot of the X-ray source and the receiving slit are the main controlling properties. The angular opening of the divergence slits have little effect except on the size of sample irradiated.

Simple modifications to the diffractometer and to its operation during data collection can improve crystallite statistics significantly, Broad focus optics and sample oscillation have more effect on increasing crystallite statistics than increased heam diverence or sample spinning. Optimum diffractometer optics are not the same as for high resolution, thus the modern diffractometer operating in most laboratories is not optimized for quantative or Rietveld analysis.
O1.3 X-RAY PHASE ANALYSIS IN TEXTURED MATERIALS. H. Oettel and P. Klimanek, Institut für Metallkunde, Bergakademie Freiberg, D-0-9200 Freiberg, BRD

The integral intensities of X-ray reflections strongly depend on the texture, respectively the pole density in the direction of investigation. Neglecting this effect, the error in X-ray phase analysis can reach more than $100 \%$ ! To suppress texture effects, it is possihle to apply special preparation procedures (powders) or to average results from different directions of investigation. For general treatment of the problem it is necessary to combine the formalism of quantitative texture analysis (description of quantitative pole figures [H. J. Bunge et al.: Cryst. Rev. 2 (1989) 65]) and the optimization algorithm of the multicomponent quantitative phase analysis [J. Fiala: J. Phys. D: Appl Phys. $\underline{5}$ (1972) 1874]. This can he realized in practice [H. Oettel, P. Klimanek: 1st Europ. Powder Diffraction Conference, Munich, 14 - 16th March 1991], if the number of the coefficients needed for descrihing the pole figures of different reflections is not too high. To keep the numher of coefficients small enough, it is useful to rotate the specimen around an axis perpendicular to the surface while measuring with different inclinations, so enforcing a fihre texture [M. Järvinen et al.: J. Appl. Cryst. 3 (1970) 313]. The uniform optimization model proposed here yields both the volume part of the phases and the texture coefficients of their enforced fihre textures. The efficiency of the procedure will he demonstrated hy experiments.

P1.1 QUANTITATIVE EVALUATION OF CRYSTALLINE AND AMORPHOUS COMPONENTS IN MIXTURES BY RIETVELD ANALYSIS OF X-RAY POWDER DIFFRACTION PROFILES. M. Bellotto, CISE, P.0. Box 12081, 20134 Milan, 1taly; and G. Artioli, Istituto di Mineralogia e Petrologia, Università di Modena, 41100 Modena, ltaly.

Accurate quantitative assessment of amorphous components in polycrystalline mixtures is a very complex and difficult task. Semiquantitative evaluations are generally carried out by combining results from chemical analysis and $\mathrm{X}$-ray diffraction techniques, but reliable estimates of the amorphous component are virtually impossible when crystalline and noncrystalline fractions in the mixture have similar chemical compositions. Such a case frequently occurs in materials having important industrial applications, as in natural zeolite-rich rocks, where the original volcanic glass has nearly the same chemical composition as the secondary zeolite minerals.

Both the Rietveld refinement and the Reference Intensity Ratio (RIR) techniques have been recently employed to successfully evaluate crystalline phase fractions in polycrystalline mixtures. We here propose a method for the quantitative determination of the amorphous component, based on a combination of the Rietveld analysis of powder diffraction profiles and of the internal standard matrix flushing technique. By successive dilution of the original sample with known amounts of a glassy component and a fixed amount of a standard material, it is possible to check for internal consistency of the extracted weight proportions. Linear regression on the concentration values obtained for the series of diluted samples leads to an independent estimate of the fraction of non-crystalline component in the original sample.

The method has been tested on two artificially prepared materials, and then applied to two clinoptilolite-rich tuffaceous samples from the Zlatokop deposit (Yugoslavia). The results obtained with both methods are in good agreement and allow a reliable estimate of the volcanic glass fraction in the rock. 
P1.2 MODAL ANALYSIS OF EIGHT MAJOR ROCK TYPES BY RIETVELD ANALYSIS OF X-RAY POWDER DIFFRACTION DATA. R.J. Hill, G. Tsambourakis and I.C. Madsen, CSIRO Division of Mineral Products, PO Box 124, Port Melbourne, Victoria 3207, Australia.

Mineral modes have been determined for specimens of eight natural rock types by multiphase Rietveld analysis of $\mathrm{CuK} \alpha \mathrm{X}$-ray powder diffractometer data. The samples include two granites, a granodiorite, adamellite, gabbro, basalt, trachyte amphibolite and a granulite. The normally severe problems encountered with preferred orientation (especially of the feldspar components) when using a Bragg-Brentano, flat-specimen configuration were eliminated by packing the samples in glass capillaries and using Debye-Scherrer geometry (Hill \& Madsen, 1991). Up to eight individual mineral components were measured in each sample, with a detection limit of 1 to 2 weight percent for the accessory minerals, depending on the mineral assemblage. The X-ray analyses compare favourably with (i) optical modes determined for the medium-coarse grained samples by point counting, (ii) normative calculations undertaken with locally enhanced catanorm and mesonorm software, and (iii) corresponding Rietveld modes determined, for two samples, from neutron powder data. The good agreement of the $\mathrm{X}$-ray and neutron modes shows that microabsorption is of little concern in these rocks. The results confirm one of the major advantages provided by Rietveld modal analysis over the more traditional "reference intensity" methods, namely, that the crystal chemistry (and thus the calibration constants) of the individual phases can be adjusted dynamically during each individual analysis (Hill, 1991). This not only provides more accurate phase analyses, but also gives important supplementary information about the unit cell dimensions, chemical composition and structural state of the major components.

HILL, R.J. (1991). Powder Diffraction, 6, 74-78.

HILL, R.J. \& MADSEN, I.C. (1991). Zeit. K'ristallogr. 196, 73-92.

\section{P1.3 SIGNIFICANCE OF PHASE ANALYSIS IN} HETEROGENEOUS CATALYSIS. S. Mehdi and M. Reddy, X-Ray Section, IICT Hyderabad, 500 007, AP, India.

Phase analysis plays an important role in the study of catalysis where the nature and state of the catalyst, its carrier and promoter greatiy influence its activity. Results of phase analysis study of a series of $\mathrm{TiO}_{2}$ supported $\mathrm{MoO}_{3}$ and $\mathrm{WO}_{3}$ and $\mathrm{ZrO}_{3}$ supported $\mathrm{MoO}_{3}$ catalysts, prepared by wet impregnation technique are presented in this paper. Variation of the $\mathrm{MoO}_{3}$ $\mathrm{TiO}_{2}-\mathrm{MoO}_{3}-\mathrm{ZrO}_{2}$ and $\mathrm{WO}_{3}-\mathrm{TiO}_{2} \mathrm{XRD}$ intensity ratio as a function of weight percent of $\mathrm{MoO}_{3}$ and $\mathrm{WO}_{3}$ respectively lead to estimates of crystalline contents in the catalyst. In the case of the $\mathrm{WO}_{3}-\mathrm{TiO}_{2}$ catalyst a good correlation was found between the intensity of crystalline $\mathrm{WO}_{3}$ loaded. For $\mathrm{MoO}_{3}-\mathrm{TlO}_{2}$ no such correlation is noted because $\mathrm{MoO}_{3}$ was found to be in an amorphous state and the intensity of $\mathrm{TiO}_{2}$ (anatase) remains unaffected by the increase in $\mathrm{MoO}_{3}$ loading. Variation of the XRD intensity ratio in $\mathrm{MoO}_{3}-\mathrm{TiO}_{2}$ is different from the other two catalysts. It passes through a minimum at $6 \mathrm{wt} \% \mathrm{MoO}_{3}$ and increases again. Formation of interstitial or substitutional solid solution could be inferred from the changes observed in the cell parameters of the active species or the support.
P1.4 POWDER DIFFRACTION IN PHARMACEUTICALS. L. Zevin, J. Bernstein, and S. Lach, Ben-Gurion University of the Negev, P.0. Box 1025, Beer-Sheva, 84110, Israel

During the past 6 years we have prepared more than 150 standard diffraction patterns for various pharmaceuticals in high demand. Though most of the studied materials are low-symmetry, large unit cell, low-absorbing, grinding-sensitive, orientation-prone and low-mass-available compounds, X-ray diffraction proved to be an excellent tool for their identification and quantification.

The experimental pattern was compared with a simulated one computed from the positional parameters obtained by single crystal structure analysis, when available. The X-ray technique is unique for the identification of the often numerous polymorphs, which have diverse physical and chemical properties crucial for pharmaceutical application, such as melting point, rate of dissolution, thermal stability, etc. Curiously, we have found that the polymorphs serving as standard pharmaceuticals sometimes vary from country to country. Polymorphs of anhydrous material are often accompanied by hydrated and solvated forms (pseudopolymorphs) which are also readily identified by powder diffraction.

Due to rapid decrease of the atomic form factor, appreciable thermal vibration and the sharp drop of the Lorentz factor, the characteristic powder diffraction patterns of the studied materials are confined to within $\sin \theta / \lambda \approx 0.1 \AA$, i.e. in the range of maximal instrumental peak distortion. Nonetheless a high degree of precision is generally achieved in the lattice constants (relative standard deviation is less than 5-10 $0^{-4}$ and $\Delta 2 \theta \leq 0.02^{\circ}$ ). Comparison of observed and calculated peak intensities usually gives an R-factor of $-25 \%$. This figure is quite satisfactory given the peak intensity measured and simplifications made in pattern simulation. In many cases we detected significant deviation between the lattice parameters reported by different authors and those observed in this study. This phenomenon, which is formally similar to solid solution effects, is probably caused by the presence of various amounts of retained solvent. Numerous examples are given of powder diffraction studies of pharmaceuticals with emphasis on polymorphism.

\section{P1.5} NEUTRON POWDER DIFFRACTION STUDY OF LEAD ZIRCONATE TITANATE CERAMICS. S. L. Town, C. J. Howard, E. H. Kisi* \& P. Bryant ${ }^{\dagger}$, ANSTO, PMB 1, Menai, NSW 2234, Australia, *Division of Science \& Technology, Griffith University, Nathan, Queensland 4111, Australia, fGEC Marconi, Faraday Park, Meadowbank, NSW 2114, Australia.

Lead zirconate titanate ceramics are widely used in piezoelectric transducer applications ${ }^{1,2}$. These materials have their best piezoelectric properties, and the samples pole more easily, near the morphotropic phase boundary. Although there have been some neutron diffraction studies ${ }^{3,4}$ on these materials, there has been little work done on and around the phase boundary. Here we report room temperature neutron powder diffraction data collected on samples with the following compositions: $\mathrm{Zr}$ $42.5 \%, 47.5 \%, 49 \%, 50 \%, 52.5 \%, 55 \%, 57.5 \%, 60 \%$ and $62.5 \%$. Details of QPA results are given.

1. P. Bryant, Ceramic Developments, Materials Science Forum Volumes 34-36 1988, p285.

2. P. Bryant, Ceramic Developments, Materials Science Forum Volumes 34-36 1988 p 249.

3. H. Fujishita \& S. Hoshino, J. Phys. Soc. Jap., Vol 53, No 1, 1984, p226.

4. A. M. Glazer, S. A. Mabud \& R. Clarke, Acta Cryst, B34, 1978, p1060. 
P1.6

SEARCH/MATCH PROCEDURES WITH WHOLE-PAT-

TER. MATCHING. Deane K. Smith, Susan Q. Hoyle and Gerald G. Johnson. Jr., Materials Research Laboratory, The Pennsylvania State University, University Park, PA 16802, USA.

Computer-based identification procedures in powder diffraction have employed numerical matching schemes relying on the $d-I$ data sets since 1962 . With the availability of digitized diffraction traces, it is now feasible to use patternmatching techniques based on the characteristic regions of the full diffraction traces of the unknowns and the reference patterns.

Databases containing digitized diffraction patterns may be composed of experimental data or data calculated from crystal structures or simulated from d-I data sets. Search procedures use matching algorithms which emphasize the probablility of the reference pattern being contained in the pattern of the unknown regardless of the other patterns which comprise the unknown. Rapid matching algorithms and sensitive figures-of-merit are critical to success.

Successful searching requires techniques compensating for sample and instrument aberrations and variations in materials such as solid solution. Successful searching yields candidates for matching and stripping from the initial data trace for further search analysis. This matching involves profile fitting then subtraction, which effectively removes the phase contribution to the unknown pattern. Sequential search/match/stripping allows more phases to be detected than are usually obtained with $\mathrm{d}-\mathrm{I}$ matching, and the levels of detection are usually lower for the minor phases. 
O2.1 ACCURATE DETERMINATION OF UNIT-CELL PARAMETERS USING AN INTERNAL STANDARD REFERENCE MATERIAL AND WHOLE-PATTERN FITTING. Hideo Toraya, Ceramics Research Laboratory, Nagoya Institute of Technology, Asahigaoka, Tajimi 507 Japan

A new technique for peak-shift correction achieved the accuracy of a few p.p.m. in routinely determining the unit-cell parameters of a sample with internal standard reference material (Toraya \& Kitamura, J. Appl. Cryst., 23, 282285, 1990; Toraya \& Parrish, PICXAM, 1991). This algorithm is based on 1) the simultaneous least-squares determination of both unit-cell parameters and unknown parameters in the calibration function and 2) the use of observed profiles with symmetric shape. The first idea in the above two has already been realized by the whole-powder-pattern fitting without reference to a structure model (Toraya, Advances in Ceramics, Vol 21, 811-819, 1987). However, the profile asymmetry usually observed in conventional powder diffractometry induced a strong parameter correlation between the peak positions (unit-cell parameters) and parameters in the function for modeling profile asymmetry, and the required accuracy could not be realized. Recent use of a high-resolution powder diffractometer and a synchrotron radiation source make it possible to obtain nearly symmetric profile and greatly improve the accuracy of determining the peak positions. The use of whole-powider-pattern fitting has a great advantage in that the method can easily be extended to the analyses of samples with low crystallographic symmetry. In the present study, the unit-cell parameters of samples of cubic to orthorhombic symmetry were measured by using whole-powder-pattern fitting on the above two bases. The procedure will have potential uses in the routine detrmination of unit-cell parameters with both conventional tube-generated X-ravs and synchrotron radiation.

P2.1 A UNIVERSAL FILE FORMAT FOR POWDER DIFFRACTION DATA ARCHIVE AND INTERCHANGE: THE IUCr CRYSTALLOGRAPHIC INFORMATION FILE (CIF) FORMAT. B. H. Toby, Air Products \& Chemicals Inc, 7201 Hamilton Boulevard, Allentown, PA 18195, USA; J. I. Langford, University of Birmingham, School of Physies \& Space Research, Birmingham B15 2TT, UK; and S. R. Hall, Crystallography Centre, University of Western Australia, Nedlands 6009, Australia.

Powder diffraction data items have been defined for use with the Crystallographic Information File (CIF) approach to data storage and exchange. These will permit the ready exchange of processed and raw powder data generated by diffractometers of every type: commercial sealed-tube instruments; CW synchrotron; neutron; energydispersive; and time-of-flight. International collaboration is vital to ensure that the definitions meet the needs of the powder diffraction community.

The Crystallographic Information File (CIF) has been developed by the IUCr for storage of crystallographic data ranging from measured intensities through structural diagrams and manuscript text [S. R. Hall, F. H. Allen \& I. D. Brown, Acta Cryst. A47, 655-685.] A ClF is composed of ASCIl characters which may edited with a normal text editor, and transmitted via electronic networks (e.g. email or ftp). A CIF is completely self-descriptive in that data items are identified by unique tags (referred to as a data name). These data names may be defined locally or globally. Global data items (i.e. those used outside the local environment) are carefully defined in a CIF data name dictionary that is also electronically stored in a CIF-like format.

Powder diffraction data are usually measured by computer-controlled diffractometer or densitometer and are stored in a data file format specific to the measurement instrument. Typically these data are then processed using computer programs modified to use data in the local format. If the data will be published or archived, the diffractogram will usually be reduced to a peak table of position and intensity values. Apart from the need to standardise the data processing soft ware, the need for the global exchange of unprocessed powder diffraction data is increasing. This need arises because of the use of whole pattern methods for (i) crystalline phase identification and quantification, (ii) ab initio structure determination, (iii) structural refinements, and (iv) characterization of materials with limited ordering (e.g. polymers and clays), where the diffractogram cannot be represented as a set of peaks.

Definitions for CIF data items used in powder diffraction are proposed. These are in addition to those data items in the proposed 1991 Core CIF Dictionary. Discussion of these definitions is to be encouraged prior to their formal adoption at the 1993 IUCr Congress.

\section{P2.2 INSTRUMENT ALIGNMENT METHODS FOR RESIDUAL} STRESS MEASUREMENTS BY MEANS OF NEUTRON DIFFRACTION. P.C. Brand and H.J. Prask, National Institute of Standards and Technology, Gaithersburg, MD 20899, U.S.A.

Neutron powder diffraction in general, and residual stress measurements by means of neutron diffraction in particular, require very well aligned instrumentation.

Unlike regular powder diffraction where the specimen is fully immersed in the neutron beam, stress measurements by means of neutron diffraction require the presence of small beam apertures that define the size of the gauge volume in the specimen. It is the gauge volume for which one determines the stress state during a stress measurement.

The correct positioning of the beam apertures is crucial for successfully applying the stress measurement technique. Therefore we have developed a sequence of alignment procedures to assure correct positioning. These employ diffraction in a small test specimen situated at a known location on the neutron diffractometer, and a new analysis method of the diffraction data taken from the test specimen. These will be presented. The presented alignment methods have proved extremely helpful in obtaining very well aligned beam apertures in a reproducible manner.

\section{P2.3 A REFERENCE SPECIMEN FOR}

HIGH RESOLUTION LINE PROFILE ANALYSIS. PREPARATION, CHARACTERIZATION AND APPLICATION. J.G.M. van Berkum, G.J.M. Sprong, Th.H. de Keijser, E.J. Sonneveld, A.C. Vermeulen \& R. Delhez. Delft University of Technology, Laboratory of Materials Science, Rotterdamseweg 137, 2628 AL Delft, The Netherlands.

High resolution line profile analysis requires a high quality standard reference specimen to characterize the instrumental line profile. A universal reference has to meet very stringent criteria, of which negligible structural broadening is the most important. Si SRM 640a/b powder has been selected as an appropriate starting material. Reference specimens have been prepared according to a standardized procedure, which includes (i) removal of the smallest particles $(<5 \mu \mathrm{m})$, (ii) sedimentation in 2-propanol of a mono-layer of particles onto a $\mathrm{Si}$ (510) single crystal substrate (for low background) and (iii) annealing for $20 \mathrm{~h}$ at $1273 \mathrm{~K}$. The sedimented powder sticks to the substrate and is very homogeneously distributed. Loose fractionated powder, annealed in a rotating ampulla, can be applied as an internal standard.

Using a conventional powder diffractometer the reference specimens yield narrower line profiles than those reported in the literature (down to $0.050^{\circ} 2 \theta$ full width at half maximum) and the breadth $v s .2 \theta$ plots show very smooth curves. As a comparison $\mathrm{LaB}_{6} \mathrm{SRM} 660$, also sedimented onto a Si substrate (but no other trearment), yields systematically broader lines over the complete $2 \theta$ range and the breadth $v s$. $2 \theta$ plot shows deviations from a smooth curve due to (hkl]-dependent structural broadening. Crystal statistics is the limiting factor for the line profile reproducibility. For a spinning specimen with $2-4 \times 10^{4}$ particles per $\mathrm{mm}^{2}$ the integral intensity and the breadth can be reproduced within $1 \%$. An improvement is possible by preparing thicker specimens, having several lavers of powder particles, but only at the cost of extra 'transparency' broadening. Essentially mono-layered non-spinning specimens are not suited as a reference.

Using the new reference specimens line profiles with a very small amount of size and strain broadening can be interpreted reliably. As an example, line profiles from ball-milled particles $(1-10 \mu \mathrm{m})$ of various materials have been studied. Although the integral breadths of the measured lines may be smaller than $0.1^{\circ} 2 \theta$ at low angles, they can be analysed reliably using Stokes deconvolution. Besides high resolution line profile analysis, the new class of standard specimens also provides a means for assessing the state of any powder diffractometer. The smallest deviations from the perfect alignment, from the ideal shape of a focus or from the average room or cooling-water temperature result in detectable breadth changes. 
P2.4 SELFCORRECTING METHOD FOR PRECISE DETERMINATION OF LATTICE PARAMETERS. M. Ya Gamamik, Institute of Geochemistry and Physics of Minerals, Academy of Sciences, Palladin Pr. 34, Kiev 252680, Ukraine

The standardless method is proposed for lattice parameters determination by $\mathrm{X}$-ray diffractometer. Goniometer aberrations, distorting Bragg's reflections, can be accounted for by the method. The displacement of the sample reflecting plane from the goniometer axis, the zero detector position, the longitudinal and transversal components of goniometer eccentricity are determined from reflections locations for both large (analytical reflections) and small (correcting reflections) Bragg angles. These aberrations are calculated from the equation systems including also the lattice parameters as unknown quantities. The method accuracy is determined by that of the angle measurement and by the positions of analytical and correcting reflections. The method can be used for polycrystal and monocrystal samples of any crystallographic system.

P2.5 USE OF AN AUTOMATED POWDER DIFFRACTOMETER TO PERFORM LATTICE PARAMETER REFINEMENT. R. A. Newman, Analytical Sciences Laboratory, 1897 G Building, Dow Chemical, Midland, Michigan 48667, U. S. A.

It is well known that carefully performed experiments using specialized powder diffraction instrumentation to analyze pure, crystalline materials can yield lattice parameters with a precision of $1 \times 10^{-5}$ or better. However, this is seldom the case when using standard laboratory diffraction equipment, especially Bragg-Brentano geometry powder diffractometers. The errors associated with the reflection geometry, sample size/preparation, etc. for typical diffractometers limit lattice refinement performed on these units to precision on the order of $1 \times 10^{-4}$ in the best cases.

At Dow, we have improved our ability to perform diffractometer-based lattice refinements by using the combination of [1] a Siemens D-500 diffractometer equipped with an incident beam monochromator, [2] the use of zero-scatter sample holders, and [3] our in-house developed profile-fitting software. With this system, we are able to obtain data from a $\mathrm{K} \alpha_{1}$ source, which greatly simplifies the diffraction pattern. The use of a shallow depth zero-scatter sample holder reduces the effect of sample transparency and flat sample errors. Finally, the ability to perform interactive profile decomposition base on a split Pearson VII algorithm improves our accuracy in peak centroid determination. Utilizing this approach, we are able to routinely obtain lattice refinement results on the order of $1 \times 10^{-4}$. The various steps in the data collection and analysis procedure will be illustrated, and examples of lattice refinements will be shown.
P2.6

ACCURACY IN POWDER DIFFRACTION: OPTIMISATION OF DATA COLLECTION STRATEGIES. W.I.F.David, ISIS Facility, Rutherford Appleton Laboratory, Chilton, Didcot, Oxon., OX11 0QX, U.K.

$\mathrm{X}$-ray and neutron constant wavelength powder diffraction are conventionally collected in step scan mode with constant time increments or with a constant monitor count. From an instrumental viewpoint these methods are clearly the simplest - additionally, of course, the resulting pattern has a Bragg peak intensity variation that is similar to that obtained by photographic methods. For neutron diffraction data this generally is an adequate procedure since the high angle Bragg peaks do not suffer from a form-factor fall-off. However, in $\mathrm{X}$-ray powder diffraction the atomic scattering factor variation as a function of $\sin \theta / \lambda$ may lead to intensity differences that exceed two orders of magnitude between low and high angle data. Standard least-squares for X-ray profile refinement is often dominated by fitting the first few Bragg peaks (which contain the least structural information!). The principle of optimised powder diffraction data collection strategy that will be discussed is similar to well-known leverage techniques that are used in single crystal data collection. Simple theoretical models will be presented and initial results discussed.

P2.7 ON THE PRECISION AND ACCURACY OF POWDER DIFFRACTION STUDIES OF KAOLINITE AND DICKITE. L̆. Smrčok, Institu te of Inorganic Chemistry; Slovak Academy of Sciences, 84236 Bratislava, Czechoslovakia.

Kaolinites are known to exist in four polytypic forms which differ only in the stacking mode of simple layers formed by $\mathrm{SiO}_{4}$ tetrahedral and $\mathrm{Al}(\mathrm{O}, \mathrm{OH})_{6}$ octahedral sheets. Since kaolinite, in contrast to dickite, has not, with one exception, yielded single crystals suitable for structure analysis, (the results are, however, of lower quality) all structure refinements carried out so far have been based on powder data. Such refinements are hampered mainly by heavy overlap of the majority of individual reflections (kaolinite is triclinic) and by kaolinite's extreme tendency to orient preferentially. In order to evaluate the precision and/or accuracy of the refined structure parameters, we compared the interatomic distances $d(\mathrm{Si}-\mathrm{O})$ and $d(\mathrm{Al}-\mathrm{O})$ calculated from the data reported in ten powder refinements of both well ordered and partially disordered kaolinite specimens. The main tool used in our considerations were median-based box-and-whisker plots. We have found, that the $d(\mathrm{Si}-\mathrm{O})$ are within the interval $<1.43,1.8 \%>\AA$ while $d(\mathrm{Al}-\mathrm{O})$ vary from $<1.64,2.12>\AA$; the expected values being 1.617 and $1.91 \AA$, respectively. A comparison of these individual refinements with two reliable single crystal analyses of dickite has shown that the spread of distances is in only one case comparable with the single crystal results. Several authors have reported rather precise but inaccurate distances, or vice versa. As far as the medians are concerned, all refinements have provided very close results, but the sizes of the boxes vary significantly from one refinement to another. Potential sources of difficulties are (not ordered according to their relative importance): preferred orientation wrongly accounted for; weights emphasizing the role of small intensities, which dominate in the region of heavy overlap and are measured frequently with very limited precision; small ratio of the number of Bragg reflection to the number of refined structure parameters; and limited ability of the method to decompose unambiguousiy profiles that are heavily overlapped. It is rather surprising that the accuracy of the interatomic distances is almost independent of the presence or absence of disorder in the structure. 
O3.1 THE FAST ITERATIVE PATTERSON SQUAFING (FIPS) METHOD - AN IMPROVED TREATMENT OF SEVERELY OVERLAPPING REFLECTIONS IN A POWDER PATTERN FOR THE APPLICATION OF DIRECT METHODS. M. Estermann and V. Gramlich, Institute of Crystallography, ETH, CH8092 Zürich. Switzerland.

Single crystal techniques have been used to solve a number of structures $a b$ initio from powder data (see, for example, refs. (I) and (2), and references therein).

Obviously, these tecbniques require a single-crystal-like data set, which has to be extracted from the powder pattern. Extraction programs cope well with partial overlap of diffraction intensity in the powder pattern, but not with severe or exact overlap. Usually the intensity ratio of tbese severely overlapping reflections is set to one (equipartitioning).

Unfortunately, direct methods, which are so successful for routine single crystal structure determination, often fail when used with an extracted powder data set. The limiting factors are tbe complexity of the crystal structure and the number of severely or exactly overlapping refections in the powder pattern.

In our laboratory, we bave recently developed a new method, the Fast Iterative Patterson Squaring (FIPS) method $(3,4)$, for unravelling the intensities of severely or exactly overlapping reflections. Starting from an equipartitioned data set, a Patterson map is generated, each point in the map is squared, this new map is backtransformed to obtain new Fourier coefficients, and these coefficients are then extrapolated to give a new set of $|\mathrm{F}(\mathrm{hkl})|^{2}$ and a new intensity distribution for the overlapping reflection (non-overlapping ones remain unchanged). The cycle is repeated until the intensity statistics of the overlapping reflections approximate those of the non-overlapping ones. Tests with powder data obtained from materials with known crystal structures proved to be successful.

Tbe molecular sieve SAPO-40 of unknown structure, which could only be synthesized in the form of a polycrystalline powder, provided a real test case for the FIPS method (5). Approximately $65 \%$ of all the reflections were affected by severe overlap. and the structure could not be solved from tbe equipartitioned data. However, after the intensities bad been redistributed using the FIPS metbod, the structure was solved by direct methods.

(1) McCusker, L. (1988). J. Appl. Cryst. 21, 305-3I0.

(2) Hiraguchi et al. (1991). J. Appl. Cryst. 24, 286-292.

(3) Estermann, M. (199I). Thesis.

(4) Estermann, M. and Gramlich, V. (1992). in preparation.

(5) Estermann, M. and McCusker, L. (1992). in preparation.

\section{O3.2 COMBINED RIETVELD AND SHORT RANGE ORDER}

PARAMETER REFINEMENT. J. Schneider, Institut für Kristallographie und Mineralogie, Universität München, Theresienstrasse 41, D-8000 München 2 Germany.

Although the Rietveld method ${ }^{1}$ has become a widely accepted technique in powder diffraction to refine structural parameters from Bragg peak profiles, less attention has been given to refinement of concurrent diffuse disorder gcattering. Substitutional disorder in binary alloys produces a modulation of monotonic Laue scattering intensity if short range order is present. For cubic polycrystalline alloys this may be written as a series expansion ${ }^{2}$ :

$$
I_{S R O}=N x_{A} x_{B}\left(f_{A}-f_{B}\right)^{2} \sum_{i} c_{i} \alpha_{i} \sin \left(k r_{i}\right) /\left(k x_{i}\right)
$$

$\mathrm{N}$ is the number of lattice sites, $\mathrm{x}_{\mathrm{A}}$ and $\mathrm{x}_{\mathrm{B}}$ are the fractions of the $\mathrm{A}$ and $\mathrm{B}$ components, respectively, $f_{A}$ and $f_{B}$ their formfactors (scattering lengths), $c_{i}$ is the number of neighbors in shell $i, r_{i}$ it's radius, $k=4 \pi \sin \Theta / \lambda$ and $\alpha_{i}$ are the well known Warten-Cowley short range order parameters. Eq. (l) $(i=1 . .6)$ was incorporated into the local PC version ${ }^{3}$ of a standard Rietveld program ${ }^{4}$.

Application of the program to neutron data ${ }^{5}$ of $\mathrm{Na}$ deficient $\mathrm{Na}_{\mathbf{x}} \mathrm{Tl}_{1-\mathrm{x}}, \mathrm{x}=$ 0.47 showed that the observed diffuse intensity can well be described by eq. (1). The major contribution arises from a term which yields $r_{1}=3.227(9)$ $A$. This agrees well with the next neighbor distance $d=\sqrt{3} a_{0}=$ 3.2250 (4) $\dot{A}$ derived from the Rietveld refinement. Assuming that the heavy Tl atoms do not take part in substitutional disorder, this result suggests short range order between $\mathrm{Na}$ atoms (and vacancies) on the $\mathrm{Na}$ sublattice.

${ }^{1}$ Rietveld, H. M., J. AppI. Cryst. 2 (1969) 65.

${ }^{2}$ Warren, B. E., X-ray diffraction, Addison-Wesley 1969.

${ }^{3}$ Schneider J., Dinnebier R. E., Material Science Forum 79/82 (1991) 277.

${ }^{4}$ Sakthivel A., Young R. A., Int. Worksbop on the Rietveld method, Petten I989.

${ }^{5}$ Schneider J., Material Science Forum $27 / 28$ (1988) 63.
P3.1 USE OF THE CSD PROGRAM PACKAGE FOR STRUCTURE DETERMINATION FROM POWDER DATA. L. G. Akselrud, P. Yu. Zavalii, Yu. N. Grin, V. K. Pecharski. Institute of Inorganic Chemistry, Lviv State University, 290005 Lviv, Ukraine \& B. Baumgartner, E. Wölfel, STOE \& Cie, 6100 Darmstadt, Germany

The CSD program package has been developed to combine the possibilities of single crystal and powder diffractometry for crystal structure research and offers to the user tools for solution, refinement and interpretation of the crystal structure independent of the data collection method. It runs on a PC XT/AT/PS2 or fully compatible computer systems under DOS3.3 and higher.

The power of this program system has been tested using the powder data of various samples with already known structures. These crystal structures have been investigated using STOE powder diffractometers for data collection and CSD as the computational system, starting from reflection indexing and followed by structure solution and integrated-intensity or full-profile structure refinement. Different kinds of data collection (transmission and Bragg-Brentano geometries) have been compared in respect to their advantages for structure solution. The samples comprise both inorganic and organic compounds with crystal systems ranging from triclinic to cubic, thus presenting usual experimental situations. These include fully overlapped peaks as a result of symmetry of the unit cell, partially overlapped peaks dependent on unit cell parameter relations, and different variation of peak-half-widths with theta for different reflection zones.

The precision and accuracy of these results are discussed.

P3.2 SOFTWARE PACKAGE PATCOM FOR SIMULATION AND MANIPULATION OF POWDER DIFFRACTION PATTERNS AND STANDARDS. Ya. E. Cherner, Institute of Physics, 194 Stachki Ave., Rostov-on-Don, 344104, Russia.

The PATCOM software package is intended for simulating, comparing, displaying, and printing powder diffraction patterns of single- or multiphase samples and solid solutions using crystal structure data for the substance or its isomorph. It consists of five programs.

SCREEN is a program for preparation of the input files. The SITES and HKL programs calculate, by means of symmetry operations, the atomic position multiplicities and coordinates of all atomic sites, as well as the Miller indices, $\mathrm{d}$ spacings, angular positions, and multiplicity factors of all lines within the desired angle range.

INTENS computes both the integrated and relative intensities of Bragg reflections. It also calculates the unit cell weight, volume, density, and the mass absorption factor. INTENS allows the creation of lattice vacancies, and can place up to 5 different atoms (ions) into a single atomic position.

PICTURE is an interactive color graphics program for:

- displaying or printing up to 5 theoretical or measured patterns, or powder diffraction standards simultaneously as a 2-, or 3-dimensional picture (line- and continuous diagrams are available);

simulating and storing the patterns of multiphase substances;

- comparing patterns of different origin by means of superposition and/or subtraction.

Five profile functions are available for peak shape simulation and the profile parameters may be changed. STRART enables crystal structure to be displayed or plotted.

Options include rotation of a structure model around any direction, shift of any atoms and recalculation of the theoretical pattern for a modified atomic arrangement, drawing of the unit cell projection on a desired plane, etc. 
P3.3 SOFTWARE PACKAGE FOR POWDER DIFFRACTION DATA PROCESSING. Ya.E. Cherner, Institute of Physics, 194 Stachki Ave., Rostov-on-Don, 344104 , Russia.

The software package PD-ASSISTANT is intended for handling measured powder diffraction patterns. It consists of four programs.

The PATTR program offers many facilities for displaying and treating patterns. It also creates the input parameters files for the BCGR and PEFIT programs.

The BCGR program for background intensity elimination is based on our own algorithm. The background is interpolated with a polynomial of degree up to 6 . The effect of spread in the raw background data is eliminated by means of the use of an intensity-deviation band. PATTR has a routine for choosing a band width directly on a display. BCGR can create output files containing a list of Bragg peak regions, refining polynomial coefficients, running protocol, and other useful information. The program runs in both autopilot and interactive mode. A user has the opportunity to mark on the pattern some areas belonging to background or peaks and to exclude areas using PATTR. This improves the result when the measured pattern is not sufficiently good.

The PEFIT program allows the separation of overlapped Bragg peaks and the refinement of position, integral intensity, and FWHM of both single and overlapped peaks. This routine employs the Powel method. The list of profile functions available for description of the peak shape includes Gaussian, two Lorentzians, pseudo-Voigt and Pearson VII. Each Bragg reflection is approximated by the $\mathrm{K}_{\alpha} 1-\mathrm{K}_{\alpha} 2$ doublet; the ratio of the intensities of its components can be set by the user (the default is 2:1).

The COMP utility program allows the comparison of two measured patterns, to shift them with respect to each other, and to display the difference diagram on a separate panel.

\section{P3.4 PDN-PROGRAM FOR RIETVELD ANALYSIS, A. B.} Tovbis and Ya. E. Cherner*, Institute of Crystallography, 59 Leninsky Ave., Moscow, 117333, * Institute of Physics, 194 Stachki Ave., Rostov-onDon, 344104, Russia.

PDR IS a program for Rietveld analysis of X-ray and neutron powder diffraction patterns. It runs on IBM PCs and compatibles (540 kB memory required). The raw pattern file can contain up to 10,000 data Points, the allowed number of Bragg reflections is less than 4,000, and up to 10 phases can be refined simultaneously. The list of profile-shape functions includes Gaussian, three Lorentzians, pseudo-Voigt, Pearson VI, and Thomson-CoxHastings pseudo-Voigt. PDR takes automatic account of symmetry dependent atomic coordinates, anisotropic temperature factors and unit-cell parameters. The program has an extremely easy and flexible way of creating and editing input parameter flies in an interactive manner and a routine for set-up of the input files using a data entry screen (for beginners).

A color graphics utility enables the display and plotting of the raw and refined patterns, the difference diagram and a structure model.

The PDR's runtime for a test data set is half the corresponding runtime of the well known program DBW 4.1 (890101).

The use of PDR together with PD-ASSISTANT and PATCOM software packages provides the best starting model and an optimal strategy for the Rietveld refinement.
P3.5

A VERSATILE PROGRAM FOR RIETVELD REFINE. MENT. J.F. Bérar $\left(+^{*}\right)$ and P. Garaier $(+),(+)$ Chimie-Physique du Solide (UA 453 CNRS), Ecole Centrale, 92295 Châtenay, France and $\left(^{*}\right)$ LURE, Université Paris-Sud, 91405 Orsay, France.

The XND program for crystal structure refinement of powder data was written in order to take into account the correlations between data obtained in real-time experiments for which the statistical qnality of each diagram is very low.

Line profiles are represented by Voigt functions, new terms have been added to describe the evolution of line width and asymmetry. Preferred orientation effects are implemented using spherical harmonic functions. These functions are also used to allow an anisotropic broadening due to size or strain effect for the sample dependent part of the line width.

This allows us to reach very good profile agreements even if data have been collected with a conventional X-ray diffractometer ( $R_{w p}=0.035, G o F=1.2$ have been obtained with various $M V_{2} \mathrm{O}_{6}$ samples).

Some other possibilities (anomalous scattering, bond length penalties and rigid bodies) allow its use for complex structural problem : for example zeolites or stearate salts.

\section{P3.6 A RIETVELD PC-PROGRAM SYSTEM ADAPTED FOR} THE GUINIER METHOD. N. O. Ersson, Solid State Chemistry, Uppsala University, Box 531, S-751 21 Uppsala, Sweden

The incomparable sharpness of the diffraction lines obtained with a Guinier film camera may give problems when the observed data, as obtained with a film scanner, ${ }^{1}$ are used in a Rietveld program. The problems in finding a profile function describing the complex angle dependence are well known. ${ }^{2}$ Another complication is the deviation from linear relationship between film distance and Bragg angle that is always present on a film. Even small distortions may cause an incomplete overlap when observed and calculated data are compared.

The use of an internal calibration substance can eliminate the latter complication. We use high-purity Si, the NIST standard SRM 640b. In the presented system an angular correction function is first calculated. This function is then used to "rubber-band stretch" the theta-scale, so that the distance between two observed points is no longer constant. In practice this is obtained by interpolating the observed intensities and maintaining a constant step-length for the input data to the Rietveld refinement.

The present Rietveld program is a modified version of DBWS-9006PC written by Young and Satthivel. ${ }^{3}$ Besides some changes in data input (to provide for different formats) functions for Lorentz factors for the Guinier geometry have been incorporated. A display program for easy graphics control of the output has also been written using the QuickBasic language, as for the correction programs. The Rietveld program is still written entirely in FORTRAN-77 language.

${ }^{1}$ Johansson, K.-E., Palm, T., and Werner, P. E., Rev. Sci. Instrum. 13, 1289-1291 (1980).

'Ersson, N.0., J. Appl.Crystallogr. 12, 295-302 (1979).

${ }^{3}$ Sakthivel, A., Young, R. A., User's Guide to program DBWS-9006PC (1991). 
P3.7 MACINTOSH POWDER DIFFRACTION SOFTWARE. A. W. Hewat, Institut Laue-Langevin, 156X Grenoble, 38042 FRANCE.

Personal computers now rival workstations for processing speed, and have the advantage of a common, user-friendly windowing interface. For example, the new Macintosh Quadra machine, using the 20MIPS 68040 processor, runs powder profile refinement programmes at typically 10-20 seconds per refinement cycle, rivalling the ILL-Vax central computer !

Standard mainframe Fortran, Pascal and C programmes can be compiled and run as easily as on any other computer, since there are no limitations to the size of the code, and a simple windowing environment is added automatically.

With a little work, more sophisticated windowing environments can be included, greatly simplifying the input of data and the examination of results. An example of this second class of programme is Mac-Lazy for the calculation of powder patterns. Much of the data can be entered simply by pointing and clicking, and the results plotted in independent graphic windows. The structure can be drawn in another window, rotated, scaled and 'animated' in real time to emphasise its 3D nature.

Personal computers can be justified if only for 'word-processing', but can in practice be used for much more. Macintosh versions of some of the 'classical' powder diffraction software will be shown, together with applications using more sophisticated user interfaces, such as Mac-Lazy.

P3.8 COMPUTER PROGRAM “CUVFIT" FOR DECOMPOSITION OF MULTIPHASE POWDER DIFFRACTION PATTERNS. S. L. Wang, Department of Chemistry, National Tsing Hua University, Hsinchu, Tajwan 30043, R. O. C.

The program CUVFIT has been developed for analyzing powder diffraction patterns which may contain several phases. Determination of the intensities of completely overlapping reflections is feasible by using this program. Up to ten peaks can be fitted and refined simultaneously, including phases that can be stipulated to have only certain reflections. The program handles various peak shapes such as Gaussian, Lorentzian, pseudo-Voigt, and Pearson VII. Angular dependence of the peak's FWHM for each phase can be best-determined by the program and used in the next fitting cycle. Either shifted Tchebychev polynomials (degree 2) or the usual tangent function can be chosen for describing the behavior of the width. Other parameters include peak position, asymmetry factor, and polynomial background. The changes, such as the zero offset of the $2-\theta$ value, and constraints between various parameters, can be made easily. The program is essentially an individual profile-fitting one which aims at apportioning observed intensities to the individual components analytically. Details of the program and its applications to several complex systems will be presented.
P3.9 EXTRACTING STRUCTURE FACTORS FROM POWDER DIFFRACTION DATA BY ITERATING FULL PATERN PROFILE FITTING. A. LE Bail. Laboratoire des Fluorures. Université du Maine, 72017 Le Mans Cedex. France.

New strategies using old formulae sometimes lead to efficient algorithms. Every user of the Rietveld method has worked with the so-called "lobs" (or - $\mid$ Fobs $\left.\right|^{\circ}$ ) either by the simple presentation of the Bragg $R$ facto: or by Fourier difference calculation or so on. Some special uses of the Rietveid's formula for "|Fobs|" extraction were made (to be classified among the twostages altematives to the Rietveld method). however the starting $\mid \mathrm{F} / \mathrm{s}$ were always the $\mid \mathrm{Fcalcls}$ corresponding to a (complete or partial) structure model Toraya. Marumo and Yamase. Acto Cryst.. 1984, 840, 1<5-150 Taylor. Miller and Bibby. Z. Kristallogr.. 1986. 176. 183-192: Taylor, Z. Krisallogr., 1987. 181, 151-160)

In the original Rietveld's work, the $\mid$ Fobs $\mid$ " are estimated by partitioning among reflections contributing to each profile point of the pattern according to the $\mid$ Fcalcl. thus they are biased. However, it will be shown that iterating the Rietveld's formula provides a nice and simple way to extract $\mid$ Fobs| as accurately as possible. starting from initial $\mid \mathrm{Fls}$ arbitrarily set to have the same value. The first study applying such an algorithm for structure determination purpose (using the unpublished program ARITB) was from Le Bail, Duroy and Fourquet. Mater. Res. Bull., 1988, 23, 447-452. Near 20 $a b$ initio structure determinations have been now performed in this way. either from conventional or synchrotron X-ray powder data. The algorithm has been implemented as an option in two other programs (FULLPROF. GSAS): any Rietveld program can be easily modified to accommodate this possibility to extract structure factors. The strategy necessary for success will be exposed. The best results are from cell and space group constrained conditions: only all equal starting $|\mathrm{F}| \mathrm{s}$ can ensure that the structure factors of strictly overlapping reflections are undifferentiated: they keep the same value after each iteration: it is strongly recommended to start from very precise cell parameters (not approximated ones) In order to avold false minima in case of complicated patterns with strong overlapping: some iterations are necessary without modifying cell and profile parameters in order to start their least-squares refinement of further cycles in good conditions. A definite advantage of the procedure is the very low number of parameters to be refined ( 15 moximum in ARITB) allowing $t$ to handle problems of any size. Extracting in one run the several thousand $\mid \mathrm{F} / \mathrm{s}$ (for instance from synchrotron powder data with 0.02"20-FWHM) implicit for large and/or low symmetrical cells is not a utopia.

\section{P3.10 MAXIMUM ENTROPY METHOD ANALYSIS OF X-RAY} AND NEUTRON POWDER DIFFRACTION DATA. M. Sakata, T. Uno, M. Takata, Department of Applied Physics, Nagoya University, Nagoya, Japan; and C. J. Howard, Australian Nuclear Science \& Technology Organisation, Lucas Heights, NSW 2234, Australia

It is often said that X-ray and neutron diffraction are complementary. In Xray powder diffraction, $X$-ray photons are scattered by electrons, while neutron beams are diffracted by nuclei in neutron powder diffraction, assuming that there are no magnetic interactions. What can be observed from X-ray and neutron powder diffraction data is, therefore, the electron and nuclear density distribution of the crystalline materials, respectively. It is, however, not known how to restore such electron and/or nuclear density distributions directly from the observed structure factors by powder diffraction without using a structural model.

Recently Sakata \& Sato [M. Sakata \& M. Sato (1990) Acta Cryst. A46, 263] have successfully applied the Maximum Entropy Method (MEM) to restore the precise electron density distribution from structure factors accurately determined by the Pendellosung method. It is possible to measure accurate Bragg intensities free from extinction effects by a powder diffraction experiment. In this study, the MEM analysis of both X-ray and neutron powder diffraction data will be given. In the neutron diffraction case, it was necessary to overcome the difficulty of negative scattering length of some atoms, such as $\mathrm{H}, \mathrm{Ti}, \mathrm{Mn}$. Both the electron and nuclear density distributions of rutile were obtained from X-ray and neutron diffraction data. In the electron density distribution, apical and equatorial bonds of rutile are clearly shown, while it is shown in the nuclear density distribution that the nuclei of $\mathrm{Ti}$ and $\mathrm{O}$ are located within very confined regions around atomic sites. 
P3.11 A BAYESIAN APPROACH TO BRAGG PEAK LOCATION IN POWDER DIFFRACTION PATTERNS. W.I.F.David, R.M.Ibberson and D.S.Sivia, ISIS Facility, Rutherford Appleton Laboratory, Chilton, Didcot, Oxon., OX11 OQX, U.K.

This paper presents an ab-initio Bayesian approach to Bragg peak location in powder diffraction patterns. It addresses the question: "Given that a region of a powder diffraction pattern is presumed to contain a few Bragg peaks and that the instrumental resolution function is known, what is the most probable number of peaks and what are their positions and intensities?". One of the major advantages of this Bayesian approach is that the relative probabilities of the number of possible peaks is quantified. Two applications are illustrated: (i) the location of Bragg peak positions in an unknown powder diffraction pattern prior to auto-indexing and (ii) the unit cell determination of pseudosymmetrical structures.

\section{P3.12}

TREOR9O: AN IMPROVED VERSION OF THE TRIALAND-ERROR INDEXING PROGRAM TREOR. L. Eriksson, P.-E. Werner, and M. Westdahl, Department of Structural Chemistry, Arrhenius Laboratory, University of Stockholm, S-106 91 Stockholm, Sweden.

Although high quality data is the most important prerequisite for powder indexing, the rate of success of a semi-exhaustive program for powder indexing is most strongly dependent on the built-in crystallographic experience. Accumulated experience from the use of the trial-and-error indexing program TREOR ${ }^{1,2}$ has been incorporated in the new version, TREOR9O. Dominant zone tests, originally used only for the monoclinic symmetry have been added for the orthorhombic and triclinic symmetries. Dominant zone tests are made prior to the conventional trials, thus preventing the most frequently occurring situation when figure of merit tests in powder indexing may fail.

Other new features included in the program are: successive reduction of trial cell volumes by statistical criteria, automatic unit cell reduction and conversion to conventional cells according to the metric symmetry, a condensed output file and an increased over-all efficiency of the indexing algorithms.

The original key-word system is retained, but at the expense of a relatively small increase in computing time the user may normally accept a default setting of parameters to run all symmetries from cubic to triclinic.

Computing times by the vectorized TREOR9O for triclinic and monoclinic patterns have never exceeded 5 and 2 minutes, respectively, on a CONVEX 210 , which is about $5 \%$ of the time required on a Micro-VAX $I$.

${ }^{1}$ P.-E. Werner, Z. Kristallogr., 120, 375-387, 1964.

2P.-E. Werner, L. Eriksson and M. Westdahl,J. Appl. Crystallogr. 18, 367-370, 1985.
P3.13 THE EFFECT OF THE THIRD ANGULAR PARAMETER IN TWOCIRCLE DIFFRACTOMETRY. Cong Qiuzi, Laboratory of Solid Lubrication, Lanzhou Institute of Chemical Physics, Chinese Academy of Sciences, Lanzhou 730000, People's Rep. China

On the basis of the $\theta$-and $2 \theta$-angles in a two-circle diffractometer, a third $\alpha$-angle between the sample surface and the incident $X$-ray beam is introduced, so that three $X$-ray scan modes of $\theta / 2 \theta, 2 \theta$ and $\theta$ are normalized into a common form of $\theta(\alpha) /$ $2 \theta$. Subsequently, based on the asymmetrical Bragg reflection geometry, equations of the diffraction intcnsities and the observed crystal-plane azimuthal angles are derived as the following common forms;

$$
I=1(\alpha, 2 \theta, t) \cdot P \quad \text { (1) and, } \Phi_{h k 1}=\theta_{h k 1}-\alpha
$$
where $t$ is the film thickness or effective $X$-ray penetration depth, $P$ is the reflection profile function, and $\theta_{\mathrm{hkl}}$ is the Bragg angle, for the given reflection.

Changing one or two parameters in eq. 1 we can obtain three groups of equations corresponding to three scan modes respectively.

a. For $\theta(\theta) / 2 \theta$ (called CBD) scan mode,

$$
\mathrm{Ic}=\mathrm{Ic}(\theta, 2 \theta, \mathrm{t}) \cdot \mathrm{Pc} \quad \text { (3) and, }\left(\theta_{\mathrm{hk} 1}\right) \mathrm{c}=0^{\circ}
$$

b. For $\theta\left(\alpha_{0}\right) / 2 \theta$ (called STD) scan mode, Is $=$ Is $\left(\alpha_{0}, 2 \theta, t\right) \cdot P s \quad(5)$ and, $\left(\Phi_{h k 1}\right) s=\theta_{h k 1}-\alpha_{o}$
(6) $\alpha_{0}$ is an angular parameter in the range from $0.6^{\circ}$ to $\theta_{h k 1}$ for $\mathrm{Cu} \mathrm{K}$ where $\alpha_{0}$ is an angular parameter in the range from $0.6^{\circ}$ to $\theta_{h k 1}$ for $\mathrm{Cu} \mathrm{K} \mathrm{K}_{\alpha}$ radiation.

c. For $\theta(\theta) / 2 \theta_{\mathrm{hk} 1}$ (used for ADA) scan mode,

$$
\mathrm{Ta}=\mathrm{Ta}\left(\theta, 2 \theta_{h k 1}, t\right) \cdot P a \quad(7) \text { and, }\left(\Phi_{h k 1}\right) \mathrm{a}=\theta_{h k 1}-\theta
$$

where $\theta$ is the rotating angle of the specimen, and $2 \theta_{h+1}$ is the diffraction angle for the given (hkl) reflection.

The effect of each scan mode in X-ray diffractometry is well known, for example, the " $a$ " mode is most used to determine phases, as is the " $b$ " mode. Furthermore, the effective $\mathrm{X}$-ray penetration depth can be controlled with " $b$ " mode by adjusting the sample-tilting $\alpha_{0}$-angle; at the same time, the film-layer or film thickness can be fitted with a combination of " $c$ " and " $b^{n}$ modes.

\section{P3.14}

RIFRAN '92: THE IBM PC SYSTEM FOR AUTOMATIC $X$-RAY POWDER DIFFRACTION PHASE ANALYSIS. T. Havlik, M. Skrobian, and F. Petricko, Technical University of Kosice, Czechoslovakia.

An efficient identification system RIFRAN ' 92 was developed for rapid qualitative and quantitative $\mathrm{X}$-ray powder diffraction phase analysis of polycrystalline materials. The system is represented by a set of interactive computer programs written in the Borland Turbo $\mathrm{C}++$ and Borland Turbo Pascal 6.0 computer languages and implemented on the IBM PC computer working under MS DOS.

The identification procedure for the analysis is based on comparison of the diffraction patterns of standards with those of the unknown sample, utilizing statistical criteria and chemical analysis for qualitative analysis, and the method of mathematical optimization for quantitative analysis.

The JCPDS database is used for qualitative analysis, as well as the specialized database for quantitative analysis in which the standards were calculated previously ${ }^{1}$.

The system enables the specialized database preparation according to the requirements of the user, using chemical composition, mineralogical name, subfiles, PDF numbers, etc. Thus, the analysis is more effective and faster. Specification of the chemical criteria with the aid of Boolean operands is used to further enhance the procedure.

The analysis takes only a few seconds in the average case. The results are sorted according to statistical criteria, or chemical analysis. Output of results is printed on the screen, as well as to the printer or a file. Both numerical and graphical output of the results are possible.

${ }^{1}$ Smrcok L., Weiss Z.: J. Appl. Cryst.-in press. 
P4.1 NONDESTRUCTIVE RESIDUAL STRESS MAPPING BY NEUTRON AND X-RAY DIFFRACTION METHODS. C.R. Hubbard", T.A. Dodson, S.A. David, Metals and Ceramics Division, and S.Spooner, Solid State Division, Oak Ridge National Laboratory, Oak Ridge TN 37831-6064, Phone (615) $574-4472$.

Advanced $X$-ray and neutron diffraction facilities to map macro and micro residual stresses have been established at Oak Ridge National Laboratory. For mapping stresses within the specimen by neutron diffraction, attachments for a triple axis spectrometer at HFIR have been built and automated which include beam collimators, an XYZ-chi specimen stage, and a linear position sensitive detector. Sampling gauge volumes of 20 to as little as $2 \mathrm{~mm}^{3}$ have been successfully used in mapping stresses in several specimens including a fernitic steel plate with a multipass weld, weld simulation test samples, and a brazed zirconia to iron joint. Results of mapping macro residual stresses in these specimens show significant stress gradients. Micro residual stresses in ceramic and metal matrix composites have been studied with both high resolution neutron powder diffraction and the triple axis spectrometer attachment. Comparison of the sensitivity and time required for measurement of microstresses will be presented. For mapping stresses at the surface a new $X$. ray mapping facility was developed which consists of an $18 \mathrm{KW}$ rotating anode generator and a Scintag PTS goniometer. Capabilities of this system will be presented, and data collected by both neutron and $x$-ray method will be compared.

Research soonsored by the Lasoratory Direcied Research and Develosment Program of Oak Ridge Natonal Laboratory anc the Office of Transporation Technolog es. Conservation and Renewadie Energy, Department of Energy, Under convact DE.AOS-6_OR21400 witn Marun Manetta Energy Systems, Inc.

\section{P4.2}

ABOUT ANALYTICAL MODELS FOR TEXTURE CORRECTION. M. Järvinen, Department of Information Tecnology, Lappeenranta University of Technology, SF-53851 Lappeenranta, Finland.

Powder diffraction is commonly used for obtaining information on the properties of materials. Accurate intensity measurements are needed, for instance, for the determination of the volume fractions of phases in a sample or for refinement of the structures of different materials.

The accuracy of results depends significantly on the treatment of sample effects. Loose samples can suffer from inhomogeneity and dense samples can be bothered by preferred orientation or texture. In practice, a sample with random orientation is very difficult to prepare. so that usually dense samples are used in measurements, and the texture effects are corrected mathematically. For this procedure analytical models are needed for presentation of the orientation distribution in the sample.

Very elegant theoretical and experimental methods have been created for determination of the texture factors of a sample, but often these methods are too complicated and superfluous for practical use. That is why simplified methods have been developed for some frequently occuring cases, for instance, a Gaussian function or March function is used for the description of fiber texture.

In the present paper the use of Gaussian and March functions are compared with the use of symmetrized harmonics by making model calculations and analyzing $\mathrm{Ni}$ and $\mathrm{Mg}$ powder data. The accuracy of these analysis methods are evaluated, and the advantages and the limitations of different models are discussed.
P4.3 SAMPLE-INDUCED ERRORS IN QUANTITATIVE TEXTURE ANALYSIS FROM DIFFRACTION POLE FIGURES. A. Mücklich and P. Klimanek, Institute of Ton Beam Physics and Materials Research, Research Centre Rossendorf, DO-8051 Dresden, Germany, and Institute of Metal Science, Mining Academy Freiberg, DO-9201 Freiberg/Sa., Germany

During the last decade many efforts have been made to improve the reproduction of orientation distribution functions ( $\mathrm{Odf}$ ) of polycrystalline materials from $\mathrm{X}$-ray and neutron diffraction pole figures. The advances achieved in this field are in contrast with a relatively unsatisfactory level of data evaluation due to the neglect of a number of systematic errors, which can significantly influence the reliability of quantitative texture analysis. In this connection two types of uncertainties have to be taken into account:

- experimental errors which, however, can be kept sufficiently small by suitable choice of the conditions of investigation, and

- sample-induced errors caused by the microscopical architecture (e.g. lattice perfection of the crystallites, grain size and orientation distribution) of the material taken under investigation.

Errors of the second type are characterized by the fact that they modify the actual absorption and/or scattering behaviour of the sample material. This is particularly important in investigations of structurally inhomogeneous polycrystalline materials [P. Klimanek in: X-Ray and Neutron Structure Analysis in Materials Science (Ed. J. Hašek).Plenum Press, New York 1989, 125; EPDIC 1 - European Powder Diffraction (Ed. R. Delhez, E.J. Mittemeijer), Mater. Sci. Forum, Vol. 79-82, Pt.1, 73. Trans Tech Publ., Aedermannsdorf/Switzerland 1991]. After a brief discussion of general aspects of pole figure evaluation, the present paper considers three effects that give rise to errors of the second type in the case of neutron diffraction:

1. absorption weighting of diffraction pole figures from polycrystalline layer structures with texture gradients or different texture components at various distances from the sample surface,

2. structure-induced extinction in materials whose texture components clearly differ in grain size and/or lattice perfection of the crystallites [A. Mücklich, P. Klimanek: Mater. Sci. Forum Vols. 79-82 (1991) 185] and

3. texture-induced secondary extinction in polycrystals with strong preferred orientation [A. Mücklich, P. Klimanek in: Cryst. Res. Technol. 23 (1988) K105; Mater. Sci. Forum Vols. 70-82 (1991) 185].

The practical importance of the effects is illustrated by computer simulation of neutron diffraction pole figures as well as by experimental results obtained with copper and iron.

P4.4

CHARACTERIZATION OF PREFERRED ORIENTATTON IN CRYSTALLINE MATERIALS BY X-RAY POWDER DIFFRACTION WITH THE MARCH FORMULA APPRAISAL OF THE LINE RATIO METHOD. H. Sitepu, Centre of Basic Sciences, Indonesian Institute of Technology, Serpong - Tangerang, Indonesia. 15320; B. H. O'Connor and $\mathrm{Li}$ Deyu, Department of Applied Physics/Centre for Materials Technology Curtin University of Technology, Perth, Australia.

Texture, i.e. preferred orientation, can cause large systematic errors in quantitative analysis of crystalline materials using $x$-ray powder diffraction (XRPD) data. Various mathematical forms have been proposed for the application of preferred orientation corrections. The most promising of these appears to be the singleparameter March (1932) model proposed by Dollase (1986).

Li et al. (1990) and O'Connor et al. (1991) applied the March model to determine the level of preferred orientation in various gibbsites using two procedures. The first involved the Rietveld (1969) least squares pattern fitting method. Each pattern was Rietveld-analyzed in two ways, initially assuming random orientation of the crystallites and subsequently with the March model. The second procedure for preferred orientation analysis, described here as the line ratio method, determines preferred orientation factors according to the intensity ratios of carefully selected line pairs.

In this study the procedures proposed by $\mathrm{Li}$ et al. and $\mathrm{O}^{\prime}$ Connor et al. for texture analysis have been evaluated with XRPD data sets for molybdite, calcite and kaolinite. The results indicate that, while the March formula improves agreement between the calculated and measured patterns in Rietveld analysis, other forms of systematic error in the intensity data appear to limit the effectiveness of the March formula in general. It has been found also that the line ratio method improves agreement between the data sets, but less effectively than the Rietveld method. It is proposed that extinction is likely to be the most influential source of systematic error competing with texture.

Dollase, W. A., 1986, J. Appl. Cryst., 19:267.

Li, D. Y., O'Connor, B. H., Roach, G.1.D and Cornell, J. B., 1990, Powd. Diff., 5:79.

March, A., 1932, Zeith. fur Kristallogr., 81:285.

O'Connor, B. H., Li, D. Y and Sitepu, H., 1991, Adv.in X-ray Analy,, 34:409.

Rietveld, H. M., 1969, I. Appl. Cryst., 2:151. 
P4.5 MICROSTRUCTURAL EFFECT ON THE CORROSION OF TUNGSTEN IN SF 6 . W. Wong-Ng, L. P. Cook, and C. S. Choi, Materials Science and Engineering Laboratory, National Institute of Standards and Technology, Gaithersburg, Maryland 20899, U.S.A.

Stored chemical energy release systems based on liquid metal combustion in general require the injection of a gaseous oxidant through a nozzle of chemically resistant material. Tungsten (W) has been the traditional metal of choice. Understanding of the kinetics of reaction of $\mathrm{W}$ with the oxidant $\mathrm{SF}_{6}$ as a function of temperature is of primary importance for predicting service lifetime. Scanning electron microscopy study of tungsten rods manufactured by a swaging process and exposed to $\mathrm{SF}_{6}$ indicated a significant orientational effect on the corrosion process, possibly originating from the considerable granular elongation parallel to the rod axis produced during the fabrication process. X-ray analysis of these rods showed preferred orientation. Texture measurement was subsequently conducted using neutron diffraction. This paper discusses the result of the pole figure study and the correlation of the relative rate of reaction with the grain alignment.

P4.6 FITTING X-RAY PROFILES IN TERMS OF PHYSICALLY RECOGNIZABLE PARAMETERS. R.W. Cheary and A. Coelho. Department of Applied Physics. University of Technology Sydney. PO Box 123. Broadway, NSW. Australia 2007.

$\mathrm{X}$-ray powder line profiles are generated by convoluting the instrumental aberration functions of a Bragg-Brentano diffractometer with the $\mathrm{Cu} \mathrm{K \alpha}$ emission spectrum and the diffraction function arising from the presence of either small crystallites or strain in the specimen. These profiles are fitted to experimental profiles using a combination of multilinear regression and Newton-Raphson non-linear least squares fitting based on numerical differentials. In addition to the $2 \theta$ values and integrated intensities of the lines. the fitted instrumental parameters include the receiving slit width. the length of the receiving slit. the $x$-ray target width, the divergence angle of the incident beam and a "top hat" mis-alignment function which can be used to represent defocussing effects or specimen tilt. The wavelengths and lifetime widths of the five components of the emission spectrum are fixed by the spectrometric measurements of Berger(1). The relative intensities of these components are calibrated with an Si crystal or a standard $2 \mu \mathrm{m}$ polycrystalline $\mathrm{MgO}$ disc to allow for differential attenuation by either a diffracted beam or incident beam monochromator. Crystallite size effects are assumed to produce a Lorentzian profile whilst strain produces a Gaussian shape: the refined parameters are the apparent crystallite size and the RMS strain. In practice each instrumental parameter is constrained to be the same for all profiles from a paricular specimen. As some of the parameters are either highly correlated with one another or do not contribute significantly to the profile shape. it is only possible to refine on two or three instrumental parameters even when diffraction data over a wide range of $2 \theta$ values are being fitted. Refining on all the instrumental parameters simultaneously normally produces results with little physical validity. The successful application of this convolution fitting procedure to a standard powder diffractometer has already been discussed(2). In this work we will demonstrate how the fitting procedure can be used for standardless line profile analysis and the measuremen of crystallite size and stacking fault defects. We will also examine the consistency of the fitted instrumental parameters when applied to two additional powder diffractometer configurations. In the first case the effects of incorporating a high resolution $\mathrm{Ge}$ monochromator ( $\mathrm{CuK} \alpha_{1}$ only) in the incident beam are considered. In the second system, the effects of replacing the receiving slit/detector with a position sensitive detecior are considered and how the broadening of the line profile under non-focussing conditions is incorporated into the fitting procedure.

(1) Berger. H. (1986) X-ray Spectrom. 15.241.

(2) Cheary. R.W. \& Coelho. A. (1992) J. Appl. Crịst. 25. In Press
P4.7 INSTRUMENTAL BROADENING DETERMINATION FOR XRD PROFILE ANALYSIS. P. Scardi \& L. Lutterotti, Dipartimento di Ingegneria dei Materiali, Universitá di Trento, 38050 Mesiano (TN), Italy; Paul Maistrelli, Dipartimento di Fisica, Universitá di Trento, 38050 Povo (TN), Italy.

A standard sample for XRD instrumental profile determination was prepared by pressing $-32+45 \mu \mathrm{m} \mathrm{KCl}$ powder to obtain a tablet. After a suitable annealing treatment, the tablet gave very sbarp profiles (FWHM $=0.07^{\circ}$ and $0.04^{\circ}$ at $2 \theta=28.4^{\circ}$ for the normal and high resolution set-up, respectively). The experimental patterns were modelled by non-linear least squares fitting using pseudo-Voigt ( $\mathrm{pV}$ ) or Pearson VII (PVII) functions, corrected for asymmetry, and the trend of fitting parameters describing the curves ( $\omega$ and $\eta$ for $p V, \omega$ and $m$ for PVII) as a function of $2 \theta$ was measured. These trends, which can be described by fitting data with a low degree polynomial, can be used for a fast and reliable $2 \theta$ dependent instrumental correction in line broadening analysis, botb using raw experimental data and modelled profiles.

The features of the experimentally determined instrumental function were studied using several diffractometers, always in Bragg-Brentano geometry; with different set-ups. In particular, the effect of using a monocbromator in the diffracted beam and changing the slits width was studied. It was found that tbe goniometer radius, beside having a remarkable influence on the resolution (peak width) of the diffractometer, also affects low angle peak asymmetry: High resolution geometry (narrow slits and large radius) is bighly advisable if sharp and fully symmetric profiles are needed, especially at low angles. In spite of the loss in intensity, involving an increase in the data collection time necessary to obtain a given precision, this kind of set up can greatly enbance tbe limit for crystallite size and microstrain determination through line broadening analysis. Also, structure refinements from $\mathrm{X}$-ray powder diffraction data give better results when the instrumental peak is sharp and symmetric.

P4.8

A PREDICTABLE DIFFRACTION PATTERN - METHOD AND SUBSTANCE. V. A. Kogan, Physical Dept. of Rostov State University, Zorge St. 5, Rostov-on-Don, 344104, Russia

The purpose of this work is to study the possibility that X-ray powder diffraction profiles can be computed independently. The problem may be divided into two parts:

\section{i) development of the method of computation}

ii) synthesis of a stable substance in whicb tbe factors of physical broadening are small and may be described on an unempirical scale.

The Fourier-syntbesis method [Kogan \& Kupriyanov, 1992] implemented in the computer program for full profile refinement, KET, was used to compute tbe reflection profiles theoretically. This programme makes it possible to syntbesize powder patterns for Bragg-Brentano geometry by taking into account tbe dimensions of the geometrical system, the spectral distribution, porosity effects and a model for imperfections in the sample. The $5 \mu \mathrm{m}$ fraction of strontium titanate was used as a sample. The crystallites obtained were of cubic habit and were not subjected to any external influences after synthesizing. It was assumed that tbe coherent scattering regions coincide with tbe geometrical crystallite sizes, and that micro-deformations of the crystal lattice are absent. During tbe experiments the diffraction profiles were recorded over a wide angular range for two different geometrical conditions. Full-profile refinement without introducing varying 'profile' parameters $\left(\mathrm{R}_{\mathrm{prof}}=.05-.07\right)$ provided adequate values of the structure parameters in different experiments.

\section{References}

V. A. Kogan \& M. F. Kupriyanov, (1992), J. Appl. Cryst. 25, 16-25. 
P4.9 X-RAY TRACING, A TOOL FOR IMPROVED ACCLRACY IN POWDER DIFFRACTOMETRY J. Timmers (Philips Research Laboratories, P.O. Box 80.000, NL-5600 JA Eindhoven, The Netherlands), R. Delhez, F. Tuinstra (Delft University of Technology. The Netherlands), F. Peerdeman (Philips IE Analytical, Almelo, The Netherlands)

The dimensions of $\mathrm{X}$-ray optical components as source, specimen and slits have a significant influence on the positions and shapes of powder diffraction lines and establish a well-known source of error (the so-called geometrical aberrations) in powder diffraction analysis. With the advancing mechanical goniometer accuracy in mind, corrections for geometrical aberrations, in order to arrive at improved accuracy of peak positions and improved interpretation of line shapes, become increasingly relevant.

The majority of geometrical aberration sources can be quantified analytically (see e.g. A.J.C. W'ilson, Mathematical Theory of X-ray Powder Diffractometry, Eindhoven Centrex 1963) in terms of centroid shift and variance of the geometrical part of the instrumental line profile. However, for the full geometrical line profile neither the shift of the peak maximum nor the shape can be obtained analytically. Therefore, a versatile computer program has been developed in order to simulate X-ray powder line profiles from the known dimensions of the diffractometer and its optical components. Diffraction geometries to be simulated include the BraggBrentano, Seemann-Bohlin and Schulz (texture) geometries as well as all non-focusing geometries.

As an example, peak shifts bave been calculated for two Philips diffractometer systems: the Integrated Powder Diffractometer (PW1800) and the new X'Pert diffractometer. Simulated and measured peak shifts for the NBS silicon powder standard (SRM1640b) are compared in fig. ure 1. A clear discrepancy exists between peak and centroid shifts in the low-angle region.

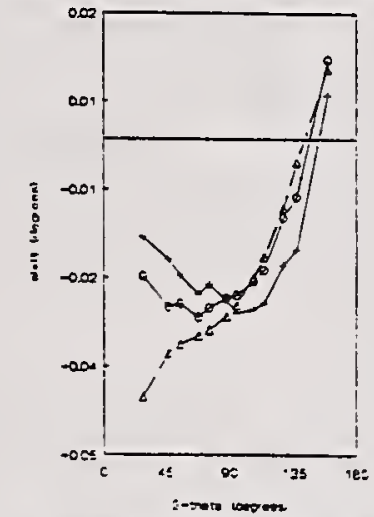

Figure 1. Numerically calculated peak (-0-) and centroid $(-\Delta-)$ shifts as well as measured peak shifts $(+-)$ as a function of Bragg angle for the NBS silicon standard SRM $640 \mathrm{~b}$. All data refer to the Philips Integrated Powder Diffractometer.
P4.10

MEANS OF X-RAY POWER DIFFRACTOMETRY. G. Berti, Dipartimento Di Scienze Lella Terra - Univ di Pisa -Via S. Maria 53 56100 Pisa - Italy

According to the symmetry rules of the crystalline system used to describe the crystallographic features of the sample, the crystallinity may be considered as a natural performance of the ideal crystalline state. The crystallinity is a feature pertaining to the sample, not to the crystalline system. The achievement of a high level performance is ruled by the crystallization conditions which act on the crystal evolution and produce mosaicity and also other observable results. Unfortunately, the effects related to the compositional variation, phase transformation, and planar defectivity are not directly visible on the diffraction pattern. They have to be deduced by reconstructing both the reciprocal average lattice and the shape of the nodes. The starting point of the reconstruction is the profile fitting of the experimental pattern by means of suitable analytical functions. The accurate determinations of positions and shapes of the peaks in the powder pattern are useful to find the proper lattices and shapes of nodes. So the crystallinity determination requires a phenomenological description as accurate as possible where, besides the many instrumental aberrations, crystallinity and structure factors are taken into account. To this aim the mathematical theory of $\mathrm{X}$-ray powder diffraction suggests simple and effective relationships.

Some samples of quartz show that the crystallinity and the crystallite volume are closely related when an equivalent average lattice is used to describe the assemblage of defective and/or disordered cells.
P4.11 X-RAY PROFILE ANALYSIS IN STRUCTURALLY INHOMOGENEOUS POLYCRYSTALS. P. Klimanek, Department of Materials Science, Academy of Mining, P.O.B. 47, DO - 9200 Freiberg/Sa., Germany

X-ray diffraction peaks of real polycrystals as, for instance, bulk materials or real powders interesting in materials science and technology have, in general, to be considered as diffraction multiplets (i.e. as reighted sums of partial reflections) due to the volume fractions of lattice disorder-statistics that are formed by crystallite groups with significantly different degrees of structural perfection. Accordingly the macroscopically observable line broadening of such reflections must be interpreted in terms of a generalized sample statistics representing the microscopical architecture of the scattering crystallite ensemble, which encloses

1. the type of the lattice-disorder statistics, and

2. the mean grain size, the orientation distribution (texture), and the spatial arrangement of the structurally different crystallite groups.

After a brief representation of the formalism needed in this case (Klimanek,P. in: Freiberger Forschungsheft B 265 (1988) 76; X-Ray and Neutron Structure Analysis in Materials Science (Ed. J.Hasek). Plenum Press, New York 1989, 125; EPDIC 1 - European Powder Diffraction (Ed. R.Delhez, E.J.Mittemeijer), Mater. Sci. Forum, Vol. 79-82, Pt.1, 73. Trans Tech Publ., Aedermannsdorf/Switzerland 1991) some important methodological aspects of the analysis of X-ray multiplet profiles are discussed and illustrated by experimental results obtained with model specimens (powders) and real polycrystalline materials.

\section{P4.12}

A STUDY OF CRYSTALLITE SIZE AND STACKING FAULTS IN EX-OXALATE ZnO BY PATTERN DECOM POSITION. D. Louër*. A. Boultif*, J. P. Auffrédic* and J. I. Langford $\dagger *$ Laboratoire de Cristallochimie, Université de Rennes I, Avenue du Général Leclerc, 35042 Rennes cedex, France, and †School of Physics an Space Research, University of Birmingbam, Birmingham B15 2TT, U.K.

Previous X-ray diffraction studies of ex-hydroxide nitrate powder $\mathrm{ZnO}$ demonstrated that the form of the crystallites was prismatic, on average approximating to cylinders (Louêr et al., J. Appl. Cryst. 16, 183-191, 1983). Recently, data were obtained for the first 27 lines of the diffraction pattern for $\mathrm{ZnO}$ obtained from thermal decomposition of the oxalate, by using the same sample as bad been previously investigated by means of electron microscopy and adsorption calorimetry (Bolis et al., J. Chem. Soc. Faraday Trans. 85, 855-867, 1989). Integral breadths obtained by total pattern fitting again have \& marked hkl dependence; an indexed Williamson-Hall plot indicated that microstrains are negligible and that there are three groups of reflections, according to the values of hkl. This is a classic example of the effects of stacking faults combined with broadening due to crystallite size and it is the first time that the former has been reported for $\mathrm{ZnO}$.

Twelve reflections with $1=0$ or $h-k=3 n$ are unaffected by structural 'mistakes' and these were used to obtain mean crystallite dimensions, based on spherical and cylindrical morphologies. From the dimensions obtained $[\langle D\rangle=453(17) \dot{A}$ for spheres and $\langle D\rangle=404(7) \dot{A},\langle H\rangle=351(9) \dot{A}$ for cylinders)], the 'size' contribution to the remaining reflections $(h-k=3 n \pm 1)$ can be calculated. This can then be separated from the total breadth for these lines to obtain the 'mistake' component and hence the stacking-fault probability. For this purpose it is customary to assume that the constituent line profiles are Lorentzian (eg. Warren, X-ray diffraction, 1969, p. 303), but here all sample line profiles, including those broadened solely by 'size' effects, have an appreciable Gaussian component. The Voigt function may therefore be a better approximation to the 'size' profiles and an alternative analysis based on this function (eg. Langford, J. Appl. Cryst. $11,10-14,1978$ ) was also carried out. From the Lorentzian model and cylindrical form. the mean probability of the occurrence of a stacking fault was found to $0.007(3)$, corresponding to a separation between fault planes approximately equal to the mean height of the crystallites. By assuming Voigtian 'size' profiles, the probability was about $0.014(9)$, equivalent to $\sim 1$ fault per crystallite, on average.

This study has also demonstrated that with high quality data, detailed self-consistent and fairly precise information about struetural imperfections can be obtained from a combination of line-profile analysis and total pattern fitting. The ex-oxalate $\mathrm{ZnO}$ analysis represents a fairly demanding case, since the fault probability is small and the crystallites are relatively large, and a significant factor in obtaining such detailed information was the use of a high resolution diffractometer. On the other hand, it revealed some of the limitations of the procedure used to model the diffraction line profiles. 
P4.13 DETERMINATION OF CRYSTALLITE-SIZE AND LATTICE-STRAIN PARAMETERS FROM X-RAY DIFFRACTION LINEPROFILE ANALYSIS BY APPROXIMATING BOTH SIZE AND STRAIN BROADENED PROFILES WITH THE VOIGT FUNCTION. D. Balzar' and $\mathrm{H}$. Ledbetter, Materials Science and Engineering Laboratory, National Institute of Standards and Technology, Boulder, Colorado 80303, U.S.A.

When the pure-specimen (physical) broadened profile can be satisfactorily described with a Voigt function, by applying the Langford (1980) method, it is possible to separate exactly the size $A^{S}(L)$ and strain $A^{D}(L)$ Fourier coefficients. The column-length distribution functions are analytically derived. It is shown that $\langle D\rangle_{s} \leq\langle D\rangle_{v} \leq 2\langle D\rangle_{s}$, where $\langle D\rangle_{s}$ and $\langle D\rangle_{v}$ refer to surface-weighted and volume-weighted average domain sizes. Distribution of average strain is evaluated. For not too large a harmonic number, mean-square strain decreases linearly with distance $L$, but generally a more complex relationship holds with a finite value for $L=0$. Errors are estimated, and the method is compared with the Warren-Averbach (1952) analysis and its modification (Delhez et al., 1980) for separation of size and strain parameters. Some problems with the application are described.

\section{References}

Delhez, R., de Keijser, Th.H. \& Mittemeijer, E.J. (1980). Accuracy in Powder Diffraction. NBS Special Publication No. 567, edited by Block, S. \& Hubbard, C.R., pp. 213-253. Washington, D.C.: National Bureau of Standards.

Langford, J.I. (1980). ibid., pp. 255-269.

Warren, B.E. \& Averbach, B.L. (1952). J. Appl. Phys. 23, 497.

On leave from Department of Physics, Faculty of Metallurgy, 44000 Sisak, Croatia.

P4.14 A COMPARATIVE STUDY OF WARREN-AVERBACH, MITRA-MISRA AND de KEIJSER METHODS FOR CRYSTALLITE SIZE AND STRAIN ANALYSIS IN NiO POWDER. L.D. Pradhan and B.S. Acharya, R.R.L. Bhubaneswar-751013, Oriss, INDIA.

Out of all the methods available for analysis of crystallite size and strain in polycrystalline materials, the three most widely used methods are WarrenAverbach (WA), Mitra-Misra, and the recently proposed method of de Keijser et al. In many cases it is difficult to use the WA method because of the nonavailability of multiple orders of reflections and the fact that the shape of the profile from Cauchy or Gaussian. The method proposed by de Keijser et al. takes into account the shape of the profile function, whether it is Cauchy, Gaussian, Voigt, Pseudo Voigt, or Pearson VII. Nickel oxide powder, the material used in the present investigation, gives an interesting case study for comparison of the above three methods. This is due to the fact that multiple order reflections are available, and the shape of the profile is Voigtian. Line profile analysis of the powder has been performed repeatedly at different temperatures, including $300,773,873$, and $973 \mathrm{~K}$, and the crystallite size and strain values have been calculated by using the WA, Mitra-Misra, and de Keijser methods. In calculating strain from the de Keijser method, the Voigt, Pseudo Voigt, and Pearson VII functions have been used. It has been observed that the de Keijser method does not yield meaningful results, whereas the WA and Mitra-Misra methods do give good outcomes. The cause of this variation is not known. However, it is tentatively recommended that even if the profiles fit the equations for Voigt, Pseudo Voigt, or Pearson VII, one should perform all the above analytical methods before arriving at any conclusion.
P4.15 X-RAY DETERMINATION OF THE CRYSTALLITESIZE DISTRIBUTION FUNCTION FOR ALKOXY-DERIVED BARIUM TITANATE POWDER MATERIALS. V. V. Zhurov, S. A. Ivanov, M. I. Yanovskaya, V. K. Lymar', Karpov' Institute of Physical Chemistry, 103064 Moscow K-64, Russia.

A detailed knowledge of the crystallite-size distribution function (CSDF) is required in many fields of materials science. A careful analysis of X-ray diffraction line broadening provides a convenient method for obtaining this information. The purpose of our work was to determine the CSDF in barium titanate powders prepared by hydrolysis is of alkoxides with or without further heat treatments.

$X$-ray powder diffraction experiments (URD-63 diffractometer, Cu-radiation, $\mathrm{T}=300 \mathrm{~K}$ ) were combined with electron microscopy (JEM-1000X) and analysis with Fritsoh laser partical sizer. Some peaks in the diffraction patterns of our samples were carefully recorded by the step-scanning technique. The instrumental and spectral broadening was obtained from a single crystal standard. The regularization method of Fourier X-ray line profile analysis was applied for the CSDF determinations. All calculations were performed by special programs adapted to an IBM-PC computer. Some of the CSDF curves obtained are shown in the figure. There is a quite reasonable agreement between the sizes obtained from $\mathrm{X}$-ray profile analysis and other physical methods up to $1 \mu \mathrm{m}$. The particles above $1 \mu \mathrm{m}$

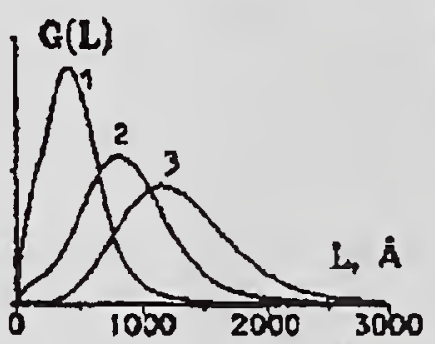
detected by electron microscopy are not monocrystalline. Values of crystallite size were computed for several crystallograhic directions. The shape of crystallites is isotropic for all tested samples.
1) initial powders
2) after heating at $800^{\circ} \mathrm{C}$

3) after heating at $1000^{\circ} \mathrm{C}$

P4.16 X-RAY DIFFRACTION STUDY OF THE EFFECT OF SiC ON THE DEFECT CHARACTERISTICS OF Al-Mg-Zn ALLOY. R. Sen, S. K. Chattopadhyay, and S.K. Chatterjee, Regional Engineering College, Durgapur-713209, INDIA

Silicon carbide was introduced into the matrix of an $\mathrm{Al}-\mathrm{Mg}-\mathrm{Zn}$ alloy (5.98 wt\% $\mathrm{Zn}, 2.91 \mathrm{wt} \% \mathrm{Mg}$, and traces of $\mathrm{Cu}, \mathrm{Mn}$, and $\mathrm{Fe}$ ) and $\mathrm{X}$-ray diffraction line profile analyses were performed to evaluate the role of SiC in the composite matrix. Peak shift peak asymmetry and Fourier line shape analysis carried out in detail in the cold worked systems have revealed that the concentration of twin faults introduced into the matrix increases with the increase in $\mathrm{SiC}$ concentration (from 0 to $20 \mathrm{wt} \%$ ). The other fault parameters, such as intrinsic and extrinsic faults, have been found to be negligible, as expected in aluminium based systems having high stacking fault energy. The observations obtained from X-ray analysis have been supported by scanning electron and optical metallographic studies. 
O5.1 A HIGH RESOLUTION X-RAY POWDER DIFFRACTION AND MOLECULAR MODELLING STUDY OF THE STRUCTURAL CHANGES ACCOMPANYING ABSORPTION OF TRANS-STILBENE INTO H-ZSM-5 ZEOLITE. J. B. Parisc, SUNY Stony Brook, NY 11974-2100, J. Hriljac and D. E. Cox, BNL, Upton NY11972, Dr. R. Corbin and V. Ramamurthy, DuPont Company, Wilmington De 19880-0262, USA

The restricted pore geometry available in molccular sievcs makes them cxcellent candidate materials for a number of selectivc chemical reaction. These have recently included the first stabilizations of the cation radicals of the trans-polyenes ${ }^{1}$ We have studied stilbene in $\mathrm{H}-\mathrm{ZSM}-5^{1}$ and have suggested the molecule absorbs into the straight channels on the basis of NMR datal. High rcsolution X-ray data collected at room tem. perature have been modellcd, using a commercial software package, and are consistent with the interpretation of the NMR data.

Sorption of trans-stilbene causes the monoclinic H-ZSM5 to transform to a pseudo tetragonal phase at room temperature with no detectable distortion of the unit cell. Upon cooling to $20 \mathrm{~K}$ a pronounced orthorhombic splitting is evident. The figure above shows scans through the (400/040) reflections from trans-stilbene/H-ZSM-5 at selected temperatures up to $300 \mathrm{~K}$ collected at a wavclength of $1.3064 \AA$.

Acknowledgements: Work at $\mathrm{X}-7 \mathrm{~A}$ supported through DOE, DMR under contract DE-AC02-76CH00016; partial support for JBP DuPont and NSF DMR-9024249

1. Ramamurthy ot al. J. Amer. Chem Soc., 1991, 113, 594)

\section{O5.2}

$X$-RAY LINE BROADENING FROM INTERGROWTHS AND ANTIPHASE DOMAINS IN BARIUM HOLLANDITE COMPOUNDS. R.W Che3ry and R. Squadrito, Department of Applied Physics, University of Technology Sydney. PO Box 123. Broadway. NSW. Australia 2007.

Barium hollandites $\mathrm{Ba}_{\mathrm{x}}\left(\mathrm{A}_{\mathrm{y}} \mathrm{Ti}\right.$-y $) \mathrm{O}_{16}$ generally form over the composition range $\mathrm{x}=8 / 7$ to $x=4 / 3$ where $A$ is normally a $2^{+}$or $3^{+}$ion such $A l$. $M g$ or a metal from the first transition series. As the tunnel sites accommodating the $\mathrm{Ba}$ are only partially occupied the $\mathrm{Ba}$ and vacant sites usually adopt an ordered configuration along the tunnels and superlattice lines appear in the $\mathrm{x}$-ray powder pattern. These compounds undergo an orderdisorder transition and above the transition temperature $T_{c}$ the superlattice lines disappear. For the hollandites $\mathrm{Ba}_{\mathrm{x}}\left(\mathrm{Mgx}_{\mathrm{x}} \mathrm{Ti} 8-\mathrm{x}\right) \mathrm{O}_{16}, \mathrm{~T}_{\mathrm{c}}$ goes from $710^{\circ} \mathrm{C}$ at $\mathrm{x}=8 / 7$ to $900^{\circ} \mathrm{C}$ at $x=4 / 3$. When these materials are first cooled below the transition temperature $T_{c}$. the superlattice lines are broadened through the formation of intergrowths and antiphase domains. On further annealing the superlattice lines become sharp and also undergo a $2 \theta$ shift. No significant changes are observed in the hollandite lattice lines on ordering. At $x=4 / 3$, Ba ions occupy $67 \%$ of the siles and the ordering sequence is $B a-B a-V$ with a $3 c_{\text {holl }}$ supercell where $\mathrm{V}$ represents a vacancy. When the occupancy drops to $60 \%$ $(x=1.20)$ the ordering sequence is $\mathrm{Ba}-\mathrm{Ba}-\mathrm{V}-\mathrm{Ba}-\mathrm{V}$ with a $5 \mathrm{c}_{\text {holl }}$ supercell. At intermediate occupancies, the structure consists of intergrowths of regions with $\mathrm{Ba}-\mathrm{Ba}-\mathrm{V}$ order and $\mathrm{Ba}-\mathrm{Ba}-\mathrm{V}-\mathrm{Ba}-\mathrm{V}$ order. For $\mathrm{x}<1.20$, the structure switches between regions of $\mathrm{Ba}-\mathrm{Ba}-\mathrm{V}-\mathrm{Ba}-\mathrm{V}$ order and $\mathrm{Ba}-\mathrm{V}$ order. In all compositions lateral ordering occurs between tunnels. This is manifested as unbroken corrugated layers of vacancies perpendicular to the tunnels. To fully describe partially ordered hollandites it is also necessary to include antiphase domains into the model with boundaries parallel to and perpendicular to the tunnels. In this work a general diffraction theory is developed for interpreting the breadths and shifts of the superlattice lines from partially ordered polycristalline hollandites. This will allow the kinetics of ordering to be studied through the time and temperature dependence of the superlattice line breadths. Analytical expressions for $x=4 / 3$ hollandites that only contain antiphase domains have recently been published(1). This work has now heen generalised to incorporate intergrowths in the model as well as antiphase domains. In all cases the diffraction profiles are Lorentzian. Results will be presented for a range of hollandites at different Ba concentrations. These include the $2 \theta$ shifts and apparent crystallite sizes corrected for instrumental effects for different superlatice Iines from both fully ordered and quenched specimens. We have been able to measure the mean dimensions of the ordered regions and identify both the boundary layer structure and the hkl planes defining the boundaries. In monoclinic hollandites the boundaries form on the 101 and $10 \overline{1}$ planes parallel to the tunnels and on the 010 perpendicular to the tunnels, but after extended annealing only 010 boundaries remain.

(1) Cheary. R.W. \& Squadrito. R. (1992) Acta Cṇ'st. A48, In Press
O5.3 A PHYSICAL MODEL FOR X-RAY ANALYSIS OF LAMELLAR SYSTEMS: APPLICATIONS TO MYELINATED NERVE SHEATHS. R. Vargas and L. Mateu, Centro de Bioflsica y Bioqulmica, IVIC, Apdo. 21827, Caracas 1020-A, Venezuela, and V. Luzzati, Centre de Génétique Moléculaire, CNRS, 91198 Gif-sur-Yvette, France.

In order to undertake the study of order-disorder phenomena in myelinated nerve sheaths, a physical model has been proposed and an algorithm developed for the fast and accurate analysis of the whole diffraction pattern of lamellar systems [1].

The model consists of planar lamellae, all stacked into crystals with a variable repeat distance. Within each crystal the lamellae are parallel to each other. The following parameters are considered in the model: the average lattice repeat and its variance, the average number of unit cells per crystallite, the continuous intensity curve of a single motif the intensities of the diffuse scattering, and the fraction of lamellae that does not belong to the coherent diffraction domains.

The algorithm has been applied to a variety of X-ray scattering spectra from rat sciatic and optic nerve myelin $[2,3]$. Such data have been recorded and analyzed in a few minutes using a position sensitive detector and a personal computer. A precise determination of the structural parameters of myelin and as evolution as a function of the age of the rats has also been analyzed.

\section{References}

[1] Luzzati, V. and Mateu, L., J. Mol. Biol. (1990) 215, 373-384.

[2] Mateu, L., Luzzati, V., Vargas, R., Vonasek, E. and Borgo, M., J. Mol. Biol. (1990) 215, 385-402.

[3] Mateu, L., Luzzati, V., Borgo, M., Vonasek, E. and Vargas, R., J. Mol. Biol. (1991) 220, 351-357.

\section{P5.1} CRYSTAL STRUCTURE SOLUTION OF MG-LEUCITES FROM SYNCHROTRON X-RAY POWDER DIFFRACTION DATA. R. J. Cernik ${ }^{\mathrm{t}}$, A.M.T. Bell ${ }^{1,2}$, P. E. Champness ${ }^{3}$, A. N. Fitch ${ }^{2,}$, C.M.B. Henderson $^{3}$, S. C. Kohn ${ }^{4}$, and S.A.T. Redfern ${ }^{3}$.

A previously unknown low-symmetry leucite structure has been determined from synchrotron $x$-ray powder diffraction data collected on a hydrothermally synthesized leucite analogue of stoichiometry $\mathrm{K}_{2} \mathrm{MgSi}_{5} \mathrm{O}_{12}$ Laboratory based $\mathrm{x}$-ray powder diffraction and ${ }^{29} \mathrm{Si} \mathrm{nmr}$ work showed that this structure is a distorted version of the known cubic (Ia3d) leucite structure and that all 12 tetrahedral $(\mathrm{T}) \mathrm{Mg}$ and $\mathrm{Si}$ sites are ordered. Electron diffraction showed that the low-symmetry structure was monoclinic $\left(\mathrm{P}_{1} / \mathrm{c}\right)$. The cubic structure in the monoclinic cell was used as a starting model for Rietveld refinement of this structure. The refinement was able to determine on which two of the $\mathrm{T}$ sites $\mathrm{Mg}$ were situated from differences in the T-O bond lengths. The synchrotron $\mathrm{x}$-ray diffraction data were able to distinguish quite clearly between the $\mathrm{Mg}$ and $\mathrm{Si}$ sites.

Rietveld refinement has also been carried out on a dry synthesized, cubic (Ia3d) $\mathrm{K}_{2} \mathrm{MgSi}_{5} \mathrm{O}_{\mathrm{t} 2}$ leucite analogue, in this structure the $\mathrm{T}$ sites are disordered.

'SERC Daresbury Laboratory, Warrington, WA4 4AD, UK.

${ }^{2}$ University of Keele UK.

${ }^{3}$ University of Manchester UK.

${ }^{4}$ University of Warwick UK.

*Now at ESRF, Grenoble, France. 
P5.2 CRYSTAL STRUCTURE REFINEMENTS OF $\mathrm{M}_{3} \mathrm{Al}_{2} \mathrm{O}_{6}$ ( $M=\mathrm{Ca}, \mathrm{Sr}, \mathrm{Ba}$ ) USING NEUTRON POWDER DIFFRACTION DATA - AN EXAMPLE STUDY FROM THE NEW HB-4 DIFFRACTOMETER AT THE HFIR, OAK RIDGE*. B. C. Chakoumakos, J. A. Fernandez-Baca, and G. A Lager ${ }^{1}$, Solid State Division, Oak Ridge National Laboratory, Oak Ridge, TN 37831, U. S. A., ' Department of Geology, University of Louisville, Lousiville, KY 40292, U. S. A.

$\mathrm{Ca}_{3} \mathrm{Al}_{2} \mathrm{O}_{6}$ is a major component of Portland cements, and the conversion of the aluminate to the hydrogarnet structure is an important hydration reaction in the setting of these cements. Detailed structural studies of isostructural alkaline earth aluminates are underway to elucidate their reactivity and to examine the systematics of $\mathrm{Al}-\mathrm{O}$ bond length and $\mathrm{Al}-\mathrm{O}$ - $\mathrm{Al}$ angle variations among tetrahedral aluminates. Room temperature powder diffraction patterns were collected for the title compounds in the form of dense ceramic rods using a step size of $0.05^{\circ}$ over the range of $11^{\circ}$ to $135^{\circ} 2 \Theta$ with a wavelength of $1.400 \AA$. These compounds are cubic, $P a 3, \mathrm{Z}=24$, and are described by 48 structural parameters using isotropic thermal vibration parameters. Their crystal structure consists of isolated 6-membered tetrahedral aluminate rings cross- linked by the alkaline earth ions. Rietveld structure refinements were completed, employing a Pseudo-Voigt peak shape function, and a total of 12 profile and background parameters. For $M=\mathrm{Ca}, a=15.2588$ (4) $\AA$, $V_{\text {cell }}=3552.7$ (2) $\AA^{3}, R_{\text {exp }}=0.0496, R_{\text {wp }}=0.0618$, for 1444 reflections. Comparison of the refined structural parameters with a previous single crystal $\mathrm{x}$-ray structure determination of the $\mathrm{Ca}$ analog (Mondal \& Jeffery, 1975, Acta Cryst. $B 31,689)$ show excellent agreement. For $M=\mathrm{Sr}, a=15.8544$ (4) $\AA, V_{\text {eell }}=3985.2(2) \AA^{3}, R_{\text {exp }}=0.0294, R_{\text {wp }}=0.0446$, for 1614 reflections. The present refinement of the $S$ r analog is essentially the same as an earlier refinement employing the Voigt peak shape function (Chakoumakos et al., 1992, Acta Cryst. $C 48$, in press). For $M=\mathrm{Ba}, a=16.512$ (2) $\AA, V_{\text {cell }}=4502$ (1) $\AA^{3}, R_{\text {exp }}=0.0497, R_{w p}=0.0955$, for 1829 reflections. This refinement is not as good as those for the $\mathrm{Ca}$ and $\mathrm{Sr}$ analogs. The assumption that the $\mathrm{Ba}$ analog is isostructural (based on the similarity of the powder pattern) may be incorrect, or the sample purity may not be as good. Both possibilities are being explored.

-Supported by the Division of Materials Sciences, U.S. D. O.E. under contract DE-AC05-840R21400 with Martin Marietta Energy Systems, 1nc.

\section{P5.3 STRUCTURE DETERMINATION OF THE LOW- AND} HIGH-PRESSURE FORMS OF $\mathrm{Mg}_{3} \mathrm{BN}_{3}$ FROM X-RAY POWDER DATA. H. Hiraguchi, H. Hashizume, S. Nakano* and O. Fukunaga*, Research Laboratory of Engineering Materials, Tokyo Institute of Technology, Nagatsuta, Midori, Yokohama 227, Japan. "Fac. of Engineering, TIT.

The crystal structures of magnesium boron nitride $\mathrm{Mg}_{3} \mathrm{BN}_{3}$ in the low- and high-pressure forms have been determined $a b$ initio from $\mathrm{X}$-ray powder data. The strategy used is to decompose the powder pattern to estimate individual reflection intensities, calculate Patterson functions and build trial-anderror structure models, which are refined by the Rietveld method. 54 and 21 reflections observed on a $\theta-\theta$ diffractometer were used to solve the structures of $\mathrm{Mg}_{3} \mathrm{BN}_{3}(L)$ and $\mathrm{Mg}_{3} \mathrm{BN}_{3}(H)$ respectively. Low-pressure $\mathrm{Mg}_{3} \mathrm{BN}_{3}(L)$ has a hexagonal cell (space group $P 6_{3} / m m c, Z=2$ ) with $a=3.54453(4)$ and $c=16.0353(3) \AA$ while the high-pressure $\mathrm{Mg}_{3} \mathrm{BN}_{3}(H)$ cell is orthorhombic (Pmmm, $Z=1$ ) with $a=3.0933(2), b=3.1336(2)$ and $c=7.7005(5) \AA$. The two structures commonly include linear $\mathrm{N}-\mathrm{B}-\mathrm{N}$ molecular anions, considered to play a role in the catalytic properties of the material in the reaction converting hexagonal boron nitride into a cubic form.

A very similar structure has been obtained by applying standard direct methods to the same $\mathrm{Mg}_{3} \mathrm{BN}_{3}(L)$ intensity data. High-quality electron density maps have been calculated for this form using the maximum entropy method and the phased structure factors. A separate application of the maximum entropy program MICE [C. Gilmore et al.: Acta Cryst. A47, 830 (1991)] to the unphased structure factors yielded a similar map, in support of the determined structure of $\mathrm{Mg}_{3} \mathrm{BN}_{3}(L)$.
P5.4 STRUCTURAL CHARACTERIZATION OF WEBERITERELATED ANTIMONATES $\mathrm{A}_{2} \mathrm{Sb}_{2} \mathrm{O}_{7}(\mathrm{~A}=\mathrm{Pb}, \mathrm{Sr}, \mathrm{Ca}, \mathrm{Cd})$. S. A. Ivanov, Karpov'Institute of Physical Chemistry, 103064 Moscow K-64, Russia.

The discovery of ferroelectricity in weberite-related $\mathrm{Pb}_{2} \mathrm{Sb}_{2} \mathrm{O}_{i}$ crystals initiated a search for similar compounds with unusual dielectric properties. The title compounds appear to be good candidates for application as ferroelectrics. Investigation of the crystal chemistry of these antimonates may be beneficial in developing a better understanding of the origin of ferroelectricity. With this in mind, the features of the crystal structure of $\mathrm{A}_{2} \mathrm{Sb}_{2} \mathrm{O}_{7}$ complex oxides have been thoroughly studied by X-ray powder diffraction. Diffraction patterns were collected with a URD-63 diffractometer at room temperature between 12 and $132^{\circ} 2 \theta$ using $\mathrm{CuK}_{\mathrm{e}}$-radiation and analyzed with the Rietveld profile refinement technique (the DBW-3.2s computer program). The structures of the $\mathrm{A}_{2} \mathrm{Sb}_{2} \mathrm{O}_{7}$ samples are basically similar: the $\mathrm{Sb}$ ions are octahedrally coordinated whereas the $\mathrm{A} 2+$ cations are B-coordinated and lie in square prisms and hexagonal bipyramids. The existence of ferroelectric properties at room temperature only in $\mathrm{Pb}_{2} \mathrm{Sb}_{2} \mathrm{O}_{7}$ can be related to the features of crystal structure. The important role played by the effective size of the $\mathrm{A} 2+$ cations in determining the structural parameters, and the electrostatic effect of the lone pair of electrons on $\mathrm{Pb} 2+$ are also discussed.

\begin{tabular}{lcccccc}
$\mathrm{A}^{2+}$ & a, A & b, A & c, A & S.G. & $<\mathrm{a}\rangle$ & $\mathrm{R}_{\mathrm{A}} / \mathrm{R}_{\mathrm{Sb}}$ \\
\hline $\mathrm{Pb}$ & $7.484(1)$ & $7.857(1)$ & $10.426(2)$ & $\mathrm{I} 2 \mathrm{~cm}$ & 5.351 & 1.93 \\
$\mathrm{Sr}$ & $7.455(1)$ & $10.369(2)$ & $7.684(1)$ & Imma & 5.295 & 1.85 \\
$\mathrm{Ca}$ & $7.298(1)$ & $10.213(2)$ & $7.459(1)$ & Imma & 5.179 & 1.68 \\
$\mathrm{Cd}$ & $7.302(1)$ & $10.207(2)$ & $7.249(1)$ & Imma & 5.131 & 1.61
\end{tabular}

\section{P5.5}

CHARGE TRANSFER AND COMPRESSION EFFECTS IN DOPED Y-123. S. Eriksson ${ }^{\dagger, \#}$, C. Strōm ${ }^{\dagger}$, P. Berastegui ${ }^{\dagger}$, L.-G. Johansson ${ }^{\dagger}$, A. Simon ${ }^{\#}, \mathrm{Hj}$. Mattausch", 'Dept. of Inorganic Chem., S-412 96 Gōteborg, SWEDEN, "MPI fur Festkōrperforschung, 7000 Stuttgart 80, GERMANY.

In most high- $\mathrm{T}_{\mathrm{c}}$ superconductors the number of positive holes in the $\mathrm{CuO}_{2}$, sheets to a great extent determines the superconducting properties. By introducing dopant atoms or by preparation of oxygen deficient samples drastic structural changes occur. The changes can in many cases be correlated with a charge transfer in the structure and thus to a change in $\mathrm{T}_{\mathrm{c} \cdot}$

The POLARIS instrument at ISIS, RAL, England has been used for collection of high quality TOF neutron powder diffraction data. In all cases solid state sintered single phase samples of doped Y-123 have been analyzed by the Rietveld method. The structural changes caused by the various dopant atoms have been correlated with the change in properties (See table 1). Compression effects $\left(\mathrm{Sr}^{2+}\right)$ can be separated from charge transfer effects $\left(\mathrm{Co}^{3+}, \mathrm{Al}^{3+}, \mathrm{Fe}^{3+}\right)$ and the two effects seem to be additive. Raman spectroscopic investigations, susceptibility and Hall effect measurements confirm the picture obtained from the analysis of the neutron data.

Table 1. Some characteristic distances in $\mathrm{Y}_{1} \mathrm{Ba}_{2-y} \mathrm{SryCu}_{3-x} \mathrm{Co}_{x} \mathrm{O}_{7-d}$. Cul is the chain copper, $\mathrm{Cu} 2$ the plane copper, $\mathrm{O} 2$ and $\mathrm{O} 3$ the plane oxygens and $\mathrm{O} 4$ the apex oxygen.

\begin{tabular}{cccccc}
$\mathrm{x} / \mathrm{y}$ & $(\mathrm{Cu} 2-\mathrm{O} 2,3)$ & $(\mathrm{Ba}-\mathrm{O} 4)_{z}$ & $\mathrm{Cul}-04$ & $\mathrm{Cu2}-\mathrm{O4}$ & $\mathrm{T}_{\mathrm{c}}$ \\
\hline $0.00 / 0.00$ & 0.269 & 0.299 & $1.853(2)$ & $2.291(5)$ & 92 \\
$0.00 / 0.20$ & 0.270 & 0.297 & $1.847(3)$ & $2.282(3)$ & 90 \\
$0.00 / 0.60$ & 0.269 & 0.295 & $1.846(2)$ & $2.250(3)$ & 86 \\
$0.00 / 1.00$ & 0.267 & 0.299 & $1.843(3)$ & $2.224-(3)$ & 81 \\
$0.05 / 0.00$ & 0.259 & 0.304 & $1.854(5)$ & $2.306(6)$ & 89 \\
$0.15 / 0.00$ & 0.228 & 0.343 & $1.836(5)$ & $2.345(6)$ & 76 \\
$0.30 / 0.00$ & 0.216 & 0.349 & $1.838(6)$ & $2.352(7)$ & 30
\end{tabular}

Data will also be presented for the materials $\mathrm{YBa}_{2-y} \mathrm{Sr}_{y} \mathrm{Cu}_{3 z_{2}} \mathrm{Me}_{x} \mathrm{O}_{7-\mathrm{d}}\left(\mathrm{Me}=\mathrm{Co}^{3+}, \mathrm{Al}^{3+}\right)$ and $\mathrm{Y}_{1-\mathrm{y}} \mathrm{Ca}_{\mathrm{y}} \mathrm{Ba}_{2} \mathrm{Cu}_{2.7} \mathrm{Co}_{0.3} \mathrm{O}_{7-\mathrm{d}}$. The introduction of $\mathrm{Ca}^{2} \mathrm{x}_{7}$ at the $\mathrm{Y}^{3+}$ site reiniroduces boles in the $\mathrm{CuO}_{2}$-planes is $\mathrm{Y}-123$ doped with cobalt. From the large number of samples investigated it has been possible to obtain a firm understanding of the structure-property relations is the $Y-123$ system. 
P5.6 APPLICATION OF HIGH-RESOLUTION POWDER NEUTRON DIFFRACTION TO THE DETERMINATION OF THE NUCLEAR AND MAGNETIC STRUCTURES OF $\left(\mathrm{Y}_{1-\mathrm{x}} \mathrm{Ca}_{\mathrm{x}}\right) \mathrm{Ba}_{2} \mathrm{Fe}_{3} \mathrm{O}_{8-\delta} . \mathrm{Q}$. Huang, P. Karen, V. Karen, J.W. Lynn, A.D. Mighell, I. Natali-Sora, N. Rosov \& A. Santoro. Materials Science and Engineering Laboratory, National Institute of Standards and Technology, Gaithersburg, Maryland 20899, U.S.A.

The nuclear and magnetic structures of $\left(\mathrm{Y}_{1-\mathrm{x}} \mathrm{Ca}_{\mathrm{x}}\right) \mathrm{Ba}_{2} \mathrm{Fe}_{3} \mathrm{O}_{8-\delta}$ have been determined as function of temperature, calcium doping, and oxygen stoichiometry, using data collected with the high-resolution five-counter neutron diffractometer at the Reactor of the National Institute of Standards and Technology. The general atomic configuration in the unit cell of this compound is similar is that of the superconductor $\mathrm{YBa}_{2} \mathrm{Cu}_{3} \mathrm{O}_{7}$. For $\delta=0$ the symmetry of $\left(\mathrm{Y}_{1-\mathrm{x}} \mathrm{Ca}_{\mathrm{x}}\right) \mathrm{Ba}_{2} \mathrm{Fe}_{3} \mathrm{O}_{8}$ is tetragonal and the iron ions corresponding to the Cu chains have octahedral coordination since all the oxygen sites on the basal plane are fully occupied. For $\delta>0$, the oxygen vacancies occur on the basal plane and in such a way that some of the iron ions remain octahedrally coordinated and some assume a square-planar coordination similar to that of the chain copper ions in the $1,2,3$ compound. This means that oxygen is eliminated preferentially only in one direction of the basal plane and, as a consequence, the symmetry of the compound becomes orthorhombic. The magnetic structure is based on a unit cell related to that of the nuclear structure by an axis transformation of matrix (1 $\overline{1} 0 / 110 / 002)$. The iron moments are coupled antiferromagnetically within each $\mathrm{FeO}_{2}$ layer. The coupling along the c-axis, however, depends on the values of $\mathrm{x}$ and $\delta$.
P5.7 A NEUTRON POWDER DIFFRACTION STUDY OF THE STRUCTURAL CHANGES IN $\mathrm{Tl}_{2-\mathrm{x}} \mathrm{Ba}_{2} \mathrm{CuO}_{6-\mathrm{d}}$ AND $\mathrm{Tl}_{2-\mathrm{x}}$ $\mathrm{Ba}_{2} \mathrm{CaCuO}_{8-d}$ RELATED TO THE OXYGEN AND THALLIUM CONTENT. C. Ström*, S. Eriksson*†, and L.-G. Johansson*, *Dept. of Inorganic Chem., S-412 96 Göteborg, SWEDEN. †MPI für Festkörperforschung, 7000 Stuttgart 80, GERMANY.

The thallium-based high $T_{c}$ superconductors with two Tl-O layers have tetragonal symmetry except for $\mathrm{Tl}_{2} \mathrm{Ba}_{2} \mathrm{CuO}_{6-d}$ (T1-2201), which exists in both tetragonal and orthorhombic form. Tl-2201 shows a remarkably wide range in $\mathrm{T}_{c}(0-90 \mathrm{~K})$ depending on preparative conditions. It is known that changes in $\mathrm{T}_{\mathrm{c}}$ and cell parameters can be correlated with changes in oxygen content. $\mathrm{Tl}_{2-\mathrm{x}} \mathrm{Ba}_{2} \mathrm{CaCuO}_{8-\mathrm{d}}(\mathrm{Tl}-2212)$ shows no phase transition but it does show a large variation in the $c$ axis length dependent on the method of preparation.

In this investigation we have studied the structural changes by neutron diffraction of samples with different thallium and oxygen content in order to simulate different synthetic conditions.

High quality neutron diffraction data were collected at the spallation source ISIS, RAL, UK, using the high flux, medium resolution powder diffractometer POLARIS. Data were collected from twelve solid state sintered single phase samples of $\mathrm{Tl}_{2-\mathrm{x}} \mathrm{Ba}_{2} \mathrm{CuO}_{6-d}$ and $\mathrm{Tl}_{2-\mathrm{x}} \mathrm{Ba}_{2} \mathrm{CaCuO}_{8-\mathrm{d}}$ with different $\mathrm{x}$ and $\mathrm{d}$, and analyzed by the Rietveld method.

Results from the analysis show that the tetragonal-orthorhombic phase transition in T7-2201 is a function of both the oxygen and thallium content. The samples with high thallium deficiency are tetragonal. Orthorhombic samples with small thallium deficiency become tetragonal after oxygen treatment. The structural changes are largest in the Tl-O layers. T1-2212 shows a shortening of the $c$-axis when the thallium and oxygen content increase. However, the changes in bondlengths are not additive. Structural and $T_{c}$ data will be presented for Tl-2201 and Tl-2212. 
K6.1 DEVELOPMENT OF HIGH PRESSURE POWDER DIFFRACTION ON SYNCHROTRON SOURCES. Y. Fujii, Institute of Materials Science, University of Tsukuba, Tsukuba, Ibaraki 305 and now Institute for Solid State Physics, The University of Tokyo, 7-22-1 Roppongi, Minato-ku, Tokyo 106, Japan.

By using pressure to directly control inter-atomic interactions, we have studied crystal structures newly stabilized at high pressures and related phase transition phenomena. At high pressures, hydrostaticity is no longer maintained because of the freezing of the pressure-transmitting fluid. This fact necessarily leads us to the use of powder specimens for diffraction experiments to obtain reliable intensity data. The relatively small aperture of a high-pressure apparatus seriously limits the accessible reciprocal space; therefore, powder diffraction has another advantage of allowing the collection of as much diffraction data as possible in such a restricted geometry. In order to enable us to obtain reliable intensity data from the powder specimen, the following systems dedicated to synchrotron radiation $\mathrm{x}$-ray diffraction experiments at the Photon Factory have been developed cooperatively by the High-Pressure Group in Japan:

(1) SR (BM, MPW on the $2.5 \mathrm{GeV}$ Storage Ring, 10-20 keV)

- Diamond-anvil cell (at low/high temperatures)

- Imaging-plate, SSD (energy-dispersive mode)

- Data acquisition software,

(2) SR (BM on the $6 \mathrm{GeV}$ Accumulation Ring, $10-100 \mathrm{keV}$ )

- Multi-anvil apparatus (high temperatures, MAX-80 \& -90)

- SSD (energy- or angle-dispersive mode)

The precise structure determination of molecular crystals and metals has enabled us to study precursory behavior, structural instability, and structural-similarity/scaling-rule uniquely driven by pressure.

\section{O6.1}

HIGH TEMPERATURE X-RAY DIFFRACTION DETERMINATION OF PHASE TRANSITIONS IN LARGE GRAIN ALLOYS. O. B. Cavin*, C.R. Hubbard, and P.J. Maziasz, Metals and Ceramics Division, Oak Ridge National Laboratory, Oak Ridge TN 37831-6064, Phone (615) 574-5121.

HTXRD studies of the phase equifibria of alloys as a function of temperature depend. upon the ability 10 accurately follow the intensity of particular lines. Large grain size introduces the additional complication of spotty patterns due to poor particle statistics yielding ambiguous or uncertain intensities. To overcome this limitation, a rocking curve method has been used which involves sample oscillation about the goniometer axis. We have studied inherently large grained intermetallic aluminides, some of which have two ordered lattice types $\left(\mathrm{DO}_{3}\right.$ and $\left.\mathrm{B} 2\right)$ and have determined the transition temperatures of each type. These temperatures are required for modeling the alloys and for predicting ideal forming temperatures. By carefully choosing the alloying additions, the properties of these alloys can be controlled. Changes in the transition temperatures have been observed which makes them more attractive for specific applications.

Research sponsored by the U.S. Deparment ol Energy. Assistant Secretay lor Conservation and Renewable Energy, Office of industnal Teehnologies. Advanced Indusuial Concepts Materials Program, under contract DE.ACO5-84OR21400 with Martin Manenta Energy Systems. Inc
O6.2 NEUTRON POWDER DIFFRACTION AT THE HIGH FLUX ISOTOPE REACTOR AT OAK RIDGE *. J. A. Fernandez-Baca', H. A. Mook ${ }^{1}$, H. S. White ${ }^{2}$, B. S. Hoffheins ${ }^{3}$, and B. C. Chakoumakos ${ }^{1},{ }^{1}$ Solid State Division, ${ }^{2}$ Computing and Telecommunications Division, ${ }^{3}$ Instrument and Control Division, Oak Ridge National Laboratory, Oak Ridge, Tennessee 37831, U. S. A.

A new multidetector neutron powder diffractometer has been installed at the HB4 beam tube at the High Flux Isotope Reactor at Oak Ridge National Laboratory. The diffractometer design currently includes a $12^{\prime}$ collimator in the primary reactor beam, a flat $\mathrm{Ge}\left(\begin{array}{lll}1 & 1 & 5\end{array}\right)$ monochromator crystal and 32 equally spaced $\left(2.7^{\circ}\right){ }^{3} \mathrm{He}$ counters, with $6^{\prime}$ Soller-slit collimators, that can be scanned to obtain a diffraction pattern between $11^{\circ}$ and $135^{\circ} 2 \theta$. A new, vertically focusing, Ge monochromator will be installed in the near future. The monochromator angle is usually fixed at $2 \theta_{M}=80^{\circ}$ to select neutrons of a ravelength of $1.4 \dot{A}$. The instrumental resolution for this configuration has a minimum of $\Delta d / d=2 \times 10^{-3}$. The instrument control is performed from a microVax computer, which is also used for data visualization and analysis. In order to allow for different users' preferences various Rietveld analysis codes are available. These include RIETAN (by F. Izumi), LHPM1 (by R. J. Hill and C. J. Howard) and GSAS (by A. C. Larson and R. B. Von Dreele). Various tests of the performance of the diffractometer have been completed successfully and this instrument is now available for routine use. These tests included the crystal structure refinements for a number of powder diffraction standards ( $\mathrm{Ni}, \mathrm{Si}, \mathrm{Al}_{2} \mathrm{O}_{3}, \mathrm{ZrO}_{2}$, etc). and a variety of oxides: $\mathrm{M}_{3} \mathrm{Al}_{2} \mathrm{O}_{6}, \mathrm{M}=$ $\mathrm{Ca}, \mathrm{Sr}, \mathrm{Ba} ; \mathrm{Sr}_{3} \mathrm{Al}_{2}\left(\mathrm{D}_{4} \mathrm{O}_{4}\right)_{3} ; \mathrm{MCuSi}_{4} \mathrm{O}_{10}, \mathrm{M}=\mathrm{Ca}, \mathrm{Sr}, \mathrm{Ba}$; and $\mathrm{PbAl}_{2} \mathrm{Si}_{2} \mathrm{O}_{8}$. The best fits were obtained using the pseudo-Voigt peak shape function.

-Supported by the Division of Materials Sciences, U. S. D. O. E. under contract DE-AC05-84OR21400 with Martin Marietta Energy Systems, Inc.

P6.1 A NEW FOURIER DIFFRACTOMETER AT THE IBR-2 PULSED REACTOR - HIGH RESOLUTION, HIGH INTENSITY, HIGH ACCURACY. A.M.Balagurov, Laboratory of Neutron Physics, JINR, 141980 Dubna, Moscow Reg., Russia.

Two neutron Fourier diffractometers are in practical operation at present: the mini-SFINKS facility/1/ in Gatchina (Russia) and FSS/2/ in Geesthacht (Germany). They use the Reverse Time Of Flight (RTOF) method $/ 3 /$ of data acquisition and have good prospects in structural and residual stress studies. It is anticipated that a new high resolution Fourier diffractometer (HRFD) will commence operation in Dubna (Russia) at the IBR-2 pulsed reactor in $1992^{/ 4 /}$. At this diffractometer the RTOF mode will be also used, and precise structural studies of powders with very high resolution and high intensity are proposed as the main area of interest. The $\Delta d / d$ resolution of 0.0005 is provided by the high frequency of neutron beam modulation $(\sim 150 \mathrm{kHz})$; a high intensity is due to the high transmission of the Fourier chopper $(\sim 0.25)$ and the large solid angle $(0.1 \mathrm{sr})$ of the time focused detector system. The additional modulation of the beam by neutron source pulses leads to a considerable (by 10 or more times) decrease in the correlation background level compared to a Fourier diffractometer at a steady state reactor. The high total neutron flux at the sample position enables studies of very small samples and time resolved experiments. The measurement time for a complete diffraction pattern is determined by the $\Delta d / d$ resolution and in the low-resolution limit $(1 \%)$ is much less than one minute. The opportunities for structural studies with the new Fourier diffractometer are expected to be similar to those of the HRPD at the RAL and, in addition, the new instrument offers the possibility of optimizing the experimental conditions with respect to resolution and intensity.

1. V.A.Trunov et al, Sol.St.Comm., 59 (1986) 95

2. H.G.Priesmeyer, J.Schroder, Mat.Res.Soc.Symp.Proc., 166 (1990) 299

3. P.Hiismaki et al, J.Appl.Cryst., 21 (1988) 349

4. A.M.Balagurov, Physica B, 174 (1991) 542 
P6.2 THE HIGH RESOLUTION NEUTRON POWDER DIFFRACTOMETER AT JRR-3M. Y. Morii, K. Fuchizaki(*), S. Funahashi, N. Minakawa, Y. Shimojyo and A. Ishida $\left(^{* *}\right)$, Department of Physics, Japan Atomic Energy Research Institute, Tokai, Ibaraki 319-11, Japan

The characteristics of the High Resolution Neutron Powder Diffractometer (HRPD) installed and studied recently at the JRR-3M are reported. Some experiments performed with the HRPD are also presented. The HRPD is an ILL-D2B type diffractometer which has the following features. (1) a fine collimation system: $6^{\prime} / 12^{\prime}-20^{\prime} / 40^{\prime}-6^{\prime}$, (2) pseudobent germanium 331 and copper 311 monochromators with take-off angles of $89^{\circ}$ or $130^{\circ}$, (3) Sixty four 3rd collimator and detector systems placed at every $2.5^{\circ}$ of diffraction angle. Typical diffraction patterns can be taken over scattering angles from $5^{\circ}$ to $160^{\circ}$ in a few hours, depending on the sample material, and analyzed by the Rietveld profile refinement method or others. For the case of a monochromator take-off angle of $130^{\circ}$, the angular resolution, FWHM, of the diffraction peaks is less than $0.35^{\circ}$ over the whole scattering angle range. The best resolution is $0.2^{\circ}$ at the focusing position, which corresponds to $0.2 \%$ in $\Delta d / d$. Various kinds of materials such as high $T_{e}$ superconductors, ionic conductors, metals, carbon clusters etc. have been studied with the HRPD. Among them, $\mathrm{La}_{2} \mathrm{NiO}_{4.00}$ was investigated between $610 \mathrm{~K}$ and $6 \mathrm{~K}$. The crystal structure below $60 \mathrm{~K}$ was determined to be orthorhombic, and the magnetic moment of $\mathrm{Ni}^{2+}$ was evaluated to be about $1.5 \mu_{\mathrm{B}}$ at $80 \mathrm{~K}$ and $1.7 \mu_{\mathrm{B}}$ at $6 \mathrm{~K}$ through a Rietveld analysis.

$\left(^{*}\right)$ present address: Department of Physics, Faculty of Science, Kyusyu Unjversity; Fukuoka 812 Japan

$\left({ }^{* *}\right)$ present address: Nuclear Plant Department, NKK corporation; Yokohama 230 Japan

\section{P6.3} THE HIGH RESOLUTION POWDER DIFFRACTOMETER AT THE NIST REACTOR. E. Prince, A. Santoro, I. G. Schröder \& J. K. Stalick. Materials Science and Engineering Laboratory, National Institute of Standards and Technology, Gaithersburg, Maryland 20899, U. S. A.

A new, state-of-the-art, high-resolution, neutron powder diffractometer has recently been installed at the NIST research reactor. Because optimum powder patterns require differently shaped curves of resolution as a function of $d$ spacing for crystals with different unit cell sizes, this instrument has provision for producing monochromatic beams with $\sim 1.5 \AA$ wavelength at three different monochromator takeoff angles. A $75^{\circ}$ takeoff angle is most useful for large unit cell crystals such as zeolites, where there is comparatively little information at short $\mathrm{d}$ spacings. On the other hand, a $120^{\circ}$ takeoff angle is necessary for crystals with small, but low symmetry, unit cells. The instrument has $32 \mathrm{de}$ tectors spanning an angle of $155^{\circ}$. Focusing monochromators allow optimum use of reactor neutrons without significant degradation of peak shape. The instrument will be used for the determination and refinement of the structures of a wide range of technologically important materials.
P6.4 NEUTRON POWDER DIFFRACTION TO $20 \mathrm{GPa}$. J. S. Loveday, R. J. Nelmes, Department of Physies, University of Edinburgh, Edinburgh EH9 3JZ, UK, J. M. Besson, G. Hamel, Université Pierre et Marie Curie, 4 Place Jussieu, 75252 Paris, Cedex 05, France, and S. Hull, the ISIS Facility, Rutherford Appleton Laboratory, Chilton, Oxfordshire, UK

The maximum pressure accessible to neutron diffraction techniques has until recently remained in practice at the $\approx 3 \mathrm{GPa}$ available with McWhan type alumina clamp cells. This has limited the study of a range of classic neutron problems (magnetism, structures containing light atoms, disorder, etc). To address this problem we have been developing an opposed-anvil cell, able to take samples of sufficient size for crystallographic studies to pressures in excess of $20 \mathrm{GPa}$. This cell is now in use on the POLARIS powder diffractometer at the UK spallation neutron source ISIS.

The cell consists of a compact $200 t$ hydraulic ram applying pressure to a sample contained between two opposed anvils. These are made either of tungsten carbide or, for the highest pressures, sintered-diamond. A toroidal anvil profile is used and allows a sample of $\approx 100 \mathrm{~mm}^{3}$ volume to be taken to $10 \mathrm{GPa}$ or $30 \mathrm{~mm}^{3}$ to be taken to $20 \mathrm{GPa}$.

This geometry has required the development of new shielding procedures to obtain the optimum signal to background and a means to correct for the complicated attenuation by the anvils. This work is still in progress, but the results of a number of preliminary structural studies will be presented, including the pressure dependence of the structure of $\mathrm{D}_{2} \mathrm{O}$ ice VIII and the high-pressure phases of bismuth .

\section{P6.5}

HIGH-PRESSURE STRUCTURAL STUDIES USING ANGLE-DISPERSIVE TECHNIQUES. M. I. McMahon, R. J. Nelmes, P. D. Hatton, R. O. Piltz, J. Crain and D. R. Allan, Department of Physics, The University of Edinburgh, Edinburgh, EH9 3JZ, UK.

During the past year, an image-plate (IP) system has been developed at SRS Daresbury with the objective of performing full Rietveld refinement of high-pressure crystal structures using angle-dispersive powder-diffraction techniques. Considerable care has been taken to reduce background scatter and eliminate contaminant diffraction lines, and this has enabled us to collect data of unrivalled quality. In our initial studies of the III-V semiconductor $\mathrm{InSb}$, we have discovered two new high-pressure phases, and have shown that the long-accepted structures of two more highpressure phases are incorrect. Furthermore, the very high sensitivity of the IP system, coupled with anomalous dispersion techniques, has enabled us to determine whether the high-pressure phases of InSb are ordered or not - a question that has remained unanswered for 30 years. The latest results on InSb will be described, along with new results from a nother III-V material, GaSb. Very recently, we have managed to obtain high-quality diffraction patterns from the low- $Z$ semiconductor $\mathrm{B}_{4} \mathrm{C}$. The effect of pressure on this interesting material will also be presented. 
P6.6 AUTORIET: SOFTWARE FOR "AUTOMATING" RIETVELD ANALYSIS OF TIME-OF-FLIGHT NEUTRON POWDER DIFFRACTION DATA. F. J. Rotella, Intense Pulsed Neutron Source (IPNS) Division, and R. L. Hitterman, Materials Science Division, Argonne National Laboratory, Argonne, Illinois 60439 , U. S. A.

In certain types of experiments (e.g., time-resolved diffraction measurements), the rate at which the data are collected far outstrips the speed with which the said data can be analyzed by standard (user-intervention) Rietveld techniques. In an effort to deal with this situation, computer software has been written to automate Rietveld analysis at IPNS by optimizing data analysis time, much in the same way that computer control of instruments has optimized data collection times and sample throughput. AUTORIET is similar to software which has been used for the automated analysis of time-resolved neutron powder data from the Institut Laue-Langevin for the past eight or so years. It is a VAX/VMS DCL command file that, with auxiliary FORTRAN programs, creates the appropriate files that will be used for input to the IPNS Rietveld analysis software, controls the execution of the software, and creates summaries of the analysis for all sets of data considered. The user analyzes the initial data set via standard Rietveld techniques; AUTORIET will analyze the remaining sets in a user-specified order, based on the assumption that the starting parameters for any given data set are the results from the analysis of the preceding set. The summaries that are produced may be collated into files from which the variation of any parameters with respect to one or more other parameters in the model may be obtained in tabular form and/or graphically using visualization software on the IPNS VAX Cluster or on a workstation or personal computer. Although AUTORIET was designed to be used specifically with software in the IPNS Rietveld analysis package, adapting it for use with other Rietveld analysis software in a VAX/VMS environment should be fairly easy. The use of AUTORIET in the analysis of in situ diffraction data from a working electrochemical cell and in the determination of therma expansion coefficients of bismuth-based superconductors will be presented.

This work was supported by the U. S. Department of Energy under Contract W-31-109-ENG-38.

\section{P6. 7}

HIGH-PRESSURE X-RAY

DIFFRACTION STUDY OF INDIUM UP TO 93 GPA K. Takemura, National Institute for Research in Inorganic Materials, Tsukuba, Ibaraki 305 JAPAN

Structural change of indium under pressure has been precisely studied up to $93 \mathrm{GPa}$ at room temperature using a diamond-anvil cell, synchrotron radiation and an imaging plate. The c/a axial ratio of the face-centered tetragonal ( $f c t$ ) structure increases with pressure, reaching a maximum around $24 \mathrm{GPa}$ ( $\left.(c / a)_{\max }=1.091\right)$ and decreases with further compression [1]. At around $45 \mathrm{GPa}$, indium undergoes a structural phase transition. The powder diffraction pattern of the high-pressure phase can be indexed to a face-centered orthorhombic cell with the lattice constants of $a=3.771, b=3.841, c=4.142 \AA$ at $93 \mathrm{GPa}$. By assuming plastic deformation of indium under uniaxial stress, the observed diffraction intensity can be successfully explained, including the asymmetric peak profiles. The model assumes a successive slip of the (111) plane in the [011] direction, which often occurs in the fcc metals subjected to uniaxial stress. The estimated volume change at the fct-fco transition is close to zero, offering a possibility that the transition is of the second order. The transition was reversible with respect to pressure.

The lowering of the lattice symmetry of indium under pressure can be understood on the basis of the universal phase diagram for the group IIIb elements calculated by Hafner and Heine with the pseudopotential method [2].

[1] K. Takemura, Phys. Rev. B44, 545 (1991).

[2] J. Hainer and V. Heine, J. Phys. F13, 2479 (1983).

\section{P6.8} HIGH RESOLUTION SYNCHROTRON X-RAY POWDER DIFFRACTION WITH A LINEAR POSITION-SENSITIVE DETECTOR. D. E. Cox and G. C. Smith, Brookhaven National Laboratory, Upton, N.Y. 11973, U.S.A., L. W. Finger, Carnegie Institution, Washington, D. C. 20015, U.S.A., and A. P. Jephcoat, Oxford University, Oxford OX13PR, England.

Some synchrotron $\mathbf{x}$-ray powder diffraction experiments are described in which a multiwire proportional detector was operated in the "escape-peak" mode with a $90 \% \mathrm{Kr}-10 \% \mathrm{CO}_{2}$ gas mixture at 4 bar. Under these conditions, it is possible to achieve intrinsic position resolution of about $30 \mu \mathrm{m}$ for $17.4 \mathrm{keV} X$-rays, compared to about $150 \mu \mathrm{m}$ for the "photo-peak" mode [1]. A gating signal can be applied to the position-sensing electronics to provide energy discrimination. The detector, which has an active length of $10 \mathrm{~cm}$, was mounted vertically on the scattering arm of the diffractometer at beam-line X7A at the NSLS about $45 \mathrm{~cm}$ from the sample axis. With well-crystallized reference materials such as $\mathrm{CeO}_{2}$ and $\mathrm{Al}_{2} \mathrm{O}_{3}$ and an incident $0.7 \AA$ x-ray beam with horizontal and vertical dimensions of 4.0 and $0.2 \mathrm{~mm}$ respectively, the angular resolution obtained in the center of the detector was about $0.03^{\circ} \mathrm{FWHM}$, although there was considerable degradation at the ends because of parallax effects. High quality data suitable for precision lattice parameter determination, auto-indexing or Rietveld analysis can be obtained in as little as a few minutes by stepping the detector at $2^{\circ}$ intervals and combining the central segments into a composite data set. Some applications will be described, including diffraction experiments in diamond anvil cells.

[1] J. Fischer, V. Radeka, and G. C. Smith, IEEE Trans. Nucl. Sci. NS-33, 136 (1986); Nucl. lnstrum. and Meth. A252, 239 (1986).

Work supported by the Division of Materials Sciences, U.S. Dept. of Energy, Contract DE-AC02-76CH00016 (DEC and GCS), National Science Foundation grant EAR-8817263 (LWF), and Nato CRG 890944 and Natural Environment Research Council grant GR3/7984 (APJ).

P6.9 TIME RESOLVED POWDER DIFFRACTION. H. Fuess and P. U. Pennartz*, TH Darmstadt FB21/Strukturforschung. *now: ENRAF/NONIUS-Solingen Germany.

Complete powder diffraction patterns were recorded at the synchrotron source DORIS Il (HASYLAB, Hamburg) in time intervals of milliseconds. Recording of the diffracted intensity was achieved by a commercial diodearray detector (EG \& G-PARC) in Debye-Scherrer geometry. A time resolution of $200 \mathrm{msec}$ for a snapshot of $40^{\circ}(2 \theta)$ was obtained by a toroidal mirror and a conventional $(n,-n)$ silicon (111) double crystal monochromator.

The detector consists of 1024 single silicon $(p / n)$ diodes each $25 \mu \mathrm{m}$ wide and $2 \mathrm{~mm}$ high. The detecting window is therefore only $25 \mathrm{~mm}$ long. In order to record a large region of the diffraction pattern the detector is mounted very close to the sample. The angular resolution of this setup is comparable with that of a standard $x$-ray powder pattern from a conventional source, but the time resolution is about 10 times higher than with conventional systems. Enlarging the detector to sample distance will increase the angular resolution and decrease the integral time resolution.

A multilayer monochromator (OVONICS, 200/W/Si double layers of 2.05 $\mathrm{nm}$ thickness each) decreased the measurement time for a whole pattern to $2.5 \mathrm{msec}$. The multilayer system, however, increased the energy-bandpass by two orders of magnitude. Thus the angular resolution is about 10 times worse than the angular resolution of conventional powder diffractometers (Pennartz et al., J. Appl. Cryst, 1992, in press).

In contrast to previous work (Wong et al. 1990, Science, 249, 1404) we aimed at the recording of a complete powder pattern and not just a small region or a single Bragg-reflection. Therefore we tested two other detectors (Pennartz et al. Z. Kristallogr. 1989, 186, 227-230), including the bladeanode ENRAF-NONIUS/INEL $120^{\circ}$ curved CPS120. The performance of these detectors has been tested using conventional sealed $\mathrm{x}$-ray tube sources and rotating anodes, and also at the synchrotron. 


\section{P6.10 A NEW METHOD FOR POWDER DIFFRACTION}

STUDIES. Mark A. Beno and Gordon S. Knapp,

Material Science Division. Argonne National Laboratory,

Argonne 1160439 , U.S.A.

Powder diffractometers are among the most widely used of all analytical instruments. Seemann-Bohlin diffractometers, the most widely used type, measure data serially, one angle at a time with many angles scanned to observe the entire diffraction pattem. Linear and area detectors lack energy resolution and so can suffer from high backgrounds particularly if materials containing elements which fluoresce are to be studied. In addition, the one dimensional detectors developed to date do not possess sufficient resolution for highly accurate structural studies particularly in the case of synchrotron based $x$-ray powder diffraction instruments.

The method we employ uses a focusing diffracted beam monochromator in combination with a multichannel detector which allows us to reduce the background and to take data in parallel, providing a large improvement over conventional diffractometers. In our technique the incident $\mathrm{x}$-rays (from a laboratory source or synchrotron) fall on a flat plate or capillary sample and are intercepted by a bent focusing monochromator which has the focus of the bend at the sample surface. The beam emerging from the bent crystal monochromator falls on a linear or 2-dimensional detector. Initial tests show that this method can be used to acquire the data much faster and with much higher sensitivity. Existing instruments can be easily modified to employ this method.

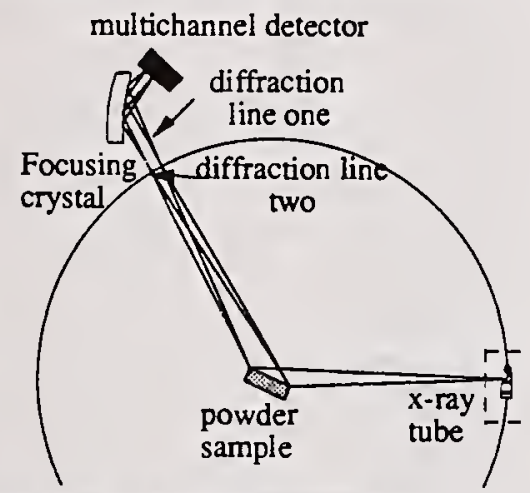

Results from laboratory and synchrotron sources will be presented for crystalline and glassy materials along with schemes for rapid data collection employing other monochromator and detector configurations.

Work at Argonne National laboratory is sponsored by the US Department of Energy, Office of Basic Energy Sciences, Division of Materials Sciences, under contract W-31-109-ENG-38.
P6.11 HIGH TEMPERATURE NEUTRON POWDER DIF. FRACTION FURNACES, S. L. Town, R. L. Davis * \& E. H. Kisi ${ }^{\dagger}$, ANSTO, PMB 1, Menai, NSW 2234, Australia, *AINSE, PMB 1, Menai, NSW 2234, Australia, †Division of Science \& Technology, Griffith University, Nathan, Queensland 4111, Australia.

A neutron powder diffraction furnace, capable of temperatures to $1100^{\circ} \mathrm{C}$ has been tested and commissioned for use on the medium and high resolution powder diffractometers at ANSTO. The design of this is discussed, and data from $\mathrm{Y}_{2} \mathrm{BaCuO}_{5}$ and $\mathrm{CaF}_{2}$ are presented; problems in refining thermal parameters are highlighted. A new furnace, capable of temperatures to $1750^{\circ} \mathrm{C}$ is currently being developed, and the design is detailed here. 
Acharya, B. S.

Akselrud, L. G.

Allan, D. R

Artioli, G.

Attfield, J. P.

Auffrédic, J. P.

Balagurov, A. M.

Balzar, D.

Baumgartner, B.

Bell, A. M. T.

Bellotto, M.

Beno, M. A.

Berastegui, P.

Berkum, J. G. M. van

Bérar, J. F.

Bernstein, J.

Berti, G.

Besson, J. M.

Bish, D. L.

Boultif, A.

Boysen, H.

Brand, P. C.

Brauer, S.

Bryant, P.

Cavin, O. B.

Cernik, R. J.

Chakoumakos, B. C.

Champness, P. E.

Chatterjee, S. K.

Chattopadhyay, S. K.

Cheary, R. W.

Cherner, Ya. E.

Choi, C. S.

Cline, J. P.

Coelho, A.

Cong, Q.

Cook, L. P.

Corbin, R.

Cox, D. E.

Crain, J.

David, S. A.

David, W. I. F.

Davis, B. L.

Davis, R. L.

Deem, M. W.

Delhez, R.

Dodson, T. A.

Egami, T.

Eriksson, L.

Eriksson, S.

Ermrich, $M$.

Ersson, N. O.

Estermann, $M$.
P4.14

P3.1

P6.5

P1.1

175

P4.12

P6.1

P4.13

P3.1

P5.1

P1.1

P6.10

P5.5

P2.3

63, P3.5

P1.4

P4.10

195,P6.4

154

P4.12

165

P2.2

189

P1.6

O6.1

P5.1

P5.2, O6.2

P5.1

P4.16

P4.16

P4.6, O5.2

P3.2, P3.3, P3.4

$\mathrm{P} 4.5$

68

P4.6

P3.13

$\mathrm{P} 4.5$

O5.1

O5.1, P6.8

P6.5

P4.1

P2.6, P3.11

P6.11

80

P2.3, P4.9

P4.1

105

P3.12

P5.5, P5.7

38

P3.6

O3.1
Fernandez-Baca, J. A.

Finger, L. W.

Fischer, H. E.

Fitch, A. N.

Freeman, C. M.

Fuchizaki, K.

Fuess, $\mathrm{H}$.

Fujii, Y.

Fukunaga, $\mathrm{O}$.

Funahashi, S.

Gamarnik, M. Ya.

Garnier, P.

Gramlich, V.

Grin, Yu. N.

Hall, S. R.

Hamel, G.

Hashizumi, $\mathrm{H}$.

Hatton, P. D.

Havlik, T.

Henderson, C. M. B.

Hermann, $\mathrm{H}$.

Hewat, A. W.

Hill, R. J.

Hiraguchi, $\mathrm{H}$.

Hitterman, R. L.

Hoffeins, B. S.

Holden, T. M.

Howard, C. J

Hoyle, S. Q.

Hriljac, J.

Hubbard, C. R.

Huang, Q.

Huang, T. C.

Hull, S.

Ibberson, R. M.

Ishida, A.

Ivanov, S. A.

Järvinen, $M$.

Jephcoat, A. P.

Johansson, L. G.

Johnson, G. G., Jr.

Karen, P.

Karen, V.

Keijser, Th. H de

Kisi, E. H.

Klimanek, P.

Knapp, G. S.

Kogan, V. A.

Kohn, S. C.

Lach, S.

Lager, G. A.
$\mathrm{P} 5.2, \mathrm{O} 6.2$

183, P6. 8

189

P5.1

80

P6.2

P6.9

K6.1

P5.3

P6.2

P2.4

P3.5

O3.1

P3.1

P2.1

P6.4

$\mathrm{P} 5.3$

P6.5

P3.14

P5.1

38

P3.7

P1.2

P5.3

P6.6

O6.2

136

P1.5, P3.10

P1.6

O5.1

P4.1, O6.1

P5.6

P6.4

P3.11

P6.2

P4.15, P5.4

P4.2

P6.8

P5.5, P5.7

P1.6

P5.6

P5.6

P2.3

P1.5, P6.11

O1.3, P4.3, P4.11

P6.10

P4. 8

P5.1

P1.4

P5.2 
Langford, J. I.

Le Bail, A.

Ledbetter, $\mathrm{H}$.

Li, D.

Louër, D.

Loveday, J. S.

Lutterotti, L.

Luzzati, V.

Lymar, V. K.

Lynn, J. W.

Madsen, I. C.

Maistrelli, P.

Marquart, R. G.

Mateu, L.

Mattausch, Hj.

Maziasz, P. J.

McCusker, L. B.

McMahon, M. I.

Mehdi, S.

Mighell, A. D.

Minakawa, N.

Mook, H. A.

Morii, Y.

Mücklich, A.

Nakano, S.

Nakhmanson, M. S.

Natali-Sora, I.

Nelmes, R. J.

Newman, R. A.

Newsam, J. M.

O'Connor, B. H.

Oettel, H.

Parise, J. B.

Pecharski, V. K.

Peerdeman, F.

Pennartz, P. U.

Petricko, F.

Piltz, R. O.

Pradhan, L. D.

Prask, H. J.

Prince, E.

Ramamurthy, V.

Reddy, M.

Redfern, S. A. T.

Root, J. H.

Rosov, N.

Rotella, F. J.

Sakata, M.

Santoro, A.

Scardi, P.

Schneider, J.
110, P2.1, P4.12

142, P3.9

P4.13

P4.4

92, P4.12

195, P6.4

P4.7

O5.3

P4.15

P5.6

P1.2

P4.7

O1.1

O5.3

P5.5

O6.1

75

P6.5

P1.3

P5.6

$\mathrm{P} 6.2$

O6.2

P6.2

P4.3

P5.3

47

P5.6

195, P6.4, P6.5

P2.5

80

P4.4

O1.3

O5.1

P3.1

P4.9

P6.9

P3.14

P6.5

P4.14

P2.2

P6.3

O5.1

P1.3

P5.1

136

P5. 6

P6.6

P3.10

P5.6, P6.3

$\mathrm{P} 4.7$

O3.2
Schröder, I. G.

P6.3

Sen, R.

P4.16

Shimojyo, Y.

P6.2

Simon, A.

P5.5

Sitepu, H.

Sivia, D. S.

Skrobian, M.

Smith, D. K.

Smith, G. C.

Smrčok, $\breve{L}$.

Snyder, R. L.

P4.4

P3.11

P3.14

O1.2, P1.6

P6. 8

P2.7

Sonneveld, E. J.

25

P 2.3

P2.3

Sprong, G. J. M.

O5.2

34, P6.3

Stalick, J. K.

Stephenson, G. B.

189

Ström, C.

Ström-Olsen, J. O.

Sutton, M.

Takata, M.

Takemura, K.

P3.10

P6.7

P4.9

Timmers, J.

Toby, B. H.

Toraya, $\mathrm{H}$.

Tovbis, A. B.

105, P2.1

O2.1

P3.4

P1.5, P6.11

Tsambourakis, G.

Tuinstra, F.

P1.2

P4.9

Uno, $T$.

P3.10

Valvoda, V.

Vargas, R.

Vermeulen, A. C.

127

O5.3

P2.3

Wang, S. L.

Werner, P.-E.

Westdahl, M.

White, H. S.

Wölfel, E.

Wong-Ng, W.

P3.8

51, P3.12

P3.12

O6.2

P3.1

P4.5

Yamanaka, T.

199

Yang, Y. S.

189

Yanovskaya, M. I.

P4.15

Zavalii, P. Yu.

Zevin, L.

Zhurov, V. V.

P3.1

17, P1.4

P4.15 
Tariq Ahmedali

McGill University

3450 University St.

Geol. Sciences

Montreal, H3A 2A7

Canada

Charles Albert

SCM Chemicals

3901 Fort Armistead

Baltimore, MD 21226

U.S.A.

David Allan

Univ. of Edinburgh

Mayfield Rd.

Edinburgh EH9 3JZ

UK

J. Paul Attfield

Univ. of Cambridge

Dept. of Chemistry

Lensfield Rd.

Cambridge CB2 1EW

UK

Neil A. Ball

University of Manitoba

Dept. of Geological Sci.

Rm 24, Wallace Bldg.

Winnipeg, Manitoba R3T 2N2

Canada

Davor Balzar

NIST

325 Broadway 853

Boulder, CO 80303

U.S.A.

Maurizio Bellotto

CISE-Tecnologie Innovative

Via R. Emilia, 39 - Segrate

(MI) PO Box 12081

Milan, 20134

Italy

Mark Beno

Argonne National Lab.

9700 S. Cass Ave.

Bldg. 223, A225

Argonne, IL 60439

U.S.A.
Jean Francois Bérar

Ecole Centrale Paris

voie des Vines

92295 Châtenay

France

J.G.M. van Berkum

Laboratory of Materials Sci.

Rotterdamseweg 137

2628 AL Delft

The Netherlands

Giovanni Berti

Univ. Pisa

Via S. Maria 53

I-56100 Pisa

Italy

David L. Bish

Los Alamos National Lab.

Mail Stop D469

Los Alamos, NM 87545

U.S.A.

Julie A. Borchers

NIST

Bldg. 235, Rm E151

Gaithersburg, MD 20899

U.S.A.

Alun Bowen

Defence Research Agency

Materials \& Structures Dept.

RAE Farnborough

Hants, GU14 6TD

UK

Hans Boysen

Inst. für Kristallographie

Universität München

Theresienstr. 41

8000 München 2

Germany

Paul Brand

University of Maryland/NIST

Bldg. 235, Rm. E151

Gaithersburg, MD 20899

U.S.A.

Bob Broach

UOP

50 East Algonquin Rd.

Des Plaines, IL 60017

U.S.A.
Xianhui Bu

Chemistry Department

Univ. of California (SB)

Santa Barbara, CA 93106

U.S.A.

O. B. Cavin

Oak Ridge National Lab.

PO Box 2008

Bldg 4515, MS 6064

Oak Ridge, TN 37831

U.S.A.

Bryan Chakoumakos

Oak Ridge National Lab.

PO Box 2008

Solid State Division

Oak Ridge, TN 37831

U.S.A.

Lawrence Chatfield

Alfred Univeristy

Inst. for Ceramic Super-

conductivty BMH-1-55

Alfred, NY 14802

U.S.A.

Robert Cheary

University of Technology

Broadway

Sydney, 2007

Australia

Yakov E. Cherner

Institute of Physics

194 Stachki Ave.

344104 Rostov-on-Don

Russia

Feng Chen

Rider College

2083 Lawrenceville Rd.

Chemistry Dept.

Lawrenceville, NJ 08648

U.S.A.

Rosaria Chiappetta

University of Calabria

Arcavacata Di Rende

87030 Cosenza

Italy 
Steve Chipera

Los Alamos National Lab. Mail Stop D469

Los Alamos, NM 87545

U.S.A.

Chang Sun Choi

NIST

Bldg. 235, Rm E151

Gaithersburg, MD 20899

U.S.A.

Jim Cline

NIST

Materials Bldg., Rm A256

Gaithersburg, MD 20899

U.S.A.

Alan A. Coelho

University of Technology

51 Stanley St. East

Sydney 2010 NSW

Australia

Palola Comodi

Piazza Universita

Dipartimento di Scienze della

Terra

I-06100 Perugia

Italy

David E. Cox

Brookhaven National Lab.

Physics 510B

Upton, NY 11973

U.S.A.

M. K. Crawford

DuPont Co.

Experimental Station

E356/209

Wilmington, DE 19889-0356

U.S.A.

Yongshan Dai

American Museum of Natural

History (Mineral Sciences)

Central Park West at 79th St.

New York, NY 10024-5192

U.S.A.

Bill David

Rutherford Appleton Lab.

Chilton, Didcot, Oxon

OX11 0QX

UK
Briant L. Davis

X-Ray Diffraction Laboratory

South Dakota School of

Mines and Technology

Rapid City, SD 57701

U.S.A.

W. Depmeier

University of Kiel

Mineralogisch-Petrogr. Inst.

Olshausenstr 40

D-W-2300 Kiel

Germany

Janet Douglas

Freer Gallery of Art

12th \& Independence Ave.

Washington, DC 20560

U.S.A.

Lars Eriksson

Stockholm University

Arrhenius Laboratory

S-106 91 Stockholm

Sweden

Sten Eriksson

University of Göteborg

Dept. of Inorganic Chem.,

Sven Hultius gata

S-41296 Göteborg

Sweden

Nils O. Ersson

Uppsala University, Inorg.

Chemistry, Box 531

S-75121 Uppsala

Sweden

Michael Estermann

ETH Zürich, Inst. of Cryst.

ETH-Zentrum NOF

CH-8092 Zürich

Switzerland

Howard T. Evans

U.S. Geological Survey

National Center 95Q

Reston, VA 22092

U.S.A.

Melanie E. Feather

Smithsonian Institution

CAL-MSC

Washington, DC 20560

U.S.A.
Jaime Fernandez-Baca

Oak Ridge National Lab.

PO Box 2008 MS 6393

Oak Ridge, TN 37831-6393

U.S.A.

Paul Fewster

Philips Research

Cross Oak Lane

Redhill, RH1 5HA

UK

Larry Finger

Geophysical Lab.

5251 Broad Branch Rd., NW

Washington, DC 20015

U.S.A.

C. M. Foris

Dupont Central Res. \& Develp.

Experimental Station

Bldg. 228

PO Box 80228

Wilmington, DE 19880

U.S.A.

Joseph Formica

Siemens Analytical X-Ray

6300 Enterprise Lane

Madison, WI 53719-1173

U.S.A.

Mario Fornoff

International Centre for

Diffraction Data

1601 Park Lane

Swarthmore, PA 19081

U.S.A.

Yasuhiko Fujii

University of Tokyo

Institute for Solid State

Physics

7-22-1 Roppongi Minato-Ku

Tokyo 106

Japan

Klaus Futterer

Technische Univ. of Berlin

Inst. für Mineral. \& Krist.

Ernst-Reuter-Platz 1

D-1000 Berlin 12

Germany 
Bill Giessen

Northeastern University

Boston, MA 02115

U.S.A.

Ganesh Golikeri

Unilever Research

45 River Rd.

Edgewater, NJ 07020

U.S.A.

Donald Goodwin

Charles Supper Co.

25 Tech Circle

Natick, MA 01760

U.S.A.

Berton Greenberg

Philips Laboratories

345 Scarborough Rd.

Briarcliff Manor, NY 10510

U.S.A.

Boualem Hammouda

NIST

Bldg 235,Rm E151

Gaithersburg, MD 20899

U.S.A.

George Harlow

American Museum of Natural

History

Dept. of Mineral Sciences

Central Park West at 79th St.

New York, NY 10024-5192

U.S.A.

\section{Richard Harlow \\ DuPont Co. \\ CRP E228/316D \\ Wilmington, DE 19880 \\ U.S.A.}

Hiroo Hashizume

Tokyo Institute of Technology

Research Lab. of Eng. Matls.

Nagatsuta, Midori

Yokohama 227

Japan

Daniel Häusermann

European Synchrotron

Radiation Facility

B.P. 220

F-38043 Grenoble Cedex

France
Tomas Havlik

Technical University

Letna 9/A

Dept. Non Ferrous

CS-04385 Košice

Czechoslovakia

Peter Heaney

Princeton University

Guyot Hall

Princeton, NJ 08544

U.S.A.

Helmut Hermann

Inst. of Solid State Res.

Helmholtzstr. 20

Postfach 6

D-0-8027 Dresden

Germany

Alan Hewat

I.L.L.

156 X Grenoble 38042,

France

Rafael Hidalgo

Northeastern University

Boston, MA 02115

U.S.A.

Roderick Hill

CSIRO Mineral Products

PO Box 124

Port Melbourne

Victoria 3207

Australia

T. M. Holden

Chalk River Nuclear Lab.

Atomic Energy of Canada

Chalk River, Ontario K0J 1J0

Canada

Mark Holomany

International Centre for

Diffraction Data

1601 Park Lane

Swarthmore, PA 19081

U.S.A.

Joe Hriljac

Brookhaven National Lab.

Dept. of Physics

Bldg. 510B

Upton, NY 11973

U.S.A.
Jingzhu $\mathrm{Hu}$

Geophysical Laboratory

5251 Broad Branch Rd., NW

Washington, DC 20015-1305

U.S.A.

Qingzmen Huang

University of Maryland/NIST

Bldg. 235, Rm E151

Gaithersburg, MD 20899

U.S.A.

T. C. Huang

Alamaden Research K34/802

IBM Research Division

650 Harry Rd.

San Jose, CA 95120

U.S.A.

Camden Hubbard

Oak Ridge National Lab.

PO Box 2008

Bldg. 4515, MS 6064

Oak Ridge, TN 37831

U.S.A.

Diane Hues

NIST

Bldg. 222, Rm 1113

Gaithersburg, MD 20899

U.S.A.

Matti Järvinen

Lappeenranta Univ. of

Technology

PO Box 20

SF-53851 Lappeenranta

Finland

William Jackson

Shell Development Co.

3737 Bellaire Blvd.

Room 210

Houston, TX 77025

U.S.A.

Ron Jenkins

International Centre for

Diffraction Data

1601 Park Ave.

Swarthmore, PA 19081

U.S.A. 
Quintin Johnson

Materials Data Inc.

PO Box 791

Livermore, CA 94551

U.S.A.

Arthur Jurgensen

Westinghouse

Savannah River Lab.

Aiken, SC 29808

U.S.A.

James Kaduk

Amoco Corporation

PO Box 3011 F-9

Naperville, IL 60566

U.S.A.

Walter Kalceff

University of Technology

PO Box 123 Broadway

Sydney NSW 2007

Australia

Vicky L. Karen

NIST

Bldg. 223, Rm A215

Gaithersburg, MD 20899

U.S.A.

Richard M. Kirchner

Manhattan College

Chemistry Dept.

Bronx, NY 10471

U.S.A.

Peter Klimanek

Mining Academy Freiberg/Sa.

PO Box 47

DO-9200 Freiberg/Sa,

Germany

Vladimir Kogan

Rostov-on-Don State Univ.

Zorge St. 5,

344044 Rostov-on-Don

Russia

Susan Krueger

NIST

Bldg. 235, Rm E151

Gaithersburg, MD 20899

U.S.A.
George A. Lager

University of Louisville

Department of Geology

Louisville, KY 40292

U.S.A.

Vratislav Langer

Chalmers Univ. of Technology

Dept. of Inorg. Chemistry

S-41296 Göteborg

Sweden

J. Ian Langford

University of Birmingham

School of Physics \& Space

Research

Birmingham B15 2TT

UK

Armel Le Bail

Laboratoire des Fluorures

Université du Maine

72017 Le Mans Cedex

France

Kurt Leinenweber

SUNY at Stony Brook

Earth \& Space Science

Stony Brook, NY 11794-2100

U.S.A.

Kenneth Liao

National Steel Corp.

1745 Fritz Dr.

Trenton, MI 48183

U.S.A.

Ulrich Loechner

Forchenweg 1

7100 Heilbronn

Germany

Daniel Louër

Université de Rennes

Laboratorie de Cristallochimie

Avenue de Général Leclerc

35042 Rennes Cedex

France

John Loveday

Rutherford Appleton

Bldg. R3

Chilton, Oxon OX11 0QX

UK
Charlotte Lowe-Ma

Naval Air Warfare Ctr-WPNS

Code 3851

China Lake, CA 93555

U.S.A.

Luca Lutterotti

Univerisitá di Trento

Dept. of Materials Engineering

Via Mesiano, 77

Trento, 38050

Italy

Jeff Lynn

NIST

Bldg. 235, Rm E151

Gaithersburg, MD 20899

U.S.A.

Paul Maistrelli

Dipartimento Di Fisica

Povo

Trento, 38050

Italy

Massimo Marezio

AT\&T Bell Labs/CNRS

IC-211

Murray Hill, NJ 07974

U.S.A.

Curtis Martin

NSWC

10901 New Hampshire Ave.

Code R31

Silver Spring, MD 20874

U.S.A.

Lynne McCusker

Inst. of Crystallography

ETH-Zentrum

CH-8092 Zürich

Switzerland

Malcolm McMahon

The Univ. of Edinburgh

Dept. of Physics

Edinburgh EH9 3JZ,

UK

Jan van Mechelen

Koninklijke/Shell Lab

Badhuisweg 3 AG-Dept.

1031 CM Amsterdam

The Netherlands 
Ron Medrud

Chevron Research \&

Technology Co.

100 Chevron Way

50-1254

Richmond, CA 94802

U.S.A.

Apurva Mehta

Princeton University

113 Guyot Hall

Princeton, NJ 08544

U.S.A.

Alan Mighell

NIST

Bldg. 223, Rm A215

Gaithersburg, MD 20899

U.S.A.

Evan Morgan

Babcock and Wilcox

PO Box 11165

Lynchburg, VA 24506

U.S.A.

Yukio Morii

Japan Atomic Energy

Research Inst.

Tokai, Ibaraki, 319-11

Japan

Arndt Mücklich

Research Centre Rossendorf

P.O.B. 19

$\mathrm{D}(\mathrm{O})-8023$ Dresden

Germany

Michael S. Nakhmanson

Comphys Lab Enterpise

NPO Bourevestnik

68 Mapookhtinsky Ave.

195272 St. Petersburg

Russia

Isabella Natali-Sora

NIST

Bldg. 235, Rm E151

Gaithersburg, MD 20899

U.S.A.
Fang-Ling Needham

International Centre for

Diffraction Data

1601 Park Lane

Swarthmore, PA 19081

U.S.A.

Diane Nelson

Air Products and Chemical

7201 Hamilton Blvd

Allentown, PA 18195-1501

U.S.A.

Robert Newman

Dow Chemical

1897 G Bldg.

Midland, MI 48667

U.S.A.

John M. Newsam

Biosym Technologies Inc.

9685 Scranton Rd.

San Diego, CA 92121

U.S.A.

Jackie Nicol

University of Maryland/NIST

Bldg 235, E151

Gaithersburg, MD 20899

U.S.A.

Heinrich Oettel

Tech Univ. Freiberg

DO-9200 Zeunerstr 5

Freiberg/SA,

Germany

Rosemary Pacalo

SUNY at Stony Brook

Earth \& Space Sciences

Stony Brook, NY 11794-2100

U.S.A.

John Parise

SUNY at Stony Brook

Earth \& Space Sciences

Stony Brook, NY 11794

U.S.A.

Damodara Poojary

Texas A\&M University

College Station

Dept. of Chemistry

College Station, TX 77840

U.S.A.
Ben Post

International Centre for

Diffraction Data

1601 Park Lane

Swarthmore, PA 19081

U.S.A.

Jeffrey Post

Smithsonian Institution

10th \& Constitution Ave., NW

NHB 119

Washington, DC 20560

U.S.A.

Henry Prask

NIST

Bldg. 235, Rm E151

Gaithersburg, MD 20899

U.S.A.

Charles Prewitt

Geophysical Lab.

5251 Broad Branch Rd., NW

Washington, DC 20015

U.S.A.

Edward Prince

NIST

Bldg. 235, Rm E151

Gaithersburg, MD 20899

U.S.A.

Angeline Rehfeldt-Oskierski

Siemens

7500 Karlsruhe 21

Germany

James Rice

Sun Co. RM/R\&D

PO Box 1135

Marcus Hook, PA 19061

U.S.A.

Lee Robertson

Oak Ridge National Lab.

Bldg. 4500, MS 6118

Oak Ridge, TN 37831

U.S.A.

Stephen B. Robie

Scintag, Inc.

707 Kifer Rd.

Sunnyvale, CA 94086

U.S.A. 
Nick Rosov

NIST

Bldg. 235, Rm E151

Gaithersburg, MD 20899

U.S.A.

Daphne Ross

Smithsonian Institution

10th \& Constitution Ave.,NW

E408/NHB 119

Washington, DC 20560

U.S.A.

Frank J. Rotella

Argonne National Laboratory

9700 S. Cass Ave.

IPNS Division - Bldg. 360

Argonne, IL 60439

U.S.A.

J. Michael Rowe

NIST

Bldg. 235/A106

Gaithersburg, MD 20899

U.S.A.

John J. Rush

NIST

Bldg. 235, Rm E151

Gaithersburg, MD 20899

U.S.A.

Makoto Sakata

Nagoya University

Chikusa-Ku, Furo-cho 1

Nagoya, T464-01

Japan

Antonio Santoro

NIST

Bldg. 235, Rm E151

Gaithersburg, MD 20899

U.S.A.

David Schiferl

Los Alamos National Lab.

MS C345

Los Alamos, NM 87545

U.S.A.

Julius Schneider

Inst. für Kristallographie

Universität München

Theresienstr. 41

D-8000 München 2

Germany
Lyle Schwartz

Materials Bldg./B309

NIST

Gaithersburg, MD 20899

U.S.A.

Julia Sigalovsky

Massachusetts Institute of

Technology

77 Massachusetts Ave.

Rm 12-022

Cambridge, MA 02139

U.S.A.

Husinsyah Sitepu

Indonesian Institute of Tech.

IL Raya Puspiptek

Serpong Tangerang

Indonesia

Ewa Skrzypczak

Univeristy of Toledo

$2801 \mathrm{~W}$. Bancroft St.

Chemistry Dept

Toledo, OH 43606-3390

U.S.A.

Deane Smith

Penn State University

239 Deike Bldg.

University Park, PA 16802

U.S.A.

Lubomir Smrčok

Institute of Inorganic

Chemistry

Slovak Academy of Sciences

Bratislava

Czechoslovakia

Robert Snyder

NYS College of Ceramics

Alfred University

Alfred, NY 14802

U.S.A.

Judith Stalick

NIST

Bldg. 235, Rm E151

Gaithersburg, MD 20899

U.S.A.
Eric Steel

NIST

Bldg. 222, Rm 1113

Gaithersburg, MD 20899

U.S.A.

Carin Ström

University of Göteborg

Dept. of Inorganic Chemistry

S-41296 Göteborg

Sweden

Mark Sutton

McGill University

3600 University St.

Montreal H3A 2T8

Canada

J. Timmers

Philips Research

Bedrokvem B/V Bldg. WY

2.22

PO Box 80.000

5600 JA Eindhoven

The Netherlands

Brian Toby

Air Products

7201 Hamilton Blvd.

RSD1 R1102

Allentown, PA 18195

U.S.A.

Hideo $\mathrm{H}$. Toraya

Nagoya Institute of

Technology

Asahigaoka

Tajimi 507

Japan

Sue Town

ANSTO PMB 1

Menai

NSW 2234

Australia

Judy Triphahn

UOP

50 East Algonquin Rd.

Des Plaines, IL 60017

U.S.A. 
Shirley Turner

NIST

Bldg. 222, Rm 1113

Gaithersburg, MD 20899

U.S.A.

Václav Valvoda

Charles Univ., Prague

Ke Karlovu 5

12116 Prague 2

Czechoslovakia

Rodolfo Vargas

I.V.I.C.

Apartado 21827

Caracas $1020 \mathrm{~A}$

Venezuela

Mary Vickers

BP

Chertsey Rd.

Sunbury-on-Thames

TW16 7LN

UK

Jan W. Visser

Henry Dunantlaan 81

2614 GL Delft

The Netherlands

Robert Von Dreele

Los Alamos National Lab.

Lansce, MS H805

Los Alamos, NM 87545

U.S.A.

Wilem L. Vos

Geophysical Laboratory

5251 Broad Branch Rd., NW

Washington, DC 20015-1305

U.S.A.

\section{Sue-Lein Wang}

National Tsing Hua Univ.

Dept. of Chemistry

30043 Hsinchu, Taiwan

Republic of China

Donald Weidner

SUNY at Stony Brook

Earth \& Space Sciences

Stony Brook, NY 11794-2100

U.S.A.
P.-E. Werner

Arrhenius Lab.

Stockholm University

S-10691 Stockholm

Sweden

Marianne Westdahl

Stockholm University

Arrhenius Lab.

S-10691 Stockholm

Sweden

Angus Wilkinson

University of California

Materials Dept.

Santa Barbara, CA 93106

U.S.A.

George Will

University Bonn

Poppelsdorfer Schloss

5300 Bonn

Germany

Eric Windsor

NIST

Bldg. 222, Rm 1113

Gaithersburg, MD 20899

U.S.A.

Winnie Wong-Ng

NIST

Materials Bldg., Rm A215

Gaithersburg, MD 20899

U.S.A.

Nicholas Wright

Edinburgh University

Edinburgh

Scotland

Takamitsu Yamanaka

Osaka University

College of Gen Educ.

1-1 Machikaneyama

Toyonaka Osaka, 560

Japan

R. A. Young

Georgia Inst. of Tech.

School of Physics

Atlanta, GA 30332

U.S.A.
Marek Zakrzewski

Procter \& Gamble

Pharmaceuticals 13815-0191

Norwich, NY 13815

U.S.A.

Pier Franceseo Zanazzi

Piazza Universita

Dipartimento di Scienze della Terra

I-06100 Perugia

Italy

Lev Zevin

Ben-Gurion University

Institute for Applied Research

PO Box 1025

Beer-Sheva

Israel

Xinyu Zhang

Northeastern University

Boston, MA 02115

U.S.A. 


\begin{tabular}{|l|r|l|}
\hline $\begin{array}{l}\text { NIST-114A } \\
\text { (REV. 3-90) }\end{array}$ & U.S. DEPARTMENT OF COMMERCE & 1. PUBUCATION OR REPORT MUMBER \\
NIST/SP-846
\end{tabular}

4. TITLE AND SUBTITLE

Accuracy in Powder Diffraction II

Proceedings of the International Conference

May $26-29,1992$

5. AUTHOR(S)

E. Prince and J. K. Stalick, Editors

6. PERFORMING ORGANIZATION (IF JOINT OR OTHER THAN NIST, SEE INSTRUCTIONS)

U.S. DEPARTMENT OF COMMERCE

NATIONAL INSTITUTE OF STANDARDS AND TECHNOLOGY

GAITHERSBURG, MD 20890

7. CONTRACT/GRANT NUMBER

8. TYPE OF REPORT AND PERIOD COVERED

Final

9. SPONSORING ORGAMIZATION NAME AND COMPLETE ADDRESS (STREET, CITY, STATE, ZIP)

JCPDS-International Centre for Diffraction Data, 1601 Park Lane, Swarthmore, PA 19081, USA

International Union of Crystallography, 5 Abbey Square, Chester CH1 2HU, UK

National Institute of Standards and Technology, Gaithersburg, MD 20899, USA

10. SUPPLEMENTAAY NOTES

11. ABSTRACT (A 200-WORD OR LESS FACTUAL SUMMARY OF MOST SIGNIFICANT INFOAMATION. IF DOCUMENT IMCLUDES A SIQMIFICANT BIBUOGRAPHY OA UTERATURE SURVEY, MENTION IT HERE.)

The proceedings of the international conference Accuracy in Powder Diffraction II present the invited papers and abstracts of the papers contributed to the conference, which was held at NIST, Gaithersburg, Maryland, during May 26-29, 1992. The conference was organized by the Commission on Powder Diffraction of the International Union of Crystallography, and was jointly sponsored by NIST, JCPDS-International Centre for Diffraction Data and the International Union of Crystallography. The proceedings contain 25 invited papers and 73 contributed abstracts. The program of the conference was divided into six topics: Phase Identification and Quantification; Accuracy and Standards; New Developments in Software and Data Analysis; Profile Fitting, Decomposition and Microstructural Effects; Novel Applications and Structural Science; and New Developments in Hardware, Including Detectors, and Studies under Non-ambient and Time-resolved Conditions. A ceremonial session was devoted to a tribute to the late William Parrish and his contributions to powder diffraction. In addition, there were two tutorial workshops organized by the JCPDS-International Centre for Diffraction Data, one on diffractometer sensitivity and one on automatic indexing methods.

12. KEY WORDS (6 TO 12 ENTRIES; ALPHABETICAL ORDER; CAPITALZE ONLY PROPER MAMES; AMD SEPARATE KEY WORDS BY SEMICOLONS)

accuracy; instrumentation; microstructure; phase analysis; powder diffraction; software; standards; structural science.

FOR OFFICIAL DISTRIBUTION. DO NOT RELEASE TO NATIONAL TECHNICAL IMFORMATION SERVICE (NTIS).

$X$ ORDER FROM SUPERINTENDENT OF DOCUMENTS, U.S. GOVERNMENT PRINTING OFFICE, WASHINGTON, DC 20402.

$X$ ORDER FROM NATIONAL TECHNICAL INFORMATION SERVICE (NTIS), SPRINGFIELD, VA 22161.

\begin{tabular}{|l|}
\hline 14. NUMBER OF PRINTED PAGES \\
\hline 241 \\
\hline 15. PRICE \\
\hline
\end{tabular}


ded 


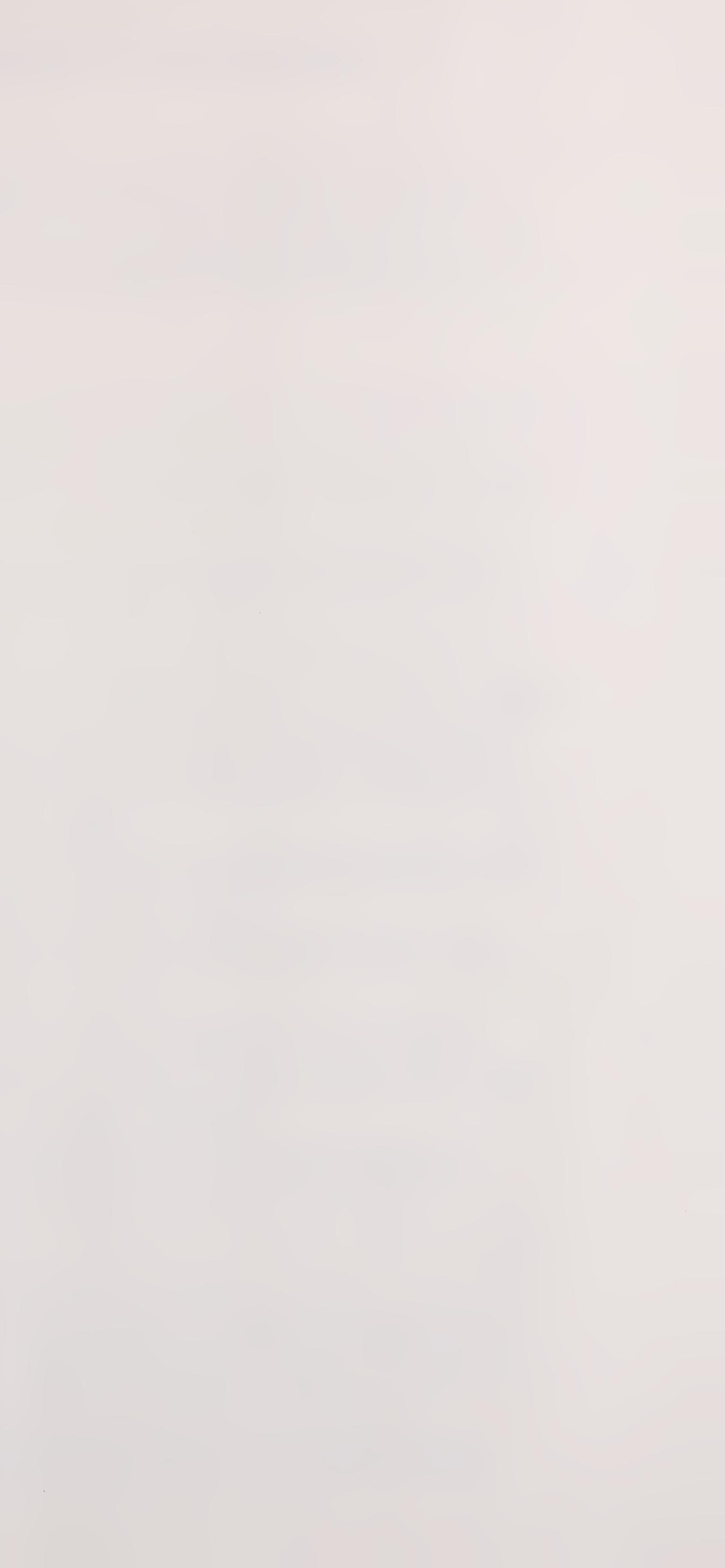




\section{NIST Technical Publications}

\section{Periodical}

Journal of Research of the National Institute of Standards and Technology-Reports NIST research and development in those disciplines of the physical and engineering sciences in which the Institute is active. These include physics, chemistry, engineering, mathematics, and computer sciences. Papers cover a broad range of subjects, with major emphasis on measurement methodology and the basic technology underlying standardization. Also included from time to time are survey articles on topics closely related to the Institute's technical and scientific programs. Issued six times a year.

\section{Nonperiodicals}

Monographs-Major contributions to the technical literature on various subjects related to the Institute's scientific and technical activities.

Handbooks - Recommended codes of engineering and industrial practice (including safety codes) developed in cooperation with interested industries, professional organizations, and regulatory bodies.

Special Publications - Include proceedings of conferences sponsored by NIST, NIST annual reports, and other special publications appropriate to this grouping such as wall charts, pocket cards, and bibliographies.

Applied Mathematics Series-Mathematical tables, manuals, and studies of special interest to physicists, engineers, chemists, biologists, mathematicians, computer programmers, and others engaged in scientific and technical work.

National Standard Reference Data Series-Provides quantitative data on the physical and chemical properties of materials, compiled from the world's literature and critically evaluated. Developed under a worldwide program coordinated by NIST under the authority of the National Standard Data Act (Public Law 90-396). NOTE: The Journal of Physical and Chemical Reference Data (JPCRD) is published bimonthly for NIST by the American Chemical Society (ACS) and the American Institute of Physics (AIP). Subscriptions, reprints, and supplements are available from ACS, 1155 Sixteenth St., NW., Washington, DC 20056.

Building Science Series-Disseminates technical information developed at the Institute on building materials, components, systems, and whole structures. The series presents research results, test methods, and performance criteria related to the structural and environmental functions and the durability and safety characteristics of building elements and systems.

Technical Notes - Studies or reports which are complete in themselves but restrictive in their treatment of a subject. Analogous to monographs but not so comprehensive in scope or definitive in treatment of the subject area. Often serve as a vehicle for final reports of work performed at NIST under the sponsorship of other government agencies.

Voluntary Product Standards - Developed under procedures published by the Department of Commerce in Part 10, Title 15, of the Code of Federal Regulations. The standards establish nationally recognized requirements for products, and provide all concerned interests with a basis for common understanding of the characteristics of the products. NIST administers this program in support of the efforts of private-sector standardizing organizations.

Consumer Information Series - Practical information, based on NIST research and experience, covering areas of interest to the consumer. Easily understandable language and illustrations provide useful background knowledge for shopping in today's technological marketplace. Order the above NIST publications from: Superintendent of Documents, Government Printing Office, Washington, DC 20402.

Order the following NIST publications - FIPS and NISTIRs-from the National Technical Information Service, Springfield, VA 22161.

Federal Information Processing Standards Publications (FIPS PUB) - Publications in this series collectively constitute the Federal Information Processing Standards Register. The Register serves as the official source of information in the Federal Government regarding standards issued by NIST pursuant to the Federal Property and Administrative Services Act of 1949 as amended, Public Law 89-306 (79 Stat. 1127), and as implemented by Executive Order 11717 (38 FR 12315, dated May 11, 1973) and Part 6 of Title 15 CFR (Code of Federal Regulations).

NIST Interagency Reports (NISTIR) - A special series of interim or final reports on work performed by NIST for outside sponsors (both government and non-government). In general, initial distribution is handled by the sponsor; public distribution is by the National Technical Information Service, Springfield, VA 22161, in paper copy or microfiche form. 


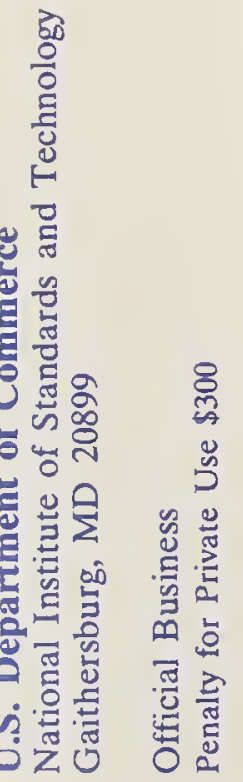

